


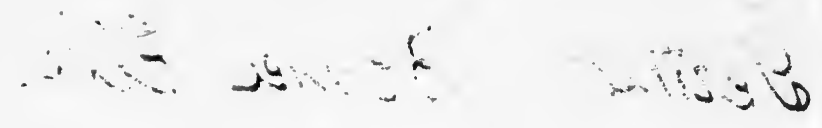

From the collection of the

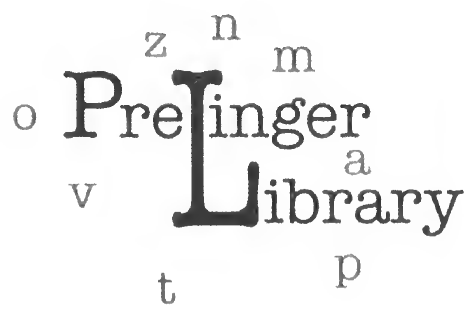

San Francisco, California 2006 
i

1292
20271

Sertude 83 lake Ellis.

5)-RP 
.

$-$ 
ans

$i^{4}$ 


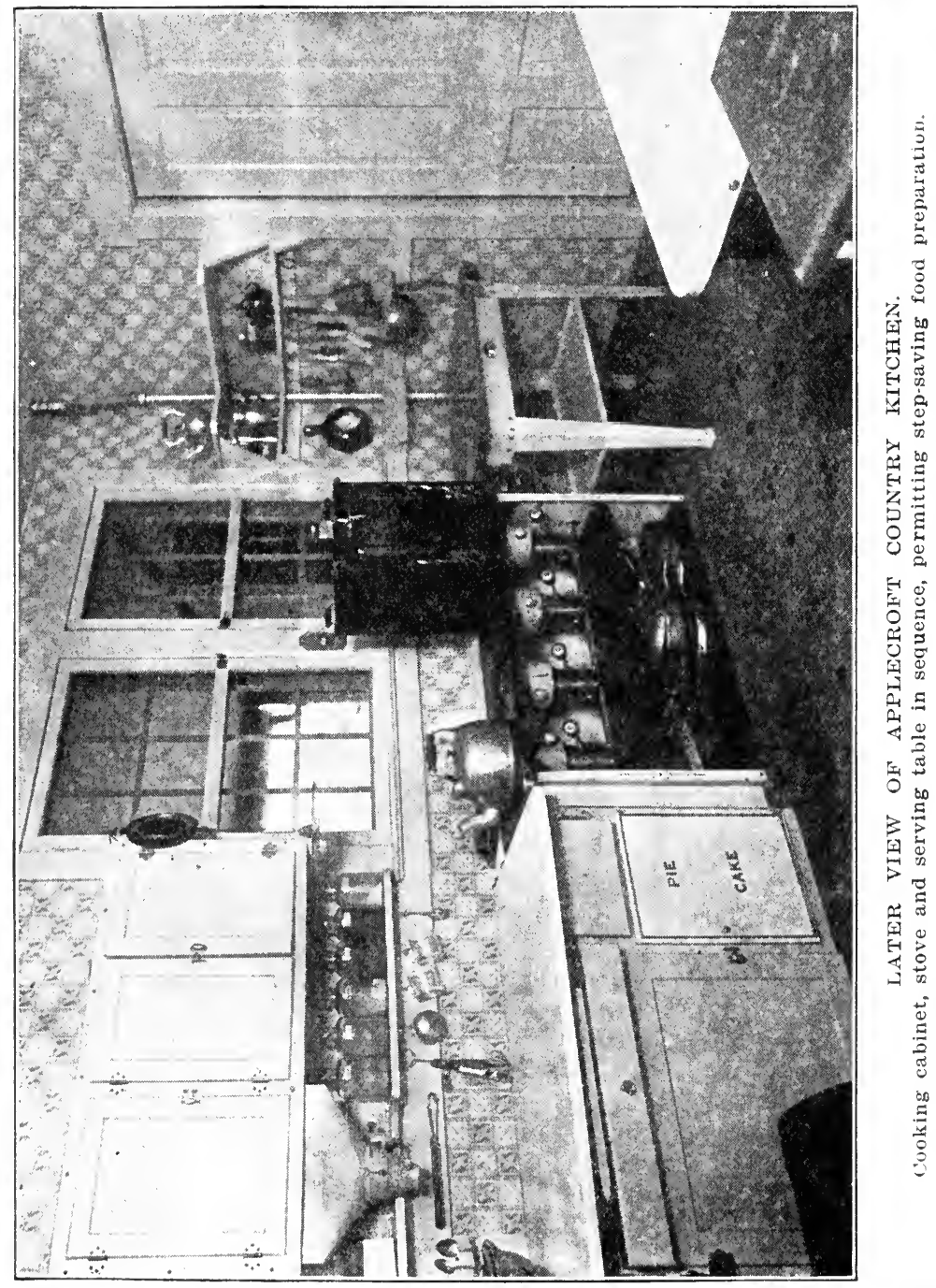




\title{
HOUSEHOLD ENGINEERING
}

\section{SCIENTIFIC MANAGEMENT IN THE HOME}

\author{
BY \\ MRS. CHRISTINE FREDERICK \\ Household Efficiency Engineer, \\ FOUNDER "THE APPLECROFT EXPERIMENT STATION" \\ CONSULTING HOUSEHOLD EDITOR, "LADIES' HOME JOURNAL" \\ chaUtaUQu lecturer, aUthor "THE NeW housekeeping"
}

\section{A CORRESPONDENCE COURSE}

ON THE APPLICATION OF THE PRINCIPLES OF EFFICIENCY ENGINEERING AND SCIENTIFIC MANAGEMENT TO THE EVERY DAY

TASKS OF HOUSEKEEPING

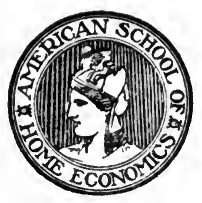

CHICAGO

AMERICAN SCHOOL OF HOME ECONOMICS 
Copyright 1915, 1919, I920, I921, I923 BY Home Economics Association 


\section{FOREWORD}

$\mathrm{H}^{\circ}$

OUSEKEEPING is not only the oldest, most fundamental and complex of all professions, but modern success in it is more difficult to attain than success in factory, warehouse, transportation or shop, because it must be attained by women working alone, and with many purposes. Men in work and play have specialized in groups along a single path, for a single end. Women have specialized not as a group, but as individuals along all paths, for many ends.

There are six distinct classes of activities: production, transportation, manufacture, storage, exchange and personal service. The boy is prepared for 15 years or more to co-operate witn otners in mastering one particular part of one of these activities. A man will give his life to the specialization and standardization of the methods and tools for a single oft repeated operation. Housekeeping, if a kitchen garden or milking is included, covers all six activities. Often without preparation a young woman working alone, without the discipline of the group, expects to be an adept in all six fields and in all parts of each field at once!

Mrs. Frederick has succeeded in specializing and standardizing the tools and methods for the many ever changing occupations of the home. This was an exceedingly difficult undertaking which she has admirably completed.

Man is irresponsible, woman is deeply responsible and, therefore, she evolved and built all the foundations of civilization and still holds onto them in her life. These foundations are home, cooking, textiles, pottery, storage, manufacturing, music, language, medicine and teaching. But man, like a boy growing beyond his mother's size, strength, experience, energy, authority, has usurped all that woman has developed, even to the feeding of babies with his modified food, and by his methods applied to her inventions has enormously 
increased and cheapened the product through group endeavor and through labor-saving equipment. Thus, man and woman have parted company industrially. Man may be at fault because he rushed impetuously ahead, woman may be at fault because she has held too long to the old.

For women to gain a grip on the new the point of view must be changed. Therefore, earnest, high-minded, self-sacrificing, progressive women who have the privilege of this course, so thoroughly covering so great a problem, should come to it with the new point of view. They must learn to waive a hundred instincts and prejudices they do not even know they possess, constantly and continuously asking themselves "Why?"-weighing the real value of what is relinquished against the gain in efficiency, time and selfexpression.

There are many ideals in homekeeping. Mrs. Frederick's methods are good for all ideals, but because she has made work easier, do not add another half dozen ideals! I remember when sewing machines were first introduced; they made the running of long tucks one hundred times easier. But this was made a reason for making seventy times as many tucks! Because Household Engineering makes tasks as formerly done much easier, do not take on a great deal more "unessential" work.

The World War was fought with woman's direct help, women doing the work of men successfully because they followed the laborsaving principles of work established by men. Let women introduce these same principles into the work of the home and, thus, similarly make a success of their work as they have so signally done with men's work. This text is the manual which will point the way to a modern, successful solution.

HARRINGTON EMERSON, Author of "The Twelve Principles of Efficiency," Etc. 
TOTHING is more worth while than bringing efficiency into the home. When housekeeping becomes a science, as well as an art, when it is based on measurement-then it becomes worthy of the best brains and highest endeavor. Mrs. Frederick has rendered a real service to this country, in that she has eliminated from housework that monotony that comes from doing uninteresting and repetitive work without an incentive, and in that she has seen the necessity for making the home a laboratory, - a training school for the women and children in it, and perhaps an example to the men.

Every reader of her book will find not only concrete directions as to how to make housework stimulating, productive and constructive, but also a method of attack that applies to all problems of any life-whatever they may be.

$$
\begin{gathered}
\text { Frank B. Gilbreth, } \\
\text { Consulting Engineer. } \\
\text { Author of "Fatigue Studies," "Motion Study," Etc., Etc. }
\end{gathered}
$$




\section{Dedicated to}

\section{EDWARD BOK}

to whose encouragement and

progressive leadership in reaching the mass of American homemakers with

the gospel of home efficiency

I owe much inspiration 


\section{CONTENTS}

CHAPTER

PAGE

A Personal Introduction •.$\quad \cdot \quad \cdot \quad \cdot \quad \cdot 7$

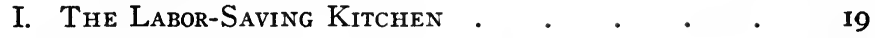

II. Plans and Methods for Daily Housework • • 65

III. Helpful Household Tools • $\quad$ • $\quad$ • 99

IV. Methods of Cleaning . $\quad . \quad$. $\quad$ • $\quad$ • 147

V. Food Planning for the Family • : : $\quad$ - 179

VI. The Practical Laundry: Methods and Tools . . 2 II

VII. Family Financing ANd Record-Keeping • . 265

VIII. Efficient Household Purchasing • • • • 315

IX. The Servantless Household . • • • • 377

X. Management of Houseworkers . • . . . . 419

XI. Planning the Efficient Home . . • . 449

XII. Health and Personal Efficiency . • • . $48 \mathrm{I}$

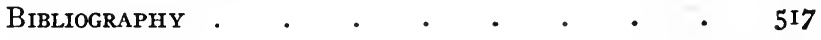

INDEX • . . . . . . . . . . $52 \mathrm{I}$ 


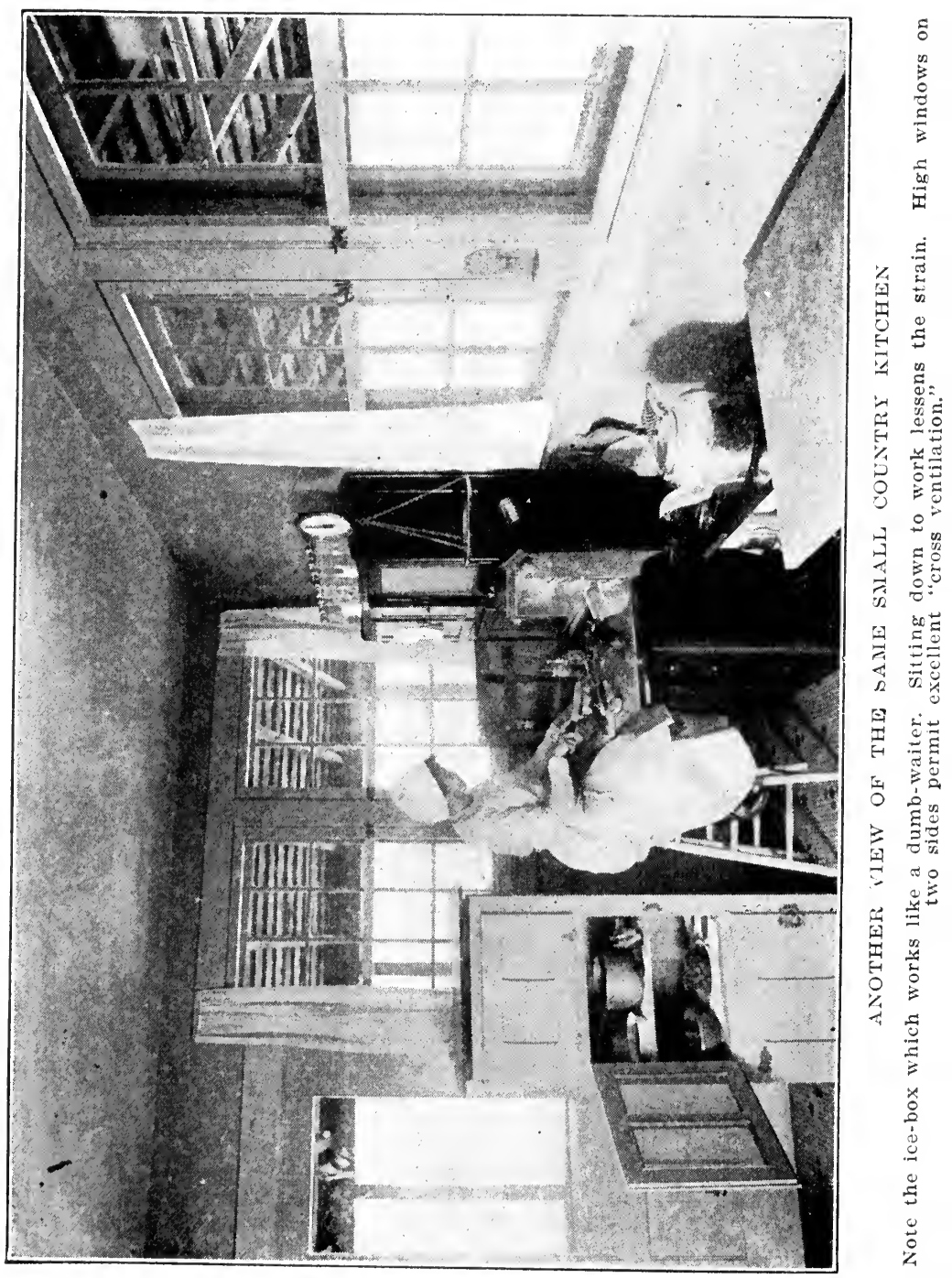




\section{HOUSEHOLD ENGINEERING}

\section{A PERSONAL INTRODUCTION}

GEVERAL years ago I faced the problem which con$\checkmark$ fronts many young mothers-how to do my housework and care for two small children, and yet have any time for myself or outside interests.

I had managed my mother's home at different periods and really liked housework, especially cooking. But now it was a daily struggle to "get ahead" of household drudgery. Try as I would, there seemed so many tasks to do, so many steps to take, and so many matters needing my attention and supervision. Just as I felt I had reduced the cleaning to its lowest terms, I found the cooking or the laundry work or the mending claiming the remainder of my time. It was a continuous conflict to do justice to all the housework tasks, and yet find enough time for the children. And between it all, I knew I was not doing justice to myself, and that I was becoming more and more tired out. Indeed, I was often without much energy to "dress up" in the evening, and when my husband came home, I was generally too spiritless to enjoy listening to his story of the day's work.

Things were dragging on in this unsatisfactory way and I was becoming more and more discouraged with what seemed my lack of ability to manage my household problem. Occasionally I was so depressed as to wish that I were not married and that $I$ was back in my teaching "harness" where I did have a grip on things!

Just about this time my husband's work brought him in 
touch with the new movement called "scientific management," and he came home with glowing accounts of what it was accomplishing in the various shops, offices and factories where it was being followed. In fact, he and his friends (some of whom were pioneers in the movement) talked nothing but this new "efficiency idea." Because I had an intuition that perhaps in this new idea was the lifepreserver for which I had been so earnestly searching in my own problem, I listened eagerly to their discussion.

\section{Purpose of Scientific Management}

I found that the purpose of scientific management was to save time and effort and to make things run more smoothly. Its object was to short-cut and reduce work to such a system that the shop or office or any business would be managed with less effort, less waste, and even at a lower cost. It seemed to me that this was exactly what my aim was in my own home, only I had all this time been helpless to carry it out! That was just what I too wanted-some plan or general guiding principles that would make my housework easier, more successful and less expensive. If this wonderful new "scientific management" brings about such result in other businesses, why couldn't it do the same in my business of home-making?

So I decided to learn all about it and understand it, and $I$ went for help to my husband and his friends who were applying the new idea every day.

"If this new efficiency idea is all you claim," I said to them, "and can be followed in work as widely different as iron foundries and shoe factories, I don't see why it can't be applied to housework as well. You men have made me so interested in it that I want to try it in my own home. But first I want you 'efficiency engineers,' as you call yourselves, to explain the idea to me in detail-the why and 
the how and every point so that I will be sure that I thoroughly understand it before I attempt to put it into practice. Will you?"

\section{Principles of Efficiency Engineering}

So my husband and other efficiency engineers made it clear to me, and I found that scientific management was nothing difficult or expensive or mysterious, but that it was a plan, or guiding set of twelve principles, as follows:

I. Ideals.

2. Common Sense.

3. Competent Counsel.

4. Standardized Operations.

5. Standardized Conditions.

6. Standard Practice.
7. Despatching.

8. Scheduling.

9. Reliable Records.

Io. Discipline.

I I. Fair Deal.

I2. Efficiency Reward.

"There is this first principle of Ideals," they explained. "When we go into a factory and try to improve the work, the first thing we ask the owner about his business is, What are you running it for? The reason so many people are not making a success of their business is because they do not know why they are running it. Yet ideals are the most important thing to have in any work. They are that 'something' that controls and guides the whole plan, a kind of chart they are trying to follow. You must know where you want to go before trying to get there.

"Many women do have a strong ideal in their homemaking. It frequently is health or the education of their children, or sometimes only a spotless house. Think of the strong ideals that the mother of Charles Wesley and his brother must have had for their education to buoy her up in all those years of poverty! The ideal can be so strong as to look beyond present difficulties and discouragements and make work a success in spite of apparently handicap- 
ping conditions. The clearer a homemakers' ideals, the more bound her work is to succeed. Homemakers, like other managers, must know what they are striving for.

\section{Common Sense}

"Then there is this next principle-Common Sense, which is sometimes only a homely term to cover some of the other principles. It is common sense to be sure your tools are sharp and in good condition before you start workand it's efficiency as well: Competent Counsel means expert advice. We efficiency engineers are one kind of competent counsel because our past experience and practice makes us 'competent' to come into a new factory and suggest better methods and plans. Other 'counsel' is found in books, and the written experiences of what has been found out in this or that field. Even the most successful business men profit by the 'counsel' of specialists and their recorded experiences in solving problems in other lines. Many firms employ such paid counsel to visit their branch offices, instruct their salesmen, help their dealers, or in some way keep the workmen on the right track."

The efficiency engineers continued their explanation while I listened attentively.

\section{Standard Operations}

"Standardized operations, etc., sounds formidable, but you will see clearly what the next three points mean. For instance, when we go into a factory, we watch the men at work, we see what motions and tools they use; then, after repeated experiments. and time studies, we try to give them standardized or definite conditions of work, and show them methods or standardized operations. This means working at the right height, with the right tools, under the best conditions of light, ventilation and comfort, with the least 
possible waste of energy and time. When we have found out this best and shortest,-or 'standardized'-way, we write it down, and these instructions of just how to do a given task are called 'standard practice.' Then all the workmen need to do is to follow these instructions and they get the best results."

\section{Despatching and Scheduling}

"The next two points of Despatching and Scheduling are very important," they continued. "You see, when we have determined the one best or standard way to do any task, we are not quite finished. We have to go still further and find the best order of work, or when to do it, as well as how to do it.

"Despatching means planning, and Scheduling means the carrying out of that plan. You know how they despatch trains on schedule time. Suppose a train leaves Chicago at 8 P. M. and arrives at St. Louis at 7 A. M. The despatching consists in running the train so that it reaches all the intervening stations-Peoria, Springfield, etc.-at a specified time. The schedule is the I I hours it takes to make the trip. Work in a factory is despatched in much the same way. The raw material enters one room and then another and so on; or the workmen take up first one task and then another after it has been laid out in definite order by the foreman. This means saved time, and orderly, unconfused work.

"There is a great deal to be explained about Immediate, Accurate and Reliable Records. It includes ways of keeping information, bills, receipts, addresses, etc., so that no time is wasted looking for a piece of information when needed.

"The last three points-Discipline, Fair Deal and Efficiency Reward-taken together, refer to the benefits that scientific management brings to the worker himself. It 
isn't enough to make work shorter and easier and less wasteful-it must mean more happiness and even more money to those who work. In shops where scientific management is in force, there have been few strikes and troubles. Applied to the home, it would refer of course to the hired worker or servant. If a mistress applied the principles of Fair Play, for instance, to her help, they wouldn't leave her in a crisis, perhaps, as they do now. And if she used the principle of Efficiency Reward, she might secure from them that something over and above mere work-that "service plus"-which makes any employee really valuable."

\section{These Principles in Operation}

After I had grasped this brief explanation of scientific management, I visited factories and places where I could see the principles in actual operation, so as to make it even more clear in my mind.

I saw the marvelous improvement this efficiency idea had brought in the commonplace task of laying bricks, which had been done up till then in the same way since the time of the Pharaohs. In all history, bricks had been dumped in a mixed pile at the workmen's feet. Then he had to stoop his entire weight, 150 pounds say, each time to pick up a 4-pound brick before he set it in place. Think of the thousands of times a day he did this useless stooping! Now, when the efficiency engineers watched bricklayers at work, they saw how many waste motions and time were lost in this senseless stooping; so they devised a little adjustable table, which brought the bricks in an orderly pile to the worker's side, and because he didn't need to stoop at all, or even take time to sort the bricks, he now laid 350 bricks an hour where before he could lay only I20, besides working with far less fatigue and effort. 
Then I was surprised to see how "common sense" and "standardized conditions" had been applied in a cash register factory. It had been the habit of the workmen to go every morning for their special tools to lockers at the end of a very long floor, and to return the tools there in the evening. When "competent counsel" efficiency men studied this factory, they immediately noticed this twice-a-day double walk across the floor, with resulting confusion, loss of time, and talking. This waste of time and steps was avoided later by having the benches of each worker fitted with small drawers and cross-strips to accommodate each man's tools. Then the moment a man came to his bench, he could start work, and at night work until the whistle blew, which meant more work and less unnecessary wasted time.

$I$ visited another instance of scientific management in the shop of a chemist who had a force of girls packing pills into boxes. Formerly they counted out a hundred pills by hand, at the rate of one box a minute. But by installing a simple little device which automatically counted a hundred pills and pushed them off in a little shovel into boxes fed to them on a belt underneath, each girl was now able to fill twenty boxes a minute with no more labor.

Again, I saw a workman in an envelope factory who had been considered the best in the shop because he could turn out the largest number of envelopes per hour. But when the efficiency engineers observed him, they found that he took four cuts to each paper, thus making a great deal of waste and expense. By finding a new way to cut envelopes with only three cuts, the efficiency engineers saved tons of paper and thousands of dollars for the firm each year. And I will never forget the increased efficiency which resulted in one foundry by the most simple little change. Formerly the workers used small shovels which meant very frequent stooping to dispose of a given pile of coal. But 
by studying to see just what weight and shape of larger shovel a man could handle most easily, and yet carry the largest load, the same number of workers were able with the new large shovel to move the same load of coal in onethird the time! And all because scientific management had studied a shovel!

\section{Scientific Management in the Home}

In every instance I saw how these efficiency principles were saving time, and effort, and money, wherever applied. The more I saw and read, the more certain I felt that they could save time and effort and money in my business-the home. There was the point of height-didn't I with hundreds of women stoop unnecessarily over kitchen tables and sinks and ironing boards as well as bricklayers stoop over bricks? Couldn't we perhaps standardize dishwashing by raising the height of the sink and changing other conditions? Did we not waste time and needless walking in poorly arranged kitchens-taking twenty steps to get the egg-beater when it could have been hung over my table, just as efficiency insisted the workman's tools must be grouped? Couldn't my housework train be despatched from station to station, from task to task, and I too work on a "schedule," or definite plan, so that I wouldn't lose time in thinking what to do next or in useless interruptions?

I came to earnestly believe that scientific management could, and must, solve housework problems as it had already solved other work problems. I began to see where I had been losing time-where I had been taking waste motions and useless steps-where I could use different tools and methods. Formerly I had been doing my work in a dead, mechanical way, but now every little task was a new and interesting problem. I found that housework was' just as interesting and more so than many other tasks of business. 
Every day I tried to find new ways, new methods and new short cuts in my home problems. If I made out a good schedule of work for one week I tried to improve on it for the week following. No housework detail was too small or too unimportant. I constantly kept in mind that "shovel" which had cut down the drudgery of coal heaving by onethird! I found that I, too, was actually doing my work in almost one-third less time, without any extra physical, and with far less nervous effort. I found that I could "despatch" my work, that I could "standardize" it to a great extent, and so have that longed-for "time to myself" some part of the day.

\section{The Efficiency Attitude of Mind}

But by far the best result of all that came was the confident "efficiency attitude" of mind which I developed. No matter how hard things were-and they did not grow perfect all at once-I had that inward feeling that they would, and should, come right in the end. I felt that in spite of any difficulty or trying conditions, that I could master my house problems-that there were solutions, and that there was no such word as "fail" in the whole language of scientific management. I cannot express how much poise and determination came from this efficiency attitude,- the attitude of being superior to conditions, of having faith in myself and in my work, to feel that it was drudgery or degrading only if I allowed myself to think so. I felt I was working hand in hand with the efficiency engineers in business, and that what they were accomplishing in industry, I too was accomplishing in the home.

I kept on studying, visiting plants and factories, and getting in touch more widely with the movement. Besides studying myself, I got friends to watch themselves at work and tell me the results. I began to test equipment and 
household apparatus in my own home so that I could tell other women what I found out. I remodeled my own kitchen and then the kitchens of friends. Before I knew it, I became a "household engineer," and was called in as "competent counsel" by other homemakers!

I was so enthusiastic over the results of my experiments that I wrote four articles called "The New Housekeeping" which appeared in The Ladies' Home Journal of 1912. The interest from them was so great that I later brought out the same material in book form. Since then the application of efficiency principles and scientific-management to the home has been more widespread than I ever dared hope or believe.

I have had literally thousands of correspondents among all kinds of homemakers. In one month only over 1,600 women wrote me for information. Sometimes I am able to help them with suggestions for a better kitchen arrangement. In many cases I lay out "schedules" of work. Again, I tell them about the new tools which are tested every month in my own home, Applecroft Experiment Station.

Not only have I been able to help these thousands of correspondents, but they have helped me with many suggestions and especially to understand more fully the problems that come to homemakers in all sections. Perhaps it is the cost of living, or the struggle with young children, or the lack of conveniences, or again, the feeling that housework is drudgery. I have tried to be a "competent counsel," a "household engineer" to all of them, and do for them what I so greatly wished someone could have done for me in my former housework struggles.

\section{HouseHold ENGINEERING}

This course in "Household Engineering" includes in greater detail everything given in my book, "The New 
Housekeeping," and all the help and suggestions gathered from constant study during the five years which have elapsed since its publication.

My correspondence has given me an exceptionally wide viewpoint; and in this course I have tried to present the whole subject of the application of scientific management to the home in such a way that any homemaker, no matter where she lives or what her home conditions, can understand and apply it to the solution of her own problems.

I want you who take this course to feel that you are not working alone in your own home kitchen. I want you to feel that when you discover new methods of housework and better ways of management that you can receive the same recognition that a scientist or business investigator receives. Do not think you are working out the problem for your own home only. You are helping solve the problems of countless other women and homes, and what you do will be passed on, and help build up a great mass of proved knowledge on housekeeping. Is not housework as worth while studying as the shoveling of coal? Is not housekeeping the biggest, the most essential industry of all?

I am confident that some of you who take this course have already been successfully meeting difficult conditions. You need only a little more assistance and the presentation of this new viewpoint to become a household engineer yourself. All my efforts would be useless if you did not cooperate with me to carry out scientific management in your own home. I want your help and interest in making this course a mighty success. You are going to be one of a great band of women investigators, working toward the splendid aim of putting housework on a standardized, professional basis. 


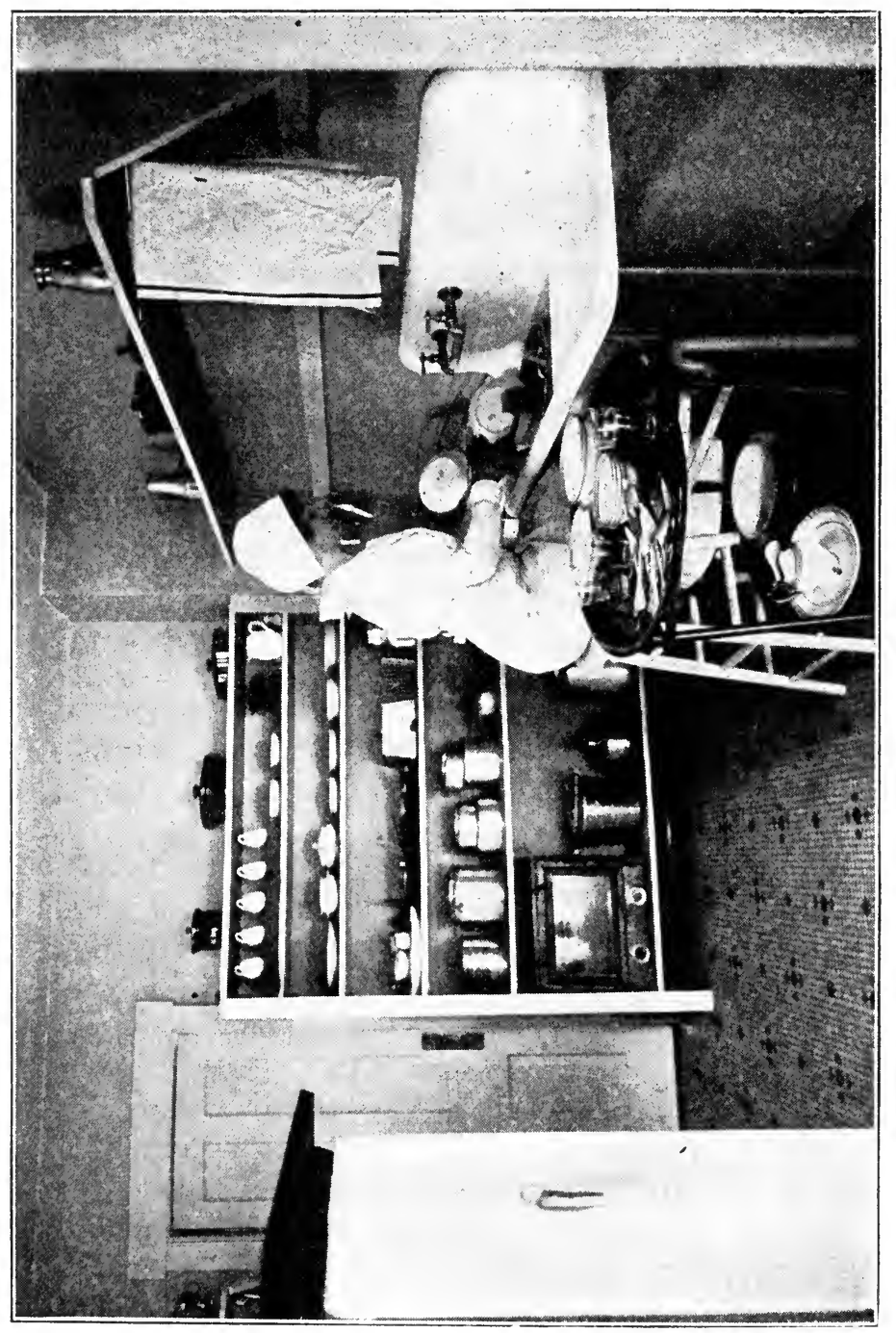

苟

苟

कृ

这

कृ

రृ

$\cong$

$+5$

自

罂

$\vec{A}$

II

यद

$B 2$

0 如

H $\Xi$ 표

乙 记

$\frac{3}{0}$ :

ค :

光 吉范

피워

स

त

巳્

$=0$

है:

$>\frac{4}{2}$

ए人

进

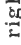

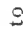

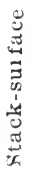




\section{HOUSEHOLD ENGINEERING}

\section{THE LABOR-SAVING KITCHEN}

W

HEN we estimate the time consumed in all the various tasks of the home, cleaning, cooking, serving meals, laundry, etc., we find that about 70 per cent of the housekeeper's day is spent in and about the kitchen. It is therefore clear that any plan for a reorganization of the work of the home on a more efficient basis must begin with a careful study of present kitchen conditions and methods of work.

What is a kitchen? It is a place for the preparation of food. All unrelated work, such as laundry work, with its particular equipment, should be kept out of the kitchen as much as possible. We see then that a kitchen, or a place merely for food preparation, can be much smaller than was formerly the case when it was used as a combined sittingroom, laundry and general workshop.

The first step towards reducing time spent in the kitchen is to have a kitchen small and compact, without loosely connected pantries and cupboards. The small kitchen costs less to build, but even more important to the worker, the small kitchen saves steps by concentrating the working processes. It should be slightly oblong, or almost square as this shape permits the most step-saving arrangement of 
the main equipment. Good sizes are 9 by II feet; I I by I 3 feet; 14 by 16 feet; and in large homes with service and large equipment, $\mathrm{I} 8$ by $\mathrm{I} 8$ feet.

Formerly much more storage or pantry space was necessary than today when more frequent marketing is possible. Also, a pantry which contains the pots, pans and utensils needed in the kitchen causes waste motion and useless steps. As will be described in detail later, the efficient plan is to have utensils and materials grouped close to the surface where they are actually used.

For this reason particularly, the detached kitchen pantry is giving place to the built-in pantry which forms an integral part of the kitchen, and to the portable kitchen cabinet which sometimes takes its place. The average pantry or cupboard with broad, widely-separated, and high, useless shelves is responsible for much of the fatigue and trotting back and forth of the worker. An improved construction plan would be to take some of the pantry space and use it for cupboards and shelves built into the kitchen itself.

If the family is small and has simple service, only a very tiny butler's pantry is needed, and even this can be dispensed with. The more direct the route between kitchen and dining room, the more step-saving and easy is the serving of meals. The butler's pantry in its most common location between dining-room and kitchen has the two good points of preventing kitchen odors and noises from disturbing the dining room, and of storing table china. But with adequate ventilation, which the kitchen should have in any case, simple service and built-in conveniences for china in the dining-room, the sole reasons for the existence of the butler's pantry are removed. In brief, the closer the connection between kitchen and dining-room, and the fewer the detached pantries and cupboards, the simpler will be the processes both of preparing and serving food. 


\section{Grouping of Large EQuipment}

When we study the steps entailed in food preparation, we find that work in the kitchen does not consist of independent, separate acts, but of a series of inter-related processes. No matter whether we are serving a six-course formal luncheon, or a simple family breakfast, each act in food preparation is part of a distinct process. There are just two of these processes: (I) Preparing Food, and (2) Clearing Away. Each of them has (or should have) definite, distinct steps, as we see if we analyze our work from the time preparation of food is started to the moment when the last dish is washed and laid away.

The steps in the preparing process are:

(I) Raw materials taken from storage, refrigerator or pantry to

(2) Preparing surface where they are beaten, mixed, or put in condition to place on

(3) Cooking surface or in cooking device. When finnished, placed on

(4) Serving surface (table or tray) on which hot food is laid and given final touches before being sent to the table.

In other words, we ( I) Collect, (2) Prepare, (3) Cook, and (4) Serve food materials according to these definite steps, even with so simple a task as boiling an egg.

The steps in the clearing away process are:

( I) Remove soiled dishes and utensils from diningroom.

(2) Stack and scrape them to right of sink.

(3) Wash, drain and wipe.

(4) Lay away in respective closets and shelves. 
In other words, we (I) Remove, (2) Scrape, (3) Wash, and (4) LAY AwAY dishes and utensils according to these definite steps, in this definite order at every meal.

It therefore follows that the equipment connected with these two processes and their respective chain of steps should be arranged in a corresponding order. This prin-

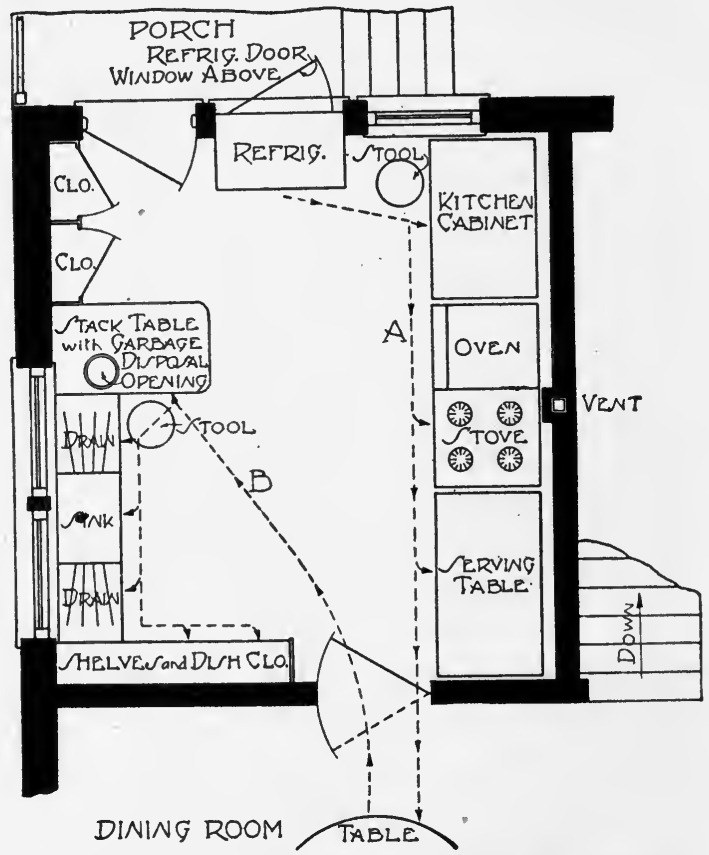

EFFICIENT GROUPING OF KITCHEN EQUIPMENT

A. Preparing route. B. Clearing away route.

ciple of arranging and grouping equipment to meet the actual order of work is the basis of kitchen efficiency. In other words, we cannot leave the placing of the sink, stove, doors and cupboards entirely to the architect. The reason 
why so many kitchens are work-making is solely because both the fixed and portable equipment are riot placed in right relation to all kitchen processes. Instead, equipment is commonly placed wherever there happens to be space left after cutting in all the doors and windows.

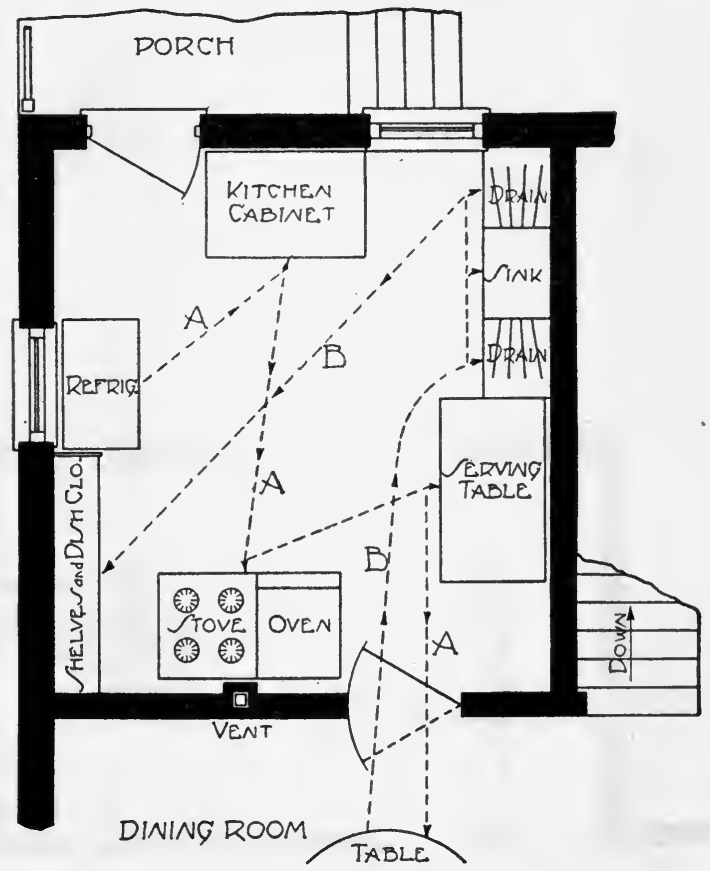

BADLY GROUPED KITCHEN EQUIPMENT

Again considering the two kitchen processes, (a) PREparing and (b) Clearing Away, we note that a definite piece of equipment corresponds to each definite step, as follows : 
(a) Preparing Process

(I) Storage,-pantry, refrigerator, ice-box, etc.

(2) Table or kitchen cabinet surface.

(3) Stove or other cooking equipment.

(4) Table, tray on wheels, or other serving surface.

(b) Clearing Away Process

(I) Stack surface to right of sink.

(2) Sink.

(3) Drain surface to left of sink.

(4) Adjacent closets and shelves to left of drain.

If the storage, stove, tables, sinks, etc., are arranged after this fundamental order, the work will proceed in a progressive, step-saving track, or "routing," as the efficiency engineer calls work which proceeds in a consecutive, orderly

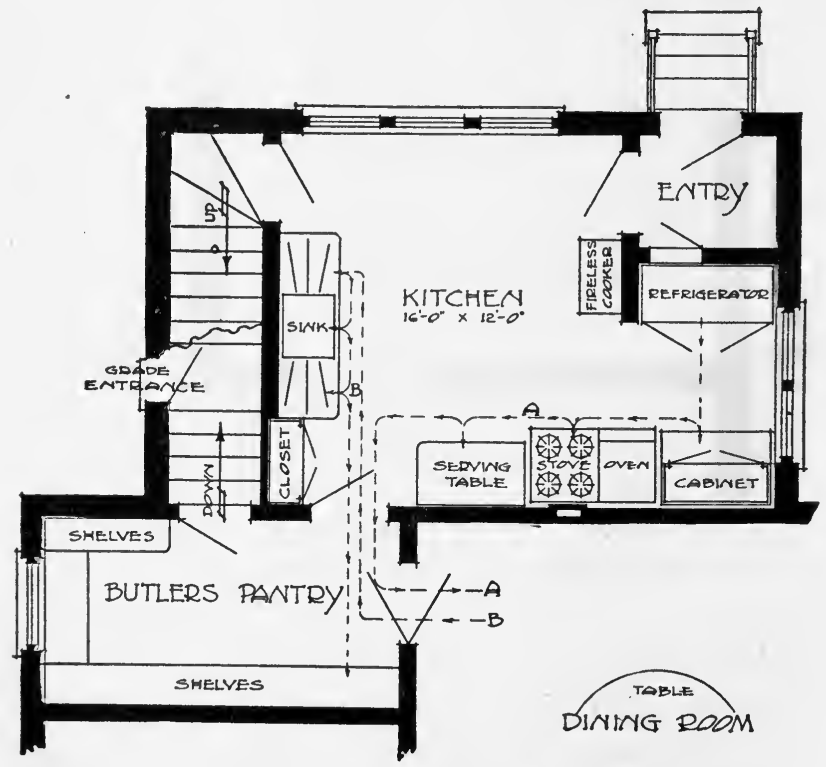

WELL ARRANGED KITCHEN WITH BUTLER'S PANTRY A. Preparing route. B. Clearing away route. 


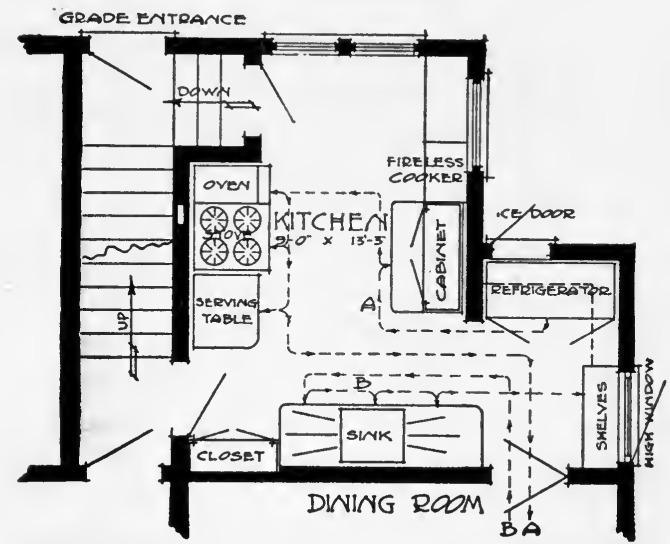

CONVENIENT KITCHEN FOR A SMALL HOUSE

manner. If the equipment is not arranged on this principle, the result will be cross-tracking, useless steps and waste energy in all kitchen work. On pages 22 and 23 are given two diagrams which clearly illustrate the efficient versus the drudgifying kitchen arrangement.

\section{Separate Surface for Each Process}

The "routing" or step-saving method of kitchen arrangement requires separate surfaces for each process. The old and commonly followed plan is to have one "kitchen table" at which preparing is done, hot dishes set down, soiled dishes from the dining-room dumped, etc. This results in a double or triple handling of utensils and dishes besides unnecessary steps back and forth.

For instance, if one kitchen table in the center of the room be used as the surface on which to make a pie and also as the surface on which to lay the hot pie when baked, and also as the surface on which to lay the soiled diningroom dishes, let us see what happens. The egg-beater, bowls, pie-tin, etc., as well as lard, flour, etc., must be brought from their respective permanent places to the table. 
After they are used, each article has to be taken back; the pie when finished, is carried several steps from stove to table. The soiled dishes brought from dining room are laid first on this table and then require a second handling to take them to the sink where they are ultimately washed.

Contrast the different handlings and walking required by this arrangement with the same work if done under the efficient arrangement given in the first diagram. At the preparing table is everything necessary for making the pie with the exception of raw materials which are kept in adjacent refrigerator, hence there is no walking to gather materials and utensils together. When the pie is finished, it is laid with a single motion on the serving surface to the right of stove. Soiled dishes, instead of being dumped on

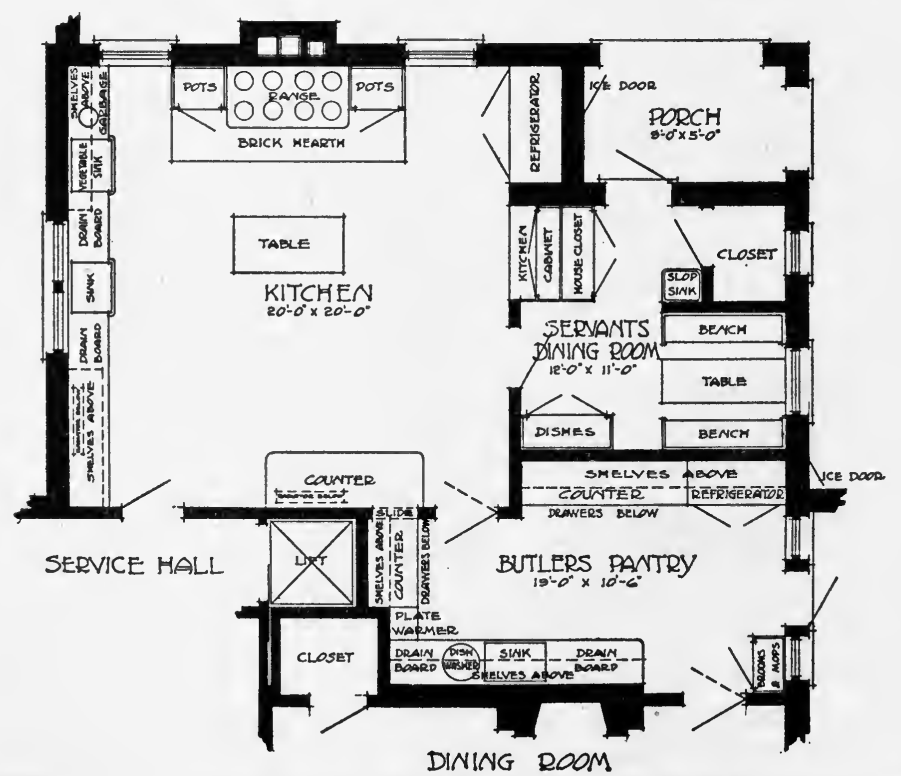

EFFICIENT KITCHEN, BUTLER'S PANTRY AND SERVANTS' ROOM OF A LARGE HOUSE 


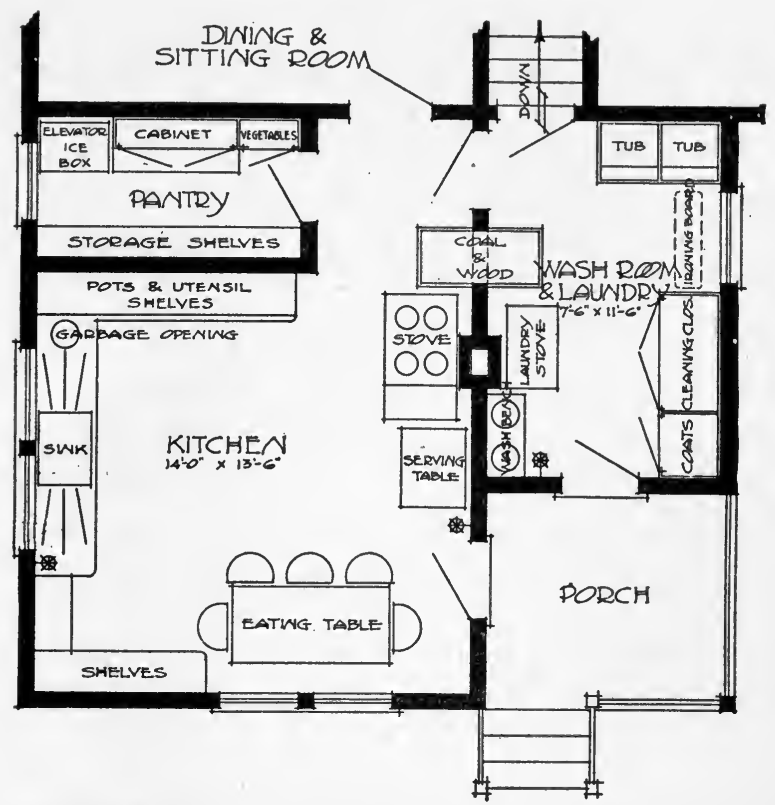

A LARGE FARM KITCHEN COMBINED WITH WASH AND LAUNDRY ROOM

center table, are directely "routed" to the right of sink with but a single trip and a single handling.

No one surface can serve for several processes without resulting in extra handling, extra walking and confused work.

In addition, the kind of material needed to cover the preparing surface or table, is generally quite different from that on which hot pots can be placed safely. The preparing surface can be made of any impervious material, but the serving surface demands a metal, heat-resisting covering.

It is much better to have several small surfaces for each different process or special work, than to have one large surface on which miscellaneous work is performed. For 
instance, it is more efficient to have a small special surface on which to clean vegetables near the sink (where they must be washed) than it is to prepare them on a general "catchall" table which will necessitate a trip with them back to the sink. If preparing is always done at one place, serving at another, soiled dishes laid on a definite unalterable surface to the right of the sink-there will be less confusion, less handling and consequently less waste time.

\section{Improving Wrong Arrangements}

Many housekeepers, even when won over to the worth of scientific grouping of equipment, seem to think it impossible to alter wrong arrangements in their present alreadybuilt or rented homes. But a little study will show that even the most inconvenient kitchen can be made step-saving.

If the stove and flue must be permanent in their present position, the stove can be used as the pivot to which the entire plan can be adapted, still keeping the right order of grouping.

The sink and plumbing connections can even be radically changed without too great expense or interference with other systems in the house construction.

Tables can most easily be moved into the right position; or a tray table on wheels can act as a stacking surface for the sink if the sink is tightly jammed into a corner, or as a serving surface to the right of the stove in cases where space is at a premium.

Sometimes a few shelves over the kitchen table, near the sink or the stove, will replace the inconvenient shelves of detached pantries; or two-inch strips on which to hang pots and small utensils will enable the idea of grouping to be followed out so that the kitchen approximates the ideal, step-saving plan. Often a portable kitchen cabinet, rightly placed, will effect a great improvement. 


\section{Grouping Small Equipment}

The same principle of grouping already applied to the fixed equipment (stove, sink, tables, etc.) must also be applied to the placing of the small, portable equipment. The old idea of keeping pots and pans out of sight, or of putting bowls and kitchen china in a separate closet from that containing groceries or utensils, is opposed to the efficiency idea which insists that bowls, pots, and all utensils shall be permanently grouped at the place where they are used. Any other plan or arrangement is step-taking and labor-wasting.

Concretely, if the egg-beater, mixing-bowl and nutmeg grater are used invariably at the preparing table, then near this surface they should be placed or hung. If frying-pans, soup-skimmers and ladles are always needed near the stove, near the stove they must be grouped. If can-opener, vegetable knife and apple corer are always needed near the sink, then near the sink they must be hung. Not until a close time study is made of the actual number of steps taken in each small kitchen task is it possible to realize the great amount of "waste motion" caused by failure to group the small equipment. Why walk ten feet across the kitchen to a distant pantry for the tea caddy when both the tea-pot and tea-caddy can be grouped near the stove where tea is always made? Why walk eight feet to a kitchen table and eight feet back again for the breadknife which is always needed near the breadbox kept on the cabinet across the room?

Articles should be grouped and placed nearest the surfaces on which they are used. Saucepans which must always be filled with water before being carried to the stove, belong near the sink to save steps in filling. Supply of clean dish-towels belong as near the sink as possible. All the distinctive dishwashing accessories and cleansing preparations also have their place near the sink. 


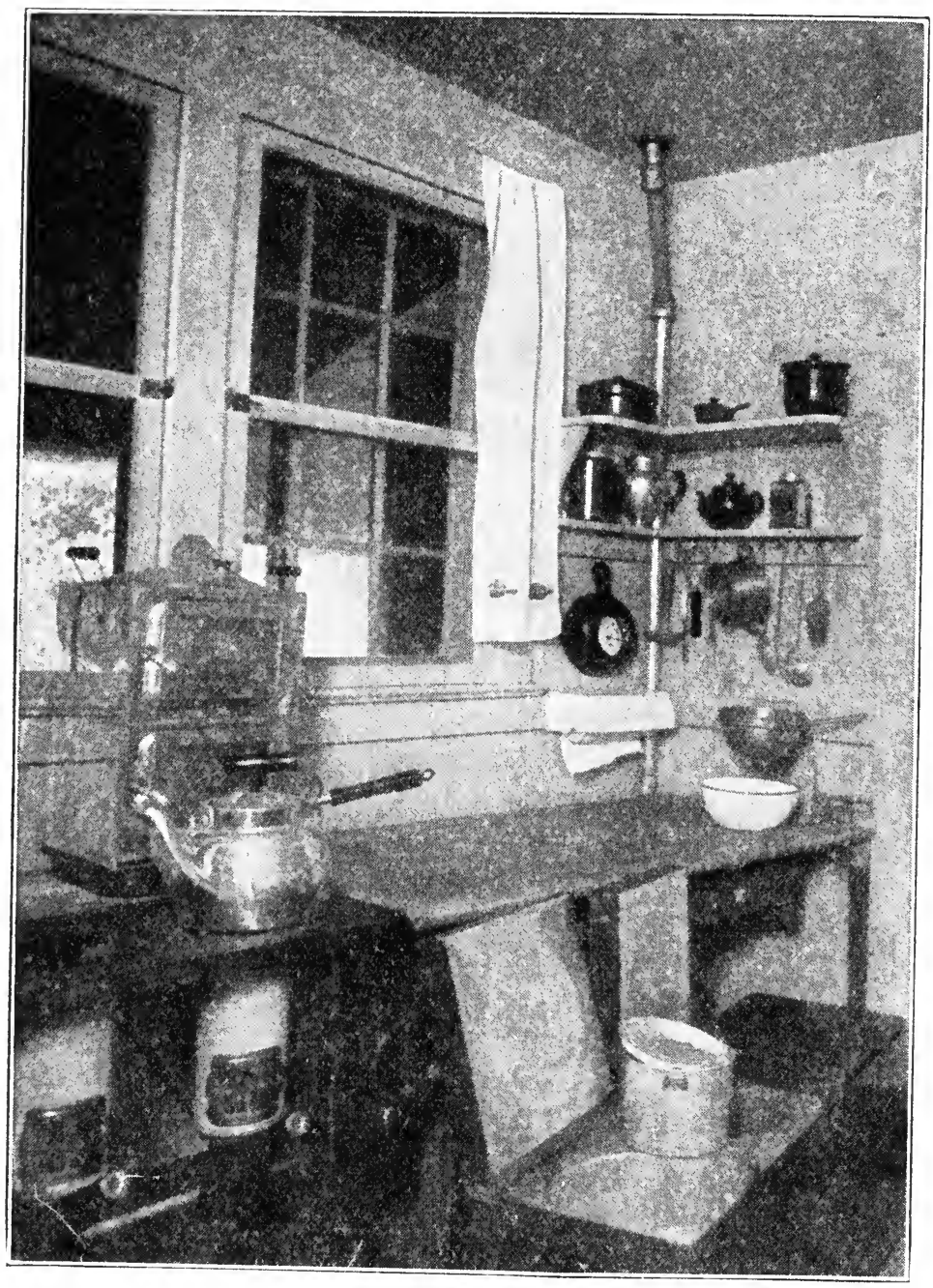

CORNER OF KITCHEN SHOWING GOOD GROUPING

Note tea-pot and tea-supply, coffee and coffee-pot grouped together, so that with one motion they can be lifted down, and eup of tea easily made are used on this table. All the small tools, spoons, etc., under the shelf 
On the other hand, there is a very large number of small pieces-muffin and cake tins, moulds, meat chopper, measuring cup, funnels, etc., etc., which peculiarly belong to the preparing table or surface.

Others belong more especially to the serving surface where the final touches of mashing, straining, etc., are given foods as they are removed from the stove.

Grouping Food Supplies

The old idea of keeping dry cereals, sugar, spices, flavorings and currently used canned goods, in a far-away cupboard is also giving way before the efficiency grouping idea. If these materials are needed in the daily work of seasoning, baking, and other cooking, they too must be grouped near the preparing table, or the stove where they are used. The exact place for every piece of equipment can easily be determined by asking, "Where do I actually use and need this article most?"

A "time-study" made of any particular task under two sets of conditions will show surprising differences in time and number of steps required. The arrangement of the main equipment and the grouping of the small tools will be found very greatly to lengthen or shorten each task. Below is given the result of such a time-study of the simple task of preparing boiled potatoes under two varying arrangements of equipment.

In Study I, the pot was kept in the pot-closet; the knife was kept in a drawer in the pantry; there was no special garbage arrangement.

In Study 2, the pot was kept on the shelf adjacent to the sink; the knife was kept on a nail at the vegetable preparing surface near the sink; garbage pail was lifted on a shelf with the circular opening above as illustrated on page 32 . Position of storage and stove was the same in both cases. 


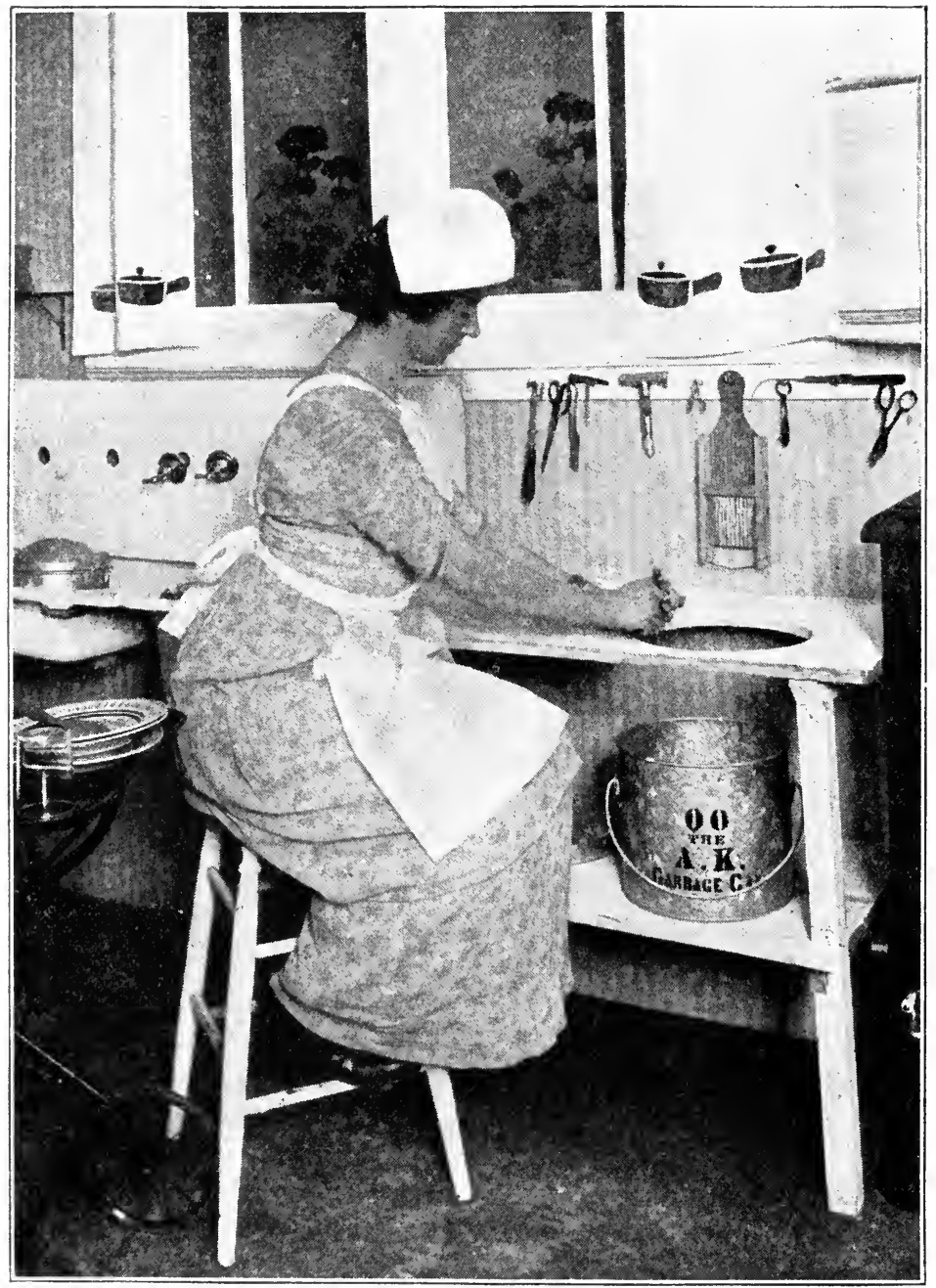

VEGETABLE PREPARING TABLE

Paring directly or scraping dishes into pail underneath saves soiling any surface. Note knives, parers, graters, etc, directly above working surface. (The opening as shown is too large; should be about eight inches.) 
Time Study Showing Saving through Correct Grouping of EQUIPMENT

Study I. I. Walk to storage.

2. Return from storage with small basket of potatoes, and lay on kitchen table.

3. Walk from table to pot-closet for pot.

4. Return from pot-closet to table, on which lay pot.

5. Walk from table to pantry drawer for knife.

6. Return from pantry with knife.

7. Peel potatoes on table surface.

8. Take pot of potatoes in hand and walk to sink.

9. Wash potatoes and fill pot with water.

Io. Walk from sink to stove and lay pot on.

II. Walk from stove to table, place refuse in basket.

12. Walk from table to sink with refuse and empty same into garbage pail on floor.

I3. Take scrub cloth from sink to table, wipe up same.

I4. Return with soiled cloth and knife to sink.

I5. Wash cloth, hang up. Wash knife.

I6. Walk from sink to pantry drawer to replace knife.

17. Walk from pantry drawer to sink to get basket.

I8. Take small basket back to storage.

19. Return from storage.

Time consumed: 5 minutes.

Study 2. I. Walk to shelf adjacent to sink and get pot.

2. Walk to storage, carrying pot, and fill it with potatoes.

3. Return from storage, laying pot directly on vegetable preparing surface near sink.

4. Pick up knife (from nail above this surface).

5. Pare potatoes directly into pail (soiling no surface).

6. Wash potatoes and fill pot with water.

7. Wash and hang up knife (on nail above sink).

8. Walk with pot and lay on stove.

Time consumed: less than 2 minutes, not counting actual peeling, which would require the same time in each case.

RÉSUMÉ:

TIME REQUiRED

Number OF STEPS

Study I

5 minutes

I9 steps

2 minutes

8 steps 
Other time studies will show equally great differences. In no way but by a careful time study can the experienced housekeeper convince herself that many of her habits and methods are wasteful of time and effort.

One of the reasons why women have been unwilling to follow the grouping idea is their belief that kitchen neatness requires the keeping of all utensils and equipment behind closed doors or in drawers. This idea perhaps was justified when the kitchen served also as the family sitting-room, or when the fuel used was so dirty as to make it necessary to protect utensils against dust and ashes. But with modern fuels of gas, electricity or oil and the complete separation of cooking-room from living-room, the kitchen can follow the efficient ideals of other workshops.

The "bench" of the mechanic can serve as a model for the kitchen. Here above the working surface, or adjacent to it, are strips, pockets and hooks for the holding of every tool and supply needed in his particular work. There are no doors to open and take up valuable floor space, no waste motion pulling out drawers, no confusing or blunting of one tool with another.

The kitchen must follow this workshop ideal. Every utensil and tool should have a definite place either on a hook of its own or on an open shelf so that it can instantly be grasped and used without waste handling. Again, the hanging of utensils prevents contact and wear. This is especially true of a large class of indispensable kitchen tools, namely, knives. They are universally "banged" into table drawers with the can-opener and the apple corer so that their delicate edges become blunted, and consequently give poor service. Yet it is just as easy to place them in the separate pockets of a chamois or wooden strip over the surface on which they are used, thus keeping them in good 


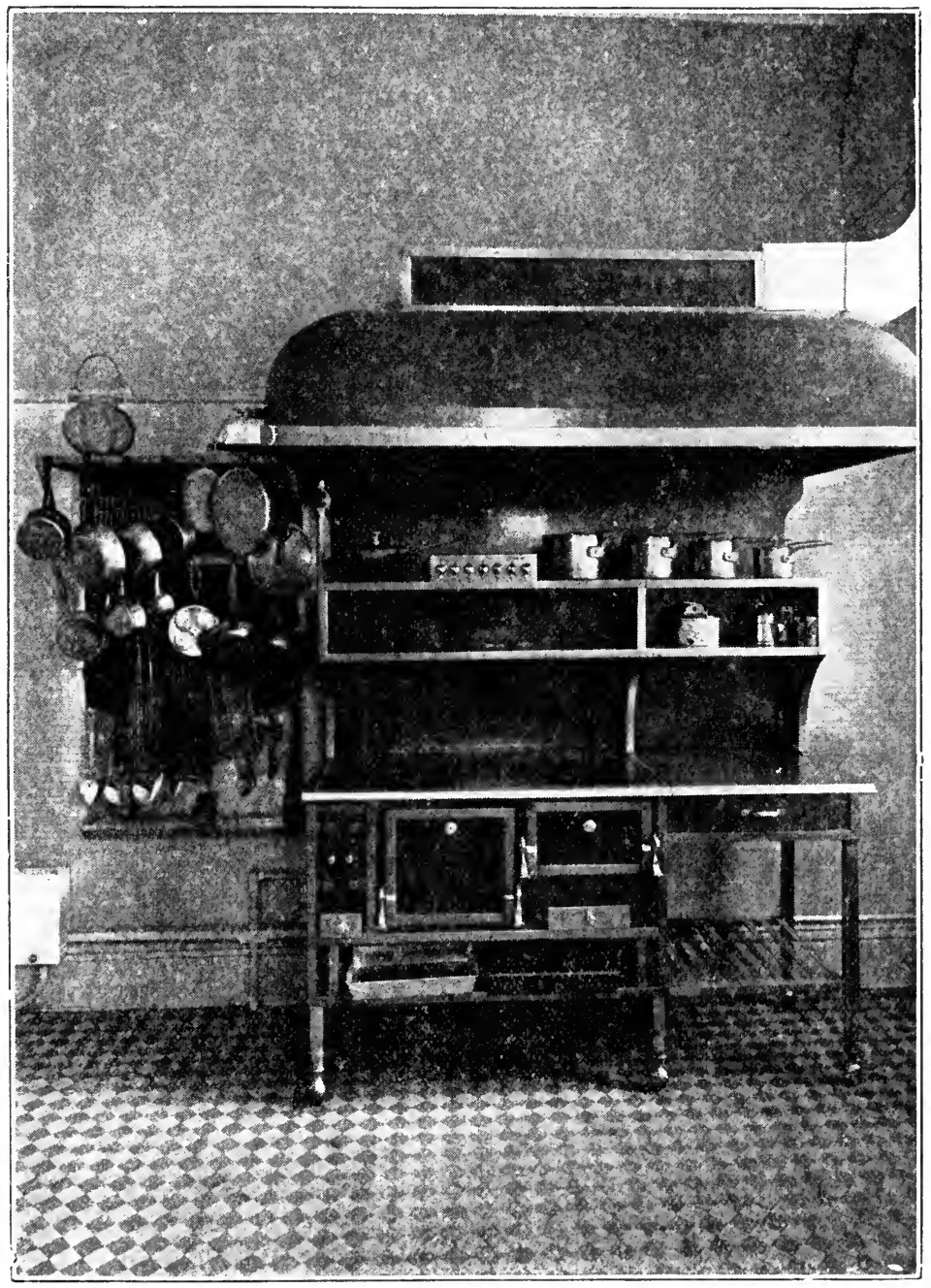

LATEST MODEL ELECTRIC COOKING RANGE WITH HOOD

On left, cooking rack of steel with excellent grouping of broilers, saucepans and small cooking utensils. Notice cook's pots on range and efficient grouping of salt, pepper and spices over tablo section of stove. 
condition, and making it easy to instantly pick out the required blade.

Racks of various kinds permit attractive, exposed grouping. There is the "cook's rack" extending over the range, from which skillets and saucepans hang neatly. A similar kind of rack is constructed specially for use at the side of

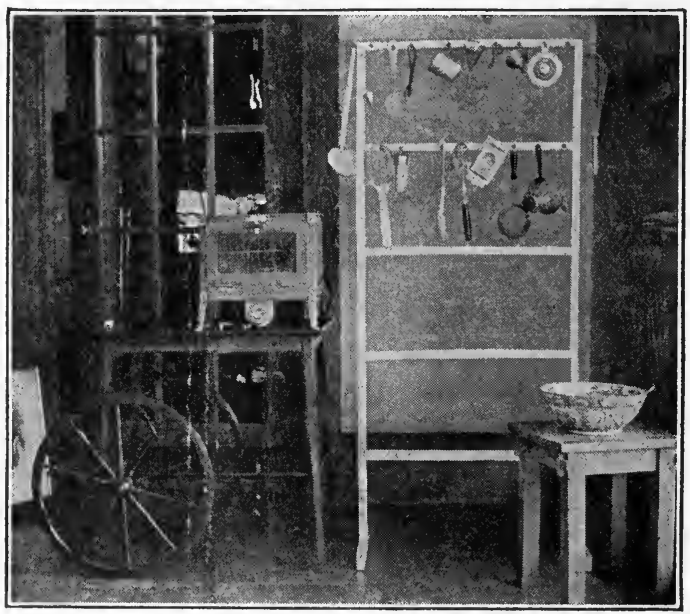

A WOODEN RACK OF NARROW STRIPS

Courtesy of Mrs. Mary Patterson.

the stove and is commodious enough to hold many of the small pots and tools needed only at this point. A wooden rack about 5 by $2 \frac{1}{4}$ to 3 feet built of narrow strips and mounted on castors will partly replace inconvenient drawers and shelves. On such a rack can be hung small saucepans, spoons, beaters, knives, etc., which can be moved up to the working surfaces, as needed. If painted to match the trim of the kitchen it is as attractive as it is useful in applying the grouping idea. Or a much smaller rack or set of strips can be fastened directly to and over a kitchen table either 
when it is in the center of the kitchen or when placed against the wall. If the straight cup hooks are placed at regular intervals in these strips, they will accommodate many groups of small tools needed at the table.

The right grouping of small equipment and food supplies can easily be made in any kitchen with little or no expense.

\section{Correct Height of Working Surfaces}

Next in importance to correct grouping is the correct and comfortable height for all working surfaces and equipment. For years women have stooped their backs over sinks and tables that were too low, strained their muscles over ironing boards that were too high, bent themselves double peering into the oven, or stretched for utensils away out of reach. No shelves should be lower than one foot from the floor nor higher than six feet; and for small women a comfortable reaching height is between $4 \frac{1}{2}$ and $5 \frac{1}{2}$ feet.

The sink, as universally installed, is several inches too low for the woman to work without bending over, thus increasing the strain and fatigue of the already fatiguing task of dishwashing.

The same is true of kitchen tables in the heights com-
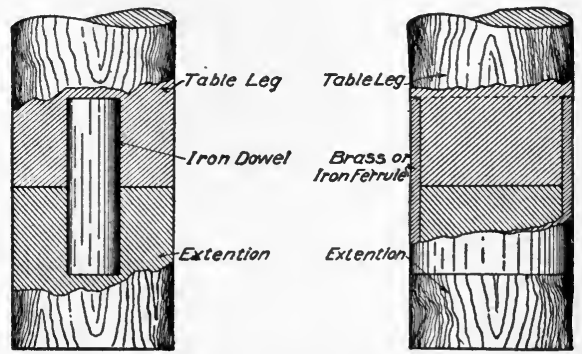

TWO MEthods OF LeNGthening Legs of table, ETC. 
monly manufactured, which are from one to three inches too low to permit standing and working at them in a hygienic upright position.

While most stoves are high enough for convenient work, many of the portable types are also beneath a comfortable working level. Although the coal range is the only stove necessitating a low oven, manufacturers of stoves using other fuels, such as gas and electricity, have been slow to place all ovens, broilers and warming-closets above the waist level. Happily, however, the latest models have ovens either at the side or above the regular cooking surface, which results in more comfort and less fatigue for the worker.

Even the fireless cooker box should be mounted on a frame or stand so that its top surface is practically the same height as the usual cooking surface of other stoves, which will prevent repeated stooping every time the cooker is used.

No absolute rule can be given for invariable heights because not only the height of the worker must be taken into consideration, but also the length of her arm, and whether she is short or long-waisted, etc. From tests made on women of varying heights, the following guide was compiled. This may be used by each worker as a basis for determining the exact height suitable to her individual needs. But she should also make actual tests herself by placing a dishpan or tray on a high stool, raising and lowering with the aid of books of various thicknesses, or some other object, until she finds the exact height at which she can work without strain either on the arms or back. If the working surfaces are to be used by workers of different heights (servants or maids) it is best to put them at the height convenient for the taller workers and use a small platform to make them the right height for shorter women. 
The height of the sinks is given separately from other working surfaces because here actual work, such as dishwashing, is performed at a level about 2 inches or more above the bottom of the sink. On other surfaces the actual work, such as peeling potatoes, is done at the exact level of the surface itself.

Table of Standard Heights for Working Surfaces

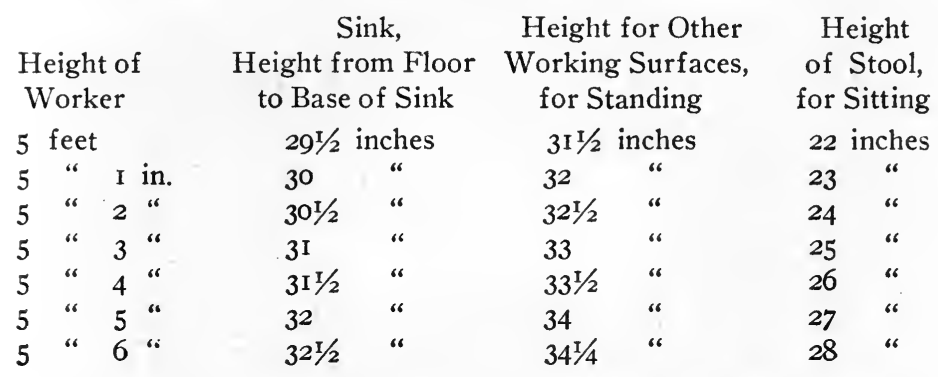

It will be found generally that there is an inch of difference in the height of the stool to each inch of height in the worker.

\section{SitTing AT Work}

Another great preventive of strain and fatigue is the practice of sitting down to many household tasks. It is only custom, or a false fear that they will be considered lazy, which makes many women object to sitting down at work. A high stool (preferably with an adjustable seat) can be used when washing dishes, peeling vegetables, preparing pastry, and many other tasks. When sitting, a great deal of strain is removed from the feet and abdomen of the worker. This lessens the possible fatigue of the task, and permits the worker to give her entire energy to it, thus resulting in quicker and better work, after the sitting habit is acquired. 


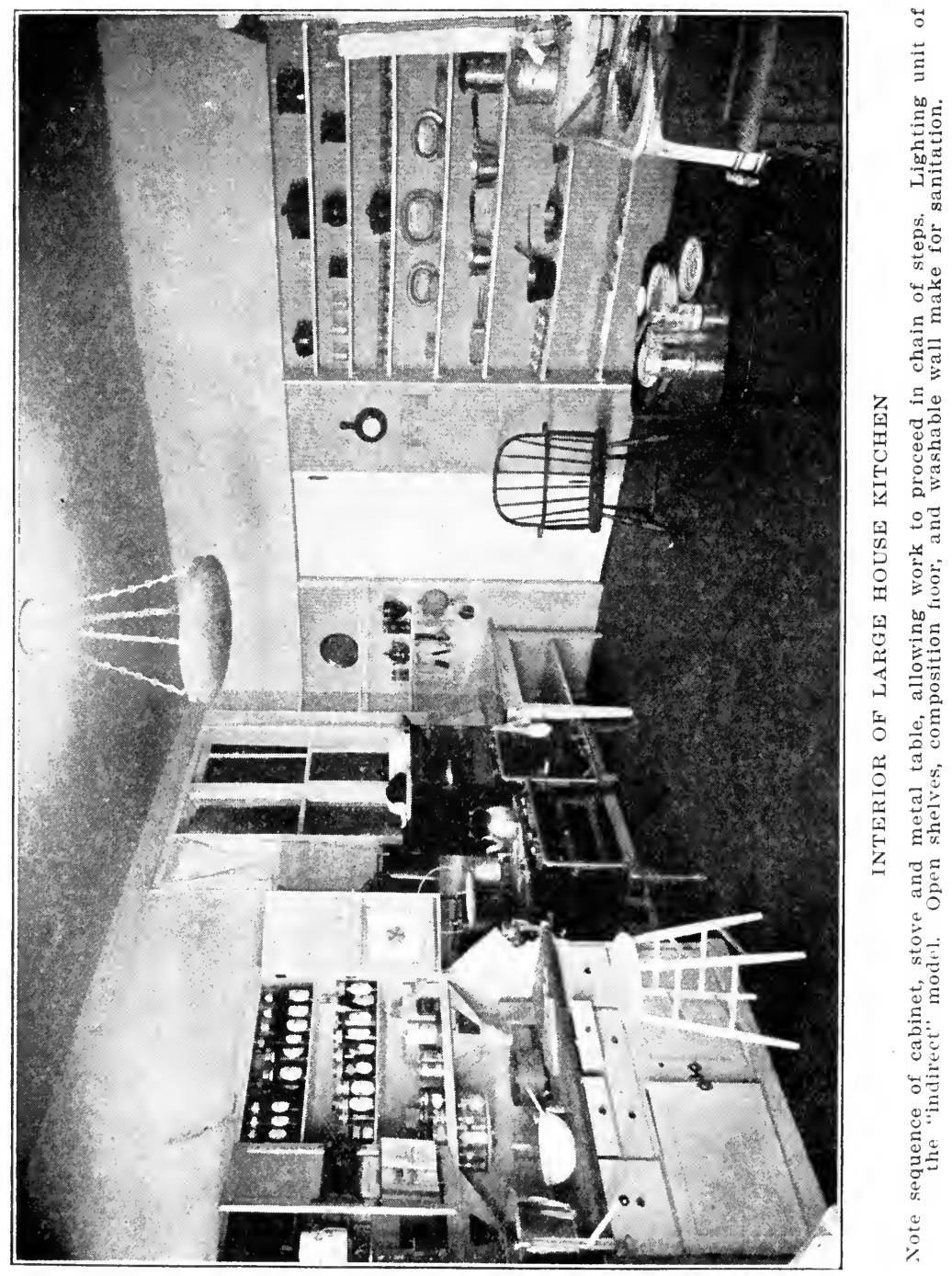




\section{Lighting}

Adequate lighting and ventilation are two further essentials to kitchen efficiency. In building, windows and doors should be placed in harmony with the working surfaces. Too frequently the good light from a window is wasted because it does not reach the equipment where it is most needed. Very intense light is needed at table, stove and sink, yet because windows are not placed so that the light falls from a proper angle on these surfaces, the worker is all too frequently standing in her own light or the equipment is in shadow.

Windows should be placed so as to give a direct light over the important working surfaces of table, stove and sink. They should preferably be placed high in the walls with the sills 42 to 46 inches from the floor. This will allow the wall space under them to be utilized to the most advantage. It will give better ventilation to the upper part of the room and the high sill cannot be used as a "catch-all" for bottles, small dishes, etc. The high sill also keeps any window curtains out of reach of working surfaces likely to soil them.

The placing of artificial illumination must also be carefully studied. The very common single "drop" in the center of the room is one of the poorest forms. It casts shadows and inadequately lights the corners of the room, and is almost universally placed so high that it is a strain to light it. If either electricity or gas is used, the bulb or burner can be enclosed in a bowl of opaque glass and the light reflected up to the ceiling in the manner called "indirect" lighting. This method diffuses the light more over the entire room and is less glaring on the eyes. However, if the kitchen is large, it is better to use separate wall brackets giving direct light at stove, table and sink. "Goose- 
neck" fixtures are manufactured especially for this purpose of focusing artificial light directly where needed. If a kerosene lamp must be used, the most efficient type is that having a "mantle" which permits a strong white light of about 40 candle power similar to that given by the Welsbach. An identical kind of light is given from lamps using denatured alcohol with special kinds of wick and similar "mantle."

\section{VENTILATION}

Cross ventilation is essential to keep the kitchen well aired and free from odors. Windows in opposite walls, or a window opposite a door frequently left open, may be sufficient in the small kitchen. Transoms above the door, and windows placed high in the walls will give additional ventilation.

But the most complete system is to have a special ventilation flue in the chimney running to the kitchen. If the natural current through this flue is not sufficiently strong to carry away odors, a small electric fan can be placed in this flue at its face and used as needed. If there is no special vent flue in the brick chimney, the best plan is to place a hood over the stove which will concentrate the drafts around the surface of the stove. From this hood a pipe can be carried through the wall of the kitchen and from there to the top of the roof. This flue should be double on the outside and about ten inches in diameter for use with a 4-foot stove. Even a gas stove should be installed with a flue connection which will lessen the amount of heated air, gases and odors in the kitchen.

\section{Floor Surfaces}

The labor-saving kitchen must meet the highest ideals of sanitation. Therefore all its surfaces-floor, walls, tables, etc.-should be as non-absorbent, non-porous and as easy 
to clean as possible. What material, then, is best for the kitchen floor which receives a heavy daily quota of dirt, grease and water? All wood no matter how treated, is absorbent of grease, and cracks collect dirt particles. Frequent scrubbing merely widens the cracks and coarsens the wood. For these reasons a wooden floor, even when painted, varnished or shellaced, is out of place and unserviceable in the kitchen. There are three groups of sanitary floor coverings with definite points of merit and fault, i.e., linoleum, "composition flooring" and tile.

Inlaid linoleum with the pattern extending through the entire thickness is more durable than printed linoleum and makes a most satisfactory and inexpensive floor covering. So-called "battleship linoleum" comes in a single color with a dull, attractive surface, and from tests made by hospitals and other institutions, has been proved the most durable and satisfactory of any linoleum.

The laying of linoleum is most important. Unless laid by a professional, it is apt to "belly" or the seams will not be perfectly joined. Care should be taken to have the baseboard joining perfect; the baseboard should be put in place after the linoleum is laid, or preferably, a rounded metal strip should be used to cover the joining and facilitate mopping, or the seams can be cemented.

Within the last few years great improvement has been made in various "composition materials" of asbestos, cement, rubber, etc., sold under various names. Generally the material comes in a powder form, and is mixed and spread after the manner of cement. Two or more coats are applied, the first one frequently being applied to metal lath and forming a continuous surface with the baseboard, which may be made of the same material. These materials come in a variety of colors, are resilient and easy to walk on, yet non-porous, without cracks and require a minimum of effort 
in cleaning. To be entirely satisfactory they should be laid by the manufacturer's own workmen. These floors are suitable for pantries, halls, porches and service quarters as well.

Although tile may seem the most sanitary and impervious surface for the kitchen floor, experience has shown that its chief defects are that it is too hard a surface on which to stand continually; it is also slightly slippery, which makes quick walking dangerous and its hard surface is fatal to dishes accidentally dropped. It is frequently desirable, however, to have a tiled area $I \mathrm{I} / 2$ to 2 feet around the stove, with the stove inset, and flush with the tile. This is a protection against fire; and it is more sanitary than the dust-catching space between stove and floor when the stove is mounted on legs.

\section{Wall Covering}

As a wall covering, however, tile is ideal because of the ease of cleaning, its impervious qualities and constantly fresh appearance. Many institutional and commercial kitchens have tiled ceilings, walls and floors on which a hose can be turned daily if necessary. But for the family home a wainscoting of tile is sufficient protection for those parts of the wall which receive the hardest use.

Another good material for wall coverings is the oil-cloth wall fabric which can easily be wiped with a cloth.

Above this wainscoting, plaster in either of its two main finishes-smooth "hard trowel" or "rough sand"-can be employed. The entire wall may be finished in hard trowel plaster if desired, but the entire surface is not to be recommended in the rough sand finish which offers too many projections for the accumulation of dust and grease. The smooth plaster finish can be painted preferably in a "flat" paint easily cleaned with a damp cloth. The sand finish 
should be treated with kalsomine and given a fresh annual coat. Paper of any kind should never be used on a kitchen wall or ceiling, because it puffs loose with heat and steam and is unsanitary to a degree.

\section{CoLOR}

The color of these floor and wall surfaces has a more important influence on the worker than is sometimes realized. If some so-called "unattractive" kitchens were carefully analyzed, it would be found that they were unattractive largely because of ugly green or hideous blue colorings. The woodwork or trim should not be dark; cherry, mahogany or even golden oak stain is too heavy and somber. Pine or birch or maple in natural finish, or painted wood in pure ivory white or such tones as "putty," warm gray, light apple green, make the kitchen lighter as well as more cheery. Similarly, light tones only should be used on walls or ceiling, and large patterns should be avoided in both floor and wall coverings. The ceiling should preferably be dead white; if there is a wainscot, the section above it should be a pale shade of the preferred tone; the wainscoting may be still darker and the floor the darkest of all. Good color combinations are: (I) ceiling white, above wainscot light warm yellow, wainscot buff, floor white and brown, woodwork old ivory white; (2) ceiling white, above wainscot pale apple green, wainscot medium apple green, floor white and green, woodwork putty. Baseboards, and a similar height across the bottom of doors, and a small circumference around door knobs may be painted the darkest shade of the color used, to conceal wear.

\section{TABle Tops}

Wooden surfaces of all kinds must yield to the pressure for more perfect sanitation. The exposed wooden kitchen 
table top or drainboard absorbs water, stains, and grease. This means wasted effort in continual scrubbing and scouring; in addition, the wooden surface is marred by having heated pots and utensils laid upon it. The working surfaces of the labor-saving kitchen must be covered with nonabsorbent, easily cleaned materials, of which there is a wide choice.

For the preparing table there is a selection of vitrified glass, porcelain (baked enamel), monel metal and plate glass, all of them sanitary, impervious to grease or water. For the serving table-or surface-on which it is necessary to lay heated objects, either galvanized iron, zinc, monel metal or German silver are desirable because they do not mar or stain badly, and can be kept clean with a minimum of effort. For drain surfaces, zinc, porcelain, German silver or copper are practical and sanitary.

\section{Built-in Conveniences}

As was said previously, it is better to eliminate the disconnected pantry and build into the kitchen itself permanent closets and shelving in harmony with the processes of work. In order to follow the chain of steps in the clearing away process (No. 2) it is necessary to have permanent shelves and pot-closet at the left of the sink. This permits dishes and pots to be laid away with no walking or carrying, since such a closet is but an arm's length from the drainboard.

Most shelves, as commonly built, have two faults: they are too wide, and too far apart. The first fault results in a broad shelf on which there are consequently placed a double or triple row of articles. Then when one article is needed, it is necessary to displace and search behind others, which means waste of time and effort. The efficient shelf, no matter whether used to hold supplies, dishes, pots or still 
larger utensils, is only wide enough to accommodate one article. That is, shelves should be graded in width according to the size of the articles or utensils they are to contain. Their width may vary from six inches for a row of pitchers or sauce dishes, glasses, etc., to eight inches for jars containing cereals and small supplies, to ten inches for plates and usual size pots and pans. Large pieces like breadmixer, steamer, preserving kettle, etc., may need a shelf twelve or fourteen inches.

Again, shelves too widely apart mean waste space and useless effort in reaching. Most shelving can be lowered so there is less space between each shelf, which will give more shelf space in the same wall area. If possible, the preparing surface should be a built-in fixture with shelves and closets above and below of these correct sizes and widths to economically use the space. Small narrow shelves such as one for the tea-pot and tea supply near the stove, or broad shelves to hold bread-box, etc., can be placed exactly where most step-saving. Two-inch strips can be fastened directly under small shelves over the serving surface or adjacent to the stove in which straight cup-hooks can be screwed at regular-intervals. On these can be hung and grouped many of the smaller beaters, cook-spoons, mashers, etc., so that the shelf and objects under it can be related to the working surface near it.

APPROXIMATE PRICES OF SURFACE AND WALL COVERINGS

Composition floorings (cork, cement, asbestos mixtures, etc.), set directly on wood or expanded metal lath, per square foot....................... $\$$.17 to $\$ .50$ Sanitary cove baseboard, per lineal foot............ .20 up Linoleum, inlaid and battleship (not laid), per square yard

Tile (floor), per square foot, laid, but not including cost of cement foundation.....................30 up 
Tile (wall, glazed), per square foot, laid, but not including cost of cement foundation..............6 up Oilcloth fabric (glaze and mercerized). Comes in rolls

48 inches $\mathrm{x}$ I2 yards, per lineal yard.............25 to

Paint, per coat, per square yard............. .04 to

Kalsomine, per coat, per square yard........... or to

Zinc (22 gauge metal), per square foot................. 30

Galvanized iron (22 gauge metal), per square foot........... 15

Copper (22 gauge metal), per square foot..............$_{50}$

Polished steel (22 gauge metal), per square foot........... .I5

Monel metal (22 gauge metal), per square foot.....\$1.oo to 1.25

German silver (22 gauge metal), per square foot......... 1.85 .

Plate glass, per square foot................... I.00

Vitrified glass, per square foot.............. .90 to 1.25

Porcelain enameled steel, per square foot.......... r.oo up

(Note.-The above are pre-war prices; present prices unstable.)

Bins AND DRAwERS

In the built-in fixture, it is best to allow for bins of various sizes for holding flour, sugar, etc., in quantity. The most improved type slide forward easily on ball bearings, and are so made as to tilt with little effort. Bins should be lined with zinc or similar metal to keep them moisture and insect proof. The point to avoid in the built-in drawer is not to have it too deep, as deep drawers cannot be kept in order, and it is more difficult to pick up any required article. Shallow drawers, three inches deep for kitchen ware, and about five inches deep for linen, are most satisfactory. Large drawers in center kitchen tables are now manufactured on ball bearings so that they can easily be pulled out and reached from both sides of the table.

\section{SinkS}

The three important pieces of fixed equipment in the kitchen are sink, stove and refrigerator. Sinks are made of various materials, iron, slate, soapstone, enamel ware. 
For the country kitchen where a great deal of work must be done, the slate or soapstone is preferable. But the needs of the modern kitchen are best met by the attractivelooking, white sink of enameled iron. In choosing any sink, these are the points to bear in mind: it should be deep enough to give ample room for the dishpan and thus avoid water splashing over (8-Io inches); it should have the back and drainboards an integral part of the sink to avoid crevices in which dirt might accumulate, and to avoid splashing

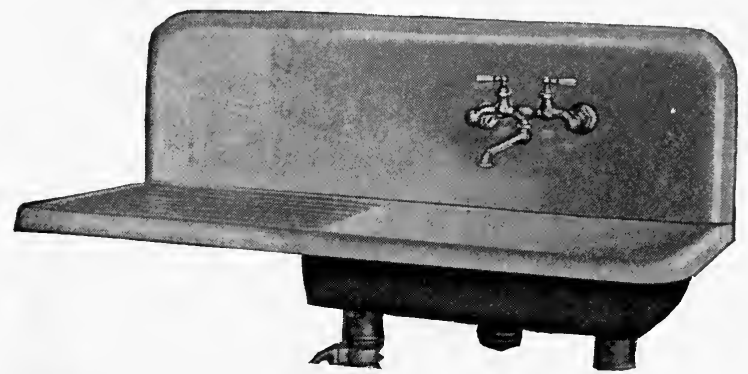

ENAMELED IRON SINK WITH MOVABLE FAUCET NOZZLE Convenient for dish washing, etc., as hot or cold water may be drawn at will.

the wall, as occurs when the drains are not protected by the splasher back of the main sink; it should have a concealed "hanger" attachment rather than be mounted on legs which prevent ease in floor mopping; it should have a movable faucet nozzle, or have the ordinary faucets protected with rubber tips to prevent china breakage.

The double sink with two compartments makes for ease in dishwashing, one being use for washing, the other for draining. If the establishment is large, it may be necessary to have a separate "vegetable sink" and a deeper, so-called "pot sink" in the kitchen, when the table dishes are washed in a separate sink in the butler's pantry. For the small 
kitchen a serviceable size is $30 \times 18 \times 8$ inches with draining surfaces 2 feet long on either side. If there is only one drainboard, it should always be at the left.

\section{Stoves}

In the past few years great changes have been made in the kinds of fuel used, and hence in the character of the cooking equipment. The familiar kitchen range was generally a combination of heating and cooking equipment, as the water back attachment heated water for household use in addition to the stove doing the actual cooking. A No. 8 stove which used 2 tons a year of nut coal for cooking only, used $4^{\mathrm{T}} / 2$ tons of coal when connected with the waterback.

Owing to other changes in our methods of general household heating, it is possible and much more efficient to separate the heating system entirely from the cooking system, and to have the latter under more exact control than is possible with a coal range.

The ideal cooking device is that in which fuel is consumed only when actual cooking is in progress, and where the fuel can be cut off instantly. This ideal reaches a high degree of perfection in stoves using gas, electricity, denatured alcohol or kerosene, because here the heat can be controlled definitely by the operator-no actual cooking, no actual fuel. In addition, modern science has proved that much cooking can be done equally well with "conserved" heat. Therefore, the modern kitchen has a choice of stoves or devices embodying this principle, such as the insulated oven of several gas and electric stoves; the fireless cooker, and combinations of the fireless idea in other stoves operated by various fuels. Perhaps the most efficient type of stove is the small gas stove with insulated upper oven which permits cooking by both direct and radiated heat, with a minimum of fuel. 


\section{Plate Warmers}

In every kitchen there should be adequate facilities for keeping dishes and foods warm. With the coal range, this is easily done because of the radiated heat from the stove to the warming shelf above. With a gas stove of the range type, the oven may be used to heat dishes and keep foods warm. If a gas hot plate is the only stove, it should be located so that a perforated metal shelf can be fastened to the wall over the stove at a convenient height. This will allow the radiated heat and steam to warm whatever dishes or towels are placed on the shelf, or a small "portable" oven or steam cooker answers the purpose admirably.

If the kitchen is heated by steam or hot water, a metal grill can be fastened over the kitchen radiator, thus serving as a shelf on which to dry utensils or keep dishes warm. Some radiators are made specially in the "pantry radiator" type with two or three decks of coils on which plates may be laid. Or the dining-room or pantry radiator may contain a small compartment. In large establishments a separate "plate warmer" may be built in any size to be heated by electricity. Similarly, one of the most improved metal kitchen tables has a plate warmer compartment underneath heated by current. A larger table which approximates the excellent "steam table," seldom seen except in institutions, is heated by gas and connected with the steam-heating system.

\section{Hot Water Supply}

When the cooking arrangements are thus separated from the heating of hot water, other provision has to be made. One of the most satisfactory and inexpensive plans of independently treating the water supply is by the installation of a small heater somewhat like a laundry stove, preferably in the basement or cellar, using pea coal. This heater can 
be attached to a boiler and connected with the pipes to the kitchen or bathroom. The boiler should always be installed vertically (water takes longer to heat in the horizontal position), jacketed with asbestos, and the heater itself covered with plastic asbestos to prevent radiation. Such a heater can be operated at a cost of $\$ 2.00$ to $\$ 3.00$ per month, and combines a laundry stove with its use as a water heater. If not installed in the laundry, one of the cylindrical type of heaters with a magazine fuel feed should be used, as these require less attention to operate.

An independent hot water plant permits of an abundant supply of hoter water both summer and winter This plan is preferable to having a hot water coil in the furnace or other house heater, which at times gives insufficient hot water, and at others causes the water to boil; then the coil may rust out or become stopped up, necessitating repairs in cold weather. Experiments show that little or no coal is saved, for the hot water coil requires as much extra coal as is needed to run the independent heater.

If gas is available and the rate is low, the water supply may be heated by one of the several types of "gas heaters" now on the market. In some models the heating coils are placed outside the boiler; in others within it, or in some a cast iron plate or burner heats the boiler by direct contact. Types where the heating coils are within the boiler are preferable because there is less loss of heat by radiation; the coils should be brass or copper in preference to save fuel. Some of the latest models are automatic in action and keep the water in the boiler at any desired temperature. This method of heating the water supply is very clean and convenient, and its cost is about $\$ 3.00$ to $\$ 4.00$ monthly in a medium sized family.

The so-called "instantaneous" heaters, of which there are several makes, operate by an automatic valve which lights 
the gas burners as soon as the hot water faucet is turned on; similarly the flame is extinguished when the valve closes, by shutting off the faucet. These heaters are most efficient, but are more expensive in their first cost, and somewhat also in the operating cost, over the simpler gas heater.

All gas hot water heaters should be connected with a flue to the outside air, as poisonous carbon monoxide gas is often given off.

Several makes of water heaters using kerosene are on the market, but, owing to the slowness of kerosene fuel, do not give as quick results as the gas heater. For homes without gas, and where it is not desired to use coal, the kerosene heater may be adequate. The best model on the market costing \$18.00 has a separate, welljacketed heater, and which is claimed by manufacturer's tests to care for "two bathrooms, kitchen and laundry, the fuel cost being one-half cent per

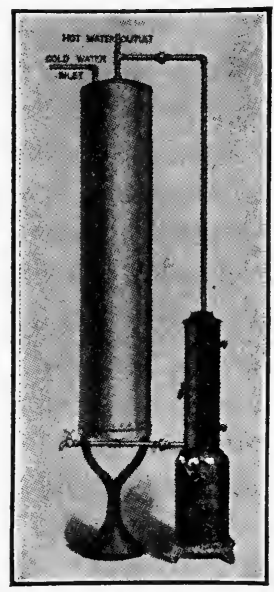

KEROSENE HOT WATER HEATER hour, with kerosene at 12 cents per gallon."

The approximate cost of the various water heating device is as follows: Coil in furnace, \$10.00 to \$12.00; coal heater, $\$$ I 5.00 to $\$ 25.00$; outside gas heater, $\$ 10.00$ to $\$ 25.00$; boiler and inside gas heater, $\$ 40.00$; automatic outside gas heater, \$50.00; automatic instantaneous gas heater, \$150.00 (no tank required). The boiler will cost about $\$ 14.00$ for a $30-$ gallon tank to $\$ 20.00$ for a 6o-gallon tank and the jacket for the boiler $\$ 5.00$. The labor will average $\$ 10.00$ additional. The local plumber will give an exact estimate. 


\section{REFRIGERATOR}

The third important piece of fixed equipment is the refrigerator. Even if a family requires a separate cold storage room for storing perishables and containing its quantity of preserves, canned goods, etc., a good refrigerator is indispensable in modern kitchen economy. The points to be carefully considered in buying are the insulation, which must consist of adequate layers of non-conducting materials (cork, mineral wool, etc.) with a dead air space between; there must be sufficient circulation of dry, cold air; the ice chamber should be situated on the side; all compartments should be one piece of sanitary glass or enamel with easily removable shelves to facilitate perfect cleansing; if possible, there should be a coil under the ice chamber and connection with the drinking supply so that a constant supply of chilled drinking water is available from a tap on the face of the ref rigerator. The refrigerator should preferably form a permanent part of kitchen construction, and be built into the wall space so that it can be iced from outside. This plan saves the tracking of ice delivery into the kitchen and makes it possible to use very little ice or none in winter months. If the refrigerator is perfectly insulated and made, the modern kitchen temperature will not affect it. Step-saving ideals demand that the refrigerator be near the preparing surface, in order that supplies can be withdrawn with little effort.

In country regions where the ice supply is scarce and where many food supplies are kept "down cellar," an elevator refrigerator will be found most step-saving. This device operates on pulleys and counterweights and can be easily raised or lowered through the kitchen floor into a cold storage closet. It has a small ice chamber and two more commodious sections screened with wire netting. 
The so-called "iceless refrigerators" operate in the same way through a galvanized iron cylinder to beneath the cellar floor. Either will keep even milk and butter in good condition in the warmest weather.

\section{Garbage Disposal}

Garbage disposal is part of the kitchen problem. In the country it may be fed to stock, or in the city it is removed by the janitor. Every effort should be made by householders to have the municipality adequately handle the garbage question. But in some detached homes, where the garbage service is inadequate, and where the house is piped for gas, it is desirable to install a device for the incineration of garbage. These appear like small portable stoves, are operated by gas, connected with a flue, and so built that they can reduce a pailful of garbage to an ash in ten or twelve minutes. It is preferable to install them as close to the sink as possible so that the sink garbage pail can be emptied into it with only a few steps of walking.

In all cases, garbage should be carefully drained and kept as dry as possible. Waxed paper bags may be used within the garbage pail, which will keep the pail clean and enable the garbage to be handled in a sanitary manner.

\section{Vegetable Preparing Table}

A small built-in fixture which has been found to save effort and waste motion is a vegetable preparing table, which can be incorporated into different kitchens in various ways. From a close study of how vegetables and other foods are prepared at the sink, the usual methods of handling refuse several times and finally stooping to throw it into a garbage pail on the kitchen floor appear wasteful of time and energy. A simple board or small table surface can be extended from the right drain. In this a circular 
opening about eight inches in diameter should be cut and the whole surface covered with zinc or other metal. Under this board a small shelf must be placed at a sufficient distance to allow the garbage pail to rest on it and permit the cover to be removed easily. The method of using it is to bring vegetables to the adjacent sink, and wash them; then instead of peeling them on a surface directly, the lid of the garbage pail can be removed and the worker peel or prepare over the opening so that the refuse falls at once into the pail without any handling whatever. If small preparing tools are hung on cup hooks over this surface, the whole will form a serviceable vegetable preparing outfit. If the garbage pail is kept in proper sanitary condition, there is no unpleasantness attached to this labor-saving method. If this refuse table is placed near the right of the sink where the dishes should be scraped, the refuse from the plates also can be scraped directly through this opening into the pail, thus saving the unsanitary handling with a sink strainer, etc., as commonly done.

\section{Choosing Small Equipment}

In choosing the small equipment, it is better to buy too little than too much. The first step taken in putting a certain kitchen on an efficiency basis was to dispose of half of the twenty-three saucepans and six egg-beaters or whips found in the pantry. Too many women mistakenly overbuy small equipment, which takes up room, requires additional care in cleaning, and duplicates itself.

Here are the points to observe in buying utensils and small equipment:

(I) Right and exact size and shape for purpose needed.

(2) Right material for purpose needed.

(3) Keep shapes and colors uniform and harmonious. 
(4) Choose utensils with handles an integral part.

(5) Avoid "seconds," "three-in-one" tools, poorly finished articles with rough edges, etc.

(6) Select tools without complicated parts, which will make washing easier.

(7) Select utensils that are comfortable to hold, to grasp and to handle; and

(8) that it will be a profitable investment for the price paid and the amount of use.

Nothing is more essential before purchasing pots and yans than to measure and find out the best sizes for the needs of your particular family. One reason for excess equipment is that the required size was not studied before purchasing, and hence a great number of sizes had to be bought. The shape is important because broad, shallow utensils have more surface exposed to the heat and hence heat faster, which helps in economy of fuel. The tall, narrow utensil should therefore not be chosen.

"Bail" handles become too hot, as they hang at the side; hollow metal or black rubberoid handles in one piece allow easiest and safest handling.

Lids should not be fitted with a ring or separate wooden knob, which may work loose from the nut; the most durable method is to have a strap-shaped metal knob welded to the lid. Saucepans and skillets should be chosen with a lip on either side to facilitate easy pouring. Utensils should be free from seams and crevices in which food particles may collect.

Just as there are different kinds of cooking methods, so there are different metals and materials which are best suited to each particular purpose. Different metals are adapted to different degrees of heat. They also affect chemically certain food elements cooked in them. The 
difficulty of cleaning each metal should also be considered.

Enamel or Agate Ware. Here a vitreous material is melted and baked on to a mould of iron or steel of the required shape. This gives light weight utensils of smooth, easily cleaned finish. It does not resist a high temperature, but "chips" when foods go dry. The gray finish seems more durable than either the white or blue and white, though different grades vary greatly. It is most suitable for small bowls, pitchers, saucepans, and for simple stewing and boiling.

Iron. Heavy in weight, but easy to clean when used for some time. Resists very high temperature. Suitable for frying, roasting and baking, and very large boiling kettles. Sheet iron is a thinner quality used for baking sheets, bread pans, etc.

SteEL. Resists high temperatures. Moderately hard to clean. Used for same purposes as iron, also tea-kettle, frying pans, etc.

Tin. Does not endure high temperature. Discolors quite readily. Is light in weight, and best for cake pans, colanders and similar small pieces. The best grades are cheapest in the end.

Aluminum is light in weight, does not radiate heat quickly, fairly easy to clean, affects acid foods slightly and is seriously affected by water containing alkalies. Made in seamless shapes, suitable for all purposes, but not best for frying, griddles, etc.

Earthenware. Moderate weight, endures moderately high temperature, easy to clean, impervious surface, suitable for slow cooking of all kinds-bowls, custard cups, pudding dishes, casseroles, etc.

Woodenware. Use confined to meat, vegetable and pastry boards, mashers and wooden mixing spoons. 


\section{Harmonious Shapes and Colors}

One of the reasons so many kitchens have a cluttered untidy appearance is that no two pots or utensils are the same shape or finish. If a saucepan of a certain style is decided on, use the same style in saucepans of all sizes. If gray agate has been the material chosen for one kettle, do not choose others of white, blue or mottled. If some mixing bowls are yellow, do not pick out others that are white or dark brown. A harmonious row of utensils as to shape and color has much to do with making attractive appearing shelves.

\section{SECONDS}

The housewife should be wary of buying apparently cheap tools. "Seconds" may be uneven so the bottom does not sit squarely, or, as in frying pans, have a raised surface in the center so that grease sinks to edges and makes unsatisfactory frying. Agate-ware bargains commonly have an exposed portion of the under-metal, which consequently greatly shortens the life of the pan. The "three-in-one" or combination tool is seldom a success. Just as there is no satisfactory combination saw, plane and chisel for the workman, so there is no practical can-opener, grater and parer or other combination. It is much wiser to buy the best grade of a particular standard tool rather than to invest money in "novelties," for which extravagant claims are made.

There is no one standard list of equipment that will fit every family, because of differences in the main equipment, in fuel used, in table standards and number in family. Here is given a list of utensils and fuels for a family of six, where all cooking is done at home on a small gas range with fireless cooker attachment. If a kitchen cabinet is part of the fixed equipment, it will contain breadbox, bread- 
board, spice jars, etc. Similarly some of the pieces may be unnecessary in certain families. The prices given are average for the best grade of materials.

\section{LIST OF UTENSILS AND TOOLS,' GROUPED ACCORDING TO USE (PRE-WAR PRICES)}

PREPARING Tools (Grouped near kitchen cabinet or preparing surface)

2 half-pint glass measuring cups..............each \$ .Io

I graduated quart measure, enamel, or............ $.35^{\circ}$

I graduated quart measure, tin.................. .

I serrated bread-knife ...................... 50

I biscuit cutter, tin........................... .10

2 case knives ...............................

2 kitchen forks $\ldots \ldots \ldots \ldots \ldots \ldots \ldots \ldots \ldots \ldots \ldots \ldots$.I5

I large sabatier kitchen knife.................. .90

I small sabatier kitchen knife.................. $\quad .45$

I egg beater and cream whip combined........... $\quad .50$

3 earthenware mixing bowls, 8,6 and 5 in. spread ,IO

I 4 -sided grater $\ldots \ldots \ldots \ldots \ldots \ldots \ldots \ldots \ldots \ldots \ldots \ldots, .25$

I flour dredger $\ldots \ldots \ldots \ldots \ldots \ldots \ldots \ldots \ldots \ldots \ldots$, .Io

I flour sifter $\ldots \ldots \ldots \ldots \ldots \ldots \ldots \ldots \ldots \ldots \ldots . ., 25$

I small funnel, enamel, or.................... 20

I small funnel, tin........................... . 08

I glass rolling-pin $\ldots \ldots \ldots \ldots \ldots \ldots \ldots \ldots \ldots \ldots, \quad{ }_{50}$

I pastry board $\ldots \ldots \ldots \ldots \ldots \ldots \ldots \ldots \ldots \ldots, \quad{ }_{40}$

I small meat board $\ldots \ldots \ldots \ldots \ldots \ldots \ldots \ldots \ldots \ldots, \quad .25$

2 large wooden spoons....................each .I5

I spatula, steel $\ldots \ldots \ldots \ldots \ldots \ldots \ldots \ldots \ldots \ldots \ldots . .50$

I each standiard tablespoon and teaspoon........... .23

I meat chopper, stationary.................. $\quad 1.25$

Preparing Tools (Grouped near sink)

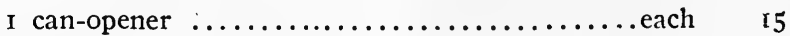

2 vegetable preparing knives................... .20

I curved blade fruit knife.................... $\quad: 5$

I glass lemon squeezer......................... 10

I apple corer $\ldots \ldots \ldots \ldots \ldots \ldots \ldots \ldots \ldots \ldots \ldots \ldots, . .15$

I corkscrew $\ldots \ldots \ldots \ldots \ldots \ldots \ldots \ldots \ldots \ldots \ldots, 10$ 
2 vegetable scrub brushes...............each $\quad 05$

I pair of scissors...................... .45

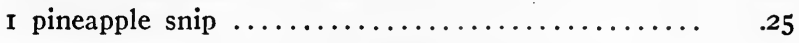

Cooking and Serving Utensils (Grouped near stove)

1 skimmer, enamel ..........................

I small deep skillet, 8 -in. spread.................

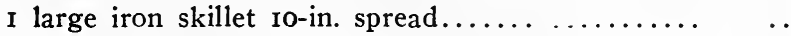

I each tea and coffee pot wîh supply jars............

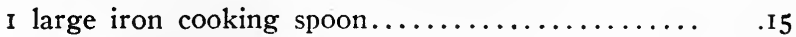

I long-handled cooking fork................ I5

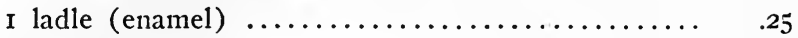

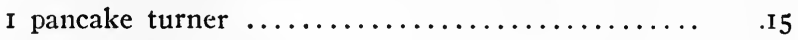

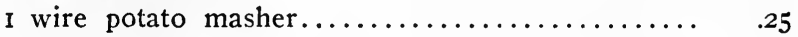

I 3 -mesh sieve or colander.............. 75

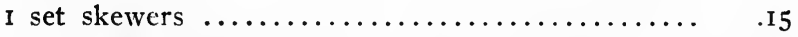

I tea kettle with boiler insert, 5 qts. (alıminum)... 5.00

I tea kettle without boiler insert, 5 qts. (enamel ware) 2.00

Cooking Utensils (Grouped near kitchen cabinet)

Alumi-

Agate num

2 hemispherical 6-hole gem pans........each $\$ .50 \quad \$ .75$

3 bread pans, $9 \times 6 \times 3 \ldots \ldots \ldots \ldots \ldots \ldots \ldots .40 \quad .70$

2 layer cake tins, square or round......... .30 .45

2 pie tins, Io inches, I deep, I shallow....... . .30 $\quad .35$

I deep earthen pie plate................... .25

I china enamel jelly mould, $I \frac{1}{2}$ pts. to I $q t . . .50 \quad .50 \quad$..

I iron baking pan, $12 \times 16 \ldots \ldots \ldots \ldots \ldots \ldots .45 .4$

I earthen baking dish, I $1 / 2$ qts., 9-inch spread. . . 30 $\quad$.. (pudding, scalloped dishes etc.)

I large earthen casserole, 3 qts., stews, soups.. $\quad .50$

6 earthen custard cups.................. .05

I small covered roaster, I5 X II .......... $3.25 \quad 4.50$

Cooking Utensils (Grouped near sink)

I handled saucepan, I $1 / 2$ qts.-cream gravies, boiling eggs, etc................... .60

I handled saucepan, 3 qts.-cocoa, warming milk, heating canned goods. 
2 4-qt. saucepans-potatoes, vegetables, cereals $\quad .85$

I 8-qt. saucepan-spinach, ham, corn, etc.... .70 $\quad .65$ Miscellaneous Equipment and Suggested Grouping

I clock ............................... I.00

I covered garbage pail (near sink) $\ldots \ldots \ldots \ldots \ldots \ldots, \quad .45$

I wire rubbish burner (near sink) ............ I. 25

I match-box (near stove) ..................... .10

2 oval flannel pot-holders (near stove)........each $\quad 05$

I cooking thermometer (near stove)........... I.oo

I handled asbestos mat (near stove)............ . Io

Kitchen salt and pepper (near stove).......... .I0

I toaster (near stove) .................... $\quad .25$

I pan-hanging kitchen scale (near cabinet)....... 2.50 Glass cereal jars, spice jars (near cabinet)...... ..

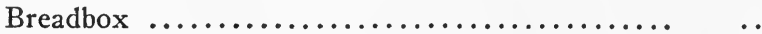

Card recipe cabinet, bill-hook (near cabinet).... ..

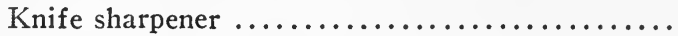
Coffee mill

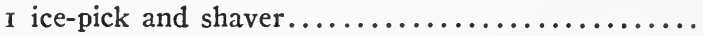

I enameled egg holder, glass butter jar.......each

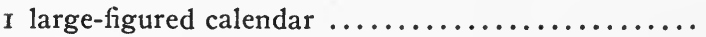

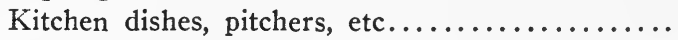
White enameled plates and dishes exclusively for

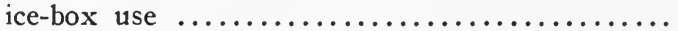

I high stool on castors................... I.50

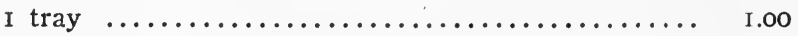

Dishwashing Equipment (Grouped near sink)

I square dishpan on feet.................. I.50

I wire dish drainer................. \$ 50 to 1.25

I large, I small dishmop.................each .05

I wire pot-brush $\ldots \ldots \ldots \ldots \ldots \ldots \ldots \ldots \ldots \ldots \ldots$.I

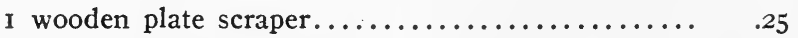

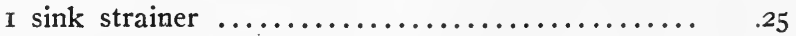

I soap-shaker $\ldots \ldots \ldots \ldots \ldots \ldots \ldots \ldots \ldots \ldots \ldots \ldots \ldots$.I

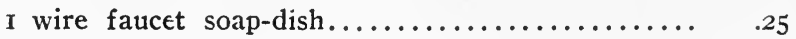

I sink-brush and scoop....................... .I

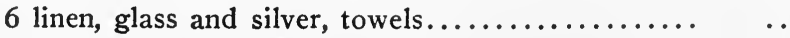

2 mesh pot-rags for wiping pots and surfaces..each .I5

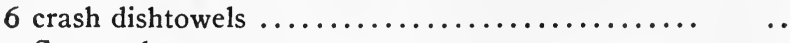

Soaps, cleansers, etc..................... 
Other pieces of equipment might be the portable steamer, bread-mixer, cake-mixer, broiler, if not in connection with the range, pastry outfit, bread-slicer, cleaver, saw, cherrystoner, etc., which would find a useful place in some family, but scarcely be needed in others. Equipment must be chosen having the needs of the particular family in mind rather than blindly following a set list.

\section{Business Equipment}

One more group of equipment must be mentioned in connection with every kitchen, and that is the more businesslike helps, of which there are several. The card cabinet filing cookbook which will be described in detail later must have a place on the shelf above the preparing surface. This makes for neatness, accuracy and ease in following recipes. A drawer containing the cards can be attached to a shelf at about the level of the eyes with a square of glass protecting the cards as used. A bill-hook will keep sales checks and other memoranda until wanted. A large envelope should be used to contain the direction tags which come with many pieces of equipment and which are so necessary to turn to from time to time. If the calendar is large and distinct enough, the daily deliveries of milk, ice or bread or other memos can be placed in the square around each date. A kitchen reminder list of some kind, and a separate pad with pencil attached to string, on which to write daily menus, is invaluable. A small "bulletin-board," possibly a slate, will also be found helpful to outline work, suggestions or reminders.

\section{Glass Containers and Labels}

It is preferable to have uniform glass jars to contain cereals, spices and other dry food supplies like powdered sugar, beans, cornstarch, etc. Glass makes the best con- 
tainer because then it is possible to see always the exact amount on hand. All containers should be air and moisture proof and have lids or stoppers that are easy to adjust. Quart, pint and half-pint jars come in various shapes, some with excellent sliding metal tops, which permit easy opening. The square glass containers used in pharmacies with solid glass plug stopper are equally excellent for coffee, tea, rice, etc. All containers and all shelves should be plainly labeled, and one can obtain attractive labels, square or oval, with gummed backs in all sizes for every need. The kitchen is now following the laboratory in its sanitary, systematic storing of supplies in glass with plainly marked labels.

\section{QUESTIONS ON HOUSEHOLD ENGINEERING I}

\section{The Labor Saving Kitchen}

I. Draw a diagram of your kitchen, pantries, etc., showing position of stove, sink, refrigerator, work table, shelves, doors, windows, etc. Let one-fourth inch in the plan equal one foot in the kitchen.

2. Draw a duplicate sketch rearranging this kitchen so far as possible in accordance with the suggestions of the text.

3. Show the "routing" on these two plans (A) for preparing and (B) for clearing away a meal. Estimate the distance and number of steps saved by the second arrangement.

4. Get estimates from carpenters, plumbers, decorators, etc., of how much the various changes and improvements would cost. Give the order in which you would like to have these changes made.

5. Tell what you have already accomplished in the better grouping of small equipment and supplies; also of any other suggestions you have carried out.

Nore. Those taking the correspondence course will be supplied with a Report Blank having cross section lines, which are of assistance in drawing the sketches. 


\section{HOUSEHOLD ENGINEERING \\ II}



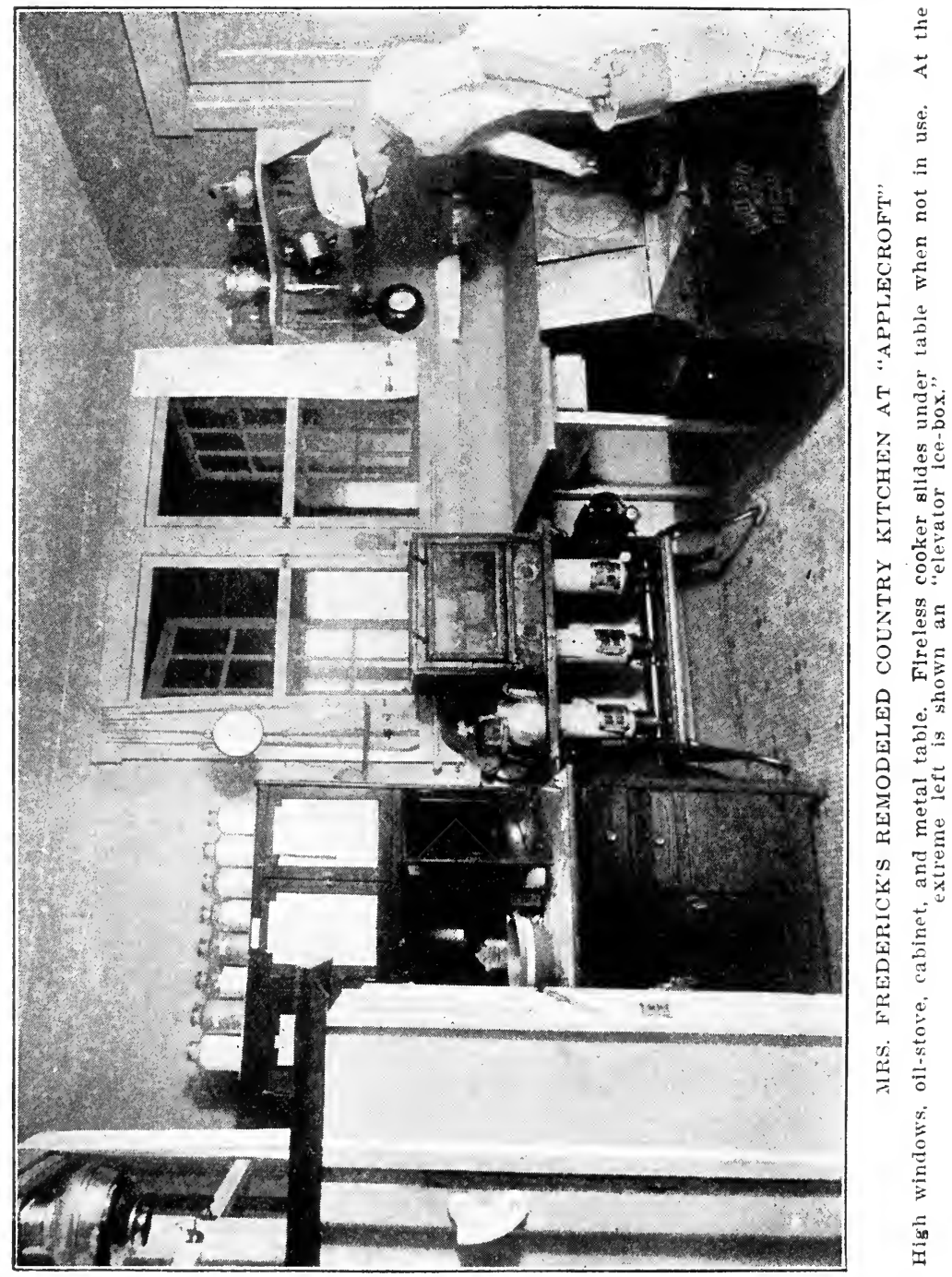


\section{HOUSEHOLD ENGINEERING}

II

\section{PLANS AND METHODS FOR DAILY HOUSEWORK}

ONDITIONS in no two homes are exactly alike; in one the family may number six or more; in another be only three. The location, whether city apartment, . detached suburban house, or isolated country farm, also greatly affects the kind and extent of the housework. The house construction itself either increases or lessens the amount of work to be done. The hours of meals; whether or not there are children or invalids in the family; all these factors have a bearing upon the plans and methods of daily housework.

Letters by the hundred come to my desk, all bearing a similar plaint that women like housework, are fond of some special branch like cooking or sewing, but that they do not seem to be able to "get done" and have any time to themselves. In other words, the woman with the small family and the woman with the large family have the same problem-not how to do any special task, but how to plan and work out a schedule of all tasks; how to relate work and apportion it so that it shall progress smoothly with as little interruption as possible.

"My work is so different every day, and there are so many separate kinds of tasks that I don't see how it is possible to 
make a definite plan of daily work, or a 'schedule,' as you call it," some women have said.

But it is just because there are different tasks that a schedule is needed. If a woman were doing nothing but the same thing without interruption from morning until night, there would be no use for a plan of work. There is only need of a plan when there are several pieces of varying work to be done at different hours with different tools. Then it becomes essential to arrange these varying tasks in order and on time, so that the worker may proceed with the least amount of friction and effort.

\section{Daily Tasks in All Homes}

While it may appear that conditions vary greatly in any two homes, when we compare all the tasks done daily, we see that no matter how large or small the home, or what the number in the family, ets., the tasks themselves remain constant.

\section{DAILY TASKS}

Cooking and serving of 3 meals a day

Dish and pot-washing

Bed-making and bedroom care Light cleaning of living-room, stairs, hall, kitchen, bath and porch
WeEkly OR Special Tasks

Laundry-washing and ironing Mending or sewing Thorough cleaning of house Window, silver or metal cleaning Special cooking or baking

Refrigerator, pantry or closet cleaning

Marketing and ordering of supplies

Every schedule or work-plan has two objects :

(I) The order of work.

(2) The time of work.

The order of 'work is by far the most important, and the thing that must be determined first. The reason for so much "nerves" and useless effort is solely to be found in the lack of order in the work-plan. The time at which a particu- 
lar task is done is secondary and can be decided only after the order is arranged and provided for.

\section{Planning the Daily Schedule}

The first thing to do in making a schedule is to follow the principle which other executives follow, namely: use the head first, and with pencil and paper write down the few absolute conditions around which the schedule must center. For instance, the first facts to be set down would be the hours of meals, as these must be definite, and on them depend the cooking and some of the other work. Next, write down the order of the regular daily tasks in the way you think they will go best in your particular home; whether, for example, it will be better to wash all the breakfast dishes, straighten the kitchen, and start some cooking for lunch, before going upstairs to make the beds; or whether to merely put away food and scrape the dishes, proceed to making the beds, doing light cleaning, and return to start lunch later, doing breakfast and lunch dishes together. What is the best order only the individual worker can determine for her individual case. By watching yourself at work, by counting how long one plan of work takes versus a second plan, and which of the two seems to save the most interruption, most trotting, the best plan can finally be worked out.

In making out the daily schedule, the schedule of weekly or special tasks must be considered at the same time, because some of the special tasks are done each day. For instance, in planning both the cooking and cleaning of Monday or Tuesday, we must consider whether or not the laundry is to be done on either of these days. Again, in planning the daily schedule for Friday or Saturday, we shall have to take into account the special thorough cleaning of the house, special cooking, etc. In other words, there is no such thing as a true 
daily schedule, but rather a schedule of every day, since the entire week's tasks must be considered at once.

Purpose of the Schedule

Much of the confusion of unscheduled work arises because too many things are crowded into the one day, while other days have too little. The schedule aims to prevent just this unevenness in work. It tries to consider all the tasks, daily and weekly, and then group and arrange them in such a way as to have the work evenly distributed over the entire week. Very often work is so poorly planned that suddenly a woman finds it "all piled up" and herself facing the task of an excessive load of work at one time. Some women, by temperament, like to work "by spurts," but it has been found that the smoothest housework is that which has its definite task done regularly so that there never are periods of overloading.

Without a schedule it too frequently happens that the worker allows an unexpected piece of work to interrupt and confuse her entire day. For instance, during her morning's work a woman might be just ready to leave the kitchen and go upstairs to make the beds. But she suddenly sees that the breadbox is surprisingly full of stale and even mouldy bread. She stops to give the box a thorough scalding. She notices then that the entire pantry seems unusually disorderly. A "spasm" of cleaning fever seizes her, and she decides that the pantry right then and there needs a thorough cleaning. One thing leads to another, and before she knows it the entire morning is given over to this unexpected task. When she notices the clock she sees that the cleaning and bedmaking have been neglected and lunch preparation entirely forgotten. It takes her the whole day "to catch up." The schedule way would have provided for a special pantry cleaning on some definite time, and never allowed the routine to be so interrupted. 
So many women have said, “Oh, I couldn't bear to do my housework like factory work. I want to rest when I want to, and to do things as I feel like it." Let it be understood that a schedule is not a treadmill, and does not mean perpetual work without rest. On the contrary, every schedule must contain a definite "rest" period. The worst opponents of the schedule plan are those women who insist on working "till they drop." Furthermore, the schedule plan is the only one which forces regular rest or recreation periods. Its whole idea is simply, plan what you are going to do, do it, and then rest; instead of not knowing what you are going to do, resting or stopping when you feel like it, and never knowing when you are going to get done. In a certain factory in Massachusetts girls test the delicate parts of ballbearings. The work is so trying that every two hours they are forced to stop for ten minutes in which they can talk, leave work, or do what they like. In another immense organization employing thousands of clerks, fifteen minutes is given during the forenoon as an intermission. Nurses and workers in many other lines have definite "time off." But only by assigning definite hours for work, can you also assign definite hours of rest. For the homemaker, the schedule should provide a short "rest period" in the forenoon, and a longer one in the afternoon.

\section{Examples of Schedules}

While, as was said, it is not possible to give one type schedule that will apply to any and all conditions, here is a work schedule carefully planned for a week for a woman who does all her own work in a family of 5 , the 3 children going to school (but coming home at noon); husband's shirts being sent to laundry. The house is a detached, 7 -room suburban cottage; the fuel used gas, with coal water heater. 
ONE WEEK SCHEDULE FOR FAMILY OF FIVE Without Labor-Saving Equipment

MoNDAY

$6: 00-6: 30$ Rise and dress; start water heater

6:30- 7:00 Prepare breakfast

$7: 00-7: 30$ BREAKFAST

7:30- 8:30 Wash dishes; straightẹn kitchen; inspect icebox; plan meals for Monday and Tuesday

8:30- 9:00 Prepare towards lunch

9:00-10:00 Bedrooms, bath and hall cleaned; sort and prepare soiled linen and laundry

I0:00-I I :00 Thorough downstairs cleaning

II :00-I I :30 Rest period

I 1 :30-12:00 Serve lunch

I2:00- I :00 LUNCH

I :00- 3:00 Lunch dishes; prepare cooking for Monday and Tuesday; mop kitchen

3:00- 4:00 Sewing and mending

$4: 00-4: 30$ Soak clothes and prepare for next day's washing

4:30- 5:30 Rest period; play with children; walk, recreation or market

5:30- 6:00 Prepare supper

$6: 00-7: 00$ SUPPER

$7: 00-7: 30$ Wash dishes

\section{TUESDAY}

$6: 00-6: 30$ Rise and dress; put on boiler

6:30- 7:00 Prepare breakfast

$7: 00-7: 30$ BREAKFAST

7:30- 8:00 Stack dishes; make beds

8:00-11:30 Washing

I $1: 30-12: 00$ Rest period

I2:00- I :00 LUNCH (prepared day before)

I :00- $2: 30$ Wash breakfast and lunch dishes; clear up laundry

$2: 30-4: 00$ Take in clothes; fold, sprinkle, lay away

4:00- 5:30 Rest period

5:30- 6:00 Prepare supper

6:00- 7:00 SUPPER

$7: 00-7: 30$ Wash dishes 


\section{WEDNESDAY}

6:00- 6:30 Rise and dress; start water heater

$6: 30-7: 00$ Prepare breakfast

$7: 00-7: 30$ BREAKFAST

7:30- 8:30 Wash dishes; inspect icebox; plan meals; start iunch

8:30- 9:00 Make beds; light cleaning

9:00-12:00 Ironing

I2:00- I :O0 LUNCH

I :00- $2: 00$ Finish ironing; put away clothes

$2: 00-3: 00$ Wash dishes; straighten kitchen

3:00- 4:00 Rest period

$4: 00-5: 00$ Market; walk

5:30- 6:00 Prepare supper

6:00- 7:00 SUPPER

$7: 00-7: 30$ Wash dishes

THURSDAY

6:00- 6:30 Rise and dress; start water heater

6:30- 7:00 Prepare breakfast

$7: 00-7: 30$ BREAKFAST

7:30- 8:30 Wash dishes; straighten kitchen; plan meals

8:30- 9:00 Make beds

9:00-I I :30 Bedrooms and closets cleaned

II :30-I2:00 Rest period

I2:00- I :OO LUNCH

I :00- 2:00 Wash dishes; prepare vegetables toward supper

2:00- 3:30 Upstairs windows cleaned (Up and down stairs windows alternately each week)

3:30- 4:00 Silver polished

4:00- 5:30 Rest period

5:30- 6:00 Prepare supper

$6: 00-7: 00$ SUPPER

$7: 00-7: 30$ Wash dishes

FrIDAY

$6: 00-6: 30$ Rise and dress; start heater

6:30- 7:00 Prepare breakfast

7:00- 7:30 BREAKFAST

7:30- 8:30 Wash dishes; straighten kitchen; plan meals 


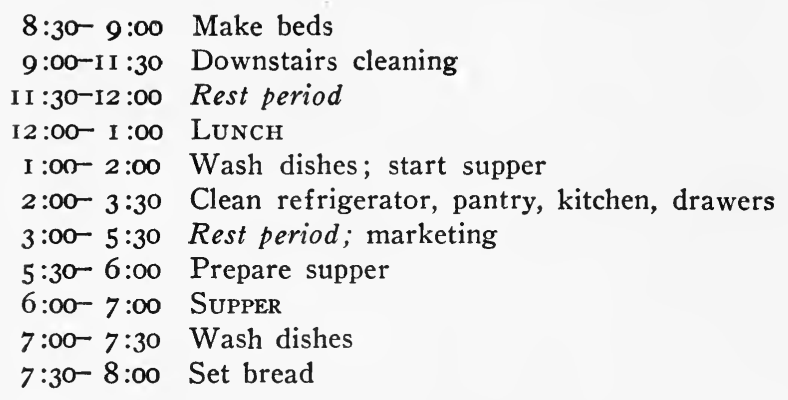

Saturday

$6: 00-6: 30$ Rise and dress; start heater

6:30- 7:00 Prepare breakfast

$7: 00-7: 30$ BREAKFAST

$7: 30-8: 00$ Make beds

8:00- 8:30 Wash dishes

$8: 30-$ I I :30 Special cooking, and baking

I I :30-12:00 Rest period

I $2: 00-$ I :OO LUNCH

I :00- $2: 00$ Wash dishes; start supper

$2: 00-3: 30$ Clean stove; wipe kitchen and porch

$3: 30-5: 30$ Rest period

5:30- 6:00 Prepare supper

$6: 00-7: 00$ SUPPER

$7: 00-7: 30$ Wash dishes

(Good hot dinner Saturday night; light or cold meals Sunday)

From the foregoing schedule it will be seen that both daily and weekly special tasks are provided for; that there is a definite rest period every afternoon and generally in the morning. It will also be noticed that there are certain units or groups of work done together. For instance, the period when the lunch dishes are washed is used for vegetable preparation so that vegetables and sometimes dessert for the night meal can be cooking and watched while the dishes are washed. This considerably lessens the time necessary 
for supper preparation and allows a longer free period in the afternoon.

It will be seen also that Tuesday and not Monday is given to washing. This plan has several advantages. Sunday visitors generally leave the house in confusion and the woman more tired. Monday washing also means soaking the clothes some time on Sunday, which is not desirable. The Tuesday washing plan allows for a thorough brushing-up of the entire house on Monday, a sorting and soaking clothes, preparing shirts to be sent to laundry, and a double cooking so that there will be practically no cooking on Tuesday.

Marketing is allowed for three times a week, but if it can be done once a week, so much the better. Notice that icebox inspection and meal planning take place immediately after breakfast, the menus then being written down on a kitchen pad. This schedule gives simply order of work without attempting any very special timing of any one task, nor does it consider the work assisted by any labor-savers.

\section{Routing Work Depends on House Construction}

In the above schedule has been shown how one definite task was taken up after another, or, as it is called, "routed." Now, this routing depends somewhat on house construction. In planning the cleaning particularly, the arrangement of the rooms should be studied. By starting work in one room and proceeding to others in a given order, saving of time and steps can be made. The following diagram will show the easier way to clean a given set of rooms and the more step-taking plan which might have been followed if the house construction had not been studied. This is true not only of the work itself but particularly in regard to the handling of utensils and tools. Lack of a definite order of work makes for double or even triple handling, which is altogether unnecessary. 


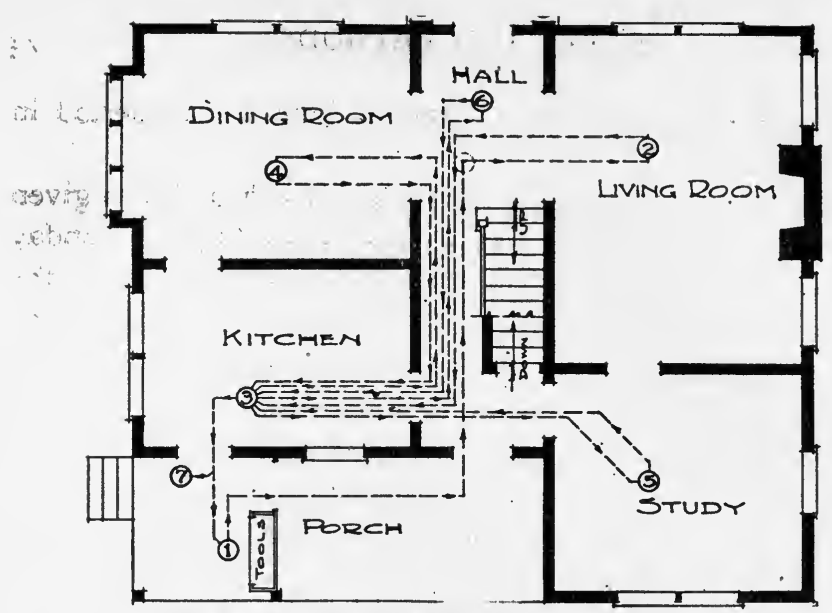

DIAGRAM 1-UNPLANNED CLEANING ORDER

Method.-Worker gets tools from tool closet (1), and walks down hall and begins on living room (2); returns with trash to kitchen (3), and walks to dining room (4); after cleaning it, again returns to kitchen with trash, and proceeds to clean the study (5); she walks back to kitchen again, and last cleans hall (6), ending by bringing back tools and last refuse to kitchen again. before taking the final walk back to tool closet (1). This is not an exaggeration, but the method used by a so-called "good worker."

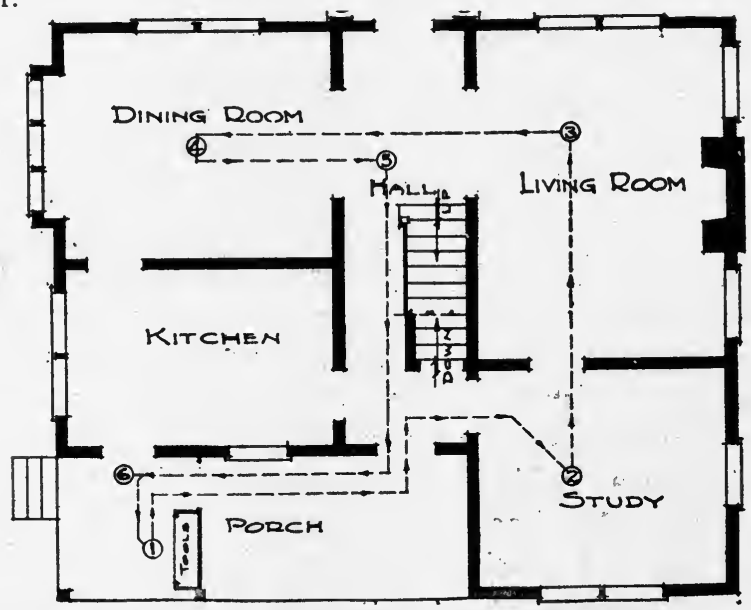

DIAGRAM 2-PLANNED CLEANING ORDER

Method.-Worker gets tools from tool closet (1), and proceeds direct to study (2) ; from study through door to parlor (3); across parlor hallway to dining room (4); she then begins at upper end of hallway (5), and cleans its length back to the door opening on rear porch, carrying all waste and tools back directly to service porch (6). Note that this method eliminates all tracking to kitchen and results in about two-thirds less unnecessary steps and walking. 


\section{Minor Tasks in the Schedule}

As was indicated, laundry to be sent away was gathered and made into a bundle Monday morning. If at the same time the bundles were carried directly near the back door, they would be right at hand when the laundry man came for them. So much unnecessary trotting is due solely to the fact of running upstairs or going after some article which should have been carried directly to the place from which it is finally to be handled. Another minor detail is the emptying of garbage, and cleaning of the pail. If this is not provided for, other work will be interrupted by this unpleasant task. In general, it seems best to empty it after the lunch dishes and vegetable preparing for night have been done, washing the pail and setting to air until night.

Similarly with lamps if their cleaning and care must be included in the work schedule. This is a definite daily task and should be fitted in and not left as an afterthought or when this unpleasant piece of work might interrupt other clean tasks.

Again, a very small task which often causes much unnecessary dirt is the emptying of waste baskets. So frequently the wrong plan is followed of carrying baskets, tools, mops, etc., and dumping them into the kitchen when their ultimate destination is somewhere else. Again and again, it has been noticed that on a general cleaning day waste, trash, cleaning tools have all been brought to the kitchen when they might just as well have been taken to their rightful closet or rubbish box without tracking through the kitchen at all. Making the kitchen a general dumping ground means that additional work and handling must be done in the kitchen, thus wasting time and effort.

Another way in which many minutes are wasted is because of a lack of definite understanding in regard to tradesmen 
and deliveries. For instance, it is most inefficient to have the iceman call every morning inquiring whether you need ice, if you need ice only three times a week. Make a point of looking at the ice supply at a definite morning hour, and hanging out a card so that the iceman can deliver without interrupting you at all. Plan definitely when you want tradespeople to call and insist that they do not call at other times. The special shelf near the kitchen or rear door on which supplies may be laid has been spoken of. Give orders that bundles, articles, etc., shall be left here with as little interruption as possible. Keep a supply of change in the kitchen. Running upstairs to a pocketbook, even once a day every day in the week, runs into enough time in a year to read several best sellers!

\section{Special Schedules}

The type schedule given above will not fit every family. There is the family with younger children or babies. Their care will have to be provided for, and the special baby washing, naps, airing, etc. Such a schedule for one day might work out as follows:

\section{ONE-DAY SCHEDULE FOR FAMILY OF THREE}

(One small baby on 3 -hour feeding period; small house or flat)

6:30- 7:00 Rise and dress; give baby morning feeding

7:00- 7:30 Breakfast. (Uncooked cereal, or cereal cooked in fireless. Table set night before)

7:30- 8:00 Clear table; stack dishes; plan meals for the day; put on water for baby's bath

8:00- 9:00 Bathe baby, feed and put to sleep; pick up after bath; straighten bedroom

9:00-10:00 Prepare baby's gruel, sterilized milk, etc.; wash baby napkins while watching food

(Baby naps 9:00-11:00)

Io:00-II :0 Clean living-rooms, hall, etc., and dress ready to take baby for morning airing while marketing

I : :00-12:00 Outdoors with baby while marketing 
I2:00- I :00 Return for lunch and baby feeding

I :00- 2:30 Wash combined breakfast and lunch dishes; prepare vegetables, dessert and meat, if possible, for evening meal; brush up kitchen; empty garbage; sweep porch (Baby awake and playing outdoors, if possible, from I-3; at 3 o'clock feeding and sleep until 4)

2:30- 3:00 Iron baby clothing

3:00- 4:00 Rest period; preferably nap with baby while it is asleep $4: 00-5: 00$ Afternoon airing

$5: 00-5: 30$ Start supper

5:30- 6:00 Put baby to sleep with night feeding

6:00- 7:30 Own supper; supper dishes washed; table set for breakfast following morning (Baby's last feeding io P. M.)

Similarly, the family with an invalid or where meals are taken at very irregular hours, as by many professional men; or the many country conditions where the care of stock, garden, etc., must be included. In each case, the schedule must be built to meet and fit the essential needs of the particular family. It must always be kept in mind that the schedule in itself is worthless; and that it is useful only as a means to an end-that the schedule must fit the family, and not that the family be made uncomfortable or be moulded over to fit an iron-clad housework plan.

\section{Standardizing and Timing Special Tasks}

We have spoken of the order of work and its importance as the backbone on which the development of the schedule rests. We now want to think about the timing of various tasks in order that we can arrange a more closely-knit, exact schedule. Every task represents a number of motions and effort, and hence time. On study we find that there is one best, shortest way of doing a task under a given set of circumstances. Finding out this best, shortest way and reducing it as nearly as possible to a habit is called "standardizing" it. 
To standardize any task we must study how we do it and then see if we cannot improve and shorten this former time of work. Bed-making, dishwashing, cleaning, especially, are purely routine pieces of work and can easily be standardized. Let us take dishwashing.

\section{Time Studies of Dishwashing}

When we say "dishwashing," we commonly think of a single household task. But when closely analyzed and made the subject of a time or motion study, we see that it is composed of several parts or steps, each with different motions, and generally performed with different tools, as follows:

(I) Scraping waste from surface of china, agate or other kind of dish or utensil.

(2) Stacking or arranging dishes on surface adjacent to sink, preparatory to washing.

(3) Actual washing with water, soap or other cleanser, with aid of cloth, mop or other mechanical means.

(4) Rinsing dishes with clear water.

(5) Wiping dishes with towel or equivalent drying.

(6) Laying away dishes on or in respective shelves and cupboards.

The efficiency of the whole process of "dishwashing" can be improved only by increasing the efficiency of each step.

From careful experiments made with dishwashing over a period of two months and analysis of each of the six steps in the dishwashing process, the following results were obtained :

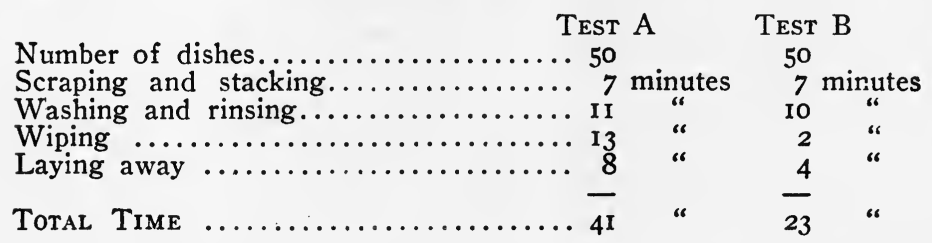


In both tests the number of dishes washed was the same. But in Test B the conditions were changed. First, a wire drainer was substituted for a tray which entirely eliminated the hand wiping; then shelves for dishes were placed adjacent to the sink instead of in a pantry $\mathrm{r} 8$ feet distant, which considerably reduced the time of the laying-away step, and thus reduced the total process from 43 to 23 minutes, or nearly one-half the time.

From many time-studies similar to the above, the following general conclusions were reached:

(I) The height of the sink must be adjusted to the height of the worker, and be sufficiently high so that she can work without the slightest stooping.

(2) The depth of the sink must be such as to allow ample accommodation for the dishpan with sufficient surface above to prevent sloshing of water over the edge and on person of worker.

(3) Stack surface must be to right of sink, drain surface to left for right-handed worker. This permits easy and rapid laying-down motion of each dish without awkward crossing of left arm over right.

(4) Thorough scraping facilities the actual washing.

(5) Wire drainer keeping each dish separate is more efficient than tray or other flat surface which does not allow quick drying.

(6) Similar shaped pieces can be washed with much greater rapidity per number than can poorly assorted ones; hence the need of stacking similar dishes in groups. 
(7) Sitting down at dishwashing does not lessen the actual time, but does greatly lessen the fatigue.

(8) Scalding dishes in drainer more sanitary and less time-taking than hand wiping.

(9) If shelves and closets for dishes and utensils are grouped near left of sink or in same relative position, the time saved in the laying away step will be considerable over that in which distant pantries are used, entailing several trips with trays, etc.

(I0) These generalizations cover washing of pots, silver and glass as well, except that all in these last three classes must be dried by hand.

This shows the method of analyzing and standardizing any particular task. The standardization includes close observation of the way the worker uses her hands, the tool and its conditions and particularly the preparatory and following steps of any given piece of work. For instance, many a woman might, by timing herself, find that she made a cake on a Io-minute schedule, which is rapid work. But if she counted up the time she spent gathering her eggs, butter, milk, etc., together, and the time she spent "clearing up" and putting her materials and pans away, she would find that the total time was not ro minutes, but possibly 25 minutes. In this case, standardization of her work would involve a more step-saving kitchen arrangement, and better grouping of tools.

Every task consists not of one single set of motions, but of a number of sets or processes linked together, as was shown above in dishwashing. The only way in which the whole process can be improved and shortened is by studying 
each one of these steps, improving and lessening the time it takes, which will thus lessen the time of the whole task.

While cooking will be discussed in a later chapter in greater detail, it may be mentioned here that every cooking task consists of these three parts:

(I) Getting materials ready for work.

(2) Actual cooking proper.

(3) Clearing-up; replacing materials and utensils.

As was pointed out, time is lost not in point 2 , but in points $I$ and 3 , and these conditions must be improved and time shortened here before we can shorten the entire time of cooking.

\section{Standardizing Cleaning}

In considering cleaning also, we find that it is not a single act, but composed of many complex processes, as Sweeping, Wiping, Dusting, Polishing, etc. Cleaning the average room includes several or all of these processes. Again, each of them is done with a separate tool.

Now, we find that much time is lost by needless handling of cleaning tools. By carefully scheduling the order of cleaning a number of rooms, less frequent handling of utensils will be necessary. The time of cleaning a bedroom daily may be cut down from 20 to Io minutes by repeating each day the definite cleaning order or schedule decided on.

\section{Time Studies of Various Tasks}

Following are a few time-studies of common tasks. It must be remembered, however, that this "time" will not apply in every case. They are only the result of work done under one given set of conditions. In your home they might be widely different, owing to the different conditions surrounding your work, or the different tools used. The amount of furniture in each room, the size of the room, etc., 
will affect a time-study of room-cleaning; or even the window washing will vary with the dirt on the windows, and the tools and method used. Because you cannot do a similar piece of work in the same time does not mean that you are not a good worker-these figures are given only to show you how you can work out your own time-studies, and use them as a basis of a schedule in your own home:

Making double bed, approximately.............. 5 minutes

Making single bed, approximately.............. 3 “

Brushing up bedroom I4xi6, approximately........ I2 “

Daily care of bathroom, approximately........... I0 "

I. Washing 50 dishes and 50 silver (entire process) by

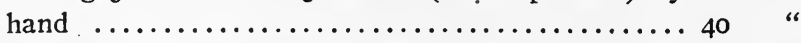

2. Washing 50 dishes and 50 silver by hand, but under standardized conditions $\ldots \ldots \ldots \ldots \ldots \ldots \ldots . \ldots 23$

I. Setting table for night meal, family of six (trips with trays $) \ldots \ldots \ldots \ldots \ldots \ldots \ldots \ldots \ldots \ldots \ldots$. 13 "

2. Setting table for night meal, family of six (tray on wheels)

Washing average size 3 -foot window, in and out.... I2

Washing 8 -light pane window............... 16

I. Mopping kitchen (IOxI2) on hands and knees..... 20

2. Mopping kitchen (IOxI2) with improved mop...... I4

I. Breadmaking, by hand, 4 loaves, including cleaning

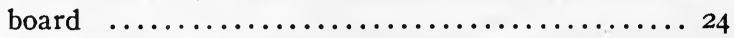

2. Breadmaking with mixer, 4 loaves, including cleaning board $\ldots \ldots \ldots \ldots \ldots \ldots \ldots \ldots \ldots \ldots \ldots \ldots \ldots \ldots$ 16

Many women still persist in thinking that by timing themselves they are holding a kind of whip of drudgery over themselves. On the contrary, no one factor makes a piece of work more interesting than that of timing it, and if possible, lessening this time in future work. Instead of making a task drudgery, the timing acts as a stimulus to do the work more efficiently and "beat the record" of a previous effort. Women who have tried the timing plan say it makes the work more fun to do it with eyes on the clock in a 
determination to see in just how little time they can accomplish it and yet do good work without "hurry."

The more closely the worker can figure the time it takes for any given task, the more carefully can she arrange the housework schedule: She may, for instance have decided this to be the order of the morning's work:

(I) Prepare and serve breakfast.

(2) Scrape dishes; lay away food.

(3) Make beds; brush up bedroom.

(4) Brush up living rooms.

(5) Lunch preparation.

(6) Rest period.

But, by timing herself at these various pieces of work, she will be able to add definite figures opposite, as,

Preparing and serving breakfast.... 6:30- 7

Scrape dishes and lay away food... 7:30- 8:15

Make beds; brush up bedrooms.... 8:15- 9

Brush up living rooms......... 9 $-9: 45$

Lunch preparation ........... 9:45-10:45

Rest period.............. I0:45-I I :45

By working out a still closer time schedule she might be able to also include in the morning other work like special cleaning, cooking, sewing, or her marketing. The more closely work is timed the more nearly perfect the schedule will be. This timing will not be a handicap, but make the work more automatic so that it requires less nervous attention.

\section{Advantages of a Schedule}

Sometimes women criticize the idea of the schedule, saying that it is impossible under home conditions of children, sickness, etc., to run the housework train on schedule time, and that it is not practical to think that at a given hour, say $8: 15$, 
the beds are going to be made, day in and day out. The answer is that we do not make any rule or helpful plan based upon exceptions. The fact that even the best "limited" express has to stop unexpectedly for accidents or because other trains are not living up to their schedule, does not interfere with the careful working out of a schedule under ordinary, normal circumstances. Because once in a week we are suddenly called from home, or because the baby is suddenly taken ill with croup and all schedules have to go by the board, is no sufficient reason why there should not be a schedule for the many regular days on which there are no unexpected interruptions, and which, after all, form the average day's work.

Even if the exact time plan for a certain task cannot be followed, the order can usually be followed and the entire schedule either swung later or earlier in the day, or the less vital work omitted entirely. For instance, if we have a carefully planned schedule which starts with scraping dishes at 7:30, but unexpectedly receive an important telephone call which takes us out of the house for an hour, we merely shift the schedule an hour ahead to 8:30 and begin later, cutting down the least important morning's work, but still following the regular order. It is just in emergencies that the value of the schedule is most fully felt; with it we have a guiding plan of work under normal circumstances. Under the abnormal circumstances, it does not permit us to become flustered and completely upset.

Again, the schedule enables us to have a better "grip on ourselves." Hundreds of women write in that they don't know where they are at; that they can't get ahead of the work, or never find time for themselves. Now, an attempt, at least, to work out and follow a household schedule gives a "grip" on one's self that is most helpful and encouraging. It makes you know what must be done and sets the problem 
before you of a certain given number of hours in which to do it. If you can plan, can arrange and master this situation, then you feel as proud and as confident as other workers in business or other fields who likewise have the assurance that comes from working under schedule conditions.

I have seen women in food factories fill thousands of bottles of mustard per day, and girls bind books, or stamp and label cartons. In all these cases, a very great amount of work was demanded, but while the workers were tired at the end of the day, they uniformly said that there was little nervous strain because they knew what they could do in a day and when it was to be done and when they were to be through. In other words, standardized work anywhere relaxes the nervous strain and gives a worker a feeling of mastery that working hit-and-miss never permits.

\section{Schedules and Servants}

While a schedule of household work most certainly helps the woman who does all of her own housework, it is just as important and necessary in a home where one or more servants are employed, or where a worker comes in for the day, as cleaning woman, laundress, etc. One of the most frequent and strongest reasons alleged by servants themselves as to why housework is not a desirable occupation is that they too "never have time to themselves," and do not work under any standard conditions. In large establishments where there are many workers this point is usually much better handled than in the small home with one general maid-of-all-work. It is actually much easier to secure help for a large establishment where there are definite assigned duties and definite work hours than it is to secure a worker for "general housework" in homes where the mistress either does not know or has not taken the trouble to schedule out the work for the one maid. 
Much friction can be avoided if the mistress will either alone or in co-operation with the worker, work out a daily and weekly schedule. How many times we hear a mistress remark, "I wonder what Katy is doing now ?" and there is a feeling that Katy is shirking her work or taking unnecessary time for herself. Again, Katy never knows what her definite rest period or "time off" will be, and the result is unsatisfaction on both sides.

There is no difference between planning a schedule for a worker and planning a schedule for one's self. All the tasks that must be done daily and weekly should be written down and arranged in a tentative order. Some definite afternoon hour or hour and a half should be allowed for the worker in which to care for herself and do as she likes. It should also take into account the special holidays or afternoons off previously arranged with her at the time of engaging her. Good workers much prefer to work under schedule conditions and appreciate the fairness of such an arrangement, which will prevent argument as to why such and such a piece of work has been neglected. The schedule enables the worker to "know where she is at," and will prevent too much work being crowded into any one day. How can a mistress expect a smooth-running household and workers to give good service if the workers are left to follow out a hit-and-miss plan, to do work as they feel like it, or to be blamed for something which was overlooked, when the mistress herself never gave definite scheduled orders that this work should be done?

Both a daily and weekly schedule can be written down for the hired worker. It should include the smallest details, as on what days tradesmen come, when to have laundry bundle ready, the exact hours at which meals are expected to be served, when special tasks like silver, window washing, pantry overhauling, etc., are to be done. Besides, a simple 
daily schedule including the best order of bedmaking, brushing up, meal preparation, etc., should be written down and both these schedules hung in the kitchen or written down in a little booklet and given to the worker when she first comes.

While such a schedule will vary in every household, the following points should be covered in a schedule made for the worker :

(I) Hour of rising for worker.

(2) Hour of meals.

(3) Hour of consultation with mistress regarding food supplies and meals.

(4) Daily work routine.

(5) Days on which special work is done.

(6) Definite rest period each afternoon.

(7) Definite arrangements as to holidays or off time.

In connection with this schedule, explicit directions can be given as to where tools are kept, how to use and care for them, and minor details of the way work is preferred to be done in this particular household. These directions will be spoken of in a later chapter, where it will be shown how a mistress can make a "practice book" which will be invaluable to put in the hands of a new worker and which will assist in "training" a servant more rapidly and enable the work to be done with far less misunderstanding and friction.

POSSIBLE SCHEDULE DIRECTIONS FOR SERVANT FOR ONE DAY

(Winter; family of adults; city conditions)

6 A. M. Worker rises

Attends to furnace and range

Prepares and serves breakfast 
Inspects supplies with mistress and plans meals, perhaps 'phoning to tradespeople

Dishwashing and kitchen straightening

Daily chamberwork

Brushing up living-rooms

Prepares and serves lunch

Luncheon dishes; supper preparation; kitchen straightening. Special afternoon task of cleaning

Afternoon rest period, generally $3: 30$ to $4: 30$ or $3: 30$ to 5 , in which worker freshens up, changes to afternoon uniform and has time to herself

Prepares and serves supper

Washes dishes and makes slight preparations for breakfast

More specific directions giving the hours and times of these tasks would have to be worked out in each special case, depending on the number in the family, the size of the house, and the hours of meals, etc., and whether some of the work was done by the mistress herself (as daily care of bedrooms), or whether some of the work was done by other hired help, as care of furnace by hired man, or laundry work done by laundress. If there are more than one worker, the schedules should show the duties of each, where one worker takes the place of the other, and other details which will prevent any clash between them.

\section{Relation of Equipment to Schedule}

In developing plans for more standardized housework it will be found that equipment as well as methods affects the schedule. As was shown in the discussion of improved dishwashing methods, every step must be studied in a given task to see where time and effort can be saved by doing it in a better, shorter way. This way may depend also on the kind of equipment used. For instance, a work scheduled in a certain home may be carefully developed and followed where the fuel is coal or gas, but by using a fireless cooker in con- 
nection with these fuels the schedule would be greatly modified, the time at which cooking was done would be altered and the rest period changed. This is shown in the following schedule:

\section{HOW THE "FIRELESS" AFFECTS THE DAILY HOUSEHOLD SCHEDULE}

A. M.

6:00- 6:30 Rise and dress

6:30- 7:15 Prepare breakfast

7:15- 7:45 Breakfast

7:45- 8:30 Wash dishes and clear up kitchen

8:30- 9:30 Make beds; brush upstairs rooms and bath

9:30-10:45 To kitchen to start luncheon. Return to cleaning of downstairs rooms

Io:45-I I :45 To kitchen to watch cooking and prepare other food for lunch

II :45-12:00 Serve lunch

I2:00- I :00 Lunch

P. M.

1 :00- $2: 00$ Wash lunch dishes; mop kitchen; sweep porch

$2: 00-3: 00$ Finish interrupted downstairs cleaning of morning

3:00- 4:00 Special cleaning; windows, or silver, stove or pantry

4:00- 5:30 Prepare roast, vegetables and dessert for dinner and watch their cooking

5:30- 6:00 Dress; serve dinner

\section{With a Fireless Cooker}

\section{A. M. (30 minutes saved)}

6:30 to $7: 00$ Rise and dress

$7: 00-7: 15$ Remove breakfast from fireless

7:15- 7:45 Breakfast

7:45- 8:45 Wash dishes and clear up kitchen; place lunch in fireless

8:45- 9:45 Make beds; brush upstairs rooms and bath

9:45-10:45 Clean downstairs rooms

I0:45-I I :45 Special cleaning; windows, stove, silver or pantry

II : $45-12: 00$ Serve lunch

I2:00- I :00 Lunch 
P. M.

I :00- 2:00 Wash lunch dishes; mop kitchen; sweep porch

$2: 00-2: 30$ Put dinner in fireless

$2: 30-5: 45$ Rest or recreation period

$3 \frac{\mathrm{T}}{4}$ hours saved

5:45- 6:00 Serve dinner from fireless

Again, the use of a washing machine might change the schedule of washday quite differently than if a boiler were used. If a vacuum cleaner is used this might quite considerably alter the amount of time necessary to a daily cleaning with broom. Many of the better pieces of equipment affect the schedule not so much in point of time saving in a single operation as in the number of times or amount of handling the method without the equipment entails. To illustrate; a twice-a-week cleaning with a vacuum cleaner might take the place of an every-day brushing up with the broom. Or the preparation and handling of a boiler, laundry stove, sad. irons, etc., would make a different schedule than if a washing machine were used with an abundant hot water supply from a central hot water heating system.

\section{Country Schedules}

The housekeeper who faces the greatest number of problems seems to be the woman in the country without "conveniences" and whose fuel requires more attention, and whose home is usually larger and home duties more numerous. In addition, the country woman has her chickens, her garden, her canning, perhaps even butter-making, and frequently many more to cook for.

Here is where the schedule meets the severest test and. where also it helps the most. As was said, if a worker has only one or two tasks to do all day long there is scarcely any need for planning, but if her hands and head must see through a dozen, yes more, tasks, then a plan becomes an 
absolute necessity, if she is not going to find herself worked to death, fagged out, with no recreation time. The schedule for the country worker must include all the tasks which fall into her particular hands. It must attempt to divide the whole week's work so that only a fair share is done each day. It too must give the worker time to attend a grange meeting, to read an agricultural bulletin or to merely sit outdoors and enjoy some of her own trees and sunshine.

The meal problem is generally the heaviest, so that the point to begin the schedule with here is careful menu planning and arrangement of cooking so as to simplify as much as possible. The use of a fireless cooker, an oil stove, or a steam cooker, will cut down the cooking time. Also cooking ahead, as is frequently practiced in the country, is the best means of having a lighter afternoon. Simple furnishings and the doing away with unnecessary care-making articles will lessen the cleaning problem, as generally the country woman has less time to spend on cleaning and the upkeep of the house. Careful planning of trips outdoors will save time, bringing in vegetables on returning from feeding poultry, etc. Washing vegetables outdoors or out of the kitchen will prevent much unnecessary cleaning work in the kitchen. Lamps can be carried down on going downstairs in the morning so that special trips will not have to be made to come up and get them.

The arrangement of the kitchen, particularly, affects the country schedule, and every means should be taken that the pocketbook affords to solve the water problem and make the kitchen as convenient a workshop as possible. One case comes to mind of a farmer who had water piped into his barn for his convenience in watering the animals, but who refused to pipe it into the kitchen for his wife, who was thus forced to carry wash water from a distant outside pump. Built-in conveniences like wood-bin, elevator ice-box 
which saves running up and down cellar, ample shelf or dresser room will make a difference of an hour perhaps in the work schedule.

\section{Hoúsework AND OfFICE WORK}

The argument is sometimes advanced that business can be run more on the sechedule plan because there are no interruptions like there are in homes. But a trip through any business office or establishment will show that this is not true. There are visitors who must be interviewed, constant calls on the telephone, demands of stenographers or clerks, letters must be written, merchandise looked over and directions given. Yet the modern man in his work has applied the schedule method with the result that he can handle twice as much business as his father with half the effort.

Today the woman in the home is called upon to be an executive as well as a manual laborer. Just to be a good worker and keep on working until you drop is not sufficient -or efficient either. The more planning, the more brains, the more management, a woman puts into her housework, the less friction and the less nervous energy she will have to expend. Housework above any other must be followed on the schedule plan so that a woman will know what she must do, how long it takes her to do it, and when she can get through and do something else.

Many women everywhere are working schedules out for their own particular conditions. As one little woman, the mother of four babies, said at the close of a lecture: "I never used to know what piece of work I had to do next, but as you said, I sat down and wrote out all the things that had to be done, trying to arrange them in the best order I could. I followed this order for a week, perhaps longer, seeing where I had made a mistake and could arrange something better in its place. It took me about six weeks to 
master the situation, but I did overcome it and have been doing housework the schedule way ever since, and thanks to this plan, I now do just as much work and have, in addition, about an hour and a half a day in which to sew for the babies. If I can't finish everything on schedule owing to interruptions from the babies, etc., I at least have the satisfaction of doing the most important work in an orderly way and knowing where I must catch up later."

No tiny piece of work or task is too small to be left out of the schedule. Indeed, the three-meals-a-day problem, or even the cleaning problem, do not have to be considered and planned for so much as the little task, the ordering, the running back and forth, the right location of tools, the deliveries, the minor details which either make or mar the housekeeping management. There is no excuse for "Oh, I forgot to order more sugar," for making four trips upstairs which could have been taken in one, or of finding that there isn't another egg in the house. Scheduled work can be proved a success no matter what the conditions, the family, or the location. You can make your housework easier and find time for yourself if you will only try to follow the schedule plan. Find out what you must do, write out when you can best do it and try to improve even this plan. Repeat common tasks in the same manner, and if possible at the same time so that they become mechanical and thus take less energy. That is, study dishwashing, or cleaning, or laundry, or any other minor tasks until you know just how you ought to proceed to do it in your particular home. Watch yourself at work and make a "time study" of the time and steps in one method and then in another. When you have found out the method that seems shortest, practice it until it becomes second nature and habitual. Time yourself, just for fun, at first, and you will see the practical value in the end. Try to go your former schedule "one better" and beat your- 
self. You will be repaid in more recreation time and in a grip on your housework that you have never had before.

\section{Actual Form of Schedule}

After finding the plan of work which seems best and after having arranged each task in its approximate time. make a permanent record of it. One way is to take sheets of paper about $6 \times 9$ and on each sheet write the outline for a separate day. Punch a hole at the top, tie loosely together and fasten on a cuphook in the kitchen either over the sink, table or other conspicuous place. Each day turn one of the sheets over to the proper day, as, Tuesday. Another way is to use large filing cards and keep them in a filing cabinet over the kitchen table, substituting a new card in place each morning. A notebook at hand will serve in which to jot down suggestions and improvements which can later be added to the permanent record.

For a servant it is best to write the schedule in a permanent blank-book so that they will not be lost. Special instructions or standards for each specific task can also be included, as, "Standard Practice for Dishwashing," for "Setting the Table," for "Cleaning Rooms," for "Laundry Work," etc. Such a "practice book" will correspond to the "instruction card" given workmen in factories where scientific management prevails. These instructions can include the exact tools to use and approximately how long it takes. This makes for accuracy and avoids misunderstanding.

For instance, a practice card on bread-making to be handed a new worker might be as follows:

\section{Milk and Water Bread}

2 c milk

$2 \mathrm{c}$ water or potato water

$\frac{1}{4} \mathrm{c}$ shortening

$4 \mathrm{t}$ salt (level)
2 t sugar

I yeast cake softened in $\frac{1}{4} \mathrm{c}$ tepid water Flour (about 3 quarts) 
Hear milk and water to boiling, add salt, sugar and shortening. Put into the bread mixer. When cooled to $100^{\circ}$ (luke warm), add the yeast which has been soaking in tepid water. Add half the flour to make a soft batter and stir vigorously. Add remainder of flour to make a stiff dough and stir till springy.

Let rise in a warm place until double the bulk (about $2 \mathrm{~h}$ ), then stir down, take out of mixer, form into loaves, let rise in the baking pans until double the bulk (about $2 \mathrm{~h}$ ) and bake about I hour. Keep the dough warm throughout, $80^{\circ}-90^{\circ}$.

Wednesdays and Saturdays-baking days.

Make 4 loaves, three plain, one with raisins, and pan of hot biscuit

Use breadmixer and agate measuring utensils

Preparation time required, about 8 minutes-soak mixer and scrape board as soon as work is finished

In a similar way, "practice instructions" can be given on each of the cleaning tasks which will be mentioned in a later chapter. The very smallest detail of work can be timed and written down. The more detailed the schedule the less chance for the unexpected to be overlooked, or for any mistake, forgetting, confusion and hurry.

Have you a small house or a large house? Jabies to care for, meals to cook, and cleaning work to be done? Then try the "schedule way" for two weeks at least. See it "things" don't come easier, and that you are less worried and tired. Determine to "master" this planning of workfor that is all a schedule is, a plan of work which shall permit the tasks of housework to be done in an orderly, smooth manner with the least friction and confusion. You, too, can have a "housework train." It is your part to decide at what stations it shall stop, and for how long at each one. It is a route from drudgery to efficiency and personal happiness. 


\section{QUESTIONS ON HOUSEHOLD ENGINEERING}

\section{II}

Plans and Methods for Daily Housework

I. Make out a schedule of your present plan of work. Study to see where it can be improved. Try the new schedule two weeks. Revise and try another two weeks, and report.

2. Time yourself for at least a week on the same task, as, washing dishes, peeling potatoes, making beds, or cleaning the bathroom. How long does it take? Do you find the time varying from day to day? Write down two complete "time-studies" on these tasks, showing the first record and the last.

3. "Standardize" some household task so that jou can do it every day in an identical manner without much mental attention. Does this not make it seem less difficult?

4. What are your worst "interruptions"? Make a schedule which will take care of them as much as possible.

5. Do the same task with two different tools, and note the difference, or do the same task with two different methods, or do it under two different sets of conditions. Find out the way that seems the best and shortest for your particular case and report. 


\section{HOUSEHOLD ENGINEERING \\ III}




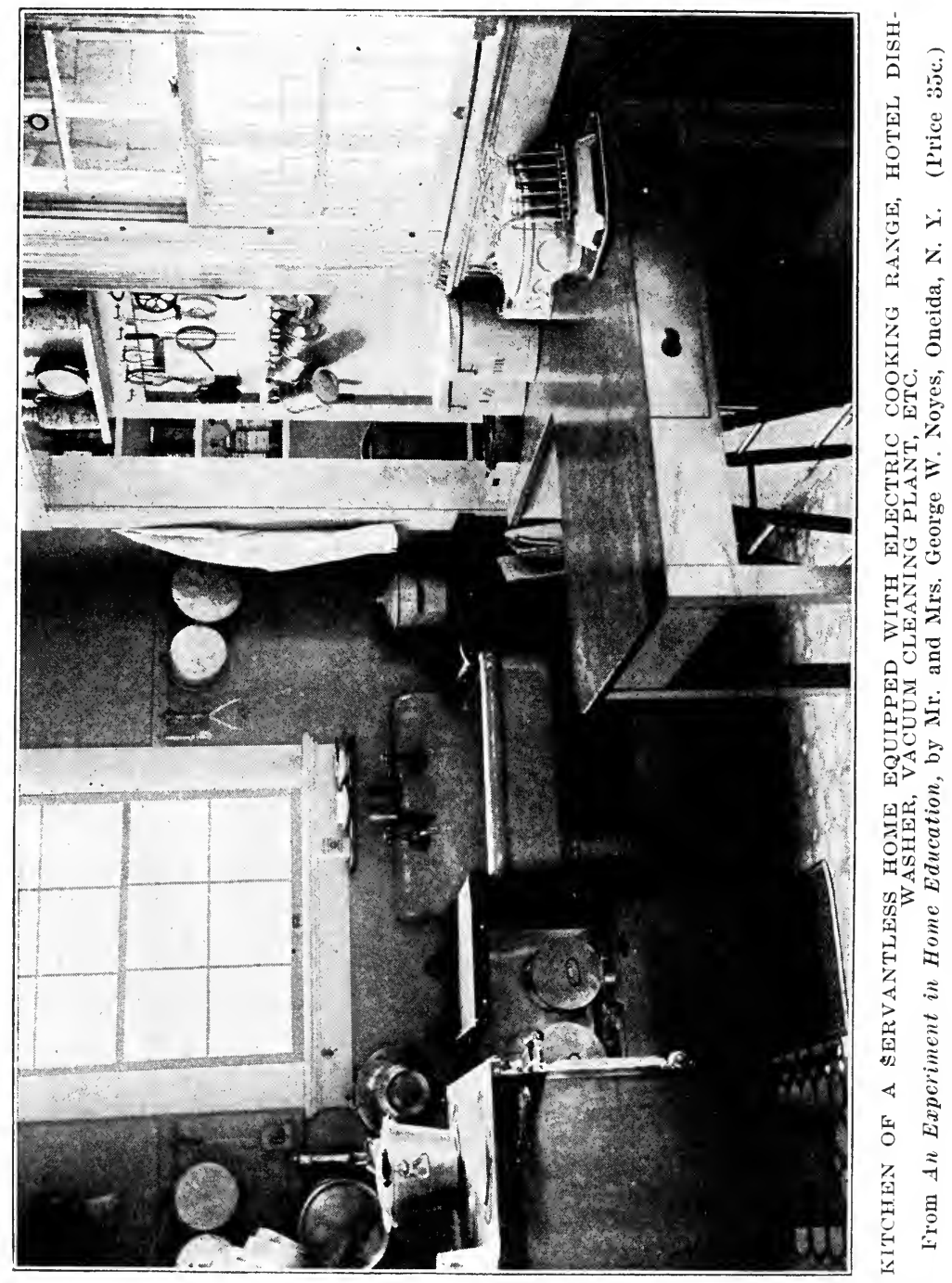




\section{HOUSEHOLD ENGINEERING}

III

\section{HELPFUL HOUSEHOLD TOOLS}

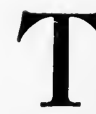

HERE is a great contrast between methods of housework in the United States and in other-countries, with the balance of convenience, labor-saving and easier methods in the American housewife's favor. The reason for this is that in no other country has mechanical invention been applied so extensively and successfully to all the different tasks of the home. The inventiveness of the Yankee is proverbial, and he has turned this quality to the making of mechanical labor-savers not only in his own shop and office, but for the benefit of the homemaker as well. There are on the market today literally' thousands of household tools, devices and equipment for every possible need of the home. It only remains for the homemaker to choose among them wisely.

Another reason for the great supply and demand for household labor savers in this country is that the American homemaker has to face the increasingly complex problem of scarce domestic help. Even today in other countries service cost has been low, and one can secure a cook for $\$ 12$ or a housemaid for $\$ 8$ a month. With such cheap labor, the need for the mechanical replacers of labor, or "mechanical servants," has not been keenly, felt there. In the 
United States, according to estimates, only 8 percent of all families employ even one servant permanently. This means that 92 percent of homemakers are performing their own household tasks. It is to this class of women who are actively concerned in the work of the home that the laborsaver and improved modern tool most appeal. The homemaker's time and effort are worth conserving by every means. She should therefore, be eager to buy and use all the household tools which will save her strength and time and liberate her from household drudgery.

\section{Need of Mechanical Knowledge}

While some women are "handy" with tools, the fact remains that most women are unfamiliar with the different principles involved in mechanical tools and devices. The boy almost unconsciously absorbs knowledge about gears, motors, force pumps, turbines, etc., in his daily work and play, but the girl neglects handling or learning about tools, believing it unnecessary or possibly unfeminine.

The homemaker, however, needs a most thorough knowledge of the principles of applied mechanics. Even many a good course in school physics unfortunately leaves a student with but little practical knowledge applied to the tools and equipment to be found in every kitchen and home. The more a woman knows about tools the more intelligent she will be as a buyer. Such knowledge will save her from the useless expense of buying worthless equipment, and make her more interested in purchasing the good tools and highclass equipment which will help greatly in saving time and labor.

\section{Equipment Buying an Investment}

The only right and economical view to assume in buying any and all equipment is to ask one's self beforehand, "Will 
this article be a permanent investment?" We cannot afford to buy tools for temporary use. They should be regarded in the light of permanent purchases whose use will be extended over a considerable period of time. Too many women buy equipment on a basis of cost only. They look at the price without considering how many times the article will be used. It is not the cost, but the number of times of use, which must be the basis of economical, efficient buying.

For instance, a woman may see an attractive cherry seeder costing only $\$$ r.oo. The ease with which it removes the pits and time it saves influences her to its purchase. She will, however, hesitate and pass by a serving tray on wheels costing $\$ 10.00$ which she can just as readily see will save her steps in setting and clearing the table, serving meals, etc. The reason that she buys the $\$$ r.oo device in preference to the $\$ 10.00$ article is not because she cannot afford either of them, but because she is wrongly buying on a basis of cost only. The cherry seeder may be used only ten times during the cherry season and never used the rest of the year. The serving tray will be used three times a day every day in the year, and on an investment basis compares with the cherry seeder as follows:

First Cost Per

Cost Use

Cherry seeder, used to times during season..... \$ I.00 \$0.10

Serving tray, used 3 times daily, 365 days...... $10.00 \quad .009$

This illustration is used not to disparage the cherry seeder or any other good device, but to show that equipment must be bought on a basis of the number of times of use, and not on the basis of first cost. In other words, the homemaker must ask herself, not "How much does it cost?" but "How many times will I use it?"

This investment point of view must be taken especially in regard to more expensive equipment like washing machines, 
dishwashers, mangles, fireless cookers, and others in which the first cost represents considerable money outlay. If her family is large and she hears of a good, labor-saving dishwashing machine costing $\$ 50.00$, her attitude must not be "Oh I cannot afford \$50.00!" She must reason to herself something like this: "This dishwasher with care will last a minimum of ten years. Allowing 6 percent interest on my money, the annual cost of such a washer would be $\$ 5.00$ depreciation and $\$ 3.00$ interest or 15 cents per week or about 2 cents per day."

The question of purchase then, resolves itself not into whether one can afford $\$ 50.00$ but whether one can afford 2 cents a day to reduce the drudgery of dishwashing. This is the investment, "long distance" view which is the only really economical one to take in purchasing all tools, no matter how small or great their cost. The chief reason why women have not still more successfully put their homes on a mechanical and labor-saving basis as has long since been done by men, is because they have taken the short-sighted view and spent most of their money on small, cheap, but seldom used articles on a cost basis.

\section{Tools Depend on Family Needs}

The second important question the homemaker must ask herself before purchasing equipment is, "Is this tool needed in my particular family?" A tool that would be an excellent investment for Family $A$ might be an injudicious and unnecessary purchase for Family B. For instance, even so very useful a device as a breadmixer might be an unjustifiable outlay in a small family where bread was made only once a week. Similarly, an excellent fireless cooker, no matter how worth-while in itself, might be questionable as an investment for a family especially fond of broiled meats, 
or with an aversion to stewed foods, and which seldom made soups at home, or followed cooking methods in which lies the chief value of the fireless. Too often women are influenced in their purchasing solely because other neighbors have bought a certain device; because it appears attractive in the store, or because they think it is a helpful tool in itself, without considering its relation to the needs of their particular family.

\section{Scientific Construction Necessary}

Again, one of the most neglected points in the mind of the woman purchaser is, the scientific construction of the tool or device. This is the most difficult point on which it is necessary to be informed, and usually her only means are the words of salesmen and descriptive circulars. But the scientific construction should be understood, especially before buying such pieces as refrigerators, stoves, fireless cookers, various kinds of washing devices, and others where the satisfactory working depends on proper insulation, convenient leverage, etc. Before buying say, a refrigerator, it is best to read some authority or some dependable pamphlet on the principles of refrigeration. This will enable the prospective buyer to question the salesman intelligently, compare the various models examined, and see if they fulfil scientific demands as to insulation, lining, air currents, etc. If this is done, there will be fewer purchases of refrigerators which waste ice and give poor service after short use.

Another reason why a woman should understand scientific construction before purchase is that there will be fewer chances of her being disappointed in the device afterward because of her own failure to understand it. For instance, a friend hearing of the widespread craze over fireless cook- 
ers, purchased an excellent make. After a two weeks' use she returned it, complaining that "it wouldn't work," and was thereafter prejudiced against all fireless cookers. The real situation was that she hadn't understood the scientific idea of cooking by conserved heat on which the fireless is based. She didn't learn exactly how to operate it before purchase, and so was dissatisfied and deprived of the service of a good device, largely because of her own failure to understand scientific principles.

The best method is to have one or several demonstrations of any device, handling it one's self before purchase. First hand information from those who have used it thoroughly is also better than trusting entirely to circulars. Another means is to know the standing of the manufacturer, insist on his guarantee, and whenever possible, buy trade-marked, identified lines of goods. Very often there is a free test offered in the home, especially with vacuum cleaners, washers, etc., which should always be taken advantage of. Every possible test should be given the device before actual purchase.

\section{Comfort in Use Essential}

Very often a device which fulfils other conditions mentioned above fails in the small but essential point of comfort in use. This is especially true of handles, levers, etc., which either by their shape, finish, or point of attachment prove uncomfortable when used in the hands of the worker. There is the case of a breadmixer with the leverage applied at the top of the pail; otherwise a fine labor-saver, it requires an awkward arm motion which would not be the case if the leverage were applied at the base and side of the pail as indeed it is, in another make. The handles of many egg-beaters, mashers, spoons, etc., are not shaped for the comfort of the hand, although there are others on the 
market which do offer this point of comfort. Sometimes the handle is too short or too long, flat instead of rounded. Or a lever would be easier to operate if several inches longer, and many other instances occur where the small but important points of comfort are not considered.

\section{Device Should Be Well Finished}

Frequently the lack of a well-finished surface, or poor construction spoils an otherwise good tool. An excellent dish drainer with a tray of galvanized iron is on the market, but the edges of the lower pan are so imperfectly soldered and so rough that the hand continually becomes scratched while working near it. Again, the hinges of a fine fireless cooker were found to be so jagged that as the cooker set out in the room, the worker tore her apron upon it every time she passed quickly. This detail of finishing should not have been overlooked in such a high-priced device, nor indeed in any other. The interior of kitchen cabinets, the trays of gas ranges, the seams and handles of many other utensils, especially those made of wire, tin or galvanized iron, are all places for the housewife's careful inspection before purchase.

\section{Ease in Care and Cleaning}

"Is this device easy to wash and keep clean?" is another important question which should be asked previous to buying. Too frequently the time and difficulty of washing and keeping a tool in good condition is entirely overlooked. Many devices have complicated parts, gears, beaters, adjustable cutters, etc. Now, the question of how long it takes to wash and assemble these parts after use must be considered, because this time really forms part of the total time that the device is being used. For instance, there is a 
most efficient stationary puree-strainer consisting of a perforated drum, stand, rotating blade, separate handle and three screws. It does most excellent rapid work in mashing potatoes, straining, etc., but it requires six or eight minutes to wash, dry, and assemble the parts ready for the next use. In short, while it does the actual work in less time than the old-fashioned strainer, the additional time required to clean it makes the total time of both equal. The value of this tool, then, cannot be estimated in terms of time, but only in terms of the superior quality of the work done by this device over some other.

It should be firmly remembered that no device should take longer to clean and adjust than the time it saves by its increased efficiency over some other method-otherwise; it ceases to be a labor-saver and must be justified on some other grounds. For the woman who does her own work, this point of the time and care required in washing and handling any tool must not be overlooked.

\section{Size Adapted to Actual Needs}

Just as it is necessary to know exact measurements in buying apparel, furnishings or other household articles, so it is worth while to consider exact sizes in buying household tools and equipment. Shall one purchase a two-hole or a three-hole fireless? Will a certain vacuum cleaner be adequate in size for the number of rooms and work demanded? How many sheets or other unit of capacity will a given washing-machine hold?

These questions of size must be asked in buying, in order to purchase a utensil or other piece of equipment adapted to the particular needs of a particular family. Unnecessarily large equipment has the disadvantages of taking up floor or storage space, being in many cases heavier to handle and care for, and particularly offering a larger surface 
to clean. If a two-hole fireless be ample, it is surely unwise to purchase a three-hole which will take up another square foot of space and be that much heavier to move ; or a "large" meat-chopper offers no advantage over a "medium" size under most conditions.

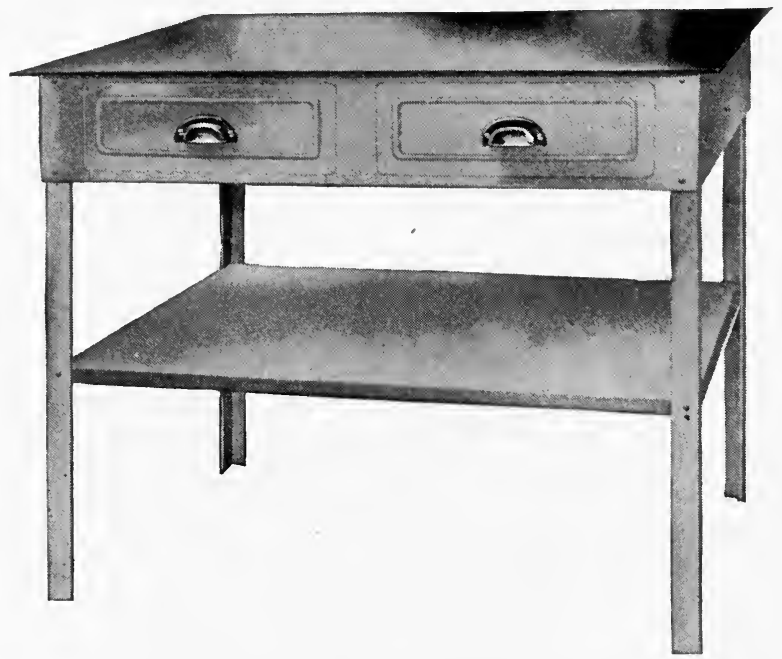

ENAMELED METAL KITCHEN TABLE

Sanitary and easily cleaned

In one pantry recently explored by the writer, no less than twenty-four saucepans and cooking utensils were found. When this was commented upon, (chiefly because it took up so much space), the homemaker replied that she had to have them. The truth was, that they were badly chosen as to size, and that the same service could have been filled by as few as eight pots, if selected with their exact purpose in mind, thus cutting down pantry space two-thirds. It is easy to measure the capacity of any utensil with a quart 
measure or to secure from the manufacturer the estimated capacity and power of other equipment. If the kitchens of the future are to be more step-saving and less costly the minimum of equipment should be chosen.

\section{The Four Main Groups of Labor-Savers}

All equipment falls broadly into two classes: (I) the fixed, like the sink, range, etc., and (2) the portable, to which the great bulk of minor tools and devices belong; and this latter class can again be divided into
(I) Labor savers.
(2) Fuel savers.
(3) Time savers.
(4) Step savers.

In addition, there is a fairly large group whose main appeal is sanitary or hygienic value; there is also a wide group of miscellaneous tools which cannot be clearly classed. In many cases, too, the same device overlaps into two or even three of the above groups, but this classification is very convenient because it helps the homemaker decide exactly what value she most receives from the tool or device.

We shall discuss in this chapter all equipment except that belonging particularly to the cleaning or laundry processes which will be taken up separately in the discussions of these subjects.

\section{LABOR SAvers}

Many of the best household tools fall under the heading "labor savers." The list is long and includes the mechanical helps which replace more laborious hand process work. Such a partial list is:

Bread and cake mixers.

Egg beaters, cream whips, mayonnaise mixers.

Ice cream freezers, butter churns. 
Coffee, spice and meat grinders.

Stationarv colanders, strainers and mashers.

Potato parers, fruit corers and parers, slicing devices of all kinds.

Stationary chocolate and cheese graters.

Stationary nut crackers.

Dishwashing machine.

Dishdraining rack.

\section{Dish Washing Machines}

The results of standardizing dishwashing by hand have been given on pages 78 to 80 , showing that it is possible to

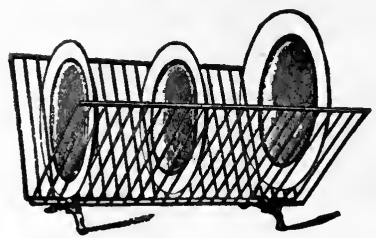

FOLDING DISH DRAINER

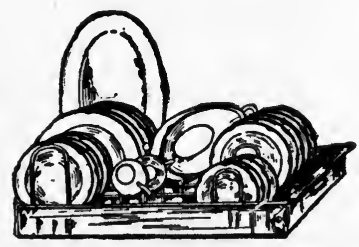

DISH DRAINING TRAT

reduce the time nearly one-half by substituting rinsing on a wire drainer for wiping and arranging shelves adjacent to the sink for laying away the dishes. The following tests were made with the most prominent portable dishwashing machines on the market.

Both hand tests and tests with four different types of mechanical commercial dishwashers were made simultaneously over a considerable period. In each of these tests the same number of dishes (50) and silver (50) were used at each test. The temperature of the wash water was I 40 degrees with the washers, and I2O degrees with hand 


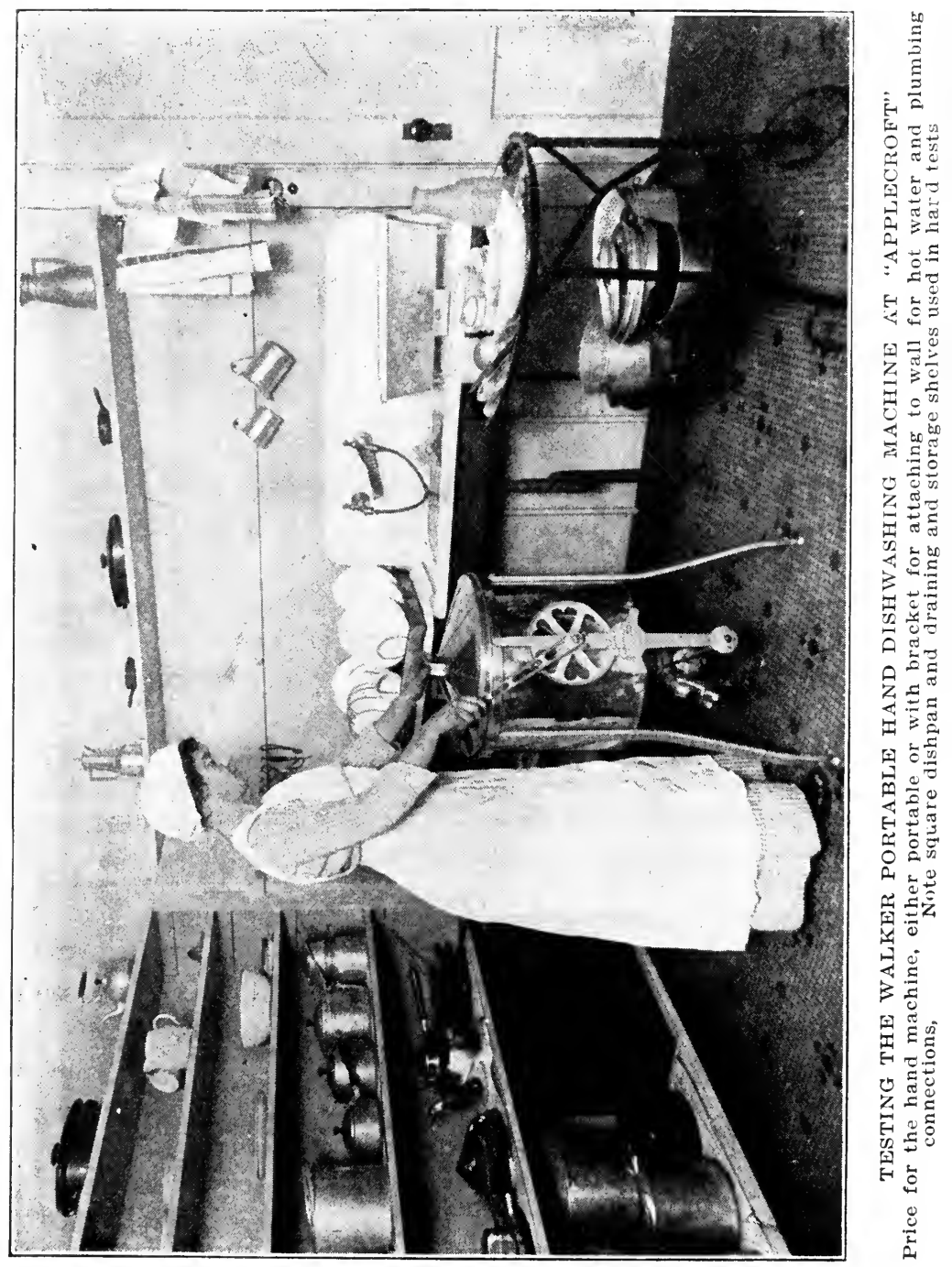


tests. The average from three tests is given below with full comments.

Dishwasher A: Tub or barrel model; in poorly finished, aluminized sheet iron. Principle of force pump; operating through a perforated cylinder in center of washer from which water is forced over the dishes by action of a hand-lever at top of lid. Two drain racks; only fair stacking of dishes possible. Very laborious method of draining water by hand from stop-cock in base of can. Can bulky and space-taking. Action easy, but force of water poor and not satisfactory for thorough cleansing.

Results: Inadequate; many dishes, especially cups, not entirely clean.

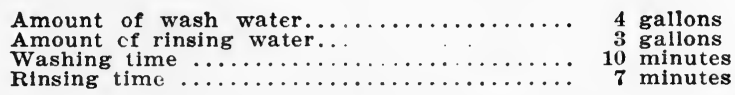

Dishwasher B: Tub model of enameled stamped metal; operating on principle of turbine by hand lever. Low gearing. One wire rack without handles, perforated upper lid. Action of lever very easy; force of water only moderate. Wastes space in upper part of tub. Difficulty in removing rack without handles.

Results: Inadequate, as custard in cups, coffee grounds, etc., not removed in given period of washing. Good points are neat, small appearance of can and simplicity of emptying water by pressure on small valve.

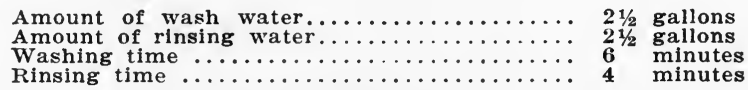

Dishwasher C: Square box model of galvanized iron; on principle of force-pump operating by hand lever on outside of box; a spray of water thrown by swinging nozzle which can be moved from side to side; water easily emptied by pressure on small valve. Height about three inches; too low for convenient operation. Maximum amount of dishes, 40; silver, 25 pieces; lield in small detachable box in center. Vertical wire racks for dishes, with separate side cup-hooks.

Results: Satisfactory, but action back-breaking owing to low height and general inconvenience of this model.

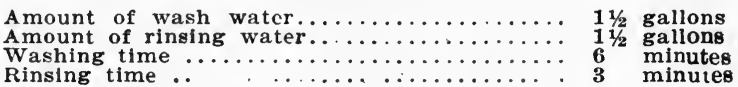


Dishwasher D: Tub model of substantial retinned metal. Power transmitted through hand lever and chain sprocket to a shaft and bevel gear at bottom of can which operates a horizontal dasher inside on the principle of the turbine; throws water with great force, adequately spraying upper and lower tray which have convenient handles. Permits satisfactory stacking odd-shaped pieces in top tray under convex lid. Action a little too difficult and lever should work much more easily.

Results: Entirely adequate, and even milkỳ and egg-soiled dishes entirely cleansed.

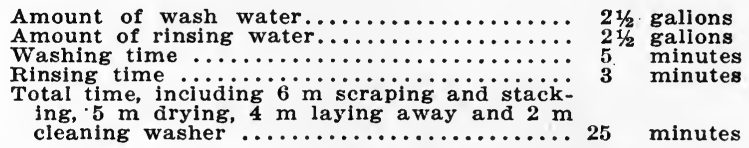

Hand Method: Stack surface at right of sink, drain surface at left of sink, rectangular washing pan, wire drainer keeping each dish separate and permitting them to dry after rinsing without wiping, long handled dish mop.

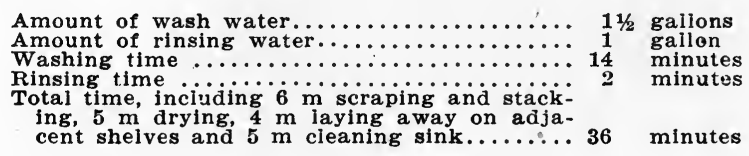

Conclusions on Mechanical vs Hand Method

The case for all mechanical washers can be summed up as follows :

(I) There is less danger of chipping and breaking.

(2) There is much greater sanitation and possible sterilization.

(3) There is no discomfort or hard usage to hands.

(4) They cut down only actual washing time, but that considerably. If operated by motor power the amount of effort as well would be greatly reduced. This was seen in several hotel dishwashers operated by power where the labor of the operator is negligible.

(5) They offer a sanitary container for the temporary storage of soiled dishes over night or until desired number accumulate, permitting washing to be done once a day in a small family. This is a very great advantage. 
The case against the mechanical washer in its present form, and under existing conditions, is :

(1) Results, except in one of the small portable detached washers, were unsatisfactory, and the amount of effort required in operation somewhat offset other good points.

(2) In every test, the portable washer "crept" about the floor with the action of the lever. The difficulty of filling and emptying these models by hand was considerable and had to be included in the total washing time.

(3) The time required for the steps of stacking, scraping and laying away is identical under both hand and mechanical methods. The only steps affected are the ones of actual washing and sometimes wiping. This saving of time and also of effort is much more marked in those machines operated by power, as was seen in close study of hotel washers.

(4) The use of the washer is strictly limited to dishes, glass and silver; no odd shaped utensils, pitchers, eggbeaters, or pots were successfully washed in all cases, and the arrangement of the racks does not permit the stacking of these articles.

(5) The time in one step, washing ( 5 minutes vs. I4 minutes) is cut considerably by the use of a mechanical device; but this saving is partially offset by the increased amount of effort required to operate the lever in all cases. In no case would it be advisable to operate a dishwasher in a beautiful street dress, as some manufacturers suggest by their circulars-in order to thoroughly cleanse the dishes considerable physical movement must be made.

(6) The quantity of exceedingly hot water required in nearly every case with a washer is a point against it in many homes under present conditions. The efficiency of all washers was found to depend very largely on the degree of heat of the water; second, the satisfactory "self-wiping" of the dishes as they rest on the racks, which is made such a strong point by the washer manufacturers, depends even more largely on this same factor. 


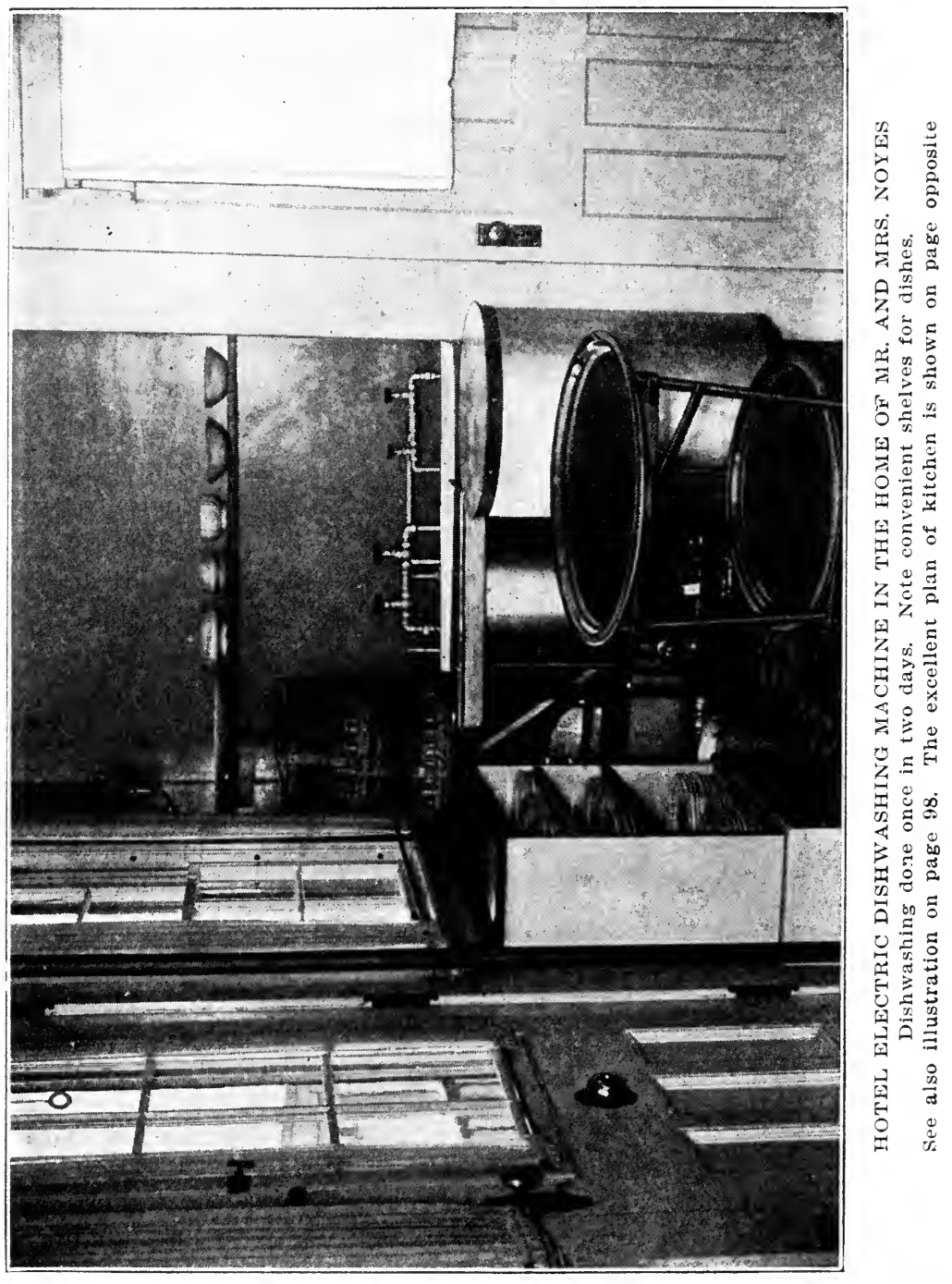




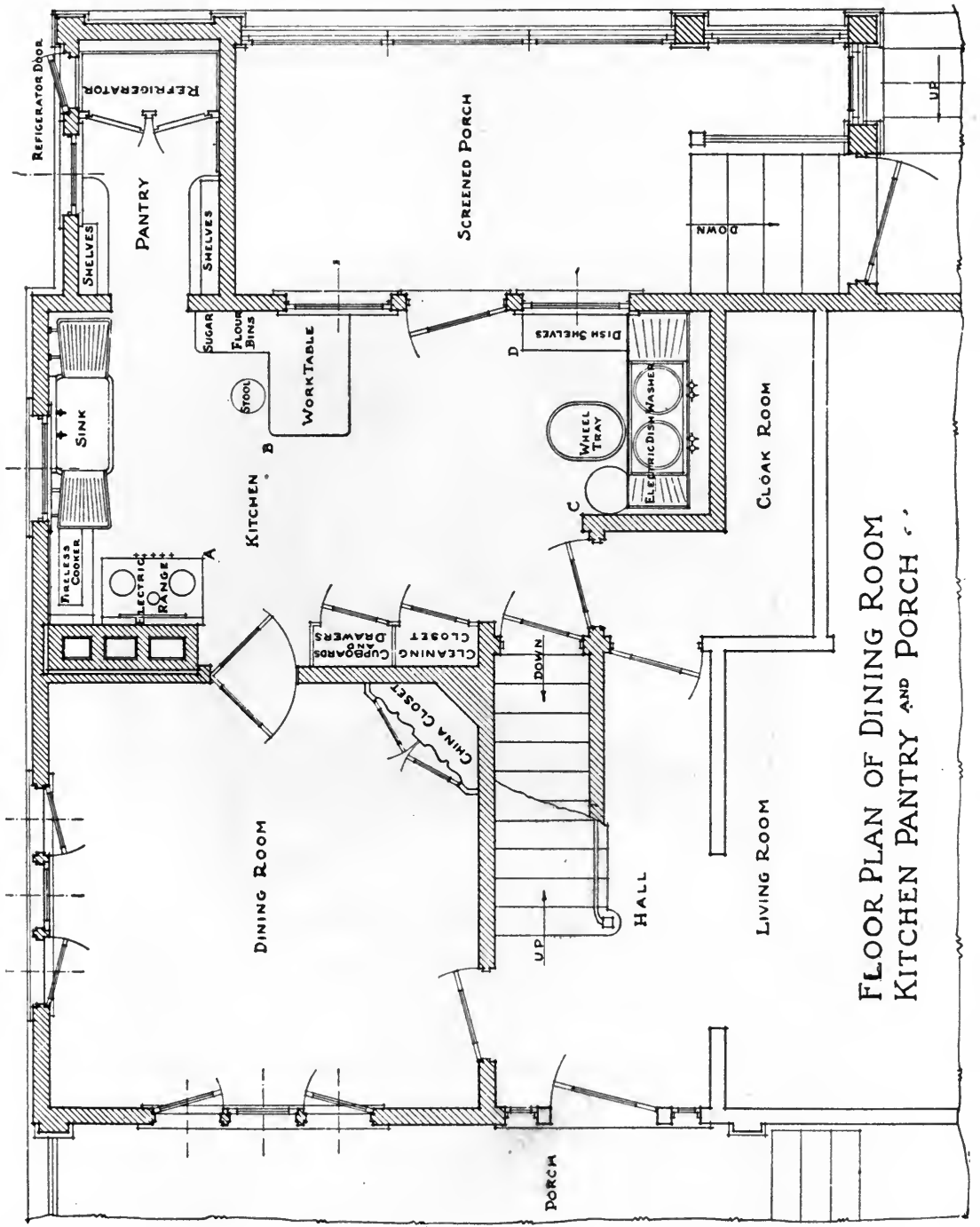


It should be borne in mind that the defects pointed out in the mechanical washer refer solely to those operated by hand, and not to dishwashers operated either by motor or other power; and to those not permanently connected with the plumbing and hot water system.

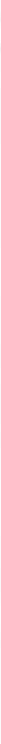

WESTERN ELECTRIC HOUSEHOLD DISHWASHER

Combines kitchen table, with white enameled top, with a dishwashing machine

In conclusion, it is the writer's opinion that no portable, detached washer which entails hand filling and emptying, has very great efficiency value. The ideal for the mechanical washer must be a permanent installation connected with an adequate water supply of scalding water, having its drain outlet connected with the regular plumbing. Such con- 
nections are not difficult or expensive to make. At present the best solution of the hot water problem is to put in one of the automatic instantaneous gas hot water heaters which can be regulated to give scalding hot water in any quantity desired. These heaters for furnishing hot water all over the house on the turn of the faucet cost about $\$$ I50, but a small "bath" instantaneous heater, for dishwashing, can be installed for $\$ 45$.

\section{Time Saving Fuels}

In the days when wood was to be had for the picking up, costing nothing but labor, and when coal was $\$ 3$ and $\$ 4$ a ton, no question arose of economy of fuel. Today the cost of all fuels has so markedly increased that the conservation of fuel, and thus the lowering of fuel cost is one of the homemaker's chief problems.

Since she must pay the prices demanded for fuels today (range coal costing in many sections anywhere between $\$ 6$ and \$Io per ton and other fuels also being high) the only way she can meet the problem is by using such cooking methods and cooking equipment as will conserve, not waste fuel. In addition, she can today command the services of three fuels practically unknown to her grandmother-gas, alcohol and electricity.

The great disadvantage of both coal and wood is that it is not possible to put them under direct control; even with the best constructed range, much of the heat generated is not actually used in a cooking process. Regarded solely in the light of a cooking equipment, both coal and wood-using stoves are wasteful of fuel. Moreover, they result in a large percent of waste products like ashes, clinkers, dust etc., which entail labor in keeping the stove and kitchen in proper condition. 
The ideal cooking fuel is one that can be directly controlled and checked, the moment it is desired to finish a cooking process. It must also be one in which waste products, offensive gases, radiated heat and other accompaniments of combustion are reduced to the minimum. Gas as a fuel has these advantages and even when the price is as high as $\$ 1.50$ per I,OOO cubic feet it costs less to use than coal, if any value is placed on the housekeeper's time. At low prices, 60 cents to 80 cents per I,000 cubic feet, the advantages are all with gas.

The newer models of the gas stove have an upper oven, which makes for efficiency by preventing unnecessary stooping. Burners of different sizes are provided and broilers and warming ovens so placed as to be in the most convenient position. Before using any gas stove it is best to have a demonstration by the agent of the local company who should explain the care of the burners, how to adjust the flame, how to detect when there is too much or too little air mixed with the gas and other details which will enable the operator to use the range most intelligently. Besides the gas range, there are other devices using gas now perfected. Such are the gas iron, chafing dish and percolator with gas connection, gas water heater and other pieces heated by gas.

\section{Alcohol as Fuel}

Within very recent years, the new fuel, denatured alcohol, has been offered the homemaker. Denatured alcohol is formed by adding wood (methyl) alcohol and petroleum benzine to ordinary (ethyl) alcohol. It is thus completely unfit for food use and is indeed dangerously poisonous. It burns with a clear, intense flame (with no by-products of carbon, odors, etc.), and there are on the market special stoves for its use which resemble the small gas "hot plate." But its cost in this country (even at the wholesale prices of 
45 to 75 cents per gallon) makes it too expensive a fuel for regular family use. Also the fuel value in a gallon of alcohol is only about $2 / 3$ that of a gallon of kerosene or gasolene. Its use at present must be confined to the occa-

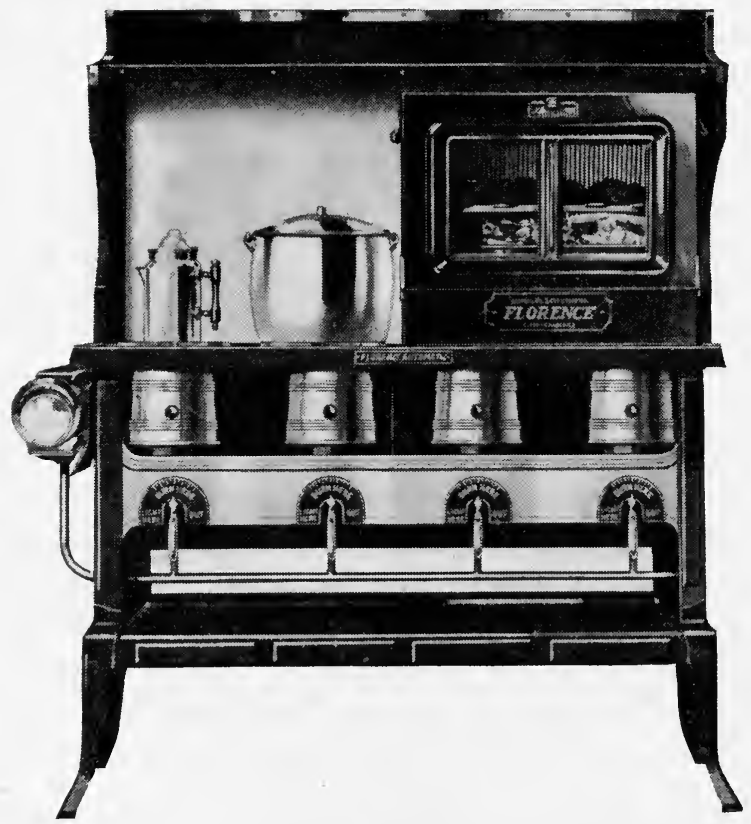

AN EXCELLENT FOUR BURNER, WICKLESS BLUE FLAME OIL STOVE Portable oven fits over two burners

sional chafing-dish, percolator, plate warmer and other small pieces for distinctly table service. There are however, several excellent one-burner stoves, easy of operation, which will fill many a place in sick-room and light housekeeping cookery where other fuels are not available. 


\section{KEROSENE}

To the rural housekeeper especially, who is out of the range of gas and electricity, and who nevertheless wishes to free herself from the labor and dirt of coal and wood ranges, kerosene offers a solution of the fuel problem. Kerosene can be bought everywhere, the price averaging 12 cents per gallon, with a saving of several cents per gallon if bought by the barrel.

Kerosene burns with a slow heat, not to be compared with gas in intensity. The chief criticisms generally advanced against it is the odor, and possibly smoke present and it radiates more heat than gas. Neither of these accompaniments however, need be present if the stove is properly cared for daily. The best type of stove is by all means that using a wick, as the "wickless" stoves are much more unsatisfactory and require more attention.

Kerosene stoves are now manufactured in two, three, or four-burner models with an upper standard, warming shelf and portable oven, which make them compare in appearance and results quite favorably with gas stoves. They require, nevertheless, daily care in wiping off each wick and attention to see that the wick is never raised too high or burns with "points" which will cause yellow, smoky flame instead of the blue flame necessary for perfect results. In the writer's opinion, a three-burner kerosene stove and a fireless cooker are the ideal country combination to supplant the laborious coal range with its waste of heat and imperfect control. In my summer home, with a family of eight, we consume between three and four gallons of kerosene a week. A fireless cooker is used.

A late type of the "Perfection" oil stove contains a fireless cooker oven with thermometer, cabinet style, four burners, shelf, etc.-in fact, a complete oil range. 


\section{Gasoline}

Gasoline is in somewhat bad repute as a fuel because of the many distressive accidents from the use of gasoline stoves. Most often, however, such accidents come from carelessness, such as filling the reservoir when the stove or

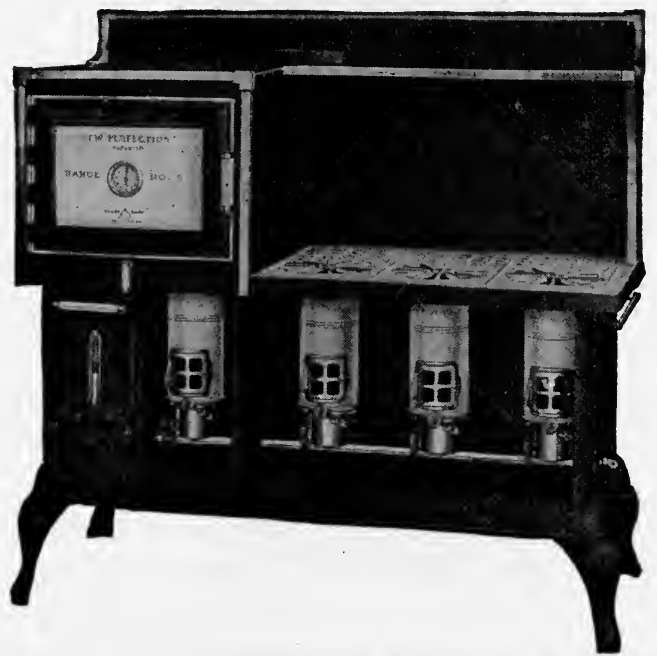

“PERFECTION" OIL RANGE WITH FIRELESS COOKER OVEN Marketed by the Standard Oil Co.

lamp is lighted, spilling the gasoline, knocking over the stove, etc. In the older styles the flame might easily be blown out by a draught of air, the gas would then continue to form and an explosion might result if a light were brought into the room.

The best types of gasoline stoves are safe if used with intelligence and care. The supply tank can be placed in a different room and piped to the stove, the stove can be secured to the floor and placed away from draughts.

A good gasoline stove gives a blue flame nearly equiva- 
lent to gas in intensity and so is a quicker fuel than kerosene. The chief disadvantage is that the generator in the burner must be heated for some time before the burner can be lighted properly. Gasoline is more expensive than kerosene, costing from 14 to 25 cents a gallon.

\section{Acetylene Gas}

Acetylene gas is still another fuel being used more and more in country homes for cooking as well as for lighting. Acetylene generators, approved by the National Insurance Board, are safe if given intelligent care. The gas burns with a very intense blue flame and must be used in stoves and hot plates especially designed for it. The expense of using acetylene for cooking is greater than kerosene but not prohibitive.

If a coal or wood stove must be used in winter, by all means have the fuel stored on the same floor level as the stove-not in the celler. Some of the steel ranges are now provided with a sheet iron pipe from the ash pit of the stove to a metal ash can in the basement, through which the ashes may be dumped as necessary. This arrangement saves much labor and dirt and could be adopted in many cases.

\section{Electric Cooking Equipment}

The most modern of all fuels is electricity, which while not a fuel proper, is a source of heat and thus a means of cooking. Several years ago there was scarcely a piece of electric cooking apparatus on the market, and the use of electricity was confined chiefly to lighting and power purposes. Today electricity, "the silent servant" is being adapted to not only the small portable cooking devices such a percolator, toaster, grill, hot plates, etc., but to the larger fixed equipment of stoves and ranges proper. 
No other fuel can be under such direct control. No other fuel equals it in entire absence of unpleasant gases, odors, soot or other products of combustion. It is without doubt the most ideal cooking fuel from the standpoint of cleanliness, direct control and absence of waste heat by radiation. Becatse it can be measured and the degree of heat so accurately obtained, electric cooking can be performed at any continuous desired degree.

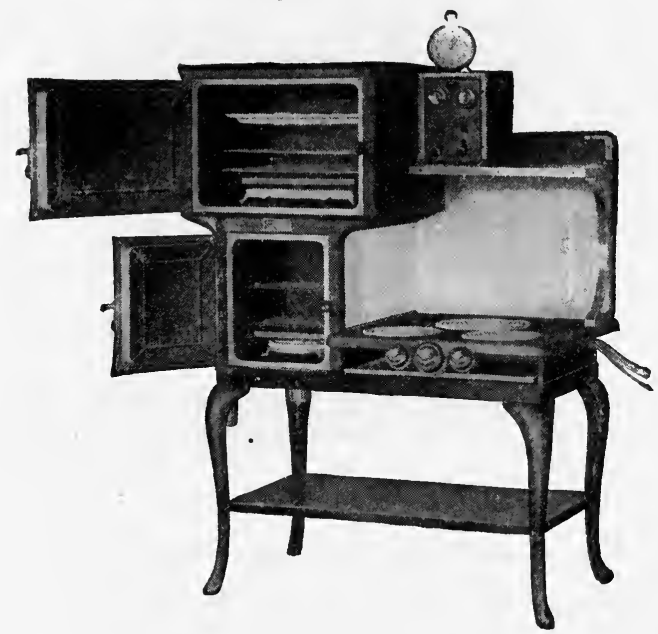

WESTINGHOUSE AUTOMATIC ELECTRIC RANGE

The fireless cooker ovens shut off the current at any desired temperature. 'The clock will turn on the current at the time set.

At present however, electric cooking is not much used for two reasons: the present high rate of current, and the high cost of the equipment itself, owing to the fact that electric equipment can be made only of the highest grade materials and requires expensive metals in its construction. Many sections of the country have not progressed far enough to make separate rates for cooking and power from the usual rate charged for lighting. Other localities, notably 
Oregon and some of the western states, have a low "flat" rate which covers cooking and the use of electricity as power to operate washers, vacuum cleaners, etc. Thus the cost of current differs widely, and can only be summed up in the extreme figures of 3 cents to I $_{5}$ cents per kilowatt hour, the average being about ro cents in medium sized cities.

\section{APPROXIMATE FUEL COSTS}

Fuel

Electric

Rate

$6 \mathrm{c}$ to $\mathrm{I} 5 \mathrm{c}$ per kilo

Burner

Cost per hour

Disc. stove,

I burner $250 \mathrm{~W}$. I I $/ 4 \mathrm{C}$ to $33 / 4 \mathrm{c}$

Denatured

Alcohol

$40 \mathrm{c}$ to $75 \mathrm{c} \mathrm{gal}$.

I burner

I $1 / 2 C$

Gas

$70 \mathrm{C}$ to $\$ \mathrm{I} .25$

per I,000 feet

Acetylene

\$I per Ioo feet

Oil

I2c per gal.

Coal range $\$ 5$ to $\$ 9$ per ton

From $1 / 2$ to $I$ ton per month

I medium top

$5 \mathrm{ft}$. per $\mathrm{h}$.

$1 / 3 \mathrm{c}$ to $2 / 3 \mathrm{C}$

I burner

$2 \mathrm{c}$ to $3 \mathrm{c}$

I medium flame $1 / 2 \mathrm{c}$

Entire stove

(without water back)

$3 / 5 \mathrm{c}$ to $2 \mathrm{t} / 2 \mathrm{C}$

\section{Electrical Terms}

There are a few terms constantly used in speaking of electric apparatus which should be familiar to the homemaker. The first of these-the "watt"-is the unit of measurement of electrical encrgy. It is estimated by the kilo or I,000 watts, usually expressed "kilo-watt" or kw.

The second term is the volt, which is the unit of electrical pressure. Electricity may be considered as forced under pressure along the conducting wires to the stove or other apparatus. If a wire of large diameter is used, the pressure or "voltage" can be lower than if a lower pressure or voltage is employed. The common high pressure voltage used in houses is generally 200 to 250 volts; low pressure being at 100 to I ro volts. 
The third term which must be understood is the "ampere," which is the unit of electrical quantity flowing through the wire. The number of amperes multiplied by the volts gives the number of watts- $(A \times V=W)$, for instance, if the pressure used is 200 volts, and 5 amperes of current are absorbed in the circuit, the number of watts used is $200 \times 5$, equals $\mathrm{I}, 000$.

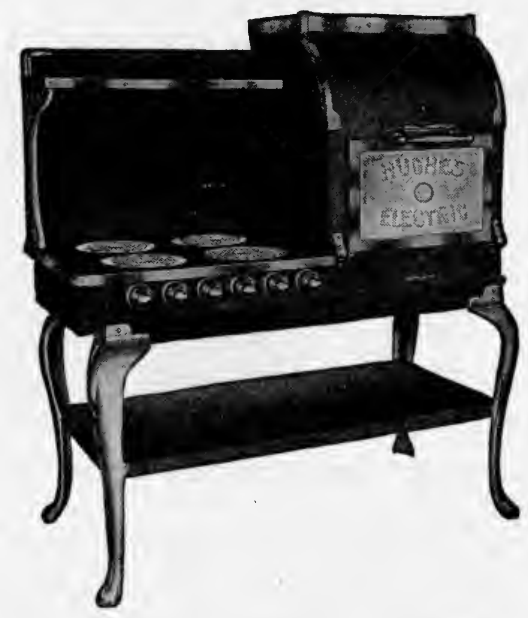

HUGHES ELECTRIC RANGE

The coils of these newer electric stoves are visible and become red hot

Stoves, percolators and small appliances are rated in "watts" as for instance, a certain kettle may be rated at 480 , a grill or table cooker at 550 , or a small hot plate stove may have two or three degrees of heat, say 600 for "high," and 300 for "low." This ability to change the degree of heat is one of the economies of electric cooking, as it is possible in many devices to change from a high degree, (perhaps 900) for full boiling, to a medium degree (600) for simmering and slower cooking to the lowest (300) 
used merely to keep foods warm: The user should always know the amount of volts supplied, as a device which is fitted for 220 volts supply will not heat when connected to a I Io volt supply, but if a device wired for a i io volt current is connected to a 220 volt circuit it will be overheated and probably ruined. Every electrical device from reputable manufacturers is marked at the bottom, as a toaster may be marked 206-2i 5 V.-2.4 A. This means that the toaster may be used on any voltage between 206

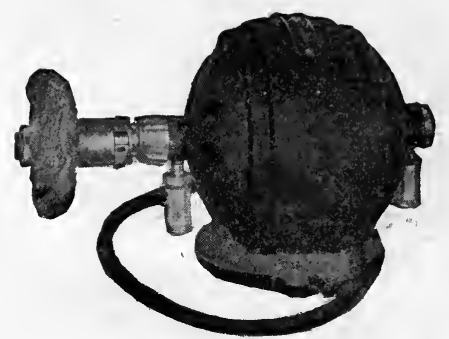

A SMALL UTILITY AOTOR WITH BUFFING WHEEL

and $2 I_{5}$ and that it will take 2.4 amperes of current. Multiplying these figures would show that the toaster will use about 500 watts an hour. If the cost of electricity is ro cents per kw.h. (I,OoO watts per hour), the cost of running the toaster will be 5 cents an hour. Both the wattage and voltage are usually given in catalogs of electric equipment, and should be noted carefully before buying.

When electric energy is to be delivered to any great distance "alternating" current of 2,000 volts or more is produced, thus permitting small conducting wires to be used. As such high voltage is very dangerous to life, a small "transformer" is put in, usually on the pole nearest the house, which reduces the voltage to the desired intensity, most commonly to I Io volts. All household heating and 
lighting apparatus work equally well with "direct" or with "alternating" current of the required voltage, but direct current motors cannot be used on alternating current, nor alternating current motors on direct current circuits, except for a few special motors which may be used on both. For an alternating current motor also the number alternations
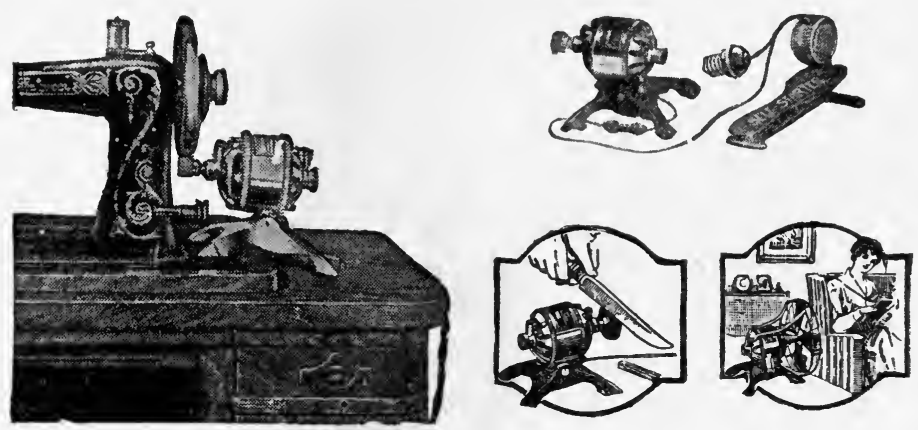

UTILITY MOTOR FOR USE ON SEWING MACHINE (\$16.)

Fan, grinder, polisher and cream whipper attachments at extra price.

or the "cycles" of the current must be known. Direct current is used for electric cars and in small plants. Nearly all electric lighting circuits use alternating current.

\section{Electricity as Power}

But while electricity has not come into its own as a fuel for reasons given above, it has more certainly entered the modern household as a source of power. In no other country are there so many household labor-savers operated by electricity. First perhaps in importance is the increasing number of vacuum cleaners. Washing machines, mangles and other laundry equipment electrically operated, are being 
put on the market in greater numbers, thus robbing wash day of many of its former terrors. Dishwashers, coffee grinders, meat choppers, and other similar pieces come fitted for power.

One piece of electric apparatus which is bound to come into more general use is the small utility motor. Such a

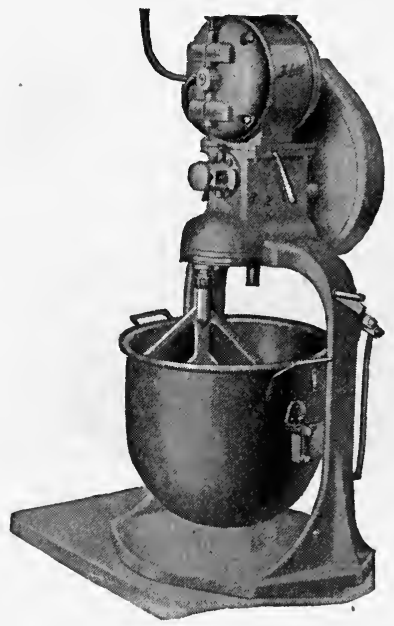

TYPICAL ELECTRICAL BEATING, STIRRING AND GRINDING MACHINE, FOR A LARGE FAMILY OR SMALL INSTITUTION

motor can be attached so as to operate a common handpower washing machine. It will also polish silver, freeze ice cream, grind meat or coffee and can be connected to a house pump, to a vacuum cleaner or many other pieces of household equipment. In one case where such a motor was attached to a simple ironing machine, all of the family ironing was done in one hour, which previously took over five hours by hand. In many rural sections electricity is being successfully used to operate churns, separators, grindstones, pumps, etc. It should be only a step to continue 
its use to help the woman on the farm, lighten her washday labors, wash her dishes, or clean her house. A last most important use of the small motor is its application to the sewing machine so that there is no treadling, and the machine and material need only be guided by the worker.

ELECTRICAL HOUSEHOLD DEVICES WITH ESTIMATED OPERATING COSTS

Watts

Consumed

Per Hour
Cost, on Basis of roc Per Kw.

Combination kettle, saucepan and disk stove

for table cooking.............. 550

Coffee percolator, 3 pts............. 440

Toaster ........................ 600

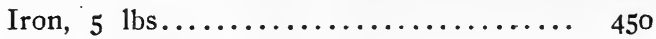

Oven, 3 heats................. 150

300

600

Electric radiator............... 1,000 or 750 or 500
$5 \mathrm{~T} / 2 \mathrm{c}$ per hour $4 \mathrm{~T} / 2 \mathrm{c}$ per hour $6 \mathrm{c}$ per hour $4 \frac{\mathrm{T}}{2 \mathrm{c}}$ per hour $\mathrm{I} / 2 \mathrm{c}$ per hour 3c per hour 6 per hour roc per hour $7 \mathrm{r} / 2 \mathrm{c}$ per hour $5 \mathrm{c}$ per hour

Small utility motor, for running sewing machine, chopping meat, polishing, ventilating, operating washing machine or mangle .................... roo

Water heater.........................

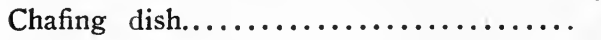
500 or $5 \mathrm{C}$ per hour r,000 or Ioc per hour 2,000 or $20 \mathrm{C}$ per hour 3,000 30c per hour 600 or $6 \mathrm{c}$ per hour 300 or $3 \mathrm{c}$ per hour $2002 \mathrm{c}$ per hour Heating pad.................. 60 or $3 / 5 \mathrm{c}$ per hour Baby's milk warmer............. $440 \quad 42 / 5 \mathrm{c}$ per hour 


\section{Devices and Utensils Which Save Fuel}

Even though the modern fuels discussed above are less wasteful of heat than coal or wood, it is nevertheless necessary to economize fuel still further by the use of devices and utensils which conserve heat or make for economical cooking. Foremost among such equipment is the muchdiscussed fireless cooker, based on the principle of cooking with conserved heat in an airtight box, instead of by direct heat.

The first cookers were merely well insulated boxes into which the container of heated food was left to cook slowly until finished. Improvements were made by adding the so-called disks or radiators of soapstone or other metal with a rack to hold them, thus permitting roasting and baking as well as boiling and stewing.

Another step in advance has been to combine the principle of the fireless in some regular cooking stove. For instance, there is the regulation gas or electric stove with usual burners. In addition, such a stove has an insulated oven or compartment corresponding to the "well" of the fireless with its heated radiators. Food is put into this compartment, heated for a short period, after which the fuel is turned off, when the food continues cooking by means of the heat radiated from the compartment walls, or from a disk or metal plate in the bottom of the compartment. In a gas-fireless stove recently used by the author, a 5-pound rib roast was thoroughly browned and cooked in the oven fireless, using only 20 minutes of actual fuel. In the ordinary oven about one hour and a quarter would have been required.

The advantages of fireless cooker ovens are that they have greater cooking space, take up no extra room, require no preheating of hot plates, save more fuel and more time. 


\section{Automatic Çookers}

In some of the later models, the cooking compartment, whether gas or electric, is under automatic control. In one gas stove, with a fireless cooker oven, the gas is turned off automatically at the time set and the cooking continues on the fireless principle (page 203). An electric stove with a fireless cooker oven will turn on the current automatically

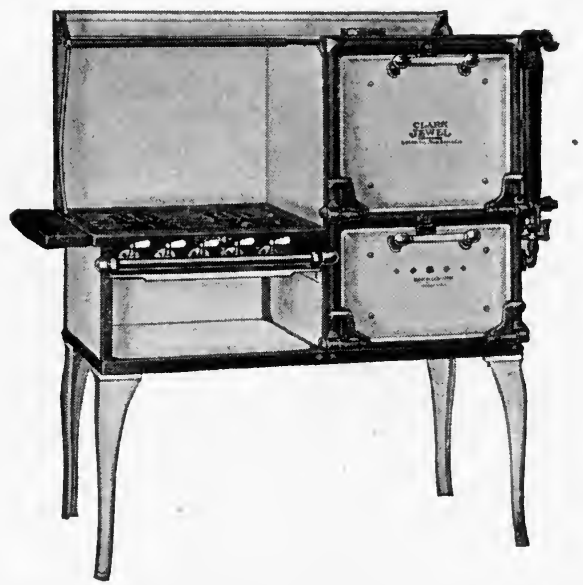

CLARK JEWEL RANGE WITH "LORAIN" AUTOMATIC OVEN TEMPERATURE REGULATOR.

at the desired time and also turn off the current when the required temperature is reached (page 123 ).

One of the best time and fuel savers is the automatic oven temperature regulator, which maintains indefinitely any desired oven temperature. This gives constant temperatures for baking, saves watching and some gas. With the regulator set for low heat, the cooking may continue for three to five hours, thus giving about all the advantages of a fireless cooker, with none of the disadvantages. Still another cooker is an electric pressure cooker, heat insulated so that it acts like a fireless cooker. 


\section{SUMMARY OF THE FIRELESS COOKER}

(I) It reduces fuel cost, sometimes as much as one-third or one half.

(2) It lessens labor by eliminating a great deal of work attendant upon the usual cooking processes, as basting, looking at food, etc.

(3) It saves time by making unnecessary so much useless potwatching and time spent in kitchen while foods are cooking, to prevent scorching, etc.

(4) It cooks food with little loss of weight, owing to absence of evaporation or drying out, as in usual methods.

(5) It brings out the juices and flavors of foods, and renders tender inexpensive cuts of meat as is almost impossible in any other way.

(6) It is especially adapted for the long cooking of cereals, soups, beans, and large pieces like whole ham, etc.

(7) The utensils used in fireless cooking do not scorch or stick; hence can be washed with the least effort.

Disadvantages

(I) Considerable planning and forethought necessary to operate one successfully. (This may be considered an advantage!)

(2) Does not brown foods as well as an oven. Flavor not so good with some foods.

(3) Unless used often the fuel or time saved does not justify the investment.

(4) Requires intelligence, care and some experience to get good results.

\section{Steam Cookers}

Another important fuel saver is the steam cooker. This consists of several round compartments fitted horizontally into each other, or a square compartment with sections, below both of which there is a water tank. Steam from this water penetrates all compartments and cooks the food. By accommodating from four to ten different dishes or foods, the steam cooker saves fuel which would be consumed if each of these were cooked on a separate burner. Steam cooking is also preferable to boiling, especially with 
vegetables, cereals, etc., because it causes less loss in weight, flavor, and particularly loss of important mineral salts which are too frequently extracted in the boiling process and thrown away.

In a recent test it was proved that odors from different foods do not contaminate each other. Boiled cabbage, rice, custard and beets were all cooked at the same time. The

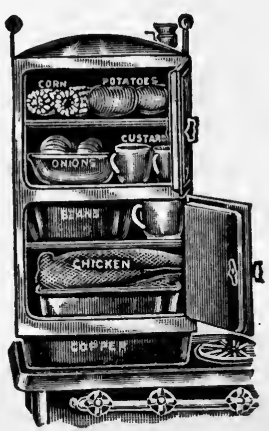

"IDEAL" STEAM COOKER No. 40

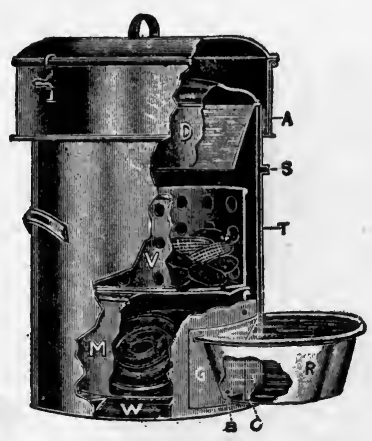

AUTOMATIC STEAM COOKER

custard and rice were entirely free from cabbage odor. This cooker can also be used excellently as a canning device, accommodating from 4 to 24 jars, according to size.

\section{Other Fuel Savers}

We have also the "triplicate pail" or the 3 or 2 interlocking pots fitted to one burner-not very useful. There are also various combinations of pots and insets which permit boiling in a lower compartment and the steaming of one or two foods above. The newest tea kettle with its "boiler inset" permits the cooking of rustard, rice, etc., at the same 
time that a quantity of water is being heated for other uses, in this way taking the place of the cumbersome double boiler.

Several radiating plates are manufactured especially for gas stove or range use. One of these is triangular in shape so that it covers three burners at once, but uses the heat from only one, thus permitting foods on the other two burners to cook slowly by radiated heat alone.

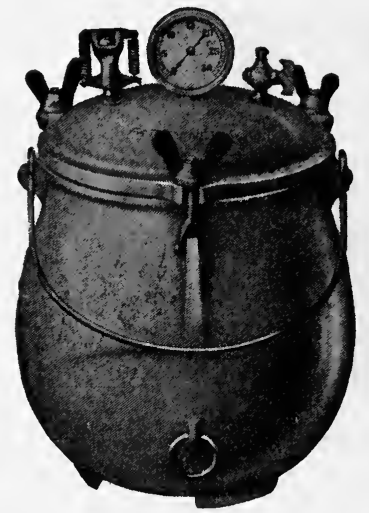

PRESSURE COOKER, WITH STEAM GAUGE AND SAFETY VALVE

A new cooking pot which is an improvement on the castiron pot of our grandmother has a rack in the bottom and a lid fitted with a steam valve. The valve is left open at

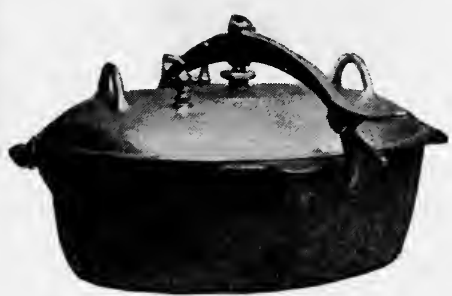

COOKING POT WITH TIGHT COVER

first while the food is being browned; it is later closed so that the generated steam falls back on the food, thus increasing the tenderness, and the pot once heated requires only a minimum of fuel. A further development along this line is the pressure cooker, fitted with a clamped cover, pressure gauge and safety valve. These save much fuel and time and give excellent results. The small portable ovens to fit over one burner of a gas stove use about onehalf as much gas as the large oven and are useful for cooking one or two small dishes quickly. 


\section{Vacuum Fuel Savers}

Another group of modern utensils which save fuel is the devices operated on the vacuum principle. These are either in the form of bottles, jars or large containers which when filled with a food or liquid of a desired temperature, retain it for a considerable number of hours. Coffee made in the morning can be poured, scalding, into the vacuum bottle and be ready to serve at a later meal. Many foods may be kept in these containers without a second fuel expense for "warming-up." Platters and dishes with hot water pans underneath also permit foods to be kept hot for a considerable time. There are, too, platters and serving dishes made of a composition metal which retain heat for several hours and permit the most satisfactory service of meat, fish, etc., at the right temperature. Ovens with glass doors are said to save fuel by allowing the

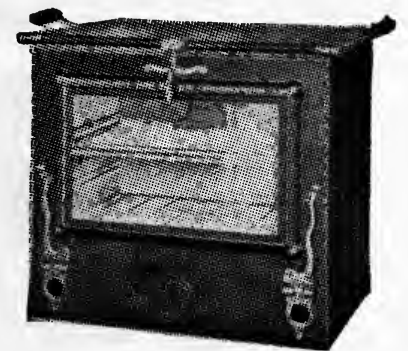

“BOLO” OVEN, ADJUSTABLE T, TWO SIYES BY SHELF IN CENTER, SAVES FUEL food to be watched without needless opening, but some heat passes through the glass as "radiant heat." This could be reflected back into the oven by a piece of bright sheet metal over the glass. Various roasting pans and other utensils are fitted with insulated hoods or covers, all of which save heat by preventing radiation.

\section{Step Savers}

While many devices in other groups also save steps there are a few pieces of equipment which may be called distinctly step savers. Chief among these is the kitchen cabinet 


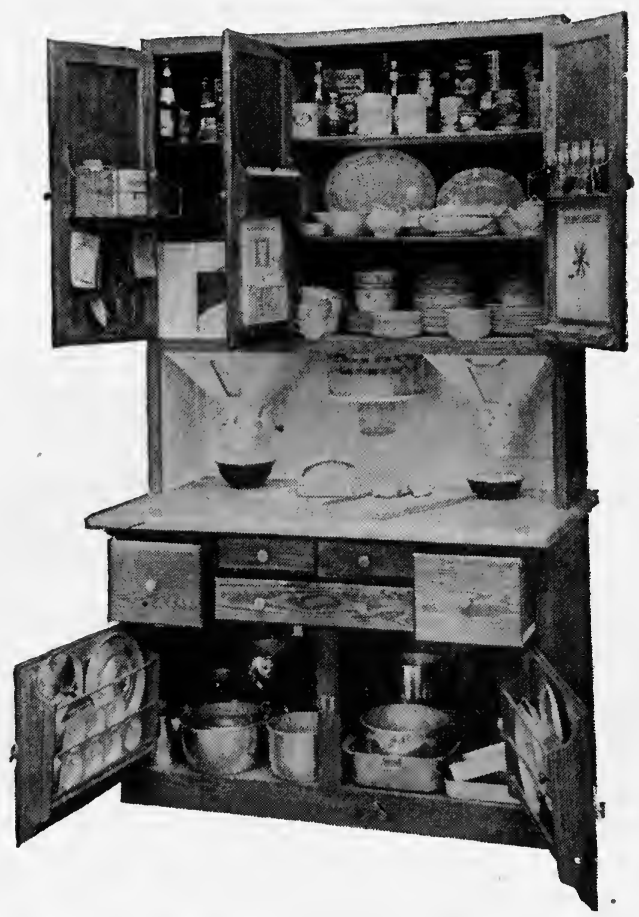

CHIEF OF THE STEP-SAVER GROUP-THE KITCHEN CABINET

which combines a pantry, table and shelf-space into one article of furniture. No one piece of kitchen equipment does more to co-ordinate utensils and working processes than the manufactured kitchen cabinet. The newest models have flour and sugar bins, cereal and spice containers, rack shelf space and adjustable moulding boards. When used with a stool, such a cabinet saves endless steps by grouping within arm's length of the worker both supplies, utensils and tools needed in many kitchen processes. 


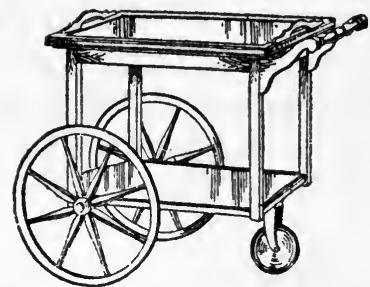

TYPICAL TEA WAGON

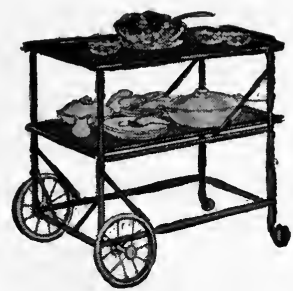

"FULTON" FOLDING CART

The serving tray on wheels is another distinctive step saver. Several models are on the market, some with single, others with double tray, mounted on rubber-tired wheels which can be steered easily. Such a tray enables the homemaker to serve a complete meal with one or possibly two carryings of dishes, or to clear the table with similar ease. This kind of tray can also be used excellently as a stacktable when there is no drain to the right of the sink, or it can be used to wheel clean dishes to the pantry, avoiding constant trips and the dangers attendant on tray carrying. Larger and more massive styles are found in the typical hotel dishcart which can be used equally well in the large household.

The so-called "Lazy Susan" or servette finds favor with the homemaker who is her own maid. This is a revolving

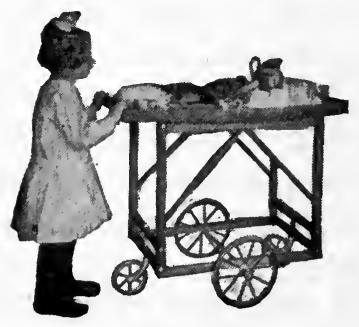

HOME-MADE WHEEL TRAY

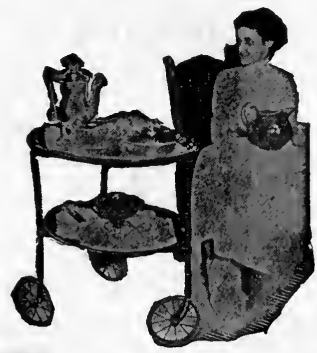

WHEEL TRAY 
circular wooden or glass disk, supported on a stand placed in the center of the table. Foods laid on the disk may be revolved to each person in turn, thus saving "passing," or frequent rising. It also saves space on the table by giving a place to bread and butter, sauces, condiments and other small dishes.

A unique refrigerator most excellent in country homes particularly is a worth-while step saver. This "elevator

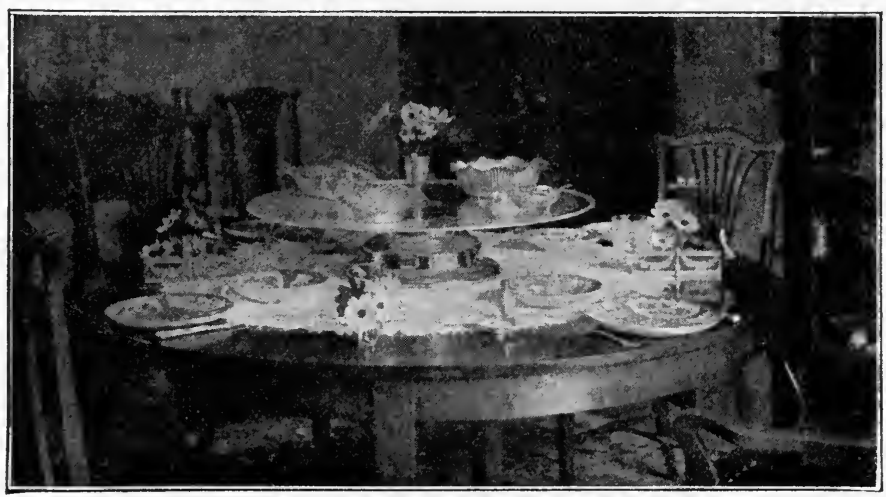

REVOLVING TABLE SERVER

ice-box" looks much like other small refrigerators, has three compartments, but is operated by clock-work pulleys. It is so installed that the pressure on a button in the floor causes the ice box to rise up into the kitchen; or a similar pressure causes its descent into the cellar. This saves the hundreds of tedious steps entailed by the country homemaker who has to keep many food products "down cellar." And if the cellar is cool, this icebox can be satisfactorily used even without an ice supply.

Any other device or equipment which co-ordinates work, such as these: a tool-basket with compartments, a house- 
maid's bucket with places for rags, soap, powder, etc., 'speaking tubes or "house" telephones, etc., can be grouped properly under the important head of step savers, and hence energy and effort savers.

\section{Knives and Cutting Devices}

There is no one more important kitchen tool than the knife, and yet no other tool is so universally abused. A cook can be judged by her knives, and it is indeed rare to go into a kitchen and find either good knives or knives in good condition. Many make the mistake of thinking they can buy a well-made knife at a low price, but it is unwise to purchase an inexpensive knife when it is the most used tool in the kitchen which cuts our bread, prepares our vegetables, slices meat, and without which no meal can be prepared.

The most efficient knife blade for general kitchen cutting is triangular in shape and is called the "sabatier" knife. In moderate size, it will cost 75 cents, and a larger size $\$ 1.00$ or more. For cake and bread slicing a special knife with serrated edge cuts quicker and cleaner than the ordinary straight edge. Even the small vegetable preparing knives should be of the best quality, firmly riveted into the handle, and with points best adapted to their use of picking out eyes, etc. As was suggested previously, the important point about knives is not only their selection but their care. No knife or cutting device of any kind should be ruthlessly banged into a drawer along with nutmeggrater, apple corer or other implements which unquestionably will dull the edge. Strips of leather or grooves of wood can be placed where convenient, and into these knives can be slipped, each having its separate pocket. Small knives and cutting devices in which the metal blade is not exposed 
between the wooden handle at the bottom can be fitted with screw eyes and hung up.

Knives must be kept in good condition by frequent sharpening. Either a "steel" or sharpening stone is excellent if the worker understands how to get the best results, but for many one of the newer knife sharpening devices seems

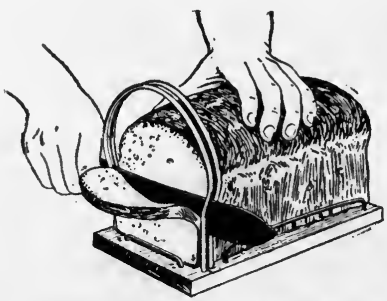

AN EFFICIENT BREAD SLICER

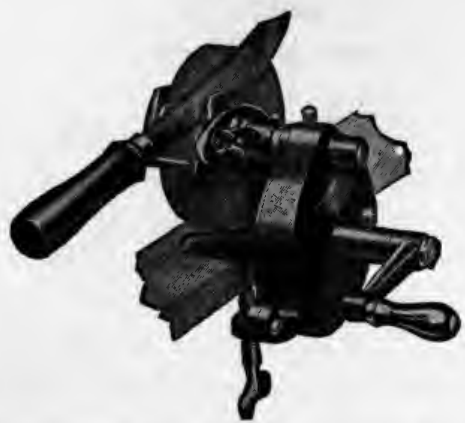

LUTHER HOUSEHOLD GRINDER

easier to use. One consists of a double set of wheels placed opposite each other. The knife blade is inserted between them, and the wheels are set revolving by means of a small handle at the side. These little sharpening devices are clamped permanently on the wall or table and make quick and correct sharpening possible.

Here is a partial list of helpful small cutting devices which save time and make easier the preparing of vegetables and other foods.

Cost.

Sabatier kitchen knife (medium) $\ldots \ldots \ldots \ldots \ldots \ldots \ldots \ldots \ldots \ldots$.. .00

Sabatier kitchen knife (small) ......................... .60

Vegetable parers ................................... $\quad .25$

Serrated breadknife .............................. $\quad .50$

Breadslicer ...................................... $\quad .50$

Medium sized shears................................ 60

Grape fruit knife with curved edge................... ${ }_{50}$ 
Vegetable Scalloper ................................ .25

Strawberry huller $\ldots \ldots \ldots \ldots \ldots \ldots \ldots \ldots \ldots \ldots \ldots \ldots \ldots \ldots \ldots . . .05$

\section{Measuring Devices}

No kitchen is complete without accurate measures. They are necessary to good cooking and scientific kitchen work, and to better marketing and purchasing because they enable the homemaker to check up and co-operate with dealers and manufacturers.

A reliable scale easy to read, should be placed in a permanent position preferably near the preparing table where most measuring is done. The best scale for household use is the so-called pan or hanging scale. This type is preferable to others because here the weight is suspended from the dial and not placed over it. This means that there is less chance of the scale getting out of order, or of being tampered with as is the case where the weight is placed above the spring. Scales of this type come with attractive white enamel pan, glass protected dial, and a convenient hook for hanging. A dial registering only ten pounds is better than one indicating twenty because the clearer lettering and more space to each division permits easier reading of the fractions of the pound.

If the stove used had no heat gauge, an oven thermometer will be required for accurate cooking. Such thermometers come fitted with an easel back so that they can be stood on either shelf or base of oven, and have large white figures on a black background for easy reading. A cooking thermometer of the tube type will also give more satisfactory results in the cooking of finer dishes. A glass graduate measure registering both ounces and tablespoons, and the familiar glass measuring cup and triplicate measuring spoon cannot be dispensed with. Quart and pint measures (liquid) with the funnels an integral part of the measure save time and the washing of separate utensils. A set of 
"dry" measures, peck, quart, pint, will assist in checking purchases and help along the cause of honest weights and measures.

\section{PAPER Products}

No one group of minor kitchen furnishings has done more to make for neatness and sanitation than the increasingly popular group of paper products. The kitchen "roller" can easily be replaced by the paper towel roll in its attractive white holder, lessening the laundry labor and making for increased cleanliness. The same roll or separate "towels" of paper can also be used for draining fried foods, making food containers, wiping up, and in general, taking the place of unsanitary "rags."

Paraffine paper is developing daily new uses in keeping foods moist, wrapping cakes, etc. It may be secured in disks cut to fit any size, square or round pan, as for jelly glasses or cake making, thus saving time. The same disks can be used in connection with paper plates. It is possible by using a fresh disk at each course, to serve an entire meal (except soup of course,) on the same paper plate. The paper plate too, is no longer confined strictly to picnic use, but can be utilized at many summer meals for children, and indeed in most attractive forms at adult meals from time to time. Each season brings new paper dishes, bowls, etc., which are convenient for icebox use. Pies are found equally delicious if baked in a paper plate.

Much has been said about paper bag cookery, and it need only be mentioned here that while not adaptable to all forms of cooking, the paper bag does save labor and pan-washing, and will be found especially helpful in cooking fish and certain meats and other dishes. The same paraffine bags used in cooking are excellent when used in the garbage pail, preventing waste from coming in contact with the 
metal can, thus corroding it, and permitting the entire bag of garbage to be easily moved. This plan was followed most successfully in Boston, and found a great preventive of the fly nuisance.

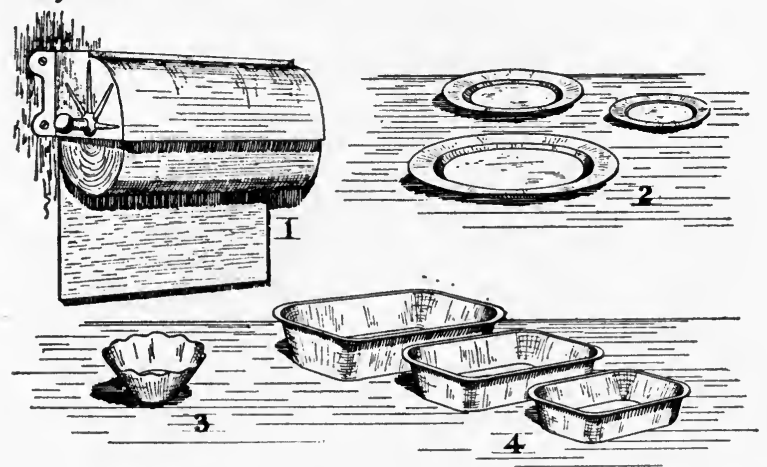

1. Roll Paper Towel. 2. Paper I'lates. 3. Paraftined Sundae Dish.

SOME PAPER PRODUCTS 4. Oblong Dishes.

Paper tablecloths are made exactly resembling damask. Paper napkins also are made in fine quality, and these and the new paper plates which look just like china can do much to reduce laundry and dishwashing for the busy homemaker.

\section{VARIETIES AND PRICES OF PAPER PRODUCTS.}

Oblong deep dishes, I-3 lbs.............. \$I.75 to $\$ 2.00$ per $\mathrm{M}$ Table plates, 5 inches in diameter.............. \$1.95 per 400 Table plates, china finish.................25c per dozen Paraffine disks, 5 to 6 inches.................... ioc per $M$ Paraffine disks, 9 to to inches..................25c per $\mathrm{M}$ Roll paper towel......................35c per 150 sheets Paper tablecloth, $66 \times 72$ inches.......... \$1.00 to $\$ 1.50$ per dozen Paper napkins $\ldots \ldots \ldots \ldots \ldots \ldots \ldots \ldots \ldots \ldots \ldots$. $\$$. 00 to $\$ 2.50$ per $M$ Paraffine paper cooking bags, I5 to 60 bags for $25 \mathrm{c}$, depending on size

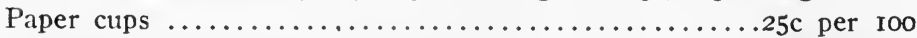
Paraffine sundae dishes......................50 per 100 Paper dish cloths $\ldots \ldots \ldots \ldots \ldots \ldots \ldots \ldots \ldots \ldots \ldots \ldots \ldots$. Paper cake pan linings, round or square.............. 5 c per 50 
There are many minor helpful tools, many of which can be justified largely on sanitary grounds. Others, like a good canner, will do much to save labor and give better results in this important part of food preparation. Still others make for neatness, for skill, or fill other demands of the efficient kitchen. The following list could be expanded indefinitely:

Canning machine.

Sanitary egg holder, prevents breakage.

Milk bottle cap, makes pitcher of bottle and keeps milk clean.

Cream dipper to remove cream from quart bottle.

Cream syphon.

Glass butter and food containers.

Gas lighters, do away with burnt matches, safer, quicker.

The subject of Helpful Tools will be touched upon further in the next chapter on Cleaning and in Food Planning, the Laundry, and elsewhere.

In the endeavor to save time and labor in housekeeping it must be remembered that correct planning, good practice and efficient head work are far more effective than the most elaborate and expensive equipment, without efficient management.

\section{QUESTIONS ON HOUSEHOLD ENGINEERING III}

Helpful Household Tools

I. In your housekeeping at present, which seems most important and why (a) to save time, (b) to save labor, (c) to save money?

2. All things considered, what fuel is best for you to use for cooking in winter? In summer?

3. Your method of washing dishes-how can you improve it?

4. Based on the study of your schedule of work, what would be your next purchase of helpful household tools, if you were free to choose?

5. Tell of your failures in the purchase of household equipment. 


\section{HOUSEHOLD ENGINEERING}

IV 


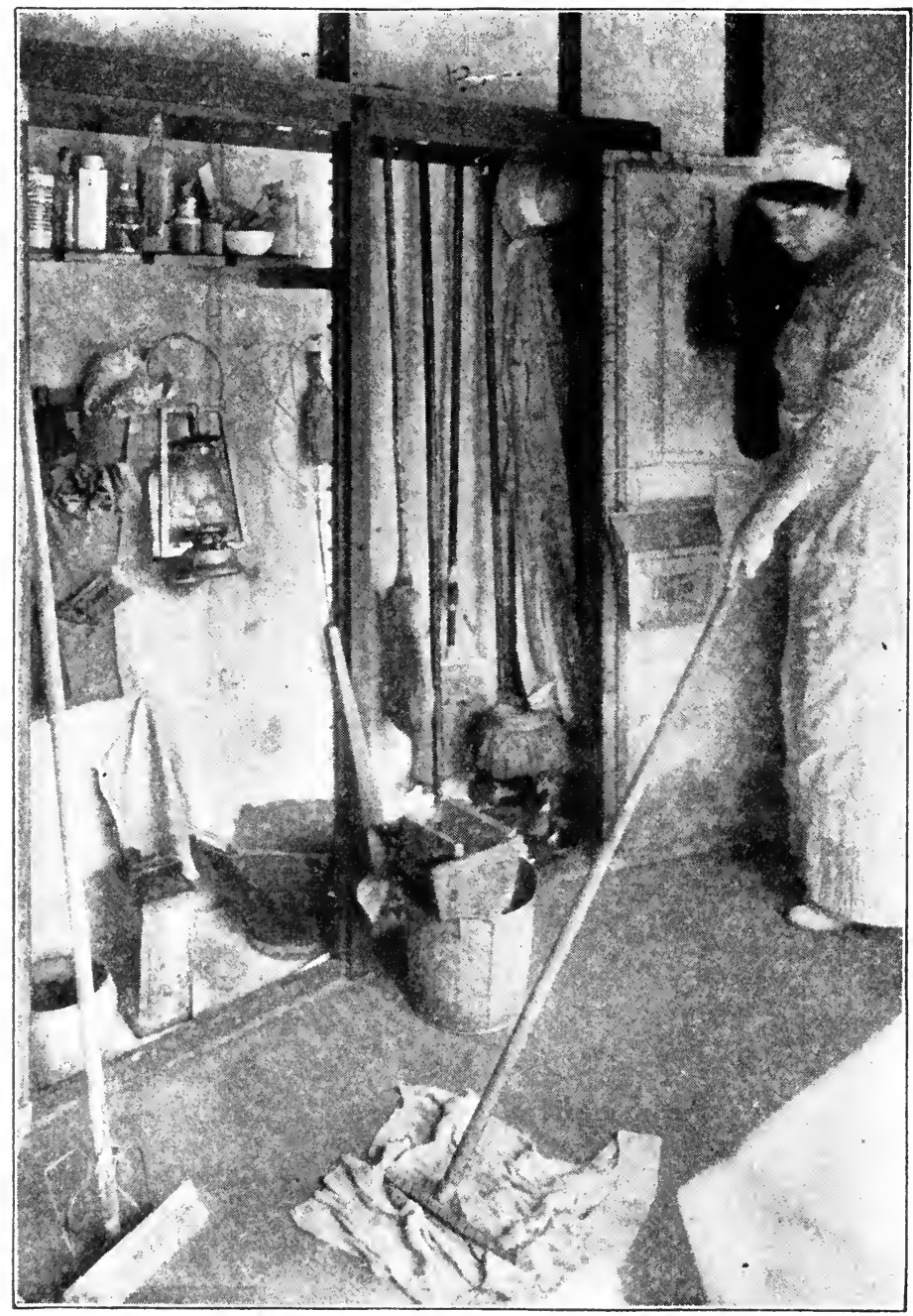

HOUSE CLOSET ON COUNTRY BACK PORCH.

Shelf for bottles and cleansers.

Brooms, mops, etc., on separate hooks.

Bag for string, paper, gloves, etc.

Labels, and a definite place for each article make it easy to quickly find the right tool. Using Oliver cleanser. Note long handled scrub brush and mop wringer. 


\section{HOUSEHOLD ENGINEERING}

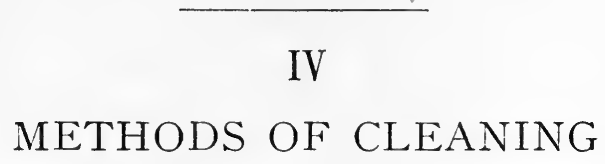

TCREASINGLY high standards of sanitation in the home have made cleaning one of the most important divisions of housework. Probably a house that was regarded as clean a century ago would not be considered "clean" in our modern sanitary sense, which disproves of large carpets, tufted furniture, and an excess of draperies and ornaments. This high cleaning standard results in a definite money expense, and in a time expense on the part of homemaker and houseworker. In many homes, cleaning is one long drudgery, consuming hours in disagreeable and fatiguing work. How can a home be kept satisfactorily cleansed without too great an expense of time and effort?

\section{Cleaning Processes}

First, a clearer idea of the term cleaning must be understood. As generally used, it would appear to be a single piece of work. But, like many other household tasks, cleaning is composed of many different processes, many of them complex and done with different tools and motions, as :

I. SWEEPING-carpets, rugs, bare floors, tufted furniture, etc.

2. Wiping or scrubbing windows, walls, tiles, porcelains, etc.

3. Dusting furniture, woodwork, walls and ornaments.

4. Polishing-silver, brass and other metal objects, utensils.

"Cleaning," therefore, may be any one of these processes, or several of them, and in cleaning the ordinary room we find all of them included. 
Again, cleaning is almost entirely muscular or physical work or exercise more than any other kind of housework. To make a broad comparison, it might be said that the several steps of cleaning can be likened to different athletic exercises, as tennis, ball, rowing, skating, etc. We know that in tennis certain bodily motions are gone through, while in playing ball other kinds of motions are performed. So, too, an analysis of cleaning and its steps shows that each different step is a different kind of muscular adjustment. That is, sweeping (I) with a common broom uses definite sets of muscles in the back and arms with a broad swing and play; again, scrubbing (2), as commonly practiced in a prostrate position, uses an entirely different adjustment of back, arms, and trunk. In the third step of cleaningdusting (3)-there is again a different motion of the body, much less violent, and done chiefly with one arm and a slight stooping. In polishing (4) the worker may either stand quietly or sit, using only the arms. Other special cleaning steps, like beating rugs, shaking draperies, or cleaning walls with a long-handled tool, will require other and differing muscular adjustment or physical motion.

\section{Change of Shift}

The usual method of "cleaning" a room is to perform several of these differing steps consecutively in the same room, changing rapidly from one to another. That is, if a group of rooms were to be cleaned thoroughly, the worker would first sweep, then dust, then wipe floors in one room, and then repeat the four processes in the next room, and so on. This would mean a frequent change from one muscular process to another, or, as it is called, a "change of shift." But a study of this method proves that it is most fatiguing and time-taking. Every time there is a change of shift (from sweeping to scrubbing or from any one step to 
another), there is waste motion and effort. The reason is that it takes time to "speed up" on any one muscular act, and for the muscles to become adjusted to any repeated consecutive motion. In tennis, ball, etc., the first efforts are never as easy, smooth and rapid as when the player has been performing for some time and "gotten into his stride." The same holds true of cleaning, and the more rapid the change of shift from one step to another, the less easy, smooth, and effortless the work. The usual cleaning methods of jumping rapidly from sweeping to scrubbing, etc., can be compared to an attempt to jump from tennis to rowing, and from that to swimming or other sport, and expect to do smooth, rapid work in each. No wonder a worker is "all fagged" when she attempts to work in a way no athlete would follow!

\section{Efficient Cleaning Avoids Rapid Change of Shift}

Efficient cleaning, therefore, depends on avoiding rapid change in shifts of work; or, to put it differently, to continue one cleaning process as long as possible before changing to another. The idea works out concretely in the following way:

Let us suppose that it is general cleaning day on an upstairs floor with four bedrooms and a bath. The usual practice would be to sweep, dust, and mop each room separately, that is, with rapid change of shift ; but let us work the new way, and sweep all rooms through consecutively, next dust them, and last mop them, continuing a similar process through four rooms without a break, instead of stopping in each to change. Similarly, any special cleaning process, like washing windows, should be continued through as many rooms as possible, and one kind of work should not be dropped and another allowed to interrupt it. 
Change of Shift Causes Extra Handling of Tools

Not only did the old method of rapid shift change cause fatigue and lose time, but its second fault lies in the frequent handling of tools necessitated. As was stated, each step of dusting, scrubbings, etc., is done with different tools -broom, bucket, cloth, etc. Now under the old method each change meant change and handling of the tools. Room A would be swept and the broom dropped to pick up the scrub bucket, and this in turn laid aside for the duster. The same handling would prevail in rooms $\mathrm{B}, \mathrm{C}$, and $\mathrm{D}$. In certain house arrangements this extra handling would be very considerable.

Contrast this handling of each piece many times with the handling under the efficient plan: In this case, the broom would be picked up with which to clean Room A, and never laid down until rooms $B, C$, and D had been swept and the broom laid down with one final handling. Similarly, a bucket used in room A could be carried straight through the other rooms. Not until work is closely watched and motions noticed does it appear how much useless time and effort is caused by change of shift in work.

\section{Time Studies in Cleaning}

These facts are clearly brought out in the following time studies of cleaning several rooms under the old and the new plan:

\section{Three Rooms Cleaned Separately}

Preparing Rooms for Sweeping................. 8 minutes Sweeping Rooms.........................21 minutes Dusting Rooms.......................... I9 minutes

Total time............................ $\overline{58}$ minutes

(Three handlings of each tool.) 
Three Rooms Cleaned Consecutively

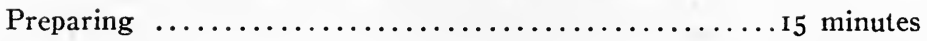

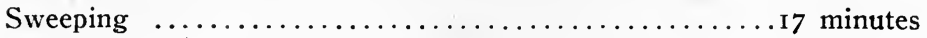

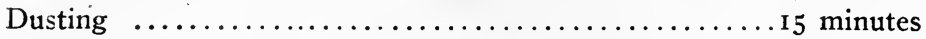

Total time.......................

(One handling of each tool.)

This rule of change of shift and its results apply particularly to such tasks as window washing, where exactly similar motions are done with each successive window. Nine windows cleaned consecutively take less time than nine windows cleaned intermittently with stops for other work. Several beds made one after the other (witness the sleeping car porter!) can be made in less time, each, than if the worker makes a bed, and then stops to brush up or do other work. It follows, then, that such tasks as windows, airing of bedding in several rooms, shaking rugs, etc., should be done in as "wholesale" a method as possible. The only exception to this avoidance of shift-change may be when rooms are so far apart, or on different floors, that the extra walking entailed might be as time-taking as the old method. The fatigue is always less when work is uninterrupted. Change also causes nervous adjustment, and it is not so much the work itself, which causes the fatigue, as it is the "jump" from one kind of work to another. This has been quite conclusively proved in various tasks by different workers. Therefore the woman who wishes to work with the least friction will avoid such planning that will force her to do too many kinds of work on the same day, or too many kinds of tasks in the same hour.

\section{"Standard Practice"}

Each cleaning task should have its own set of directions or instructions, since each step of cleaning is done with different tools and in a different way. This "standard" or 
best way, these rules, can be called "standard practice," just as in industries we have written rules for the way this piece of work or that should be done. We have long used "standard practice" or definite instructions in cooking, as in recipes and the kind of bowl, spoons, etc., to use. The reason why our meals are so often better cooked than our rooms are cleaned, is almost solely because there have been no written directions or "practice" for the latter, while there was for the former. There cannot be a properly cleaned room if some one step is forgotten, any more than there can be a properly made cake if some needed ingredient is forgotten.

Standard practice means, then, written directions as to method and tools, and time. While these directions may vary slightly owing to the different construction and furnishing of homes, these following few "standard practices" will help in making such as will exactly suit the particular needs of any special home.

\section{Standard Practice on Bedroom Care}

Tools-Carpetsweeper; long handle dustpan; dustless duster; long handle string mop.

Methop-I. Pick up clothing, shoes and soiled linen (beds thrown back in advance, windows open).

2. Make bed, leaving valance, if any, tucked up.

3. Remove burned matches, papers, etc., from bureau, table, etc.; arrange and dust toilet articles, placing waste in dustpan box.

4. Sweep rug with carpet sweeper.

5. Dust furniture, window and door trim, and baseboard edge.

6. Use string mop around rug edges and under bed, replacing chairs, etc., at same time; let down bed valance.

7. Pull shades to half, allow sufficient ventilation.

It would be easy to modify this "standard" if there were a fireplace in the room and a hand washstand; in that case the "standard" could be amended: 
Remove ashes and lay wood for fresh fire (to precede step 3). Scour basins and bring fresh water (to follow step 3 ).

In this way a "standard" for any given set of conditions can be worked out simply by setting down in the best order the various steps which are needed to do the entire work in the most satisfactory manner.

\section{Standard Practice on Bathroom Care}

Tooss-Long-handled toilet brush or tongs.

Impregnated metal polish cloth.

Cleaning cloth and cleansing powder.

Wet mop and bucket.

Disinfectant, soap, linen.

Mетнор-I. Remove soiled linen and bring new supply, also soap, paper roll if necessary, and.lay on convenient chair.

2. Clean windows and medicine closet mirror and light fixtures.

3. Wipe window ledge and exposed woodwork, including baseboard.

4. Rub faucets, towel bar and other metal parts with impregnated metal cloth.

5. Clean bathtub, then washbasin with cloth and cleanser, and last, the stand supporting toilet.

6. Wipe floor with mop, being sure to get under tub.

7. Empty pail into toilet, flush; clean with toilet tongs, flush again; pour down disinfectant and replace lid.

8. Lay linen, soap and other supplies in place.

Standard Practice for Cleaning Several Rooms (On one floor)

Conditions-Large rugs, hardwood border, 4 rooms opening on central hall.

Tools-Carpet or vacuum sweeper; long-handled dustpan, string mop; dustless duster for furniture; flannel duster for ornaments and glass.

MEтHOD-Open windows top and bottom about one foot, carefully pin back draperies. 
TRIP :-I. Assemble all tools at entrance of room A; take both dusters and dustpan in hand; pick up waste into dustpan while dusting and replacing ornaments and tops of tables, bureau, etc., and dusting baseboard, door and window trim and exposed woodwork. Do this in rooms A, B, C and D, returning to Room A entrance and exchanging for sweeper tool.

TRIP 2-2. Use sweeper in rooms $\mathrm{A}, \mathrm{B}, \mathrm{C}$ and $\mathrm{D}$, returning and exchanging for string mop, using this on all rooms as before. Return with sweeper and exchange for string mop.

TRIP 3-3. Use string mop in rooms $A, B, C$ and D; return to entrance $\mathrm{A}$ (arranging furniture if necessary).

LAST STEP-Gather all tools from entrance A and carry direct back to house closet.

Under these conditions, dusting precedes using the suecper, so that any dust or waste from shaking and handling books and ornaments may fall on the floor and be swept up.

Sweeping precedes mopping with oil or string mop, so as to avoid any tracking on the nicely oiled floor. Either with carpet sweeper or vacuum cleaner this is the best order:

I. Dusting.

2. Using sweeper.

3. Using oil mop.

If window cleaning must be included on this day (although it should not be included, if possible) it should be (lone after dusting; if the walls and pictures need thorough cleaning, they should be done before dusting. In other words, it is only common-sense, as well as efficiency, to perform first those processes which cause moving and shaking of objects. In general, the work must be done from the ceiling down, as:

I. Ceiling.

2. Lighting fixtures.

3. Pictures and mirrors, hangings. 
4. Ornaments and books on tables and horizontal surfaces.

5. Furniture (including window, door and baseboard).

6. Rugs or carpets on floor.

7. Exposed wood on floor.

If a wet process like scrubbing must be done, it should follow the rule for floor cleaning, and thus follow the carpet cleaning.

It is thus seen how easy it is to make "standard practice" instructions for any given set of conditions. This "practice" had best be written down when once worked out, and not left to the memory. It must include, as shown above, the tools, the method, and the time. This last element can easily be found after the method has become mere routine. It may be, "Bathroom, 25 minutes," or bedroom, "I5 minutes," or whatever the case may be. In this way the "standard practice" becomes a means of developing a good schedule.

Copies of this "practice" may be written on cards to be pasted or tacked in an inconspicuous part of the room, or included in the instructions to a hired worker in her instruction book.

\section{Place for Cleaning Tools}

Just as a special place like a kitchen cabinet is needed for keeping kitchen tools in order, so there must be a definite location for the equally important tools of cleaning. Such a place is the so-called "house closet." This may be small or large, specially built or developed from the waste space in a back stairs, etc.

In country homes an excellent place for such a closet is on the back porch or at the head of cellar stairs or between kitchen and washhouse. The closet should be high enough to accommodate long-handled brooms and mops hung up, with additional space above for a small shelf. Good dimen- 
sions are 6 feet high by 4 feet wide by I foot deep. This permits the floor space to conveniently hold coal hods, scrub buckets, etc. (See page 146.)

Each tool should have a screw eye of the right size put in the handle; then in the strip under the shelf right angle up-hooks can be placed at convenient distances apart, on which the tool can be hung by its screw-eye. On the shelf can be placed bottles and boxes of cleaning preparations.

The closet is not complete without a label marking the right place of each tool. If these are pasted on and then shellacked, they will stay in place for years. A shoe-bag with different sized pockets is excellent for holding dusters, twine, newspapers, cleaning gloves, etc. Even in such a closet the grouping of tools can be carried out, and duster, dustpan, and mop, or other combination, hung together so that they can easily be picked up at once.

\section{New Versus Old Idea in Cleaning}

If one general term could be applied to the manner of all cleaning up to the present, that term could well be "scattering"; for in all the various steps of sweeping and dusting, the work was done in such a way as to scatter and spread the dust particles (and bacteria) from one place to another. The corn broom swept the dust out of the carpet, only to raise it in the air so that it lodged on pictures, mouldings, etc. Again, the feather duster removed the dust from these same pictures only to have it fall on the floor, and thus went on a continuous cycle of dust which was never entirely eliminated from the house after all.

The new sanitary ideal today has for its watchword "absorption." The broom is being largely replaced by the suction method of the modern vacuum cleaner, and the $d u s t-$ less duster holds dusts as it cleans. No one invention is so responsible for new cleaning methods as is the so-called 
vacuum cleaner. The principle on which these are built is that of suction, or the intake of air. This suction is developed in various ways, and takes with it the dust as well as air from whatever surface the cleaner is operated on. There are four broad types of cleaners as follows:

(I) Large portable vacuum cleaners, dustbag within the cleaner; hand or electric.

(2) Small vacuum cleaners, dustbag outside the cleaner; electric.

(3) Carpet sweeper, box model vacuum cleaner (hand type).

(4) Stationary machines, located in basement, with pipes to all floors.

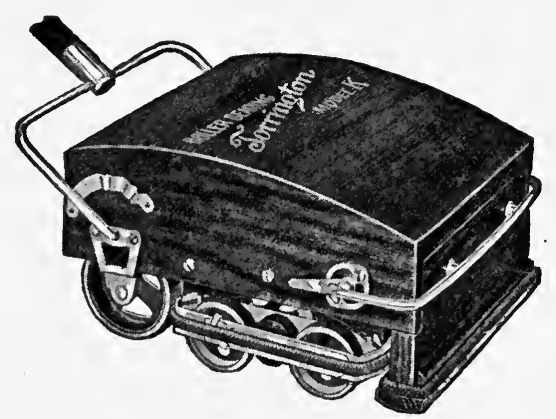

SWEEPER TYPE HAND VACUUM CLEANER COMBINED WITH CARPET SWEEPER.

There is a slight distinction between vacuum cleaner and suction cleaner. In the vacuum cleaner, a diaphragm or rotary pump is used to create a partial vacuum in each stroke. The surrounding atmosphere then rushes in to fill this vacuum, bringing in dust. At the end of each stroke, when operated slowly, the vacuum model loses "pull" for an instant, but this is not noticeable at full speed. This type of cleaner can make a very strong pull. 
In the suction type of cleaner using power, the suction is created by means of fans or turbines operated by a small motor encased in some portion of the cleaner. These fans in revolving cause an intake of air, bringing with the air dust, small fragments of paper, matches, etc. In this type of cleaner the "pull" is not very strong, but a large volume of air can be moved, permitting the use of a wide opening. In the suction type of cleaner operated by hand, the suction is created generally by a pair of bellows which, in being shut, caused a similar but less violent suction of air and intake of dust. In the hand type, however, the effort of the operator is needed to make the necessary power. In the suction type also there is a continuous flow of air, while in the vacuum type there is a distinct "pull" for an instant, as mentioned above, at the end of each stroke.

The large portable vacuum cleaner (electric) is made with a powerful motor and is particularly suited to cleaning large houses or where there are many thick, all over rugs, carpets, and a quantity of draperies. This type of cleaner is always used with a hose attachment inserted in the intake opening. At the other end of the hose is what is called the nozzle opening, which is the actual part of the cleaner moved over any given surface. This nozzle tool may be a tool for the floor, for mattresses and tufted furniture, for draperies, etc., etc. The price of such a large portable cleaner is about $\$ 75.00$ because of the cost of a powerful motor.

The large portable vacuum cleaner (hand) is operated by an air pump and lever, and preferably is worked by two persons, one to pump and one to move the hose attachment wherever necessary. In both of these portable type machines, the dustbag is within the body of the cleaner. This hand portable when operated by two people does almost as good work as any electric machine, but the labor is consid- 
erable. It comes fitted with all similar attachments, and costs from $\$ 20.00$ to $\$ 30.00$.

The small portable cleaner (electric) is made in many models and seems the best type suited for average use in homes wired for electricity. With this type, the dustbag is on the outside, the machine is light in weight, and can be

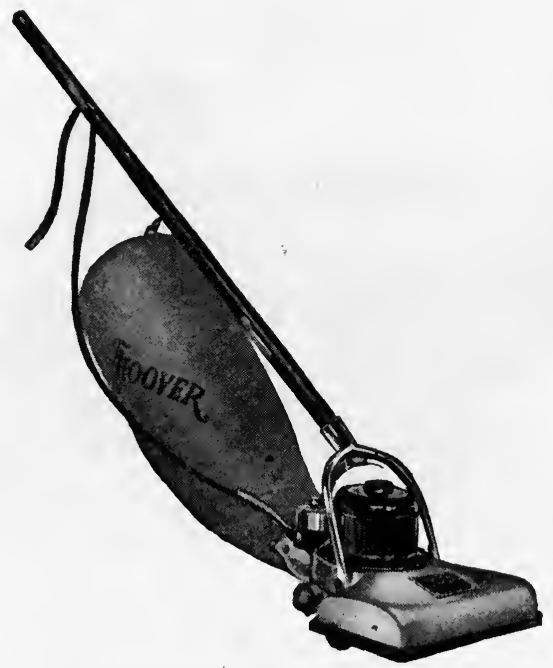

SUCTION SWEEPER WITH MOTOR DRIVEN BRUSH.

operated on carpets and floor without a hose attachment. The hose is necessary here only to clean draperies, mattresses, etc., and anything off the floor level. The advantages of such a machine are that it can easily be taken up and downstairs by a woman, that it rolls easily, that it takes little storage space when not in use. The price of such a cleaner is from $\$ 30.00$ to $\$ 45.00$. 
The sweeper type of cleaner is operated by hand. It consists of a box built like a carpet sweeper in which is contained a bellows. These bellows are operated whenever the cleaner is moved backward or forward over the carpet, the suction created drawing the dust from the carpet. No attachments can be used with this type, which is strictly for floor use and is suitable only for carpets and large rugs.

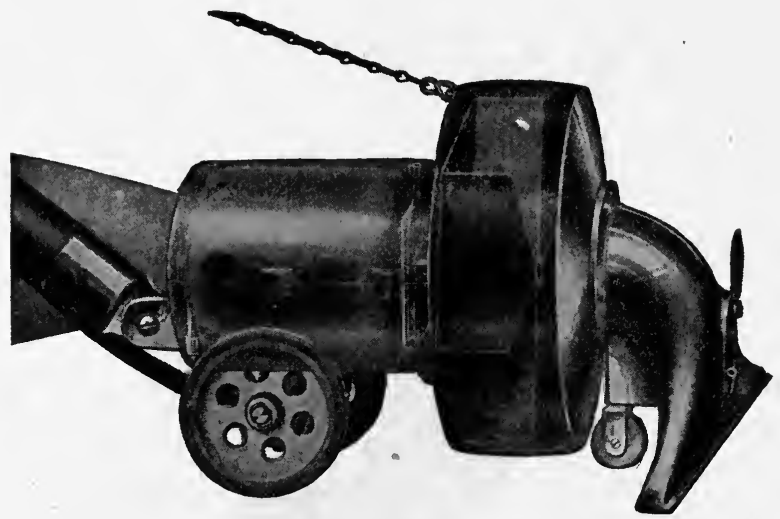

ELECTRIC VACUUM CLEANER, SHOWING FAN

It does not clean bare floors well. This type is somewhat heavy and fatiguing to work in comparison to the usual carpet sweeper. In some models of this box type a sweeper just like a carpet sweeper is combined so that the carpet is swept at the same time that dust is sucked out of it. They cost from $\$ 5.00$ to $\$ 12.00$.

While the powerful electric machine and even the small, light machines suck dust from a given surface and pick up lint, matches, etc., most of them do not pick up threads, crumbs, or, in other words, brush the carpet at the same time that they clean it pneumatically. In order to provide 
for this picking up of threads, many cleaners, both large and small, are fitted with brush attachments either within the body of the cleaner or mounted without on a small platform. It is well to note this point in a cleaner before purchasing.

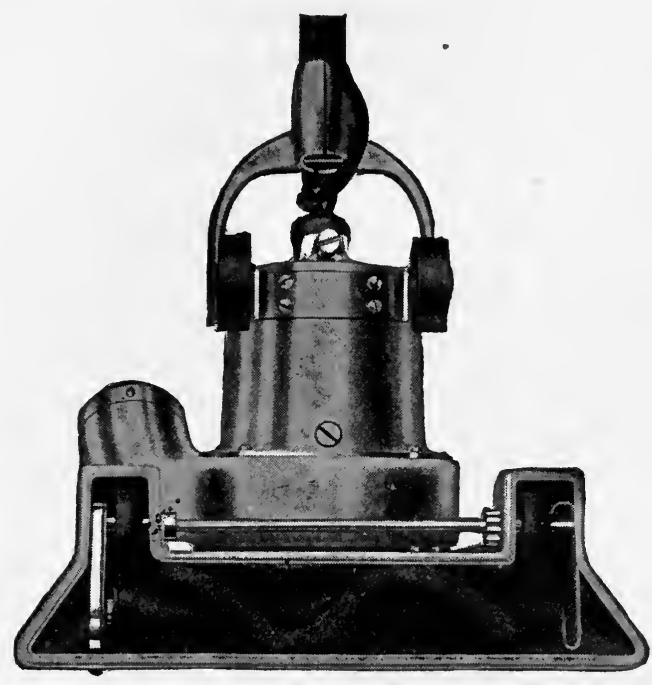

SHOWING REVOLVING BRUSH OF ELECTRIC CLEANER

\section{Suggestions on Buying Vacuum Cleaners}

The first thing to decide before purchasing is the conditions in the home in which the cleaner is to be used. If there are large areas of all-over carpet, many draperies, etc., a powerful portable type will be needed. If small rugs, matting, and bare floors, the light-weight electric cleaner can be used. If it is more important to have the draperies, tufted furniture, etc., cleaned by this method, select a machine that has a direct inlet to the fan-chamber instead 
of an attachment that clamps to the floor suction nozzle. In all cases, select a cleaner having a wide suction nozzle, about Io or 12 inches, so that there will be enough width in the cleaner nozzle to clean a sufficient width at one time. Aluminum parts and case make the lightest cleaner to move and use. The cleaner should be mounted on rollers so that they can be moved readily. The electric machine should be equipped with a small switch on the handle so that it is not necessary to reach up to the light socket to turn the current on and off.

Note the details of bellows construction, the materials of the dustbag and general construction before purchasing. It does not pay to buy a "cheap cleaner" which will become worthless in a short time. The cost of the electric models depends on the power and quality of the motor. Know also the voltage, for while most cleaners come fitted to a IOO to I2O-volt socket, the voltage should be known before buying.

Never attempt to run an electric model over a damp place or allow it to suck up water, as this will spoil the motor. In operating the cleaner, be sure to elevate the suction nozzle at the right height from the surface. This may make all the difference between fair and excellent cleansing.

\section{Permanent Vacuum Systems}

There are two types of permanent vacuum systems, one operated by electric motor, and the other by a motor operated by water pressure. In this case pipes are laid in the walls, having openings near the baseboard of each room, and connected with the motors in the basement. It is then only necessary to attach to these floor openings the hose, and thus clean all rooms easily. The present cost of such systems is fairly high; but in new houses they are worth considering, as such systems certainly make for "dustless 
homes." Many hotels and institutions are so equipped, and the cost of installation is balanced by reduced worker's and cleaning women's cost.

The water motor system costs much less than the electrically operated motor system. It, however, needs a pressure of about 40 pounds to the square inch in order to be satisfactory. The cost of installation of electric systems is from about $\$ 200$ up, depending on the number of rooms.

Time Study on 9xi2 Rug by Different Methods

Minutes

Broom sweeping (outside on porch) $\ldots \ldots \ldots \ldots \ldots$. Io

(Moving to porch and replacing, 6 minutes)

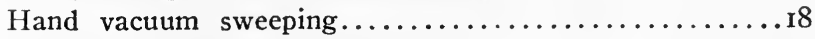

Electric vacuum sweeping..................

On Small 4x6 Rugs

Minutes

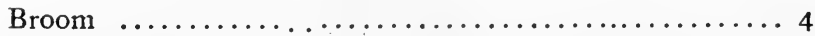

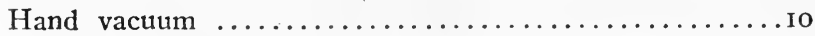

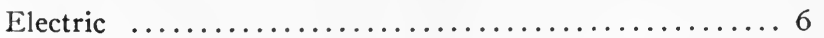

From the point of time, the vacuum cleaner gives little if any saving, the chief advantage being only in the absence of the scattering of dust, and in the somewhat greater thoroughness of the work, and the fact that the rugs need not be lifted from the floor. It is the most labor saving where there is a large rug, or stair treads covered with thick carpet which it is almost impossible for a broom to get at. On bare floors the hand vacuum cleaner is almost totally useless; the electric models, even when fitted with a floor tool, do not do the work as easily as a long handled string mop. It is on carpets only that the vacuum cleaner is most worth while. Small rugs where there are large bare floor areas can be as 
easily cleaned of surface dirt with a carpet sweeper. The thorough suction cleaning given by a vacuum necessitates less frequent cleaning, which is the great advantage.

In the time studies given above, the amount of effort is important; it took less effort to clean the rug by broom than by hand vacuum cleaner. In the electric cleaner test, the effort was much less than in either the hand vacuum or the broom method.

\section{The Handled Versus the Short Tool}

Another great broad difference between the tools of to-day and yesterday, is that the modern ones are generally mounted on handles. The scrub brush, the hair brush, the many kinds of fibre duster tools, even the dustpan, have at last been elevated by being placed on handles. Why stoop to the dustpan, when it can come to you? Why use a bundle of coarse cloths on a floor to mop with, and bend prostrate over the task, if you can fasten the same cloths on a stick and get better results?

"But I can't scrub so clean if I don't get right down on the floor," some women may say. We believe this is only imagination, and a habit of working, rather than the facts. There is practically no cleaning tool which cannot be mounted on a handle and give better results than the same tool used in the hands. This is because the "handle" comes under the laws of the lever. By test it has been proved that a handle, even a short one, acts as a fulcrum or point of leverage, so that greater pressure is exerted by means of a handle than if the hand were used direct.

Some interesting experiments were made on lengths of handles of cleaning tools. Three women of varying heights, $5 \mathrm{ft} .3$ in., $5 \mathrm{ft}$. 6 in., and $5 \mathrm{ft} .8$ in., were tested and grasped the handle as follows: 


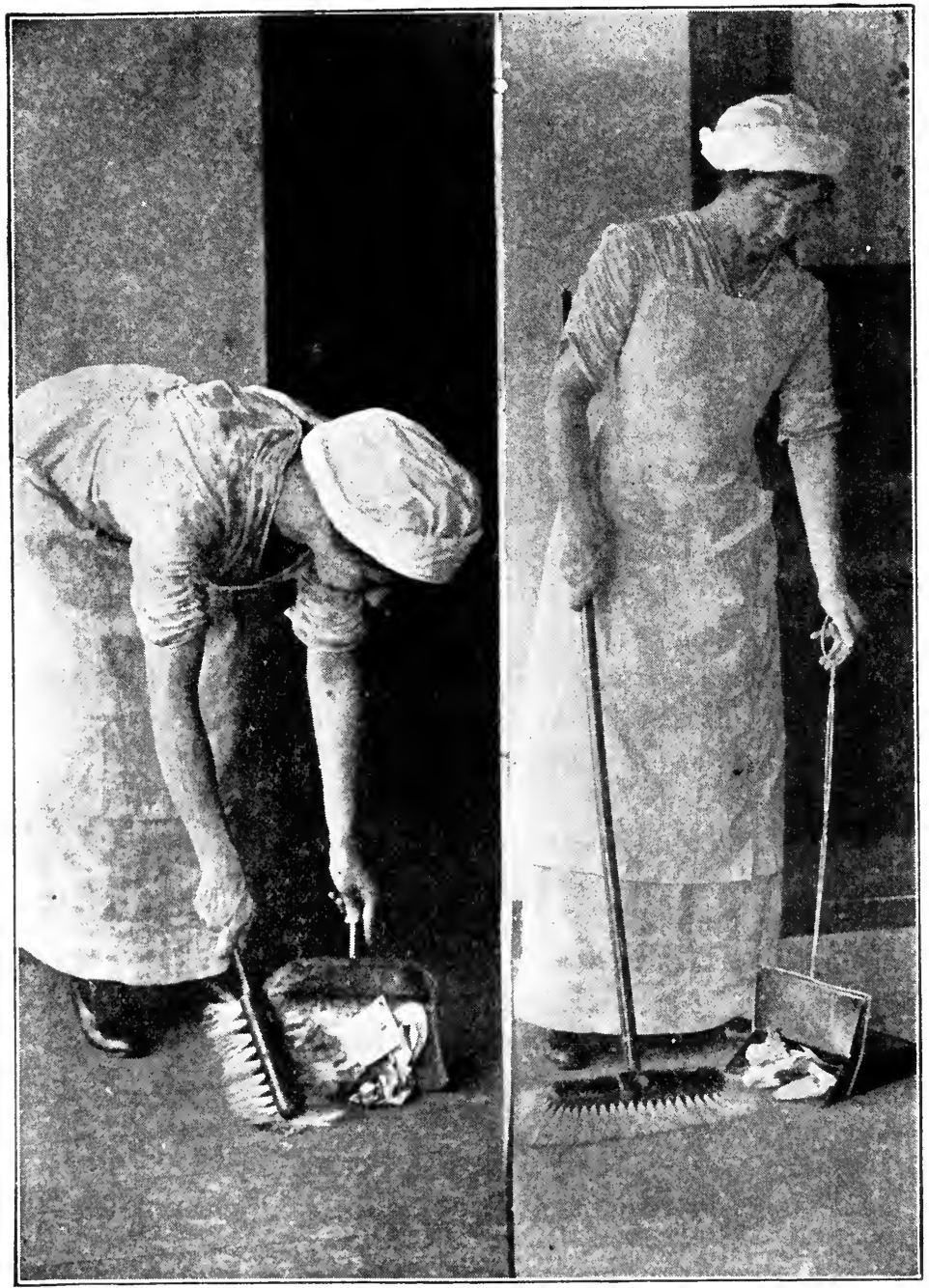

USING THE SHORT HANDLED AND LONG HANDLED DUSTPAN "Why stoop to the dustpan when it can come to you?" 
WoMan A5 feet 3 inches

Long handle scrub

brush ........... I foot $I$ inch from end
WOMAN B5 feet 6 inches I foot 6 inches I foot 9 inches from end
Woman C5 feet 8 inches from end

Short handle scrub brush .......... I foot 4 inches I foot 9 inches I foot 10 inches from end from end Oil mop $\ldots \ldots \ldots \ldots$ I foot 5 inches I foot 6 inches from end

Broom .......... I foot 5 inches
I foot 6 inches from end I foot 9 inches from end I foot 8 inches from end

From these figures and other data in regard to the position they took as they worked, it appears that each height of worker has a fairly constant distance apart at which she uses her arms, no matter what the length of the handle of the tool. Now if the handle is short, the worker has to stoop over to get into the particular position in which her arms remain the proper distance apart; if the handle is long, her hands assume this comfortable distance without stooping.

Again, the angle at which a long-handled tool is used has much influence on the amount of pressure exerted on the mophead or whatever it may be. If the mop or broom could be held straight down, the same amount of pressure exerted at the handle would be given to the mophead. The further the mophandle inclines from the vertical, the less its pressure. Now we see where the real benefit of a long handle comes in ; it permits the worker to exert at a standing height the same amount of pressure that she would have to bend greatly to exert, if the handle were short.

The facts to remember in order to make cleaning more efficient are:

I. Any handled tool should preferably be 5 feet or over in length. 
2. The straighter the handle is held to the vertical, the greater the results with any given amount of pressure.

3. Grasp handles with one hand as far down the handle as possible-this gives a longer "force-arm" to the handle, and hence greater pressure on the mop itself.

One hand is used chiefly to direct the handle, while the other, sometimes right and sometimes left, depending on individual cases, gives the real force.

By the use of the handle, then, it is possible to sweep, mop, dust, and scrub, upright and with less effort, the same work done with a short handle or none at all. The longer the handle, the straighter the position of the worker, the further down the force is applied-the easier and more efficient will be the work.

\section{Importance of Proper Clothing}

As has been pointed out, "cleaning" is exercise to a greater or less degree. Why not, then, dress, as if it were exercise, in order to receive the maximum benefit from the work? No heavy skirts, "pull" at the arms, tight waists or shoes can result in comfortable work. While much may be said in favor of the one-piece dress, because of its neat appearance, it has been found that a two-piece garment gives more freedom if planned right. Such a dress is a modified "jumper" consisting of a plain, short, gored skirt and a jumper waist like a middy blouse. This allows very great freedom of movement to both waist and arms such as needed in wiping, stooping, etc. Since the waist of this dress extends four or five inches below the natural waist line, it permits a great deal of bodily motion without exposing the belt. It should have elbow sleeves and low collar, and can be made in seersucker, chambray, or similar materials.

No detail is more important than the kind of shoe, and 
while it may seem economy to wear "cast-off" best shoes at work, it generally brings poor results in causing sore feet. Only a broad, low heel, preferably of rubber, or a rubber soled "athletic" shoe or.a "nurse's" shoe should be worn by one doing much cleaning, and who does not wish to risk a "turned ankle" at rapid work.

A cap is also a needful accessory, keeping the hair from coming loose about the face, as well as serving as a dust protector. Emphasis is here put upon the kind of clothing because right, neat clothing affects one's self-respect. The reason why many women think of cleaning and similar tasks as degrading, even, is because in the past the worker looked like a "slavey," and was so uncomfortable about her appearance that it made the work drudgery in her mind. It seems, too, that a worker does neater, more careful work if she is neatly attired and avoids doing careless, sloppy work; while if dressed in a slovenly manner, her work is slovenly too, because she "doesn't care how she looks." It is possible to do even mopping and cleaning without becoming a "sight." Training in working in an efficient manner results in a neater appearance, and vice versa. It is not how much we get done, but how well we get it done, with comfort to ourselves and others, that means true efficiency.

There is also the "bungalo" dress, which is merely a large allover apron with kimono sleeves, buttoning in the back. This has been found very neat and serviceable as a cleaning garment, because it permits little clothing used under it, and a great deal of arm movement. Another dress found desirable is a "reversible" dress which fastens with only one button! This is made in one piece, of a comfortable short sleeve type, and buttons at the belt. It can be reversed and worn on the other side, if desired. Its advantage is the easy way in which it is fastened. 
So many women say, "I hate housework because it is so hard on my hands!" It is, however, possible to preserve the nice looks of the hand considerably by the use of gloves of various kinds. The following have been proved worth while:

Rubber gloves-dishwashing, toilet cleansing, washing baby napkins. (Avoid using with grease; good pairs cost \$1.)

Large white cotton "teamster's" gloves-for sweeping and dusting; for silver and metal cleansing; while using many kinds of devices in the hand. (Cost Io cents a pair.) Grease the hands with cold cream before using.

Yellow oilskin-may be used instead of rubber as they last longer. For same purposes and also as sweeping gloves. Cost about 5o cents.

Rub cold cream on the hand before beginning heavy cleaning, slip on gloves. After work, rinse hands, and rub in an astringent like benzoin water.

\section{Importance of Posture}

Cleaning is exercise, but even exercise must be done properly. It is possible to twist and contort the body unnecessarily. The use of long handle tools will allow the worker to stand more often than stoop. But there is even a best way to stoop, and that is from the waist and not with the back. Go upstairs on the ball of the foot, and not the heel. Expand the chest even while using the arms with broom, mop, etc. Never prostrate and shake the body as in usual floor scrubbing, but choose some tool that will permit standing work. Pails can be carried with less effort if the body is rightly balanced. Tool handles can be grasped more handily, so that the hand is not made misshapen and awkward, just as there is an easy, graceful way of handling a table fork and knife, and one that is awkward. Try and 
assume comfortable positions. Train the hands to quick, deft action, even in picking up floor cloths, grasping handles, levers, etc. In this way the highest results and exercise will replace drudgery.

\section{What Is a Good Cleaning Outfit?}

The needs of homes differ owing to their differing furnishings; the following list covers the needs of many homes in a complete and yet not expensive way. If certain tools, as vacuum cleaner, are included, this will dispense with tools doing equivalent work. (Pre-war prices are given.)

\section{SWEEPING AND DUSTING TOOLS}

I. VACUUM or Suction Cleanfr for thorough carpet, Approximate floor and drapery cleaning.

Price

Electric models ................... \$25.00-\$75.00

Hand models ( 2 persons).............. 20.00 and up

2. Carpet Sweeper for threads, and surface cleaning

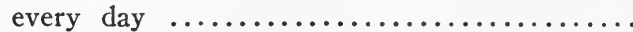

3. Long Handle (5 feet) HaIr Broom for exposed wood, tile and linoleum sweeping.......... I.50

4. Short Handle (3 feet) Hair Broom for sweeping fireplace, picking waste into dustpan.........

5. Dry or Oil String Mop for dusting or oiling wood floors $\ldots \ldots \ldots \ldots \ldots \ldots \ldots \ldots \ldots \ldots \ldots \ldots \ldots$.

6. CoRN BROom-only for sidewalks or coarse porch floors, etc., or rugs swept outside............

7. Wall Brush of Fibre or Pulled Cotton-long handle, to clean walls, mouldings, ceilings, pictures etc. when no vacuum cleaner is used...

8. Short Handle Fibre Brush for dusting stairs...

9. Dustless Dusters for furniture and woodwork.

Flannel or silk duster for ornaments, piano,

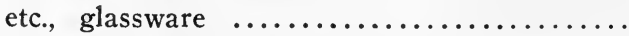

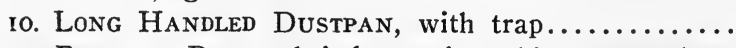
3.50 and up

.75

.75 and up .45 and up

I.25 and up .50 .25 each .50

I1. RADIATOR BRUSH, bristles set in a thin, narrow handle for cleaning between radiator pipes...... 


\section{MOPPING AND WET CLEANING TOOLS}

1. Oliver Sanitary Cleaner-long handle with rubber grip at base to hold mop cloth; excellent for cleaning tile, linoleum, etc.........\$ 1.25

Approximate Price

2. String Mop for coarse work on porches, cellars, etc.; not needed generally if the "Oliver" is used

3. Combination Scrub Pail and Mop Wringer. One motion of foot wrings the mop head. Only needed if string mop is used...............

4. Common Papier Mache Bucket, light weight.

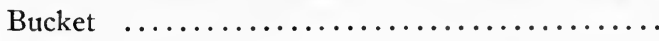

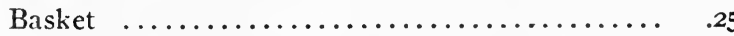

Use sponge and soap basket on edge.

5. Scrub Cloths of knitted heavy crash-22x22 is a good size

(Such cloths are much better than "cast-offs" and rags, which are hard to clean, and do uneven work.)

6. Handled Scrub Brush-regular scrub brush on 4 -foot handle $\ldots \ldots \ldots \ldots \ldots \ldots \ldots \ldots \ldots \ldots$

7. Window WASHER-contains water, and is fitted with both rubbeir edge and felt dryer-mounted on long handle. Useful for many high, outside window work................... I.00

8. Windowclean Cloth-inside work, needs no water, and replaces chamois for mirrors, globes, etc.

9. Bathroom Tongs or Brush. The tongs are more sanitary and, used with paper clean the toilet bowl. A long handled brush is necessary if tongs are not used.

Brush

Tongs

\section{POLISHING AND CLEANING TOOLS}

I. Silverclean PAN - cleans flat ware and small

Approximate shaped pieces in shorter time.........\$ 1.00 and up 
2. Impregnated Cloths for Metal-one kind for brass, another for nickel. Use instead of pastes and powders. Always wear gloves when using

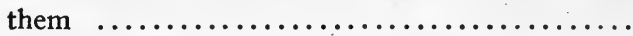
.25 each

3. Polishing Mitr-for use on stoves and other dirty work. Shaped to the hand, and white wool on one side

\section{ACCESSORIES}

Approximate

Price

I. Combined Stepladder and Chair...........\$2.00

2. BROOM-HOLdERS-small devices which permit han-

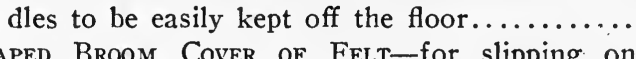

3. Shaped Broom Cover of Felt-for slipping on corn broom in place of string mop..........

4. Paint Brushes-of several sizes for getting into stair corners, brushing wicker, etc...........

5. Putty KnIfe-This triangular tool is helpful in cleaning baseboards, angles, and for general

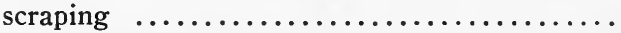

6. Galvanized Iron Strip, $6 \mathrm{x}_{3}$ inches. If this is held flush with baseboard or window trim it will prevent paper from being soiled while wood-

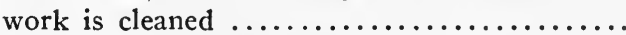

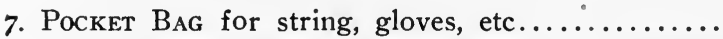

8. WATERPRoOF Apron, for doing heavy or unpleas-

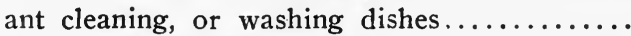

9. RUBbish BURNer of wire.

.1o each

.15 and up

\section{SUMMARY OF CLEANING VARIOUS FINISHES}

LINOLEUM-Wash with tepid water and naphtha suds; rinse with clear tepid water and dry thoroughly. Avoid water seeping under edges, which causes rotting. Do not use brush, but soft cloth. It can be successfully waxed, which preserves it and makes it easier to care for.

MATTING-Sweep with hair broom; vacuum cleaner is particularly good on matting; to cleanse thoroughly, wipe with cloth wrung out of strong warm salt solution (one-half cup salt to one gallon). There is a matting made of woven paper which looks identical, but wears better, as it does not split. 
TILE OR BRICK-Use scouring powder and hot water. Avoid sloshing, and do only a small section at a time; successively scrub, rinse and dry small sections.

ENAMELED WOOD-Woolen cloth wrung out of ivory soap suds. Wipe with clean, damp cloth. Do only a small section at a time. Use no scouring powder which will remove the finish, but whiting paste if very soiled. Polishing with dry flannel restores the high finish.

WAXED WoOD-Sweep with hair broom. Mop with dry mop or bagbroom covered with flannel. Do not oil or wash with water; use only mop moistened with turpentine, then clean dry mop to polish. Wax small spots as they appear, and polish with weighted brush about once a month. The well waxed floor is, of all, the easiest to keep clean and in repair.

VARNISHED WOOD-Sweep with hair broom; use dry or oil mop daily. Never use water if possible, as this removes varnish and coarsens wood. Repair small worn places before they wear entirely through; if a varnished floor is waxed it stands wear better and is easier to care for. Light stains show dust less than dark ones. Be sure no excess oil is left on floor to soil rug edges. Varnished wood is hard to care for, shows heel marks and "wears through" quickly unless care is taken.

SHELlacked WoOD-Use hair broom and string mop without oil. Never use water. In beginning, use equal portions of white shellac and grain alcohol applied with a brush. Shellac gives a hard finish temporarily, but must be renewed frequently. Never use shellac on linoleum.

OILED WOOD-Use hairbrush and dry or oil mop. This finish looks well, but shows footsteps readily. It should not be wiped with water, but reoiled with good boiled linseed oil every three months or so, being sure to "rub out" the excess oil.

PaInted Wood-U'se hair broom and string mop; wipe with moistened flannel cloth, but never scrub or slosh with water. Shellacked paint is good in inexpensive bedrooms where there is little wear, but not where there is much treading, as the paint wears off in ugly splotches.

Painted Plaster-As for enameled wood.

BURLAP OR FABRIC WALLS-Use stiff small whiskbroom or vacuum cleaner too? 
Cork Carpet or Floor-It should first be oiled when laid; then use hair broom and oil mop; water scours it but spoils the soft coloring. Not suited for kitchen or pantry, as it is too porous and absorbs grease readily.

Composition Floors-As tile; only, being without cracks, coarse brush can be used.

An excellent polish for all wood except light maple or mahogany is :
$1 / 3$, benzine
$2 / 3$ crude oil

Mix thoroughly, and use on cloth or mop to both clean and polish. It costs about 30 cents a gallon.

Briefly, avoid water on all wood; use damp cloth but not sloppy; use cloth or soft string mop, but not brush.

From the point of view of care, only, and not upkeep, floors may be graded in the following order:

I. Waxed.

2. Stained and oiled.

3. Varnish or shellac.

4. Painted.

5. Linoleum or tile.

\section{LIST OF CLEANSERS AND THEIR USES}

I. Soaps-strong for coarse work bare boards, cement walks, etc.; mild for fine woodwork, china, glass, etc.

2. BoN AMI-powdered and brick, for porcelain, windows, tile, etc.

3. EleCtro-silicon-for cleaning silver.

4. Barsum's Putz Polish-brass polish, aluminum.

5. BRILlo and steel wool-aluminum.

6. LIQUid VENEER or other good furniture polish.

7. Old English Floor wax for floors and fine woodwork.

8. Washing soda for cleaning drains, traps, toilets, etc.

9. C-N Disinfectant, or Platt's Chlorides for disinfecting bathroom. 
I0. Bathbrick for scouring knives and other steelware.

II. Kerosene for outdoor disinfectant, pouring down drains and cleaning coarse woodwork.

12. Parson's ammonia for washing windows, linoleum, etc.

I3. Borax for softening water, for washing glassware, etc.

14. Whiting for cleaning enameled paint and nickelware.

I5. Impregnated metal cloth for polishing nickelware.

16. French chalk for cleansing spots in fabrics-blotting paper.

17. Linseed oil for wiping woodwork and polishing cast-iron ranges, etc. Burn rags used with linseed oil; danger from firespontaneous combustion.

18. "Sapolin" in aluminum, gold, black, etc., for finishing stoves and radiators.

19. "Porcela" for bathtubs and porcelain.

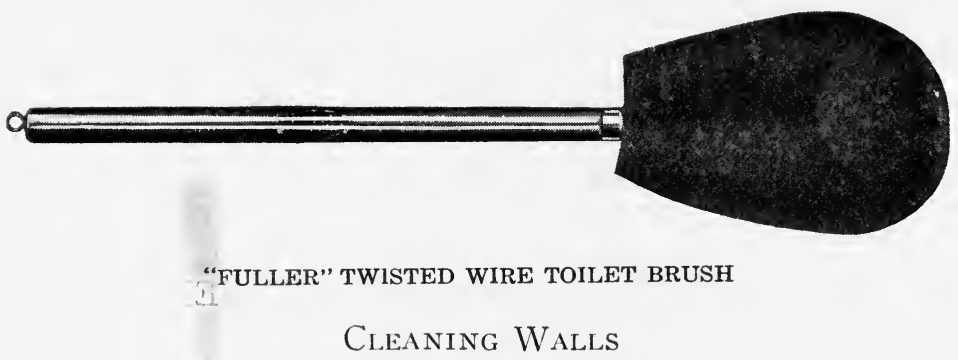

Wall surfaces for the kitchen have been already discussed in Part I. Without doubt painted walls are the easiest to care for, and retreat, as well as being the most sanitary. A painted wall may be treated with a thin coat of starch paste at the time of painting; this may be washed off when soiled, taking the dirt with it, and a new coat applied, which is as easy to do as scrubbing the paint. Paper is the surface to show the greatest marring, as well as proving fairly unsanitary. Grease spots in paper can be removed by holding over the spot a clean white blotter, and holding a hot iron over this, which will draw the grease into the paper. Watercolor paints and a fine brush can be used to "tint" mars and small abrasions. 
One of the most satisfactory wall surfaces is a material called "lincrusta," which comes in definite sized sections, and resembles a thin plasterlike material on cardboard. This material comes in different patterns, imitating wood and burlap, and can be either painted or stained after it is nailed to the wall. It can then be scrubbed, if necessary, and as it is practically part of the wall, it is most durable and sanitary. It can be applied before the "smooth finish" coat of plaster, in a new house, thus saving expense. In homes with children, especially, lincrusta proves the best wall coating, as it is practically lifelong in wear and upkeep.

Various kinds of "beaver board" also make more easyto-care for wall surfaces, as they are sanitary, and need renewing far less often than paper, paint, kalsomine. Burlap and fabric coverings, although they may look well, shrink and "bubble" if not very well put on, and are quite unsanitary and difficult to clean.

\section{QUESTIONS OF HOUSEHOLD ENGINEERING} IV

\section{Methods of Cleaning}

I. (a) What cleaning tools have you at present? (b) Do you think it advisable to add any and what? (c) Where do you keep these tools?

2. If possible, report time studies on cleaning rooms separately and again without "change of shift."

3. Write out "Standard Practice" for your most unsatisfactory or difficult cleaning task.

4. What is your experience (if any) with vacuum cleaning?

5. From experience, do you agree with the text in the use of long-handled tools? 


\section{HOUSEHOLD ENGINEERING}

V 


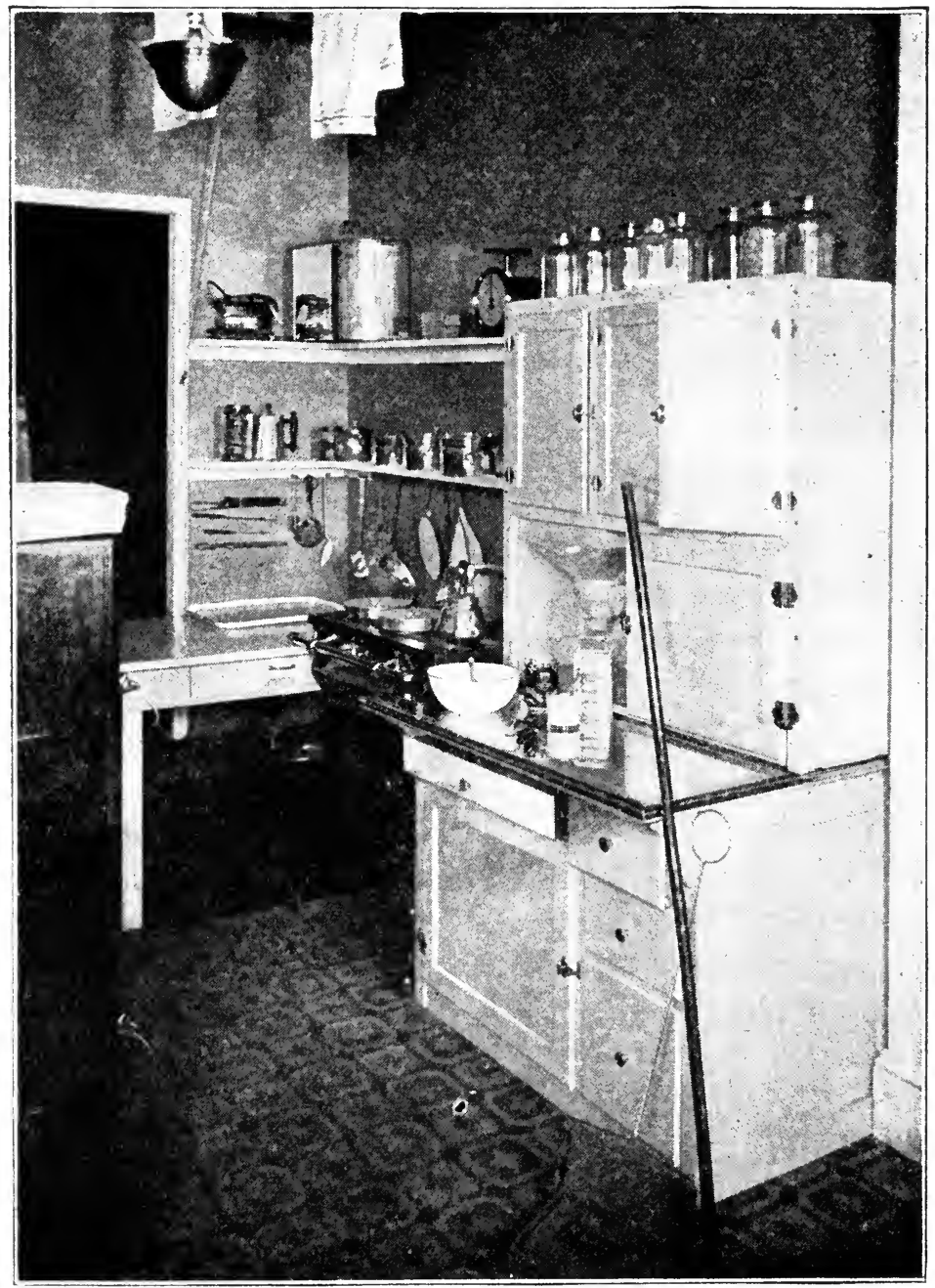

A WELL ARRANGED KITCHENETTE, $7 \times 9$ FT.

Contains a small refrigerator, cabinet, 3-burner gas stove, steel table (fireless underneath), combined laundry tray-sink and shelves for supplies, pots and utensils for a family of four on the "light housekeeping" plan. 


\section{HOUSEHOLD ENGINEERING}

$\mathrm{V}$

\section{FOOD PLANNING FOR THE FAMILY}

$\mathrm{M}$

ANY women admit that while cleaning takes a great deal of time, still it is one of the tasks of the home which can be glossed over, or quite neglected in extreme need. But the three-meals-a-day problem seems the one from which there is no escape. We can leave the windows unwashed if we don't get time or are too tired, but no matter what the circumstances or how the homemaker feels, the family must eat and so food must be prepared regularly. It is estimated that 70 per cent of the total time spent on all housework is devoted to meal planning, cooking, serving, and dishwashing, whether the family be rich or poor.

Is it necessary that this large proportion of time and effort be spent by the homemaker in order that her family is properly nourished? Even though three meals a day must be prepared every day in the year, it is possible to reduce the work involved:

Ist: By studying and understanding food values.

2nd: By following cooking methods that are wellplanned.

3rd: By using fuels and utensils that cut costs and save effort and time.

The question must be asked first, "Why do we cook?" And the answer is naturally, "To nourish the body and 
maintain it at the highest point of health and power for work." But again and again it is found in many homes that a great amount of cooking is done that does not fulfil this aim. Cooking is all too frequently unthinkingly done to feed the family, without planning to nourish it. For instance, an individual might eat two or three large dumplings which would undoubtedly "feed" him, but which would not nourish him properly. Cooking is also done according to the whims or tastes of the members of the family, who do not understand what their body requires, but who desire food that merely gratifies the palate. It is therefore the all-important duty of the homemaker herself to make a study of food values and to supply her table with rightly-chosen foods, cooked intelligently.

The first step toward simpler, easier cooking is this true understanding of food values. The practice of cooking alone never gives this, and someone may "cook for twenty years" and still not be preparing food according to the real needs of the family. While the knowledge of foods is a study in itself (which can only be touched on here), nevertheless there are certain principles of nutrition on which the easier and better preparation of meals depend.

\section{NUTRIENTS OF FOOD AND THEIR USE IN THE BODY}

(I) Protein: Lean of meat and fish, white of egg; abundant in cheese, beans, peas and other legumes; in milk and many grains like oatmeal, wheat and barley and in nuts.

(Protein is the only nutrient which can furnish the material to replace old or grow new tissue.)

(2) Carbohydrates: Starch, sugar, etc. Starch found in all cereals, flours, meals and their products; in many vegetablespotatoes, beans, peas, etc.; in nuts and some fruits. Sugar as cane sugar, beet sugar, and present in most fruits, some vegetables and in milk.

(Carbohydrates furnish "fuel" for heat and muscular - energy to the body. They are the quick fuels.) 
(3) Fats: Fats of meat and fish, butter, cream, lard, suet; also vegetable oils like olive, cottonseed, nut oils and shortening preparations made from them.

(Fats furnish over twice the "fuel" for heat and energy as same weight of carbohydrate or protein. Excess of food supply is stored in the body as fat.)

(4) Mineral Salts: Salts found in all vegetables and fruits and in the outer coats especially of various cereals; also in milk and eggs.

(Mineral salts form the greater part of the bony structure and are a very small, though necessary, part of every tissue in the body; they are necessary for the digestive secretions and for the blood.)

BUlK or Waste Products: Tissues of many vegetables and fruits which do not contain nutrients, but which serve to give "bulk" to the meal, and act as "brooms" to the system, stimulating the intestinal and muscular walls, as the cellulose in cabbage, celery, beets, carrots, spinach, etc.

WATER: This forms a large portion of all foods and body tissues and is necessary in dissolving the food, carrying away waste products, and in regulating the temperature of the body through the blood.

Every food contains one or more (sometimes all) of these "nutrients." For the average person the best meal is that which is planned after what is called the "balanced ration."

\section{What Is the "Balanced Meal"?}

$A$ "balanced" meal is one in which the various food principles are combined in a proper proportion. The "balanced" meal must contain some protein, some carbohydrate, some fat, some mineral salts, some water and some bulk. This combination or "balance" should be present in all meals, both for the needs of the body and for good digestion. In other words, it will not do to eat nearly all starch at one meal, nearly all protein at the next. A meal should not contain roast beef, beans and a rich custard dessert, or there will be too much protein; a meal should not contain corn on 
cob, mashed potatoes and rice pudding, because this would give too much starch, and a meal should not consist of pork, fried potatoes and rich pudding with butter sauce, for it would contain too much fat.*

A glance at these two sets of meals will show which is "balanced" and which is not:

\title{
BALANCED MEALS
}

( I)

BOUILLON
POTROAST WITH PARSNIPS AND CARROTS
BAKED APPLE OR OTHER ACID FRUIT

(2)

ROAST PORK

WHITE POTATOES CABBAGE SLAW

LEMON GELATIN

(3)

VEGETABLE SOUP

POTITO SALAD
CHOCOLATE LAYER CAKE

MEALS NOT BALANCED

(I) CREAM POTATO SOUP

POTROAST WITH POTATOES, PARSNIPS, CARROTS

LEMON MERINGUE PIE

(Too much starch)

(2)

ROAST PORK
SWEET POTATOES APPLE FRITTERS
STEAMED PUDDING WITH HARD SAUCE
(Too much fat)

(3)

\author{
BOUILLON \\ ROAST BEEF KIDNEY BEANS \\ CUSTARD AND SPONGE CAKE \\ (Too much protein)
}

* Bulletins of the School, "Food Values," "Freehand Cooking," "Five Cent Meals" (10 cents each) are a help in this connection. The book "Lessons in Cooking, Through Freparation of Meals" gives 267 well balanced menus, with recipes and directions for preparing each of the meals, $500 \mathrm{pp}$. illustrated. Price $\$ 2.00$, postpaid, on approval. 


\section{The Balanced Meal Is Less Costly}

Not only does the "balanced" meal furnish the proper proportion of nourishment, but it lessens the cost of the menus. The homemaker who understands the principles of the balanced meal will not, for instance, have an expensive egg dessert when the first course is a substantial meat dish, or vice versa. She will not have ham and eggs and cheese and a floating island dessert. She will know that many of the so-called cheaper cuts around the flank, etc., furnish as much nutriment as porterhouse or expensive loin. She will know that a simple meal of cream soup, bread and butter with a custard furnishes as much nourishment as an elaborate luncheon of sardines, sliced meat, potatoes, canned peaches and cake. In other words, the balanced meal makes for real economy.

\section{The Balanced Meal Saves Time in Cooking}

But the balanced meal truly makes for efficiency in cooking, because the housekeeper who follows this plan does not need to spend the time in preparing the balanced meal that she does usually on an elaborate meal. When she knows that the simple balanced meal, well cooked and served will satisfy the actual needs of her family, she need not spend hours over unnecessary pot-watching, making complicated desserts and taking several hours to prepare the food for one meal. Most of the 70 per cent of her time spent in cooking results because the housewife attempts to serve too many kinds of food or too many courses at the same meal. Cooking can be simplified largely by having a simpler standard.

\section{Planning Meals for Taste}

Another important thing to remember in planning meals is, to consider the taste and appetite as well as the nutritive 
values. Even the best dinner, arranged on the most nourishing plan, would not necessarily be appetizing if certain rules are not observed. Every meal should have contrast-sweet with sour, light with heavy, crisp with soft, strong with delicate. The ideal meal is that in which one passes from one pleasurable taste to another. Every dish in the menu must be considered with relation to every other from the point of view of taste alone.

It is not only what we eat, but the pleasure we enjoy when we eat that makes meals satisfactory. Science has proved that the appetizing meal is more likely to be well digested. Therefore the housewife's planning must include the appearance and daintiness of each dish, and its contrast with the others. We do not want so many courses as in the so-called "formal" meal, but we do need to follow the dexterous nanner in which each course opposes the other, and, as it were, "plays up" the succeeding one.

Two creamed foods should not generally be served at the same meal or there will be too much "sloppy" foods. Dry meats should be served with creamed or scalloped potatoes, dry potatoes with gravy. A meal should not contain two strong flavored foods like cheese, onions, cabbage, cauliflower, turnips. Rich puddings should have acid sauces, light cakes go with rich desserts, and rich cakes with plain and fruit desserts. Not even a good appetite can enjoy monotonous unrelieved foods.

\section{Planning Meals Ahead}

Nothing wastes time more or is more inefficient than to let the choosing of a meal go until an hour or two hours before it is to be served. If left in this way until the last moment it is quite sure not to be a "balanced" meal, but one hastily put together, of anything that happens to be in the house or that can be obtained quickly. 
Planning meals ahead has definite advantages:

(I) It permits economical marketing in advance, and purchase in larger quantities.

(2) It cuts down the time necessary in marketing, as instead of shopping every day for a small amount, marketing is done once or twice a week.

(3) It permits cooking for more than one meal at a time and saves in the use and washing of kitchen tools.

(4) It permits food preparation many hours in advance of the actual meal.

If meals are left until the last moment it is likely that someone may have to "run to the store," or telephone or pay the highest price for some article which is to be included in the meal. The most extravagant way of purchasing household supplies is to purchase in small quantities "by the bag" or by the box from day to day. On the other hand, by planning meals in advance, the materials for these meals can be carefully chosen, a list made, and bought in quantity. Staples should be estimated and bought in quantity, and by weight. Each time an article is divided in a smaller and smaller unit, as a pound, a quart, it costs more proportionately than an equal amount bought by the bushel, the case or the ten pounds.

It has been estimated that potatoes which cost $\$ 1.00$ per bushel for a 6o-pound bushel, or at the rate of 25 cents a peck, cost the consumer as high as 45 cents a peck when sold by small bags or Io or I 5 cents' worth at a time. Rice, cornmeal, sugar and other bought groceries should be purchased in 3,5 or Io-pound quantity. The menus can be so planned as to get the greatest advantage from a plan of wholesale buying. 
How to Make and Use a Purchasing Sheet

In all institutions and hotels meals are planned considerably in advance and a "purchasing sheet" made out for these articles. This makes for economy because by seeing what several meals are to consist of at one time the housekeeper can apportion the materials to the best advantage and arrange the meals so that meats, vegetables, etc., are planned for two or more servings.

A purchasing sheet can be made out for one week or for two weeks, and should include every itcm necessary for the satisfactory completion of all the meals in a given time.

This does not mean that every item for the whole week must be bought at one time, but that it be known in advance what every item is which is needed to develop these meals satisfactorily. Thus the "purchasing sheet" fulfils the second benefit of meals planned in advance; it prevents the possibility of being "out" of any product needed in the preparation of the meals.

To make a purchasing sheet, proceed as follows :

First write down the menus desired. Then estimate the number of eggs, the pounds of tea and coffee, the amount of potatoes, meats and vegetables, etc., needed. For instance, in the meals following, by looking at the desserts and other dishes which call for eggs, we see that 30 eggs are needed. By measuring a pound of coffee it is found that it contains 50 tablespoons. On this basis, as 5 tablespoonfuls are used each morning, I pound of coffee will suffice for Io mornings. Beets are used twice and 2 cans of tomatoes, and it is always noted what foods are in season before making up the menus in the first place. Suppose about 8 potatoes are used every meal, and that 8 potatoes weigh approximately $2 \mathrm{lbs}$. By having potatoes at 9 meals, $18 \mathrm{lbs}$. will be needed for this one week. Since it is further known 
that a bushel of potatoes weigh 60 lbs. a further estimate shows that a little over $1 / 4$ bushel is needed each week. In a similar way every item, number of pounds, bunches or dozen, can be worked out to prepare the menus for any given family.

The menus following and their corresponding purchasing sheet are for simple, average family meals, particularly seasonable for the cold winter months. They are arranged to give "balanced combination of foods," and yield a large amount of heat and energy so that the body can withstand the cold. That is why the menus contain much fat and starchy foods, hot cakes, baked or casserole dishes. But it will be noted no starchy vegetable is used at the same meal with a starchy pudding; a heavy meat is relieved by an acid salad or a fruit dessert; poor combinations like rice, potatoes and macaroni are avoided, and each meal has a proper "balance" of protein, starch, fat and bulk.

\section{SUNDAY (I)}

Breakfast-

IRISH OATMEAL

STUFFED BAKED APPLES

FRENCH TOAST AND MAPLE SYRUP COFFEE

Dinner-

BREAST OF LAMB

$\begin{array}{llc}\text { CREAMED POTATOES } & \text { MASHED RUTABAGAS } \\ \text { LETTUCE AND PIMENTO SALAD } & \\ \text { LEMON GELATIN } & \text { CHOCOLATE WAFERS }\end{array}$

Supper- CREAMED FINNAN HAdDIE BROWNBREAd SANDWICHES

FRUIT LOAF

COCOA WITH MARSHMALLOW WHIP

\section{MONDAY (2)}

Breakfast-

STEWED APRICOTS
POACHED EGGS
BUCKWHEAT CAKES AND SYRUP
COFFEE

Luncheon- 
Dinner-

Breakfast-

Luncheon-

Dinner-

\author{
LAMB CHOPS \\ SCALLOPED POTATOES BEETS \\ RAISIN PUFFS WITH FOAMY SAUCE
}

\section{TUESDAY (3)}

BAKED BANANAS

OMELET

TOAST

COFFEE

SPAGHETTI WITH CHEESE AND PIMENTO CELERY SALAD
JELLY ROLL

SLICED ORANGES

CASSEROLE OF LAMB, POTATOES AND CARROTS

CABBAGE SLAW

COLD COCOANUT CUSTARD PUDDING WITH LEMON SAUCE TEA

\section{WEDNESDAY（4）}

Breakfast- BAKED PRUNES

DROPPED EGG ON TOAST
CRACKED WHEAT COFFEE

LuncheonBRAN MUFFINS

CROQUETTES COCOANUT PUDDING WITH HOT CHOCOLATE SAUCE

DinnerVEAL CUTLETS

CREA MED CABBAGE MASHED POTATOES PINEAPPLE WHIP

\section{THURSDAY (5)}

Breakfast-

$$
\text { ORANGES }
$$

CEREAL

CREAMED DRIED BEEF AND EGG ON TOAST COFFEE

Luncheon-

CREAM OF TOMATO BISQUE

$$
\text { POPOVERS }
$$

POTATO CAKES

COCOA FRUIT LOAF

Dinner-

MOCK CHICKEN POT PIE

FRENCH FRIED POTATOES

CRANBERRY BETTY

\section{FRIDAY (6)}

Breakfast-

Luncheon-
APPLE COMPOTE

WAFFLES AND SYRUP

COFFEE
STEWED FLAGEOLETS TEA

TOMATO ASPIC

(1)


Dinner-

Breakfast-

Luncheon-

Dinner-

\author{
CASSEROLE OF HALIBUT WITH TOMATO SAUCE \\ BAKED PARSNIPS \\ DRESSED LETTUCE
}

\title{
SATURDAY (7)
}

\author{
STEWED APRICOTS \\ GRILLED SAUSAGE AND BACON \\ TOAST \\ COFFEE
}

\author{
CREAMED FISH ON TOAST \\ FRUIT TAPIOCA \\ LEMON SNAPS \\ COCOA
}

\section{BAKED POTATOES \\ BAKED LENTILS WITH TOMATO SAUCE \\ BAKED APPLE WITH MINCEMEAT STUFFING \\ TEA}

\section{PURCHASING SHEET FOR FOREGOING MEALS}

$1 / 2$ peck of apples

$1 / 3$ pound each of Irish oatmeal, cracked wheat and other cereal

$1 \mathrm{I} / 2$ pints of maple syrup

$3 / 4$ pound of coffee

Io pounds of forequarter of lamb

$\mathrm{I} 1 / 4$ pecks of potatoes

2 medium sized rutabagas

I small can of pimentos

I box of gelatin

I $1 / 2$ pounds of smoked finnan haddie

$1 / 2$ pound box of marshmallows

I $1 / 2$ pounds of dried apricots

$21 / 2$ dozen eggs

I quart jar, or a large can of beets

I pound box of raisins

2 pounds of sausages

$1 / 2$ pound of rice

8 bananas

$3 / 4$ pound of spaghetti

$1 / 4$ pound sharp American cheese

2 bunches of celery

I bunch of carrots

I large cabbage

I box of grated cocoanut
I pound of dried prunes

5 pounds of veal

2 cans of tomatoes

I small can of pineapple (or the fresh fruit)

$1 / 2$ pound of dried beef

$1 / 8$ box of cocoa

$1 / 4$ cake of chocolate

$1 / 2$ pound of flageolets

I pint of cranberries

I quart of sweet potatoes

I quart of oysters

I box of oyster crackers

4 pounds of halibut

I quart of parsnips

$1 / 2$ pound of bacon

$1 / 2$ pound of tapioca

I box of lemon snaps

2 pounds of lentils

2 cupfuls of mincemeat

2 quarts of milk daily

I large loaf of bread daily

$1 / 2$ small bag of salt

I $1 / 2$ pounds of lard

$1 / 4$ pound of tea

$1 / 8$ box of cornstarch

6 pounds of flour for rolis. cakes, gravies, etc.

$2^{\mathrm{T}} / 2$ pounds of butter

2 very small heads of lettuce

3 lemons 
Looking at the menus it will be seen that the meals were planned so that: Tomato from Dinner No. 4 made soup for Luncheon No. 5 ; potatoes from Dinner No. I made potatoes for Dinner No. 2; veal from Dinner No. 4 made potpie for Dinner No. 5 ; fish from Dinner No. 6 made creamed fish for Luncheon No. 7; pudding from Dinner No. 3 made Luncheon No. 4; the forequarter of lamb made four meals (explained below); cabbage for slaw No. 3 made creamed cabbage for Dinner No. 4; egg.yolks from dessert No. 4 made Breakfast No. 5 ; left-over lamb from dinners Nos. I and 3 made croquettes for Luncheon No. 4.

The fifth strong argument in favor of scheduled meals is that it saves endless time and nervous energy. Under the old way one had to stop and think about "What shall I have?" at least every other day. Poor planning means that a suddenly needed article is "out," and the whole meal has to be replanned to fit this condition. Poor planning also makes for "hit-and-miss" results, and it is impossible to estimate how certain quantities will last. With "scheduled meals" there is no such fussing and readjusting.

Such menus and purchase sheets should be preserved for reference. With changes and improvements they can be used many times, thus saving time in planning.

\section{Time Schedule of Meals}

Still another advantage of planning meals ahead in this way is that it permits a more accurate "time schedule" to be made and followed, as outlined in Chapter II, Plans and Methods of Daily Household. Indeed, it is impossible to make a practical schedule unless the meals are planned in advance and the cooking fitted in with the other household tasks.

It is also advisable, especially for beginners, to put down the order of preparation and time at which the cooking of 
each dish must commence, so that the whole meal may be done and ready to serve on schedule time.

\section{How Careful Marketing Saves Money}

It is difficult to say just where to draw the line between the saving made by careful planning and by careful marketing, as they dovetail so much. Careful marketing depends on careful planning, and on the other hand successful, efficient marketing is based on exact planning.

A reference to the menu shows that dinner on Sunday consists of breast of lamb, while the second dinner is larnb chops ; Dinner No. 3 is of Irish stew ; and Luncheon No. 4 is croquettes. All these meals are obtained from the same piece-the forequarter of lamb or mutton, bought at one time in one section. As a whole, this forequarter, when it weighs ro pounds would cost $\$ 1.50$ for the piece; but if bought in sections it would cost considerably more, as the following table, based on pre-war prices, shows:

\section{SAVING IN BUYING MEATS IN QUANTITY}

Io $\mathrm{lb}$. forequarter of mutton, at $15 \mathrm{c}$ per pound

If divided: 4 lbs. shoulder roast @ I8c......\$ \$0.72 23/4 lbs. neck for stew@i3c.......36

$3 \frac{1}{4}$ lbs.rib chops@20c.......... .65

or 23 cents saved by buying the entire forequarter.

Io $1 \mathrm{~b}$. hindquarter of mutton @ I8c............

If divided: $7 \mathrm{lbs}$. of roast @ 22c...........\$1.54 3 lbs. of chops @ $28 \mathrm{c} . \ldots \ldots \ldots \ldots \ldots . .8 \%$

or 58 cents saved.

ro lb. shoulder of veal @ $@$ 18c. $\$ 1.80$

If divided: 7 lbs. roast $@ 22 c \ldots \ldots \ldots \ldots \ldots \ldots \$ 1.54$ 3 lbs. of stew @ $18 \mathrm{c} . . . . . . . . .5 .54$ or 28 cents saved. 


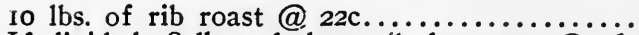

If divided: $8 \mathrm{lbs}$. of short rib for roast @26c $\$ 2.08$

2 lbs. of long ribs for soup @ 18c .36

or 24 cents saved.

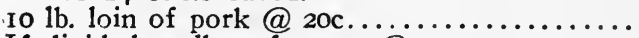

If divided : 5 lbs. of roast $@ 20 c . . . . . . . . . . \$$ 1.00

5 lbs. of loin chops@22c....... I.10

or ro cents saved.

It would seem from these figures that there is a decided saving in buying certain meats in quantity enough for two meals or more. This is possible when the family is large enough and there is adequate storage facility to keep the uncooked portion until needed. However, this saving is made only on the better meats on "prime" cuts. There is no saving in buying quantities of chuck, brisket or other of the cheaper cuts, because no matter how much is bought there is no more to be obtained at a less price than the small quantity price.

The saving is on large "prime" sections which, when cut up, make chops, roasts and choice pieces, and on which there is also more waste in handling for the butcher. If he can sell a whole loin at once he is willing to sell it for four cents a pound less in order to save the waste there is in it to him when he divides it up into separate sections of chops, shoulder, etc.

Another reading of these menus will show that this several-meal-buying idea was followed out with the vegetables and fruit. Enough beets were bought to do two meals, a hot vegetable and a cold relish; apples and oranges were bought with the double meals they would serve in mind; cabbage was planned for two meals; enough fish was bought at once to do for the warm dinner and the creamed fish for luncheon; the same was planned for the veal and the mock chicken pie it made the following day. This idea of overlapping the same material for two or more meals and mar- 
keting with this in view results in a big money-saving, because it forces the housewife to buy more closely and less lavishly and less in the hit-or-miss way which is always more costly.

\section{COMPARISON OF "POUND" AND QUANTITY BUYING}

Cost by the Pound Cost in Quantity Saving

Coffee $\ldots \ldots \ldots \ldots$ I pound $\$ 0.30 \quad 5$ pounds $\$ 1.25 \$ 0.25$

Baking powder..... $1 / 2$ pound $\quad .222^{1 / 2}$ pounds $\begin{aligned} \text { r.OO } & \text {.IO }\end{aligned}$

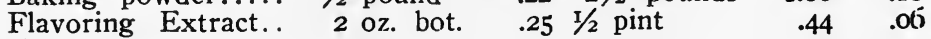

$\begin{array}{lllll}\text { Canned soups...... I can } & \text {.10 } 12 \text { cans } & \text { 1.09 } & \text {.II }\end{array}$

$\begin{array}{lllll}\text { Canned vegetables.. I can } & \text {.15 } 24 \text { cans } & 3.40 & .20\end{array}$

Canned fruits...... I can $\quad .20$ I2 cans $\quad 2.30$.IO

$\begin{array}{llll}\text { Olive oil............ I small bottle } .46 & \text { I quart } & .75 & .17\end{array}$

Whole wheat flour.. Io pounds $\quad .45 \mathrm{r} / 2$ bbl., $98 \mathrm{lbs} .3 .85 \quad .56$

Rice, beans, lentils, tapioca, etc.......

Packaged jellies, cocoanut, etc.......

Tea $\ldots . . . . . . . .$. .

Biscuits $\ldots . . . . . . .$.

Dried fruits..........

Laundry soap.......

Toilet paper..........

Laundry starch......

White soap..........

Potatoes......... .

Lard

$\begin{array}{lllrr}\text { I pound } & .10 & 5 \text { pounds } & .42 & .08 \\ \text { I package } & .10 & \text { 12 packages } & \text { I.12 } & .08 \\ \text { I pound } & .60 & 5 \text { pounds } & 2.75 & .25 \\ \text { I package } & .05 & \text { 12 packages } & .55 & .05 \\ \text { I pound } & .15 & 2 \text { pounds } & .25 & .05 \\ \text { I bar } & .05 & 10 \text { bars } & .45 & .05 \\ \text { I roll } & .09 \text { 6 rolls } & .50 & .04 \\ \text { I pound } & .05 & \text { 10 pounds } & .42 & .08 \\ \text { I cake } & .05 \text { 10 cakes } & .45 & .05 \\ \text { I peck } & .40 \text { I bushel } & 1.25 & .35 \\ \text { I pound } & .14 & 5 \text {-pound pail } & .60 & .10\end{array}$

\section{How Planning Meals Saves Time in Cooking}

Many women will say, "Oh, I always use up left-overs in salads or in various soufflé or scalloped forms." But there is a difference between the "left-over" and the deliberate planning to prepare sufficient of the same material to last two meals, with only one "long" cooking. For example, in Dinner No. I creamed potatoes are used. Now, without the several-meal idea in mind, perhaps a generous quantity sufficient for one meal would have been prepared, and if some happened to be left over, it would have been fried or prepared in any accidental way that struck the fancy. But by planning ahead, enough was prepared at one long cooking 
to reheat with a short cooking the second day,-part of creamed potatoes on Dinner I were scalloped for Dinner 2. This plan makes a decided saving in fuel, for it takes from 25 to 40 minutes to cook any potatoes or other vegetables for one meal. But it takes only ro or $x_{5}$ minutes to reheat or scallop the potatoes-which is called "short" cooking. In other words, by planning meals ahead in this way, instead of there being two "long" cookings, there is necessary only one "long" cooking and one "short" cooking. This is illustrated in the cooking of potatoes for Dinner No. I and Dinner No. 4; croquettes of Luncheon No. 4; veal of Dinner No. 4, fish of Dinner No. 6 and at various other times. This method shortens the time of preparing and saves money in fuel. In many a home potatoes are boiled half an hour for every dinner, and a hot vegetable for every meal for 40 minutes, and never a thought is given to the many times this second "long" cooking could be saved.

In the cocoanut pudding, Dinner No. 3, two distinct dishes were made at one time, one in a mold for dinner and the other in individual cups for luncheon, which, served with chocolate sauce, made a distinct dessert. In Dinner No. 6 all the halibut is cooked until done, then the second portion is removed for the next day's luncheon before the tomato is added to the first part. In any case where there was a first "long" cooking of both potato and vegetable, they were both cooked on the same burner in a steamer.

In summer it is easy to use in a salad vegetables which have had the first "long" cooking, because string beans, carrots, beets, etc., when cooked, make just as attractive vegetables served cold with French dressing as they did hot the first day. In winter, potato, cheese and egg "planned-overs" can be scalloped quickly or served in a cream sauce. Dinner No. 7 is a "baked" dinner, and dinner No. 3 a boiled one- 
all foods being baked in one case, and boiled in the other. The greatest waste in fuel occurs when frying, boiling, and baking are all attempted at the same meal.

Aside from using such helps as fireless cookers, steamers and portable ovens, the housewife can cut down fuel expense by planning left-overs or extensively developing this idea of giving the "long" cooking only once to either meat, vegetables or fruit. There are women who claim that left-overs can never, be more than what their name implies, but in France the use of small pieces is an art, and many of the most successful dishes are made from what American housewives would call worthless. The sauce, and the daintiness with which they are served are the secrets of making leftovers successful, whether they are meat, vegetable or cheese.

A novel way of utilizing left-overs is to use them with canned soups, like tomato, mulligatawney, oxtail, tomato and vegetable, costing $\mathrm{I}_{3}$ cents a can or 60 cents a half dozen cans. The contents are diluted, then thickened to make a pleasant sauce, and the beef, mutton, fish, etc., are heated in the sauce and then served either at once on toast or scalloped for a few moments in the oven and dotted with breadcrumbs. The smallest left-overs can be made into appetizing dishes in this way.

Mulligatawney soup as sauce for portions of cold meat, beef, mutton, pork or veal. Makes a delicious curry, shepherd's pie, baked peppers and rice, collops on toast, flank steak, etc.

Oxtail soup as sauce to spaghetti, rice, peppers, left-over meat of any kind, hard-boiled eggs, etc., chopped Hamburger, chopped tail of steak, pickings from any meat, made into croquettes or forcemeat.

Vegetable soup as sauce for left-over soup meat, brisket, roast or pork, in individual casseroles, etc.

Mutton and beef broth as stock, the basis of croquettes and many other made dishes, in every case where stock is generally used, which causes the great expense of the "long" cooking and straining of stock.

Tomato soup, very diluted, as sauce to portions of fish, cheese and canned salmon to make mock lobster, rabbit, Venetian eggs, etc. 


\section{Cooking Plans Which Save Time}

When any cooking is analyzed it is found that it consists of the following three steps:

(I) Grouping food materials and utensils.

(2) Actual preparation, or work.

(3) "Clearing up."

It is also found that while the time spent in actual preparation (2) is nearly constant, the time spent in grouping (I) and clearing away (3) varies considerably. The first help, then, to efficient cooking is efficient grouping of utensils and materials, as described in Chapter I. Only when tools are grouped, when materials are conveniently arranged and the kitchen step-saving, can cooking be done easily and quickly.

Much time that is often wasted in clearing up (3) can be lessened by a more dexterous, neat manner of working. For example, if a recipe calls for both dry and liquid materials, the dry materials can be measured first, in this way using only one container or cup, whereas if it is the liquid that is measured first, a clean one would be needed to measure the dry. The "wipe as you go" adage is a good one; but another saving plan is to cook at the same time dishes needing similar tools.

For instance, a Spanish cream, a prune whip and mayonnaise are types of dishes that "overlap" and use the same kinds of utensils, bowls, beater, etc. If made at the same time, there is a saving in the number of utensils and also the time of preparation over these same dishes if made at different times with separate groupings and handlings. Here is where planning ahead will permit a saving which would be impossible with haphazard meals. 
TIME FOR FOUR COOKING TASKS DONE SEPARATELY

TIME REQUiRed to

Group Prepare Clean Up

NUMBER

Spanish cream ..........4 min.

Boiled salad dressing......3 min.

Cake ..................4 min.

$7 \mathrm{~min} .5 \mathrm{~min}$.

8 min. 5 min.

Prune whip .............. 3 min. 5 min. $4 \mathrm{~min}$.

6

$9 \mathrm{~min}$.

I4 min. $\overline{29 \mathrm{~min} .} \overline{20 \mathrm{~min} .}$

Total 63 Minutes.

TIME FOR FOUR COOKING TASKS DONE SIMULTANEOUSLY

TIME Required to

Group Prepare Clean Up

Number

Spanish cream ..........4 min. 7 min.

Boiled salad dressing....... I min. $8 \mathrm{~min}$.

Cake $\ldots \ldots \ldots \ldots \ldots \ldots . . .1 \mathrm{~min} .9 \mathrm{~min}$.

Prune whip ............. I min. 5 min.

Total 45 Minutes.

$7 \mathrm{~min} . \overline{29 \mathrm{~min} .} \overline{9 \mathrm{~min} .}$

UTENSILS

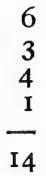

The points brought out are that the amount of time spent in the actual preparation is about the same whether the tasks are done together or separately. But if the tasks are done together, the time spent in grouping materials is cut down about one-half, from I4 to 7 minutes. Also, the time spent in cleaning up is cut down over half, from 20 to 9 minutes. The number of utensils saved is appreciable, and in general the amount of time saved is greater the more similar the kinds of materials and tools used in each case. That is, briefly, it takes much more time to clear up after separate tasks or cooking than if several dishes are made successively and only one "clearing up" performed. Thus there is a distinct saving by planning to cook dishes using similar utensils and material at one time.

\section{Cooking in Advance}

Probably the usual method of preparing three meals a day is first to cook breakfast, then proceed to other cleaning 
and housework, come back to the kitchen for lunch preparation, then spend several hours for preparing the evening meal. No wonder so many women feel that most of their life is spent in the kitchen! The planning of meals in advance has this chief value-that it permits cooking and preparation in advance. Many women now follow this plan either consciously or by instinct, and practical tests prove that it is the one thing to make cooking less complicated.

If the breakfast dishes are washed in the morning this time will give a half hour or more in which to start lunch preparation, either preparing a cup custard, starting a pot of soup, or doing some other "advance" cooking so that the actual time needed to serve lunch will be considerably lessened. Similarly, while washing the lunch dishes and being present in the kitchen for an hour or more after lunch is just the occasion to give the dinner an "advance" start. Many cases were noted in which women required an hour and a half previous to supper for its preparation of vegetables, meat, etc. Now, a great part of this time might be saved by giving a preliminary cooking to some of the supper foods while the worker must stay in the kitchen to clear up after lunch. The writer's personal plan is to prepare vegetables, arrange meat in pan, clean salad, and if possible cook the dessert in the hour following lunch. She has then a house dress on and can wash up the tools and utensils used for dinner preparation along with the lunch dishes. This makes it necessary for the worker to spend only a minimum of time in the kitchen at night when she is dressed for the evening, and greatly lessens the number of pieces to be washed at the evening meal. This is the one plan which above all permits a longer, more definite "off time" in the afternoon for calling, club meetings or rest.

That this "advance" cooking method is worth while is shown by the following letter: 
"On receipt of your letter I sat down and decided to put your good advice into practice. Since then my.husband's two brothers have come to me, I have had to take three more rooms, and yet despite this additional care I get through my work more easily than ever. I rest for an hour every day and take Sonny for a walk-all due to having followed your rule. The purchasing sheet is a fine scheme and planning a week's menu is a great nerve-saver. I do as much cooking

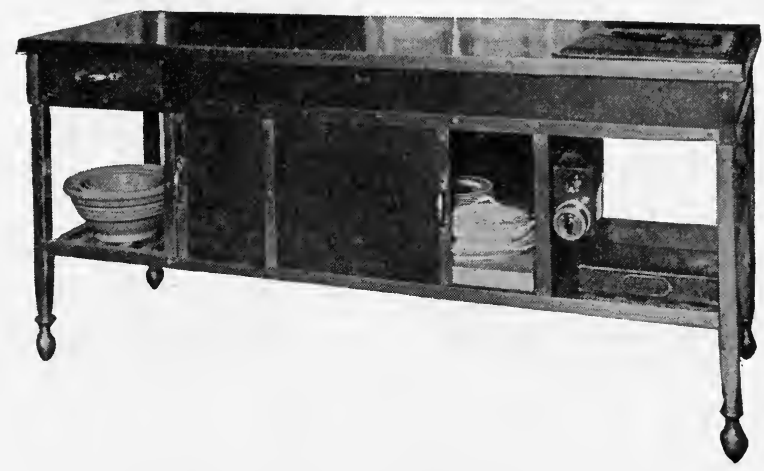

ATTRACTIVE STEEL KITCHEN TABLE.

Dish warning closet below heated by electricity.

as possible in one period. I am writing now Monday morning, I2:IO. I have my potatoes peeled in a pan of water, my carrots are scraped, I have an apple pie baked for tonight's dinner, a loaf of sponge cake and a chocolate charlotte for tomorrow's six o'clock dinner. Today I have no extra work, so I cook all I can for tomorrow. My house is in order and $I$ have a whole rainy afternoon in which to sew."

MARY F. S.

-Cooking for Several Meals at One Time

Mrs. S. said in her letter that at 12 o'clock one day she 
had cake and dessert ready for the following day's night meal. She had followed out another practical plan-that of cooking for several meals at once. It is only custom which keeps alive the idea that every single article of food needed for a meal must be cooked just previous to that meal. Many desserts, stewed fruits, can all be prepared hours in advance. Vegetables too can be cooked long before they are needed without losing flavor, if care is taken. For instance, if creamed carrots, celery or cauliflower is to be used at night it could better be given its "long" cooking during the after lunch hour while the worker is watching the stove at the same time she is washing dishes. Then at night there would be needed only a quarter of an hour's "short" cooking standing over the stove to make the cream sauce and heat the vegetable in it.

Even many roasts and similar pieces can be given a twothirds cooking during the early part of the day. A turkey is improved in taste if cooked once and given its final cooking and heating just before serving. A leg of lamb for a night meal may be given an hour-and-a-half cooking in the forenoon when both cook and kitchen are in working order and need only a half hour's heating previous to its final serving. The practice of cooking for several meals at one time proves scientifically to save the constant recurring, trotting to and staying in the kitchen, and standing over the stove at every separate meal.

Our grandmothers unconsciously frequently followed this plan when they had a "baking day" or a "roasting day" in which six or a dozen dishes were cooked which were to be distributed over two or three days. The continental people follow much the same plan, especially with their large ovens which are heated and cook a very large quantity, then served in smaller containers at separate meals. This running into the kitchen to give an hour's boiling to every vegetable, and 
a two-hours' roasting for every separate meal is unnecessary and disastrously time-taking.

\section{Similar Methods at the Same Meal}

Another chief reason for unnecessary time spent over the cook-stove is the quite general practice of following two or three different cooking methods in the preparation of a single meal. A boiled soup, a fried meat, a boiled vegetable and a baked dessert is an example of a dinner requiring too
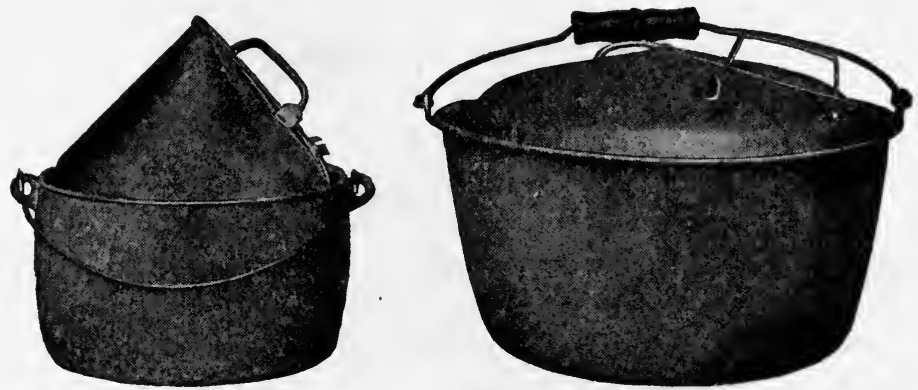

ADJUSTABLE COVER.

Fits inside any medium sized utensil, condenses steam and saves fuel. Particularly useful with a frying pan.

many different processes. If one article is to be baked, have several dishes baked-or an "all baked" dinner. If one vegetable and a soup are to be boiled why not instead make an all-boiled dinner with meat, vegetables, after the New England "boiled dinner" or the many delicious "stews" of foreign countries, so that the same method is used?

To have many cooking methods going at the same time makes it more difficult to serve and much more time-taking. Again, even if one vegetable is preferred boiled and a roast and dessert are cooked in the oven, it is possible to cook even that boiled vegetable inside the oven, if space permits. With the exception of cabbage and cauliflower, which need 
air to keep them white in cooking, other vegetables may be cooked in water in the oven with an increase in flavor. This does away with the objection of having too many burners going, or having so many pots to watch on top of the stove. Fuel is saved as well as time. (See Dinners 3 and 6, pages 188, 189.)

\section{Casserole Cooking}

Another means of enabling the worker to cook in advance is by the use of earthenware or glass casserole dishes.

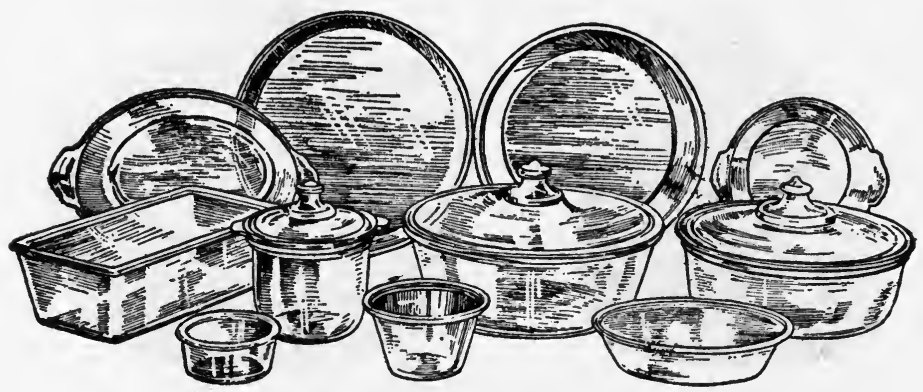

GLASS BAKING DISHES.

They withstand heat, permit foods to be cooked and served in the same dish and are easily cleaned.

Meats, soups and vegetables can in these earthenware dishes be cooked slowly or given a preliminary cooking so that only a final warming is needed just previous to the meal. The newest casseroles are of heavy but beautiful glass which stand the long continued heat of any kind of oven. By this slow cooking flavors are developed, meats are made tender and none of the juices can escape. Another advantage they possess is their sanitary superiority over the usual iron pot or roasting pan. Last, the casserole permits the food to be cooked and served in the same dish, which greatly lessens the dishwashing needed by the usual method of cooking in one dish and then serving on a table platter or dish. 
Fireless and Steam Cooking Methods

In this connection must be mentioned again the fireless and the steam cooker, which are the greatest aids in this method of "advance preparation" of meals. As is well known, foods can be placed in the fireless at night for use the next morn-

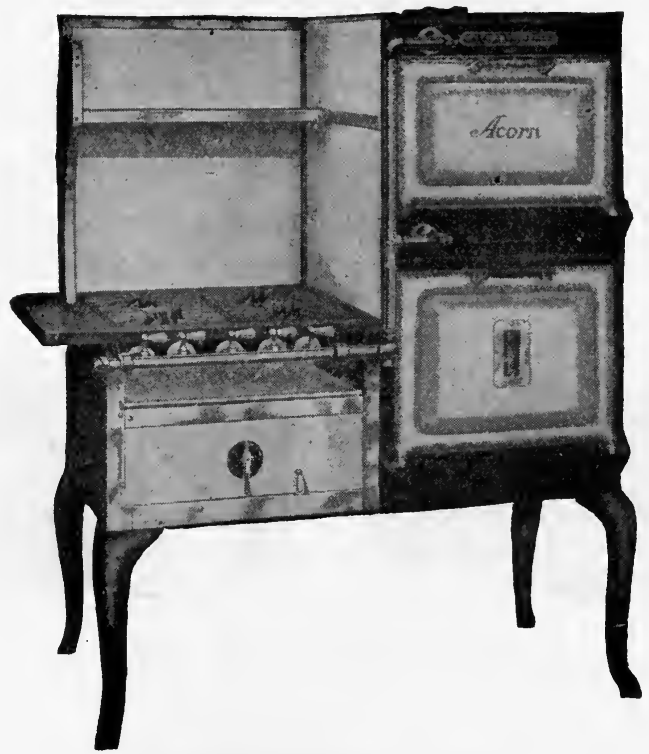

GAS RANGE WITH AUTOMATIC FIRELESS COOKER OVEN.

ing, or in the morning for use at night, etc. The steam cooker has these advantages-it operates over one burner, it permits the use of baking dishes which can also be used at the table, and foods can be placed in it and cooked hours in advance of the time they are actually served. The newest gas stove on the market is a combination gas-fireless. It is a complete gas stove with an insulated oven which is also 
operated by an automatic attachment. For instance, a roast, pot of string beans, apple bread pudding and pot of soup can all be placed in this insulated oven at one time. The gas is then allowed to burn for a short time, at the end of which it turns off automatically and the cooking proceeds on the fireless principle. In this stove probably the height of modern cooking economy and efficiency is reached. The time saved by cooking with such a stove over the usual cooking method may amount to three or four hours. (See Chapter II, Plans and Methods of Daily Housework, page 89.)

\section{Dismissing the Frying-Pan Habit}

If there is one method above all others that we, as a nation. seem to be addicted to, it is the use of the frying-pan and
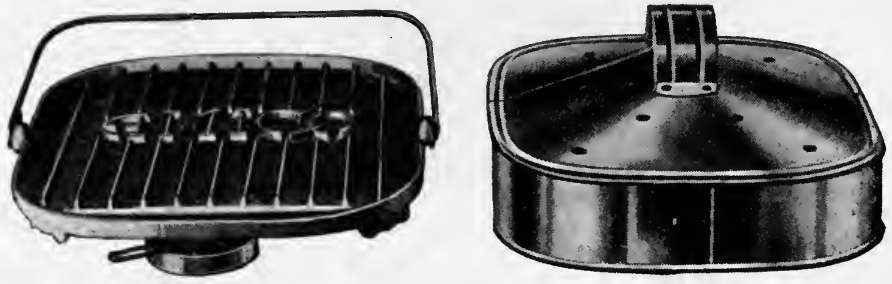

"AMICO" BROILER AND COVER.

Used on top of stoves. Drippings run into the small pan as fast as formed.

"fried" foods. An excess of fried foods is most unwholesome, but from our point of view, the frying-pan is, one of the greatest labor-makers in the kitchen. Frying, as practiced with a very small quantity of grease in the pan, creates smoke, odor and adds to kitchen cleaning. Many foods ordinarily fried can be prepared otherwise. For instance, bacon or ham taste better broiled or baked in the oven over a little bacon grid. There is no smoke and far less odor and none of the unpleasant sputtering attendant on the 
frying-pan. Similarly, chops and other foods fried can better be laid in a roasting pan and cooked in the oven.

A new broiler on the market can be used on any stove and is more efficient than the usual wire type. It consists of a corrugated iron plate with bail handle, which will fit over any burner or range opening. Broiling on this grid is far more satisfactory than cooking with the usual frying pan. All of the baking, casserole, and stewing methods and their respective equipment result in more wholesome cookery and in less labor for the cook than the frying-pan way.

\section{Standardized Amounts Prevent Waste}

No food planning can be economical and efficient which is not based upon a knowledge of weights and the various amounts needed in any particular family. Many housekeepers naturally or by practice have learned to judge how far a certain amount of food will go. But it is better to supplant this haphazard information by tested, accurate figures. For instance, how much round steak is needed? How long will I pound of coffee last? How many pounds of sugar are used a week? More exact knowledge on these and other estimates will make for better purchasing and easier cooking. For example, there are seven medium-sized meat-balls in $\mathrm{I} / 2 \mathrm{lbs}$. There are 50 tablespoonfuls in $\mathrm{I} \mathrm{lb}$. of coffee, and possibly $3 / 4 \mathrm{lbs}$. per week is an average. These facts written in the housekeeper's notebook would enable her to judge the family's needs and to buy more accurately.

\section{Planned-overs, or Overlapped Meals}

The general way in cooking is to buy a generous amount and use what you wish and if there is any left utilize it for the following day in a "left-over.", The newer idea is to purposely plan for a left-over which will be large enough to be of really practical use, as illustrated in the menus which 
we have considered. Thus, mashed potatoes might be made for one meal and so few left over that nothing could be done with them. Little scraps of meat might be either thrown out or merely tolerated. But with the "plannedover" method the estimate of what the family will eat at a given time is so close that, say, a double portion of mashed potatoes is made, one to eat hot and the other purposely sufficiently to make potato balls for a second entire meal. Instead of a few scraps of meat enough is bought so that the second portion will make a thoroughly satisfactory and adequate dish. It is not permitted to have only a saucerful of a vegetable left, but either none at all or enough for a second helping. For instance, twice as much carrots, beets, peas, etc., are cooked so that one serving can be hot and the other serving re-heated in a different manner or used cold as salad. In other words, foods are so gauged in their amounts that there is no waste and that the economical "planned-over" replaces the frequently wasteful "left-over."

\section{Other Small Economies in Cooking}

One of the most common wastes in cooking is to throw away the water in which a vegetable has been cooked. All vegetables contain-and some greatly-valuable mineral salts which are their chief value as food. But the common way of boiling carrots, spinach, etc., in large quantities of water and pouring this off merely throws away the valuable dissolved salts. The best cooking method for vegetables (with the exception of the cabbage tribe, old turnips and onions) is to steam in no water, or to boil in a very small quantity and then utilize this small amount of liquor in serving the vegetable or as a basis for a sauce.

Frequently fuel is wasted by keeping a pot boiling furiously. Once it is at a boiling point the temperature cannot increase nor the cooking time be lessened, no matter how 
rapidly the watter is bubbling. This is a common mistake, especially on a gas stove, as it is not necessary to keep water bubbling $\left(212^{\circ} \mathrm{F}\right.$.) to cook food. Inside a double boiler the temperature is about $192^{\circ} \mathrm{F}$. The correct degree of heat for stewing is about $106^{\circ}$ F. to $180^{\circ} \mathrm{F}$. Cooking over the "simmerer" burner of a gas stove can be done using only twc or three feet of gas an h o u r. Unnecessary degrees of heat are used in cooking with the result of wasted fuel, unnecessarily hot kitchen and often

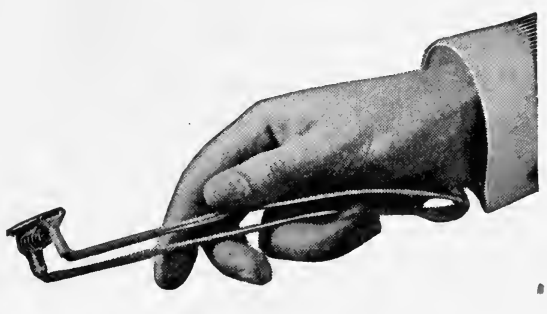

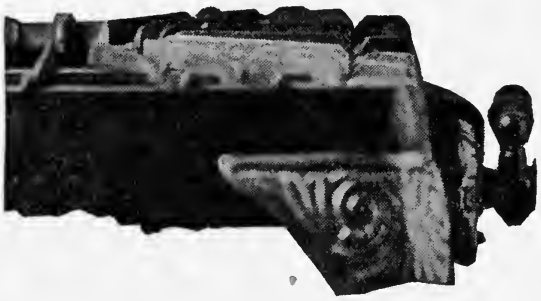

SAFETY GASLIGHTER.

Saves time, matches and gas. "Round File Populus," Price 25 cents. poorly cooked food.

The following table for baking may be helpful:

\section{OVEN TEMPERATURES}

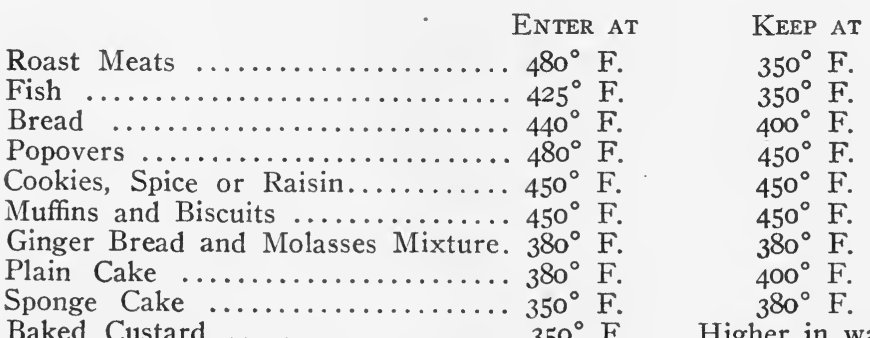

Baked Custard ................. $350^{\circ} \mathrm{F}$. 
A good cooking thermometer should be in every kitchen and may be obtained for 50 cents. A satisfactory oven thermometer which stands up in the oven costs \$1.0o.

The three-meals-a-day problem can be solved- some or all of the methods suggested in this chapter will help. Have you any other methods? What do you think of the suggestions given here?

\section{QUESTIONS ON HOUSEHOLD ENGINEERING}

\section{$\mathrm{V}$ \\ Food Planning for the Family}

1. Write out a day's menu, giving well "balanced" meals. A day's menu with poor combination of foods.

2. Give a simple menu for a week for your own family, based on what your market affords at the time of writing. . Include "planned over" or overlapping meals; similar cooking processes in the same meal; desserts, etc., cooked before the time of serving.

3. Make out a "purchase sheet" for these meals.

4. Write out a time schedule for preparing the dinnersthat is, when to start cooking each dish and the order of cooking, so that the whole meal may be ready to serve at the desired time.

5. Prepare and serve these meals to the family if possible. Report mistakes and changes you would make if the menus were repeated.

Does this plan make the work more interesting? Is it time, labor, money and worry saving? 


\title{
HOUSEHOLD ENGINEERING
}

\author{
VI
}




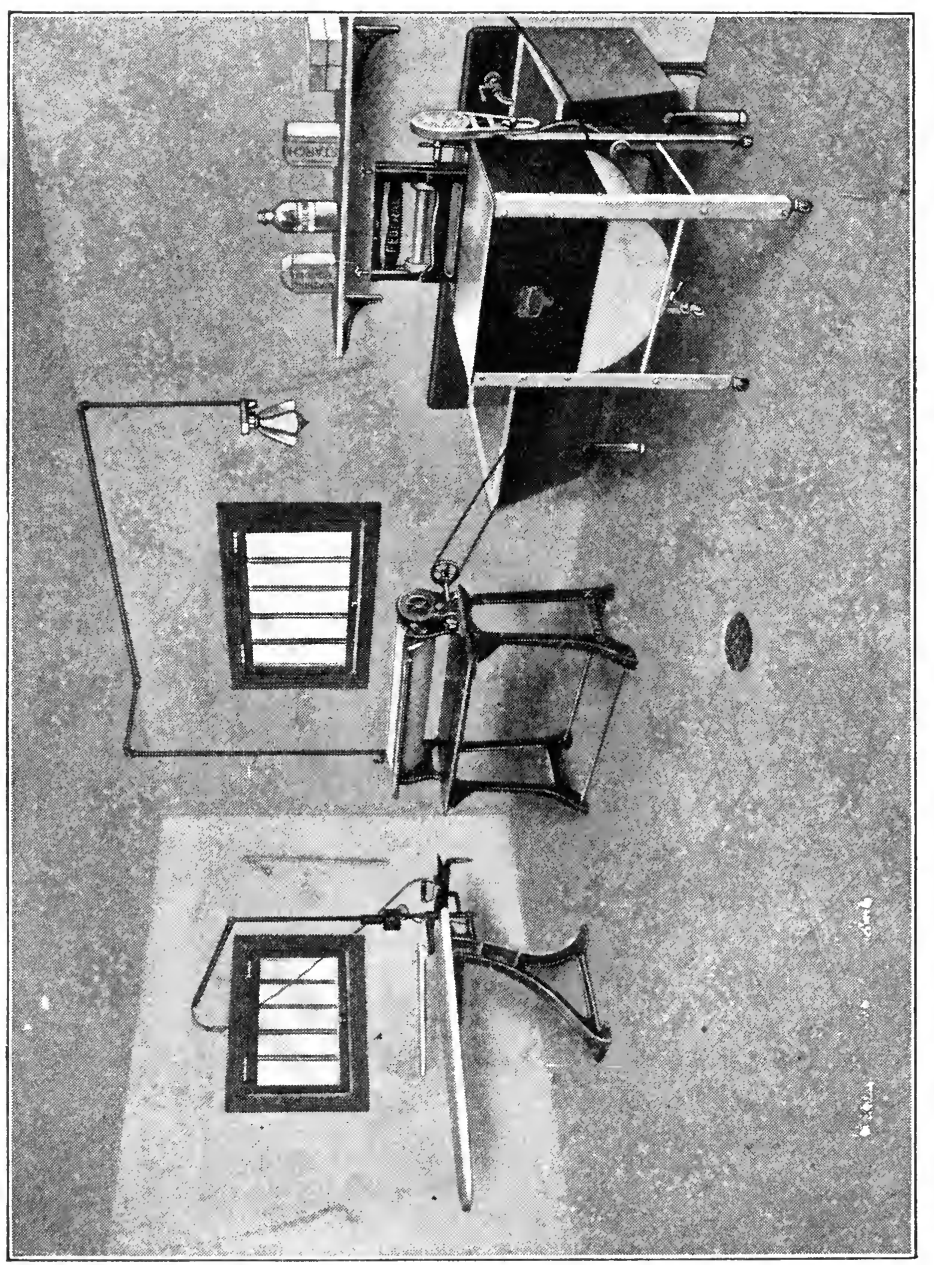

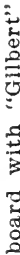

足

Di

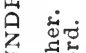

至

A $B$

A 혼

团

E

品

ज्ञ $=\stackrel{\Phi}{\Phi}$

मे तु

D- 엉

今 G

ठ भ

때

(1) +뎡

国骂

외

乙

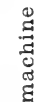

告

㻤 


\section{HOUSEHOLD ENGINEERING}

\section{VI}

\section{THE PRACTICAL LAUNDRY: METHODS AND TOOLS}

T $\mathrm{N}$ many homes the cooking and daily cleaning have been so well planned that the work proceeds smoothly and easily, but the task that frequently throws the whole week into confusion and upsets a careful daily plan is the once-a-week laundry day. "Blue Monday" is not merely a cartoonist's joke, but the most trying day in the week-the most fatiguing and the one likely to be the least organized. But the laundry problem need not be such drudgery. In fact, while it is extremely difficult to set down rules for cooking that will apply to the many different homes owing to the variations of taste even among people of the same income; and while cleaning processes vary owing to different furnishings in homes, it is encouraging that laundry work lends itself most easily to "standardization."

Cooking too often depends on the caprice of the family, the season, varying hours of meals, etc., and requires constant variety and adjustment of plans. The housekeeper must learn new dishes and adapt meals to the changing needs of a growing family.

But laundry work is the one set of tasks which can be planned and followed year after year after the same identical method, once that method is established. Here the housekeeper can simplify her laundry work and reduce it to the easiest form of standard practice in her particular 
family. Once the technique of washing flannels, removing stains, ironing, etc., is understood, she knows it for all time, and should be able to do it week in and week out with lessening strain.

The origin of the word "launder" is from the Latin, "lavander," "to wash" or "to bathe in water" ; laundry work can therefore best be defined as the cleansing of fabrics by a water method, in contradistinction to "dry cleansing," which is the cleaning of fabrics by substances other than water, like gasoline, benzine, carbon tetrachloride or other solvents of grease. The first steps, therefore, to easier, more efficient work in the home laundry are knowledge of :

I. Various textiles from which garments and furnishings are made.

2. The effect of water and temperatures upon differing textiles.

3. The action of various solvents and chemicals like soap, borax, soda, etc., on both water and textiles.

4. The effect on textiles of various processes like rubbing, wringing, pounding, starching, ironing, etc.

The two distinct classes of fibers from which all textiles are made are animal fibers-wool and silk-and vegetable fibers-cotton and linen.

The animal fibers are more easily affected by heat and by alkalies. Each wool fiber is covered with small overlapping scales lying all in one direction, which when the fiber is wet and warm, expand and tend to interlock. Rubbing wool fibers directly with the hand, or with any mechanical means causes the scales to interlock, the fibers to shorten, and thus the whole wool garment to shrink. Silk does not shrink but its fibers are weakened and its lustre lessened by either alkalies or too great heat.

Vegetable fibers of cotton and linen are tougher than silk 
and wool and resist friction, heat and alkalies better. They can be treated with strong friction, high degree of heat and with dilute acids, alkalies and bleaching powder if these are thoroughly rinsed out and neutralized. Wool resists acids well, but bleaching powder harms it. In washing any material which is a combination fabric, it is best to follow the method safest for the weaker of the two fabrics; i.e., if an undershirt is part wool and part cotton, it should be washed according to the method for wool.

The degree of heat or cold of the water also greatly affects the cleansing process. Heat tends to expand the threads of the fabric, and the dirt caught in the threads is then more easily removed. But if the cloth is again cooled during the washing, the thread contracts and the dirt is still retained. It is therefore best to soak clothing in cold or tepid water, if the soaking plan is followed, so as not to first heat the fibers and then have them contract later, when the soaking water is cooled. The principle of temperatures to follow is, to begin with warm water, and to keep the water the same, or even a rising temperature, until the clothes are clean.

Wool fibers, because of their peculiar formation, must not be soaked, or treated with temperatures of either extreme, but in "lukewarm" water of about $100^{\circ} \mathrm{F}$., and likewise pressed with a medium hot iron. Silk, also, needs both water and heat of medium temperature. Cotton and linen being stronger can be treated with both cold and very hot water and very hot irons.

\section{WATER IN LAUNDRY WORK}

Water is spoken of as "hard" or "soft," depending on the amount of lime (calcium) and magnesium salts it contains. Rainwater is best for laundry work; it is "soft" because it will quickly form a good lather with common 
forms of soap. "Hard" water contains so much lime or magnesium salts that these combine with soap and prevent it from forming suds and doing its work; instead in hard water, "lime soap" is formed, which is insoluble and which appears as a white, curdy mass floating on top and through the water. White clothes which have been washed repeatedly in unsoftened hard water are apt to have a gray appearance due to the lime soap formed on the fibers when the clothes, saturated with dirty soapy water, are rinsed in hard water. If there is any iron in the water, "iron soap" will be formed which may form iron rust stains on the clothes. Water containing much iron will give a red rust stain on bowls or water closet from a leaking valve.

Experiments show that about 2 ounces of soap is wasted in softening one hundred gallons of water for each "degree" (grains per gallon) of hardness. Lake Michigan water, which is considered fairly soft, has a hardness of 8 degrees, and as 40 or 50 gallons is a usual quantity of water for an ordinary washing, 8 ounces of soap would be wasted in softening the water.

Because of the waste of soap and the undesirable effects of lime soap, hard water should be softened for all laundry work. The most common and cheapest water softener is washing soda. If only the correct amount of washing soda is used, no washing soda is left in the water. When washing soda, borax or ammonia is added to hard water, the salts of lime and magnesia unite with it, forming carbonates which are not very soluble and so come out as a fine white powder, giving the water "milky" appearance. This powder will settle in time, but does no harm. Any iron present is precipitated also. It is using too much washing soda or throwing it in "by the handful" that does damage.

There is no very good household method of telling exactly how much washing soda to use. If the degree of 
hardness of the water can be learned from the local water department, about $2 / 3$ ounce of washing soda per 100 gallons for each degree of hardness is the correct amount. For example, if the water has 20 degrees of hardness and ro gallons is used in the tub, then $1 \mathrm{I} / 3 \mathrm{oz}$. of washing soda will soften the tubfull.

Always use washing soda in solution-two lbs. dissolved in a gallon of hot water makes a convenient strength; then for each measuring cupful of the solution will contain I ounce of washing soda. When the degree of hardness of the water cannot be learned, add the soda solution $1 / 2$ cupful at a time, mixing well, until a little of the water will give a suds with a small amount of soap solution. If sufficient soda has been used to give the water a soapy feeling when rubbed between the thumb and fingers, too much has been added for softening; then reduce the quantity.

Borax as a water softener has the advantage that using too great a quantity is not so harmful as too much washing soda. It has less softening power than washing soda, $\mathrm{I} / 3$ $\mathrm{oz}$. being equivalent to $\mathrm{I} \mathrm{oz}$. of soda. A safe proportion to use is I oz. to Io gallons of water with a hardness of Io degrees. Two level tablespoonfuls make about an ounceor better, make a solution of $2 \mathrm{lbs}$. to the gallon ( $\mathrm{I} \mathrm{oz}$. to the cup). All softeners should be dissolved and well mixed with the water and the softening finished with a little soap solution before the clothes are put in.

Use the correct proportion of washing soda or borax solution in all soaking, washing, boiling and rinsing water; bluing water, unless very hard, need not be softened.

Hard water cannot be made as soft as rain water, for a trace of lime salts and an appreciable amount of magnesium salts cannot be removed by any household methods, except by adding soap solution.

Lime soap will dissolve in gasoline, kerosene and other 
like solvents, which probably accounts for the whitening effect of the "kerosene boil" and similar methods of cleansing cloths.

\section{LAUNDRY SOAP}

Soap is the resulting product of combining fats and oils with forms of alkalies. Old-fashioned "soft soap" was made from the lye of wood ashes and melted fat. Modern "hard" or cake soap is made from caustic soda and various fats; in order to be satisfactory, the combining should be so perfect that there is no "free alkali," which is particularly harmful to wool and to colored clothing, or free fat in the soap. Also there should be no adulteration. Resin is nearly always used in the manufacture of common yellow soap because it reduces the cost ; but an excessive proportion of resin soap will cause trouble in ironing if not completely rinsed out or if used with hard water. Naphtha, kerosene, borax, etc., are sometimes added to laundry soap to aid in cutting grease and cleansing.

Soaps are classed as mild, medium or strong, and should be used correspondingly on delicate, durable and coarse fabrics. Only white soap should be used on wool and colored clothing; yellow soap on very soiled pieces and rough work.

Various soap powders are also on the market, which are mixtures of soap and some alkali, as washing soda, borax, etc. It is a better plan to use a good plain soap and the additional material uncombined, as then one is more sure of the ingredients and the cost is less. Washing powders are to be avoided, as many of them are dangerous and all of them are expensive. A great many defects in laundry work can be traced to their use. Soap chips, however, are merely regular soap cut in small pieces, but this can be done at home, using a Io-cent grater such as commonly used for shredding vegetables. 
Soap solution has the peculiar property of taking up the fine particles which make up "dirt." It also will dissolve or emulsify the grease and oil which usually holds the dirt. Thus while water is nearly a universal solvent, soap and water will take up many more substances. The more grease present in or on clothing, the stronger the soap or the "cutting" properties needed. Soap has antiseptic qualities and acts as a partial disinfectant.

\section{WASHING AND WRINGING}

The removing of soil and grease from fabrics is greatly facilitated by kneading and twisting of the fabrics which will naturally assist in loosening it from the fabric. Therefore one of the most important steps in washing is the application of a mechanical means for moving the garment, pounding it, forcing water through it, and thus loosening dirt from the fibers. This friction or movement has been accomplished by varying methods in all countries, from the primitive rubbing of the garment on a stone to the modern washing machine. The time-honored washboard on which the knuckles were rubbed as well as the clothes is slowly becoming obsolete, and in any truly efficient laundry, must give place to a more improved method of forcing the soapy water through the fabric.

Water must also be squeezed or wrung out of the clothing, and for this purpose wringing, preferably by a mechanical wringer, is the next step, as wringing both extracts the water and smoothes the article so that it can be handled more easily at the next step, which is starching. It is not necessary that clothes be starched, but it is done for the sake of appearance, and so that they will keep clean longer; as the starched surface does not absorb dirt as readily as the unfinished material. Ironing also may be spoken of as 
a luxury in the cleansing of clothes, but it makes clothes appear better and feel more smooth to the touch as well as making the use of starch possible.

\section{Stains}

Stains should be treated before washing for the reason that they may be spread or "set" during the process. Remove as follows:

GrEase Spots: Gasoline, kerosene.

Axle Grease and Old Grease Spots: Rub on lard, let stand over night and put through the machine.

PaINT: Turpentine or lard.

Coffee, Fruit, Wine Stains: Pour boiling water through.

Chocolate-Cocoa: Borax, soap and cold water.

MILDEw: Lemon juice and sunshine; bleaching powder solution. Mildew is a growth of mold and if advanced cannot be removed. SCORCH: Water and sunshine, repeatedly.

Grass Stains: Alcohol; or rub on molasses and wash.

IRON RUST: Lemon juice, salt and sunlight or dilute hydrochloric acid for bad stains; rinse and neutralize with ammonia.

INK: Cold water before it dries, followed by lemon juice and water. Small spots use "ink eradicator" (25c at drug store). Large stains, bleaching powder solution (cold) and vinegar; rinse and neutralize with ammonia.

\section{SoAKING}

Whether to soak the clothes or not depends upon conditions; it undoubtedly loosens the dirt and saves time in the actual washing, but it takes time, soap and water. Soaking in unsoftened hard water gives unfavorable results. Hard water always should be softened before the clothes are put in it. The method of wetting the clothes, rubbing soap on the soiled portions, then filling up the tub with hard water will deposit lime soap directly on the goods; the water will 
not penetrate well and more harm than good will be done. If very dirty clothes are soaked with clean ones the dirt may become distributed on the clean parts and a general grayness result which is hard to wash out.

When a power washer is used and the clothes are not very much soiled, labor is saved by omitting the soaking; or soaking for a short time only in warm water and washing for a longer time in the machine. Soaking for half an hour in warm water is as effective as over night in cold water. It is a good plan to wring the clothes out of the soaking water, to get rid of as much loose dirt as possible, for this saves soap in the washing.

Handkerchiefs which are much soiled should be soaked in salt water ( I cup of salt, 2 quarts of water) to remove mucus.

\section{BoILING}

Even with the washing machine, many clothes can be boiled to advantage, such as face towels, bed linen and underwear. Boiling also sterilizes the clothing and should always be done to handkerchiefs, etc. The clothes are usually wrung out, and placed in the boiler with cold water (softened) and a quantity of soap solution or soap chip and brought slowly to boiling; then the clothes may boil, not more than ten minutes. Long boiling with soda or yellow soap tends to yellow the clothes. A brass or copper wire grating fitted with hoop-like handles may be placed in the boiler previous to laying in the clothes. Then when it is desired to drain the clothes, these handles may be lifted up and the clothes allowed to drain on the rack before being lifted out. Never pack the boiler too full.

Various substances like a cupful of turpentine or kerosene, a half-pound of shaved paraffine, may be added to the boiler with the soap to increase the cleansing effect. The percolat- 
ing device spoken of elsewhere is excellent used in the boiling process. Except in the "paraffine boil" only clean clothes should be boiled, for boiling will serve only to still further "set" the dirt of dirty clothes.

\section{RINSING}

Much of the poor quality of laundry work is due to inadequate rinsing. One cannot rinse too much, and two, or even three, rinsing waters are better than one. The first rinsing water should be hot in order to remove the soap and dirt; and the second may be warm or cold so that there is less transition from the rinsing to the bluing water. Rinsing must be well done before bluing; otherwise the clothes may become spotted.

\section{BLUING}

Bluing is added to cover up any yellowness of the white clothes. When the clothes appear blue too much bluing has been used. Well washed and rinsed clothes which are dried in the sunshine in clean surroundings do not need bluing.

Practically all of the liquid bluing and some of the solid blues on the market are made of "Prussian Blue," which is a compound of iron. Hot soap or alkali solution will decompose this compound and iron rust stain may be deposited on the fabric. This can be shown by bluing a piece of cloth a deep shade, drying and boiling in hot soap or soda solution. One advantage of soaking is that most of the bluing is removed.

Indigo blue and ultramarine blue contain no iron and can be obtained as "ball bluing," though with some difficulty. The commercial laundries use aniline blues, sold by laundry supply houses. A substitute is to dissolve one of the roc packages of blue aniline dyes sold by nearly all drug stores, in a gallon of hot water. This will make a strong blue, less expensive and better than the liquid blues commonly 
sold. It will not give streaks of bluing and cannot make iron rust stains on the clothes.

If ball bluing is used, enclose it in a small square of muslin and test the amount of bluing in the water by bluing a small sample. Some fabrics, such as loosely woven mesh underwear, absorb more bluing than others. Clothes should not be allowed to stand in such bluing water but should be moved about either by hands or wooden paddles to prevent the blue from settling and the clothes from becoming streaked.

\section{Starching}

The consistency of the starch depends on the thickness of the fabric to be starched. Starch is known as thick, medium, or thin, and garments requiring the thickest starch, such as cuffs, shirt bosoms, etc., should be starched first, as the water squeezed from clothing gradually thins the starch. Garments treated with boiled starch should be most thoroughly dried before being dampened, and dampened several hours before being ironed.

\section{DRYING}

Drying clothes, especially in the sunshine, serves also to disinfect them. Clothes must be pinned properly and carefully, either on the line or dryer, to get them back into normal shape; i.e., stockings must be hung by the feet, shirtwaists by the collar with the two sleeves pinned up. The better the pinning the more satisfactory the drying and also the ironing. Great care must be taken to have the line or dryer perfectly clean and the clothes so well pinned that they will not blow down. In stormy weather it is a good plan to pin the small clothing, such as children's underwear, napkins, etc., on to the line while it is piled on the laundry table, and then carry it out piled in a basket, which will 
save the worker standing and pinning so long a time outdoors. All things of a kind-towels, napkins, underwear, etc.-should be kept together in a washing and drying, as this saves time in ironing and putting away.

A cheap wheel tray of wood, homemade, and mounted on small baby buggy wheels, is useful for wheeling the basket about, if the lawn is smooth; or even an "abandoned" gocart will be found useful for the same purpose, to save stooping and lifting. A simple stand on which to place the basket near the revolving "umbrella" dryer, if used, will serve the same purpose.

\section{SPRINKLING}

After the clothes are dry, they are taken from the dryer or outdoors, sprinkled, rolled smooth and then made into a tight roll and allowed to stand several hours or all night before ironing begins. The longer they stand the more evenly will they be dampened. Always use tepid water for sprinkling, and either the bottle spray which will fit any bottle opening or a whisk broom, or a special clothes sprinkler, but never the hands, which make the work uneven. Table linen needs to be sprinkled most; bedding requires little dampening; starched pieces, especially flat starched pieces, should be very damp. 


\section{THE LAUNDRY ROOM}

The washing and ironing should be done in a room separate from the kitchen whenever possible. This for sanitary reasons, and also because nothing causes more confusion than to try and wash in the kitchen while carrying on the work of meal preparation three times a day. There is much to be said in favor of having the laundry situated on the first floor with sunny exposure, avoidance of running up and down, and good ventilation; in many continental cities laundries are located on the flat roofs which permit steam and odors to escape and clothes to dry in the sun, as is done in many city apartment houses in our own country. But in most cases a well planned section of the basement is the preferable location in detached homes.

If possible, the laundry-room should be considered before building and located as far away as possible from a heating plant, coal and ash containers. An entry directly from the laundry to the yard is desirable so that no waste steps are taken. The surface of the walls may be the original unfinished or smooth plaster, painted or unpainted, or tiles. The floor should be of cement, linoleum or one of the new composition materials that permit perfect, easy flushing-wood is not to be tolerated. It should have a floor drain if possible, with floor slanting slightly to it.

The laundry needs very adequate light for thorough washing and perfect ironing. High-silled windows are preferable, and enough and large windows. Transom windows, especially if the ceiling be low, will assist in letting out steam, and keeping the worker cool without causing a direct draught on the worker. A small electric fan blowing air out of the highest windows will help to get rid of steam if ventilation is poor. 
If artificial light is needed, it should be so placed as not to be directly in the eyes of the worker, but come from the side, in an adjustable "drop." Set tubs are best or washing

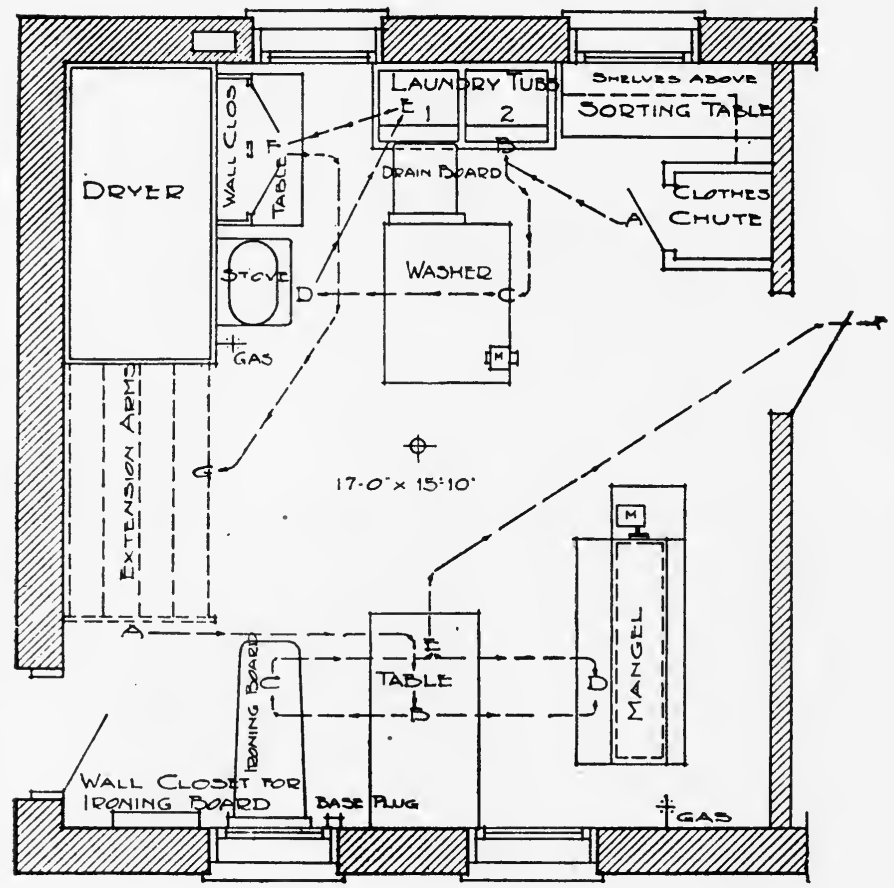

A WELL PLANNED LAUNDRY.

Washing group compact and separate from the ironing group.

machine placed at right angles to a window for the same reason. It is possible to make the laundry attractive in appearance by using color combinations like blue and white, gray and white, tan and green, etc. Tables, stool and bench of unfinished maple are neat and attractive.

A built-in closet will be helpful for keeping supplies. One 
side may be narrow, for storing the ironing board and bench; the other side may contain narrow shelves for the various soaps, bleaches, irons, and needful holders, pins, etc., as well as the utensils which should be kept especially for laundry use (see list).

\section{Grouping Laundry EQuipment}

The steps of different processes of laundry work are:

I. Sorting, mending, and removing of stains.

2. Soaking.

3. Actual washing.

4. Rinsing.

5. Bluing.

6. Starching.

7. Hanging up and drying.

8. Ironing and laying away.

Naturally, each of the steps depends on the other, and the efficiency of the whole laundry work depends on the high quality and skill with which each part is done. For instance, good ironing cannot be done on clothes that are either poorly washed or improperly starched or proper bluing on clothes poorly rinsed.

Just as we found in the labor-saving kitchen that the stove, sink and table must be arranged according to the order in which food preparation and clearing away were done, so we find that laundry equipment must be arranged as nearly as possible according to the way that the laundry processes follow each other. Although the mending is generally done in some room other than the laundry, it is in the laundry that the first step-sorting-begins. For greater convenience, a laundry chute, built in the studding, will permit soiled clothes to be conveyed from any story of the house direct to the laundry. This saves carrying a bundle 
of wash or throwing it downstairs. A sanitary basket may be placed in the base of the chute and the chute itself should be well made and preferably lined with zinc, at least in the base, for sanitary reasons.

The next step after sorting will be the soaking, which can be accomplished best in a permanent set tub. Since a large washing cannot all be soaked in one tub, it is best to have two or even three set tubs so that time may be saved in washing and rinsing without the effort of stopping to empty and re-fill. The actual washing may be done either in the set tub or in some kind of a washing machine. For ease of cleaning and convenience, the washing machine should be movable or at least placed in such relation to the set tubs that the worker can walk all around it. Boiling enters into the washing process, and so the stove on which the boiling is done must be considered, and must be so placed as to be in a step-saving relation to both the washer and the set tubs. When the clothes are wrung, they pass from the washer to a basket or container and here the washing process actually ends.

From the time the clothes are dried, through the various steps of sprinkling and ironing, they do not need any of the washing equipment. Therefore it is preferable to plan the laundry so that these two processes can be carried on independently and yet make use of commonly used equipment. Briefly, then, any laundry should be arranged so that these two processes and the special equipment of each be kept separate, i.e.,

(I) Washing—sorting, soaking, washing, rinsing, bluing, wringing, starching, hanging up.

(2) Drying - sprinkling, folding, ironing or mangling, airing and laying away. 


\section{ROUTING LAUNDRY WORK}

A study of Diagram A will show that by the proper placing of laundry equipment it is just as possible to route laundry work along a connected chain of steps and to keep the two divisions of work separate. This will prevent double handling, cross-tracking and retracing of steps. Even though the equipment of many homes be simpler than given in the diagram, the same idea holds true in laundry as in kitchen :

(a) Group related equipment together.

(b) Divide the room so that the two different processes has each its separate chain of steps.

\section{Permanent Plumbing Essential to Easy Work}

No one other household task can be so easily affected by equipment and installation as can laundry work. In fact, it might be said that 50 per cent of the drudgery of laundry work vanishes when permanent plumbing connections and tubs are installed in place. That is, much of the so-called drudgery of washing has nothing whatever to do with the actual washing of the garments and the removal of dirt, but is concerned entirely with lifting and emptying pails of water, lifting tubs, emptying the boiler, etc. Permanent plumbing at once removes the need for this effort and strain. Also a worker often condemns a good washing machine solely because of the trouble she has carrying and emptying the machine. As one prominent dealer said the other day: "Fully one-half the time is required for work which is unnecessary when hot and cold water and drain connections are provided."

The most efficient installation is that in which there is one or more permanent set tubs connected with hot and cold water, and a washing machine properly placed and sim- 
ilarly connected. But even when there is no hot and cold water supply, it is easy to install the simplest set tub of slate or composition to take the place of the frequently seen (portable) tubs, which usually means emptying and filling with a pail by hand.

In country sections, cistern water can be stored in a high tank, connected with plumbing or forced through these pipes by a compressed air tank. There is no excuse for the back-breaking work attendant on washing due to the mere lifting of tubs and heavy buckets of water when tubs can be installed permanently at so little cost. Even if there is no running water, stationary tubs are better because an easy way of draining them other than by hand can be arranged. They can be filled with water for rinsing and bluing directly from a pump, connected with hose or chute.

When no running water is present, very careful arrangement must eliminate every extra step and effort. In the diagram shown, a hand pump is mounted on a concrete base, with a trap drain underneath, the floor gently sloping to this drain. A 2-inch pipe with elbow carries the waste water from the tubs to the drain, and another similar pipe is attached to the washer outlet, and similarly.carried to the drain to avoid lifting heavy pails of dirty water. Cold water is pumped directly into the boiler for heating and into the tubs for soaking and rinsing through a short length of hose. Only the heated water need be carried by hand from boiler to washer and tubs. A boiler with a faucet outlet saves dangerous bailing of hot water.

The tubs shown are of the "portable" galvanized iron kind, mounted on a washbench 26 inches high, to avoid stooping. They are fitted with "basin plugs" and rubber stoppers which cost about $25 \mathrm{c}$ each. It will be seen from the arrangement that pump, tubs, washer and boiler are so placed as to avoid every extra step in the work. In a sep- 
arate group is a "pullman" ironing board which fits back into a shallow closet when not in use, a table for sorting and for ironing large pieces, and a closet for laundry supplies. A hand mangle could easily be mounted on this table. Ironing board is 33 inches high, table same height. A high stool is used for work. Light is given from two sides, and exit directly on drying yard.

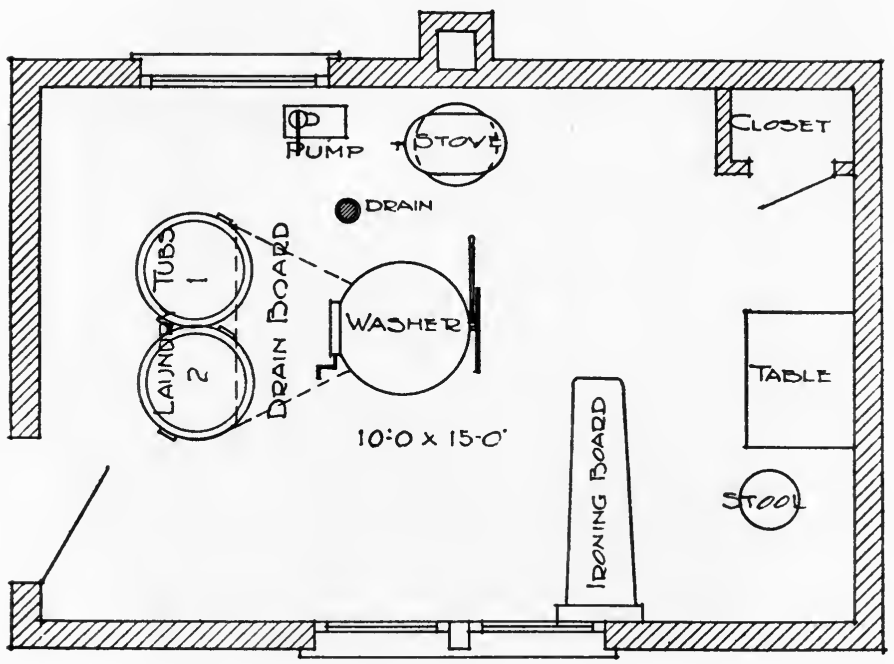

AN INEXPENSIVE COUNTRY LAUNDRY WITHOUT RUNNING WATER. (See page 256. "Standard Practice for Washing, No. 1," for method of use.)

It cannot be urged too strongly that the country laundry be fitted with running water as well as a drainage system. In many country homes some form of high tank or compressed air system supplies water to barn and kitchen and it means only a slight additional expense to supply the laundry. Even the simplest kind of a high tank filled with a force pump operated by "man" power, with drainage system 
will do away with carrying buckets and hand filling and emptying which will reduce the labor at least one-half.

When the laundry work is heavy, as is often the case in the country, a power washer and wringer, operated with a small gasoline engine, will reduce the work still more. A reliable $\mathrm{I} / 2$ horse-power gasoline engine can be purchased as low as $\$ 30.00$ and will have many other uses in the country home. A power operated washer and wringer costs about $\$ 25.00$, or there is now on the market a washing machine and wringer with a small $1 / 2$ horse-power gasoline engine geared directly to it, which is easily started and operated; price $\$ 65.00$. No investment will pay better dividends in the saving of health and strength.

\section{Frices of Set Tubs}

Composition granite set tub, $48 \times 24 \times 16,2$ compartment....... $\$ 5.50$ Composition granite set tub, $60 \times 24 \times 16,2$ compartment........ 7.00 Composition granite set tub, 60x $24 \times 16,2$ compartment, with back ..................................... 10.00

Composition granite set tub, $72 \times 24 \times 16,3$ compartment, with

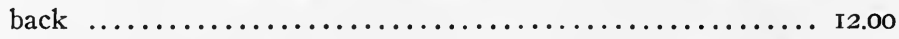

Composition granite set tub, 90x24xi6, 3 compartment, with

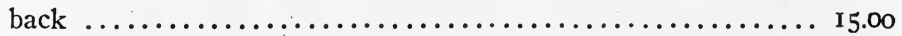

Enameled, 2 section tubs, back in one piece, with pedestal....45.00 Enameled, 3 section tubs, back in one piece, with pedestal... 65.00 Enameled, 2 section tubs, no back, iron legs............. 20.00

The height at which the tub is placed is most important. Fully as much backbreaking is done over the laundry tub as over the kitchen sink. A convenient height for a woman 5 feet 6 is to place the tubs so that their bottoms are about 22 inches from the floor, or the edge of the tub 38 inches from the floor in a straight line. The regulation iron legs are generally three or four inches lower than this, but they can easily be placed on wooden blocks so that the tub can be used by the worker without stooping, but as she is standing erect. 


\section{The Laundry Stove}

Unless an unlimited amount of water is heated by means of a coil in the furnace, or by a separate gas heater, some kind of laundry stove will be needed to heat water for washing for the boiler and possibly for the irons. The best type is the so-called "drum" stove of iron, with depressions around the drum in which to heat the sad-irons. Such a stove will heat water in the pipes, heat the boiler and irons

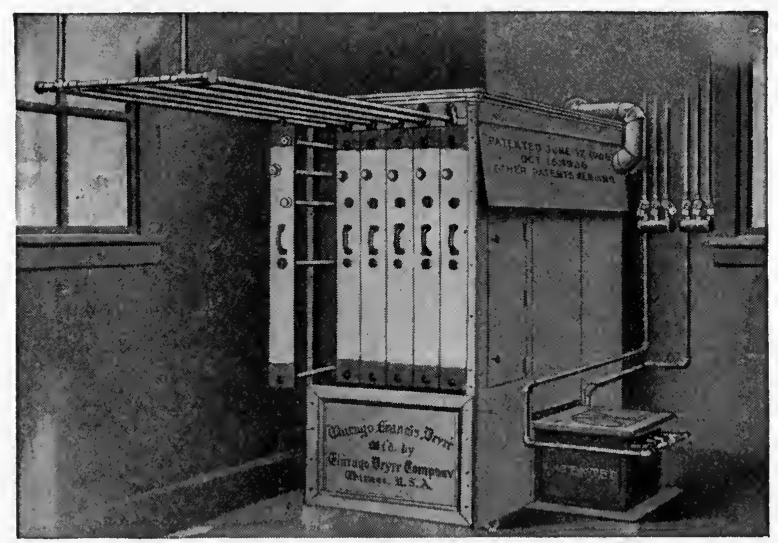

CHICAGO-FRANCIS DRYER HEATED BY WASTE HEAT FROM THE GAS STOVE.

at the same time. But if gas is available, the laundry stove may be dispensed with entirely and its place taken by a simple, two-burner hot plate. A piece of clean sheet iron. should be used under the irons used on a gasplate to keep them clean. This may be mounted on a table or stand; one burner will do for heating the boiler and the other for heating the irons and making starch.

But probably the most efficient arrangement of all is the combination clothes dryer and gas-operated heater. Here 
we have a portion of the laundry room fitted with dryingracks which come specially made and can be fitted to any size corner or room. These racks, or more properly, the enclosed drying room, are heated with an individual gas stove, and this in turn can be used for the boiler, starch and

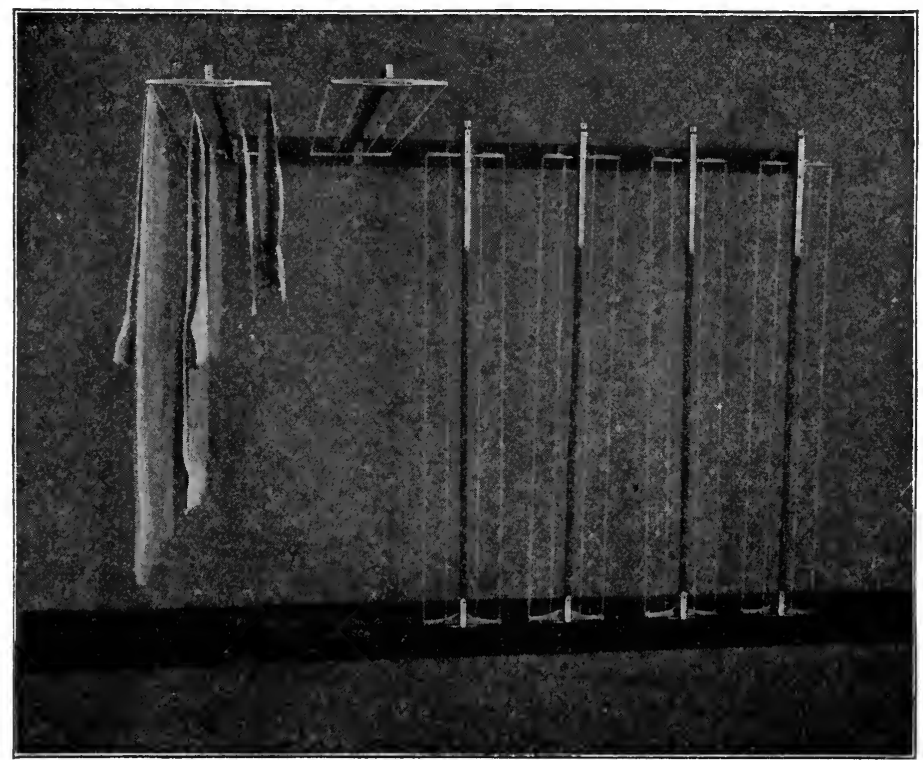

ARMS OF CHICAGO SUN DRYEP USED INSIDE.

iron so that the heat of one stove will dry the clothes, boil them and heat the irons. In a permanent house of any pretensions such a dryer should be installed because it permits a very even quality of drying without danger of windwhipping and freezing, especially in winter and rainy weather. When yard space is scarce, this is by far the best permanent plan. The objection that this indoor methor does not keep the clothes white is not true, if the drier is sufficiently ventilated, and the clothes adequately rinsed. 
In small homes where such a built-in dryer is not possible, another plan is to use the combination outdoor-indoor "umbrella" drier. This consists of a pole fitted with eight or twelve radiating arms which can be used as an outdoor fixture, especially suited for sunny days. It is so made that the arms can be detached and fastened into sockets prepared for them along the laundry wall so that they can also be used for indoor drying.

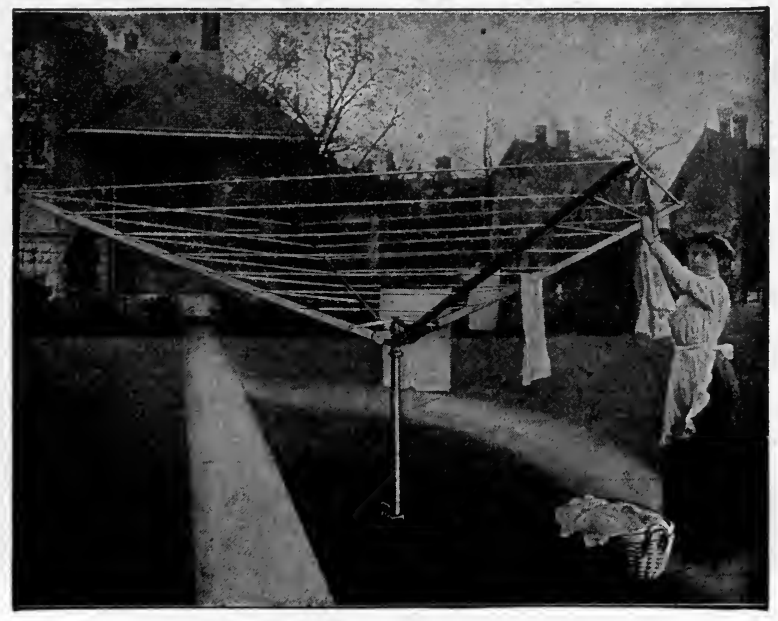

FOLDING UMBRELLA DRYER FOR LAWNS.

Still another dryer is one especially suited for very small homes and apartments. This consists of a light, wooden rack fitted with pulleys so that it can be raised and lowered from the ceiling, bearing the clothes to be dried out of the way. Smaller racks on the umbrella type are excellent for use in the laundry, on which to lay the freshly ironed clothes, and are much better than the old-time "horse" which was so easily knocked down.

A few special drying devices will make the care of clothes 
easier. One of these is the wooden or metal stocking stretchers which keep the socks in shape and prevent them from shrinking; another is the wire garment stretcher on which shirts and bodies may be kept shapely. These cost only $5 \mathrm{c}$ each, and a half dozen will save much mussing and

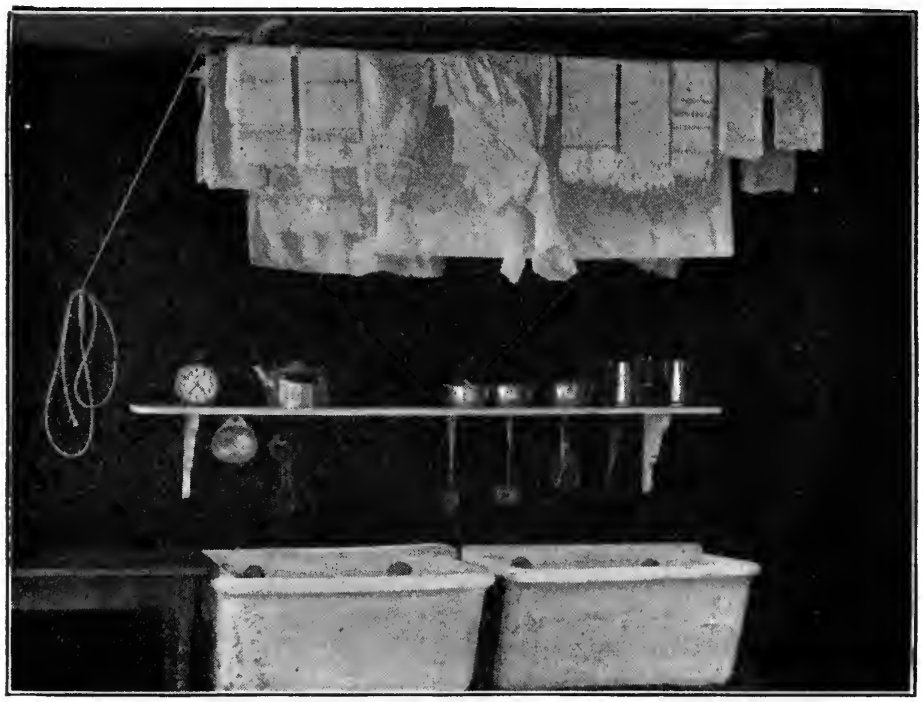

O. K. CLOTHES DRYER

Lowered for hanging on clothes, then raised for drying.

crumpling. A clothes reel which can be stretched across the room, will assist in indoor drying.

\section{The Laundry Table and Ironing-board}

A table is as necessary in the laundry as in the kitchen because on it clothes are sprinkled and sorted and its broad surface offers the most excellent space for ironing doilies and other large pieces too wide for the board. A hard maple table is best, with a separate fitted ironing quilt. There 
is also an excellent new combination table board on the market. This is a table whose top lifts up and discloses an ironing-board which can then be lifted out and put in place; and kept there when not in use so that it will stay clean.

The one point to increase the efficiency of an ironing-

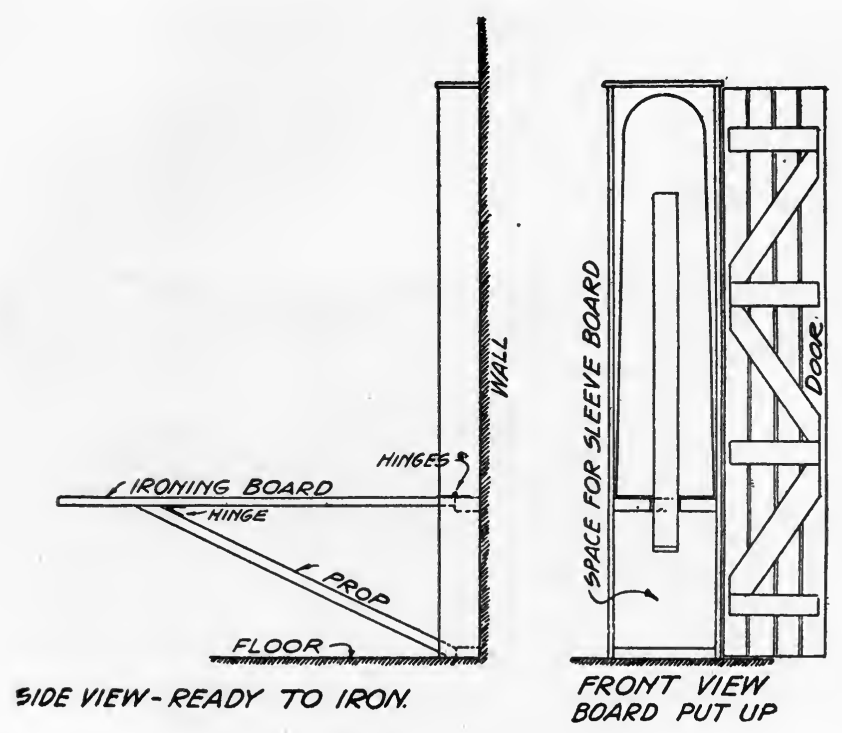

A PULLMAN IRONING BOARD.

From Housekeepers Conference Report, University of Missouri.

board is that it must be steady and of the right height to allow the worker to exert pressure with comfort. Far too many ironing-boards are wobbly, are even dangerously inclined to slip, and nothing is more inefficient than to rest the board on the back of a chair.

If the laundry room is permanent, the ironing-board can also be made permanent by being fitted to a heavy iron leg screwed into the floor. This is the type of board seen in 
conmercial laundries and institutions and will give far better service than the common, collapsible wooden board and stand. If the board cannot be clamped to a leg, it can, at least, be put on hinges and fastened permanently to a wall. It can then be laid up against the wall or in a shallow board closet when not in use, and let down at a moment's notice, and will be much steadier than the board mounted on a stand. This is called the "pullman" board.

Broad, blunt boards give wider ironing surface than the frequently too narrow board used in the home. A permanent attachable metal ironing stand can be fastened to the
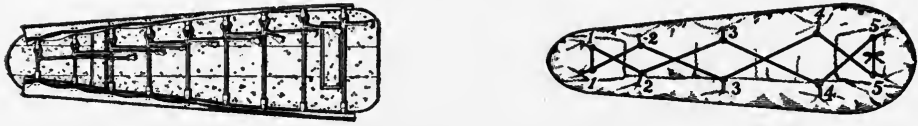

TWO TYPES OF IRONING COVER STRETCHERS.

board to take the place of the awkward ready-to-fall-off "stand" of wrought iron commonly used.

Then comes the question of the cover or pad for the board. Another poor method followed in the home is to tack the cover on each week. This causes tearing and is a great deal of trouble. There can be bought several kinds of ironing cover fasteners. One is a set of strong, steel hooks to clasp the under side of the cover together. Another is a set of pins and tape which permits the cover to be laced up and fastened securely. Even the simple plan of sets of tape at intervals is preferable to the untidy habit of using tacks.

The ironing cover may be of table padding or felt and the cover should be hemmed and neatly finished. A special felt pad and cover come fitted with a fastening device; the whole pad can be removed easily and laid away in a moment. 
The height of an ironing board for a woman 5 feet 6 with the usual arm length is preferably 34 inches. If a table is used for ironing, it can be from 32 to 34 inches. The point of height must be kept in mind fully as much in laundry equipment as in kitchen equipment.

\section{Kinds and Weights of Irons}

With either coal or gas as fuel, the usual type sad iron can be used. For the sake of economy, three of the irons may be covered with an inverted pan or a cover made for the purpose to prevent radiation of heat. The point against

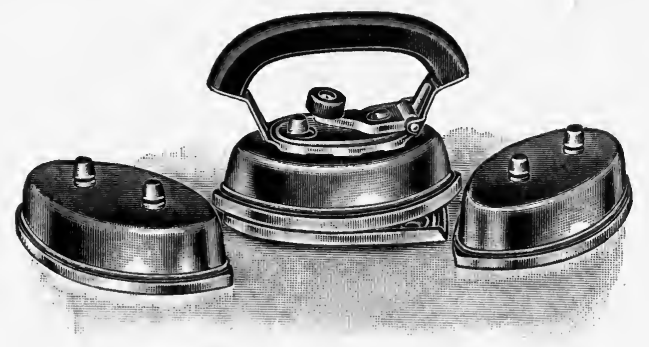

THE BEST TYPE OF SAD IRONS.

all irons not self-heating is that the worker must make frequent trips to and fro for fresh irons, thus wasting time and steps. This waste can be cut considerably by placing the worker near to the stove and its iron. If electricity is available, by far the most individual labor-saving piece of laundry equipment is the electrically heated iron. Here the heating unit is in the base of the iron so that it is possible for the worker to take and use it anywhere, even on a porch, or wherever a connecting "cord will permit; also, as in all electric equipment, the heat of the electric iron is under direct control, and it is also possible to regulate it in some 
irons to low, medium or high. There is little radiation, no excessive heat around the worker, and clothes are uniformly pressed. The working surface of the iron can be kept in perfect condition with no trouble.

The heat unit in any electric iron should be so constructed that the heat is evenly distributed, and not concentrated at

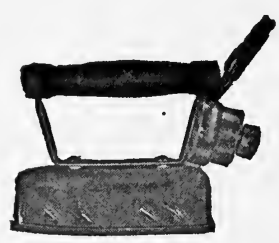

Electric

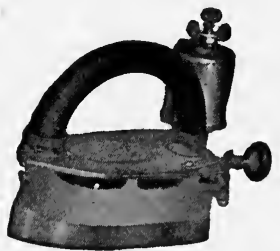

SELF HEATING IRONS Gasoline

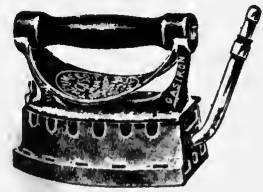

Gas

the tip only. There should be a "cut-off," and preferably a "swinging crane" connection to keep the cord taut and up out of the way of the worker, to permit more rapid work and prevent sagging. A less expensive arrangement, though less effective, is to suspend the cord with a stout string and flexible spring (like a bird cage spring).

The gas irons are a little more clumsy to operate than electric irons, though with the new metal flexible gas tubing, the difference is not great. They have all the advantages of the electric irons and cost much less to run-less than heating sad irons on a gas stove. The tubing should be suspended by a spring as suggested.

Following are the proper weights of irons for different kinds of work:

2 to 3 lbs. ...................... Baby clothes

4 to 5 lbs. $\ldots \ldots \ldots \ldots \ldots \ldots \ldots \ldots \ldots \ldots \ldots \ldots \ldots$.

6 to 8 1bs. ......................... General ironing

8 to Io $\mathrm{lbs}$........................ Flat work, tablecloth, etc. Io to 12 lbs. ..................... Pressing men's suits 3-lb. flounce iron....................... Petticoats and ruffles Troy irons $\ldots \ldots \ldots \ldots \ldots \ldots \ldots \ldots \ldots \ldots$. Polishing shirt bosoms 


\section{Alcohol and Gasoline Irons}

Another type of iron especially practical in the country where no current is available is the portable iron using gasoline or alcohol. This type of iron contains a small reservoir for the heating fluid. In the base of the iron is a small burner or generator which is pre-heated before the outer iron itself becomes hot. This generating is done by pouring a few tablespoons of alcohol or gasoline under the generator, lighting it and then turning on the air valve which permits the mixture of air with the gasoline, thus securing a hotter flame. Such irons are perfectly safe, come in several sizes and are as satisfactory as any electric iron, except that they are a little more difficult to operate. They are far preferable, however, to the ordinary sad iron in every case where the worker wants to avoid a roaring stove and the need of standing near it in order to get hot irons. By regulating the valve on such an iron, a very intense degree of heat can be secured.

\section{OPERATING COST OF VARIOUS IRONS}

Electric (light) household iron, weight $5 \mathrm{lbs}$. with 6-foot cord and lamp-socket plug; operating cost, less than $2 \mathrm{c}$ per hour.

Electric (heavy) laundry iron, $7 \mathrm{lbs}$. with cord and plug; operating cost, $2 \mathrm{c}$ per hour.

Specially heavy electric laundry iron, 9 lbs. with cord and plug; operating cost $3 \mathrm{C}$ per hour.

Self-heating gasoline iron, weight $5^{1 / 2}$ lbs.; operating cost, $1 / 3 \mathrm{c}$ per hour.

Gas iron, weight 6 lbs. with 6 feet of flexible steel tubing; operating cost, $1 / 3 \mathrm{c}$ per hour.

\section{IRONING MACHINES}

Mangles, or ironing machines, are of two types; one, the cold mangle, the other the heated ironer. The cold mangle 
resembles a wringer and has rolls of hard wood with springs which control the pressure. Clothes are slightly dampened and folded, and put through the mangle. They are pressed without gloss, but not dried. Some cold mangles clamp

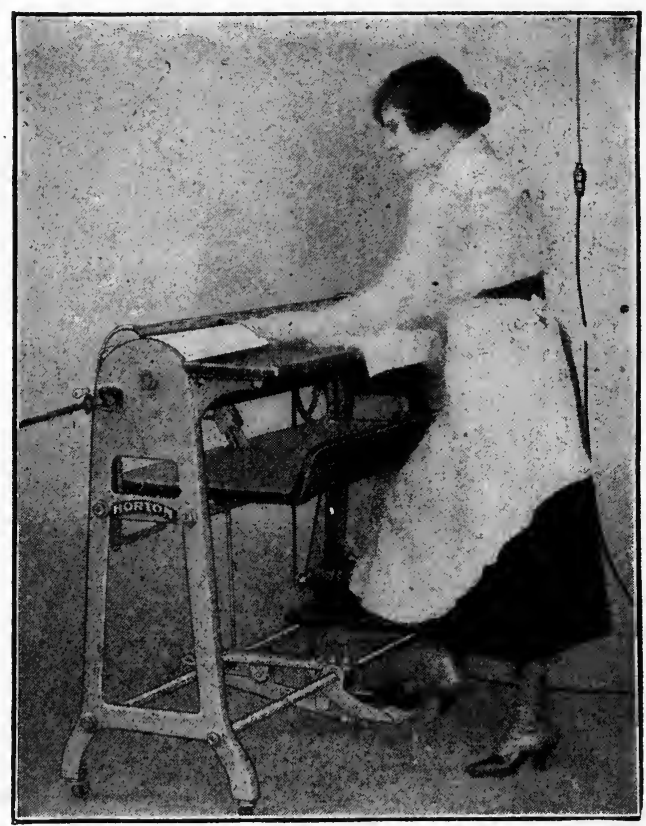

IRONING MACHINE WITH SHOE OPERATED BY FOOT LEVER

readily to the table; others come with a bass or frame of their own. The heated ironer may be run by hand, or by power. In either case, the "shoe" (which is a steel cylinder and which corresponds to the ironing surface of a hand iron) must be heated either by gas, gasoline or electricity. This steel "shoe" is outside an inner cloth-covered cylinder which corresponds to the ironing-board. Pieces are laid over the padded roll, and pressed against the hot "shoe," thus 


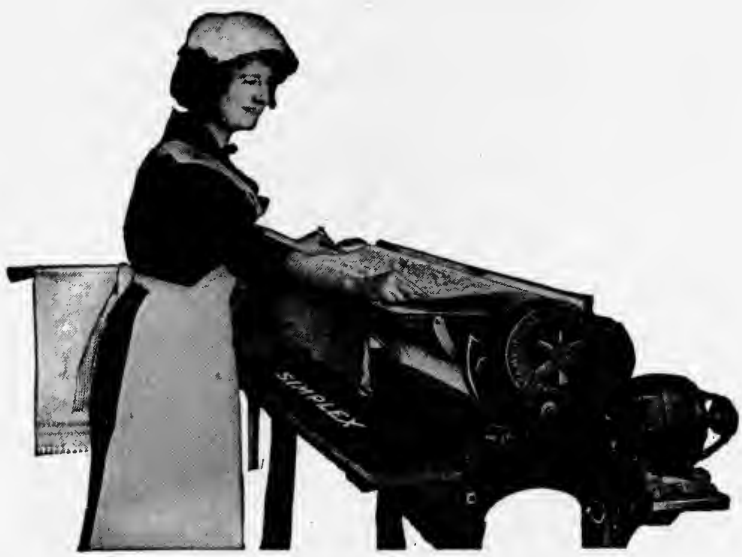

"SIMPLEX" IRONING MACHINE

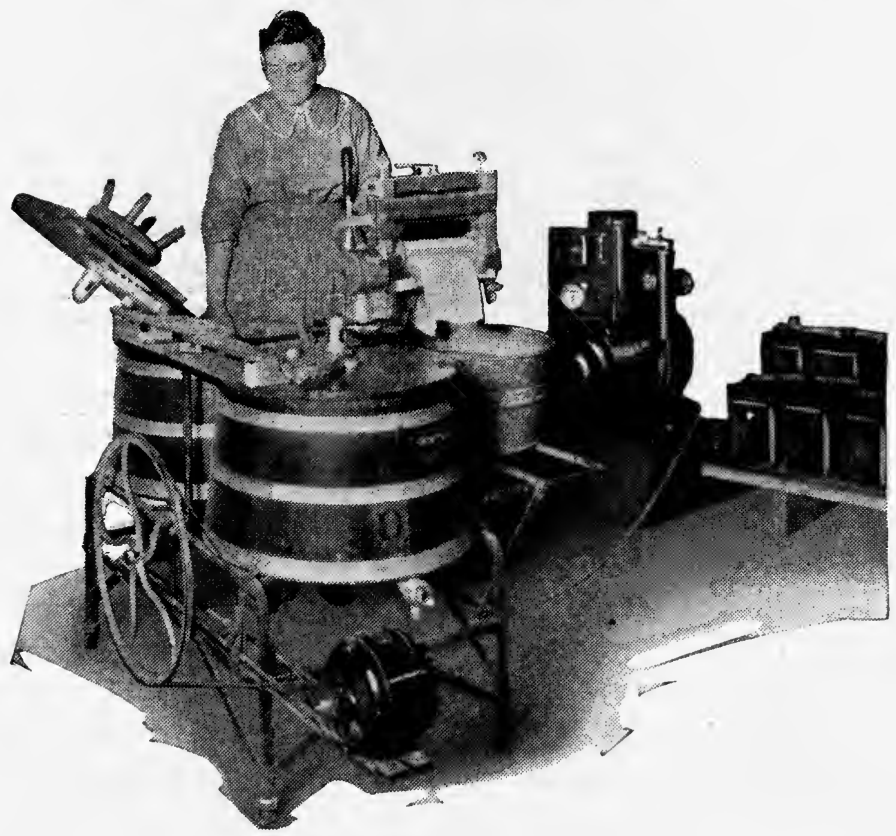

A FARM ELECTRIC LAUNDRY 
both smoothing and drying the article at the same time. These ironers come in several types, as:

( I) Gas-heated, hand operated, price $\$ 20$ to $\$ 35$.

(2) Gas-heated, electrically operated; price $\$ 60$ to $\$ 125$.

(3) Electrically heated and electrically operated; price $\$ 200$ up.

(4) Gasoline heated and operated; price $\$ 35$ to $\$ 45$.

(5) Cold roll mangle, hand operated; price $\$ 6$ to $\$ 20$.

Ironers can be used for "flat" pieces or garments, i. e., table and bed linen and towels, and also on many ordinary garments, as night gowns, rompers, aprons, etc., by practicing how to avoid buttons or hooks. The ironer saves a great deal of time, since the larger surface is equal to a surface of 6 or 8 hand irons. Ironers can be fitted for a power drive, and operated by the gas engine which is used to pump water, run the washing machine, etc. In order to heat an ironer thus driven, it is necessary to use the type that has a small gasoline tank and generator attached to it. While such a tank is fairly safe it should be used with caution. There is also another method, that of storing or burying a tank of gasoline in the ground and making the necessary connections. This removes considerable of the fire risk. Such a power operated laundry is most successful in rural homes, especially where there is a man to take the responsibility of cranking and operating the gas engine, as in general a gas engine is too much of a strain for a woman. An exception is the washing machine made by the Maytag Company, which has a very small engine directly geared to the machine. It will also operate an ironer.

Wherever there is a large family, and artificial gas, the value of the ironer is very considerable, and its first cost would easily be covered by a few months' use. The hand power ironing machines usually require two persons to 
operate them well; the motor or power machines are easily operated by one person.

Other advantages of an ironer are the uniform heat, the saving in trotting back and forth with the usual method of heating irons, and the great amount of time saved over the

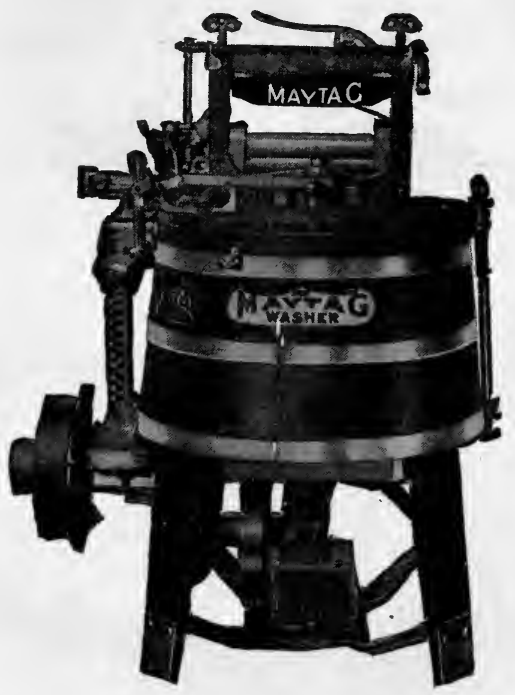

THE "MAYTAG" MULTIMOTOR WASHER.

A dolly type washer with small engine direct connected. Will run with gasoline, gas or alcohol. The engine is started by pressing the foot on the flanged wheel.

method of using an individual iron. A good family size is a 48-inch machine that will iron a tablecloth once folded. The hourly output of ironers varies according to their size, but the capacity of gas-heated models per hour is about: 25-30 bedsheets, tablecloths or centerpieces per hour. (Depends on size and thickness.) (20 inches by 40 inches size) I50-I80 towels per hour. (These estimates do not include folding which must be done by hand.) 


\section{4

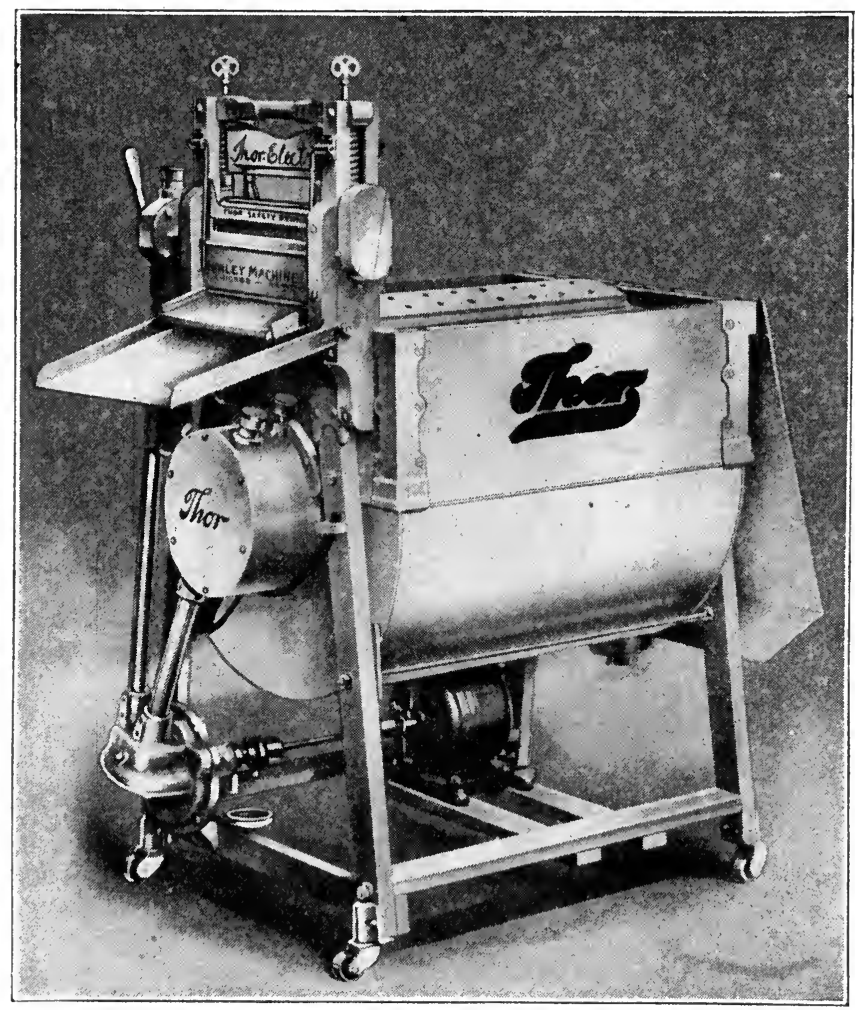

THE “THOR” CYLINDER TYPE ELECTRIC WASHER

Different Types of Washing Machines

Probably the one most important piece of equipment in the modern laundry is the mechanical washer. As was pointed out, the chief cause of fatigue in the hand method of washing clothes is due solely to the physical effort of rubbing and pounding the clothes by hand on some type of washboard. The washboard is the extreme example of a 


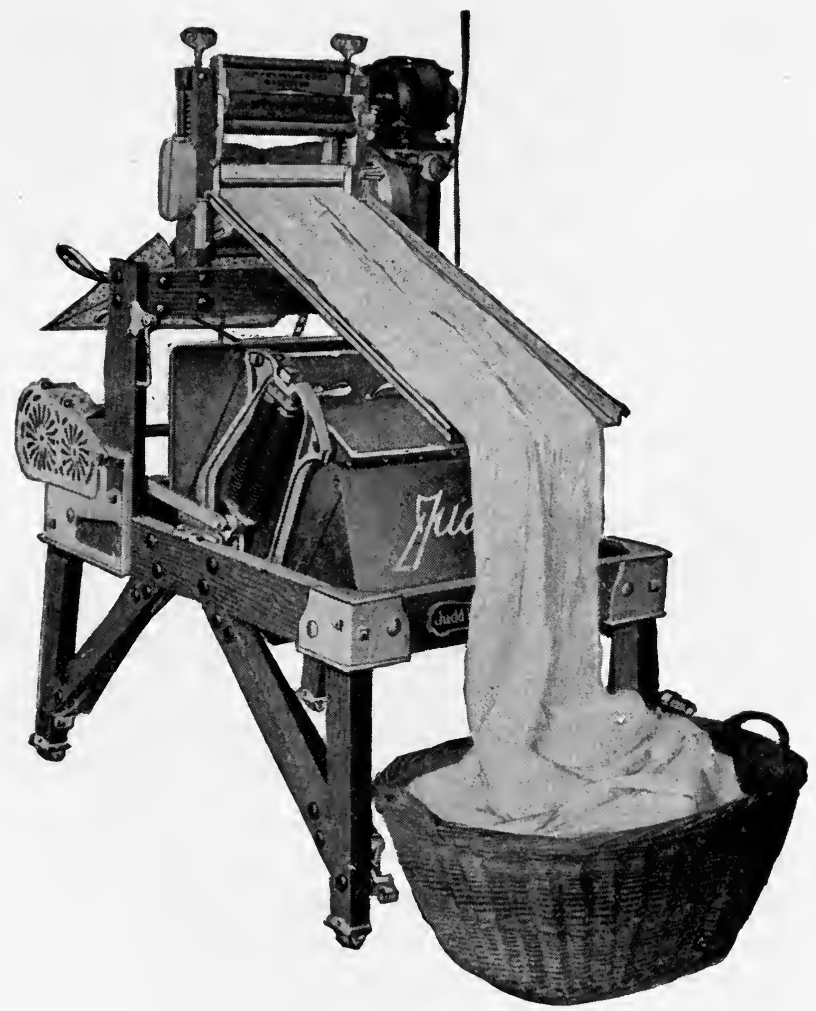

"JUDD" OSCILLATING TYPE WASHING MACHINE.

household device which causes waste motions and physical drudgery. No one has had the courage to count the number of rubs done by the hands of a woman in the usual family wash! The mechanical washer is the greatest of laborsavers because it truly replaces entirely hand labor. No matter in what other direction one must economize, money should be expended for some type of washing machine. No 
other process is so mechanical as the actual rubbing of clothes, which requires no attention from the mind. Therefore it is the one process that should be given over entirely to a mechanical servant, and thus relieve the woman of what is only pure manual labor.

An inquiry recently showed that there were 125 different manufacturers of washing machines in the United States, but these machines with few exceptions fall clearly into one of four types. Before purchasing, the principles of each of these types and its method of operation should be understood. The various types are as follows:

I. The "Dolly," or agitator type, frequently called the "rotary." Here a "wooden milkstool" or dasher revolves and reverses in the center of the tub while churning the clothes. This type has many modifications, such as corrugated boards around the side of the tub; or the lid and tub both may be grooved, thus adding to the friction exerted on the clothes.

2. The Cylinder type. This consists of inner and outer drums either of metal or of wood. The inner drum is perforated and has shelves which lift the clothes and drop them back into the water as this inner drum revolves. The action is then reversed, after a few revolutions, thus forcing the water through the clothes with a strong force.

3. The rocking or oscillating type. This consists of a metal or wooden box-like tub which rocks back and forth, cleaning the clothes by throwing them rapidly from side to side. Sometimes this box is corrugated, which adds friction to the process.

4. The vacuum type. Here both pressure and suction is exerted directly on the clothes by means of one or several metal cones, which alternately press and release the clothing. 
Any one of these types may be operated by hand, by power (gasoline), or by motor (electric). The mistake should not be made of choosing the washer on the basis of the power used to operate it, but choice should be based on the principle of the washer, and its action and effect on the clothes; - that is, not whether it is an "electric" washer or a "hand" washer; but how it washes..

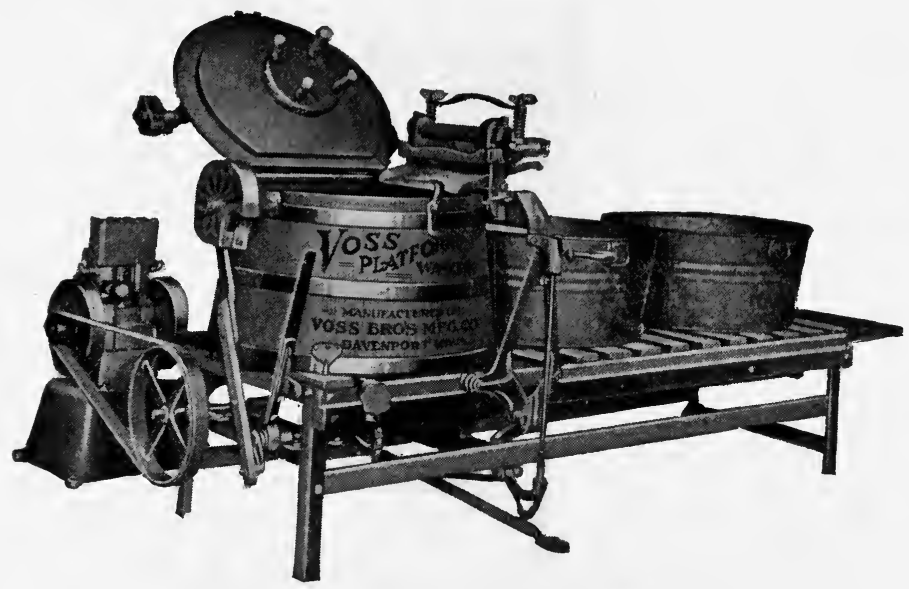

THE "VOSS" POWER WASHING MACHINE.

Cover of the machine tipped back showing the agitator or "dolly."

In the "dolly" type, the clothes are rubbed somewhat, so that this type is most suitable for heavy coarse clothing, as on the farm where overalls, aprons and heavy bedding form a large part of the wash.

In the "cylinder" washer, the clothes are not touched by any rubbing device, but are cleansed entirely by the water being forced through them, and by the clothes themselves being rapidly moved. This type is used in commercial laundries. The load drains well and can be rinsed and even blued in such a machine. 
In the "rocking" type there is also no direct friction, as the clothes are cleaned by being thrown rapidly from one side of the washer to the other, thus forcing the soapy water through and through the clothes.

Both of these types are good for all general family use, except that in these types operated by motor, the action is too strong to safely wash in them lingerie, fine baby clothes or

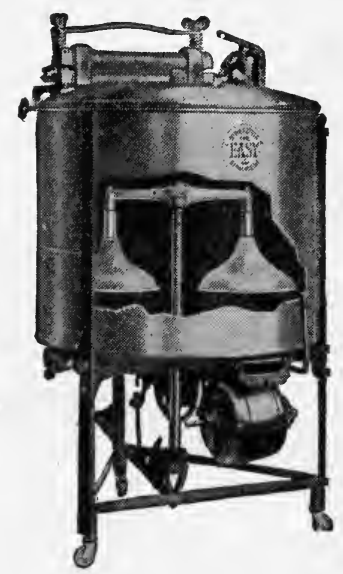

MOTOR OPERATED VACLUM TYPE WASHER.

other very dainty garments, which should not be placed in the washer, or should be enclosed in cheese cloth bags, which will take the strain but not interfere with the cleansing.

In the "vacuum" type there is considerable pressure directly on the clothes as well as suction produced by the various bells. It is probable that the vacuum type is easier on fine clothing than any other type, especially if it is a hand model.

The following representative washing machines are arranged according to type: 


\section{Dolly}

The Maytag Co., Newton, Iowa (hand, electric, gasoline or gas engine).

Horton Manfg. Co., Ft. Wayne, Ind. (hand, water, motor, electric).

The American Washer Co. (hand, water, gasoline, elec-

\section{Cylinder}

Hurley Machine Co., Chicago Pittsburgh Gauge \& Supply Co., (electric).

Gilespie-Eden Mfg. Co., Paterson, N. J. (electric).

Western Electric Co., Chicago (electric). tric), I 8 Sidney St., St. Louis, Mo.

The Grinnell Washing Machine Co., Grinnell, Iowa (hand and power).

Voss Bros. Manfg. Co., Davenport, Iowa (hand and power).
Pittsburgh, Pa., "Gainaday" (electric).

Apex Electric Distributing Co., Cleveland, O. (electric).

\section{Oscillating}

The Judd Laundry Machine Co. Apex Appliance Co., Chicago, (electric), Chicago, Ill.

Boss Washing Machine Co. I900 Washer Co., Birmingham, (hand, etc.), Cincinnati, Ohio. N. Y. (electric).

\section{Vacuum}

Syracuse Washing Machine Co., Almetal Mfg. Co., St. Louis, Mo. Syracuse, N. Y. (hand and (hand and electric). electric).

\section{Vacuum and Centrifugal Dryer}

Klymax Mfg. Co., II5 N. Des- Laundry-ette Mfg. Co., Cleveplaines St., Chicago, Ill. (elec- land, Ohio (electric). tric).

Note.-For fuller list, see "Laundering at Home," bulletin of Am. Washing Machine Mfgs. Assn., Io S. LaSalle St., Chicago.

The kind of power available in the home will also partly determine the machine purchased. Without doubt, the electrically operated machine of any type does quicker, more thorough work than the best type of hand machine possibly can do. In choosing an electric type see that the 
motor is located in a position where there is little danger of the motor becoming wet. Washers with enclosed gearing and simplicity of construction are to be preferred to those with belts which must be tightened; open gearing, however, is dangerous to the operator. Although the best tubs of cedar give excellent service, the preference may be given to the tub of copper or nickel alloy, which is the most sanitary and will give the longest service.

Further, in choosing an electric washer, that style should be selected that has a reversible wringer and drain chutes. This makes it possible to wring the first load of clothes into a basket while the second load is being washed independently in the washer. There should be a "cut-off" on the wringer so that it can be stopped quickly if clothes are caught. Before purchasing an electric machine, the woman should be sure that she understands the operation of the motor, the care of the parts, and if possible, should use the machine under the guidance of a demonstrator before she purchases it.

The water-motor operated machines are usually of the dolly type and require at least $20 \mathrm{lbs}$. of water pressure at the faucet. The wringer cannot be run by water power. Such machines use 200 to 300 gallons of water an hour.

-In selecting a hand-power type, the following points should be noted. Convenient height of washer, as machines are frequently placed too low and thus cause unnecessary stooping. Legs should be fitted with castors to permit easy moving from place to place and the washer should never be too heavy to move easily. Lever, wheel or handle should be long enough, and so placed as to allow work without strain or back bending. A satisfactory outlet for waste water should be provided other than the common "bung," which empties badly.

It is much better to have even a hand power machine 
connected with a permanent drain, and with pipe and cock of its own so that the draining and filling will be easy. Hand washers, unconnected with drain or water pipes, require so much "pailing" that their efficiency is greatly lowered. A hose connection (at least $I$ inch) from the water outlet to floor drain is almost always possible, as well as a hose from hot and cold water. A piece of brass or galvanized iron pipe with elbow and nipple in the end of the hose will hold the hose in place and direct the water where desired. There is no excuse for lifting any water in a laundry having running water.

In addition to the four types given above there are many mall washing appliances. The vacuum principle is applied to a cone mounted on a broom handle, which is useful for cleaning small rugs and sanitary cloths and baby napkins. Another cone with perforations is made to set in the middle of the boiler and sprays the clothes after the manner of a coffee percolator. Several other modifications of the funnel type are fitted to set tubs or to be attached to the ordinary galvanized tub, but the writer is frank to say that any of these is useful only for washing a few small pieces at a time and cannot take the place

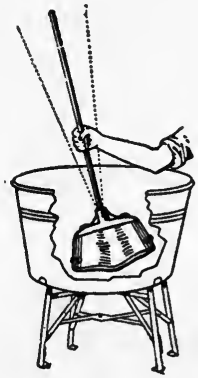

Ingram Vacuum Washer. of a regular, full-sized washing machine. There is also a cylinder washer which fits inside an ordinary set tub, but the capacity is less than that of a good machine.

\section{BoILER}

Even with a good washing machine, a boiler is necessary for the boiling of certain pieces, such as very soiled undergarments, children's diapers, etc. A boiler should be made with a copper bottom which will give longer wear and con- 
duct heat more quickly. The ordinary lifting of a boiler to empty its contents can be entirely avoided by having a regular faucet soldered in the end of the boiler. (A faucet costs $75 \mathrm{C}$, work about $5 \mathrm{Oc}$.) Then the water can easily be run off without danger and labor. Arrangements should be made for filling the boiler from a hose pipe or faucet.

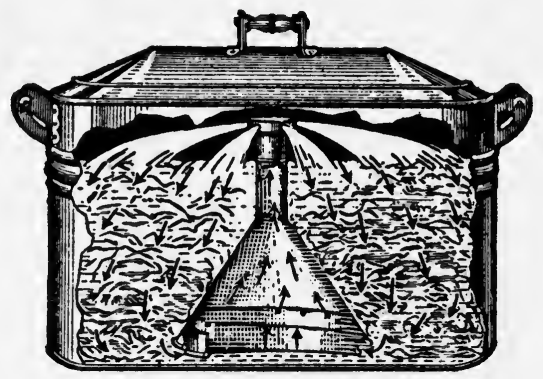

A WASH BOILER SPRAYER.

The boiler should always be well dried and aired after use.

\section{Care of Laundry Equipment}

As laundry equipment represents often a very considerable money outlay, it should be given good care, that it may not deteriorate and its value lessen. Such pieces as mangle, washer and table should have specially made covers of ticking to keep them clean and dry. Irons also should be put into a closed closet or covered with vaseline if used but occasionally. Great care should be taken with electric irons and connecting cords for all devices, as if the cord is bent the insulation is impaired. A special clothes-pin bag and ironing-board cover bag made of ticking should be used to keep them clean. Washing tubs should be kept perfectly clean and left dried to avoid rotting. The motor should never be allowed to get damp. Care must be taken that the oil used on various parts is sufficiently wiped off so as 
not to soil the clothes. Wringer rolls must be unscrewed when not in use to avoid flattening them. All minor equipment, starch-pot, etc., should be left clean and laid away and not allowed to become rusty, as rust is one of the worst foes of the laundry.

\section{Additional Laundry Equipment}

A good laundry should contain its own necessary small tools, like starch pot and spoon, quart measure, etc. Never use tin articles, as they rust. As described in the efficient laundry plan, these articles should be kept in a closed cupboard out of the way of dust and soil. Although the needs of each family vary owing to size, climate and standards of clothing, the following list of small laundry equipment will be found helpful in purchasing:

\section{LIST OF FAMILY LAUNDRY EQUIPMENT}

\section{Washing Outrit}

Approximate Pre-

Set tubs WAR Cost

Washing machines:

Dolly, hand

8.00 to 14.00

Dolly, motor 35.00 to 75.00

Cylinder, electric 85.00 to 150.00

Cylinder, hand 8.00 to 15.00

Oscillating, hand I2.00 to 20.00

Oscillating, motor 95.00 to 150.00

Vacuum, hand

1.00 to 10.00

Vacuum, motor 85.00 to 150.00

Boiler, copper bottom 2.00 to 5.00

Boiler, copper bottom, with faucet..............

Wringer, hand I.00 extra

Wringer, power 3.00 to 6.00 Clothes basket 14.00

I.5O up

Small scrubbing brush for rubbing soiled spots.... .I5 4 to 6-quart agate Berlin kettle for starchmaking.. $\quad .40$ 
Washing OUTFIT

Approximate Cosi

Long-handled agate spoon for starch........\$ .I5

Agate quart measure..................... ${ }^{25}$

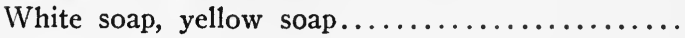

Borax

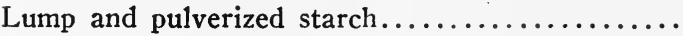

Oxalic acid (2 ounces).

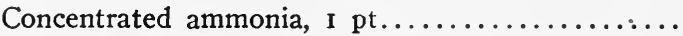

Washing soda, salt.

Wax or paraffine, turpentine.

Javelle water (made from chloride of lime, receipt on package)

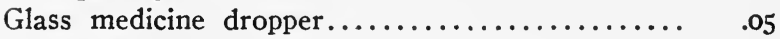

Small agate funnel..................... .I0

Wire strainer for $\operatorname{starch} \ldots \ldots \ldots \ldots \ldots \ldots \ldots \ldots$.

Galvanized iron pail..................... ${ }^{.5}$

\section{DRYING EQUIPMENT}

Clothespins, per box of $200 \ldots \ldots \ldots \ldots \ldots \ldots \ldots . .30$

Clothesline, white braided fiber, per roo feet.... .40

Umbrella clothes dryers, outdoor............ 8.00 to $20.0 \mathrm{C}$

Folding umbrella clothes rack for ironing...... I.50

Built-to-order drying closet.............. 50.00 up

Overhead moveable clothes dryer............ 5.00

Bottle stopper, aluminum sprinkler........... .I0

\section{IRONING}

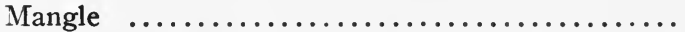

Irons

20.00 to 200.00

Iron holder $\ldots \ldots \ldots \ldots \ldots \ldots \ldots \ldots \ldots \ldots \ldots . .05$

Folding ironing stand and board............ 2.50 up

Permanent metal standard and board......... 12.00

Sleeve board ......................... $\quad .75$

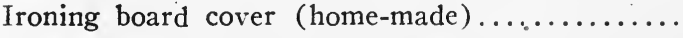

Ironing board clamps or fasteners............ .25

"BoIler-Roller"-Very useful, especially in a country laundry; easily made from a stout box a little larger than a boiler bottom. Turn box upside down and nail legs; about $3 \times 3$ inches, securely in each corner; put on heavy castors. The table when completed should be just the height of the laundry stove. To use put end of roiler close to stove and pull on the boiler, push to laundry tubs or washing machine. Also useful for holding basket of wet clothes and moving same to dry-room or elsewhere. Saves much lifting 


\section{Good Planning for Washday}

The work of washday will be greatly simplified by careful planning and preparation. On no other day is it so easy to get the house in confusion, to serve poor and hasty meals, and for the worker herself to become thoroughly tired out. In most homes the plan of a weekly washday must be followed. But if the supply of clothes is large enough, there is a great gain in having the washing done only on alternate weeks, as this gives one free week for sewing, special cleaning, etc.

Let us suppose that all the washing of a family of four is to be done by the worker herself; only shirts and collars being sent to the commercial laundry. Plan the other days of the week so that the washing is done on Tuesday, contrary to the time-honored custom. This permits the house to be given a good brushing-up after Sunday's confusion and the cooking of practically all of Tuesday's meals on Monday. It also gives opportunity for the thorough sorting and soaking of clothes and removing stains the day before, which is most inconvenient when done on Sunday. When the clothes are sorted they should (except stockings) be mended so that the washing will not make the rents larger, and to obviate the more common plan of mending the garment after it is freshly laundered, which has the disadvantage of crushing the newly ironed article. Also it gives more time for the treatment of stains which, if left to the regular washday, are likely to be overlooked and neglected.

The standard practice for any washday will differ somewhat owing to whether a washing machine is present, the method of heating the water, etc., etc.

Here follow five different "standard practices" for various conditions and methods. They may be modified or 
combined to suit your conditions. When you have determined your "standard practice" it should be written down, with its "time schedule."

STANDARD PRACTICE FOR WASHING, No. I. (Without Set Tubs. See Diagram, page 229.)

Conditions: Two portable galvanized iron tubs with basin plugs and rubber stoppers on wash bench; hand washer; pump; washer mounted on stand higher than tubs, and connected with them by double galvanized iron drainboard. Wringer on washer between tubs and washer; lever of washer to extreme right. Stove and boiler used for heating washer.

I. White clothes soaked in Tubs I and 2 over night (or for half an hour in warm water).

2. Fill boiler from pump.

3. Fill washer with boiling water from boiler, and add soap solution.

4. Drain Tub I and place Load I in washer; operate washer by hand ten minutes.

5. Remove Load $\mathrm{I}$ and run through wringer back on to drainboard.

6. Add more soap and boiling water to washer, refill boiler from pump.

7. Drain Tub 2, and place Load 2 in washer; operate by hand ten minutes.

8. Remove Load 2 and run through wringer back on to drainboard.

9. Drain soiled water from washer; refill with clean hot rinse water.

Io. Fill Tubs I and 2 with cold blue water; start starch preparation at stove.

II. Fill washer with as much of Load I and Load 2 as possible; rinse in washer (by lever) about six minutes.

12. Wring back on to drain, then put into blue water.

13. Repeat Steps II and I2 with remaining clothes.

I4. Finish starch preparation and lay pot on drainboard.

15. Wring clothes loosely from blue water by hand, and starch necessary ones on the drainboard (basket underneath).

16. Hang up all white clothing.

I7. Repeat above steps for colored clothing, of which there wiil be probably only one load, hence shorter time. Do stockings separately by hand in last soap water, and rinse in clear (never blue) water. 


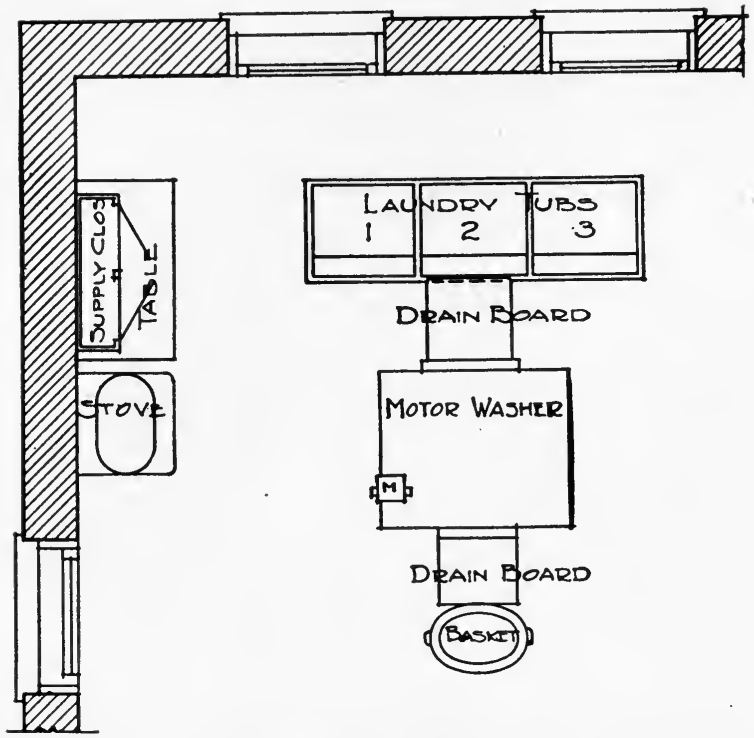

OUTFIT USED IN STANDARD PRACTICE FOR WASHING, NO. 2.

STANDARD PRACTICE FOR WASHING, No. 2.

Condirions: Three stationary tubs; motor operated washer with reversible power wringer and drain chutes; permanently installed hot and cold water and waste connections.

I. Separate all white linen and clothing into two groups, Load A requiring boiling (sheets, cases, towels, etc.), and Load B requiring no boiling (tablecloth and napkins, centerpieces, scarfs, etc.).

2. Cold soak over night, Load A in Tub I, Load B in Tub 2.

3. Drain both loads from soiled water; place Load A in washer with hot water (softened) and soap solution; wash by motor I5 minutes.

\section{LOAD A}

4. Wring and deliver Load A directly into boiler. Boil not over 5 minutes after water comes to a boil.
LOAD B

5. Add more soap solution and hot water to washer; fill with Load B; wash I5 minutes.

Use this time to prepare clear warm water in Tub 3 , blue water in Tubs 2 and $I$, and attend to boiler. 


\section{LOAD A}

I0. Refill washer with clean hot water (softened).

II. Remove Load A from boiler and place directly into washer of clean hot water. Rinse Io minutes by motor.

14. Wring and deliver Load A into $\mathrm{Tub} 3$; rinse by hand.

15. Run through blue water.

16. Run through power wringer into basket. Starch. Hang out.
LOAD B

6. Wring and deliver Load B on to drain chute. Empty washer.

7. Refill washer with clean hot water (softened).

8. Refill washer with Load B. Rinse ro minutes by motor.

9. Wring and deliver Load B into Tub 3. Empty washer. * * * * * *

12. Rinse (by hand) Load $B$ in Tub 3 ; then run through blue water in Tub 2 .

13. Reverse Load B from Tub I through power wringer into basket. Starch on Table $\mathrm{T}$; process ended. (Hang out Load B before finishing with A.)

Repeat steps with colored clothing according to practice for Load B; no boiling. Add hot water and soap to rinse water in Step II. If only one load, drain the washing water off with the clothes in the machine; run in warm rinsing water; run the machine; drain and rinse in the machine with cold water twice; wring and hang out.

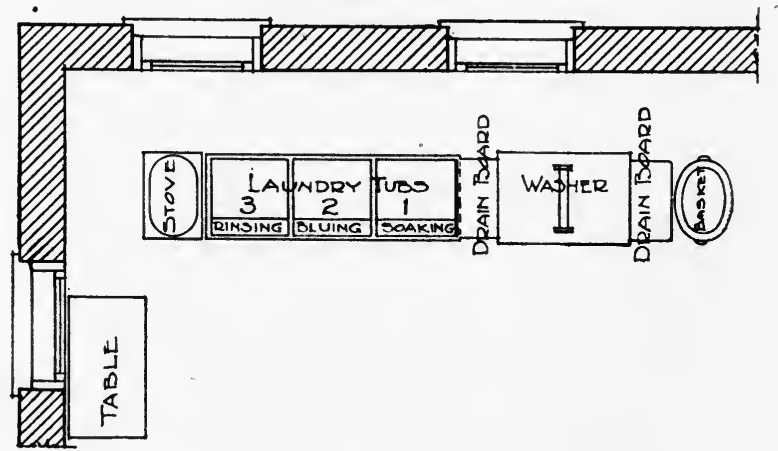

THE "JUDD" LAUNDRY LAYOUT.

For Standard Practice No. 3. 
STANDARD PRACTICE FOR WASHING, No. 3

\section{With “Judd” Rinsing Tray}

Conditions: Three tubs in line with power washer, drain chutes, reversible wringer, Judd Rinsing Tray; machine next to Soaking Tub I; Bluing Tub 2 in center; Rinsing Tub 3 on the end; boiler at same height with side close to end of Tub 3; Load A to be boiled, Loads $B$ and $C$ not boiled, Load D colored clothes.

1. Soak Load A in Tub I, $1 / 4$ to $\mathrm{I} / 2$ hour, in softened warm water with half a bar of dissolved soap.

2. While soaking Load A, oil machine, fill with hot water, soften; add $I^{T} / 2$ bars of shaved soap or equivalent of chip soa?

3. Wring Load A from soaking water through power wringer into machine; run Io minutes to half an hour, till clean.

4. W'inile Load A is being washed, soak Load B in Tub I. Fill boiler, soften, start heating.

5. Wring Load A out of machine on to Judd Rinsing Tray and slide into beiler of boiling water (no soap added). Buil s to Io minutes.

6. Wring Load B from soaking water into the machine; add a little more soap if necessary and start washing.

\%. Start Load C soaking in Tub I.

8. Take Load A from boiler to rinsing tray, let drain; slide load into warm (softened) rinsing water, Tub 3; rinse well; toss on to tray placed on Tub 2 ; swing tray and slide load into warm bluing water; take load over tray; placed on Soaḱing Tub I, through machine wringer into basket; hang.

9. Run Load B, now washed, through power wringer on to Judd Rinsing Tray; slide tray to one side.

Io. Wring Load C from soaking water into machine; add more soap and hot water and start washing.

II. Put Load D (colored clothes) into soaking water (fresh, if necessary).

I2. Rinse Load B from tray in Tub 3, a piece at a time, throwing on to unoccupied part of tray as fast as rinsed; slide into bluing water, Tub 2 ; wring over tray through power wringer into basket; starch; hang.

Repeat process No. I2 with Loads C and D, give second rinsing to Load D in place of bluing. With only two tubs, rinse and blue in the same tub. Rinsing is more thorough if done a piece or two at a time in the whole tub of water as directed. With the Judd Rinsing Tray, wringing between rinsing and bluing water is not necessary.

The Judd Rinsing Tray can be used to advantage in any type of laundry; price $\$ 3.00$, Judd Laundry Machine Co., Chicago. 


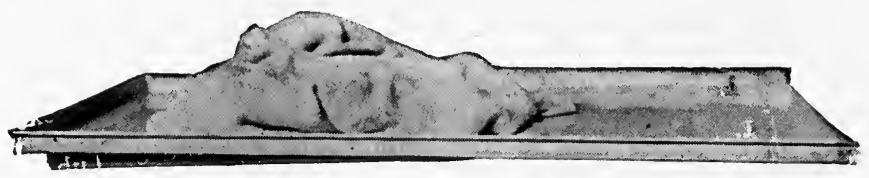

THE "JUDD" RINSING TRAY.

\section{STANDARD PRACTICE No. 4 (PARAFFINE BOIL)}

Conditions: Two portable or stationary tubs; hand power washer wringer; boiler (white clothes only).

I. Soak clothing over night in cold (softened) water in Tubs I and 2 or for $1 / 2$ hour in hot water.

2. Fill boiler with cold soft water and bring to boiling point.

3. Add to boiler $1 / 2$ of the following mixture made previously:

I $\mathrm{lb}$. bar yellow soap, shaved fine.

I cup best quality white paraffine, shaved fine.

I qt. boiling water.

Melt together thoroughly.

4. Drain clothes; wring, and place Load A in boiler.

5. Boil Load A $1 / 2$ hour.

5. Deliver Load A into washer of clear, very hot (softened) water.

7 . Refill boiler with Load B, adding another cup of paraffine mixture and boil $1 / 2$ hour.

8. Rinse Load A in washer, by lever, about ten minutes.

9. Fill Tub I with clear cold water, Tub 2 with blue water.

zo. Wring Load A into Tub I, then place into Tub 2; starch and hang.

II. Repeat steps Nos. 8, 9 and ro with Load B or Loads C, D, etc., as there are about 5 boilerfuls in a large family wash of white clothing. Clothes which are not much soiled need not be soaked but may be put directly.

\section{STANDARD PRACTICE No. 5 (NAPHTHA SOAP)}

Conditions: Two stationary set tubs and water motor washer, with permanent drain and hot and cold water connections. No boiler; hand wringer.

I. Soak clothes (white) over night in softened cold water and naphtha soap solution.

2. Fill washer with tepid water (softened) and naphtha soap solution.

3. Drain clothes from Tub I; wring and fill Load A into washer; operate washer 20 minutes. 
4. Empty washer; refill with clean tepid water; operate washer Io minutes.

5. Empty washer; refill with clean cold water, and operate for a second rinse of 8 to Io minutes.

6. Fill Tub I with blue water.

7. Wring and deliver Load A into blue water of Tub I. Starch and hang.

8. Repeat above steps with all following loads, including colored clothing.

(This cold water and naphtha soap method needs no boiling if a motor washer is used which will give the long-time and strong friction and rinsing. This is the easiest of all methods for only moderately dirty clothes.)

\section{Naphtha Soap Solution}

There are two naphtha soaps, the "white" and FelsNaptha. The latter is much the stronger. Slice 4 bars of soap and place in a two-gallon jar of water. Allow to stand over night. Then beat up the soap with a strong egg beater to thoroughly dissolve. Add about 2 cups of this jelly to each washer of clothes. (Naphtha soap cannot be used with boiling water, hence the plan of beating the jelly until it is perfectly dissolved.)

\section{STANDARD PRACTICE OF WASHING WOOLENS}

I. Avoid change in temperature either of water or drying or pressing.

2. Wash and rinse in water of the same temperature, about $110^{\circ} \mathrm{F}$.

3. Use rain water, if possible. Soften both washing and rinsing water, if hard, with correct quantity of borax or ammonia and finish softening with a little soap solution before the goods are put in the water. Leave a little soap in the last rinsing water to give a soft finish, or add glycerine.

4. Never rub soap directly on, but use soap solution of mild white soap.

5. "Squeeze" rather than rub, and avoid all possible twisting and stretching and rubbing.

6. Handle quickly, "pat" into shape, and dry in warm room or out of doors in warm weather; freezing is harmful.

Woolens may be washed in a washing machine but should be kept separate. 


\section{Washing Colored Clothing}

Colors in fabrics may be either "heavy," as deep brown, blue, red, etc., or "delicate," as pale pink, lavender, green, blue, etc. The latter wash better than the former, which have more tendency to "bleed" or run; but all colored or parti-colored materials need to be washed with great care as even the best dyes are not always to be trusted. It is best to test a sample of the fabric with hot water and soap to see if it will "bleed." If the soapy water shows color, "set" the dye by soaking in salt solution, $\mathrm{I} / 2$ cups to a quart of water, for an hour or two, then dry.

Again, articles are not generally made all of the same colored material but are composed of two or more materials and colors, i.e., a pink striped chambray dress with solid pink collar and cuff of linen; or, overalls of blue denim with red straps, or a centerpiece of white linen embroidered in colored silk. Each kind of material absorbs the same color differently, hence the extreme care needed in washing and also in the later drying and pressing under heat. Colors are affected by all the conditions of alkalis, acids, sunlight, soap and heat.

But all colored materials can be washed under the same general rules, as follows:

\section{STANDARD PRACTICE FOR WASHING AND IRONING COLORED ARTICLES}

I. Set the color, if necessary, by soaking in salt solution.

2. Avoid soaking usually.

3. Wash in water about $110^{\circ} \mathrm{F}$; never hot.

4. Use mild white soap in solution, but avoid rubbing soap directly on material.

5. Wash, rinse and dry quickly.

6. Never boil; generally avoid bluing.

7. Hang wrong side out away from sunlight, as shaded by a sheet or trees, etc. Sunshine and moisture fade nearly all colors.

8. Iron on wrong side with cool iron, using muslin or where necessary to press on right side to avoid "shine". 


\section{Washing of Baby Garments}

In many homes the washing of baby garments is a daily and time-taking task. A few words about it will not be amiss. First, a baby's clothes should never, for hygienic reasons, be mixed and washed with the family clothes. The bands, sox, shirts and petticoats of flannel can be washed as for above directions of woolens. The white dresses, unless very elaborate, need no starching, and only a good boiling each time. The diapers need especial care. The faeces should be at once removed, and the diaper placed in a metal covered pail, like a new garbage pail painted white, or a white enameled pail, until time for washing. If a few drops of Lysol solution are sprinkled in the pail it will remove odors. It has not been found a good plan, in the writer's experience, to soak the napkins that are washed every day as this makes the work more unpleasant.

A small, stiff scrubbing brush should be used for further cleansing; then they may be placed in cold water, rubbed slightly, and then put on to boil in cold water without any chemical, merely white soap chips. Reserve for this use solely a galvanized or white enamel bucket holding 2 or 3 gallons. Boil ten minutes after the water boils. Rinse in clear hot water and hang out. Never use bluing, as this yellows them and causes dangerous chafing on the tender skin. Although many small devices and washers are sold exclusively for baby use, none have proved practical or as simple as the boiling in the pail, as even with a washer they must be boiled in order to be strictly sanitary. Wash hands in alcohol after handling such articles.

\section{SOAP SOLUTION FOR WASHING MACHINE}

I lb. soap chips to 6 gals. water added directly to the water in the machine, or $I$ large cake soap to $21 / 2$ qts. water. Cut soap fine and melt over slow fire; do not cook after it is dissolved.

Soften the water by adding the correct proportion of washing soda solution before adding soap. 


\section{WHITE SOAP SOLUTION FOR FLANNELS}

I large bar (I-lb., 2-oz. size) Ivory soap.

4 qts. cold water.

Cut soap fine, add to water and heat and dissolve. One-half cup wood alcohol can be also added to very soiled woolens. Use half of this in each of the two wash waters. Add I tablespoon glycerine to 2 qts. of the tepid rinse water to give a soft finish.

I qt. water.

\section{COMMON STARCH FORMULA}

3 tblsp. starch (corn).

$1 / 2$ tsp. each borax and paraffine or clean lard.

Make a paste of some of the cold water and the starch to separate the starch grains. Have the right amount of water boiling, and stir in the starch paste slowly, stirring well to avoid all lumping. Boil gently for about fifteen minutes, using an asbestos pad under to prevent scorching. This is a "heavy" starch; thinner quality is made by using less of the starch powder.

\section{QUESTIONS ON HOUSEHOLD ENGINEERING VI}

\section{The Practical Laundry}

I. Make a sketch of your present laundry. What changes would make it more step- and labor-saving? Is your present equipment "grouped" so that work can be easily "routed"?

2 Make out a "standard practice" of your iaundry work, stating what conditions you have to work with, $i$. e., washer, tubs, installation, etc. Do you consciously follow such a "practice" on wash days?

3. What is the chief reason why your present laundry work is hard? Please compare your experience in hand (washboard) rubbing and any washing machine you may have used.

4. How large is your family washing (number of persons), and how many hours does it take to do it, both the washing and the ironing?

5. Have you used motor-operated laundry equipment, and do you like to use machinery, or are you somewhat afraid of it? Is it the first cost that prevents you from using more machinery? 


\section{HOUSEHOLD ENGINEERING VII}




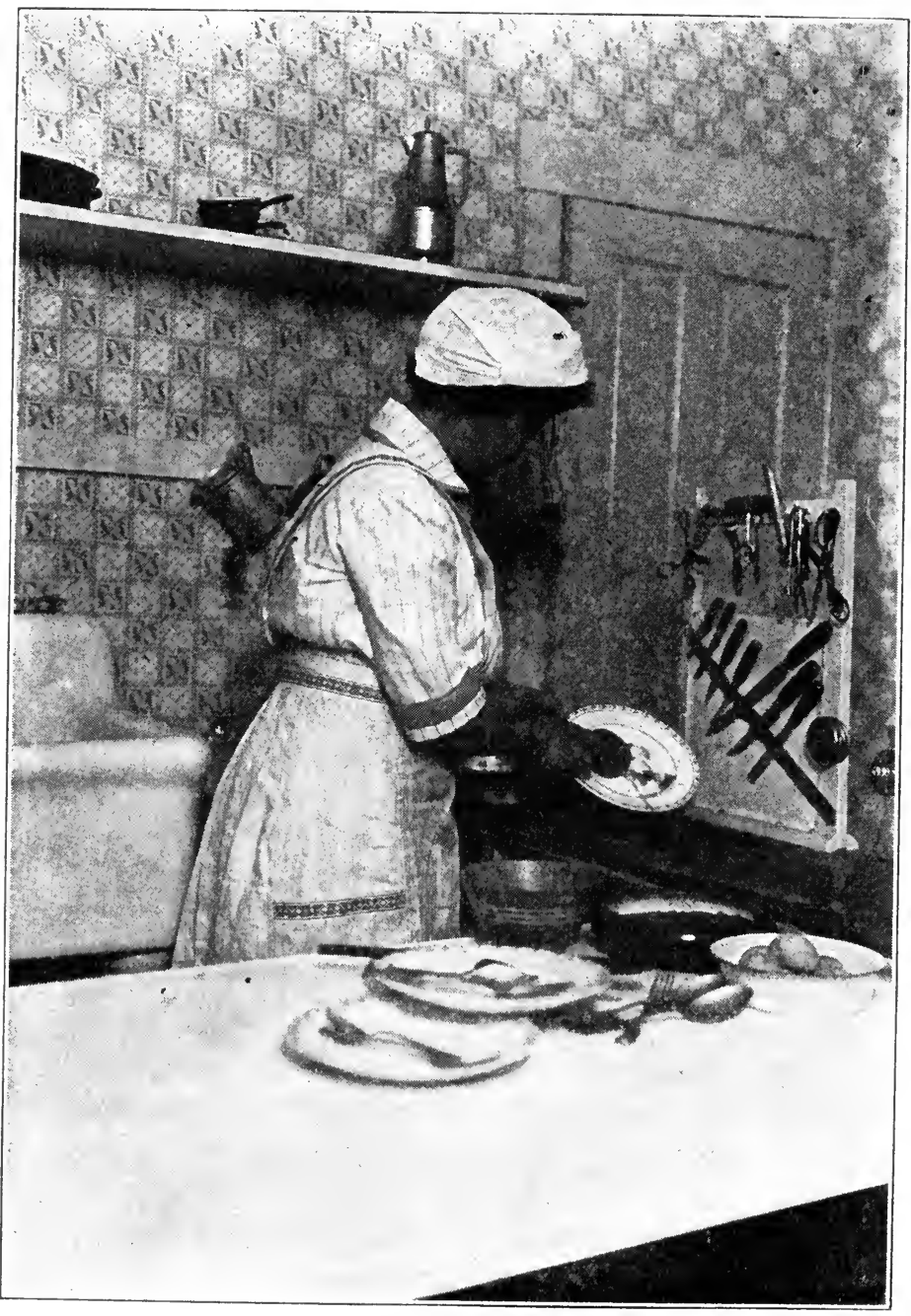

CORNER IN MRS. FREDERICK'S KITCHEN.

Showing rack for knives and shelf with holes over garbage can, where dishes are "scrapped," vegetables prepared, etc. 


\section{HOUSEHOLD ENGINEERING}

\section{VII}

\section{FAMILY FINANCING AND RECORD KEEPING}

“T Y HUSBAND earns \$200 a month; we are five in the family. My husband pays the rent, the coal and his lodge, and I bave a weekly allowance to run the house. I do all my own work, and yet have nothing saved. Do you think keeping accounts would help me? What do you mean by the 'budget' plan? Will you please help me get this money problem straightened out, and tell me how to manage better?"

This letter is typical of hundreds which have come to the author's desk, all asking some questions, about the handling and disbursement of money in the home. "Efficiency" means not only prevention of waste energy in steps and labor, but prevention of waste in money and materials as well. Therefore no discussion of new methods in home management would be complete without seeing how they apply to that most important part of homebuilding-the plans for income spending and saving, and the means of making those plans easy to follow.

In former days there was less need for care in spending or accounting of money first because less actual money was handled. Even as late as $1890,63.9 \%$ of the popula- 
tion was rural, and only $36.1 \%$ was urban. Today $53.7 \%$ is rural and $46.3 \%$ lives in cities. That is, twenty-five years ago $64 \%$ of all the families of the nation lived in the country and raised and manufactured the bulk of its products, for which no money was paid. But today almost half, or over $46 \%$ of all families live in cities, and hence are not producers, but consumers who must buy and pay with money for the countless articles and foods that each home requires.

Therefore it was then not so important for the woman, or the wife and husband together, to follow some definite plan of expenditure. In this present age, too, there are also other urgent reasons why a family must have a conscious plan of spending and saving. These reasons may be grouped as follows:

I. Greater social demands and higher standards of taste and living generally.

2. Longer periods before children become self-supporting, due to modern insistence for education and child labor laws.

3. Each individual today adds a definite cash amount to family expense without so much chance to render back value as in the past.

4. Men are considered economically "unfit" for work and are forced to "retire" from occupations at a much earlier age than formerly (i. e., the man of fifty today finds his place usurped by the young man, and many employers refuse to hire men over a certain age limit).

5. Therefore parents have to provide more savings with which to support themselves during this lengthened period without work opportunity; whereas in the past, when living was simple and rural, there were more kinds of unskilled labor at which even a very old person could "earn his keep." 
6. Increased cost of maintenance in "the rainy days" and the higher cost of medical attendance and nursing in illness.

The health of the family, its education, its pleasures, its savings, are determined, not by the amount of that income, but by the distribution, or the spending of that income. Many families who today may complain of their low income are suffering frequently, not from a low income, but from inis-spending of that income. The whole standard of family living depends only on the apportionment or spending of what the family earns. Two families may, as often happens, have identical incomes; yet one manages to own its own home and send its children to college, while the other will be always on the brink of debt and unable to afford its children advantages. To save, to diminish the expenditure, is just the same as having an increase in income.

\section{Women Hande the Finances of the Home}

For the $18,000,000$ families in the country today over nine billion dollars is spent annually, mainly by women. Women alone buy $48.4 \%$ of all merchandise for family use, and have an important voice in buying $23 \%$ more-buying a total of $7 \mathrm{I} \%$ of all products used in the home. Further, women buy:

$$
\begin{aligned}
& 48 \% \text { of all drugs, } \\
& 96 \% \text { of all dry goods, } \\
& 87 \% \text { of all raw and market foods, } \\
& 48.5 \% \text { of all hardware and house furnishings.* }
\end{aligned}
$$

As most of this sum is spent without keeping records, the proved experience of business. men shows that there

* Figures from investigation by Prof. H. Hollingworth, of Columbia University, N. Y. City. 
must be an attendant waste of at least $3 \%$, probably much more, which means that each year American women by slipshod spending waste at least one hundred million dollars! How important it is, then, not only to each family, but to the nation, that some efficient plan of family spending and record keeping be followed. All too often homemakers complain that their work inside the walls of the home is "uninteresting," and that it has not such "opportunities" as outside "business" affords. Young women who maintain this attitude of disparaging the possibilities in homemaking are frequently the very ones to devote their best intellect to keeping in order the accounts and books of a business firm. Why should not the same intelligence and care be given to the accounts and expenditures of the home-which is the center for which all other businesses exist?

\section{The Whole Family Must Plan Together}

No other question is so important to the happiness of the home as the mutual understanding of finances by all members of the family. If only the wife makes the plans, it is difficult for her to impose them on the other members of the family. If the husband handles the money, doling out a niggardly sum to the wife, no large general plan of all expenditures and savings can be made. The ideals, standards and plans must be shared equally by both husband and wife, and understood by the children, in order to have happy united "pulling" toward a definite aim. Again, if all are equally informed, and agree to the same plan, each will feel a responsibility in seeing that the "plan" is lived up to and carried out. Nothing draws a husband and wife closer together than a frank, businesslike coöperation about their finances-to have a mutual aim to buy a piece of property, pay for a house, put away an educational savings fund, etc. 


\section{Disadvantages of "Allowance” Systems}

While the plan of a personal or household "allowance" to the wife is often followed, the author is frank to say that it is, in her opinion, a relic of some past time when women were supposed to be too inexperienced to handle money, and to whom therefore it was "doled" out by the husband, who was always the financial support. These are the strong objections to the "allowance" plan as we see them: FIRST, it prevents the woman from seeing and understanding more than her own petty allowance expenditures. In handling and having a voice only in this part of the income, she is bound to be inexperienced and fail to grasp the broad view of all the family finances which it is most important she should take. SECOND, it sometimes results in extravagance and onesided expenditure; because, satisfied with her personal allowance and spending it all, perhaps selfishly, the woman loses sight of the other family needs, or does not realize that the personal allowance is an unfair proportion compared to all other items of family expense. THIRD, this plan seems to force a woman into the undignified necessity for begging or skimping for every penny she should have by right. FourTh, the greatest criticism of all, the allowance scheme is unbusinesslike, and makes impossible the satisfactory carrying out of any uniform "budget" plan of expenditures for the whole family.

Housekeeping is a business; husband and wife are equal partners. The entire income (from whatever sources) should be mutually shared and directed by both partners. Any other plan fails of self-respect, and cannot be called true business management. The allowance scheme, either for husband or wife, can only be compared to two partners in a commercial business (having a joint income of $\$ 200$ per month), one of whom gave to the other a small sum, 
say $\$ 20$, and said to him, "Here, Brown, is an allowance for your cigars and neckties; don't worry your head over any other money matters, leave the management of the other \$180 a month to me." We cannot imagine any two actual partners doing such an absurdity; nor can we imagine modern progressive husbands and wives dividing the responsibility of their joint income in any similar silly way. "But don't you believe in a 'household' allowance?" many women will ask. If by "household allowance" is meant all items of family expense, we do; but as generally used by those who write the author for advice, and by other persons, "household" seems to refer only to food, clothing, and a few extra expenses, and is not used to include rent, savings, investments, education, and the really larger items of expense. For instance, one woman writes: "I have a household allowance of $\$ 20$ a week to run the house on; my husband pays the coal, rent and insurance. I buy my own and baby's clothes out of the house money," etc. This kind of "household allowance" plan lets the woman share in the spending of only a few items-while the husband is entirely responsible for others. Such "household allowance" does not permit any carefully thought proportioning of expenses, one item to the other. Neither does it require both to share the responsibility for the spending of the whole income. The wife should share the responsibility for the financial success or failure of the family. And that is why such an "allowance" does not make for good management or efficiency in family spending, for if she does not feel responsible she will not keep the accounts on which the "budget system" of spending depends.

\section{The Old-Fashioned "Household Accounts"}

"I get $\$ 20$ a week to run the house on," again repeats my friend of the above letter, "and I keep track of every 
penny I spend. My husband is economical, too, and yet we never know where we are at from one month to the other. Some months we save; but the next month it seems as if we had to meet a big bill which leaves us 'in the hole.' And there always seems to be something unexpected turning up to use up the little we do get ahead. I can show where every penny went, but I still don't seem to be able to manage-"

An inquiry into the details of the spending in this family, and a look at their "account book" showed this fact-that they were economical to the extreme-they noted every single penny and wrote down a record of it afterward; but they didn't in the least have any general planned-in-advance scheme of spending. They knew what they had spent; but they didn't know what they would spend. And so, while they were "careful" they just met bills as they came along, took out this sum or that as the need seemed to arise, and let their expenses master them, instead of mastering those expenses! When I wrote and asked this correspondent to tell me what her income was, what per cent of it she spent annually on food, on clothes, on amusements, on savings, on running her home,- she didn't know, even with that carefully written account book before her! From all the hundred details, she had no large general percentages which would give the amount spent for one group of needs as contrasted with another, or which would be a guide to future spending. Thus, unfortunately, she had been "keeping accounts" largely to no purpose.

\section{The Budget System}

The old-fashioned "accounts" is to keep track of what has been spent; the new-fashioned "accounts" is to plan what will be spent. That is, instead of one partner having 
an "allowance" for two or three items, or instead of either spending hit or miss, just as the need seems to arise, the new way is to make a spending plan in advance, to be carried out equally by both partners.

This definite, well-thought-out plan of spending money in advance is called a "budget." This term is taken from the bag, or "budget," of financial estimates for the coming year laid before the British House of Commons by the Chancellor or Exchequer. It may be said that a "budget" is "spending on paper." Instead of taking an income, say $\$ 100$ a month, and spending it just as each need seems to arise- $\$ 30$ for food one month, $\$ 20$ the next, "squeezing" down the food some other month because the entire family needs winter clothing, etc., etc.,-the "budget" way is to plan, and particularly to apportion in advance how much can be spent for food, for clothing, and for all other needs throughout the entire year. Its view is annual, not monthly or daily. Its object is not so much skimping, economy or saving, as it is proportionate, balanced spending.

City and national governments, every business worthy of the name, estimate their divisions of expenses for the coming year. "The annual budget," reads the daily newspaper, "for improvement this year in this state will be dollars, to be divided as follows between the counties," and then follows a detailed estimate of the exact proportion of money which can be spent on roads, bridges, harbors, etc., in that state for one year. The "budget" plan can be followed just as satisfactorily in the equally important spending of home finances-how much shall we spend for food, how much for rent, how much for every need and want during the coming year?

Although the needs and wants of any two families may differ widely, it has been found that the following general divisions cover those of all families: 


\section{TYPICAL DIVISIONS FOR ALL INCOMES}

I. Shelter.-Rent, or its equivalent in interest on investment if property is owned; property taxes, fire and burglar insurance; water tax; repair and upkeep of the house; railroad or carfare incidental to situaton of house, etc.

2. Food.-All meats, groceries, vegetables, dairy products, husband's or children's lunch, meals taken away from home.

3. Clothing.-All materials and articles of clothing, mending supplies, dressmaker or tailor, clothing repairs, pressing, etc.

4. Operating.-Light, heat, ice, telephone, wages of maid, laundress and service of all kinds; house-furnishings, labor-saving devices.

5. Savings.-Payment on property, endowment or life insurance, bonds, savings account, etc., beneficiary society or lodge, etc.

6. Luxuries.-Cigars, barber, hairdresser and similar personal luxuries. All extra food, clothing, candy and other indulgences and amusements which are neither necessities nor advancement.

7. Advancement.-Education, music, books and periodicals; club dues, church, charity, gifts, recreation, vacations, health (physician, dentist, medicine), postage, toilet articles, telegrams, etc.

\section{Variation in Family Budgets}

What per cent of any given income shall be spent on each of these seven divisions? There is no one universal answer, because the percentage to be spent in each case and for each family is determined by a number of different causes or conditions. Just as each state cannot blindly follow another state in making an annual "budget" for improvement, but must base the per cent to be spent on the number, location and peculiar needs of its own roads, harbors, etc., so the "budget" for each family is influenced by many factors, chief of them being the following six:

I. The temperament, taste, and especially the "goal" or aim to which the family is striving. 
2. The climate and place where the family is located (city, country, temperate, cold, etc.).

3. The social or professional standing of the group to which the family belongs or with which it wishes to be classed (artisan, doctor, farmer, clerk, etc.).

4. The proximity or remoteness to markets and other sources of supply.

5. The number of persons in the family, and their age.

6. The amount of the annual income (this includes not only the sum earned by the father, but money earned by the children and mother, or received from investments, or the equivalent of a garden, stock, gifts, fees and other sources of revenue).

\section{Budgets Must Suit Needs of Individual Family}

What is called a "statistical family" consist of two adults and three children under fourteen years of age (actually, 4.6 persons). For greater convenience and uniformity, all theoretical budgets prepared by experts, or those budgets based on actual concrete family experience, are estimated on the basis of this theoretical family. But if your family is not the "statistical" one, but contains besides a grandmother, or has three children over fourteen instead of under that age, allowances for increased expenditures will have to be made in modeling your budget after the "typical" one from which you are trying to get help.

Now, as said before, no one family can adopt and follow the "budget" worked out by another family in exact detail; because no two families are identical, owing to the variations made by the six main causes of difference listed abovegoal, climate, number, income, etc. All that a "typical" or "model" budget can do is to act as a guide or pattern which you may follow in outlining your own budget. It is as if you were going to make a new dress from a pattern as 
nearly fitting as possible, but in which you would have to allow extra fullness at the hips, or more pleats in the back, or take in a few darts at the waist-to suit your particular figure!

Again, it is a serious mistake for persons about to make out a "budget" to base it chiefly on income. No budget can be determined by your income, but by what you want your family to get out of that income. Before you plan any budget at all, you must think what objects and aims your family wishes to realize, what values you count high. est, and therefore put first. Because the Smiths have \$200 a month, and your income is also $\$ 200$ a month, is no reason you must spend the same percentage for each item that the Smiths do. This point is emphasized because the author has all too frequently found correspondents blindly copying the per cent spent by some other family with the same income. Each "budget" must be a law unto its own family.

\section{The Family Aim-Goal-Ideals}

You recall that the first "efficiency principle" given and discusssed in the first chapter of Household Engineering was the point of "ideals." "What are we running the home for? What is the goal of this business of homemaking? What do we wish most to secure from life for ourselves and our family? This question of "ideals" or goal must be definitely answered before any budget can be satisfactorily worked out. The aim, and taste, and striving, of a given family must determine the working budget which they are going to follow. Otherwise it would be just as if a ship were to start on a voyage without a port toward which to sail. The budget is to the family what a charted course is to the navigator with a settled harbor in view and definite sailing directions to guide. 
Where do you wish to sail? What port does your family wish to make? Is it saving to send the children to college, or money spent to increase your husband's business, or more æsthetic richness in your life, or opportunity to do more good to the community? Let us repeat that a clear idea of the goal (or there may be several) toward which a particular family strives, is the first essential of budget making. On this choice depends the apportionment of all the seven divisions given above.

Before discussing several "typical" budgets, and how we can get help from them in developing our own, let us see exactly why it is not safe or wise to blindly follow the budgets of other people, or those regarded as "typical." Suppose two families have the same income of $\$ 100$ a month. One, Wheeler, lives in California, where the climate is warm and food prices low. The other, Mr. Morehouse, lives in an eastern suburb, with a rigorous winter, at a long haul from the metropolis to which it is a satellite. In one family the three children are aged 2,4 and 6 , and the mother has a helper in the home; in the other family the children are $8, \mathrm{II}$ and $\mathrm{I} 3$, and the mother and children do all the housework. The father of the California family is a skilled employe of a railroad; the father in the eastern family is a teacher in the high school, anxious to attain a better position.

Although both of these families are the "statistical" family of two adults and three children under 14 years of age, it will be seen instantly how different the per cent each must spend for the seven divisions of their income. In the first case, we will suppose that Wheeler, of California, has no great ambition or chance to ever earn much more in his occupation than $\$ 100$ a month. On the other hand, Mr. Morehouse is willing to spend his evenings on study and courses which will enable him a few years later 
to take a small college position. He wants his children, too, to have the best education. This goal alone possibly determines that a large per cent of Mr. Morehouse's \$100 monthly shall go to his children's educational fund, to books and expenses while he attends courses at a nearby university.

Again, food in the locality being cheap, and the Wheeler chindren being small, a less per cent will need to be spent for food in that family than in Mr. Morehouse's, where he has to feed three older children, and pay the long haul plus the city price for all his food supplies. Further, Mrs. Wheeler feels she cannot get along without help when her children are so small, so she has a helper who costs about $\$ 4$ a week, while the Morehouse children are themselves old enough to assist their mother in the housework before and after school. In addition, Mr. Morehouse, being a school teacher, has to dress fairly well and live in a good district, while Wheeler, who is working in a railroad office, can wear less expensive or work clothes, and lives in a section where rents are low. Also, Mr. Morehouse is expected and wishes to assist certain "causes"-he has the dues from various societies to which he must belong, certain school and social expenses which, being a teacher, he must meet. Mr. Wheeler's reading comprises the daily newspapers and his obligations are optional.

The "budgets" of these two families would therefore differ widely, even though they had the same income and the same number of children, principally because they ivanted to do different things and achieve different results. The author has given these two opposing cases in this detail to illustrate the point that a budget must not be based blindly on "typical" budgets or the experiences of others, no matter how excellent or helpful they may be. The budget of each family, as said before, must stand or 
fall alone, and be based exactly on what that particular family wants to achieve most out of its life.

All that other budgets can do is to suggest the apportionment of a given income for a given number of persons. It has always been a source of disagreement for the author to hear other authorities say, "Given \$Ioo a month, then \$25 a month must be spent for food;" or, "If the annual income is $\$ 2,000$, then $20 \%$ must be spent for clothes," etc., etc. From the two contrasts given above, it will be readily seen that differing families have different needs.

General Laws or Estimates Which Will Help Make INDIVIDUAL BUDGETS

From thousands of actual budgets studied by experts a number of helpful generalizations have been deduced. For instance:

I. The lower the annual income, the higher the per cent that will have to be spent on food. Conversely-

2. The higher the annual income, the lower the per cent necessary to be spent on food. (Example-on an income of $\$ \mathrm{I}, 200$, 40 per cent must be spent to maintain family in health, while on an income of $\$ 6,000$ only 16 per cent will need to be spent on food.)

3. The lower the income, the less per cent that can be spent for savings, luxuries and advancement. Conversely-

4. The higher the income, the greater the per cent that can be spent on these three divisions. 
5. The per cent to be spent on the four divisions, food, advancement, luxuries and savings varies with the size of the income.

6. The per cent to be spent on the three divisions, rent, operating and clothing remains more nearly fixed, whatever the income.

\section{Shelter}

The amount spent for shelter should be determined by convenience of the house, its nearness to business or school, and its sanitary and healthful situation. Too frequently rent is based on a mistaken social pride. Many families spend on rent a sum out of proportion to the other divisions in their budget. A family may rent an elaborate house for their children's sake-an expense which may not be justified, as the children might be just as well and happy in a smaller, less pretentious house. Or a young couple want to "keep up appearances" by living in a fashionable neighborhood, when they should be putting more in their own pockets and less in the landlord's.

If "shelter" includes heat, this extra sum will be balanced by lower operating expense. Distance from school or business must be figured, as rightly all transportation costs must be considered a part of "shelter." If the house has a yard and there are children, the additional expense of the yard may be offset by decreased need for a nursemaid, as the yard will enable children to take care of themselves. Again, the size and style of a house greatly affect the item of "operating," a point too little considered. The number of windows, the amount of service time and labor needed to clean the rooms, the fuel to heat it, the upkeep of lawn, etc., will either increase or keep normal the "operating" item. Even a supposedly "low" rent for a roomy o!dfashioned house may be "high" if the fuel and additional 
upkeep are considered. Shelter, then, must not be considered without "operating," and even some of the other divisions; for instance, if too large a per cent is spent on "shelter," certainly less can be spent on advancement, savings, etc., which would be of more permanent value.

Whether to rent or own is a big question to be only determined by the individual family, the location and the profession and permanency of the work of the father. If the home is owned, the amount of investment that it represents should be divided monthly, and entered under "shelter" in the budget. For instance, if house and land represent an outlay of $\$ 5,000$, then $6 \%$ of this sum annually will be $\$ 300$, the amount that $\$ 5,000$ would bring at normal interest. To this $\$ 300$ should be added taxes, insurance and repairs. The sum divided by 12 will give about $\$ 30$, or the monthly rent of which the house is the equivalent. This amount of $\$ 30$ a month should be entered in the budget. Many times a family, such as minister or supervisor of a farm, has the rent or parsonage free. This approximate value must be set down in the budget, otherwise there will be wrong estimating, such as has been found in many budgets handed the author for inspection. That is, if the income of a country minister is $\$ \mathrm{I}, 200$, and "parsonage free," his actual income is equivalent to $\$ 1,500$, basing rent of parsonage at $\$ 25$ a month. The budget would then be made out, not on a $\$ \mathrm{I}, 200$, but on a $\$ \mathrm{I}, 500$ income.

\section{Food}

The per cent necessary to keep the statistical family in good health has been approximately figured for average city prices as at least $\$ 587$ per year, or about 35 cents per day per person. If there is a garden, the equivalent of this should be credited in the budget as added to the regular income. A large garden will represent about $\$ 100$ to $\$$ I 50 
a year, especially if canned products raised in it is included. If there is one place where good management shows, it is in this item of food. The skill and training of the homemaker in nutritive values and cooking will bear greatly on this point, and one woman may feed her family for twothirds or even one-half of another. However, the amount of food should never be stinted, especially with growing children. But the amount of money spent gives little indication of the nutritive value obtained. Low doctor's fees are an indication of right nourishment and if there is no sickness, one may be sure the food supply is being cared for properly.

\section{Operating}

The percentage spent here will depend on size and style of house, the standards and taste of the family. The cost of a maid or other service by the day, plus the cost of the maid's food, must be charged in "operating." Some make a mistake when they write "We have three in family, and a maid," and then estimate the percentage of food on three, and leave the maid out of calculation. The kind of heat, its control, the kind of furnishings, etc., will all affect this item. Many "skimp" on this division, and put an excess on clothes or other item. As much labor-saving equipment should be bought and counted here as possible. Laundry, or laundry equipment, linens, furnishings and their repair, and all upkeep are included in this item. Simple furnishings, modest houses and good equipment can do a great deal to reduce expenses in this group.

\section{Savings}

While under this heading have been given payments on property, etc., there are other kinds of savings which may be entered as such by some families. Vacations, travel, 
continued expense at one's profession may be a kind of potential savings which will be realized later in increased strength or efficiency. Many families make the error of thinking that "savings" must always be concrete cash. The line between savings and advancement is slight, and many items under both divisions may be interchangeable. Instead of putting money in the bank, one young doctor expended it on further training so that he would become a specialist-a personal savings for the future when his investment in himself would be justified by the returns in a larger income.

In a similar sense, children can be looked on as "savings" or investment, and not as an expense. Healthy, efficient children represent a sentimental investment to their individual families; they are also the greatest social investment which can be made by the state and nation. We are just beginning to take this view as a community, that better babies, more sturdy and intelligent children, are investments to society. We are finding out that a state can "save" in no better way than by encouraging such conditions and investing money in such work as will produce the highest types of children.

In other words, some families fail to invest in themselves, so. that they will be able to do more important work, be more socially valuable, etc., but instead concentrate wrongfully on the kind of savings which are only represented by a bank book and which are often mistakenly made at the expense of present comforts, or even necessities-sufficient food and adequate standards.

The immigrant, who on his farm at a pitiable wage, went back to the old country with $\$ 2,000$ in his pocket after four years, may have saved-but at the price of personal growth and value to the community.

The couple without children, that has never given equiva- 
lent in service to the community, but that has instead amassed commercial savings, has certainly lived by the letter and not the spirit of this meaning.

\section{LUXURIES}

The list of these items is long, and the decision as to which are luxury and which are not, must be determined honestly by the individuals. Autos may be advancement, or the equivalent of transportation, but frequently are pure luxuries. Personal services like those pertaining to the toilet, flowers, gifts and numbers of expenses can be justified on no other ground than the head of "luxury."

\section{Advancement}

This division seems clear enough, except that many correspondents and others fail to see the connection between. health and this division. The care bestowed upon the family's teeth, eyes and health, all items for sanitary use, mark the difference between the family with a low standard and one with a higher view. All spiritual needs, all educational expense, or the means of increasing knowledge, training, development or the making of the members more efficient to themselves and to society, must be classed here.

\section{Where Children's Expense Is Estimated}

"How shall we class the expenses of our children?" write some. It is just as easy to estimate their food, clothing and education as that of the adult. Roughly, the following have been estimated as the cost of children for different ages, omitting the birth expenses, which vary most widely:

Child 3- 6 years $\$ 150$ annual cost, without nurse or attendant

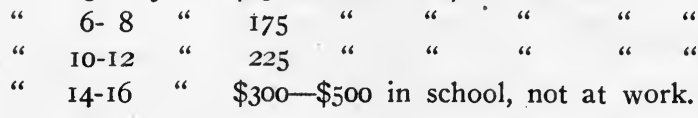


But two children do not double the cost of one. The same equipment will do for two or several, and the same fuel and time to cook for two as one. Also, older children can be taught to help the younger, and thus lessen any "operating" expense of the first child, which might have needed a nurse.

The coming of children is often used as the occasion to raise the whole standard of living, moving to a larger house and employing extra help, when, by applying more efficient household plans, and reducing the elaborateness of the home, this expense could be easily avoided. Fuel, laundry and doctor's fees usually increase the budget when there are small children.

\section{Typical Budgets}

Standard or ideal budgets are of little value except in giving averages from which to vary according to circumstances, as already suggested. In this big country with widely different conditions and prices, it is difficult in ordinary times to give average figures and percentages, but with

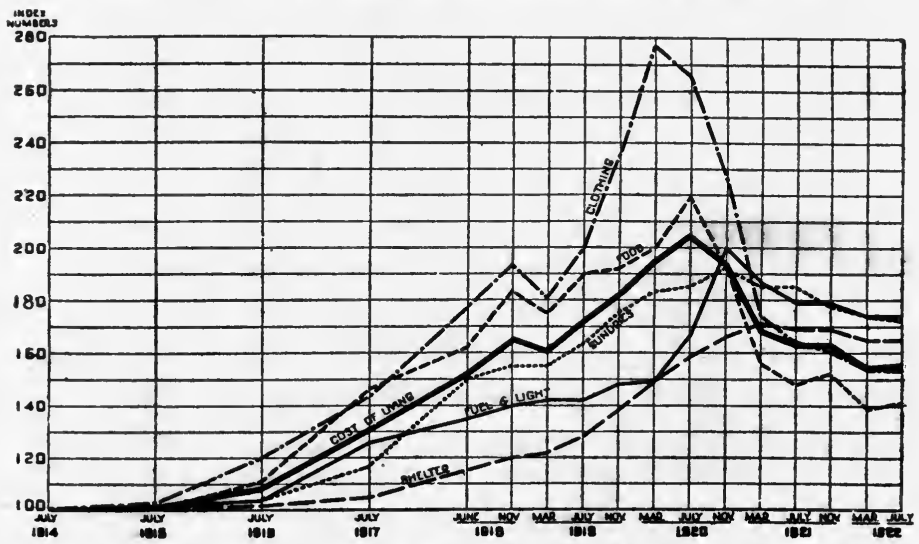

COST OF LIVING, WAGE WORKERS' FAMILIES, 1914-1922.

From Report No. 54, National Industrial Conference Board, New York. 
such violent fluctuations of prices as the past few years have shown, this becomes increasingly difficult.

The diagram gives some idea of the rise and drop in prices from July, I914, to July, I922. The price levels of July, I9I3, are taken as the pre-war standard-there was little change in 1914. These standard prices are higher than those of former years. For example, the average cost of food in 1897 , the low point for some years previous, was about 65 on the 1913 basis of 100 ; by 1907 food prices had gone up to 82 and in I9I I to 92 . In general, prices and the cost of living are now $2 / 5$ to $2 / 3$ higher than pre-war levels. It is probable that succeeding years will show a downward trend, although it may be many years before pre-war price levels are reached, if ever.

The diagram is based on average expenditures in city wage earners' families, pre-war standards, as follows: Food, 43.1\%; Shelter, $17.7 \%$; Clothing, $13.2 \%$; Fuel and Light, $5.6 \%$; Sundries (Advancement and Luxuries), $20.4 \%$. These percentages are quite different from the suggested budgets following and consequently the diagram only in a general way represents the increased cost of living for families with other standards.

STANDARD BUDGETS FOR INCOMES FROM \$100-\$500 MONTHLY. (Statistical family of two adults and three children under 14 years).

\begin{tabular}{|c|c|c|c|c|c|c|}
\hline $\begin{array}{l}\text { Monthly } \\
\text { Income }\end{array}$ & Food & Shelter & Operating & Clothing & Savings & $\begin{array}{l}\text { Advance- } \\
\text { ment and } \\
\text { Luxuries }\end{array}$ \\
\hline$\$ 100$. & $40 \%-\$ 40$ & $23 \%-\$ 23$ & $10 \%-\$ 10$ & $14 \%-\$ 14$ & $5 \%-\$ 5$ & $8 \%-\$ 8$ \\
\hline 150. & $30 \%-45$ & $23 \%-35$ & $13 \%-19$ & $14 \%-21$ & $10 \%-15$ & $10 \%-15$ \\
\hline 200. & $25 \%-50$ & $22 \%-44$ & $13 \%-26$ & $15 \%-30$ & $12 \%-24$ & $12 \%-24$ \\
\hline $300 \ldots \ldots \ldots$ & $20 \%-60$ & $22 \%-66$ & $14 \%-42$ & $15 \%-45$ & $15 \%-45$ & $15 \%-45$ \\
\hline $400 \ldots \ldots \ldots$ & $18 \%-72$ & $20 \%-80$ & $15 \%-60$ & $15 \%-60$ & $12 \%-48$ & $20 \%-80$ \\
\hline $500 \ldots \ldots \ldots$ & $16 \%-80$ & $20 \%-100$ & $15 \%-75$ & $18 \%-90$ & $11 \%-55$ & $20 \%-100$ \\
\hline
\end{tabular}

The above figures represent averages for small cities and towns, family living in a house or cottage. In large cities in heated apartments, the rent would be more, operating less. The food allowances are low, and, to be safe, would require knowledge of food values, with expert purchasing and management. Monthly allowances are $41 / 3$ times weekly allowances.

As indicated in the text, few families are "statistical" and for figuring actual family budgets the best procedure is to subtract the fixed expenses from total income and apportion the balance according to experience. See tables in "The Art of Spending" (10c) and "How the Budget Families Save and Have" (5c). Am. School of Home Economics, Chicago. 


\section{Account Keeping}

Once this "budget" or estimate is made, it must be followed, and some system kept which will keep record of the expenses and see if the actual budget conforms to the theoretical budget. The author's chief objection to most "systems" of keeping household expenses, is their elaborateness, which would prevent the busy housemother from having time to keep them correct and up-to-date.

In order to follow out the budget plan, we will have to use some of the knowledge and terms of regular bookkeeping. But the system should be as simple as possible, the idea being not to make a bookkeeper out of the homemaker, but to permit her to have the most accurate record and also the best "short-cut" method to serve her purpose.

There are two separate methods of keeping a budget or any other form of household account. One is by means of a book, or set of books, and the other by means of a set of cards. The book plan is the simplest for a beginner. It is a proved fact that American women, as opposed to those in England and France, know little about keeping accounts, or records of money. In those and other foreign countries this is made a part of the education of girls-an ideal to be striven for here.

All transactions have two sides; each person is always in the joint position of debtor and creditor. This makes every account of expense have two sides,-the debit and the credit; we are debtor to the cash account for all the money or income received. This cash account must include not only salary of father, but money earned by other members of family, or through investment and the equivalent of garden, free rent, etc. The credit account will include all sums paid out for every item of the budget.

Now, to make this very easy to keep track of, let us rule 
a large blank book about Iox 8 in. Let us use two pages for each month, and divide both pages into similar columns, about twenty in number. Write days of the month in the first column, and in the second write all the cash receipts, or amount to our deposit in the bank. Continue to the right, and label each column for each item of the budget (see illustration), keeping items of one group, as "food" (groceries, meats, vegetables, etc.) together and connecting them over the top with a bracket labeled with the main division of the budget. That is, there will be seven main divisions divided into about twenty columns straight across both pages.

The extreme right column is for "total daily balance." At the foot of each column is ample space for the total of each column and also for other figures. We will suppose that we are paying bills both by check and by cash. We will have a check book, and draw on it from the monthly sum deposited there to our credit which we think will be enough to pay the bills we desire to pay by check, as monthly butcher and grocer, light, etc. We will keep all sales checks or slips, and use them as a basis of the sum entered in its proper credit column. In this account book only the general total sum spent is entered to save space and time, i. e., instead of writing under "Vegetables-cabbage, .Io; carrots, .20, apples .40," we will write the total, "Vegetables .70," as that is sufficient for accuracy and too much detail is complicating. If there are details that we do wish to preserve a record of, they may be kept separate in a small book which corresponds to the "day-book" in business. It may be said in passing, that the household account book now being described for use with the budget plan of expense, is what in bookkeeping or business would be called a "journal-ledger," in which both receipts and expenditures can be seen at a glance. 


\begin{tabular}{|c|c|c|c|c|c|c|c|c|c|c|c|c|c|c|c|}
\hline DATE & & & & 51 & HEI & LTE & $E R$ & & $F C$ & $O C$ & $D$ & & $\mathrm{CLO}$ & DTHII & $N G$ \\
\hline $\begin{array}{c}\text { JAN. } \\
\end{array}$ & RECIEPTS & & & RENT & $\begin{array}{l}\text { Re. } \\
\text { cinas: }\end{array}$ & Taxes & $\begin{array}{l}\text { FIEE } \\
\text { INS. }\end{array}$ & $\begin{array}{l}\text { Davry } \\
\text { PEOD }\end{array}$ & $\begin{array}{l}\text { Frut } \\
\text { vea }\end{array}$ & neoc & $\begin{array}{l}\text { MEAT } \\
\text { MSSM }\end{array}$ & $\begin{array}{l}\text { MEALS } \\
\text { autson }\end{array}$ & Baran & 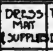 & \\
\hline 1 & 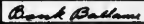 & 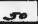 & $1 / 2$ & $20=$ & $y=$ & & & & & 120 & & & & & \\
\hline 2 & beal on hand & 7 & 59 & & & & & & & & & & & & \\
\hline 3 & dalay & 100 & . & & & & & 2 & & .96 & & & & & \\
\hline 4 & & & & & & & & & & & .67 & & & & \\
\hline 5 & & & & & & & & & 25 & .12 & & & & & \\
\hline$\leftarrow$ & & & & & & & & & & & & & & & \\
\hline 1 & & & & & & & & & & .60 & & & & & \\
\hline$\circ$ & & & & & & & & & .57 & & & & & & \\
\hline$=$ & & & & & & & & .92 & & & & & & & \\
\hline 10 & & & & & & & & & & & & 100 & & & \\
\hline & $=-n$ & & m & $\Rightarrow$ & & $\sim$ & & $=7$ & 1 & $=$ & $=$ & & & 工 & \\
\hline 30 & & & & & & & & & &.$/ 2$ & & . & & & \\
\hline 91 & & & & & & & & 2.20 & & & & & & & \\
\hline $\begin{array}{l}\text { MONTHYY } \\
\text { TOTALS }\end{array}$ & & 157 & is & 2000 & 9 & & & $1 \infty$ & $5: 42$ & 1215 & 6.20 & 100 & & & \\
\hline $\begin{array}{l}\text { GROUP, } \\
\text { TOTALS }\end{array}$ & & & & SHEL & ITER & $=\$ \frac{2}{2}$ & 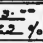 & Fa & & & & $\begin{array}{l}32.25 \\
30-\end{array}$ & & & 8 \\
\hline
\end{tabular}

RULING FOR THE ACCOUNT BOOK-LEFT HAND PAGE.

How the Account Book Follows the Budget Plan

Now let us begin to keep the accounts of January or the new year accounts. Let us suppose that we had a bank balance of $\$ 50$, and $\$ 7.50$ in cash from December. The first column would be as per illustration. Now as we pay cash for meat, groceries or what not, we merely enter the total sum in its proper column. We also pay other weekly bills by check. If so, we will write the sum under its proper column, and note on our check stub the firm to whom paid, the date and number of check. Every week it will take us perhaps forty-five minutes or less to "balance the book." To do this we add the figures of the daily expense and place in total weekly expense column. Supposing the total amount spent, according to your account book, is \$27. Your check book shows a total of $\$ 24$ paid by check. That would mean you had paid out the difference between $\$ 27$ and $\$ 24$ in cash, or $\$ 3$. You began the week with $\$ 7.50$ in cash and spent $\$ 3$ in cash. Therefore your purse should contain $\$ 4.50$. It takes longer to explain this than to actually 


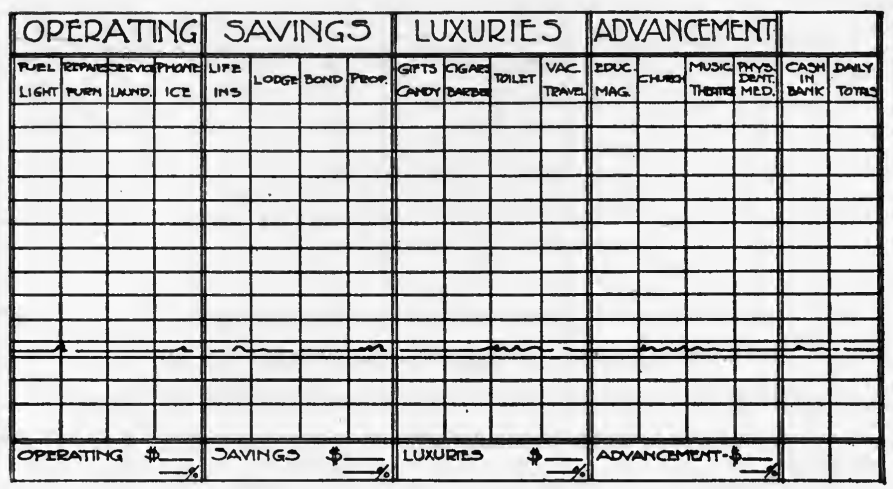

RULING FOR THE ACCOUNT BOOK-RIGHT HAND PAGE.

do it. In many cases a monthly balancing would be sufficient. At the end of the month total each of the 20 columns under the seven large divisions given above. We may find that the total sum spent in some one division is beyond our budget allowance. For example, "operating" in January may run away beyond the \$I4 per month limited by our budget. But if we look over the operating column carefully we may find that the bill for the winter's coal has been paid in January and thus brought up operating expenses. But as this is a cost to be distributed over several months, we will find that we have really not exceeded our appropriation.

Perhaps, however, we find that our food bills have been exceedingly large, and upon close scrutiny we see that we really have been extravagant in supplies. So we promise ourselves to "hold down" food expenses in February and keep within our budget.

In the back of the account book should be a record of the budget as planned for the first of the year. At the end of the year the totals of each of the seven divisions should be copied in the back alongside of the estimated totals for 

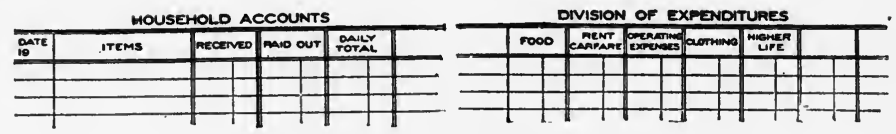

A SIMPLE ACCOUNT BOOK

Published by Am. School of Home Economics, 64 pages, cloth bound, Price $\$ 1.00$, postpaid.

quick comparison. This will be a simple kind of "balance sheet" and the failure or success in living up to the budget can be seen. The budget of this year will be nothing more or less than information for future spending next year. Indeed, no budget is perfect the first year. It takes some time to make one which will be smooth running and adjust automatically to the increased demands on it of constantly growing older children, etc., etc. But it is only by having some basis, some first budget, that future ones can be made more nearly perfect.

\section{Account-Keeping by the Card System}

This plan is a little more complex, or at least more difficult for the beginner to handle. Instead of a book we use $4 \times 6$-inch ruled cards, which can be bought at stationer's or of office supply stores for about $75 \mathrm{C}$ or $\$ 1.00$ per thousand. In addition, there will be needed about 20 cards with a special tab on the top called "guide cards." In this plan one card will take the place of one column in the book. The number of cards to be used will vary with the subdivisions or needs of each particular family. The author has found the following plan with twenty cards satisfactory for an average family: 
Materials Needed: Guide cards with tabs, of stiff cardboard, size $4 \times 6$ inches; 250 plain ruled $4 \times 6$ inch cards; small cloth or wooden box with cover to fit cards. (These can be bought for about 50c.)

Rule twenty of the plain cards, like the "Groceries" card illustrated. Write in the upper left-hand corner of each card the subject, or division of the budget, as follows (from I to 20 ) :

I. Shelter..... $\begin{aligned} & \text { 6. Rent, railroad and carfare } \\ & \text { 7. Taxes, fire and burglar insurance }\end{aligned}$

II. Food......... $\left\{\begin{array}{l}\text { I. Dairy products } \\ \text { 2. Fruit and vege } \\ \text { 3. Groceries } \\ \text { 4. Meat and fish } \\ \text { 5. Meals outside }\end{array}\right.$

III. Clothing.... $\left\{\begin{array}{l}\text { 8. Bought garments } \\ \text { 9. Dress materials and supplies } \\ \text { I0. Tailor and dressmaker }\end{array}\right.$

IV. Operating... $\left\{\begin{array}{l}\text { II. Fuel and light } \\ \text { I2. Furnishing and repairs } \\ \text { I3. Service and laundry } \\ \text { I4. Telephone and ice }\end{array}\right.$

V. SAvings..... $\begin{aligned} & \text { 19. Life insurance, real estate, beneficiary so- } \\ & \text { cieties, bonds, etc. } \\ & \text { 20. Cash in bank }\end{aligned}$

VI. LUXURIES.... $\begin{gathered}\text { I8. Gifts, candy, toilet articles, vacation and } \\ \text { travel }\end{gathered}$ VII. Advancement $\left\{\begin{array}{l}\text { 15. Education and periodicals } \\ \text { I6. Church, music, theatre } \\ \text { 17. Physicians, dentist, medicines }\end{array}\right.$

These twenty divisions or cards seem to cover the needs of an average family, but special cards can be made out for Garden, Automobile or Buggy, Chickens, or for each mem- 
ber of the family if it is desired to keep separate records for each of the children.

Arrange all twenty cards under their proper guide card, as from I to 5 under Food, I I to I4 under Operating, etc., and place upright in their box. As a purchase is made simply write the total amount, on its proper date, on its proper card. Each of the cards contains room to enter the total spent on any one item for every day in the month, with a space at the bottom to write the monthly total“Groceries, \$12.46," or "Fuel and Light, \$4.60."

In addition, prepare a recapitulation card (see illustration), on which to enter the sum total spent on each division of the budget for the entire year.

This one card alone, then, will at a glance show exactly the total spent on all the divisions of the budget, and can be compared and used as a basis for succeeding budgets, just as the simple "balance sheet" was used with the book plan.

\section{Advantages of Payment by Bank Check}

It is surprising how few housekeepers use a checking system to pay household bills. A checking account will be helpful in either the book or the card plan. It is much the best to pay as few bills by cash as possible, and to keep little cash around the house. The monthly sum which it is estimated will cover all bills payable by check can be deposited in a local bank, where it is safe, and where it may even get a low interest. Every check stub is a receipt in itself, and thus is one good means of "keeping track" of what has been spent. In business, the word "voucher" is used to mean "any document which bears witness to the truth of a statement made in the credit side" (of the account book); now, either a receipted bill or sale slip is a voucher when signed by the person to whom the money is 


\begin{tabular}{|c|c|c|c|c|c|c|c|}
\hline \multirow{2}{*}{ Grocerles } & \multirow{2}{*}{\multicolumn{2}{|c|}{$\begin{array}{l}\text { Jan. } \\
\text { Month }\end{array}$}} & Carried fwd. & 693 & Carried fwd. & 9 & 54 \\
\hline & & & 11 & & 22 & & \\
\hline 1 & 1 & 29 & 12 & 84 & 23 & 1 & 28 \\
\hline 2 & & & 13 & & 24 & & 43 \\
\hline 3 & & 56 & 14 & & 25 & & \\
\hline 4 & & & 15 & 119 & 26 & & \\
\hline 5 & & & 16 & & 27 & & 59 \\
\hline 6 & 4 & 93 & 17 & & 28 & & \\
\hline 7 & & & 18 & & 29 & 1 & 62 \\
\hline 8 & & 15 & 29 & 58 & 30 & & \\
\hline 9 & & & 20 & & 31 & & \\
\hline 10 & & & 21 & & Month's Total & 12 & 46 \\
\hline & & & & & & & \\
\hline
\end{tabular}

ONE OF THE CARDS FOR HOUSEHOLD ACCOUNTS.

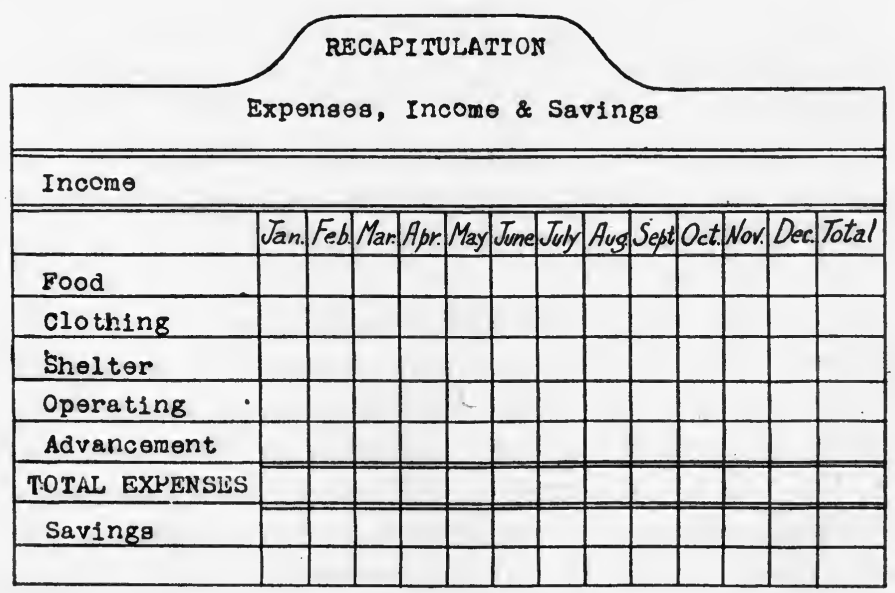

THE SUMMARY CARD OF THE HOUSEHOLD ACCOUNT FILE. 
paid; or the endorsed check paid to a person is also a voucher. These endorsed checks come back from the bank the first of the month, and should be kept as receipts that bills have been paid. It is a good plan to keep receipts of every kind at least one year, and important ones even longer.

\begin{tabular}{|c|c|c|c|c|c|c|c|c|c|c|}
\hline \multicolumn{4}{|c|}{ MONTH OF Ott hav. 1920} & \multicolumn{7}{|c|}{ EXPENDED FOR } \\
\hline \multirow{2}{*}{$\begin{array}{l}\text { DAY } \\
\text { AND } \\
\text { NO. } \\
\end{array}$} & RECORD OF CHECKS & $\begin{array}{l}\text { A.MT OF } \\
\text { CHECK }\end{array}$ & \begin{tabular}{|l} 
BANKS \\
BLAANCE \\
\end{tabular} & SAVINGS & 5000 & сLотиime & 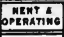 & KDYARCE- & LuXunies & pestomas \\
\hline & TOTALS AMD BAL FORWARO & $407 \cdot 11$ & 260.86 & 50.00 & 46.86 & 87.27 & 94.90 & 41.91 & 49.53 & 36.64 \\
\hline$\frac{26 \text { ta }}{10.27}$ & $\begin{array}{l}\text { ro mins addie marten } \\
\text { ron Areas mikg (2) }\end{array}$ & 12.30 & 248.56 & • & - & $12 \cdot 30$ & • & - & - & - \\
\hline $30^{20}$ & To S.S.mevonald & 25.08 & 223.46 & • & 25.08 & • & • & • & - & - \\
\hline 10.28 & ron 13.06 meat $12.02 \mathrm{Vag} .1 \mathrm{tt}$ & & & & & & & & & \\
\hline $30^{24}$ & $\begin{array}{l}\text { To fleming oros } \\
\text { ror sroceries }\end{array}$ & $\begin{array}{l}18.81 \\
46.3 .30 \\
\end{array}$ & 204.65 & 50.00 & $\begin{array}{l}18.81 \\
90.75 \\
\end{array}$ & 99.57 & 94.90 & 41.91 & 49.53 & 36.64 \\
\hline navel & Deposir 200.00 & : & 404.65 & - & - & - & - & - & - & - \\
\hline 155 & To E. Srate Bank & 50.00 & 35465 & 50.00 & - & - & - & - & - & • \\
\hline $11 \cdot 1$ & ron Educational Fund & & & & & & & & & \\
\hline 2nd & $\begin{array}{l}\text { ro a. H. Whitley } c_{0} \\
\text { ron Rent }\end{array}$ & 60.00 & 294.65 & - & - & - & 60.00 & - & - & - \\
\hline ites & 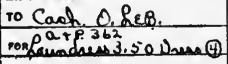 & 10.00 & 284.65 & - & 3.62 & 1.40 & 3.50 & - & - & 1.48 \\
\hline & DEPOSIT & & & & & & & & & \\
\hline
\end{tabular}

PORTION OF AN INTERLEAF OF THE HOUSEHOLD ACCOUNTING CHECK BOOK

The end of October record and beginning of November record shown: column at left gives total spent, the seven columns at the right, "where the money goes." The (2) in the first item indicates Wife's Clothing according to the "Key"; the (4) in the last item Child B Clothing. Note distribution of this cash item.

It is a much better business to pay a four weeks' bill to the home office by one check than to have the interruption of paying a collector at the door four times a week. The less money handled, the fewer mistakes and the more business-like the account can be. Efficiency in keeping money is judged by the small amount of cash carried in the homemaker's own purse. Indeed, the simplest and surest method of keeping a record of family expenditures is to deposit in the bank all money received and check it out for all expenses-even for "petty cash." 
Automatic Budget Accounting

Recently this bank accounting system has been perfected by the invention of the "Self-Accounting Check Books" which have been adopted by many progressive banks.

In the new check book are bound special interleaves having classified columns as shown in the illustration. The same classification as recommended in Household Engineering is used, with an additional column headed PersonalPersonal Allowances. When writing the checks the amount is entered in the column at the left and in the proper classified column or columns. Simple addition at the end of the month gives the total expenditures for the month and the total in each division. No other accounts need be kept, though if desired the small "Weekly Allowance Book" may be carried in the pocket book for recording personal allowances.

The reverse side of the interleaves provide for a classified record of receipts. A table at the bottom of this page is intended for sub-division of expenditures under the main divisions. The "key" in the "Directions" suggests sub-divisions which may be kept; for example, under Clothing: (I) Man's Clothing, (2) Wife's Clothing, (3) Child A Clothing, etc. ; under Food: (I) Groceries, (2) Meats, etc., (3) Fruits and Vegetables, (4) Milk, Cream, Butter; (5) Outside Meals. These sub-divisions may be varied at will and kept or not as desired.

When the small sized checks are used, the new check book is of very convenient size, 7 inches wide by $81 / 2$ inches long. It contains 300 checks as usually made up, sufficient to last most families for about a year. If your bank has not adopted this system, for a small sum you can obtain a set of the special interleaves, with Directions, table for Yearly Summary and table for drawing up the family budget. Your local bank can then have the new check book 
made up for you, or may obtain the record sheets bound together and use checks from pocket check book "fillers," keeping the records in the bound "Check Record."*

This new system overcomes the bug-bear of household accounts which, it must be confessed, are somewhat difficult, and require much persistence. The Self-Accounting Check Book is really no more difficult to keep correctly than an ordinary check.book. Best of all it can be kept easily from month to month and year to year so that it makes the family budget a thoroughly practical program.

Further description and suggestions on division of income, etc., are given in the booklet, The Art of Spending, How to Live Better and Save More.

\section{The Budget Plan with Irregular Incomes}

Frequently letters come from those who say that the budget plan is practical for those with steady incomes, but not for the doctor, lawyer, or others who receive irregular incomes. A plan for such families is to establish at once a "reserve fund" or "pool" in the bank which will carry them and run current expenses. For instance, a dentist may receive $\$ 150$ in fees one month, $\$ 300$ the following, and so on, or $\$ 2,400$ the whole year. The way to make up a budget in such a case is to make a monthly average of the total income for the previous year and base the budget on that. Another method is to use an "Office Accounting Check Book" for the business and send a "salary," say \$200 a month, to the "Household Accounting Check Book" account for detailed budget recording.

\section{How Other "Records" May Be Kept Efficiently}

But records of family expenditures are not the only facts or data which every homemaker should be able to put her

* "Self-Accounting Check Record"-Household or Office, 76 pp. $\$ 1.00$; "Weekly Allowance Book," 10 cents; "The Art of Spending," 10 cents. Am. School of Home Economics. Drexel Ave. at 58th St., Chicago. 
fingers on instantly. Is there any other "business" outside the home which has more different kinds of information, papers and bills to keep track of? While many women are orderly and most careful in this regard, it is true that countless other women managing homes spend untold hours of energy hunting, pulling out and searching for this or that paper or information when it is suddenly needed.

Now, it is just as inefficient to waste energy looking for a recipe to give a friend, or for the month-before's gas bill, or for a set of heavy underwear, as it is to allow "waste motion" in the kitchen or in cleaning tasks. Just as we have shown the need of a definite system or routing of cooking and other tasks to save time and effort, so there is equal need of a definite system or plan of keeping in one easily accessible place the different information which the housekeeper is constantly using. Some woman may say, "Oh, but I do keep addresses in a book and have recipes pasted in my cook book, and put clippings into envelopes." The fault with these plans is their lack of uniformity, and the fact that separate books and envelopes are just as likely to be "lost" or mislaid as the important facts they contain!

Another thing is that the quantity of information is constantly growing in proportion to the family's expansion; therefore some plan must be followed which will not only keep all information together, but allow for future increase for a number of years.

Now, after several years' struggle with separate books, "pigeonholes" in a desk, and other common means of keeping household records convenient, the author has, for a number of years, successfully followed an adaptation of the "filing system" used in every modern library and office, and which is only a step beyond the method of keeping accounts described above. 


\section{A “Time-And-Worry Saving” Household File}

The basis of this plan is again the $4 \times 6$-inch filing and guide cards spoken of in connection with the system of keeping household accounts above. It is so simple, inexpensive and satisfactory that it is worth a six months' trial by every housekeeper. Its advantages are:

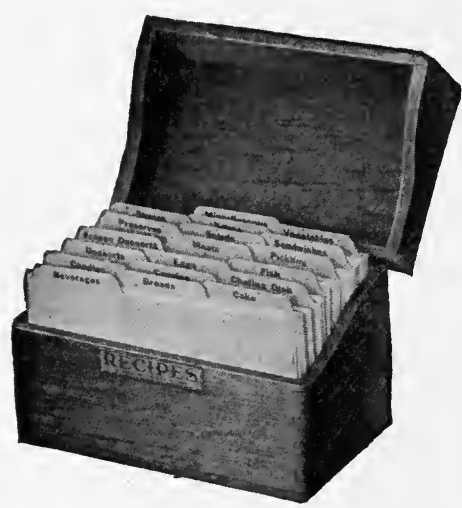

SMALL RECORD FILE

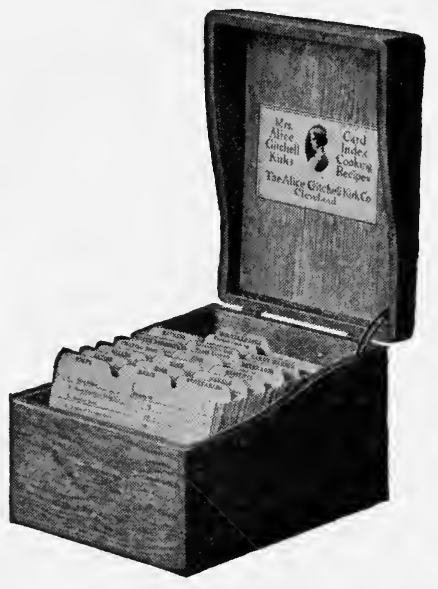

FILE WITH PRINTED RECIPES

I. It keeps all records and data in one readily accessible place.

2. It is expansive, so it can grow with the family's needs.

3. It is uniform, thus doing away with separate books and papers.

4. Changes or mistakes can be rectified with a minimum of trouble.

5. It cannot be "lost," but is easily available to every member of the family. 


\section{THE HOUSEHOLD RECORD FILE}

Materials Needed: $6 \mathrm{x}_{4}$ filing cards (about 500); guide cards with tabs, about 60 ; small cloth or wooden box; 2 sets "alphabet" guide cards. (The $5 \times 3$ in. cards may be used and are cheaper, but the larger cards are somewhat better.)

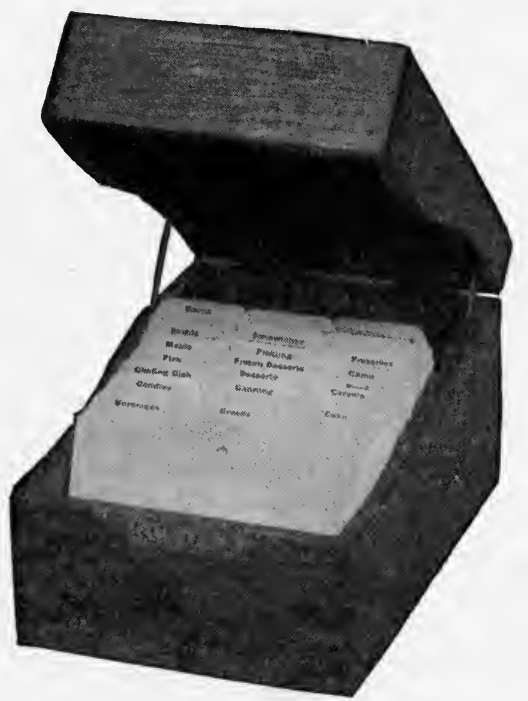

A LARGER RECORD FILE

First, decide what are the subjects or "heads" under which you need or which you want to keep information. These will naturally differ with each person or family, some having certain interests on which they want to keep information, others different ones. The following outline is given only as suggestion which will apply to all average families, and additional héadings can easily be added. In the writer's own plan the twenty-eight cards for household accounts are kept in the same box with the household file and is therefore included here: 


\section{DIVISIONS OF THE HOUSEHOLD RECORD FILE}

I.-Housenold Accounts (as given and subdivided above)

2.-ADDRESS RECORD-
(a) Social
(b) Commercial
(c) Special

3.-Medical ReCoRD-
(a) Physician
(b) Dentist
(c) Oculist

4.-House ReCoRDS-
(a) Family sizes
(b) Clothes storage
(c) Sheets, bedding, linen
(d) Supplies and pantry
(e) Gift and occasion
(f) Housefurnishings costs
(g) Repair dates
(h) Special supply firm addresses (stove dealers, home equipment, manufac- turers, etc.)

5.-LIBRARY ReCORDS-
(a) Fiction
(b) Poetry
(c) Reference
(d) Books to read or buy
(e) Music list (sheet, pianola, victrola)
(f) Music to buy

6.-Financial Records-
(a) Taxes, real estate
(b) Document record
(c) Bank records
(d) Income
(e) Bills receivable
(f) Bills payable
(g) Personal financial accounts
(h) Organization dues, etc. 
7.-House Hints-

(a) Baby care and hygiene

(b) Garden and plant care

(c) Toilet suggestions

(d) Entertainment, games, party, etc.

(e) Jokes, stories, etc.

8-General Inventory-
(a) Silver and jewelry
(b) Furniture
(c) Clothing
(d) Furnishings and miscellaneous

\title{
The Address Record
}

Arrange the two sets of "alphabet" guide cards in their proper order. Use one card for each name, writing the surname first and given name last, and the address on a lower separate line; use balance of space to write other points to be remembered, as :

\author{
Walker, Mrs. Susan J. \\ 1409 Emery Av., \\ Springfield, Ill. \\ (Pres. Club for Business Girls) \\ Mem. Gen'l Fed. Women's Clubs \\ (Interested in hotels for working women)
}

J. J. Boch \& Co., ro Barclay St., New York City.

Cut glass, imported china and novelties

(Repair broken china, ivory and shell articles-reasonable price) 


\section{The Medical Record}

Use a separate card for each member of the family. This is an especially helpful division where there are children, and where the schools require specific information; it also assists physicians by giving them a statement of the child's past health, as :

David, born Sept. 18 th, 1908

Adenoids removed Feb., I9I2

Vaccinated Sept., I9I4 (see certificate, V-receipts)

Chickenpox, March, 1915; eyes examined May, 1915; very farsighted glasses for close work.

The "Dentist" card may contain the date and cost of fillings, etc. The "Oculist" card should give date of eye examination, and prescription of glasses if they be used.

\section{House Records}

The first group here is that of family sizes (a); this means a card for each member of the family, giving all sizes, so that the cards may be slipped into the shopping bag and used as a guide. How many times one has seen a "bargain" in men's shirts which could not be bought because we did not "remember" whether the neck size was I4 or $15 \frac{1}{2}$ ! The card for one child gives sizes of underwear, suspender waist, stockings, shoes, neck measure.

\section{Clothes Storage}

While many women carefully wrap and' tag articles of clothing to be laid away, not always is there a written description of the place where they can be found. Useless "rummaging" and energy follow a vain search to locate a 
sweater, etc., when the need comes unexpectedly. After tying and labeling each bundle, any plan may be used of writing on the card, as "Full dress shirts and mufflers-hall window seat;" "children's summer nighties_right lower of cedar chest."

\section{LINEN RECORD}

While marking linen in embroidered initials is decorative, there should be some plan of more practical use to assist the housekeeper to know how many and how long her linen lasts. A simple plan is to write the date of purchase on the sheet, together with some distinguishing letter, and the sheet number. For instance, if there are three sizes of sheets used, let A be used on wide sheets, B on narrow ones, and $\mathrm{C}$ on crib or baby sheets. Letter each sheet of its class, A-I, A-2, B-2, B-3, etc. The following written carefully on the lower hem of a sheet with indelible ink, . C-5, I9I3, means that this is one of the crib sheets bought in I9I3. The date of purchase is valuable because it gives a basis on which to figure how long that quality at a given price wore. The letter is a means of accuracy in making out the laundry list. On the card itself should be written the cost, size and firm from which bought, if a really accurate account is desired. If a sheet has worn badly, why purchase the same grade and price of the same concern? Only a card record of this kind will give the facts for future purchasing.

\section{Other House Records}

The card with pantry supplies gives a list of the stock articles used in a particular home, the sizes of the cans, and the cost. For instance, one of the items reads, "Pineapple, No. $2 \frac{1}{2} 2$ can, contains eight slices, cost .I6:" it is used as a basis in quick ordering, because it contains the exact kinds and styles of products used. It has saved time 
over and over again in making out orders of quantity grocery supplies. A duplicate card of canned goods particularly can be made out and hung in the kitchen. Then, as a can of peas or peaches is used, it should be crossed off the list, so that at a glance the number of cans on hand of any particular product can be seen without poking around the storage and actually counting the cans.

A card stating the article received or given as gift is often a help at holidays or occasions. The card of house furnishing costs contains such items as the cost of painting the porch and entry; the price of bed pillows for a given size and weight; the cost of garden tools, etc., or any cost which will in future be a guide in buying. This point is emphasized over and over, because it has been noted that much unnecessary time is wasted making new estimates and measures because no one can "lay hands on" the ones made previously. Too much time is devoted to shopping, in the zeriter's opinion, because there is no accurate record of previous costs. This is one of the places where industrial efficiency is most marked, as all costs are most carefully kept for instant reference.

The other subdivisions of the file can be understood easily. The object of financial records (6) is to give, in concise form, a statement of all money, property and valuables which the family possesses. Then, in case of unexpected death, say of the head of the family, a record would be had at home of the various investments and matters, the valuable papers of which should be in a safety vault out of the house. Too many times a suddenly bereaved family has not the least idea of the condition of affairs in which the father has left it, and only long legal search discovers it. The inventory (8) is necessary so that in case of fire, or burglary, a record can be had of the actual belongings and furnishings. Such a permanent inventory is the basis 
of house, fire and burglar insurance. Other divisions can be made at will. The best point about this household filing plan is that it.expands exactly as one wishes. One small box may do to begin; a larger one can easily be added when necessary. If desired, a more permanent wooden drawer can be used, with a wire rod down its center, on which to fasten the cards, so they cannot be lost or taken away. The writer, who began five years ago with a small box, has now four drawers of cards for household and professional use.

If a card should be incorrect, or when new facts need to be added, all that is necessary is to write a new card to replace the old one. The card plan becomes an easy "habit," and anyone who has tried it long enough has become enthusiastic over its ease, compactness and quality of being in one place. As laid out by Mr. Harrington Emerson, it will be remembered that "immmediate, reliable and accurate records" is one of the chief principles of efficiency. Even many a housekeeper who now keeps "accurate" records has not them so arranged that they are "immediate" to get at or read; the system of household filing suggested above will, more than anything else we know, or have seen tried, help the housekeeper to keep all her important household information "immediate, reliable and accurate."

\section{The Card File Idea Applied to Cookbook}

One more department of housewifely knowledge was omitted from the above discussion, and that was, all information relating to cooking, recipes and household practice, because it seems better to discuss it separately. But here again is another great interest of the housekeeper-recipes and cooking methods. How shall she arrange it all? The usual cookbook contains much that is only reference; again, the interested cook is always clipping new recipes from 
periodicals, and exchanging them with her friends. What shall she do with this new material which is supplementary to the regular cookbook? Again, the usual way of laying a cookbook on the table and following the recipe results in spattered and soiled pages, as well as being difficult to follow, as it lies below the eye level. All these faults are remedied by making a cardfile recipe cabinet, as follows:

\section{Card File Recipe Cabinet}

Materials: $6 \times 4$ cards (about 500); 20-30 guide cards.

Write on each guide card the divisions into which you prefer to classify your recipes. Following is a practical arrangement, arranged alphabetically:

r. Beverages

2. Breads, yeast

3. Breads, quick raising

4. Cocktails, fruit, shellfish, etc.

5. Beans, peas, lentils

6. Cakes and icings

7. Candies

8. Cheese, rarebits

9. Desserts-

(a) Without eggs

(b) With eggs

(c) Cake

(d) Fruit

(e) Pudding

(f) Frozen

ro. Eggs, omelets

iI. Fish, salt

12. Fish, fresh, shellfish

13. Fruit, fresh, stewed.

14. Fritters, waffles, pancakes
I5. Game, poultry

I6. Jelly, preserves, canning

I7. Lunchbox

18. Macaroni, rice, curries

I9. Meats-

(a) Beef

(b) Brains, sweetbreads

(c) Mutton, lamb

(d) Pork

(e) Veal

20. Menus

21. Pastry picnics

22. Pickles and catsups

23. Potatoes

24. Salads and dressings

25. Sauces

26. Sherbets and punches

27. Soups

28. Vegetables

Special cards may be made up giving, Unusually Successful Dinners, Children's Dishes, Formal Luncheons, Afternoon Tea, etc., etc. Another classification might be according to the qualities of the foods, as "Proteins," "Starches," 
etc. Other cards might be, "meat substitutes," "fireless recipes," "milk dishes," "invalid dishes," etc., etc. Five points should be covered with each recipe:
I. Approximate cost
2. How many does it serve?
3. How long to prepare?
4. How long to cook?
5. Nutritive value

This file of recipes should not be kept with the other cards in the household file, but in a separate small box in the cabinet, or over the kitchen table. Or the cards may be placed in a sliding drawer to pull out under the shelf. A piece of glass the same size as the cards can be built into the front of the drawer. All these cards should be punched with a small hole in the center upper edge. Then a small hook should be screwed in the shelf at about eye level, and as a card is taken from the box, it is to be hung on the hook, where it can be easily seen and read. The newest guide cards for this file have celluloid tabs so that they will not soil or become bent with constant thumbing.

\section{What Shall We Do With Our Receipts?}

The receipted bills themselves are of course too bulky to be placed in the card file box. What is called a "vertical letter file" is excellent of this purpose. This is a pasteboard box, with envelope partitions, numbered alphabetically. It is an easy matter to have this file stand upright on one's desk, and quickly slip into it receipted gas bills under "G," butcher on "B," etc.

\section{Special Arrangement for Clippings}

Sometimes much of the material the housekeeper wishes to keep is in the form of clippings on this or that favorite topic. These can be handled best in a series of large envelopes such as are used in libraries and commercial 
offices. Each envelope measures about 8xi2 (they come in different sizes). To use them, place them upright (opening at back). Paste a label in the upper right-hand corner. Decide on the different divisions or subjects you wish to make, as "Women and Civic Work," "Club Programs and Study," "Montessori and Child Training," "Furniture and

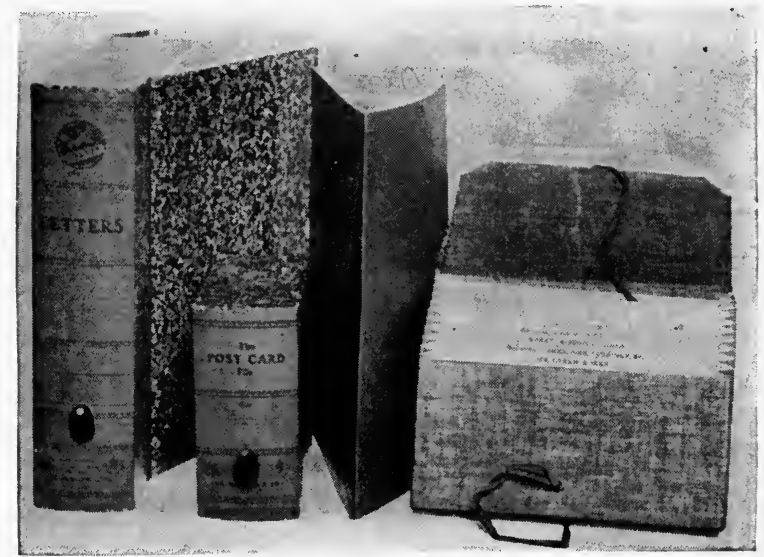

USEFUL FILING DEVICES.

Letter File, Postcard File, Manila Envelope, Recipe File.

House Decoration," etc. The following method of labelling them is the author's own adaptation of the "Dewey Decimal System" and is so simple and helpful that it can be applied to any set of household clippings, or those for other uses.

Decide on the main subject on which you wish to collect clippings and information. Call this envelope No. I. Allow ten envelopes to this one subject. Decide on another general head, call this envelope Io, and allow ten envelopes to this group. The third envelope of the general subject will be 20 , the fourth 30 , and so on. The plan works out as follows: 
ENVELope No. I-Food clippings

2-Menus for special occasions

3-Food for children

4-Lunchbox plans

5-9- to be added later

ENVELope No. 10-Child education

I I-Prenatal care

12-Nursery devices

I3-19-to be added later

ENvelope No. 20-Club programs

\section{I-Music}

22-29-to be added later

ENVELOPE No. 30-Fashions

3I-39-to be added later

"The "key" to the whole plan is to allow ten envelopes to each subject, whether they are in use or not. This brings the number of each general subject ten and multiples of ten, so that grouping is very easy. If one wishes to carry the plan to full completion, use a set of $3 \times 5$ cards in connection with the envelopes, and "index" the cards by subject to correspond with the numbering on the envelopes. That is, if envelope I is "Foods," and envelope 50 is "Garden and Sprays," the cards to correspond will have a large guide card, stating:

\section{Foods .................... I}

(with nine separate cards each with its subject behind it)

Garden and Sprays.............. No. 50

(with its nine cards behind it).

It is thus possible, by looking through the card file first, to locate instantly a clipping on a given subject. The plan may sound complicated, but it would not take more than an hour to label and arrange both envelopes and cards, and it will be a permanent way to quickly and systematically file all kinds of clippings, recipes, booklets and others which come with many household furnishings. 
The Filing Idea Is Not Complicated and Difficult

Now, after we have described these plans and the way to keep all houshold records "immediate, reliable and accurate" by means of a filing system, the author hears some women instantly look up from reading the chapter and remark, "Well, that may be efficient all right, but it sounds too long and difficult for me to try. I don't have the time

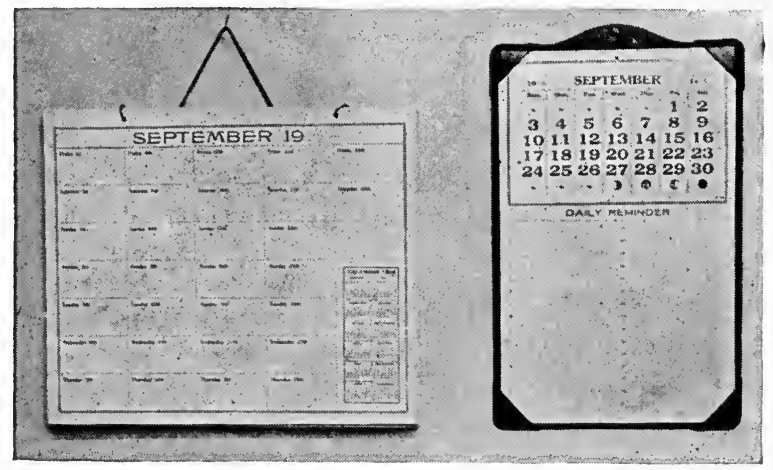

MEMORANDUM CALENDARS.

to write all that stuff on cards, and keep such a plan going. I'll have to stick to the old-fashioned way." Now, dear reader, do not condemn any plan until you have given it a fair trial. Second, the author wants to say, emphatically, that even if you don't use any such system you are taking time "to keep track of things," even in your present oldfashioned way. Think of the hours you spent "hunting" for an important address which you must have immediately. Think what an amount of energy you waste running around the house for a commercial receipt that you are "sure you paid," when the collector calls for his bill. Think how you take the time of the family asking them 
"Where is this?" or "Did you see Mama's

or

that?" Or, "I'm so sorry, I've hunted just everywhere and thought I had it all tucked away in my desk, but I can't find it," etc., etc.

Now, the author doesn't claim that any filing system needs no time, but that it at least doesn't take any more time than any present hap-hazard method, and that its results are 80 per cent more efficient. The time you spend hunting and searching and asking and "getting flustered," etc., could be spent equally well in sitting down calmly and writing on a card information which will save you a "fluster" next time. It would not take as long to write on a card that the painter charged $\$ 5$ for painting the porch last spring than it would this year to try and remember, and make a telephone call or write for a new estimate this season when the porch needs to be repainted. If housekeeping is to be a "business,"

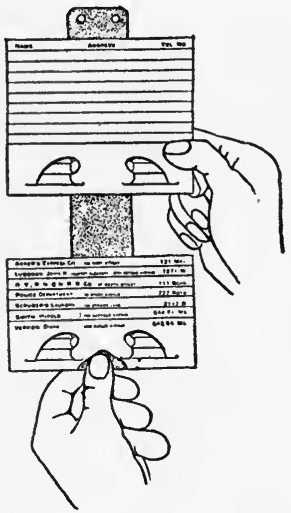

THE VISIBLE INDEX. then the least we can do is to follow the business practices in caring for money and information on which the successful management of the home depends.

\section{The Use of the Index Visible}

A small device used in many offices is equally helpful in the home. This is called the "index visible," and the author has adapted it in several ways. It consists of about fifty $3 \times 5$ cards, with a special cut on the lower edge which permits the cards to slide up and down a thin metal hanger. Only the top edge of each card is visible, but other material may be written on the body of the card. Cards can 
instantly be slipped out and changed. It can be used excellently for:

Telephone list (with alphabetical cards)

List of phonograph records

List of linen closet

List of appointments

List of standard house supplies and other subjects

Every Homemaker Can Have a "Business Corner"

One thing that contributes to being businesslike is to have the right "business" atmosphere. There should be some kind of "office" in every home, where the above files and papers, etc., can be permanently arranged. A writing desk is not so good as a flat-topped table, such as even a stained kitchen table in the corner of some room. If the homemaker does her own work, some convenient kitchen shelf and drawer would be excellent. Just as the busy executive needs a place where he can have his papers and materials untouched, so the homemaker needs an "office" corner, no matter how humble, where she can go to plan her menus, write out her orders and make up her accounts. A few shelves over such a table, to hold her reference cook books, government and state bulletins and other publications on homemaking would all tend to increase her system and pride in the "business" end of homemaking.

\section{QUESTIONS ON HOUSEHOLD ENGINEERING}

\section{VII}

Family Financing and Record Keeping

I. With what system of family financeering are you most familiar? What are its advantages? What its defects?

2. Give the budget of your own family as nearly as you can. Criticise.

3. What system of household accounts seems best to you?

4. Household records-do you keep any? 'Is it advisable?

5. Can you arrange for a "Business Corner"? 


\section{HOUSEHOLD ENGINEERING}

VIII 


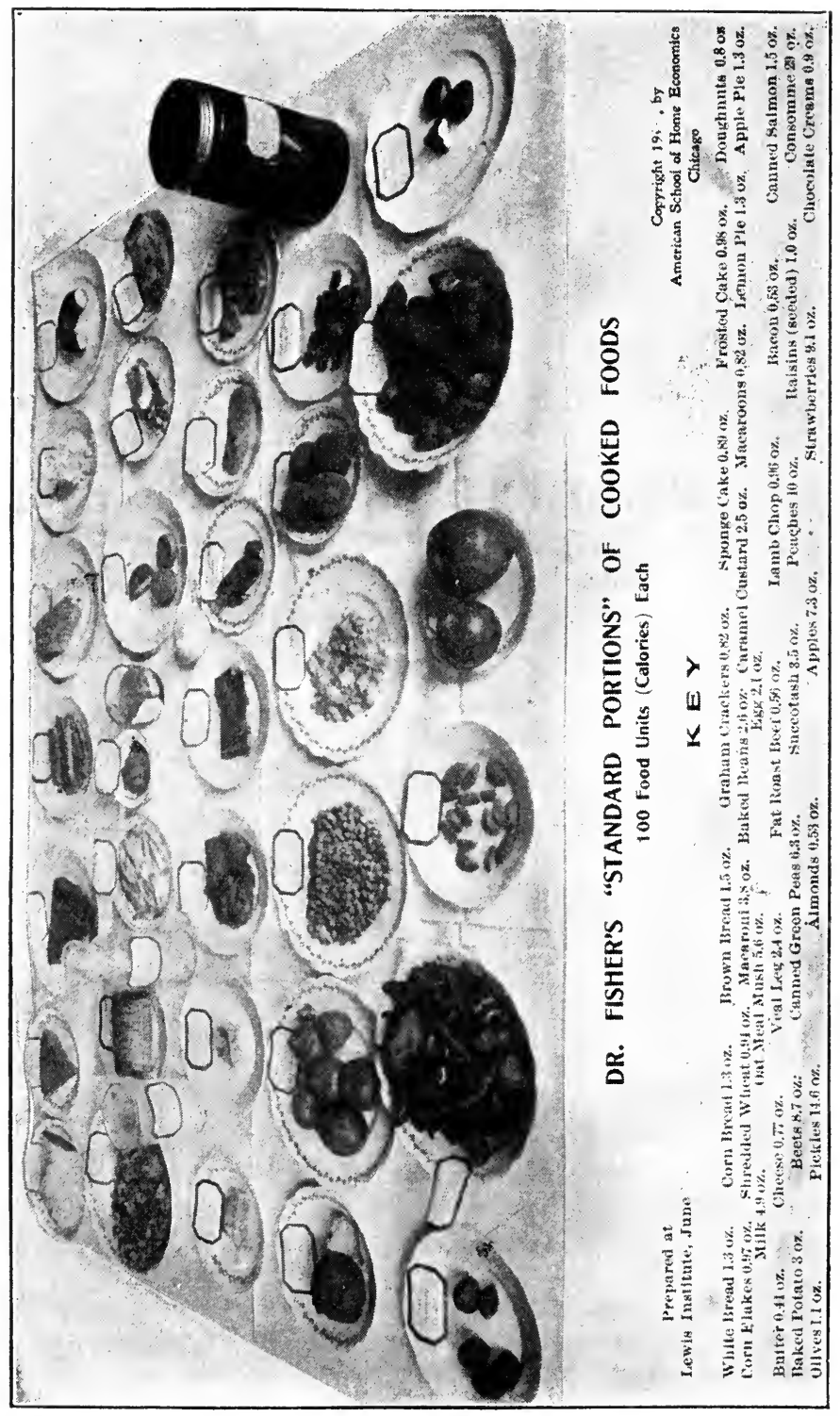




\section{HOUSEHOLD ENGINEERING}

VIII

\section{EFFICIENT HOUSEHOLD PURCHASING}

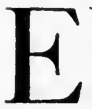

VERY large business and factory recognizes that the purchasing of its supplies and equipment is one of its most important and responsible departments. Therefore we find business firms employing persons called "purchasing agents," who are trained, informed on market conditions, and able to buy to the best advantage for their particular firms. Such "agents" enable their firms to save many thousands of dollars which otherwise might have been wasted in misguided or poorly planned purchasing. The woman in the home must occupy a similar position as the "purchasing agent" of the family, because in her hands lies the spending of the family funds. She must therefore know exactly what the family has to spend, what it wishes to spend for according to its prearranged "goal" or aims, when is the best time to buy, and what she should pay. In other words, every woman running the business of homemaking must train herself to become an efficient "purchasing agent" for her particular firm or family, by study, watchfulness, and practice.

The first basis of efficient purchasing is to plan the spending according to the budget plan given in the previous chapter. Definite amounts of the income must be set aside and known in advance for food, clothing, replacement, etc. These amounts must be known not only for the week, or the month, 
but for the whole year, or otherwise no systematic buying, no economies, or truly wise investment-buying can be practiced. Unless purchasing is so planned, then the home "purchasing agent" will not be able to take advantage of the right seasons for buying, or be able to buy in quantity, or find money enough set aside when the time comes to purchase. The larger the income, the more easy it is to waste and spend haphazardly. And with the increasingly high cost of all living, still greater responsibility is placed on the home "purchasing agent" to buy on a small or moderate income, the necessities of food, clothing and furnishing, and still have left enough money to supply the family's needs of education and advancement.

Never before in the history of the family have the burdens of purchasing been placed so heavily on woman's shoulders. This is because today the modern woman is chiefly a consumer, and not a producer. The housewife of a century ago made soap and candles, wove and fashioned all of the garments, and with her own hands produced not only food for daily use, but also "jarred" and dried fruits and vegetables, and preserved meats for winter use. Today even the good housekeeper finds it profitable to buy many articles prepared in a "food factory," some clothing that is "ready made," and to avail herself of countless articles which make for comfort and sanitation which are produced commercially.

It might at first seem that by being able to buy the article instead of having to make it, that the modern consumer is at once relieved of all responsibility. Some women take this view, but it is a mistake. Because, in letting go the work of her own hands she is instead faced with entirely new responsibilities. When food, garments, or other articles were formerly made in the old-fashioned home, the woman herself determined by personal supervision, that the quality, purity, and cleanliness of these articles produced under her 
roof, were of the highest. Just so, she today must (personally or collectively), assure herself of equal standards of purity and sanitation in these articles wherever they are made-in factory, shop, or mill. In other words, some of the time saved by having these articles manufactured outside the home must be taken by the housekeeper in learning to understand how to buy commercially made products. What standards to demand, what price to pay, what adulterants to avoid, are some of the things which must be learned in order to become a trained consumer, or a responsible "purchasing agent" for the family.

To become a trained consumer is therefore one of the most important demands made on the housekeeper of today, because, whether she is conscious of it or not, the woman in the home does the bulk of the family purchasing. The figures in the last chapter will bear repeating:

Women buy $96 \%$ of all dry goods, $87 \%$ of raw and market products, $48.5 \%$ of hardware and housefurnishings, $48 \%$ of drugs, Ir.2\% of men's clothing. The broad conclusion is that women alone buy $48.4 \%$ of all merchandise for family use, and help in selecting $23 \%$ more, thus buying practically $71 \%$ of all the products used in the home. How essential, therefore, that with this great responsibility placed on her, she teaches herself to become a wise, trained consumer, equipped with knowledge and warned against pitfalls and inefficient buying methods. If she does not train herself, this large percent, representing annually billions of dollars, will be spent for waste and not value. Her family will suffer; or the husband will be compelled to increase his income with no corresponding saving; or he may, indeed, be forced himself to take up the burden of the family purchasing agent, thus overtaxing his time and strength and taking the time needed to make his own business a success.

Still further, women, by failing to understand how to buy 
in the most efficient manner, are frequently responsible for many of the evils or high costs present in our modern selling methods. The women blame the retailer or the manufacturer, whereas, it can be proved clearly in several instances that the blame should be laid directly at their own door. By undue use of the "charge account," by excessive delivery demands, the "return goods" habit (by vagaries in buying and other errors, women make the cost of doing business higher than necessary. Also it may be said here, that every woman should be a trained consumer, whether she has a family (i. e., husband and children) or not. The waste of inefficient buying of independent unmarried women creates just as much disorder as does the poorly planned buying of the homemaker, particularly as the former. having frequently only themselves to support, are inclined to even greater carelessness in their buying habits.

\section{The Housekeeper as a Trained Consumer}

Increased skill in buying will come, like every other kind of proficiency, only from continuous trying, from experiment, and study. Any woman (or man) can become a trained consumer, if they consciously train themselves:

I-By knowing that efficient buying is based not only on price, but also on considerations of value; quality, wear, future investment.

2-By knowing the merits of various kinds of distribution methods, as parcel post, mail-órder, co-operative buying, public market, etc.

3-By knowing the nutritive values of food.

4-By knowing and co-operating in the enforcement of state and national laws governing the standards and handling of raw and manufactured products.

5-By knowing existing weights and measure laws and checking up weights in the home kitchen.

6-By knowing adulterants found in products and malpractice among dealers.

7-By knowing how to identify manufactured products, their trademarks, labels, advertising, sizes of cans, etc. 
8-By knowing the manufacture and qualities of various textiles.

9-By personal experiment and test, keeping definite records of results in all purchasing.

Io-By co-operation with organized consumers, as housewives' and consumer leagues, boards of health, etc.

\section{Value vs. Price}

The first lesson in all efficient buying is to distinguish between price and value, and to learn to make all purchases on a basis of value, and not on price alone. Prices may fluctuate because of economic conditions, but the value of an article can be fixed only in the consumer's mind. Value is the "long-distance," investment view which considers many factors and results in wise expenditure. For instance, a comfortably upholstered Morris chair at $\$ 20$ would give more value than a brocaded gilt settee at $\$ 50$. Or, a lunchcloth of excellent linen, with scalloped edges, which cost $\$ 8.00$ that can be used every day, would give more value than an elaborate one of Madeira embroidery costing twice that amount, but so fragile that it can be used only once or twice a year. Again, a house is valuable as a residence in proportion as it offers sunshine, dry cellar, reliable plumbing, and satisfactory heating, and not in proportion to the details of finishing and decoration of the exterior or the interior. The price of the second house, however, might be double that of the first, but the former is more valuable if it gives greater comfort and health to the family. Or, a small talking machine, which all the family could use, at $\$ 20$ would probably give more value than a piano at $\$ 400$ if no member were a musician.

The point of value versus price applies to all branches of expenditure. A simple recreation plan for the family, alive and interesting, such as camping, may have value far and above the actual money expended. Sight-seeing trips to city, 
factory or mine, which cost only carfare, may prove more interesting and valuable than ten times that sum spent at the theaters. Or, Mrs. B. may be willing to pay two cents more for a loaf of bread wrapped in paraffin paper, a cent more a pound for butter protected by a carton, because she believes the additional value of the increased sanitary handling is worth it. If price only were her basis, she would buy the cheaper loaf, and the bulk butter. A conception of value is of the utmost importance, for value is always greater than price and must control price in the consumer's mind in order to have efficient purchasing.

\section{The Cost of Distribution}

"Distribution" is the term which covers handling, or means of getting products from the grower or manufacturer who raises or makes them, into the hands of the consumer who uses them. This handling cost must, of course, be added to the actual production cost of the article and paid by the consumer. The price paid for eggs in a city store, or for shoes or a suit of clothes, includes not only the price paid to the farmer or manufacturer, but also includes the fee charged by a respective chain of commission men, wholesalers and retailers, for packing, handling, and bringing the article to a location or store conveniently near the consumer. In other words, "distribution" includes the service of bringing an article from a distant territory, or factory, so that it can be easily placed at the disposal of the buyer, thus saving her time, effort and personal handling. In the case of manufactured articles, particularly, the cost of distribution includes the risk taken by each one of the distributors that the consumer will actually buy this product; i.e., the manufacturer who makes women's suits, let us say, sells the suit to a wholesaler or jobber, who then assumes the risk or chance 
that he can sell this suit to the retailer; next, the retailer accepts the risk that he can sell the suit to the woman in his town, and that she will like to buy it, so that he will not have it on his hands. This risk is added to the cost of distribution, as is also the cost of storage, which, in the case of some products, is very high.

There is nothing wrong with the theory of the consumer paying for this delivery, service, and convenience; it is only that in many cases distribution costs have been excessive, due to no regulation or control by law. This has been particularly true in the case of the commission houses, that have been exposed, and proved guilty of such offenses as steaming potatoes in order to hold up the price, buying up orchards and dumping the fruit, etc., etc. The remedy here is not to do away entirely with the central wholesale place of exchange which the commission house offers, and which is absolutely necessary for the retailer and consumer in a large city, but to regulate such centers by law. Some such regulation, after the manner of public service commissions, state industrial commissions, etc., with uniform laws and minimum distribution charges, should be enforced by each community in the interests of both consumer and producer.

In most cases where the consumer evades the distribution cost, she herself must take some of the time and effort of distribution, which in the former case she avoided, but for which she paid. For instance, prices are universally lower in a public market than in a retail store. However, the market necessitates that the consumer take her own two hours of time walking a long distance, or, perhaps, paying carfare, and carry her packages home herself. In many cases it will be a real saving to do this; in many other cases the convenience and delivery of the neighborhood retailer will be worth the additional cost. Similarly, while the "catalog" house in a large center may save some money, it gives no 
service, the consumer cannot see the goods before she buys, and in most cases she must pay the delivery cost.

\section{Links in the Chain of Distribution}

I-A manufacturer is the original source of production of a product made in factory, mill, mine, etc. A producer or grower is the original source of production of farm or livestock products. A manufacturer sells to both jobbers and retailers. A producer sells generally only to commission houses.

2-A jobber buys manufactured articles from the manufacturer and sells to the retailer, but never manufactures himself.

3-A commission merchant, or "house" is a jobber in food products who buys from the grower and in turn sells to the retailer.

4-A retailer buys from either manufacturer, jobber, or commission merchant, and sells to the consumer.

The consumer may try to break through these links in the chain of distribution, and in some cases be successful, as with the parcel post, which is a direct means of selling between the producer and the consumer, or by the mail-order house, which is a jobber. But it must be said emphatically that the retailer has a gemuine place to fill, and service to give in every community. Even if the parcel post were far more universal and satisfactory than it is at present, such a system would not be satisfactory for all consumers in large centers. All buying cannot be done at public markets, or from out-oftown mail-order houses, owing to distance, time involved, etc. In other words, no matter how we may save money once in a while on buying a suit by mail, or going six miles to a market, or having eggs sent direct by parcel post, we need the retailer to give us service in providing necessities and perishablesfruit, milk, butter, bread, elastic; we need the retailer to mend our sudden plumbing leaks, to supply us with arnica and toothpaste, to let us quickly get another eggbeater, or badly needed shoes and stockings. 
We may be able to save money on buying the large, anticipated, occasional purchase, like a rug, or a suit, from a mailorder house or manufacturer direct. But it is only on such occasional, almóst luxury-purchases that the retailer makes his profit, since he makes almost none on selling us the necessities. Therefore, unless we do buy from him the luxuries or occasional purchases on which his profit is larger, he may not be able to remain in business, and we will no longer receive the convenience, delivery and service on the daily necessities.

There are many inefficient retailers, and often the cost of doing retail business is far too high. In many places there are too many retailers. On the other hand, the woman consumer herself is actually to blame for much of the excessive costs of retail selling. How many of us run up charge accounts, which increase the cost of doing business over $5 \%$ ? How many of us ask our grocer to send the boy over with a yeast-cake and a loaf of bread, or demand as many as six daily deliveries? How many of us fail to pay our bills, or are guilty of the telephone habit, the C. O. D. habit, or the "returned goods" evil-all of which can be shown to increase the retailer's cost of business unfairly. One delivery per day in a store makes the delivery service only about $5 \%$, whereas many deliveries increase it to as much as $13 \%$. The grocery store which does a "cash" business has a cost of about $14 \%$ of doing business, whereas the store which "carries" customers must pay nearly $20 \%$. Similarly in all retail lines, delivery, bad debts and inefficiency on the part of the consumer increase the cost back to herself.

The first thing is to reform herself, and insist that the retailer carry out these reforms with her. For instance, induce the retailer to make one price if goods are delivered, and another if the customer carries them home herself. This has been done in a large bakery, which, for example, asks 
I I cents per doz for rolls sent home, but only io cents if they are taken. A large milk company has announced that milk of a certain grade will be ro cents per quart if delivered as usual, or 8 cents if the consumer comes to certain depots with her own container. This is the right saving principle: to separate delivery cost from actual cost. Again, the cash customer should receive a percentage off on his purchases, or be entitled to lower prices. The "cash-and-carry" stores always can undersell stores with charge accounts.

Frequently, too, the customer is under the illusion that when she buys from manufacturer or jobber direct, that she is getting a "wholesale price." This is seldom, if ever, the case, for the manufacturer who advertises that he will sell "direct to you" has to pay the cost of advertising and printed matter; he has to make separate deliveries, take care of the risk, the collections, etc. In a word, he must assume some of the expense of the retailer. He cannot possibly sell you one chair for as low a price as 100 chairs to a retailer. Thus the consumer never gets the wholesale price, though the price may be less than charged by the local retailer for goods in stock.

The distinction between "quantity" buying and "wholesale" buying should be kept clear. Oftentimes so-called "wholesale prices" can be obtained through the local retailer, on a special order, at no more than the "mail-order" price.

It is a wise plan to co-operate with the local retailers and to understand their difficulties. The greatest saving in purchasing staples comes through quantity buying, as was shown in the purchase of meats, pages I9I-I93.

\section{Use of Newspaper Quotations in Marketing}

The consumer can keep check on the retailer, especially in the case of market or fresh produce, by reading the market reports or quotations which tell her from day to day the 
fluctuations in food prices. The prices quoted for vegetables, fruit, flour, etc., are for carload or Ioo-pound lots, and are the prices paid by the commission men, or wholesalers. These reports do not give the retail price because the desirability of goods, even in the same lot, varies, and thus no definite invariable increase can be added to the wholesale price, and the retail price estimated by the consumer. But the reports do help her by telling her the headlines, which state whether a certain commodity has risen or fallen in price. If the rise or fall is steady for two successive days, the retail market will be affected on the second or third day. War and labor conditions, the reports from the government's "volunteer crop reporters," who tell Uncle Sam about local crop conditions-all these will be straws to show which way the market winds blow, and which can be interpreted by the observant consumer so that she can buy to advantage. For instance, if weather conditions have been such that the potato crop is scarce, then it would be wise to put in a supply early, before the prices rise, due to general shortage. Or, if the new wheat crop is affected by blight, buy a barrel of flour at once (flour held in mills), before price advances on the new milling.

\section{How Shall the Consumer Market?}

It has been pointed out that quantity buying is preferable to "bag" or package buying, wherever possible. But to practice quantity buying, there must be adequate storage space, of the right temperature, and such inspection that a large portion of the goods will not waste, and thus undo the original saving.* Such staples as matches, candles, sugar, flour, etc.; are always best bought in quantity, if space permits. On the other hand, it is not generally advisable to buy perishables in quantity unless the needs of the family are

- Send for U. S. Bulletin" No. 375, "Care of Food in the Home." 
well estimated ; also, such articles as soda biscuit, spices, coffee, etc., involving crispness and flavor, must be well protected from air and moisture. Too frequently a large quantity is bought because the price is low, and then the food must be served so frequently as to tire the family palate, in order to "use it up." In buying packaged goods or small jars of such foods as potted meats, fish, etc., it is economy to buy, say one jar or package at 25 cents, rather than two jars, one at 15, and the other at 10, which, together, will not contain as many ounces as the larger jar at the same price.

If, however, the family is small, it is greater economy to buy in small quantity. All buying should, indeed, be based on the "purchasing sheet" (see page I86), and on the experiments the housekeeper herself makes and finds out in regard to her own particular family. Seasons also affect all buying, as in summer, less coffee may be used and more fruit syrups, or less cereals, and the heavier meal, beans, etc. Never keep cereals and a quantity of flour over the warm weather, as insects or mold are likely to develop. Daily shopping is not necessary; twice or, at most, tri-weekly ordering makes for efficiency and the "advance preparation" of meals. In the author's home, five miles from fresh supplies, and 40 miles from a city, meat and perishables are bought once a week; monthly a large grocery order is sent to a city dealer-and that ends all the marketing!

\section{Food Budgets}

All marketing should be primarily on the food budget, which gives the total sum to be spent for food during the year, based on a general average for each month. If staples are purchased in quantity, the expenditures of one month may greatly exceed the average. This excess should be 
apportioned over the succeeding months; thus, $\$ 24.00$ spent for winter vegetables to last six months will reduce the allowance for food $\$ 4.00$ a month for six months. Such seasonable buying should be provided for somewhat as follows:

January-Linens, reduced price winter clothing and undergarments.

February-Canned goods.

March-Kitchen utensils.

April-Barrel sugar, preserving and canning jars and accessories.

May-Coal.

June-Box of soap.

July-Reduced price summer garments and textiles.

August-Furniture, reduced price summer furnishings, blankets and bedding.

September-Winter apples, root vegetables, onions.

October-Canned goods, potatoes, cereals, dried vegetables.

November-Lard, smoked meats, syrups.

December-Holiday gifts and extra delicacies.

\section{Co-operative Buying}

Another means of lowering distribution costs, is for the consumer to buy co-operatively with other consumers. Considerable money can frequently be saved if four to ten families make up an order together and get the quantity price, and pay only one charge for delivery, instead of many. There are, however, frequently marked disadvantages, especially if the people ordering do not live closely together, or do not have the same tastes, as each family is more limited to choice and selection when nine other families must be con- 
sidered. Again, some one member of the group must take charge of collecting the money, be responsible for it, and take her own time to go to market, see to the delivery, and attend to the partition of the order into ten different deliveries. It often seems a fallacy to suppose that nine persons are going to be able to get a tenth most intelligent consumer to buy for them, and yet not pay her for the time and intelligence and effort she puts into that shopping. Further, if this tenth person is paid for the time spent, then the articles bought co-operatively may cost about as much as if bought at the high individual price. However, two or three neighbors can club together to buy a barrel of apples, crate of oranges, 3o-dozen crate of eggs and the like with a considerable saving in price and not too much trouble.

Co-operative stores are successful in some parts of the country, but are not nearly so common as in other countries.

\section{The Parcel-Post System}

In a small and sporadic way, the parcel-post system helps the consumer by enabling her to get products from the farm to the city. The disadvantages of the method are that sometimes the containers devised are not satisfactory and too much breakage occurs. Also, the shippers themselves complain that the deliveries are not sufficiently prompt to satisfy their customers, much discontent being found with delayed orders. Vegetables, fowls, or cured meats pack and carry the best.

One of the most successful developments in this line is the well-known "hamper" deliveries of the L. I. R. R. Agricultural Experiment Station at Medford, L. I., where different sized hampers of assorted vegetables are sent twice weekly to city customers. Now that autos are in such common use, it is quite possible for city people to make a weekly trip to a 
farm, with which a standing order is placed for specific products.

On the other hand, it is a not uncommon experience to find that the country producer charges exactly the city quotation, and expects his customers to carry home products themselves-or that he even refuses to sell retail at a good fair price, and prefers to sell all his produce at a low figure to a food contractor. The situation differs widely in different sections, but is worth trial. The U. S. Department of Agriculture is encouraging parcel-post marketing, and postmasters usually have a list of producers. Some of the express companies also have lists.

\section{The Telephone Habit}

The telephone habit, as generally practiced, makes for extravagance, encourages hand-to-mouth buying without advance buying, and increases the cost of doing business to the retailer. In every case greater economy will result if the housekeeper picks out the desired cut and amount herself than if left to the dealer over the 'phone. By marketing personally, a better idea of what is seasonal is obtained and the chance to substitute the cheaper vegetables or fruit than those intended. By choosing a time of day, preferably before Io a. m., and by having a tentative purchasing sheet in hand, little time will be lost. Some market days are better than others-Tuesday, Thursday and Saturday in most cities-for the selection of perishables. The telephone habit encourages that lack of knowledge of conditions and prices as exampled by the woman who ordered celery in May and complained that it cost 35 cents a bunch and that she was being robbed. If she had shopped personally, she would have, doubtless, found young carrots and spinach at a third the cost. 
Food Purchasing According to Nutritive Value

The purchasing of food supplies is the most important of all buying, since from one-fourth to one-half of the income is spent for foods, and the smaller the income, the larger the sum that must be spent. There is no relation, fortunately, between the cost of food and its nutritive value. The cost as paid by the consumer is determined by many other factors, such as the cost of production, especially with animals eating grains, which today is much higher than formerly; by the cost of transportation, as with the citrus fruits brought the "long haul" from Florida or the West; by when the food is "in season"; by the way it is packed, in bulk, or in fancy basket or carton; by whether it is "selected," all of one grade, appearance and size; by the degree of its perishability, especially with such crops as lettuce, berries and other fruits.

Broadly, there are two bases on which to buy foods: (I) a basis of nutritive value, or how much the food will be actually worth to the body as fuel and building material, and (2) a basis of taste and costhetic appeal of flavor, color, texture, shape, etc. On a generous income, there is less need to think of the nutritive value, and more scope to buy foods which please and attract the eye or palate. But on any income, every housekeeper should think, not in terms of market price, but in terms of nutritive value first, in order that her family shall be well nourished. There is far too strong a tendency to think that foods which we like are good for us, and to let appearance and flavor determine selection, when, so far as real nourishment goes, they are secondary.

\section{How Many "Food Units" Are Required Daily?}

An ordinary diet must contain from 2,000 to 3,000 or more "food units" (calories), depending chiefly on size of body 
and amount of muscular exercise. The calories give the measure of the "fuel value," or the "heat and energy value" of the food. Thus, perfectly dry starch, sugar or protein yields the body $I, 820$ calories per pound, while fat gives 4,040 calories per pound.

The U. S. Department of Agriculture has found by experiment the average calorific requirements of the body under different conditions as follows:

FOOD UNITS USED BY THE BODY PER HOUR

\begin{tabular}{|c|c|c|c|c|c|c|}
\hline Veight of Body (lbs.) IIO & 120 & 130 & 140 & 150 & 160 & $I 7$ \\
\hline Asle & 52 & 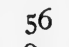 & $6 r$ & 5 & 63 & \\
\hline & 80 & 87 & 94 & 100 & 105 & \\
\hline 125 & I36 & 148 & I59 & 170 & 178 & \\
\hline ctive & 152 & 165 & 177 & 190 & 200 & \\
\hline evere & 360 & 390 & 420 & 450 & $47 \mathrm{I}$ & \\
\hline
\end{tabular}

Thus, a woman of about I 30 pounds, sleeping for 8 hours, doing light housework to hours, reading, etc., 6 hours, would require $(8 \times 56)+(10 \times 148)+(6 \times 87)=2,450$ calories. A boy of about the same weight with 8 hours' sleep, 8 hours' active exercise, 6 hours' playing tennis (severe exercise), and 2 hours' quiet would require $(8 \times 56)+(8 \times 165)+$ $(6 \times 390)+(2 \times 87)=4,282$ calories. He would need a little more food to provide for growth. No wonder he has such a ravenous appetite! A nervous, restless person will require somewhat more calories than indicated in the table; a thin person will need about as much food as if of normal weight ; and fat above the average should be disregarded.

The subject of balanced diet has been considered briefly (pages $180-183$ ). On a calorie basis a balanced diet means that out of each 100 calories, Io to 14 should be protein, 20 to 40 fat, and 30 to 60 starch and sugar. A mixed diet, unless badly one-sided, will come within this range. 
Food products have high "fuel value" when they contain but little water. As fats give $2 \mathrm{I} / 4$ times the food units of carbohydrates, or protein, foods which contain much fat have very high fuel value.

The following table gives the general average of calories per pound of classes of food as eaten, i. e., without waste, and also the number of calories which come from the protein in 100 calories of each food. Commit it to memory.

\section{"FOOD UNITS" IN EDIBLE PORTION OF FOODS}

\section{(Approximate)}

Cereals-flour, meals, breakfast foods, macaaroni, etc......................... 1650

Bread ............................ I200

Beans, peas, lentils (dry) ............... 1500

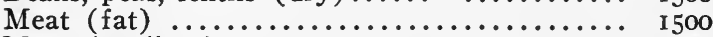

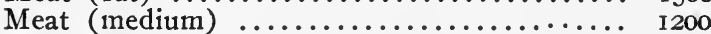

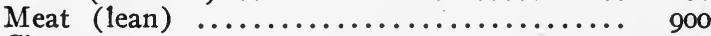

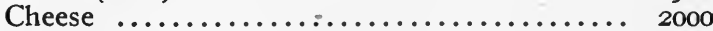

Eggs $(8$ or 9$) \ldots \ldots \ldots \ldots \ldots \ldots \ldots \ldots \ldots \ldots \ldots, 6,6 \ldots \ldots$

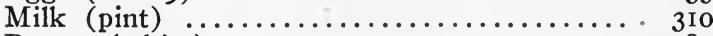

Potato (white) ........................ 385

Root vegetables ...................... 200

Green and watery vegetables...............

Fruits (fresh) $\ldots \ldots \ldots \ldots \ldots \ldots \ldots \ldots \ldots \ldots, 300$ to 400

Fruits (dried) .................... I300 to I500

Nuts (shelled) $\ldots \ldots \ldots \ldots \ldots \ldots \ldots \ldots \ldots \ldots, 3000$

Butter-oleo ........................ 3400

Lard, crisco, salad oils..................... 4000

Sugar

I750

12

13

25

20

30

40

25

32

I9

IO

IO

I 2

3

3

I3

0.5

00

$\infty 0$

Note. Meats vary so much in composition that it is difficult to give general averages; e.g., veal may have only 600 calories per pound and have 70 calories of protein in 100; fat pork may give over 2,000 calories per pound and have only 12 protein calories in 100.

In a diet containing 3,000 calories per day, for example, 300 calories or somewhat more should come from protein, and the balance from starch, sugar and fats. As will be 
noticed from the table, all food, except the fruits and manufactured food like butter, lard and other fats, corn starch and sugar have Io or more calories of protein per Ioo calories of the food, so that it is not difficult to plan a diet containing sufficient protein. But feeding the family is also a matter of satisfying the appetite of the different members and here cooking and manner of serving is more important than the chemical composition of foods.

The fuel value alone is not the only consideration. Cheap fuel calories may be dear protein calories, and vice versa. Also many foods which have low fuel value, such as the watery vegetables may be of sufficient value, even at high prices, when we consider the mineral salts and bulk or "roughage" qualities they offer. Spinach, lettuce, string beans, celery, etc., must be purchased considering the value they give in the necessary iron and calcium. Milk and eggs are especially valuable for children, for, in addition to their protein and salts, they contain "vitamines" and "lipoids," which are necessary for growth. Vitamines are also present in meats, vegetables, fruits, and the outer covering of grains.

When prices go up we are apt to feel that some common foods become too expensive to use. For example, a quart of milk at 12 cents seems high, but is equivalent in fuel value to about a pound of lean steak, which may cost 25 cents, or eight eggs costing 30 cents, or a quart of oysters costing 40 cents. Sugar and oatmeal have practically the same calorific value ( $I, 75^{\circ}$ per pound) and cost about the same price per pound, but 300 of the calories of the oatmeal come from protein, while the sugar has none. The oatmeal also furnishes iron, phosphorus and calcium salts, all of which are necessary to the body. In comparison, then, oatmeal gives more value than sugar, though not more fuel value. 


\section{"ioo Calorie Portions" of Food}

The easiest way to become familiar with the "fuel value" of different food is by examining a table of "Ioo calorie portions," as devised by Dr. Irving Fisher. This table is reprinted in the bulletin Food Values, and also shows the distribution of the calories of protein and fat and carbohydrate in the 100 calorie portions, which gives a better understanding of the real composition of food than percentage by weight. For example, shelled almonds by weight have a composition of $21 \%$ of protein, $55 \%$ of fat, I7\% of carbohydrate, $2 \%$ of ash and $5 \%$ of water-apparently a high protein food. On the calorie basis of each roo calories of almonds I 3 come from protein, 77 from fat, and ro from carbohydrate. Entire wheat flour gives about 15 of protein, 5 of fat, and 80 of carbohydrate from each roo calories. On a percentage by weight basis, the wheat flour shows only $14 \%$ protein. Thus, wheat is really a higher protein food than almonds, though the opposite might be inferred simply by examining a table of composition by weight. The contrast is even more apparent in comparing foods containing much water with dry foods. For example, milk contains only about $3 \frac{1}{2} \%$ of protein by weight, but of each 100 calories, I9 or 20 are from protein.

It so happens that a I00-calorie portion of food corresponds in many cases to a serving. For example, there are Ioo calories in a large slice of bread, an ordinary pat of butter, $I / 2$ cubic inches of cheese, $2 / 3$ of a glass of milk, small serving of beef, 2 apples, a large banana, 2 small oranges, 3 heaping teaspoons or $\mathrm{I} / 2$ square lumps of sugar, etc., etc. After weighing out Ioo calorie portions of food and actually secing the quantity, it is easy to estimate closely the amount of food being served.

" "Food Values; Practical Methods in Diet Calculations." Published by American School of Home Economics. Price, 10 cents. 
It would be helpful if all cook books gave the calorific value of recipes. This is happily being done in some books.*

It is not difficult to figure out the calories in recipes by using the following approximate values:

\section{FUEL VALUE OF FOODS IN HOUSEHOLD MEASURES}

,Measure Weight Calories

Flour, wheat (sifted) .......... I cup

Flour, wheat (sifted)......... I tbsp.

$4 \mathrm{oz} \quad 450$

Corn meal ................. I cup

Butter-oleo ................ I cup

Butter-oleo ................ I tbsp.

..... $\quad 30$

Lard $\ldots \ldots \ldots \ldots \ldots \ldots \ldots \ldots$ i cup

5 oz. $\quad 500$

Lard $\ldots \ldots \ldots \ldots \ldots \ldots \ldots \ldots$ I tbsp.

$8 \mathrm{oz} . \quad \mathrm{I} 700$

.... IIO

Crisco, cottolene, salad oil....... I cup

Crisco, cottolene, salad oil....... I tbsp.

$8 \mathrm{oz} . \quad 1900$

.... $\quad 120$

Sugar (granulated) ........... I cup

Sugar (granulated) $\ldots \ldots \ldots \ldots \ldots$ I tbsp.

$61 / 3$ oz. $\quad 1600$

Milk .................... I cup

$7^{2} / 5$ oz. $\quad 850$

…. 50

$8 \mathrm{r} / 2$ oz. $\quad 170$

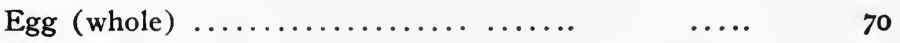

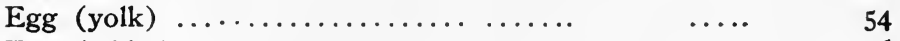

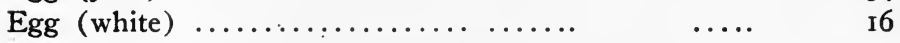

Note. Complete and detailed tables, together with food units in many standard recipes are given in Feeding the Family, Rose, price, \$2.10.

It is not a very complicated matter to plan menus for a given sum with the required number of calories for the family, as is the practice in scientific feeding in hospitals, sanitaria, and in the army and navy.

"“The New Cookery," Lenna Francis Cooper, \$2.00. “A Guide to Invaiid Cookery," Fanny Merritt Farmer. 


\section{Waste or Refuse in Food Buying}

The amount of waste or refuse must always be considered in buying food. This is particularly necessary in purchasing meat, for the amount of bone and trimmings varies greatly in the different cuts and to a considerable extent in the same cut of different grades of meat. The tables in U. S. Bulletin No. 28, "Chemical Composition of American Food Materials," give the percentage of waste in detail. For example, the average for ribs of beef is $20 \%$, and for shank of beef, $55 \%$; in the ribs, the variation of refuse is from $13 \%$ to $29 \%$, and in the shank, $50 \%$ to $68 \%$. It is an excellent plan to actually weigh at home the amount of bone and trimmings and figure the percentage of edible meat being delivered. It will be found that bones and trimmings frequently amount to half the purchased weight. The amount of trimming before weighing varies in different localities and by different butchers. All trimmings and bone paid for should be delivered, as the fat can be tried out and utilized, and the bones are of value in the soup kettle.

It will be noticed from the tables in Bulletin No. 28 that lean meats contain considerable more water than fat meats, even in the same cut. As an example, the edible portion of lean round is shown to contain $74 \%$ of water and $3 \%$ of fat, while the edible portion of very fat round is shown to contain $56 \%$ of water and $26 \%$ of fat. The first has a fuel value of 475 calories per pound, while the fat round gives I,I 45 calories per pound. The lean round has only a little higher percentage of protein than the fat. Medium fat meats give more value for the money, as well as being of better flavor.

The price of the cheaper cuts of first quality meat is but little higher than second and third quality, while the nutritive value is greater and the flavor far superior. The follow- 
ing newspaper quotations of wholesale price of beef brings out this point:

\begin{tabular}{|c|c|c|c|c|}
\hline \multicolumn{5}{|c|}{ WHOLESALE PRICE OF BEEF } \\
\hline & & No.I & No. 2 & No. 3 \\
\hline Ribs & & $2 \mathrm{I}$ & 20 & I3 \\
\hline & & 27 & 23 & 15 \\
\hline ounds & ........... & 17 & 16 & 13 \\
\hline Chucks & $\ldots \ldots \ldots \ldots \ldots \ldots \ldots$ & - $\quad 15$ & I4 & I3 \\
\hline Plates & $\ldots \ldots \ldots, \ldots \ldots \ldots$ & I3 & 12 & II \\
\hline
\end{tabular}

It will be noticed that there is a difference of only 2 cents per pound in the price of first quality chucks and third quality, while there is a difference of 12 cents between the corresponding grades of loin. City retail butchers usually have to buy extra loins and ribs, in addition to sides of beef, to fill the demand for choice cuts. Oftentimes the cheaper cuts of meat can be purchased at the high-class stores at a better price than shops catering to poorer trade, with the additional advantage of getting first quality meat.

The economy of buying meats in quantity has been shown (page I9I). Marketing for meat is the most difficult and important part of food purchasing, for one-quarter to onethird of the money spent for food usually goes to the butcher. Considerable study and experience are needed to become an efficient purchaser of meat. Then the skill of the cooking has much to do with the money that need be spent in this division. There are a number of excellent U. S. Government bulletins on meats.

No. 39I-"Economical Use of Meat in the Home." (Free.)

No. 526-"Mutton and Its Value in the Diet." (Free.)

No. 34-"Meats: Composition and Cooking." (Free.)

No. 467-"The Food Value and Use of Poultry." Price, 5c (coin). No. 469-"Fats and Their. Economical Use in Home." Price, 5c No. 28-Chemical Composition of American Food Materials. Price, Ioc.

Send to Superintendent of Documents, Washington, D. C., for bulletins "for sale." Stamps not accepted. The "free" bulletins are distributed by the Department of Agriculture and Senators and Representatives. They cost $5 \mathrm{c}$ each if sent to Canada, etc. 
The following table, figured on fairly high retail prices (but not "war" prices), will be useful in giving the relative costs of I,OOO calories of food. A similar table based on your local current price will well repay the trouble of making it.

PRICE PER POUND OF EDIBLE PORTION, AND COST PER I,000 CALORIES OF SOME COMMON FOODS (EASTERN RETAIL PRICES)

Foon

Market Percentage Price Calories Cost per price of edible perlb.as 1,000 perlb. waste portion purchased calories

\begin{tabular}{|c|c|c|c|c|c|}
\hline Porterhouse & .35 & 12.7 & .40 & 1,100 & .32 \\
\hline Chuck ribs (medium) & .20 & 16.3 & .23 & 910 & .22 \\
\hline Flank (rib) $\ldots \ldots \ldots$ & .18 & 10.2 & .20 & I. 105 & .16 \\
\hline Dried smoked beef... & .50 & 4.7 & .52 & 790 & .63 \\
\hline Forequarter veal ..... & .30 & 24.5 & .39 & 535 & .56 \\
\hline Mutton, loin ........ & .27 & 16.0 & .32 & $\mathrm{I}, 415$ & $.2 \mathrm{I}$ \\
\hline Leg of lamb......... & .24 & 17.4 & .29 & 860 & .28 \\
\hline Chicken (fowl) ..... & .24 & 25.9 & .32 & 765 & .32 \\
\hline$\ldots \ldots \ldots \ldots$ & .16 & 44.7 & .29 & 370 & .44 \\
\hline Canned salmon.... . & .25 & 0.0 & .25 & 9 I 5 & .27 \\
\hline Oysters (pint) ...... & .25 & 0.0 & .25 & 225 & I.II \\
\hline Eggs $(8$ eggs $) \ldots \ldots \ldots$ & .20 & II 1.2 & .22 & 635 & $.3 I$ \\
\hline Butter .............. & .35 & 0.0 & .35 & 3,410 & .10 \\
\hline Lard, etc. $\ldots \ldots \ldots$ & .22 & 0.0 & .22 & 4,220 & .05 \\
\hline Whole milk ( 1 pt.)... & .04 & 0.0 & .04 & 310 & .12 \\
\hline Cheese $\because . . \ldots \ldots \ldots$ & .25 & 0.0 & .25 & $\mathrm{I}, 885$ & .13 \\
\hline Macaroni...$\ldots \ldots$ & .12 & 0.0 & .12 & $x, 645$ & .07 \\
\hline Rice & .10 & 0.0 & .10 & 1,620 & .06 \\
\hline Potatoes ( $\$ \mathrm{r} .50$ bu.).. & .025 & 20.0 & .032 & 295 & .08 \\
\hline White bread $\ldots \ldots \ldots$ & .08 & 0.0 & .08 & 1,200 & .07 \\
\hline Whole wheat bread.. & .15 & 0.0 & .15 & I,I 30 & .13 \\
\hline Wheat flour...$\ldots \ldots$ & .04 & 0.0 & .04 & $\mathrm{I}, 635$ & $.02 \frac{1}{2}$ \\
\hline Honey $\ldots . . . \ldots \ldots$ & .30 & 0.0 & .30 & 1,420 & $.2 \mathrm{I}$ \\
\hline Sugar $\ldots \ldots \ldots \ldots \ldots$ & .07 & 0.0 & .07 & $\mathbf{x}, 75^{0}$ & .04 \\
\hline Dried pea beans...... & .08 & 0.0 & .08 & $\mathrm{x}, 520$ & .05 \\
\hline Mushrooms ......... & .50 & 0.3 & .50 & 185 & $\cdot 2.70$ \\
\hline Tomatoes & .04 & 0.0 & .04 & 100 & .40 \\
\hline Bananas ( 3 to 1 b.).... & .06 & 35.0 & .09 & 260 & .23 \\
\hline Oranges ( 4 to $1 \mathrm{~b}.) \ldots$ & .10 & 27.0 & .13 & 150 & .67 \\
\hline English walnuts ..... & .25 & 58.1. & .59 & $\mathbf{I}, 250$ & .20 \\
\hline$\ldots \ldots \ldots$ & .15 & 10.0 & .16 & $\mathbf{1}, 275$ & .12 \\
\hline Chocolate ............ & .40 & 0.0 & .40 & 2,625 & .15 \\
\hline Raisins & .12 & 10.0 & .14 & 1,265 & .05 \\
\hline
\end{tabular}


Note. The simplest method of figuring the price of edible portions of food is to consider $100 \mathrm{lbs}$.; thus, in $100 \mathrm{lbs}$. of porterhouse steak there will be $12.7 \mathrm{lbs}$. of bone, etc., and $87.3 \mathrm{lbs}$. meat. $100 \mathrm{lbs}$. would cost $\$ 35.00$ and $\mathrm{I}$ lb. would cost $\$ 35.00 \div 87.3$ or $\$ 0.40$. The cost of 1,000 calories is found by multiplying the price paid by 1,000 and dividing by the number of calories per pound as purchased in the table.

The figures given in U. S. Bulletin No. 142, "Principles of Nutrition and Nutritive Value of Food" (price, 5 cents), were used for this table.

\section{Knowledge of Sanitary Standards Necessary}

The trained consumer should be familiar with at least the most common laws in her state, passed for her protection in regard to the standards and handling of products, and must co-operate in their enforcement. The national government has taken some steps to enforce high standards in the manufacture and handling of products; but the consumer must generally depend on the laws of her particular state and city to insure her purity, quality and sanitation, especially among such products as meats, ice cream, fish, milk, oysters and other perishables. Each state has some well-organized department located at the state capitol; each city has a board of health and various officers who oversee weights and measures, detect adulteration, unsanitary conditions, etc., and prosecute the offending dealers.

Both these state (or county) and city departments have issued definite laws on how much certain commodities must weigh, what constitutes standard scales, measures and infringements of the law. The consumer should send for copies of these bulletins (to the state capital): "Specifications for Weighing and Measuring Devices," issued by the Board of Agriculture of Ohio, or the Bulletin "What Every Housewife Should Know," issued by the New Jersey State Department of Weights and Measures; or the "Sanitary Code" of any city, which gives the laws governing the han- 
dling of foods, as cold storage chickens, loose milk, "basement bakeries" or open markets. Armed with the information contained in the bulletins of her respective state (and city), each consumer will be able to detect violations of the law and insist on honesty and freedom from adulteration, etc. For instance, there has been a strong fight for more sanitary handling of all products in all sections. The intelligent consumer will no longer tolerate bakery goods exposed to flies, "loose" milk opened a dozen times daily and kept in foul ice chests, or ice cream factories where the floor is filthy and the workmen themselves unclean.

Economical purchasing does not mean getting foods and products which are merely "cheap," but those which have been made under or handled in a way to safeguard family health. Foods made under filthy conditions or treated, adulterated or processed with chemicals cannot be "cheap" at any price! It is a large share of the modern consumer's work and training to detect such frauds, boycott them and bring them to the attention of the proper inspectors.

How a Knowledge of Adulterants and Frauds ForeWARNS THE CONSUMER

A discussion of the common adulterants found in manufactured foods, or various fraudulent practices, is taken up here, not to prejudice the consumer against all dealers, but merely to warn her as to possible deceit. "Forewarned is forearmed," and knowledge of wrong only protects the right. Naturally, in all manufacture there are a few firms who try to make additional money out of the ignorance of the consumer. Therefore it behooves the consumer to be doubly intelligent! Most of the dealers in manufactured foodstuffs are trying to give the consumer clean, unadulterated products made in sanitary factories by clean workers. The 
National Food and Drug Act passed in 1907 has helped conditions greatly. However, there is still a great deal of adulteration in many classes of foods in which there is profit when inferior materials can be substituted.

\section{What Is a "Pure" Food?}

A pure food must fulfill these requirements :

I. It must not be positively adulterated with foreign substances, as ground hulls for the true ground spice buds.

2. It must not be treated with chemical preservatives like benzoate or salicyclic acids, as in some catsups and canned vegetables.

3. It must not be made of "substitutes," as starch and lard for true cream in ice cream, or as starch in chocolate. 4. It must not be artificially colored with dyes or flavored with chemical flavors, as in candies or "soft drinks." 5. It must not be handled in a dirty, unsanitary way, or by unclean workers.

6. It must not have some of the valuable elements or parts of the food removed, so as to make it a "devitalized" food, as polished rice or bleached flour.

These may seem like impossible demands. Yet unless the consumer insists that these pure food standards be lived up to, she cannot be sure that her family, and especially her children, are eating manufactured or bought foods that will nourish them or that will positively not harm them. If health depends on food, then surely that food must be as pure, clean, and as rich in nourishment as nature originally made it.

\section{Consumers Ignorant or Complacent}

It is often remarked that Americans will "stand" anything; and certainly it is either because they are overly complacent, or else actually ignorant, that the consumer 
allows startling and disgusting practices to be followed. Let us quickly list some of the food practices which she should not tolerate:

I. "Basement bakeries," no light, unsanitary, moldy conditions, unhealthy workers.

2. Artificial coloring and flavoring of candies, cakes; soft drinks with chemicals.

3. Substitution of low-grade materials in supposed highgrade products.

4. Artificial preservatives to "keep" foods, as benzoate, "preservaline," etc.

5. Exposed meat, bakery goods, or permission of sale of same in open carts, wagons or stalls.

6. "Devitalizing" of flours and "bleaching," which lessens nourishment.

Space does not permit going into the details of all these frauds; but many times the consumer is quite responsible for these frauds, although she may not realize it. For instance, flour is now "bleached" very white, and rice grains given artificial polish solely because women want or have made a market for white foods, and refuse to buy darkcolored grains and flours! Again, mothers who give their children soda water which is only water sweetened with saccharine and colored with coal-tar dyes, cannot expect to blame the dealers. They must refuse to purchase such foods, and then the dealers, finding trade unprofitable, will be forced to change their ways.

At a recent Farmers' Insiitute in a large western state the author was conducting a week's series of lectures. One morning, before going to the lecture hall, she visited a dozen shops and purchased about thirty samples of "penny line" candies. She had a beautiful (!) assortment of candy made. of paraffine, starch and glucose; .varnished, brightly colored 
with dyes, flavored with artificial products, and in all quite an exhibit. The samples were on a table and brought to the attention of the mothers present. Many would not believe that such candies were purchased only two blocks from their homes!-or that such vile candies were made! The exhibit was passed on to the parent-teacher association, and effort made to educate children to buy other and purer uncolored kinds.

The Importance of Being Informed on Weights and MEASURES

No one not directly connected with investigation in this line would believe the extent of fraud and deception practiced in connection with all kinds of weights and measures. Owing to recent campaigns for greater honesty, almost nation-wide, and a demand that false measures be confiscated and dishonest dealers be prosecuted, conditions today are much better than formerly. There are still, however, many dishonorable practices or causes for faulty weighing. The consumer must be on her guard against them.

I. Scales may be faulty because-the scale is out of balance, and is too heavy on the scoop side; or the scale may balance when equal weights are placed in the center of the pan, but not when the weights are shifted right or left; the scale may be "insensitive" owing to poor construction, worn or broken parts ; false weight may be given by attaching small wads of iron, lead, hooks or some small article under the scoop, or on the cross under the scoop; the "nested" iron weights may be stacked as 8 oz., 4 oz., 2 oz., thus totaling I 4 oz. instead of the required I6; the "poise" may be light, thus registering more than is true on the scoop; the weights themselves may be worn, drilled, or "filled" with lighter 
substances; or even in the beginning they may never have been accurately adjusted so as to weigh correctly. 2. Dry measures may be faulty because-of "false" bottoms, as in wicker baskets, barrels and measures; they may have "false" sides or be so cut down as to reduce the depth and hence the true amount; they may be broken, dented, or originally of wrong or "short" capacity, especially wicker or berry boxes holding less than the standard amount; or six-quart measures may be used illegally for peck or eight-quart measures; or liquid measure, which is less, may be used for dry measure.

3. Liquid measures may be faulty because-the measures are bent or dented; or they have a "cupped" bottom; or they are leaky; or they were originally manufactured "short."

4. Linear measures may be faulty because-the yardstick is warped or "short"; counter tacks may be wrongly placed; the cloth tape measure may be shrunk or inaccurately divided.

The department of weights and measures of each state employs both county and city "sealers"-men whose duty it is to periodically test all scales and measuring devices. If the measure passes the test, the dealer is allowed to continue its use, and the scale is marked with a large seal, generally red, so that the consumer may know that it will register correct weight. If it does not pass the test, it is "condemned" and its use forbidden. Sometimes several thousand inaccurate or false measures are "condemned" and burned by state authorities.

While the laws of different states vary, it is almost universally conceded that the older type arm or balance scale with scoop and iron weight is least trustworthy. The pre- 


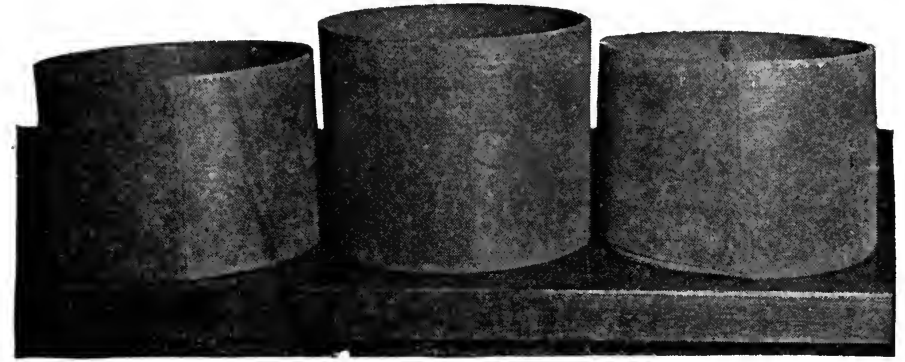

EXAMILES OF SHORT PECK MEASURE.

Condemned by The Chicago Bureau of Weights and Measures.

ferred scale is that called the "pan" or hanging type, or the excellent glass protected "computing" scale. Scales carried by ice men or platform scales on which coal and large amounts are weighed are most frequently faulty. Again, the

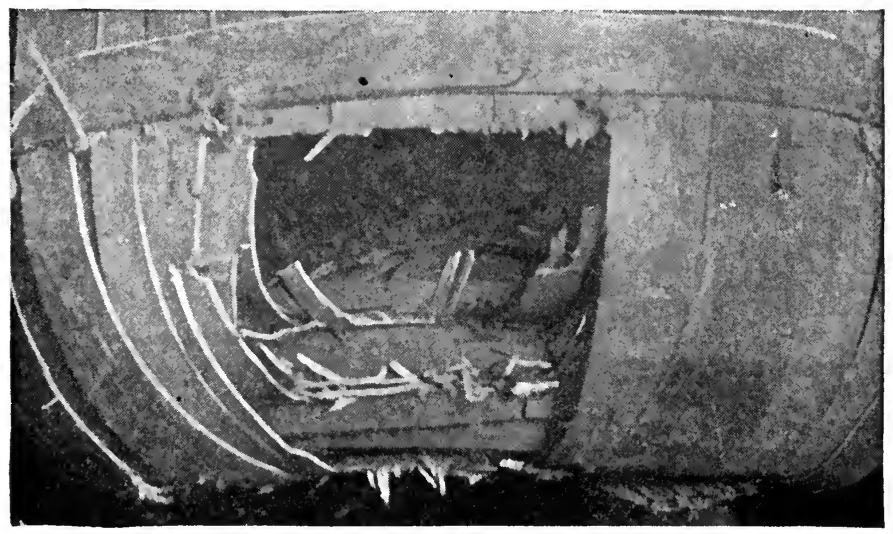

A PEDDLER'S BUSHEL BASKET.

Three Baskets Nested and Concealed Under the Top Band. Courtesy of Illustrated World. 
dealer is not supposed to rest his hand on the scale, place the package unevenly, take it off before the consumer reads the dial, or, for instance, weigh butter in a wooden container unless a similar container, empty, be placed on the opposite side. In the case of hams and other foods covered with burlap, a certain legal allowance must be made, of about four ounces. Meat dealers are allowed to sell meat at the untrimmed weight, but these trimmings belong to the housekeeper. If left with the butcher they will be resold by him to rendering plants. On a five-pound leg of lamb there may be as much as a pound and a quarter of trimmings. It will pay, and pay well, to check up the weight of all food coming into the house. Tests have shown as much as $20 \%$ shortage. A good scale is necessary (see page 6).

It is a rather common custom to sell dry beans and peas and cranberries by the liquid quart (one-quarter gallon), which contains about one-sixth less in volume than the dry measure quart. This practice is not legal, even if it is the custom.

In all of this discussion, let it not be thought that every dealer is unscrupulous. The majority of all dealers in all lines intend giving dependable measures, but frequently scales become inaccurate without the dealer knowing it; or he may have happened to buy a cheap, unreliable measure from some unscrupulous manufacturer. The consumer should insist on honesty, not so much to prosecute the dishonest dealer as to protect herself and give a fair deal to the man who is trying to be fair to her. The reliable dealer who has nothing to fear will not be incensed if the consumer investigates his measures, the sanitary conditions of his store, or other of his selling methods, but will welcome investigation, as it will only serve to bind the intelligent consumer more closely to his establishment.

All purchasing should be by definite weight or amoun', 
as bushel, peck, quart, gallon, etc. Instead, for instance, of buying a bushel of potatoes, the consumer will be assured of getting her money's worth if she asks for. sixty pounds of potatoes, or whatever the legal amount is in her particular state. Nothing makes for more wasteful, inefficient buying than this mistake of buying by the "bag" (how much does it contain?), as no two dealers sell the same kind of "bags," and even the same dealer might at different times be using different kinds of measures, so that the consumer can never be sure of always getting a definite quantity for a definite sum, unless she asks the price per weight of a definite standard, as a bushel, or fraction of a bushel, or gallon.

State laws are by no means uniform; each state has, for its own limits, passed standard weights per bushel for different articles, as shown in the table on the next page, taken from Measurements for the Household, Circular No. 55, U. S. Bureau of Standards.

\section{Net Weight of Contents LaW}

An amendment to the National Food and Drug Act passed in I9I4 requires the net weight or volume of contents to be printed on the label of containers or packages distributed in interstate commerce. A number of states have also passed a similar law, so that practically everything in the food line except bulk goods is so labeled. For example, the various brands of popular breakfast foods contain (at present writing) the following weights:

Quaker Oats......... lb. 4 oz. Cream of Wheat.... I lb. $12 \mathrm{oz}$. Pettijohn .......... i lb. 8 oz. Ralston ........... I lb. 8 oz. Wheatena
Shredded Wheat ....... 12 oz. Grape Nuts .......... I4 oz. Corn Flakes .......... 8 oz. Puffed Wheat ........ 4 oz. Puffed Rice .......... 4 oz. 


\section{LEGAL WEIGHTS PER BUSHEL}

\begin{tabular}{|c|c|c|c|c|c|c|c|c|c|c|}
\hline$\nabla$ & $\Delta \bar{T}$ & $\infty$ & U & 0 & Q & - & 2 & $\breve{2}$ & $F$ & $f$ \\
\hline Alabama .... & 24 & $\cdots$ & .. & & - & $\cdots$ & 60 & 55 & -. & 55 \\
\hline Arkansas ........ 50 & 24 & $\cdots$ & 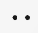 & $5 \%$ & - & - & 0 & 50 & . & 57 \\
\hline Colorado $\ldots \ldots \ldots \ldots$ & & $\dot{8}$ & & & $\dot{2}$ & $\cdots$ & 0 & & $\bullet$ & \\
\hline Connecticut $\quad \ldots \ldots \ldots 48$ & 25 & 60 & 0 & & 45 & $\ddot{0}$ & 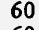 & 54 & & 5 \\
\hline Florida ........... & 24 & $\cdots$ & $\cdot$ & & $\cdots$ & 54 & & 60 & $\cdots$ & \\
\hline Georgia $\ldots \ldots \ldots \ldots$ & 24 & $\ddot{\dot{c}}$ & 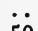 & & & $\ddot{0}$ & & & & \\
\hline daho $\ldots \ldots \ldots \ldots \ldots 48$ & 24 & 56 & 50 & & & 48 & 60 & & & \\
\hline Illinois $\ldots \ldots \ldots \ldots \ldots 50$ & 24 & 60 & 0 & & & 48 & 6 & & 3 & \\
\hline Indiana $\ldots \ldots \ldots \ldots \ldots$ & 2 & & & & 5 & .. & & & . & \\
\hline Iowa $\ldots \ldots \ldots \ldots \ldots 48$ & 24 & 56 & 50 & & 4 & 48 & & & 50 & \\
\hline Kansas .......... 48 & 24 & 56 & 50 & & 52 & 48 & & & & \\
\hline Kentucky $\quad . . \ldots \ldots \ldots$ & 24 & $\ddot{0}$ & $\ddot{0}$ & & 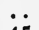 & $\cdots$ & 6 & 55 & - & \\
\hline$e \quad \ldots \ldots \ldots \ldots$ & 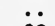 & 60 & 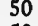 & 5 & 45 & 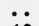 & & $\ddot{\theta}$ & 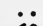 & \\
\hline land $\ldots \ldots \ldots \ldots$ & 28 & $\ddot{\theta}$ & 50 & 5 & $\therefore$ & 40 & 60 & 60 & 6 & ( \\
\hline chuetts $\ldots \ldots$. & 25 & 60 & 50 & 5 & 45 & 48 & 60 & 54 & 56 & \\
\hline $\operatorname{san} \ldots \ldots \ldots$ & 22 & $\ddot{0}$ & . & & : & - & 60 & 56 & - & 58 \\
\hline sota $\ldots \ldots \ldots$. & 28 & 50 & 45 & & 42 & $\cdots$ & & & 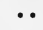 & \\
\hline ippi $\quad \ldots \ldots \ldots$ & 2 & - & & & : & $\ddot{0}$ & & 54 & • & g. \\
\hline Missouri $\quad \ldots \ldots \ldots \ldots$ & 2 & & & & 44 & 48 & & 56 & 4 & \\
\hline ana $\ldots \ldots \ldots$ & $\ddot{i}$ & 50 & 5 & & 50 & . & & $\ddot{i}$ & 8 & J \\
\hline raska $\ldots \ldots \ldots$ & 24 & $\begin{array}{l}56 \\
56\end{array}$ & 5 & & & 48 & & & 5 & \\
\hline Tevada $\ldots \ldots \ldots \ldots .48$ & $\begin{array}{l}24 \\
25\end{array}$ & $\begin{array}{l}56 \\
60\end{array}$ & 50 & & $4 J$ & $\begin{array}{l}48 \\
48\end{array}$ & & 54 & 56 & J \\
\hline ew Jersey......... 50 & 25 & 60 & 50 & &. & 50 & & 5 & & \\
\hline Jew Mexico........4 4 & 24 & 56 & 50 & & 42 & 48 & & 5 & 5 & \\
\hline York........4 48 & 25 & .. & 50 & & .. & .. & & 54 & .. & \\
\hline orth Carolina......4 4 & .. & .. & . & & . & - & 5 & 56 & $\bullet$ & \\
\hline Torth Dakota....... 5 & & 60 & & & & - & & 4 & & \\
\hline ......... & 24 & 56 & 50 & & 50 & 4 & & & 5 & \\
\hline Oklahoma $\ldots \ldots \ldots 48$ & 24 & 60 & 50 & & 44 & 48 & & 55 & 45 & 50 \\
\hline$\ldots \ldots \ldots .4$ & 28 & 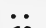 & $\ddot{0}$ & & . & . & & & • & \\
\hline ennsylvania $\ldots$ & 25 & 6 & 50 & & 5 & 4 & & 5 & 6 & \\
\hline hode Island......... & 25 & 5 & 50 & & 50 & 4 & 60 & 54 & 56 & \\
\hline outh Dakota ....... & 24 & 56 & 50 & 5 & 42 & 48 & 60 & 46 & 5 & \\
\hline Tennessee $\ldots . .$. & 24 & 50 & 50 & & 50 & 50 & & 50 & 5 & \\
\hline Texas ........ & 28 & . & 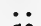 & & . & 5 & & & 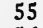 & \\
\hline zont $\ldots \ldots \ldots \ldots 4$ & 0 & 60 & 50 & & 45 & 48 & & & 56 & \\
\hline . $\ldots \ldots \ldots$ & 28 & .. & . & 5 & .. & . & & 56 & - & \\
\hline nington...$\cdots \cdots$ & 28 & .. & - & - & - & . & & •. & - & \\
\hline t Virginia....... & 25 & $\ddot{0}$ & $\ddot{0}$ & en & $\ddot{i s}$ & - & & $\ddot{\ddot{\theta}}$ & & \\
\hline Wisconsin $\ldots \ldots \ldots 50$ & 25 & 50 & 50 & 57 & 44 & .. & 60 & 54 & & \\
\hline
\end{tabular}

The legal weight for a bushel of wheat, a bushel of dry, white beans, and dry peas, is 60 pounds, of corn and rye, 56 pounds, of oats, 32 pounds. Arizona, California, Delaware, District of Columbia, Louisiana, South Carolina, Utah, and Wyoming have few or no legal weight laws.

A bushel contains 4 pecks, and a peck 8 quarts. 
This law is a great help to the consumer in comparing prices of package goods with bulk goods or different brands of competing products, as in canned goods, and in keeping informed as to the price per pound of package goods. The "puffed" breakfast foods, for instance, at I 5 cents a package cost 60 cents a pound.

As the price of staples increases, the manufacturers have reduced the weight of contents, in self-defense. This fact is not advertised. For instance, some years ago a package of

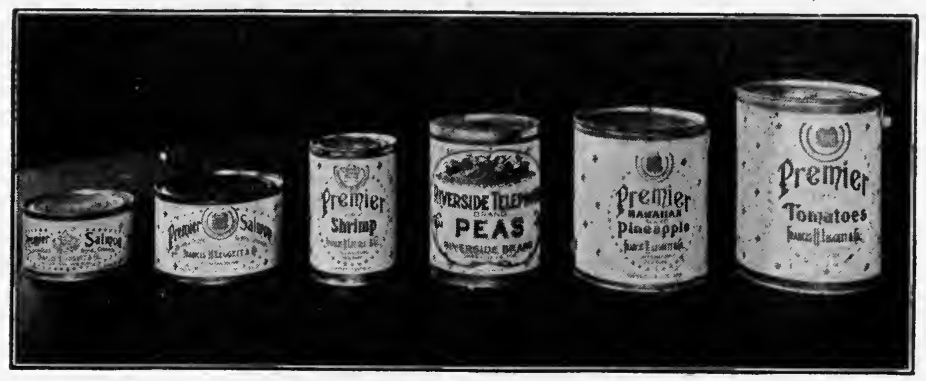

No. $1 / 2$ No. 1 Short No. 1 Tall No. 2 No. $2 \frac{1}{2} \quad$ No. 3

THE COMMON SIZES OF CANS

Quaker Oats contained 2 pounds-the price remains the same (or more) but the contents of the package has been gradually reduced to $\mathrm{I} \mathrm{lb} .4 \mathrm{oz}$. Uneeda Biscuits when first put on the market contained nearly half a pound; the package now contains $45 / 8$ ounces.

\section{Knowledge of Can Sizes}

So many of the foods used in the home today come in packages and cans that it is necessary for the consumer to be familiar with the sizes and other facts of identification. She may have noted that peas, for instance, come in cans smaller than tomatoes; but the first fact to learn is that all 
canned products, whether they be soup, vegetable, fruit, or other foods, are supposed to be packed according to a series of standard sizes, which contain corresponding weights, though the weights will vary somewhat for different products.

\section{TABLE OF STANDARD SIZES OF CANS}

Size Number

KIND of Food

Wt. of Contents

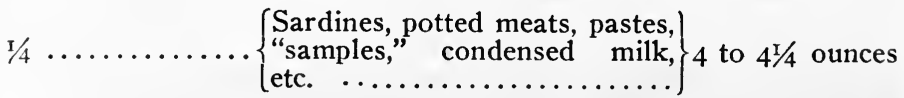

$1 / 2 \ldots \ldots \ldots \ldots \ldots\left\{\begin{array}{l}\text { Potted delicacies : shrimp, lob- } \\ \text { ster, clams, condensed milk, } \\ \text { "flat" salmon (small), etc.... }\end{array}\right\} 8$ or $73 / 4$ ounces

I (short) $\ldots \ldots \ldots\left\{\begin{array}{l}\text { Canned soup, potted or boned } \\ \text { meats, tomato or other purees, } \\ \text { condensed milk ................ }\end{array}\right\}$ Io ounces

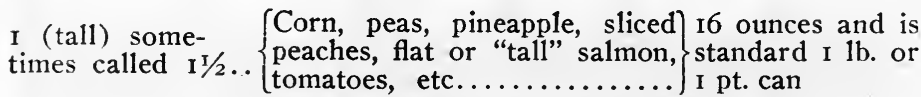

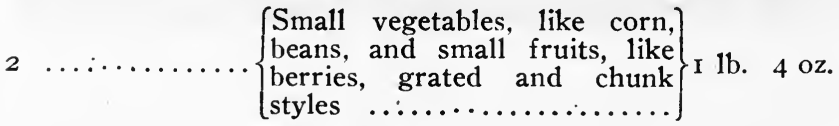

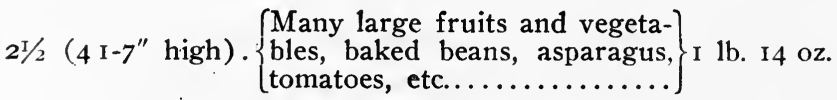

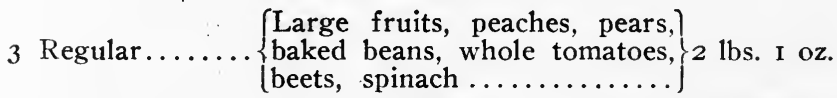

3 Tall (51/2" high). Same as above........... 2 lbs. 6 oz.

I0

[Corn on cob, sauerkraut, soup

and large quantity for trade 6 lbs. 6 oz.

distribution

There is no number on the can, as "No. 2," to indicate its size, but this size can be easily determined by noting the weight, which is always printed in small numbers, generally at the base of the face or the reverse of the can. If the label 
reads, for instance, "I lb. I4 oz.," it is probably a No. $2^{1 / 2}$ can. The same product may be packed in any one of three or four sizes. As an experiment, the author asked for "A can of tomatoes" in several stores. She was handed five different sizes of cans, varying in weight from ro ounces to 2 lbs. 6 oz.- - or from the No. I "short" to the No. 3. Five different size cans all in answer to the same request for a "can"! The grocer knows the various size cans by number. Therefore if you ask for them by number you have a better chance of getting the exact size you wish. Then note carefully the "net weight of contents" on the label.

\section{Family Needs Determine Can Sizes}

In one brand of pineapples, for instance, a No. $2^{1 / 2}$ can contains ten large slices and costs 15 cents. The No. 2 can of the same grade contains six small slices and costs Io cents. In one case a large slice costs $\mathrm{I} / 2 \mathrm{c}$, but buying the apparently cheaper can each small slice costs $I^{2} / 3 \mathrm{c}$. Therefore by estimating how many slices or portions are contained in different sized cans the housewife will be able to purchase more economically, buying the exact size can that suits her particular family's needs. It is generally better economy to purchase one large can and make it cover two servings than to purchase two small cans separately, because the smaller the size the higher, proportionately, is the price.

Many kinds of fish and other food come in flat as well as tall cans. The tall No. I weighs exactly the same as a flat No. I, but the flat can generally costs $10 \%$ or $15 \%$ more. This is because, as in salmon, for instance, a solid piece of fish is cut right out of the best portion of the salmon, whereas the tall can is filled with one or two smaller pieces, which makes the packing less expensive.

Sometimes the can appears partially empty at the top, as 
in corn. This is called "slack fill" and is contrary to law. It does not always mean that the can is short in weight. A certain amount of space must be left between the top of the can and the material in it, so that the red-hot "capping iron" will not scorch the contents. Corn especially swells in "processing," and extra allowance must be made.

It is economy to purchase canned goods in quantity, as there is a reduction in goods bought by the case, or 24 cans. Fall is the time to purchase fresh season stuff. It is easy to estimate how many cans of the various products will be used per week, month or season, then to make up an order and purchase by the case ( 2 doz.), or at least the dozen. This is not only a money but a time saving, as all too frequently unnecessary time is wasted running to the store for a can of this or that.

It is helpful to write in the kitchen note book the consumer's experience with various brands, sizes and grades of canned goods; also the number of portions, slices, the quality, trade mark or other facts which will enable her to do future buying most economically and easily. No law compels canners to use uniform sizes, and as cost of materials and labor increases there is a tendency to decrease the size of the cans instead of increasing the price. It is impossible to distinguish by the eye a can slightly under standard size, but the net weight on the label will reveal it. Again, the weight may be falsely kept up by the addition of extra water, as in tomatoes, which is an adulteration; therefore those standard brands should be bought which have proved the test of honesty and full measure and quality.

At retail city prices it is not always good economy to can vegetables and fruits at home, but near the source of supply or from the home garden a very considerable saving can be made by "putting up" canned goods. There are many Government Bulletins on the subject which will be sent on 
request from the Department of Agriculture. The "cold pack" method seems easiest and is successful.

\section{How the Trademark Protects the Consumer}

It may be asked, if there are as many methods of deceit and adulteration as have been pointed out, how is the consumer to protect herself except by constant and continued investigation? Will not every purchase be an experiment as to quality and purity? The one means of protection the consumer can rely on is the "trademark" on the package or product she buys. The "trademark" is some kind of label, emblem or other means of identification bearing the manufacturer's name, place of factory, weight, size, or other words leading to rapid identification on the part of the consumer. For instance, before buying a can of peas or a pair of hose, how can the consumer be sure of the quality and wear of these articles? Only by selecting goods which bear a "trademark" or the name of a manufacturer who has come to be widely known as a maker of articles of quality and value.

\section{Bulk Versus Package Goods}

There has been a great deal of discussion as to the best kind of products to buy-those which are "packaged," wrapped or in some kind of container bearing a trademark -and those which are merely "loose" or "bulk," and unwrapped or untrademarked, and which sometimes, as in "loose" tea, may cost a few cents less a pound than a packaged tea. Now, in the author's opinion, all "bulk" goods are open to the same criticism-namely, that they are unidentified, and thus the consumer is unable to tell the quality in advance, and thus has no means of safeguarding her purchase. Identification is the only means of protecting 
the consumer as to the quality of what she buys. In other words, some kind of trademark saves the consumer's time by enabling her to pick out an article of known quality quickly, as well as being the sole means of identifying quality, or lack of quality. Not all trademarked goods are superior to bulk or unidentified merchandise; but the mere fact that they are "trademarked" and identifiable, protects the consumer either the one way or the other. Trademarked goods are generally superior to bulk goods for the following reasons:

I. They furnish a means of identification either for or against quality.

2. They give the manufacturer's name and location of factory.

3. They inform the net weight or quality standard of the product.

4. They permit more accurate weighing.

5. They insure that the goods were made under sanitary conditions by sanitary workers.

The man who is willing to put his name on his product, and the place where it is made, is generally the manufacturer who has no fear of the investigations of the consumer. Also, if he has put out a trademarked product for years, the chances are strongly that the product has a high quality, or else consumers would long ago have found out its inferiority and put it off the market. And, last, the price of the identified products of wide distribution is generally as low as that of a similar "bulk" article; because its wider distribution and long continued sale will have enabled the manufacturer to install such machinery and methods in his factory as to lower the producing cost, and thus sell his identified article at as low a price as the unidentified "bulk" article can be sold. Examples of this are Ivory Soap and Walter Baker's Chocolate, sold under trademarked labels 
for many years, yet which are as low in price and of higher quality than similar competing unrecognized brands.

\section{Private Brands Versus Trademarked Brands}

There is another distinction in brands which the consumer must know. That is, the difference between the "private" and the genuinely "trademarked" brands of goods. For instance, here are two cans of tomatoes; both apparently have a label and name. But if we look closely we see that Can A says, "Queen Tomatoes, packed for J. Jones \& Company"; Can B will say, "Smith's Tomatoes, S. \& S. Smith Bros., Vineland, New Jersey." What is the difference between these two labels? The first is the label of a "private brand." It does not state the firm that packed it, only the name of the firm for whom it was packed. Also, it generally does not give the location of the factory where the product was packed. On the other hand, the label of can $B$ does state the name of the packer and the place of the factory. In the first case, we have a "jobber" who wants to sell tomatoes. He gets any number of small factories, even in several states, to pack tomatoes for him. Then he has these cans sent to his wholesale house, and there puts his own labels on them. In the second case we have the original packer himself putting his own name on the goods and where they were packed.

The "private" brand goods is put up in different factories, from different sources each year, and there is no guarantee of a permanent quality behind it. Since the name of the packer does not appear on the container, there is no way for the consumer to find out what kind of a factory or conditions it was packed under. On the other hand, the honest name of the packer himself insures the consumer that she can look up this man and this factory, and that he stands back of his goods, since he is willing to put his name on them. 
In every case, the trademarked brand carries more integrity or guarantee. It is now almost universal for the trademarked brand to carry a refund guarantee of giving the consumer another article or refund of original purchase price if she is dissatisfied. If, for instance, the Kayser gloves do not wear as represented, or Holeproof sox develop holes within a certain time, the manufacturer "makes good" with duplicate articles. Some jobbers, it is true, have also established the high grades of their brands by years of fair dealing, and their goods can also be relied on in such cases.

\section{"Read the Label," the Consumer's Watchword}

The label is the one protection of the consumer, if she only learns to always read it intelligently. Every label should declare the contents of the article; but often we may find labels that are deceptive, or which at least are far from straightforward. It is common on canned meats to find the words:

\section{"POTTED meat HAM flavor"}

so printed as to make the consumer think she is buying potted ham, when the contents are really only inferior meat scraps flavored with ham. Or she may think she has bought a bottle of vanilla, when really the label, closely read, states:

\section{"Extract of Vanillin"}

Now, vanillin is an artificial flavor made from the oil of cloves; even if it were as wholesome as true essence of vanilla from the tonka bean, it is not fair that the consumer should pay vanilla prices for vanillin.

Similarly, "MAPLE flavor SYRUP," or other wording, reveals the fact that the possibly expensive syrup which the consumer thought was true maple syrup is only a sugar syrup flavored and colored artificially. The labels on cat- 
sup, as "preserved with $\frac{1}{10}$ of $1 \%$ of benzoate of soda, artificially colored," etc., are familiar; often the words are purposely made misleading, as a cough syrup may say "Tincture of Poppy, 21/2"; but this "tincture" is really nothing but morphine or laudanum, if read intelligently. Drugs need most careful reading, as often headache powders which are claimed to be "harmless" contain acetanilid or other dangerous drugs. The consumer should be especially wary of the words "compound," "artificial" and "harmless."

\section{Advertising ANd the Consumer}

Modern advertising in periodicals, on billboards, cards, etc., is another means of bringing goods of all kinds to the consumer's attention. The costs of advertising must be included in the general cost of distribution of an article, and do not add any more to the price of an article than any other means of display, such as store window exhibits, circular letters and the older forms of traveling salesmen which were practiced in the days before periodical publication made modern advertising methods possible. Advertising generally raises the standard of production (since it does not pay to advertise a poor product), at the same time that it makes possible wider distribution; and wider distribution enables larger volume of production with consequent greater economy. Because it has brought so many thousand articles of furnishing, comfort, and luxury before the consumer, it has, naturally, tended to raise the standard of living. At the same time, our daily papers and periodicals would be impossible if it were not that the advertising they carry pays largely for their printing. Since most advertised articles are also trademarked articles, they insure the consumer guarantee, identification and time-saving shopping. 


\section{The “Cut-Price" Lure and the Consumer}

There are some forms of advertising practice, however, which should not be encouraged, chief of these the "cutprice" lure, especially on a trademarked article. These "specials," as "three cakes of Ivory Soap for a quarter," or Fownes' Gloves, \$1.00 grade, only \$o.79," are used solely to get the woman into the store, where they hope that she will buy other unindentified merchandise on which the dealer makes more profit but which are of poorer quality than the trademarked goods. In other words, the "cut" is merely "bait"; that it is not a sincere reduction is shown by the fact that it is offered "for this morning only," or "only one to a customer," or in some other way which has a "string" attached. The reason that such buying is demoralizing is, first, that it traps women into buying other goods of lesser quality; second, it gives them a false idea of the price which they should pay for the article-a price which their own local retailer cannot meet, so that, therefore, after a time, he may refuse to carry the article because he cannot meet the "cut" and still make a profit. This may eventually drive good products off the market; third, all juggling of price, and discrepancies, are bad, because they create in the consumer's mind false ideals and ideas of value, and encourage her to demand "bargains" to such an extent that merchants are forced to offer her inferior quality in order to satisfy this "bargain" appetite.

The sister evil of the "marked up" price is also a result of this pernicious demand on the part of women. This means that the advertised price of an overcoat, say, is stated as " $\$ 40.00$ overcoat, now only $\$ 19.50 . "$ The truth is that the overcoat was probably only a $\$ 25.00$ grade in the first place, but that it has been falsely "marked up" in order to make the "bargain" seem more of a drop in price. Such 
practices must not be confused with legitimate reductions which occur at seasonal times. But it cannot be too strongly said that women must stop encouraging the "bargain habit" if they wish dealers to make conservative, dependable claims and statements. Before John Wanamaker developed and enforced his policy of plainly marked prices, the buyer did not know whether he was being cheated or not. Today the cut-price, the marked-up price, and misleading statements are still a few surviving mal-practices which the woman consumer must help eradicate.

\section{PURCHASING OF CLOTHING AND TEXTILES}

Next to food, clothing is the most important division in household purchasing. The efficient consumer must learn to distinguish, test and judge the wearing qualities and value of all articles of clothing and textile fabrics. The market carries so many grades and variously named novelties in fabrics that it is somewhat difficult for the buyer to judge intelligently. However, the number of "standard" fabrics are not very great, and the novelties are but variations of "standard" goods.

The expert judges textiles by the appearance, feel and weight of the fabric and the knowledge of the various fibers, yarns and methods of weaving and finishing the goods.

A knowledge of the common textile fibers is the first essential. Briefly, cotton fibers are very fine and comparatively short-3/4 of an inch to an inch. Flax fiber is larger and much longer than cotton. Linen cannot be bleached by. chemical means as easily as cotton or without losing strength and luster, nor can it be dyed as easily or are the colors as fast. It launders well but shrinks more than cotton. Linen has superior wearing qualities.

As raw flax costs two or three times as much as cotton, and as the loss in finishing is much greater and the process 
more expensive; there is a strong temptation to adulterate linen with cotton to make goods less expensive. As is well known, cotton in table linen detracts from the wearing qualities, as the short fibers of the cotton give the pieces a fuzzy appearance after being used a short time.

Silk fibers are very long, are strong, and have high luster. Soft silks wear better than stiff ones which are "weighted" with salts of tin and iron. This weighting decreases the strength of the fiber but makes it "go farther." Taffetas are examples of heavily weighted silk.

Wool is the important animal fiber. Its scale-like surface gives it special felting and spinning qualities. It is strong, elastic, and when spun and woven the elastic properties give a great number of air spaces in the fabric, rendering clothing made from wool very warm and light. It will absorb up to 30 percent of its weight of water without feeling damp to the touch.

Wool fabrics are divided into "woolen" and "worsted" materials. The woolens are made from the short wool fibers obtained by carding the wool fleece. Woolen yarns are soft and fluffy. Worsteds are made from the long fibers which have been combed and carded until they lie smooth and parallel. They are then. twisted more tightly than woolen yarns, thus making it more regular and lustrous. Woolen materials are soft, elastic, of soft finish and blurred pattern. Worsted materials are of harder finish, show the weave more plainly, and have a clearer pattern.

The expense of manufacture of worsted yarn is greater than for woolen yarn, consequently worsted materials cost more than woolens of the same weight and width.

\section{Adulterations and Tests}

The mixing of fibers is legitimate, but the consumer has a right to know what percent of each textile she is pur- 
chasing, and not, for instance, pay "all wool" prices for material containing a large proportion of "shoddy" or other adulterant. The consumer often encourages adulteration by demanding very low-price materials. Generally, the brands of "trademarked" textiles, like "Skinner's Satin," etc., long established, can be best trusted. There should be a national "pure textile" law which would enforce labeling that would inform the consumer exactly what percent and grade of each textile she is buying.

The following gives some of the common adulterations and simple home methods of testing:

Cotton in Linen. Linen threads break off with sharppointed ends, while cotton threads break off short with fuzzy ends. A spot of oil or glycerine makes linen more translucent than cotton. A drop of water on linen spreads rapidly; on cotton it absorbs slowly. Singed ends of cotton thread spread out, while singed linen threads look close together and uniform.

Cotton in Woolen and Worsteds. When cotton threads make up part of the warp (running threads) or the filling of the fabric, the cotton is easily detected by picking the threads apart and burning-cotton burns quickly, with little odor or ash; wool in burning gives the odor of burning feathers.

When cotton fiber is spun with the wool, detection is more difficult. Boil a sample of the goods in a solution of washing soda, 2 tablespoons to a pint, for twenty minutes. The wool becomes jelly-like and may be separated from the cotton by rubbing in warm water. Or boil the sample in caustic soda solution (a two-inch stick of caustic in a cup of water); the wool will be dissolved completely and the cotton left.

To remove cotton from woolen, boil sample in oxalic acid solution (one tablespoonful to a cup) and dry without 
rinsing. Then wash out the acid crystals, dry, and rub the goods-compare with original sample or with sample that has been simply boiled in water and dried.

Weighting of silk may be detected by laying a sample on a tin plate and leaving in a hot oven for about an hour. The pure silk will be burned away and the weighting remain in the form of the sample; if it burns to a heap of ash, it is pure silk and not heavily weighted.

Silk is sometimes adulterated with mercerized cotton, which is somewhat difficult to detect. Mercerized cotton is made by treating cotton threads with strong caustic soda solution while the cotton is under strong tension; then the caustic is removed. This process swells the cotton fiber and gives it luster. The process strengthens the goods and makes a wear-resisting fabric, but it should not be sold at the price of silk. Artificial silk is made by dissolving cellulose (cotton) and forcing the pasty substance through small holes, thus giving the structure and somewhat the appearance of silk, but lacking its true softness and elasticity.

To test any woven material, pull sharply between the two thumbs, first one way, then the other. If it gives much or tears apart easily, the fabric shows lack of strength. Close, firm weaves are more enduring than loosely woven ones. By holding a sample of cloth to the light, imperfections may be detected. Rubbing the material between the hands or thumb and first finger will often detect "sizing" in the goods.

Wash goods may be tested by taking a sample home and heating a part of it in soap and water. If the color "bleeds" it will be quickly apparent. Moistening a sample and exposing it to direct sunshine for a day or two will show its fastness to light. A part of the original sample should be kept for comparison. Washing will also remove starches and gums used in finishing to conceal defects and 
give an attractive appearance. The author recalls some yards of Swiss with a beautiful "dot," which in the first washing, disappeared!

The word "shoddy" is often heard and not as often understood. "Shoddy" is the technical term of "regenerated" wool, made from old woolen and worsted garments, rags and tailors' scraps. These are pulled apart and the fibers respun. "Shoddy" is coarse, inelastic, and short-fibered; material made from it will crease easily, and have a dead "sticky" feeling; it cannot be depended upon for wear. A certain amount is unobjectionable as it gives warmth, if not wear, and indeed is a commercial necessity, as there is not enough pure wool in the world to go around! But the consumer must learn to protect herself and not pay a "wool" price for "shoddy."

What the Efficient Buyer of Textiles Must Know

The woman who can fulfill the following requirements may be classed as an intelligent buyer of textiles:

I. Know the appropriate kind of cloth to be used for the occasion, considering weave, color and design.

2. Know what she can afford to pay for it.

3. Know what she should be able to get for that sum of money.

4. Know whether the material she purchases is what it is represented to be.

There is a great tendency to be lured by the swiftly changing fashions into spending more for clothing than the income justifies. Some women prefer a number of inexpensive garments following the fashions and designs of the hour, while others prefer a few well made garments of beautiful material in conservative style to use for two or three years. 
The first class of women sacrifice quality of material and workmanship to faddish styles-few have enough money to extravagantly squander beautiful material on extreme gowns; the second class sacrifice the latest skirt and sleeve to the beauty of color and line so pleasing to the individual and to others. Needless to say the second class of women on the whole appear better dressed. Where growing children have no brothers or sisters to wear their outgrown clothes, inexpensive materials are justified.

Having decided the kind of clothes wanted (inexpensive, up-to-the-minute in style, and many of them, or expensive, conservative, and few in number), consider next economy in clothing from the standpoint of time and money which must be spent in construction or workmanship. It may be economy for mothers and daughters to make their own clothing, or have the clothing made at home with the seasonal aid of a visiting dressmaker, paid by the day. In estimating the true economy of these plans one must consider the free time and plus energy of the individual. Can the schedule be planned so that the household will run smoothly for the other members of the family during this period? Is the time required, justified by the results and money saved, or could it be more profitably used in other lines?

Many busy women who do not find it profitable to sew rely entirely on the department stores or mail order houses as a means of saving time and nervous energy. If they can be fitted in the regulation sizes all is well but much refitting defeats their purpose, is unsatisfactory as a rule and also expensive. Those who can not be fitted easily at the department stores turn to high class tailors or dressmakers for two or three gowns a year which last several seasons with little repair or alteration. Based on the number of years of wear of the garment the average expenditure of such a plan is not excessive. Some combine all of these methods work- 
ing out their own budget of money and time expenditure to suit their particular needs.

\section{Dressing on the Budget Plan}

Whatever the plan followed it is extremely desirable to work out a dress budget as it alone helps one to see the dress problem clearly and see it as a whole.

A dress budget is best based on a three-year average. The expenditure for one year may exceed the allowance but thru continued use of the articles in successive years the average may be maintained. In this connection the importance of keeping the clothing repaired and pressed for results in long service, can be easily seen. According to the budgets in Part $7,14 \%$ is the average amount for clothing. As women's clothes usually cost more than men's assume that $2 / 3$ of this amount may be used by the women and $1 / 3$ by the man.

\section{How to Make a Dress Budget}

After determining the amount of money which can be expended, consider next the social or business demands which must provide for; then, list the occasions for which clothes must be planned-such as church, afternoon and evening affairs, theatre, office, street, afternoons at home, morning work dresses, etc. That dress is the most economical which is appropriate to the greatest number of occasions. For instance a dressy waist with a tailored suit forms an acceptable costume for church, luncheons, theatre, informal afternoons; a light summer silk or lingerie gown can continue in use thruout the year as an evening party frock. Having listed the garments needed your present wardrobe must be reviewed, and the garments on hand checked off. The following dress budget is based on the 
purchase of all articles ready to wear. If the clothing listed here be purchased between seasons, better quality can be had for the same money or a greater number of garments can be supplied.

\section{TYPICAL CLOTHING BUDGET FOR THREE YEARS}

Income, $\$ 1,500$. Family, 2 adults. Clothing (14\%), $\$ 210 ; 2 / 3$ for woman $=\$ 140$ per year.

\section{ITEMS}

Suits, coats, waists, gowns.

Suit for all seasons.......\$25.00

Under jacket for extra

warmth .............. 2.00

Waist, dressy ......... 500

Waist, tailored ........... 3.00

Coat

Reception gown (silk)..........

Winter dress ........... 12.50

Summer waists-

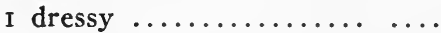

I common ............ 2.50

White wash skirt.............

Summer silk (light) or lingerie gown ........... 20.00

Summer coat ........... I5.00

Summer dresses ......... 5.00

Summer dresses ......... 8.00

House dresses-

I at $\$ 1.50 \ldots \ldots \ldots \ldots \ldots, 3.00$

$I$ at $2.00 \ldots \ldots \ldots \ldots \ldots \ldots$

Aprons-

2 at $\$ 0.50 \ldots \ldots \ldots \ldots \ldots$ I. 1.00

Underclothing, winter underwear -3 at $\$ 1.00 \ldots \ldots \ldots \quad 3.00$

Summer vests-

4 at $\$ 0.25 \ldots \ldots \ldots \ldots \ldots . \ldots . . .1 .00$

Combination suits-

2 crepes at $\$$ I.00........ 2.00

I muslin at $1.50 \ldots \ldots \ldots . \quad 1.50$

Nightgowns-

2 at $\$ 1.00 \ldots \ldots \ldots \ldots \ldots 2.00$

Brassieres-

2 at 50 cents.......... I.OO

Corsets-

$I$ at $\$ 1.50 \ldots \ldots \ldots \ldots \ldots \ldots$

$I$ at $3.00 \ldots \ldots \ldots \ldots \ldots, 4.50$
Probable Yrs.

2D Year 3D Year of Wear

$\begin{array}{rcl}\ldots & \$ 25.00 & 2 \text { to } 3 \\ \ldots & \ldots & 3 \text { to } 4 \\ \ldots \ldots & 5.00 & 2 \\ \ldots \ldots & 3.00 & 2 \\ \$ 25.00 & \ldots \ldots & 2 \text { to } 3 \\ 25.00 & \ldots \ldots & \\ \ldots . & 12.50 & 2 \text { to } 3 \\ 4.00 & \ldots \ldots & 2 \\ \ldots . & 2.50 & 2 \\ 5.00 & \ldots . & 2\end{array}$

$\begin{array}{lll}\ldots \ldots & 20.00 & 2 \text { to } 3 \\ \cdots \ldots & \ddot{3} & 3 \\ \cdots & 8.00 & \\ 6.00 & 5.00 & 2\end{array}$

I.50

2.00 I to 2

.50

$.50 \quad \mathrm{I}$ to 2

I.OO 2 to 3

.50

$.50 \quad 2$

I.0O

I.50

I.OO

2.00 I to 2

I.00 2

$4.50 \quad 4.50$ 
Probable Yrs.

ITEMS

Petticoats-

Silk for suit.......... 3.50

Muslin-

2 at $\$ 1.00 \ldots \ldots \ldots \ldots \ldots \ldots . . . . .2 .00$

I at $2.00 \ldots \ldots \ldots \ldots \ldots \ldots \ldots$

Shoes-

Oxfords ............ 3.00

Street shoes .......... 5.00

Dress shoes .......... 8.00

Room slippers ......... . .75

Rubbers

Slippers

Gloves-

Winter (heavy) ........ 1.00

White kid ............ I.50

2 pr. white washable at $60 \mathrm{c} \quad \mathbf{1} 20$ Hats-

Winter, street (new or re-

modeled) $\ldots \ldots \ldots \ldots \ldots \ldots . .$.
Dress (new or remodeled)

Summer, street .........

Dress

Hosiery-

Winter, 3 at 50 cents...... I.50

Summer, 4 at 35 cents.... I.40

I at $\$ 1.00 \ldots \ldots \ldots \ldots \ldots \ldots$ I.00

TOTAL

$\overline{\$ 158.60}$

$\begin{array}{ll}\ldots . & 3.50 \\ \ldots .00 & 1.00\end{array} 2$ to 3

$\begin{array}{ccc}\ldots . . & 3.00 & 2 \\ 5.00 & 5.00 & 1 \\ 8.00 & 8.00 & 1 \\ \ldots . & \ldots . & 4 \\ \ldots . & .75 & 2 \\ 4.00 & \ldots . . & 2\end{array}$

$\begin{array}{lll}\ldots & 1.00 & 2 \\ \cdots & \cdots & 3 \\ .60 & .60 & 1\end{array}$

5.00

I.00

5.00

1.00

$\begin{array}{ll}1.00 & 5.00 \\ 8.00 & 1.00\end{array}$

$\begin{array}{lll}1.00 & 1.50 & \text { I to } 2 \\ 1.05 & 1.40 & \text { I to } 2 \\ 1.00 & 1.00 & \text { I }\end{array}$

Total of three years..............\$408.00

$3 \times \$ 140.00=\$ 420.00$, leaving $\$ 12.00$ or $\$ 4.00$ a year

for handkerchiefs, repairs, cleaning and sundries.

Note. Prices in this budget have no special significance-the idea is to show the plan.

\section{Buying Ready-to-Wear Clothing}

The value of many ready made garments can be readily enhanced by adding a bit of hand embroidery, fine lace or a different collar. The woman who must economize on her clothing and yet wishes to dress well, must buy either garments or materials between seasons. A great deal of time can be saved in shopping if the housewife can identify herself with one clerk in the department, as the clerk knows 
the stock and soon will learn to fit it to the customer's taste and pocket book. Time of usual legitimate seasonal reduction in clothing are as follows:

After Thanksgiving - fall articles on sale.

First of the year-winter articles on sale.

First of February-between seasons-period of greatest reduction. Decoration Day-spring articles on sale.

Fourth of July-summer articles on sale.

First of September-between seasons-period of greatest reduction.

\section{Home-Made Clothing}

Where the clothing budget of the mother must be divided with little children, or where she desires to have many garments at less cost saving must be effected by making some articles at home. In general it may be said that outside clothing as suits and coats should be purchased, for their style depends entirely upon lines and tailoring. The more skilled the housewife is in designing and sewing fine clothing, the greater is her saving, as shown by the following table based on the clothing budget given.

ITEMS-

Wash waists (which have hand work) .....

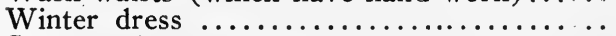

Summer dresses

Light summer silk or lingerie dress.........

Hats

House dresses

Aprons

Machine-made waists

Underclothes
Saving where nothing is paid for time

$$
50 \%
$$

$20 \%$ to $30 \%$

$5 \%$ to $15 \%$

Standard garments, such as housedresses, aprons, and underclothing which have no particular style, can be cut out by the hundred, and made by unskilled workers. As the style is not pronounced, a merchant can carry them over several seasons (unless soiled) so there is less loss in handling. All factors combine to make them cheaper, so that the busy housewife can little afford to spend her time on such items. 


\section{The Sewing Corner or Room}

Ready or home made as the garments may be, all need attention at some time in repairing and alteration, so every home needs a sewing corner, just as it needs a "business

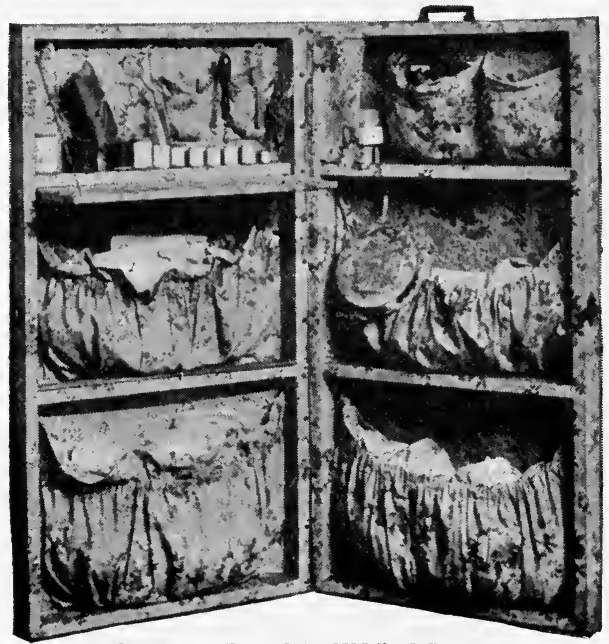

HOME-MADE SEWING SCREENS

A $2 \mathrm{~T} / 2$-inch Wood Frame Covered with Cretonne.

corner." If repairs and simple alterations alone are planned for, the simple equipment of a home-made sewing screen such as illustrated may be sufficient. The hooks and spindles provide places for all tools as scissors, hem gauge, darning ball, pin cushion, beeswax, and thread of different shades and numbers. The many pockets are arranged to hold findings, patterns, remnants, current work, and in one corner of the top shelf, pill-boxes of sliding variety with collar button punched through the front, make useful little drawers for buttons, snappers, hooks and eyes. Where home sewing is a regular part of the seasonal schedule, the following equipment should be added: 
I. Machine.

2. Cutting board or table.

3. Chair (right height).

4. Lap board.

5. Ample scrap basket.

6. Dress form.

7. Yard stick.

8. Shelves for fashion magazines.

9. Electric iron.

Io. Pressing board and cloth.

II. Dress file.

I2. Good light.

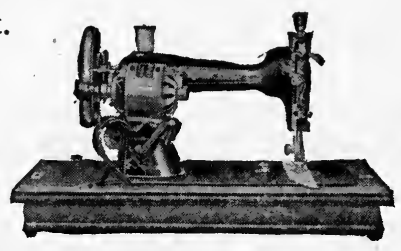

PORTABLE SEWING MACHINE.

Electric Motor $\Lambda$ ttached; Made by Western Electric Co. Price, \$45.00.

\section{Dressmaking Records}

The following form for dress file will be suggestive and prove particularly valuable where sewing is done for many members of the family:

I. Samples of each standard fabric on a card with information of width, cost, guarantee, manufacturer, and dealer's name.

2. Classified list and location of findings, kept on hand.

3. For each member of the family:

a. Dress budget plan.

b. Measurements with date of taking.

c. Sewing cost of each article.

I. Cost of materials.

2. Time required. 


\section{Dressmaking in the Schedule}

Sewing is a seasonal occupation in many households and the complaint arises that sewing and housework do not fit together, and that the many interruptions make progress in the work difficult. True the regular schedule would have to be reduced to a minimum of simple meals, bed-making and simplest putting of the house in order, and the time of different sewing operations will have to be planned according to frequency and nature of interruptions, such as:

Evening for cutting-

I. Least interrupted time, and

2. Work does not strain the eyes.

Morning for machine work-

Fewer unplanned for interruptions.

Afternoon for handwork-

Can be continued during unexpected calls.

\section{Standard Practice in Dressmaking}

Making a number of like garments at one time such as underwear, dresses, waists, and having a standard practice of procedure simplifies the work and reduces the total time.

A. Preparatory.

I. Test samples.

2. Select pattern.

I. Open each piece.

2. Mark with make and number.

3. Measure for required amount of material if allowance on pattern seems large.

3. Buy material and finishing.

4. Put pattern, material and finishing in a labeled box.

5. Note costs in dress file. 
B. Cutting.

I. Prepare equipment.

Board, scissors, weights (better than pins), yard stick, French chalk.

2. Lay goods and pattern.

3. Cut.

4. Baste or mark as necessary.

C. Replace pattern and pieces in respective boxes.

D. Work on all like articles at one time without change of shift as skirts, waists, sleeves, etc.

I. All basting.

2. All fitting on form.

3. All seams.

4. Remove basting.

5. Final fitting.

6. Do all like finishing, as buttonholes, snappers, hems, etc.

7. Pressing.

E. Replace each article in respective box as done and avoid confusion.

The time study for each step will vary with the speed of the individual and the amount of work done, but making a study of dress making as with other work will give not only a valuable gauge for schedule calculations, but will show also whether the time spent in sewing is profitable in proportion to the saving of money.

\section{The Buying of Housefurnishings}

The buying of household tools and equipment has been taken up fully in a previous chapter (see Chap. III, pp. Iooro8; also VI); the other needs which must be purchased for the home, may be divided into Furniture and Room fur- 
nishings; Medical and Sanitary; China, Glass and Silver; Art and Musical Objects.

Space does not permit going into each group in detail. It may be said, however, in regard to furniture:

I. Buy a few pieces of good model, and the best workmanship rather than an assortment of poorly harmonizing ones.

2. Avoid those pieces which have high polish, and an excess of carving, "turned legs," etc., as they show soil and scratches more easily and require more work to keep them clean.

3. Avoid buying "sets" or "suites" or "period" furniture when the home is neither large enough nor has the right setting for such furniture.

4. Buy separate mirrors and drawers rather than typical "bureaus," because they can be more easily placed in different positions and rooms; have as much of the furniture "built in," rather than of the portable type.

5. Choose such models which permit easy cleaning under them, and which have such pillows, seats, etc., which are easy of care and inexpensive in restoration.

6. In choosing beds a "box-spring" covered, is the most permanent and sanitary, and less likely to catch on bedding.

7. The cost of an iron or enameled bed depends on the width and weight of the iron supports, and on the arrangement of the spring. Those beds with woven wire spring attached permanently to the framework, are more satisfactory than using a separate wire spring. See that the spring is reinforced by strands of cross wire so that it does not sag.

8. Mattresses of laminated cotton are more sanitary, and distribute weight more evenly than those of horsehair. Ask to see a cross section of the filling before purchase. 


\section{Medical and Sanitary}

In every home there is need of certain sanitary articles and comforts bought from time to time. The point is that very few of these articles are planned in purchase, but are bought suddenly when emergency demands, with a result of either extravagant or inefficient buying, whose total is quite a sum out of the annual expenditure. A comfort like a hotwater bottle is best developed in an electrically heated pad if there is current in the home, or at least by an aluminum bottle which cannot leak, or burst like the rubber bag. Grey agate ware sanitary utensils give better service and cost less than the same articles made of white enamel. Medicines should be kept away from light; the tri-sided poison bottle should be used to avoid danger. "Quantity" buying of gauze, absorbent cotton, etc., by the half or pound is much less expensive than buying small Io and 15 cents' worth; large sizes of witch hazel, alcohol, bought by the pint, etc., toilet rolls bought by the dozen, and soap by the box where it can be unwrapped and laid to harden. In families with children the medicines or "emergency shelf" should be kept well stocked, where it is instantly accessible. All rubber tubing, etc., must not be used or left with oil and must be hung away to avoid bending and breakage. Care in keeping contents protected from air and dust, well corked, etc., will save as much as original careful estimation of purchase.

\section{China, Glass and Silver}

In buying chinaware it is wiser to buy from "open stock." This means a stock or pattern which the manufacturer and retailer have constantly on hand, as opposed to a "set" which is a pattern of which only a few "sets" are manufactured, and then discontinued. "Open stock" permits buying oniy 
a few dishes at a time, and allows easy replacement for breakage. Indeed it is always better to avoid the lure of all "sets of 108 pieces complete," etc., because many of the pieces of such standard sets are used so seldom as to be poor investments; $i$. $e$., the large turkey platter, the soup tureen, etc., can best be replaced by a more serviceable chop-plate or a less expensive casserole which can be used for other foods than soup alone. Staring or large and brightly colored patterns are tiring to the eye, do not set off the food attractively, and do not harmonize with the other table appointments. Avoid large handles and ornate knobs, which break easily and excess of gold-leaf, which comes off in the washing after hard usage. Porcelain is the finest quality of ware, almost transparent when held to the light, and the most fragile. China is the medium weight grade of which most sets are made. Pottery is the coarsest ware, of which, however, beautiful pitchers, bowls, etc., are made.

It is also not necessary to buy a "complete dozen" of each kind of plate, saucer, etc. For a small family, eight of each kind of flat dish seems to be adequate. A plate which will be suitable for both breakfast and supper, and salad can be chosen, and so avoid the endless number of sizes of plates with which the average "set" is burdened. One size sauce dish, or a shape that will do both for soup and cereal, again saves a multiplicity of dishes. The same points are true in regard to silver-that simple pattern, good lines, and few pieces, give the table a better appearance than does an excess of elaborate encrusted ware, which also means more cleaning labor for the housekeeper.

\section{Art and Musical Objects}

So many are the articles which might come under this head, that merely the general suggestions may be given, first, not to have too many of such extraneous objects as pictures, 
hangings, ornaments, since most American homes (and women buyers) delight in an excess of superfluous objects in the rooms-objects which mar the general harmony, which are often of doubtful quality and ephemeral value, and which also lessen the amount of air in the room, not to mention the great labor they entail in cleaning. Longtrailing lace curtains, portieres, fringe, doilies, bric-a-brac are generally not wise investments. "Ten-cent store" buying is a cause of unwise spending, and overcrowded, inartistic homes-simplicity and permanent quality should be two watchwords in all buying of housefurnishings.

\section{QUESTIONS ON HOUSEHOLD ENGINEERING}

\section{VIII}

\section{Efficient Household Purchasing}

I. Investigate (by telephone or better by visit) the price of flour, sugar, eggs, butter, potatoes, bacon and flank steak in all your local stores and sources of supply, on the same day, and report.

(b) How do the sanitary conditions in the different stores compare?

(c) What are your local laws, if any, on sanitary standards? On weights and measures?

2. Tell of any experience you have had in purchasing food by (a) paicel post or express, (b) co-operative buying or wholesale, (c) at public markets.

3. Make out a table of at least ten items, giving the cost per I,O0O calories of food "as purchased," using your current prices.

4. Give a recipe and calculate (a) the total number of calories it contains, (b) the total cost and (c) the cost per I,Ooo calories.

5. The dress problem-what seems the best solution under your conditions? Have you ever used a "dress - bu 'get?" 


\section{HOUSEHOLD ENGINEERING \\ IX}




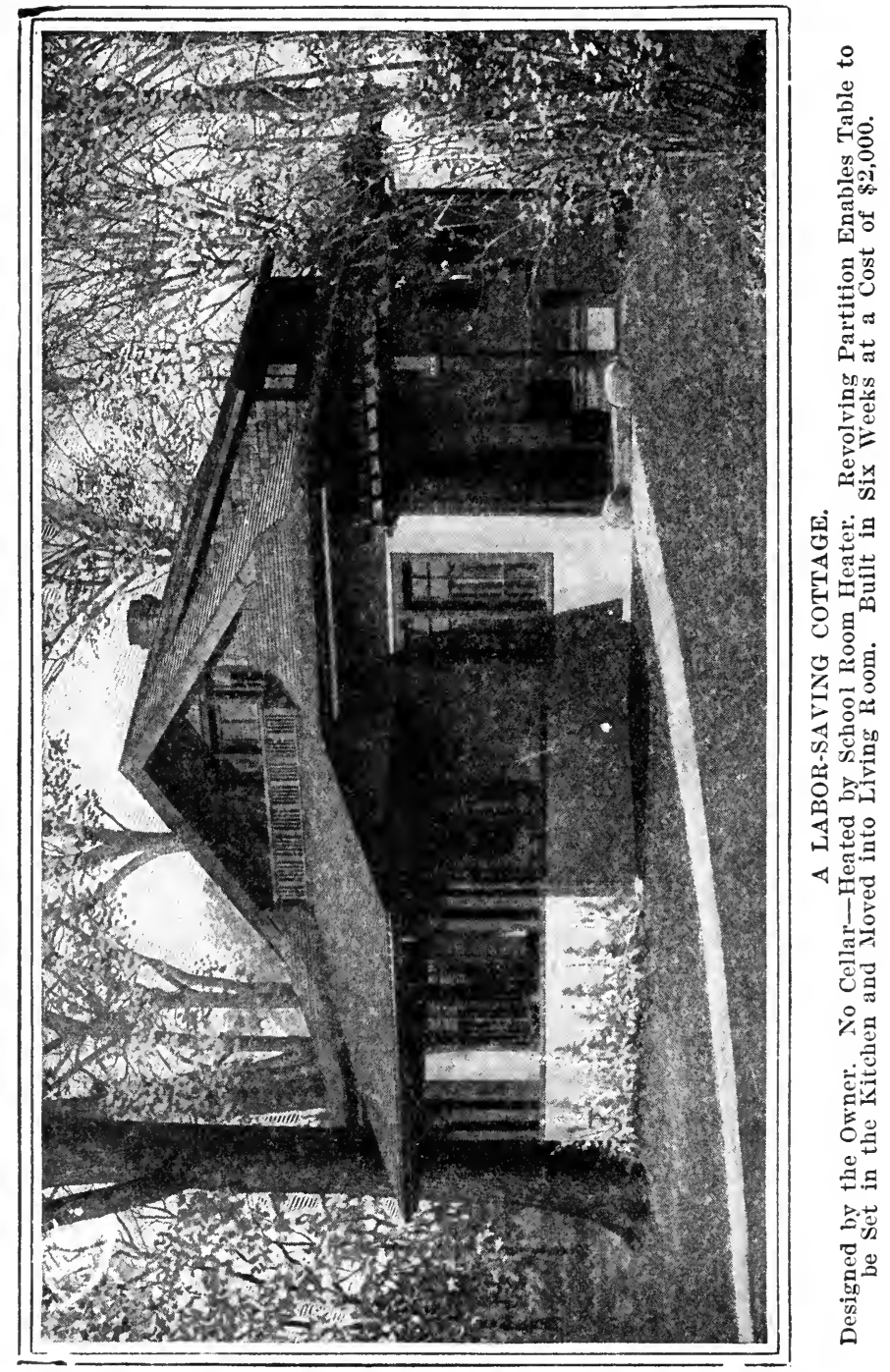




\section{HOUSEHOLD ENGINEERING}

IX

\section{THE SERVANTLESS HOUSEHOLD}

7 HERE are approximately 22 million families in the United States; of this number, $92 \%$, according to I9Io estimates, employ no permanent servants. Figures compiled now would undoubtedly show an even higher percentage; war conditions abroad have almost entirely cut off the stream of immigrant labor from which servants were formerly recruited; war conditions in this country have so disorganized industry that women are replacing men in shops and factories which offer such high wages as to tempt many professional servants away from all branches of housework into these wartime occupations.

Employment agencies in Eastern states have requests for four times as many applicants as they can find even at wages a third to a half higher than at a period "before the war." The demand is so much greater than the supply that even responsible workers are shifting and unreliable. It is even more difficult-almost impossible, to secure household help on farms and in isolated suburban sections, as workers prefer town amusements and city living conditions.

It is probable that the situation will not improve, but that there will be a still greater shortage of professional resident servants during the succeeding years. 
The average homemaker is therefore faced with the necessity of doing all her own housework, or depending on such outside assistance and agencies as will enable her to manage her home without permanent or "resident" service. But this should not be regarded by her as a situation to be deplored, but as an opportunity to manage her home according to her own highest standards of thrift, efficiency, sanitation, and family happiness.

Disadvantages of a Permanent Servant in the Home

There is a very strong case against the presence of the permanent worker in the home. First, there is the responsibility and psychological adjustment which she forces on the homemaker, and on the entire family. Even if she is a trained worker (and how seldom she is!) the employer, in addition to the strain of seeing that the worker carries out her directions and plans, feels responsibility as to whether the worker is "satisfied," if she has sufficient private life after hours, if her goings out and her comings in are as they should be, if things in the house are adjusted to please her. In other words, there is usually considerable tension between the worker on one side and the family on the other.

In many cases, the standard of the home is consciously or unconsciously made less simple or adapted to the expectations and demands of the worker. This is particularly true in the case of meals; it is a common occurrence to find the cook, hired man or houseworker dissatisfied with the simpler kind of food with which the family itself would be quite content. Again, hundreds of intelligent, progressive housekeepers have ideas of continuous efficiency, thrift, and management which it is impossible for them to get their servants to follow. The result is that the home is 
not managed so much according to the standards the home- . maker would like to have set, as according to the inefficiency, waste and lower standards of a constantly shifting and generally low-grade worker.

Further, what is the actual cost of a permanent worker? She receives a cash wage; in addition she represents (which many homemakers fail to include) a cash expenditure for food, room, light, furnishings, heat, breakage, wear and tear. She also creates more work in the family, merely by living in it-i. e., her dishes, the washing of her clothes, etc., all of which must be estimated in her exact cost. It is a fair average to figure that a servant costs double her wages; a worker costing $\$ 25$ in wages costs actually $\$ 50$ in cash, and that each additional worker costs $20 \%$ more. A professor of nutrition writes that each servant represents a cost of at least $\$ 4$ weekly for food alone.

Added to this, her general lack of thrift brings her total cost startlingly high $-\$ 600$ to $\$ 1,000$ or more a year. In other words, whether she likes to admit it or not, the homemaker is paying a high price (perhaps even more than the price of efficient day or hour help) for transient, inefficient permanent help.

\section{Estimated Cost of Each Resident Servant}

Wages (general housekeeper, to housekeeper) .... \$25-\$45 a month Board (men servants, about one-third more)...... I6- 25 Room (weekly, \$I-\$3).................... 4- I2 “.. “ Light and fuel................................ $3^{-} 5$ "

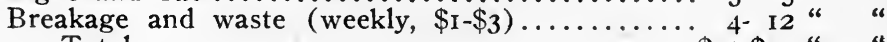
Total $\$ 52-\$ 99$

\section{EQUiPMENT}

Bed, mattress, blanket, etc................ \$25

Bureau or chiffonier .......................... Io

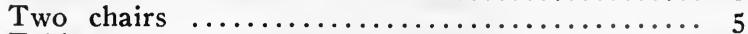

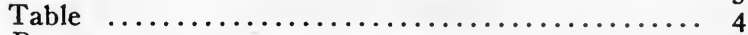

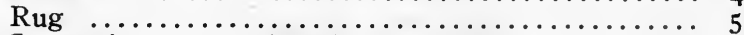

Servant's room and bath.................. $\$ 2,000$ 


\section{Advantages of the Servantless Household}

The servantless household (by "servantless" is meant without resident workers) offers the only real opportunity. for a family to follow the exact standards it wishes and to carry out its ideals regardless of adjustment to any other persons. Again, it offers the homemaker the one chance of putting into practice her progressive sanitary knowledge, the use of improved machinery, and efficient home management. Further, it enables her to practice thrift and economy, and to know that every dollar she spends for service goes for actual work done, and not for waste or additional "overhead." Last, and most important of all, the servantless household enables a family coöperation and a chance for the training of children that makes for the highest value, and which can never possibly be secured under the constant presence of hired workers

Necessity for Right Point of View for the Homemaker

It may be well for the woman who is the executive head of the servantless household to turn back to the "Introduction" (page 9) and re-read the twelve principles of efficiency. There she will see "Ideals" given as the first principle. To make a success of managing a home by herself it is necessary, above all, to have the right point of view about her work. She must want to run a home, and see that its various tasks offer scope for her best intelligence, effort and study.

The reason, perhaps, why many women "doing their own work" regard housekeeping as distasteful, is that they merely "married into housekeeping," and are constantly regretting that they can't afford a servant, or thinking of teaching or office work which they would prefer to do, or 
envying women who do nothing except try to amuse themselves. No one ever made a success of any enterprise which he was constantly disparaging in his own mind. It is most important that the homemaker of the servantless home see all its advantages for family coöperation and child training, and the chance it is for her to show to the community in her homemaking, her executive ability, her expression of what a home in the highest sense should be. Here is her best chance to be "A productive citizen of the State, not a social debtor."-Ellen H. Richards.

One of the first elements of this right point of view will be to get rid of the idea that her particular family or house must be run according to arbitrary standards, set up by friends or the community. Another is to refuse to attempt to run a home without service in the same way or on the same scale as a house which has permanent help.

If in a particular family it seems best only to wash dishes once a day, or if it should make for family happiness to do the laundry work on Thursday, instead of Monday; or if certain kinds of meals are preferred other than the conventional ones, let the homemaker follow these or whatever methods conduce to the efficient management of her particular home, regardless of tradition, or what is supposed to be the "proper" way. Too many women doing their own housework are slaves of routine and tradition, and put the work itself first, and the true comfort and development of the family, and justice to their own health and interests, second.

One woman with the right viewpoint has her week so planned that she neither cooks nor cleans on Saturday, but devotes it to her three small boys who are home from school, taking them to museums, the zoo, and other places of educational interest, since this is the only day they can go. How much better than the plan which "must" put 
baking day on Saturday! (and leaves the children to run loose around the neighborhood).

Another wise woman arranges her Sunday with buffet or tray meals pre-cooked the day before, so that she, also, has a "day of rest" and can spend more time with husband and children than if she follows the usual hot mid-day dinner which means incarceration in the kitchen the whole forenoon. The woman managing the servantless household has the widest field for ingenuity, for originality, for running a home with the greatest freedom and service to her family.

\section{Enlisting the Co-operation of Children}

The servantless household offers a wide opportunity for training and educating children. Unfortunate indeed are those children in homes of wealth where the cook will not allow them in the kitchen, or where the children are so waited upon that their initiative and responsibility are not developed. As a very prominent and successful man said to the author recently, "The greatest loss to society is the disappearance of the family woodpile." $\mathrm{He}$ meant by this that the child who has chores to do and who shares in the many small tasks of the home will receive training in the most important point of co-ordinating motor and mental tasks, as in making beds, learning to cook, doing small cleaning tasks, etc. Indeed, the modern educator with his Montessori and similar systems is only trying to give back to the child in school what he formerly (and still could) learn in the various small manual tasks of the home. But, further, if there is one thing more than another that will teach children the ethics, the spiritual side and beauty of home life, it is to enlist their co-operation with the mother in doing home tasks, and making them feel "this is my home, too."

Each child in the servantless home should have its defi- 
nite daily and weekly tasks. Older boys may care for furnace or stove, clean rugs, and similar work; girls can cook and clean, and even the smallest empty a wastepaper basket or " pick up." Children of eight or over can be held accountable for making their own bed, picking up their night clothes and leaving a room "tidy."

If housework is presented to them with the right point of view, children regard it as a privilege, rather than a duty, and are eager to "help." No mistake is more fatal than to keep children from doing work because the mother can "do it so much better myself." How will she ever expect skill if she does not permit a period of inexperience and practice? How many girls there are who have said that they did not know anything about housekeeping, because when they were small their mothers prevented them?

It is the author's feeling that there should be no sex discrimination in such tasks-that boys should cook simple dishes, and at least darn and sew buttons, and that girls should share in the mechanical jobs with hammer and saw.

Older children taking manual training in school can assist by making simple labor-savers like shoeblacking or sewing stand, coal box on castors, etc., the making of which will round out their school instruction and be an incentive because they will see their handiwork actually used by the family.

But whatever the task or work, it should be definite, provided for in the schedule with its due reward or reprimand. It should not be a constant nagging to do all kinds of running and errands so that the child comes to hate his share instead of like it. A child should not be made a "body servant"-he should have his rights as well as his duties. Here as in all child training "An ounce of example is worth more than a pound of precept."

From present indications it would seem as if the struggle 
for living is going to be even much harder for our children than it is for ourselves today. Economists and moralists point out to us that many of the serious modern problems of extravagance, divorce, etc., arise largely because young people, girls especially, have not been trained in home management, or in ideals of thrift and home life. No "domestic science training," however good, taken in later years, can replace this early dexterity in manual tasks, or the instilling ideals as to the point of view of the worthwhileness of normal, inter-co-operative home life of parents and children.

\section{What Is the Father's Share?}

In some households where there is no permanent worker, it often happens that the homemaker looks to the husband as a kind of nursemaid, choreman or kitchen assistant. The author's feeling is very much against this view, - that the moment a man comes into the house he should be asked to carry out the slops, hold the baby or wash the dishes. If the father works hard and faithfully at his task of earning money during his work day, it is not more fair to ask him to turn choreman as soon as he comes home, than it would be to ask the woman who has cooked and cleaned all day to turn around and do office or business work after five o'clock. It is not fair to put on a father any housework duties; his hours at home should be hours of recuperation, or so that he can study his own work, become more proficient, and thus secure advancement or a better economic position.

There comes to mind the case of a gifted man starting a profession, who, because of his wife's poor management, spent his time after office hours caring for the children and doing chores. He never seemed to "get on " as far as people had expected. Would it not have been better to use his 
spare time studying and improving in his own profession and thus be eventually able to pay for more service to help his wife, than to neglect his own opportunities by doing the housework?

There are other ways in which father can be of more true assistance,-one is in being satisfied with simple standards, and especially simple meals and table service. It has been found too often that unnecessary work for the housekeeper has been made because the tastes of the men of the family were capricious. One husband, for instance, always refused to eat "made dishes" of any kind; another did not think he had a good meal unless pie of some sort was included; other brothers wouldn't eat anything but roasts, demanding constant watching at the stoves, etc., etc. Sunday is the hardest day in the week for many women largely because their families demand (or are in the habit of having) an extra fancy hot noon dinner, when such a dinner could easily be taken Saturday night, and only simple buffet meals taken Sunday which would allow the housekeeper, like the others, to enjoy one day of rest.

Many of such habits on the part of the family make the housekeeping harder, but can easily be changed by a little helpful co-operative spirit and willingness to see a new viewpoint. In one home where Sunday breakfast usually was dragged on from seven until nearly ten by different members on whom the mother waited, a newer plan was followed by setting four separate trays on the dining table complete for each one with fruit, prepared cereal, and glass of milk. As each came down, he ate his trayful, then carried it out and washed and laid away those few things himself, thus doing away with any long-standing and untidy table and waiting. The ideal should be not to do anything or leave anything untidy that will make some other member of the family do needless personal service. 
Again, he may by suggestions from his business, assist her in her finances and budget making. If he is a "handy" man he may occasionally turn his tools to make some shelf, repair, or device to save her labor. Some men enjoy cooking and doing other household tasks once in a while, and any man may be expected to help out in case of illness or special emergency, but father's share is not in being a choreboy-housekeeping the woman's job and she has no right to burden the man with it.

\section{Practical Management of the Servantless Home}

The first step in managing the servantless household is to sit down and study carefully the conditions you must meet in your particular family and home. Get them down in writing! What are the "must-be's" of the problem-the maximum money you can spend for outside service, the unalterable construction of the house you live in, the inexorable needs of a baby or small children? At what hour, and what kind of meals must be served, when can you best do the marketing, when will be the best time for a "rest period" for yourself, or for outside or social interests? How can you save steps, time, effort and fuel and run the home at the lowest expenditure and yet carry out your ideals?

This takes us back-particularly to what is given on "Schedules." All that has been said in previous lessons will bear re-reading. For instance, it is of the first importance that the woman doing her own work have her kitchen arranged in the most step-saving manner, with heights of table, sink, etc., to suit her own comfort, and utensils placed and grouped where they will entail fewest steps in assembling or laying away. A high stool for work, adequate light, ventilation and both floor and work surfaces that are easy to keep clean will make the kitchen work done in shorter 
time with greater pleasure. Extra touches of decoration in plants or bowl of flowers, in curtains with stencil on them, in the use of ornamental as well as useful casseroles, will make the kitchen as charming a place to stay in as any other room in the house.

In addition, the woman doing her own work should have some sort of "comfort corner" (or "business corner") near the kitchen, preferably a screened portion of a porch or hall, where she can have an easy chair, a shelf for books, etc., where she can sit down while in work clothes and take a few minutes' relaxation. One such corner was developed near a window seat and a wash basin in the rear hall. Here was kept a shelf with mirror, cold cream for hands, and means of "freshening up" before going to the door. Clean aprons, a few magazines, a purse of money, the telephone and also the household account books and bill file made this just the corner to sit down and take a few minutes' rest while at the same time entering some bill or other item.

What is said on planning and dispatching (Part II) on marketing and planning meals in advance (Part V) will be especially helpful. For after making the thorough "survey" (as one might call it) of the special conditions in any particular home, as suggested above, the next step is to work out such schedules as will best suit those conditions, both weekly and daily, experimenting with them until they approach a "standard" that seems to do the greatest justice to the work and to the family's comfort. Buying in quantity, marketing seldom, cooking in concentrated periods for several meals, are all ways to short-cut waste time and motion, and the chapters dealing especially with these points should be studied over again and applied.

It will be distinctly "up to" the housekeeper herself whether she saves steps or the reverse. Too much energy supposed to be spent in actual housework is quite commonly 
dissipated in running up and down stairs (how many times a day?), hunting for the needed receipt, walking about the back yard, or just pure sitting around or neighborhood "visiting." You must work to have leisure!

To save running up and down, have enough money downstairs to pay all small amounts that may be presented, lay articles to be carried up on the stairs to be taken up by the first goer, and not run up especially. Don't make a special trip to carry out the garbage, unless you can also do some other outside chore, etc. Read most carefully the pages on grouping of tools, foods, and sitting down to work (page 29 et seq.). If you have a 'phone, kindly ask friends or local calls to be made only within definite most convenient periods or have an extension 'phone upstairs. In many houses a most startling amount of time is wasted in useless 'phoning. Above all, work on schedule, as it will save you more energy and time than you will believe.

Remember, too, that you should have definite rest periods as well as work periods (see Part II). Emphasize the importance of "beating your own record" and making time-studies of each particular task. The housekeeper in the servantless home has widest opportunity to make original kitchen and housework time-studies. Where does the time go? How long does it take you to make the beds? How long to clean the downstairs rooms? Do you wash dishes three times a day, or only once? Why? There is a peculiar prejudice among some women that dishes must be washed after every meal. Now, with a family of not more than two to four, it is far more efficient to wash the dishes of two meals together. The time covered by the whole work of dishwashing is very largely consumed, not in actual washing, but in the "clearing up," the scouring the sink, etc. If dishes are well scraped, and stacked, in the dish pan or even in the sink itself, fitted with a broad 
stopper, and covered with warm or tepid water, the dishes of two meals (except the solid silver) can be done in onethird less time than if two batches are made of it. When a dishwasher is used, it is even practical to wash the dishes of all three meals at once, having stacked and soaked them in the washer previously.

Many of the schedules sent in by students of this course have been particularly interesting and excellent, but no two are alike. You are the only one who can draw up a successful working schedule for yourself, under your special conditions, and you cannot plan your best schedule in a week or a month or one year even. Also, you will have to constantly modify and adjust your schedules to changing conditions, if need be.

The general tendency is to make the schedule too elaborate- to raise standards of cleanliness, complexity of meals, etc. This is often a wrong standpoint, especially for the servantless household. It is quite possible to keep the house too clean, when there is only one pair of hands to do the work, and to neglect other more important interests. Housekeeping should never be an end in itself. Work for a minimum schedule; try to simplify, not to complicate. For example, if each member of the family leaves the bathroom as he found it, daily cleaning should not be necessary; in some localities and in some families, weekly cleaning of the living room will keep it in good condition.

While some of the special cleaning needs to be done every week, much other work needs to be taken care of only once a month, such as window cleaning, high dusting, beating of rugs. These things should be provided for in the schedule, but divided so that no one week is overcrowded. Mothers with little children find it difficult to plan a working schedule. Here a margin of time over that required in the "time studies" must be allowed. One 
mother with children of two and five finds that a margin of ten minutes on the hour is sufficient to meet their many small demands.

One housekeeper writes: "I have found my schedule a great help until the spring sewing, gardening and yard work all came at once to upset it. Then everything had to be brushed aside and I hurried from morning till night in the old way." Here is just where a schedule should prove most useful. The minimum schedule, even, should be simplified, especially the meal preparation, so that free time is cleared each day. This free time may then be given to the planned seasonable tasks.

The following suggestions may be helpful in planning seasonable work which, of course, will vary somewhat in each family:

January-Household linens, supplies or furnishings.

February-Undergarments.

March-Spring clothing.

April-Spring clothing and gardening.

May-Garden and housecleaning.

June-Garden and canning.

July-Garden and canning.

August-Canning and vacation.

September-Fall clothing.

October-Fall clothing.

November-Housecleaning.

December-Holiday preparations.

A busy mother finds that her sewing progressed easily if she planned and cut at night, did all possible fitting and machine work in the morning hours and saved the handwork for the afternoon. By this plan she was able to give about six hours a day to sewing for a few days.

A number have written that they find it easier to plan menus and to market twice a week rather than only once. One, for example, markets Friday for Friday dinner, thru Sunday to Monday night and on Tuesday thru to Friday. 


\section{EXPenditure for LAbor-SAving EQUipment}

Labor-saving equipment is more justifiable and profitable in the servantless household than in any other. It is right that the woman without permanent service should invest in every device she can afford which will really save her own manual effort and time. Also, the woman doing her own work is so much more intelligent than the average hired worker, that she can get far better results with equipment requiring skill and understanding. Indeed, as a general rule, labor-saving equipment is almost useless in the hands of the servant of whom mistresses constantly complain, "She will not use a bread-mixer," or a "fireless," or some other device.

The author confesses, regretfully, that in her own home an excellent ironing machine, gas iron, fireless cooker, dishwasher and washing machine stand unused by any save herself-more than one worker (and that, too, of education, and more than 15 years' experience in managing homes of their own) refusing to be "bothered" with "new-fangled" ideas, even preferring a hand washboard and knuckle rubbing hours to an excellent rocking type washer, and asking if she could lay aside the most efficient dish drainer and substitute an old tin tray on which to lay the dishes!

Too many housekeepers have somewhat this same attitude-a feeling that " mother's way (or grandmother's way) is good enough for me." What success or progress would a storekeeper or professional man or farmer make who has this attitude? It does take some time and patience and study to learn to use labor-saving household equipment effectively and it is easier to continue in the "old ways," without progress; but is this not an indication of mental stagnation and a sign of old age?

Other housekeepers spend much money for new equip- 
ment in the vague hope that it will make the housework go easier and are disappointed to find that it does not help much. Perhaps the appliance is not suitable, perhaps not enough time and patience is given to mastering it and often the appliance is not cared for properly; it gets "out of kilter," so it is discarded and the money spent for it is wasted. Time studied will show clearly what appliances and changes are most needed. (Read Part III "Helpful Household Tools" again.)

The purchase of such equipment may be looked upon as so much money expended for service. For instance, if a washing machine enables two days' work to be done in one, then its cost may be balanced up or credited against the wages of a day laundress. The cost of a dish-washer, breadmixer or other useful device can all be bought with whatever appropriation the homemaker has for "temporary" or day service. The exact labor-saving tools which should be bought will depend on the particular home, fuel, and conditions. But it may be said generally, that the servantless household will get the most benefit from such equipment as the fireless or pressure cooker, or stoves having ovens based on the fireless or insulated principle; from the dishwasher, from a power washing machine, from the ironing machine, from paper products, from some form of portable tray on wheels for serving and clearing meals, and from a utility motor with various attachments.

A resident servant costs $\$ 600$ a year or more; if with labor-saving equipment and part time expert service, onehalf this expense is saved, that will amout to $\$ 300$. This represents an investment of $\$ 2,000$ with interest and depreciation at $\mathrm{I} 5$ per cent. This sum is very much greater than need be spent for all the equipment that could be used, so there is no question of the economy in replacing the human by the mechanical servant. 
"But," you say, "I have no money to spend on equipment." Little money is needed to make a good beginning. The U. S. Department of Agriculture has an excellent Bulletin which will be sent on request- " Home-Made Fireless Cookers and Their Use"; also descriptions of home-made wheel tray, iceless refrigerator, etc. More often it is lack of enterprise rather than lack of money which stands in the way of a more conveniently arranged kitchen and laborsaving equipment.

Electric Devices Greatest Service in the Servantless Hoмe

The device or cooking equipment operated by electricity is also most worthwhile where the intelligent homemaker herself uses it. It is generally unsafe to trust the common grade of household worker with the costly and delicate apparatus of electric cooking, or expect her to understand and use it economically. On the other hand, in the servantless household much labor may be saved by using extensively the percolator, table stove and table warming units in meal preparation; and by depending entirely on electric cooking (where the current cost justifies it), as it will mean a clean, cooler kitchen with no waste heat or combustion products as ashes, soot or smoke, to make further cleaning labor for the homemaker.

The expense of a general utility motor with its many attachments such as coffee grinder, parer, silver buffer, mixer, etc., would be excellently justified, and act as a "silent servant" for innumerable uses. There is now on the market a fairly low price motor which may be attached to bread mixer, chopper, etc., thus replacing much hand labor. Other electrically operated equipment, such as vacuum cleaner, washer and dishwasher, will replace a large share of the work usually done by a permanent servant. Indeed, 
it may be said, that "the one way out" of the servant problem in the future is the much wider use of power and machinery in the home. The servantless household will have to become more of a mechanical household, where every possible purely manual task is done by arms of steel or knuckles of copper.

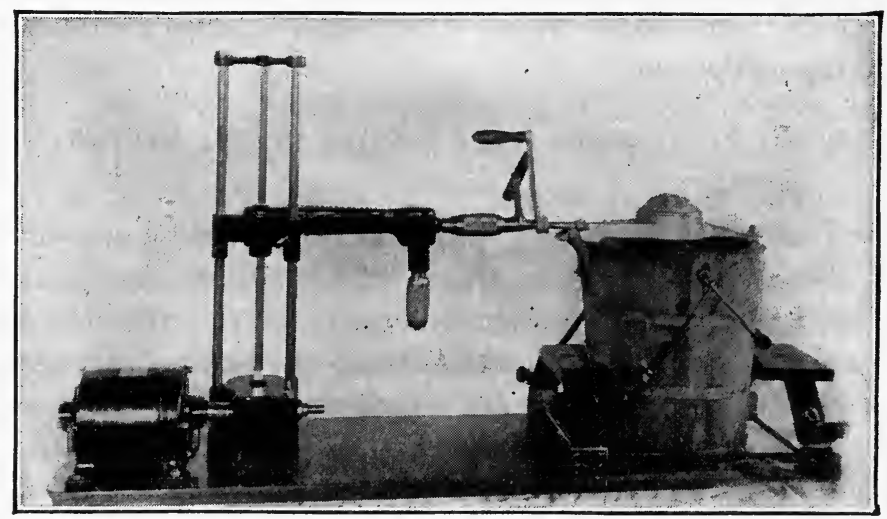

A EITCHEN UTILITY MOTOR

Will Operate all Ordinary Appliances on Hand. Made by Reynolds Electric Co.

And in the future it is believed that such machinery will be far more unified than at present. That is, instead of such small devices made by different firms and bought separately, there should be a larger installation or "system" (scientifically) planned for a specific kitchen, with the various pieces related to one another. No efficient lunchroom or hotel kitchen today, for instance, is fitted out by the manager by buying a kitchen table here, a stove there, sink there, a potato-parer off in another corner; the equipment he buys is (if up-to-date), all related, and made accordingly as it shall be placed scientifically to permit of the best "routing" of work from step to step. It is generally 
also of the same finish, same design and so matched as to avoid any grooves, cracks, etc. Similarly the home kitchen will have to be made efficient in the future, with labor-saving equipment standardized and related by a definite system of work, not placed as at present, according to the whim of the owner, or accordingly as some architect happens to leave space for it.

Standardizing Special Work in the Servantless HOUSEHOLD

After having made out a general workplan, with schedules for day and week as suggested above and in Part II, according to the conditions of your particular family, after having installed such labor-saving devices as seem to be most useful in those particular conditions, the next step is to work out standard practice or the best and shortest way to do certain kinds or divisions of work. The most frequently recurring work is the preparation and clearing away of meals-how can this be made more efficient for the woman doing her own work? Briefly, by the following suggestions and summary:

I. Understand food values so that as nourishing and attractive meals may be obtained from a few courses as from many complicated dishes.

2. Market in quantity and plan meals in advance, thus saving being "out" of articles, and wasted time in too frequent marketing.

3. Cook in concentrated periods for more than one meal at a time. (See page I93, Part V.)

4. Prepare bulk of evening meal in the forenoon.

5. Reduce time spent in "pot-watching" by use of fireless, pressure cooker, or insulated oven stoves. 
6. Adopt a simple table service without service plate and elaborate usages.

7. Make use of table and self-service cooking devices; $i$. e., electric table stove, percolator, grill, etc.

8. Use a portable tray on wheels for easy service and removal of dishes.

9. Use "self-server" table device for easy serving at table.

Io. Make frequent use of paper products to save dishwashing labor; also casseroles, glass dishes, etc.

II. Have many "tray" or buffet meals, especially at lunch, and in summer. (These can be set, individual style, in the kitchen and carried to any room or porch without setting formal table.)

12. Set table at night for breakfast, without putting dishes back into pantry.

I3. Always utilize the time spent in dishwashing, in overseeing some form of "pot-watching" or cooking, thus lessening the time needed for staying in the kitchen for cooking only.

14. Wash dishes but once daily, if possible, or at most only twice.

The serving of meals can be accomplished gracefully and with ease, even without a servant. Some form of portable wheel tray and either warming disks or electric plate warmer or disk stove turned at "low" heat, and platters with covers are needed. The tray should stand at the left of the hostess. On its lower surface, place the warming disks on which the hot roast, vegetables, or other hot dishes may be kept covered and warm until needed. On the top tray place salad, cold dessert, extra silver, water and small accessories. Reserve the middle tray for setting on the soiled plates as they are taken from the table. By having the table all set, accessories ready, the serving tray so planned, 
and the first soup or meat course on the table when the meal is announced, it will not be necessary for anyone to rise from the table to serve during the course of the meal, unless the family is very large; then it is often possible to have the removal of plates done by older children, one taking one course, the other the next. The reason meals without a servant are frequently interrupted with rising and confusion is not that it must be so, but because there has not

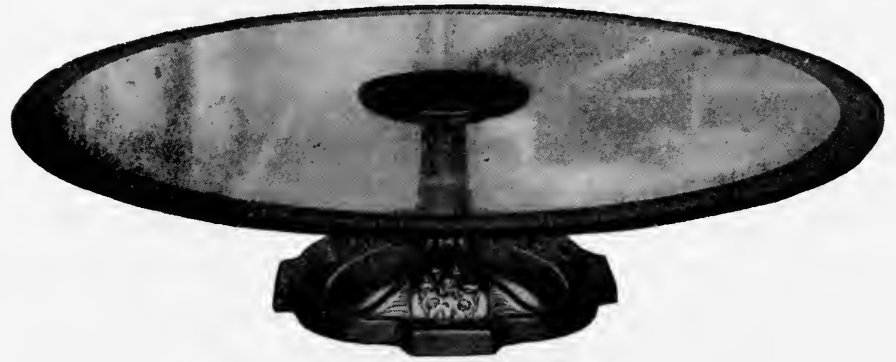

THE "SERVETTE" OR SILENT SERVANT"

been sufficient planning, and "standardization" of the servantless meal in advance.

In many cases, where the dining table has a large enough diameter it is practical to use in the middle of the table a "revolving susan"-or circular glass tray mounted on a revolving stand, which will accommodate butter, relishes, etc.; but its greatest value lies in assisting the host to pass dishes to each person to be served. Set the plate of food on the server, give slight touch, and it will revolve to the person desired, thus doing away with awkward passing from one to another. Similarly the server may be used for removing the soiled plates, by each person laying their soiled plate in turn on the 
server and whirling to the hostess, who will then remove them unobtrusively to the lower tray of the portable tray at her left.

If a crumb brush and tray are placed at hand on the tray, it is an easy and also graceful matter for the hostess to remove crumbs from her own place, and pass to each in turn. After the meal is finished, every article but the linen may be piled on the portable tray and wheeled to the kitchen to sort and wash.

\section{Dispatching Cleaning Work}

The first requisite, perhaps, in making cleaning work as simple as possible is for the homemaker to have a new point of view about the furnishings in her home. Dozens of letters have come to the author bewailing the amount of cleaning necessary and the time taken, and how tired out the homemaker became because of the daily recurring work. Now actual visits (on lecture trips) into many such homes have disclosed the fact that altho the homemaker was doing all or most of her own work, the furnishings in her home were such as to require the daily upkeep of one parlor maid, if not two! It cannot be said too plainly that no efficiency methods will help, if, in the first place, the home is cluttered and crowded with ornaments, portieres, bric-a-brac and excess furnishings. If there is only the one pair of hands to do all of the housework, it is physically impossible for them to be adequate to a heavy cleaning burden, without resulting in overtaxed strength.

Long lace curtains merely catch dust and require more time to "pin" than scrim or net curtains of sill length; platerails loaded with dishes and ornaments make the room hotter and smaller as well as being a joy to the Dust Demon; portieres, pillows with fringe, elaborate doilies and scarf covers, too many small articles lying around on the tables 
and sideboards-all make for confusion and unending work. Too often rooms have a profusion of small novelties and curios scattered on mantel, shelf, or tables, which rightly belong only in a glass-doored closet, where they will be both safe and clean, and not cause such excessive handling. One of the greatest of American failings is to purchase too many "things" which are often neither truly beautiful or useful. The homemaker doing her own work, must first of all incorporate into her efficiency point of view, that the house with few and simple furnishings will not only be, after all, the truly most restful, attractive and artistic home, but from the work point of view, the home that requires less time, less labor and less cost in upkeep and care. Get rid of the junk.

It will be well to re-read Part IV on cleaning and pay special attention to the point of trying to "route" the cleaning work to the best advantage in your particular home. Properly "routed" work will save at least 20 per cent of waste effort and time. Then again comes the need, mentioned before, of having a definite place for cleaning tools, and for keeping them in efficient condition.

It may also be said that here is one of the places where family co-operation may score heavily-much of the socalled cleaning is actually only endless "pick-up" of clothing, toys, and other articles. If each person, and child especially, is responsible for and shares in this work of "straightening" whatever newspapers, table top, playthings, etc., have been used, 50 per cent of the cleaning will be done. One very charming family is recalled, where before leaving the living-room for the night, each member "picks up" the library table in order, straightens the pillows, brushes in the hearth ashes, and sets the chairs straight; it is but the work of a moment for each, but how much better than for all to leave the room upset and unsightly, where it 
might require twenty minutes' time of one worker (if there is one) next morning.

If the income provides for outside service, part of it may be spent with the greatest advantage on forms of day cleaning service. A competent cleaning woman in one day of eight solid hours of work, will go thru a seven-room house, thoroly and completely, except the fine dusting or bureau top arranging, which would take the permanent worker most of two days, and then not be done so well.

An electric vacuum cleaner may be rented for about 50 cents per hour, to be operated by the homemaker or one of the family; or less frequently a man and his own large apparatus may be employed to give the rugs, draperies, and walls a thoro going over, at from $\$ 3$ to $\$ 5$ a day, depending on the locality. Windows may be washed by professionals at about Io cents each. By thus expending a small amount on expert cleaning service at stated intervals, the daily cleaning in the servantless household should be reduced to a minimum, especially if the family co-operates well in "picking up after itself."

When there are small children in the household, they should have their own playroom, if possible, with the rule not to bring toys into the living-room. If they have their own play-table, shelves and window seats, they will less likely be blamed for upsetness in other rooms.

There should be a good footscraper, place for rubber shoes, and rear entry where muddy boots and outside wraps may be hung without taking into the house. It is often merely habit which makes the family "track" thru the kitchen or living room when there is another entry provided. Keep the other door locked.

Children can be taught to pick up, and to eat tidily, to hang up their clothes, and not keep the whole house in one huge disorder-no training is more valuable. 


\section{The Laundry Work in the Servantless Home}

In most homes where there is no permanent servant, the washing and ironing may be done by the housekeeper herself, or by a day laundress, or part of it sent to commercial laundries. It is, however, possible in any case to cut down the amount of laundry in the following ways:

I. Use crepe materials for much personal underwear, housedresses, for children's dresses and rompers-crepe needs no ironing. Use Turkish towels only; knit goods and cotton flannel "nighties" need not be ironed. Cotton blankets in place of sheets in winter are warmer and save laundry work. Small boys much prefer jerseys and flannel shirts to starched linen.

2. Simplify table service by discarding long allover white tablecloths, and use instead separate doilies, "runners" of crash or toweling, or small colored cloths of Oriental or foreign type.

3. Use paper napkins frequently, especially for children and during the fruit season; use paper cloths for luncheon, and paper towels as hand towels in kitchen and lavatory.

4. Individualize towels, wash cloths, napkins, etc., giving out an "allowance" weekly for each person and child.

5. Avoid ruffled, be-laced articles, especially for children's wear.

6. Standardize and schedule the washing; know how many sheets, cases, bedspreads, towels, napkins, shirts, dresses, etc., there should be each week, and do not overburden any week and upset the whole weekly schedule.

7. Hang up clothing after use on proper stretchers and hangers, thus keeping clean longer by avoiding crushing. 
The need of good equipment in the laundry has been dwelt on in Part VI. The price of a washer should be counted as an investment which will save on the wages of a day laundress, or on the health and strength of the homemaker herself.

It is often advocated that the housekeeper without permanent help should take advantage of the commercial laundry to the exclusion of the day worker to whose wages must be added the cost of equipment, fuel, soap, etc. But from experience it appears, that as yet, even with all the disadvantages of inefficient help (especially in country sections) the results of laundry work done at home are preferable to that done by commercial laundries, both on a basis of wear and tear and of price. The only exception to this may be the low rate of 35 cents to 45 cents per dozen for "flat" work (sheets, cases, towels) made by some good city laundries, in which case it is most wise to take advantage of such price and have the "flat" done outside the home.

In other sections there is a "wet wash" service, meaning that clothes are washed (but not starched or ironed) and brought home wet at once. Again there is the "rough dry" plan which washes and starches and dries the clothes, but leaves them to be ironed at home. The success of these plans depends largely on the particular laundry. The "wet wash" method may not be sanitary, the "rough dry" method and "flat" or mangle work are usually safe, because of the high temperature used in drying. It is worth while to make a personal investigation before using a laundry.

A recent experiment was made in the author's home, sending to an average laundry all of the washing and ironing usually done in one day by only an average laundress. Counting the labor of the worker as $\$ 2$, and fuel, soap and interest on equipment as 50 cents, there was a cost of $\$ 2.50$ as balanced against $\$ 6.35$ which the laundry asked for the 
same bag of wash, each pair of socks counting as 5 cents, and many of the children's rompers, 20 cents and 30 cents each, etc. The cost of collection and delivery, rent, interest, bookkeeping, profit, etc., and lack of volume of household laundry makes the cost too high for ordinary use.

\section{In the Country Home}

"Yes, that's all very well, but I live in the country and have no electricity nor gas, have a small baby and halfgrown children. I can get no help at all-what shall I do?” This letter is typical of many homemakers in isolated sections, and the author herself has been in exactly such a situation. The answer is, first, simplify furnishings, service, meals, clothing, laundry. Perhaps it will be best to use a small table for meals in the kitchen and make the dining room into a first floor nursery and playroom, where the children may be watched, and yet not be right under the feet. Cut down the amount of cleaning and handling necessary by laying away ornaments and extra furnishings, leaving only a minimum of articles to care for. Do concentrated cooking ; plan one or two-dish meals instead of elaborate ones; cut down laundry as suggested; bring up into the kitchen or back porch enough vegetables and the necessary canned materials for several days to avoid constant trotting; make every step count.

And, further, pick out the essential things and do them first, and, if necessary, let the other details slide. Thomas Edison told a friend that "I always do the hardest things first." His advice is as applicable to the home as to the laboratory. The woman with little children must let some things go neglected; if it is a choice between excessive cleanliness and artistic surroundings, on the one hand, and a. woman's strength on the other, the health of the mother should come first. She has no right to sacrifice her health 
and youth for small children and housework; the children will need her much more a few years later.

Third, train the older children to help one another, pick up and watch the baby intelligently. Train the baby to a strict feeding and sleeping schedule, and arrange your work according to the baby's schedule. For instance, plan to do the most exacting work, such as cooking, when the children are good or early in the day, and save the purely mechanical work like dishwashing for the time when they

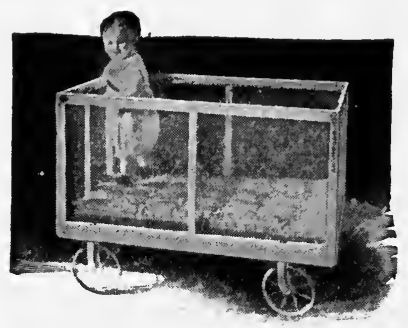

THE KIDDIE-COOP

may be fretty and you will have to be interrupted. Let the baby sleep outdoors, so that it will be less nervous and fretty. When the baby naps, take a nap or at least a complete bodily relaxation for yourself instead of foolishly utilizing that time to "dig in" and completely use your nerve force up. Train yourself to manage several tasks at once without letting it fatigue you.

Even in the country there are many labor-savers. The hand operated washing machine and dishwasher are better than doing work all by hand. A small gasoline engine will operate such equipment as washer, ironing machine, churn, separator, freezer, etc. The newest oil stoves are nearly as efficient as gas and only need a little more care in clean- 
ing and upkeep-use one for cooking the year round. The fireless, and the hand suction sweeper are available. But most important of all is the planning, the picking out the essentials that must be done, and having that efficient attitude of mind that does not permit small details to annoy and create nervous tension.

\section{Value of Co-Operative Schemes}

It is frequently urged by certain groups and persons, that co-operative living will still better solve the problems of the family which cannot afford or does not care to employ permanent help. Why, they ask, should ten families struggle with ten kitchens and ten laundries with ten secondgrade workers, when, if they all co-operated they could equip one kitchen and one laundry with the most perfect equipment and first-class cooks and workers, thus relieving each homemaker of responsibility, and giving her better service than she can possibly now alone procure? Many experiments have been tried along these lines; but it must be said regretfully, none have been a success for more than a few months.

No one questions but that such a plan would greatly remove the burdens of management and result in better service with less friction than the present plan of having ten separate homes struggling with ten inefficient workers, etc. But it seems strange to the author that all these advocates of co-operative living fail to see that it is not necessary to start any new scheme to attain exactly this end-it is only necessary to move to a high-class apartment hotel or boarding house and obtain exactly these benefits. For does any co-operative plan, such as these persons suggest, differ from living in an apartment hotel where one may have as many rooms as one chooses and eat in a common dining room? Or live even in a detached cottage and use 
a central eating place, as is so well established in many sections?

The chief obstacle of every co-operative plan is its expense; in spite of what its advocates may say, it can be proved that co-operative living is far too costly for even the average family of moderate income. Without doubt a highclass apartment hotel or cottage eating system relieves the individual homemaker of responsibility and permits better management and higher class service, than that found in the individual home, but how many families, especially with children, could afford it as a permanent method of living? The advocates of co-operation, in their estimates, count the cost of materials and wages only-they do not include the cost of management. They say, "if ten of us bought our supplies together, and we had five efficient workers instead of ten low-grade ones, how much more efficiently we could all live." But who is to buy the supplies, and who is to manage the five workers? In other words, they entirely leave out of account the cost of managing any co-operative plan. Now the whole point of a co-operative scheme of any type is this-if it is well managed, from the business side, it will be a success, if it is poorly managed it will be a failure; and in order to have it zell managed, some person or persons must be well paid for his services; and, as soon as these services are included, then the whole co-operative scheme becomes more costly than the average family can afford.

In many large cities there are apartment house hotels where it is possible to rent several rooms and then take meals in a basement or roof restaurant of the building. There are even other apartments where one may have meals cooked to order and sent up to an individual family dining-room for the family to serve themselves. In Cleveland there is a large and beautiful suite of apartments of this type which do, indeed, thus make the "mechanics of living" very simple. 
But-the price! A suite of four rooms in such an apartment costs from $\$ 60$ to $\$ 70$ monthly, unfurnished, and the meals for only two persons amount to about $\$$ Ioo. Such modes of living, then, are beyond the reach of the average home, and entirely out of the question for families with growing children on a basis of price only, even if they best filled the requirements of family development in other respects.

In nearly all cities the tendency is for more and more families to live in apartments or "flats"; the expense is less than for detached city houses, and the janitor service and heat furnished, and small rooms simplify housekeeping somewhat, but conditions are not most favorable for children nor ideal for family living.

There is also a second, and almost equally important reason why co-operative living plans (such as Montclair, New Jersey) have not been a permanent success. And that reason is that families are, and prefer to be, individual in their taste and living habits. Co-operation would be very easy if every one of us is willing to become "standardized"that is, eat just what the rest do, be served the same way without preference, choice or personal taste.

But this is not the case; we prefer our own privacy, we want certain food that others may not vare for, we have marked likes and dislikes, and any co-operative plan to meet these varying demands and tastes, must cost more than a plan where all tastes are standardized the same. Concretely, it is possible to feed thousands of men in the Army with abundance of food at the low cost of 32 cents for food per day because every one of the thousand is eating the same ration of beef, beans, potatoes, and plum duff ; on the other hand, the cost of serving thousands in the dining cars of our railways is nearer $\$ 2$ per day, because the dining car caters to individual preference. It may be said, then, 
finally, that co-operative living plans can be cheap only when those participating are willing to have their individual tastes and preferences set aside in favor of one, universal "standard" service and kind of food. The truth is, however, that most of us still prefer inefficiency in service and management to being deprived of our love of privacy, individual preference and choice-this is the real reason why co-operation has, and possibly will always continue to fail.

\section{“Part-Time” Service Is Successful}

Since such co-operative plans are neither possible or practical for the great mass of families, and since permanent service is either of low grade, or too costly or impossible to secure, the housekeeper will find her solution in her own efforts rightly aided by "part-time" service of different sorts. As has been pointed out, a skilled day worker can, in one day, do as much work as a general houseworker can do in parts of two or three days. Again, in many homes a schedule can be so arranged, that a few hours of work each day will amply assist the housekeeper. Just what service will be needed, depends on the size of the family, whether there are children, the style of house, whether the members stay at home all day or are at work, etc.

In large cities, there are many agencies which make a specialty of "part-time" workers. For instance, they will furnish a cleaning woman, a nurse, or a cook by the hour at all times. Again, in smaller towns there are many women who would like to use some of their time beyond that needed for their own family, to earn extra if the hours could only be arranged for both parties. The number of such mature and practical women who want to housework on a part-time basis has greatly increased since the war has forced up living costs, and many instances are known of where such arrangements have worked out most successfully. An advertise- 
ment in the local paper, attractively worded, will bring replies.

This is one work arrangement: For example, in a family of four adults, all of whom are employed out of the house all day, one day a week a woman comes to thoroughly clean the entire house of six rooms; one day a week a worker does the washing and ironing; the family gets its own breakfast, leaving the dishes. At 4 p. m. every day a woman (with family of her own) comes and works until 9 p. m. During this $4 \frac{\mathrm{T}}{2}$-hour period she washes the breakfast dishes, cleans silver or pantry, prepares, serves and clears up after a several-course supper, and lays the table for breakfast. The cost of this service is $\$ 4$ weekly for cleaning and laundry, well done, and $\$ 5.50$, at 20 cents hourly (for six days) for the cooking, or a total of $\$ 9.50$ weekly. This is about the same as a general houseworker receives in a small town -if she can be found! But remark that there is no cost of meals for the worker but once daily, no room, light, heat, etc., less chance of waste, and all of the service is first-class, without any responsibility or friction for the housekeeper.

Another actual case is of a woman who comes to work at Io a. $\mathrm{m}$. and stays until $3 \mathrm{p}$. $\mathrm{m}$. In this period she washes the breakfast and lunch dishes, serves lunch, bakes bread or cake, and prepares greatly toward the evening meal which the housekeeper serves and clears up after herself. The housekeeper does her own cleaning, and the ironing for a family of three. The service here costs $\$ 6$ weekly for a period of five hours daily for seven days, and \$I weekly for the laundry, with a minimum of waste, fuel, and "overhead" expense. This arrangement has been working most successfully for about four years-the woman has a family of her own that she sees off to school before she goes to work, and to whom she is back before they are out of school, and for whom she has the whole latter half of the day, and yet 
earns as much as many permanent workers, with none of the mutual disadvantages.

Such part-time workers receive from 15 cents to 30 cents per hour, depending on the kind of work, the skill, and the iocation. Generally, 20 cents or 25 cents an hour will cover any part-time service, even including mending or dressmaking. Men cleaners are able to do heavier work, and are worth the additional 5 cents or Io cents hourly they may receive. One friend told how she had the local painter clean her kitchen frequently in his spare time; old men can take care of furnace or yards, or even a boy of ten, if the family does not possces one, may do some of the work formerly done by "Mary Ann," on an hour basis.

\section{College Students and Y. M. C. A. Helpers}

Although relatively few families live in college towns, the success of student workers as helpers to the housewife should be noted. In most college towns, there are many students who for room and board, or on a straight hour basis, will, if men, care for furnace, yard, windows, beat rugs, etc. Young women can assist with the cooking, or serving, or certain specific cleaning or mending. In the house where the author lived one year in a college town, one of the women students had her room and board in exchange for getting the family breakfast, waiting on the family dinner, and doing about four hours of cleaning on Saturday. A boy student washed the windows weekly, and did the heavy rug cleaning, and cared for the lawn, by the hour. The mother of the family did the cooking, sent the laundry out, and was able, even with a family of six, to run her home on a minimum of service cost. At a western state school recently, the wife of one of the professors told how she never had a servant problem in her life, because she always arranged the work between three college boys, on 
the hour basis, and that she would never go back to any servant girl again!

Many Y. M. or Y. W. C. A. homes can furnish names of those who would be only too willing to do housework, if on a dignified part-time basis. One girl is known, who came at $8 \mathrm{a} . \mathrm{m}$. and stayed until noon in one home, and then went to a neighbor's, working from I to $5 \mathrm{p}$. m., buying her own lunch independently, and having her every evening free, and yet giving some tired housekeeper just the necessary daily "lift." It is among such girls that nurses by the hour are commonly found, or what may be called a "mother's helper." To the housekeeper with children, this is one of the most important part-time services. She may be able and want to do all her housework, if she can only find someone to take the children for a few hours in the afternoon. In large cities, this work is definitely established. One may go to a good agency bureau, and have sent a reliable young woman who will take children for a walk, to a museum, or chaperone them trustworthily anywhere. There are other grades of workers, also, who will come in and do the rougher work, such as mending, napkin washing, etc.

Every housekeeper should do all she can to encourage "part-time" household service because it will only be on a part-time or hour basis in the future, that our detached, individualized homes can continue to exist. Effort should be made to locate women of mature experience who can give three or five hours to outside household service without foregoing their own homes; to young women graduates of domestic science, who on an hour basis can find in housekeeping as dignified a work as teaching or demonstration work; to the many other young women who have a natural bent toward housework, but who enter the store or the office because housekeeping is on an unstandardized, indefinite hcur basis. 


\section{Municipal Co-operation with the Housekeeper}

In cities, of course, some parts of house or homekeeping are performed by the municipality. Such common ones as garbage and ash collection should be insisted on everywhere. In one progressive suburb, the ash man comes to the very basement door of the detached house, and takes a specified can out daily; how much better than the householder's constant struggle and overseeing of a privately engaged person! In one other progressive street, the entire care of fifteen furnaces is handled by two men employed by the owners of the houses collectively, instead of by each house individually. Such an arrangement permits more wholesale buying of coal and other advantages over separate management.

Municipal housekeeping in the way of clean streets makes a great deal of difference in the amount of cleaning and laundry work required in the individual home. Dusty streets should be oiled by the town or by the "Neighborhood Improvement Association."

In a few cities steam for house heating is distributed through the streets like gas, but only in the closely-built-up portion, as the loss of heat and expense of construction is prohibitive on long runs. The cost is not less than for individual house heating; the saving is in labor, trouble and dirt.

A few co-operative steam laundries have been started in the country in connection with dairies, but with no great success, because the housekeepers do not place a cash value on their time and labor.

\section{The Cost of the Servantless Home}

As has been pointed out, the cost of running the servantless household is much less than the upkeep of the house with permanent help. Even if the money expended for serv- 
ice by the hour is identical with the sum paid for service by the month, the saving lies in the lower "overhead," and particularly in less waste of fuel and food. As one woman put it, who dispensed with a servant, and started in to do her own work, assisted by day labor, "I see now where all my magazines and theatre tickets kept going out of the kitchen garbage can." With present food prices, the food of an additional adult is certainly worth $\$ 5$ weekly, and furnishings and room rent are higher than ever, thus increasing the upkeep over the figures of past years.

While no general percentages can be given, we can take the estimates of families who have actually tried the two plans, and who have given their opinions as follows:

Mrs. M.-Location, Boston, family of two adults and two children; over period of five years from I912-1917:

"Formerly one general houseworker, \$28 monthly; one nursegirl, $\$ 18$ monthly; estimated total cost of both workers, including their food, lodging, etc., $\$ 75$. Present plan: Laundress, I day, $\$ 2$; cleaner, I day, $\$ 2 ; 3$ hours' special nurse daily on hour basis of 50 cents day, $\$ 3.50$; Sunday dinner at hotel for four, $\$ 3.50$ (of which only about $\$$ I could rightly be charged to service); and boy, half Saturday at 50 cents to clean basement, etc. Total, $\$ 9$ weekly; no food for any worker except two lunches for laundress-cleaner, which you might say was 50 cents, thus bringing the total cost of service to not more than $\$ \mathrm{IO}$, as against $\$ 18$ weekly in the first case. Housekeeper (Mrs. M.) herself prepares the meals, but often assisted with them and did other work even with a general housekeeper."

The B. Family.-Location, Chicago, 3 children and two adults; from 1915-1917:

"I used to keep a cook and a nursemaid, and had a laundress; this cost me in wages alone, $\$ 68$. I reorganized my household this way: Had a man come in one day every week for cleaning at $\$ 2.50$; hired a more capable woman who could both cook and do the laundry at $\$ 40$; the children were older now, and one at school; I bought an electric washer, and we did our wash in half the time as before; then I did the upstairs, and paid the cook extra when she kept the two 
smaller children when I wanted to go out at night. This way the plan costs me about $\$ 55$ in wages, but I have only one person to feed and care for, and all the work runs more smoothly and is better done than before."

Mrs. S.-Five adults, near Cleveland, Ohio, I9I4-I9I8:

"I used to keep one general girl of all work at \$24, and do all upstairs myself and finish the ironing as well as special cooking. I couldn't hire a girl now for $\$ 35$, as they all work in factories. Now I send all washing out for $\$ 2.50$ a week. Every other week I and a colored woman at $\$ 1.75$ do the thorough cleaning. I get breakfast and am alone at lunch. My daughter in high school gets the dinner with me. It costs \$I5 for our service now, without any cost of food for the girl."

\section{Mrs. N.-Family of 3 adults in New Jersey:}

"In I9I5 we kept one general worker who did everything. We haven't been able to get anyone for some time, as we are in a somewhat lonely 'suburb. I can't even get a good local worker. I find this plan the best in my family, and at less expense after all. Every other week I have a crackerjack colored woman from the city come out for three days. Then she does our wash, irons, and cleans the whole house. We wash only every other week. She gets $\$ 2$ a day and her car fare, which is almost a dollar each time, or $\$ 7$ every other week. Then once a week, generally Sunday, we all have dinner at the club house here, which I don't call service, but our pleasure. One other night I try to go into town and meet $\mathrm{Mr}$. $\mathrm{N}$. and my son and have dinner with them. My son takes care of the heater. I should say that it costs me $\$ 18$ for service only. I buy home-made bread from a neighbor twice weekly, and a cake on Saturday, as my talents run to sewing and not cooking. We all like the freedom of the arrangement very much. It seems to me that there need be no servant problem for the able-bodied woman who has no small children."

The C. Family in a Pennsylvania Small Town.-Three daughters, 2 younger sons, father and mother and grandmother, I9I4-I9I8:

"You ask me how we manage such a big family without help. Well, we girls are all over twenty, and tho two of us work every day, we manage nicely. The boys cut wood for the stoves and do 
the ashes, and in summer take care of our big lawn and hose the porches. B- is a school teacher and takes Saturday morning for her work, which is baking, which she likes well, and in that time she bakes bread and pies and cake enough to last more than half the week. Grandmother makes the beds and tidies the rooms. Mother gets the other meals with me, and does the washing; we have a power washer and mangle, and it takes us about two days to get it all done along with the other work. We take turns at supper dishes between the four of us, or if we all happen to go out, father or the boys will help. Each of us girls gets a little money for our share and so do the boys. I can't say how much it costs, but of course less than any servant which we couldn't even afford."

To sum up, we can say that the servantless house is by far the more preferable method of management. The chief secret of doing housework daily, and yet not becoming overfatigued or dulled, is to use the old suggestion of "more head and less heels." Free time must be planned for. If necessary this planning must run counter to what is commonly accepted as what "ought" to be the house routine. If there are children, they should share in the responsibility of small tasks so as to make the division of work more equable. Each child should "lift his own weight" in the housework at as early an age as possible. Part-time outside service will give more efficient, less wasteful assistance than any permanent worker. A simpler standard for the home, both in food and furnishings, will make the work less laborious. Finally, the servantless housekeeper should install and use very possible true labor-saver which will save her time or energy. 


\section{QUESTIONS ON HOUSEHOLD ENGINEERING}

\section{IX}

\section{The Servantless Household}

I. How many hours per week (one person) are needed to keep your home in running condition: (a) For meal preparation and clearing; (b) For cleaning; (c) Laundry work; (d) Other work?

2. What have you done since beginning the course to "shortcut" this time? What more can you do?

3. (a) Enumerate the tasks on which you have made "time studies" and give your results. (b) How many examples of "standard practice" have you written record of? (c) How does your present "schedule" differ from the first one you worked out?

4. Count the number of "things" in your house that are neither useful nor beautiful and report. What is the most time-wasting factor in your home?

5. Give your experience (a) with resident servants, (b) part-time service. How do they compare in cost and in satisfaction? 


\section{HOUSEHOLD ENGINEERING $\mathrm{X}$}




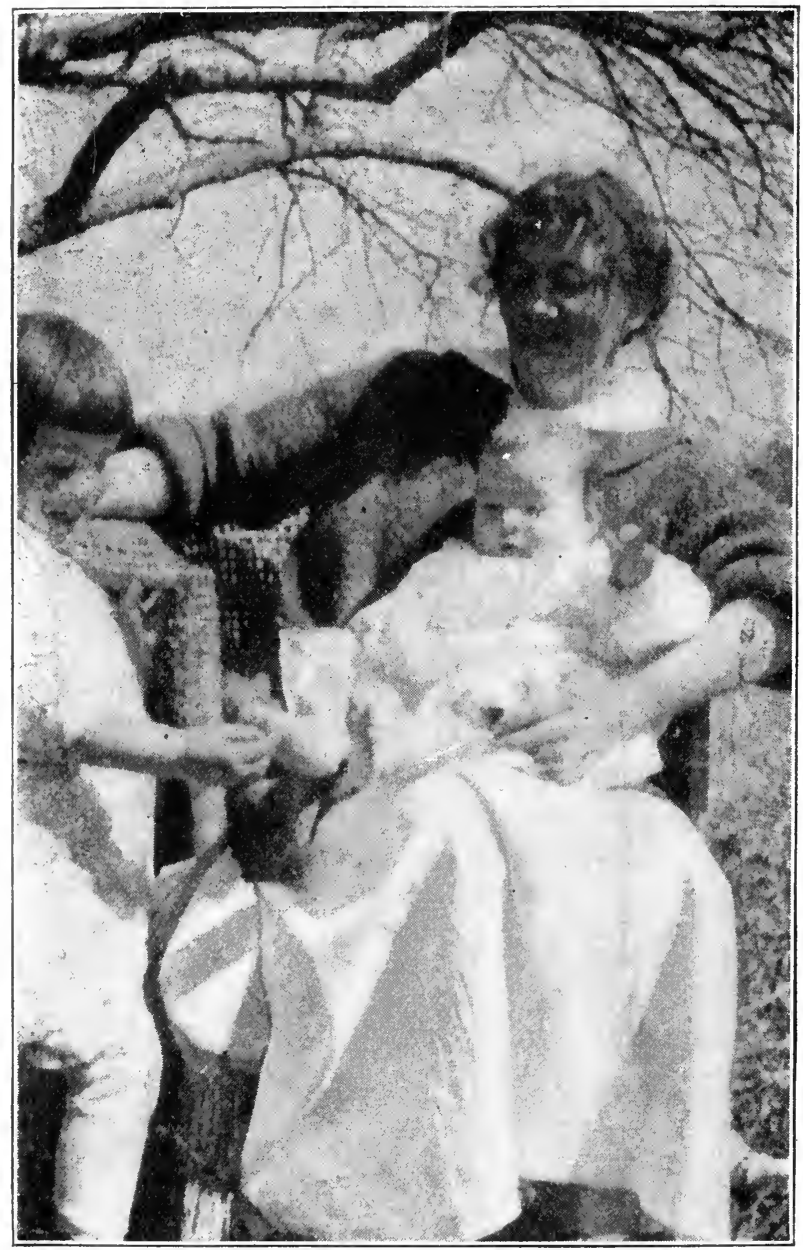

A LOVED AND RESPECTED HOUSEKEEPER-NURSE, WITH MPS. FREDERICK'S TWO YOUNGEST CHILDREN 


\section{HOUSEHOLD ENGINEERING}

\section{$\mathrm{X}$}

\section{MANAGEMENT OF HOUSEWORKERS}

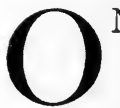

$\mathrm{NE}$ of the problems in management which many housekeepers face at least during some part of their housekeeping experience, is the handling of household workers or "servants." As has been pointed out, the "servant problem" is more acute than ever owing to the fact that women formerly engaged in household service have entered industries and prefer these conditions with high pay and freer hours to the more confining conditions which govern housework at present. In many other cases, especially among colored workers throughout the entire Southern states at period of the war, men were receiving such high pay either in Government service or at "boom" war industries that their wives or families who formerly did housework, cleaning, laundry, etc., no longer needed to work and so stay at home. As one colored cook said to the family where she had been employed for years, "Jeff am makin' so much money by de Gov'ment dese days dat I thinks I'll just set at home and help him spend it."

Even with normal industrial conditions, there is every indication that service for the home will be increasingly more difficult to obtain-and also to keep. It is therefore worth while for any employer of household labor to study the "servant problem," understand its causes, and particularly give attention to the relation and attitude between herself and any employee she may engage. 
Recently the national Y. W. C. A. undertook a report on household employment. They interviewed women workers themselves in different occupations; some girls were at the time working as house servants, others as clerks, factory hands, office help, etc. And the girls themselves (who certainly must be regarded as the best judges) summed up their opinion briefly that it is the conditions surrounding housework, and not the work itself which are today urging women into factories, stores, offices-any place except the kitchen of another woman. Prominent industrial and social welfare authorities who have studied the "problem" concur in the same view and even urge women away from domestic service because of the following reasons against it:

(I) Social stigma of "servant" both from employer's class and from other members of worker's own group in other occupations, as factory workers, shopgirls, etc.

(2) No standardized hours of work.

(3) No independence or private life after work hours.

(4) Too much loneliness and confinement and lack of stimulus from other workers.

(5) No chance for advancement or professional progress.

(6) Often lack of bodily comforts.

(7) Housework offers fewer chances of marriage.

It is significant that neither the girls themselves (this report was based on a consensus of 500 girls) nor social workers make any mention of pay in connection with the problem. All based their objections on the conditions or psychological objections involved. In other words, even if the pay of the houseworker and the office worker were identical (at the present time housework is even better paid!) a girl would prefer the latter occupation. The most important of these objections, because it is the most difficult to con- 
trol and eradicate, is the social stigma which commonly attaches to the appellation "servant." Naturally, "servant" still carries with it the old world idea of an inferior, a dependent or subordinate. And in this country of democracy, whose very air breathes the idea that "all men are created free and equal" (meaning with equal opportunities), neither men nor women like to be in positions of implied inferiority to the people for whom they work. It is only fair to acknowledge that household service is still the only occupation for women where this inferiority is implied, or often keenly felt by the worker.

\section{The "Mistress-Slave” Attitude}

It must be admitted by many women or mistresses themselves that they are largely to blame for this relation. While other work for women has been put upon a business-like "employer-employee" basis, the servant still is part of the old feudal "mistress-slave" basis long since discarded by modern industry. They may not outwardly admit it, but most women who are able to afford servants to assist them in the home, want those other women workers to stay just servants and to remain on a subordinate plane where they can be bossed and talked down to from her platform of the superior mistress. They want the "servant" as well as the service.

It would be an excellent idea if women employers of household labor could visit a modern large office or factory and see how employees in modern business are treated. The factory may hire the services of the most humble vegetable preparers, dish washers, label pasters, etc., and yet each of those employees is treated not as an inferior, but as a worker free and equal with the higher overseers and managers. The true basis of the success which modern industry has in deal- 
ing with employees and the reason why even the most inferior and low-wage factory entices girls away from domestic service, is the principle of "team work between equals." Each employee under "scientific management" feels that he is part of the company, and this team work basis elicits their loyalty and co-operation. The most successful corporations and employers of labor are those who are doing everything possible to make each employee feel his importance, instead of crushing him by browbeating and treating as a subordinate.

Whetl er she likes to or not, the housekeeper of today who employs labor will have to revolutionize her own attitude along the lines of modern treatment of employees-or she will find no other women willing to work for her. It cannot be quite explained why women who do often assume this progressive attitude in regard to a trained nurse (who does similar acts to a houseworker), or to a stenographer, or a sewing woman, or even to a day laundress whom they employ, nevertheless refuse to assume it to a woman who cooks their food and dusts the chairs. Why should she treat a day sewing woman or laundress with respect and fairness, while she so often feels it is her right and privilege as a mistress to browbeat, scold and tyrannize over a household worker? The reason is, that in her own mind, the mistress commonly does think the servant "inferior." And thus thinking, she naturally shows it in her attitude and treatment.

Let it be repeated then, that the first step in solving the servant problem, is to solve the "mistress problem," and for the mistress to place her relationship with the worker on a straight, dignified, employer-employee basis. The old feudalslave relation was possible as long as workers did not have any other occupations from which to select, and as long as they remained unintelligent and uneducated ; but today workers will not put up with such medieval treatment. In other 
words, the mistress of today is trying to impose a wornout, archaic relation upon a worker who in many cases has become more educated and who will not submit to it. Adjustment must be made before it is too late.

\section{The Employer-Employee Basis}

The adoption of the modern employer-employee basis means, first, that in her own mind the mistress will not think of the houschold worker as an inferior. Second, instead of calling her servant, why not think and speak of her as a household assistant, a household helper, or "houseworker" seems about the best term, which will counteract the present social stigma? If even the youngest $\$ 6.00$ a week office girl is called Miss Smith, why not Miss Smith in the home? If the worker is really reliable and intelligent enough to trust with the preparing of food for the family, with attending to its comfort and keeping the home sanitary and attractive, is she not worthy to be called by her surname instead of addressed by the familiar first name which we reserve either for animals or for our loved ones? If each mistress would sincerely follow out this attitude, then gradually public opinion would come to place household work on a higher plane and the worker's own friends in other occupations would not longer slightingly call her "pot slinger," "kitchen mechanic," etc.-an attitude which does much to prejudice a self-respecting girl against household service.

\section{Standardized Hours of Work}

The second chief reason given against household service by all the workers questioned was "no standardized hours." It is my firm personal belief that all household service should be based on an hour system and that workers should live and eat in some other place than the home where they are 
employed. I feel strongly that the adoption of this livingout-plan would do more to solve the problem and change the conditions now surrounding the work than any other one step. I believe, and have preached for years that housework, like any other occupation, should be placed on a day basis and permit the worker to have a home life of her own after the hours of service she gives to her employer. Such a living-out-plan, of course, would at once solve the point of standardized hours, for then arrangement can easily be made about the exact hours that a worker will give to her task. (See page 408, Chap. IX.) It will also in one sweep solve the objections of "private life after work," "loneliness" and "confinement," chances of meeting people, etc. And the author cannot too strongly emphasize her conviction and experience that the final solution of the servant problem can come only by placing housework in all its branches on a day basis of definite hours, which will permit the worker to have her own life after that specified work is done, just as is now followed by factory hands, clerks, laundresses and sewing women.

But if the mistress still wishes to cling to the present method of having workers (if she can find them!) sleep in the home, she nevertheless must arrange for them definite schedules of both the work they are to do for her and the rest or free periods they are to have for themselves. Over and over again has come the story of a worker "having no time to herself," and not knowing all day long at what time she will be "free." Mistresses say in reply that they do not require the worker to be busy all of the time, merely that she "be there" when wanted. But the mistress must surely see that it is just as fatiguing to "be there," as it is to actually work, and must provide and allow some definite period every day which the worker can use as she pleases. 
How many mistresses permit a girl an hour or two every afternoon in which she can unquestioningly go shopping, to the library, or do exactly what she likes? When this point of a definite rest period for the worker was mentioned by the author at a lecture before a prominent woman's club, several immediately exclaimed, "But who will answer the bell in the afternoon while she is out ?" It was hard to convince these dear ladies that the situation is so serious today, that it is a choice betwcen answering the bell oneself during a couple of afternoon hours or not being able to secure a worker at all!

Further, let any mistress ask herself, can she expect a worker to remain in good health and yet never leave the house except the conventional "Thursday afternoon off" and "every other Sunday"? The majority of mistresses in the past, too, have always exacted that their cooks and maids "stay in," in the evening, unless special permission is given to leave. Can any mistress imagine any factory or business man asking an employee to stay in the office or factory in the evening as long as she has finished her stipulated work? Why then, if the cook has served the supper and washed the dishes, should she not be free to leave the house every night if she wants to?

\section{Privileges Versus Rights of Houseworkers}

But no, the average mistress usually regards "time off" and free evenings as "privileges" or something for which the worker must beg and which is a favor if it is granted. In their own hearts many mistresses resent employees being sufficiently independent to leave the house without special permission. But from the employee's point of view, it is this constantly having to ask permission that creates the feeling of no independence- - feeling that they keenly resent. It is 
a mistake for any mistress to thus patronize her workers and grant them "privileges" which frankly should be the employee's business-like rights.

It may be set down as a rule and as an essential part of the new relationship that a worker should be free to leave the house and go and do what she chooses in the specified afternoon rest period or when she has finished her evening's allotted tasks.

When the author strongly stated this point at the above mentioned club audience, she was instantly met with the objection, that permitting workers to leave the house without greater supervision would result in immorality! Several of the finest club women rose and said that in restricting the girl's time away from the house and in supervising her friends they were doing her a favor and looking out for her best interests. No doubt these fine club women had the best of intentions. But in these days of independent women workers, no girl of intelligence or spirit will stand such supervision. Such a supervision implies on the face of it that the employer doubts the conduct of her employee and is therefore treating her like a child instead of a responsible adult. Let any mistress think for a moment of an office manager who would question his filing clerk as to where she had been the night before or whom she had seen. Under the new relationship, no employer has a right to question a worker as to where she has been, where she is going or what she does outside of the hours of her stipulated duty and work. The only exception to this is that she should not go places from which she might bring health contamination to the household. The old slave attitude (which so many mistresses still wrongly persist in following) meant paying for $a$ girl's life; but the new business attitude means paying for a girl's work. 
To return to the point of supervision by the mistress in protecting the girls from immorality, which was made by several audiences addressed on the domestic service problem -the facts are, that there is more immorality in the ranks of household workers than in any other class of women workers. This statement comes from such workers as Miss Grace Abbott of the Protective League, by matrons in rescue homes and from Miss Jane Addams of Hull House, who says: "In spite of the fact that domestic service is always suggested by the average mistress as an occupation of safety for girls the Federal report on Woman and Child Wage Earners in the United States, gives the occupation of the majority of girls who go wrong, as that of domestic service, and in this it confirms the experience of every matron in a Rescue Home. Many indeed are the instances of fallen girls who only a few months before had been honest girls, cheerfully working in the household of a good woman mistress, whose sense of duty expressed itself in dismissing the outcast as soon as she knew her situation. Is it not significant that the girls who chiefly supply the demand of the "White Slave" trade are drawn most largely from the one occupation which is furthest from the modern ideal of social freedom and selfdirection?"

In other words, the only result of the mistress's attempts to incarcerate the worker in her kitchen except on a specified Thursday off, and to oversee her goings out and comings in, leads to nothing but the license which comes from restraint.

How much better it would be if the mistress encouraged the worker to have wholesome social ties. She knows that for her own daughters mutual interest and recreation with young men of good standing are necessary, that every girl needs a chance to express herself socially, otherwise she will 
become dull and degenerate. Yet, in many instances, she allows her young worker possibly only in her teens, no relaxing evenings away from work, and especially resents her taking advantage of neighborhood social life. If the mistress would only see how inconsistent it is to expect the highest morality and conduct from an individual she persists in shutting up like a chicken in a coop, or from one whom she does not allow the privilege of attending church or other ethical service!

Again, why is it that mistresses expect the highest performance and knowledge from their workers and yet refuse to grant them the stimulation of attending meetings or groups where home subjects are discussed? It is not unreasonable to think of a houseworker as a member of a food club, or as an attendant at meetings where topics of diet, sanitation, economy, etc., are discussed. Some women even resent their workers reading books and magazines about the house. Indeed, the author knows of but few families outside of her own, where the worker visits food demonstrations, reads Government pamphlets or other matter along home lines. Yet, why not?

\section{SCHEDULES FOR THE HousewORKER}

This brings the discussion back to the point mentioned at first, that the mistress must prepare a definite schedule of both off time and work time for each houseworker. It will assist any mistress to re-read Chapter II, on Scheduling and Despatching, because whatever principles of work are helpful for the housewife herself are even more helpful for any employee. She should bear in mind that planned work is work done with less friction. And it is almost necessary for each employer first to try to follow the schedule she herself has made, before she exacts it of her employee. For it is 
one of the frequent complaints made by workers, especially in a household where only one is employed, that too much work is laid out for a required amount of time. It must be admitted that many mistresses are guilty of this fault or overloading work which cannot possibly be done in the required time; the result is that even the best worker becomes discouraged and resentful and doesn't do the overload imposed on her.

In households where there is but one worker, the housewife herself must assume some of the duties or hire extra help by the day, unless the house or apartment and the family are very small. It is just because so many mistresses in the past have expected anything and everything and overloaded the one worker, that we now have no longer a "general houseworker," and that girls who formerly were general houseworkers have turned to some specialized branch such as waitress, parlor maid, or cook. The only result of the mistress's unfairness has been to deprive the one-servant household of today of the unspecialized help which is so badly needed.

Careful planning of the schedule should limit it to a definite number of hours. There is no reason why the average housework in a typical family with modern conveniences cannot be compressed into 8 working hours or at most Io, instead of the 12 to 15 hours of which some servants have justly complained in the past. Of course, in every family the schedule will need to be interrupted because of occasional sickness, special guests, etc. But the regular everyday routine should be studied, systematized and made as nearly definite as possible.

The schedule should, as has been pointed out, provide for work hours, and rest hours. It should specify each task and approximate time; it should include the special outside 
help such as laundress or cleaner, and state the days on which tradesmen come, hours of delivery, etc. If there is more than one worker, then their hours also should be carefully scheduled with relation, one to the other, providing that when one is on duty the other is off. The schedule for houseworkers will depend, as for the housewife herself, on the various factors of hours of meals, size of family, number of rooms, etc. Following are some simple outlined schedules for one worker :

\section{No. I. Schedule for One Worker ( $91 / 2$ Hours)}

7:00 A.M.-I2:00 M. Breakfast, dishes, cleaning, prepa:ing lunch.

12:00 M. - I:00 P.M. Lunch.

I : o0 P.M.- 3:30 P.M. Dishes, special silver, window or pantry cleaning. Prepare towards dinner.

3:30 P.M.- 5:00 P.M. Worker's off time.

5:00 P.M. - 7:00 P.M. Dinner and clearing up.

No. 2. Schedule for One Worker ( $81 / 2$ Hours)

7: 30 A.M.-II : 30 A.M. Breakfast, dishes, etc., as above.

II : 30 A.M.- I : 00 P.M. Off time.

I : 00 P.M. - 2:00 P.M. Lunch.

2:00 P.M.- 6:30 P.M. Special work, cleaning, preparing and serving dinner, housewife clears up after evening meal and stacks dishes.

No. 3. Schedule for One Worker (9 Hours)

7:00 A.M.- 8:00 A.M. Housewife gets own breakfast with aid of toaster, percolator, etc.

8:00 A.M.-12:00 M. Houseworker begins work, as above.

I2:00 M. - I:00 P.M. Lunch.

I: Oo P.M.- 4:00 P.M. Special cleaning and preparing toward dinner.

4:00 P.M. - $5: 30$ P.M. Off time.

$5: 30$ P.M. - $7: 30$ P.M. Serve and wash up dishes of evening meal.

No. 4. Schedule in Country Home (io Hours)

6:00 A.M.- 7:00 A.M. Breakfast ; pack children's school lunches.

7:00 A.M.- 8:00 A.M. Dishes; start soup, etc., for dinner.

8:00 A.M. - 9:00 A.M. Garden or butter making.

9:00 A.M.-10:00 A.M. Beds, brush up rooms; lamps filled. 
10:00 A.M.-I2:00 M. Baking, dinner preparation.

I2: $00 \mathrm{M}$. Noon dinner.

I : oo P.M.- 3: oo P.M. Clear up after dinner, clean kitchen. porch, etc.

3:00 P.M. - 5:00 P.M. Off time; or mending.

5:00 P.M.- 6:00 P.M. Supper preparations; feed stock.

6:00 P.M.- 7:00 P.M. Supper and clear up.

The above schedules are, of course, only suggestive; they would vary from day to day according to the special tasks (see page 70 ).

While a schedule is an excellent plan for most workers, it must be admitted that there are others who will show greater efficiency if allowed to plan their own work or do it in the method that suits them best. And it is not wise for the employer of this kind of woman to lay down an hour by hour scheme,-rather write down the essential tasks that must be done and allow the worker her own license to do it.

\section{The Efficiency Reward}

The fifth charge against housework on the part of women workers themselves, was "no chance for advancement." It must be admitted that, compared even to clerking and the lowest office positions, housework suffers at this point. There is chance that the $\$ 8$ a week stenographer will before long receive $\$ 12$, or maybe $\$ 20$, if she increases her efficiency; there is the chance that the dry-goods clerk may some day become head of the department, buyer, etc., but what hope is there for the house assistant to advance? It is a well known psychological principle that no worker increases his skill or interest or loyalty without some hope of financial gain, or some other reward, emotional or otherwise. This is as true of the cook or maid as it is of the stenographer or shop girl. Remember that the twelfth principle of scientific management, and perhaps the most important one, is the "Efficiency Reward." 
But how very few housewives ever think of raising their worker's wages or offering her any incentive to increased efficiency, until she comes and announces she is going to leave and work for Mrs. Brown across the street, who is willing to pay her more? Would it not be a far better plan at the time of hiring an employee to tell her that if her work is satisfactory and she proved permanent, that she would receive a raise at the end of a certain period? One good plan is to offer an increase of $\$ 1.00$ per month after the first 6 months of service are ended, up to 25 per cent of the original salary. For instance, a worker receiving $\$ 28$ at time of engagement, would after six months receive an increase of $\$ 1.00$ each month for seven months or until she had advanced to $\$ 35$, and similarly with other wages.

This may seem an unwarranted plan to many women. But it is a plan followed with great success by business firms. The theory is that at the end of the time of increase, the worker will certainly be worth 25 per cent more than when she began. In some cases, a raise of only ro per cent is given. But business men affirm that if a worker isn't worth a certain per cent of increase at the end of a definite time of service, she isn't worth retaining. The other theory which some employers follow is to employ only the cheapest help work them hard until they demand more pay or want to leave, then discharge them and get other cheap help and repeat the process. But the more successful plan is to offer the competent worker a substantial wage incentive for increased efficiency, so that when she has become efficient, she will not leave but give her employer the benefit of her increased skill and training.

"But I cannot afford to raise the worker's wages," complain many women. Yes, but if they stop to think, they will see that when they lose trained help they do afford the ex- 
pense of new advertisements or agency fees, the cost of breaking in new help, etc., which in the end amount to as much as the increased wage would have done-not to mention the cost of nervous strain and worry.

It must be remembered, also, that wages are only a part of the expense of a resident worker and that an efficient helper can save on food, fuel, breakage and wear and tear much more than the few dollars a month which may be needed to hold her. A full time houseworker is now a luxury and when the demand is greater than the supply, a high price must be paid.

\section{How to Hold Houseworkers}

The industrial experts speak of the leaving and re-hiring of workers as "labor turnover," and they have estimated the per cent of this turnover in many industries. But no one has yet been brave enough to estimate the per cent of houseworker turnover in the home, although from the facts it would seem to be over Ioo per cent a year! Now it is true that a certain amount of change in positions will always occur; but the modern housewife must take it as one of her responsibilities, as an employer of labor, to lessen the per cent of turnover of house employees by all the methods she can. Beside granting wage increase as above, she can-

(I) Give two weeks' vacation with pay to all employees having served one year.

(2) Allow legal holidays or their equivalents.

(3) Bonus for performing special tasks; like bread and pastry making, fine ironing, pressing clothes, etc.

(4) Extra pay for extra work beyond stipulated time.

(5) Bonus for six months' or a year's service.

(6) Give percentage of saving on food and fuel bills.

(7) Promote worker from lower position to higher. 
This last point is possible if more than one worker is employed ; or sometimes a girl who is first employed as a general houseworker may become proficient and show such initiative and responsibility that she can advance to the higher position of housekeeper, and be relieved of the more fatiguing manual work by the services of a day worker.

\section{Providing Incentive For Workers}

It is true that the worker in the confinement of a private home has no stimulus from a group, such as obtains in offices, factories, etc., and which exert such a strong influence toward increased efficiency and advancement. But why does not the housewife herself act as a stimulus on her employees? Surely, if she were sufficiently interested and enthusiastic about food values and sanitation and methods of work, she could at least do something to stimulate an employee, especially if she does part of the work along with her. Praise is another means of increasing interest and skill and should be often given when it is deserved. But above all, the mistress should try to give the worker room to develop her initiative-the one practice which will surely increase efficiency and keep up the worker's interest in her work.

Far too many mistresses advertise for "responsible" and efficient help and then surround them with such constant surveillance and hold them in such rigid subordination that the worker cannot become anything but a mechanical drudge. In how many cases does not the mistress always want the worker to do things "her way"? In how few cases does she ever allow leeway for the ability of the worker to count? As someone has said, the average mistress gives her worker "responsibility without authority." Case after case comes to mind, where the mistress has actually killed the spirit of the worker by refusing to let her "have her head" as the 
saying goes. The author had in her employ for a number of years an exceptional Scandinavian woman who made this point clear: she said in her country, when a mistress employed a housekeeper or worker, she then "put it up to" that worker to make a success of her management. But in this country, the American homemaker was constantly interfering and refusing to give the authority sufficient to make the woman develop her best effort. And the author has herself found that the really capable and efficient housekeeper must be allowed to develop her initiative, to create and to have sufficient independence in planning her own work, to make her happy in doing it.

There are some exceptions, but as a rule women in the home are exceedingly poor employers. The same housewife who flatters herself that she handles her houseworkers well would doubtless not be given the position of office manager or factory forewoman by any manner of means. Also there creeps in at this point the old preference that many women have for working for men employers rather than for a "woman boss." Part of this may be due to natural sex preference, but undoubtedly part is also due to the poorer executive which the average mistress really is. When one hears tales of the petty nagging to which workers have been subjected, the interference of the mistress in the worker's private affairs, the unnecessary giving her work just to "keep her busy," it is small wonder that women prefer the freer regulations of modern industry where most frequently they are dictated to by men.

\section{Providing for Bodily Comfort}

The lack of bodily comforts often mentioned by girls who have worked in homes has also some foundation. There are many housewives who do provide sufficient bedding and fur- 
nishings; yet it has often happened that the room devoted to the houseworker is generally the least desirable in the house, hot in summer and cold in winter, furnished with cast-offs, or is the attic room, devoid of any attempt at decoration or beauty. The housewife may say in defense that workers do not take care of attractive furnishings. And it is not unusual to have an ignorant Lithuanian woman who never even heard of built-in plumbing in her own country, demand a private bath in a position here. Still, it must be remembered that a large reason why the servant girl so much featured by the comic papers, looks frowsy and untidy is because she has neither had time nor opportunity for her personal toilet. If a housewife wishes to have the worker neat and clean, she must provide the means of realizing it and the time for doing it. In the writer's own home it is plainly stated at the outset, that the attractive hangings and bedspread of blue figured cretonne will be taken away unless cared for and the room kept worthy of them.

If possible, there should be an extra bath for the worker near her own room or she should be granted the use of the family bath at certain times. In all service portions of the house, hardwood or composition floors insure the greatest sanitation. A single size enamel bed of the hospital type is most suitable; and it has been found that blankets which can be washed frequently or with each change of occupant are more sanitary than padded comforters. Beside adequate closet space a worker should have a chiffonier, table or desk and comfortable rocking chair. Generally, it is best to have the walls of light colored tones of paint and to permit each worker to "decorate" her room as she prefers. The rooms of all workers should be open to inspection at stated times and always required to be kept neat.

There should be, if possible, some provision for the 
worker's meals, other than snatching a "snack" standing, or cold food in the dining room after the family meals are finished. If there is a "dining alcove" in the kitchen, as described in the following chapter, that solves the difficulty. If not, perhaps one can be arranged, or at least a "drop leaf" in front of a window, preferably away from a view of the more or less cluttered kitchen. If the family is not too large, the worker's hot food should be served to her from the dining room. From the standpoint of health and efficiency the eating-on-the-run habit of many houseworkers must be discouraged.

There seems to be no reason why a high grade worker should not take at least the noon meal of informal luncheon with her employer. It is a little thing, but its psychological influence is great. Indeed, in many families there is no reason why the intelligent worker should not sit at the same table at least part of the time. In the writer's home, the following plan is followed with pleasant results, both to the dearly beloved "nursie"-housekeeper and the children. Every Friday this worker has time off, the whole afternoon, and does no planning or work whatever toward the evening meal on that day.

It is the children's "cooking day," and as soon as they come home from school, they go with their mother into the kitchen to prepare the supper. Generally each child is allowed to choose the dish that he prefers and great interest is taken in vying with each other. At the supper hour the nousekeeper is called and sits with the family at table, being pleasantly surprised at the children's efforts and enjoying a meal about which she has not had to concern herself. This plan trains the children and also gives a personal touch to the relation to the houseworker.

Then there ought to be some place besides the kitchen 
where the houseworker can receive her men callers. This is often possible when the right is not granted. In large households, where a number of workers are employed, a servants' dining and sitting room is usually provided and sometimes a porch. To keep a general houseworker nowadays, conditions must be made attractive, and the "human element" respected.

All the foregoing does not mean that the houseworker shall be "coddled"- the "servant must be worthy of his hire" - the employer has a right to faithful service and must insist that it be given with dignified firmness. It is continual petulant fault-finding that is so destructive to all authority.

\section{The Worker's Social Life}

"Housework offers fewer chances of marriage," is another objection raised by the girls interviewed. But even this can be overcome if provision is made for after hours recreation and the social life mentioned above. In every case, it should be realized that a short period of employment as a household worker (in a well organized household) will teach her habits of economy, experience and management which she will later find exceedingly valuable in conducting a home of her own. As soon as the social stigma is removed and more freedom for social life given, there is no reason why the worker at household employment should not meet and attract the most steady young men starting out to make their way just as they do and have done for years in the old countries of Europe.

Speaking of marriage brings up the point of wondering why more homes do not avail themselves of the mature middle-aged woman or widow instead of the young "girl," as has been done in the past. The young girl naturally, if unconsciously, is still seeking a mate and this makes her 
more eager for movies and other city amusements where she can display her clothing and attract the opposite sex, while the mature woman, possibly a widow, with certainly more experience, will not demand such amusements so constantly and will thus make the problem easier, especially for the suburban or country home.

On the other hand, many mature women apply for positions, who "kept house for 20 years," but to which this is only a handicap, and who continually lay more stress on the home they used to have, the social position they once held, and whose efficiency does not warrant their boasts. It is nearly always the case that such applicants have had "every comfort in their own home," and the difficult point seems to be to make them feel that the employer's home is as superior as what they were accustomed to. Indeed, the author has had in her employ several able and refined married women, all of whom have had more "elegant" homes and more "handsome husbands" than the author ever has had or ever hopes to have!

Mature women of the right type always are more responsible and dependable. There are two chief points to be considered in selecting them; first, they may be those who have had their own comfortable homes and who will feel very sensitive if the contrast between their former position as superior and their present one as subordinate, is too keenly made. In other words, mature workers must be "handled" differently from young girls; if competent, she should be the "housekeeper." And second, the mature woman, with experience which would be intensely valuable to her employer, often does not have the strength to do the rougher kinds of work. The combination to make here then is to hire a mature woman for efficiency, responsibility and management and to have the rough and harder work done by a day worker. 
In a somewhat wide experience, the author feels it is safe to say that no woman over 50 should be taken for active housework. But women between 35 and 50 are excellent as housekeepers, children's nurse or any executive capacity, while the active work should be done by those between the ages of 20 and 35 .

There are also numbers of women with children who offer the most permanent dependable kind of help, especially for the suburbanite or in families with other children. Such a woman, having her own child with her, will be less lonely and more satisfied away from city amusements, and also more responsible with children. In pay they receive from $\$ 10$ to \$r 5 less per month than a worker doing the same work, without a child. Such workers may be secured from the Children's Aid Bureaus or Mother and Child Department of City Charities and other social organizations. Here the only problem is to allow the mother to discipline her own child and to allow in the work schedule sufficient time for her to clean and bathe the child as well as herself.

\section{How to Obtain Household Help}

There are two most common ways of securing labor for the home. One, the use of a commercial employment agency, and the other, inserting advertisements in local newspapers or nearby metropolitan papers. Mistresses should know the advantages of each method. The employment agency is at present operated by private individuals, who in return for a fee put those desiring help in touch with applicants. The agency is supposed to be responsible or to guarantee the honesty of the workers and looks up references of past employers as to efficiency. In most agencies, however, no faith at all can be put in this kind of reference. Also, since the agency receives a fee from the applicant when she reg- 
isters, as well as from the mistress, it can easily be seen that it is to the advantage of the agency to have workers change positions as often as possible!

The one advantage the agency offers is the chance to interview many applicants quickly and at once. But anyone who has sat for an hour in the typical employment office only becomes sickened both at the casualness with which mistresses engage workers and at the class of labor offered.

Even when an agency cannot secure and says there are no workers for a position, it is possible to secure a much higher grade of worker by inserting a well written ad. The fee of the agency ranges from $\$ 2$ to $\$ 5$ for each worker, allowing one month of service, or if the worker leaves before that time the agency will replace. It is to be hoped that in the improvement in social and industrial relationships the private agency will be changed into some form of municipally operated agency, run at cost; or it has always been the writer's thought that the women's clubs all over the country should supervise locally run employment agencies and insist on higher standards of work and more dignity among the mistresses in regard to housework as a profession. It seems futile for women's clubs to discuss "Browning" and the "early Aztec pottery," while they neglect to solve or make any progress in the great problem of woman as an employer of labor in the home; or hard to understand how they can reconcile their extreme solicitude for securing an 8-hour day and the highest working conditions for the labor of women in industry and outside occupations, with their refusal to conduct their own personal homes so as to conform to such fair demands.

As was pointed out, generally a higher grade of labor can be secured through a newspaper advertisement in the classified section. The following advertisement, based on an 
8-hour day is said to have brought 120 replies at each time of insertion:

WANTED-A young woman to do light housework, 8 hours a day, 6 days a week, sleep home. Apply by letter only.

Another excellent advertisement is :

WANTED-Dependable, efficient household helper, treated as family, good wages, advancement. Apply Mrs. Smith, Riverview, N. J.

Much more can be learned by studying the applicant's face and appearance than by reading the dingy "recommendations" she carries folded in her pocket-book. Previous employers should be phoned or written to and questioned, especially in regard to honesty and habits.

Another alternative is to secure through advertising or otherwise an assistant of one's own education and social standing and make her one of the family, sharing family meals and other activities, like the farmer's daughter who acts as "help" for a neighbor. It would simplify matters to adopt her, for the time being, as distant niece, cousin or aunt, and keep the matter confidential. In such a case it is especially needful that there be a trial period and a detailed, written agreement as to duties. The ad might read:

"Competent high school graduate of good family, between 18 and 20 , wanted as mother's helper in the home of a domestic science graduate, to share family meals and have the status of a relative. Convenient and attractive suburban house. Salary $\$ 25.00$ per month, with increase up to $\$ 35.00$. Send small photo." Or

"WANTED-Working housekeeper between 35 and 40, normal or high school graduate, to be one of a family of 6 , two small children. Large, comfortable room, no heavy work. Salary \$35.00 per month. Give education, experience, and send photo."

The success of such an arrangement depends, of course, on the personalities and temperaments of the worker and the employer, and requires tact and forbearance on both sides. 
It is one way out of the difficulty if resident help must be kept. There are many thousands of women everywhere who want and need to earn money and who would prefer domestic work under the right conditions.

\section{The Square Deal for Houseworkers}

One of the chief requisites to success in all management of labor, is a clear understanding at the outset of the duties required, time off and pay. It is a good plan to have written down in black and white in duplicate what is expected and to read it to the worker and secure her approval before engaging her permanently. Too often mistresses engage a worker, glossing over some of the work they expect done, and then later resent the fact that the worker is unwilling to do it. As many of the specified points should be made clear as possible, as for instance:
(I) Hours of rising.
(2) Hours of work.
(3) Hours and time off.
(4) Extras for which extra pay is given.
(5) Pay, whether monthly or weekly.
(6) Amount of notice required before leaving.

(7) Whether medical care is paid for by mistress or worker.

(8) Understanding as to the care of the baby or young children.

(9) Understanding in regard to breakage.

(Io) Complete list of detailed duties.

The more definite the understanding at the beginning, the less likelihood there will be of friction later. In regard to the wages, it should always be specified as "two weeks notice given, or wages forfeited before leaving and two weeks' pay or notice given on discharge," or even a month's notice. It is 
usually advisable to take a worker on a week or two weeks' trial, during which time it will be seen if the position is mutually satisfactory.

\section{Worker's Manual or Book of Rules}

One of the best methods of securing definiteness in the execution of orders is to prepare a Worker's Manual, for the particular household. Any typist will make a couple of copies of these rules for a small sum. The idea is to write or paste in such a book the "standard practice" of the household, so that a worker can most easily know what she is to do and when it is to be done. The headings of such a book might include:

\section{Kitchen and Dining Room}

How to Prepare for Cooking.

How to Clear Up.

How to Make Bread.

How to Wash Dishes.

How to Set and Clear the Table.

How to Serve Breakfast, Din- ner, Supper.

How to Operate and Care for the Kitchen Stove.

How to Care for and Where to Store Supplies.

Whsn Tradesmen Call.

\section{Cleaning and Latindry}

How to Clean Living Room, Chambers, Bath, Kitchen. How to Clean Porches, etc. How to Clean Windows and Mirrors.

How to Polish Silver.

How to Clean Refrigerator. How to Change and Make Beds. How to Fill and Clean Lamps.
How to Manage and Care for Washing Machine and Wringer. How to Do the Washing.

How to Do the Ironing.

How to Care for and Where to Store Tools.

Practice in Spring Cleaning.

Inventory of Linen, Silver, Glass, China.

In other words, the Worker's Manual aims to set down in black and white the directions which otherwise the mistress will constantly be giving and reminding about. If the collars and shirts are called for Friday and this day is set down in the Manual, then the worker will have less danger of forgetting them and the mistress less need of dictating about 
them. Each household has special rules which can be thus systematized, made simple and put into the hands of the worker that she may read and follow.

\section{Management of Several Employees}

While the average mistress may employ only one or two servants, it is well for her to know distinctions of work which have come to be generally accepted as follows:

Cook-Prepare meals for family or other servant, keep kitchen and pantries clean, sometimes do own laundry. Kitchen Maid-Under cook, prepares vegetables and does rougher work of kitchen and pantry cleaning.

Waitress-Serves meals to family, possibly preparing salads, etc. Washes family dishes in Butler's Pantry and sometimes has the duties of a parlor maid.

Parlor Maid-Cares for cleaning and dusting of first floor, answering door, etc.

Chambermaid-Making beds, cleaning bath, also mending. Sometimes combined with child's nurse.

Child's Nurse or Mother's Helper-Care of young clsildren, washing their garments, mending and sewing.

General Houseworker-(If not extinct) combines duties of plain cook, cleaning and chamber work.

Managing Housekeeper-Oversees other servants, in large household, markets, has full charge over food, linens and house in general; keeps account. Does no manual work.

Working Housekeeper-Buys and markets and has some responsibility, but also does cooking and other work in small family.

No men servants are included in this list, because men employees are generally employed in only the wealthiest 
homes; they consist of butler, second man, foot man, chauffeur, etc.

In such home, with three or more employees, the mistress has the opportunity to show what she can do as a real business executive. Or the same problems confront the housekeeper in a sanitarium, club or other large establishment where she has several working under her. In order to be successful she must remember first and always to be fair, to apportion each employee's work so that the work is evenly distributed and each "carries his own weight," rather than that one employee is shown partiality at the expense of another.

Further, the manager of many people must have: (I) Clear plans as to what she wants done; (2) Be absolutely specific in issuing those instructions and commands; (3) See that the orders are executed. Nothing makes for friction so much as a misunderstanding of orders. For this reason the orders should be clear in the manager's own mind first, and after she has given them she must not change them. Again, she should try to find out the peculiar failings or excellencies of each worker and the stimulus which will cause each to do better, more efficient work. She should understand that there are broadly two types of minds, the "detail" worker, who is excellent at the small repeated tasks, but who has little judgment, initiative or power to take responsibility. The second class is more of the "executive" type of mind, those who generally dislike detail and routine, but who can be relied upon to act in emergencies or take charge and carry plans through. The more the manager or employer learns to understand these two types and give them the work that is suited to each, the more successful she will be as an executive.

Frequently, in a large household it is wisest to secure 
workers all of one nationality, or one religion, so that there will be more harmony. For instance, if all workers are Irish and Catholic, they are likely to be more congenial than one of this faith and country thrown with another of Scandinavian and Protestant origin. While there are exceptions, it seems to be true that workers of these nationalities have the following characteristics: Irish (good hearted but often untidy, inefficient, little responsibility). Scotch-English (great dependability, sense of duty, well trained). German (thrifty, hard-working, capable of much manual work). Scandinavian (self-reliant, sometimes tricky, often extravagant, excellent as laundresses and cleaners). Polish-Lithuanian, etc. (emotional, little responsibility, inefficient, but frequently good cooks). Italian (not dependable or take responsibility, sloppy at work, but thrifty and excellent cooks). French (very neat, thrifty cooks and sewers, sometimes unreliable or looking to their own interest, but excellent managers; not capable of heavy work).

There is a constantly increasing demand for trained housekeepers in sanitariums, hotels, clubs and semi-private establishments. There is also a great demand for housekeepers in Y. W. C. A. and similar social agencies. Such a person should have the experience of actual practical work, and be able to do any branch of housekeeping in case there is such a necessity. But in addition she is supposed to have the executive responsibility, and to buy foods, running her accounts on a budget system, arranging meals and overseeing servants under her. Such positions demand exceptional ability, coupled with training, and above all, the knowledge of handling subordinates. The task can be compared to the position of sales manager or office manager in the business world. Such positions pay fairly well, not usually any more than the position of housekeeper in a wealthy private 
home. Any position of executive housekeeper demands good appearance and intelligence. There are many women who have had practical experience in their own home, who could easily with a little training qualify for such resident institutional positions. They must know food values, cuts of meat, marketing and arranging of meals, economical buying of equipment and supplies, and usually have knowledge of laundry, cleaning, sewing and care of linen.

In closing this chapter it may be said that much of the solution of the "servant problem" rests with the mistress, and that it lies in her hands to become the fair-minded modern employer instead of remaining the capricious medieval mistress. Only by this adjustment and by doing all in her power to make the conditions surrounding housework on a par with the conditions surrounding other work, will she be able or should she expect to secure and retain high-grade women to give her efficiency and loyalty in the management of her household.

\section{QUESTIONS ON HOUSEHOLD ENGINEERING}

\section{Management of Houseworkers}

r. If you were compelled through circumstances to earn your living as a houseworker, what sort of treatment would you expect from your employer? How much pay?

2. Give a general daily schedule of what you would expect from a worker and show the total time per week.

3. What success have you had (or know of) in giving bonuses with the purpose of keeping houseworkers?

(b) What result for giving extra pay for extra work? 4. Under present conditions and wages figure the cost per hour for a resident worker, taking account of the extra cost of food, fuel, light, laundry, breakage, wear and tear.

5. With what ideas in the text do you disagree? 


\section{HOUSEHOLD ENGINEERING \\ XI}




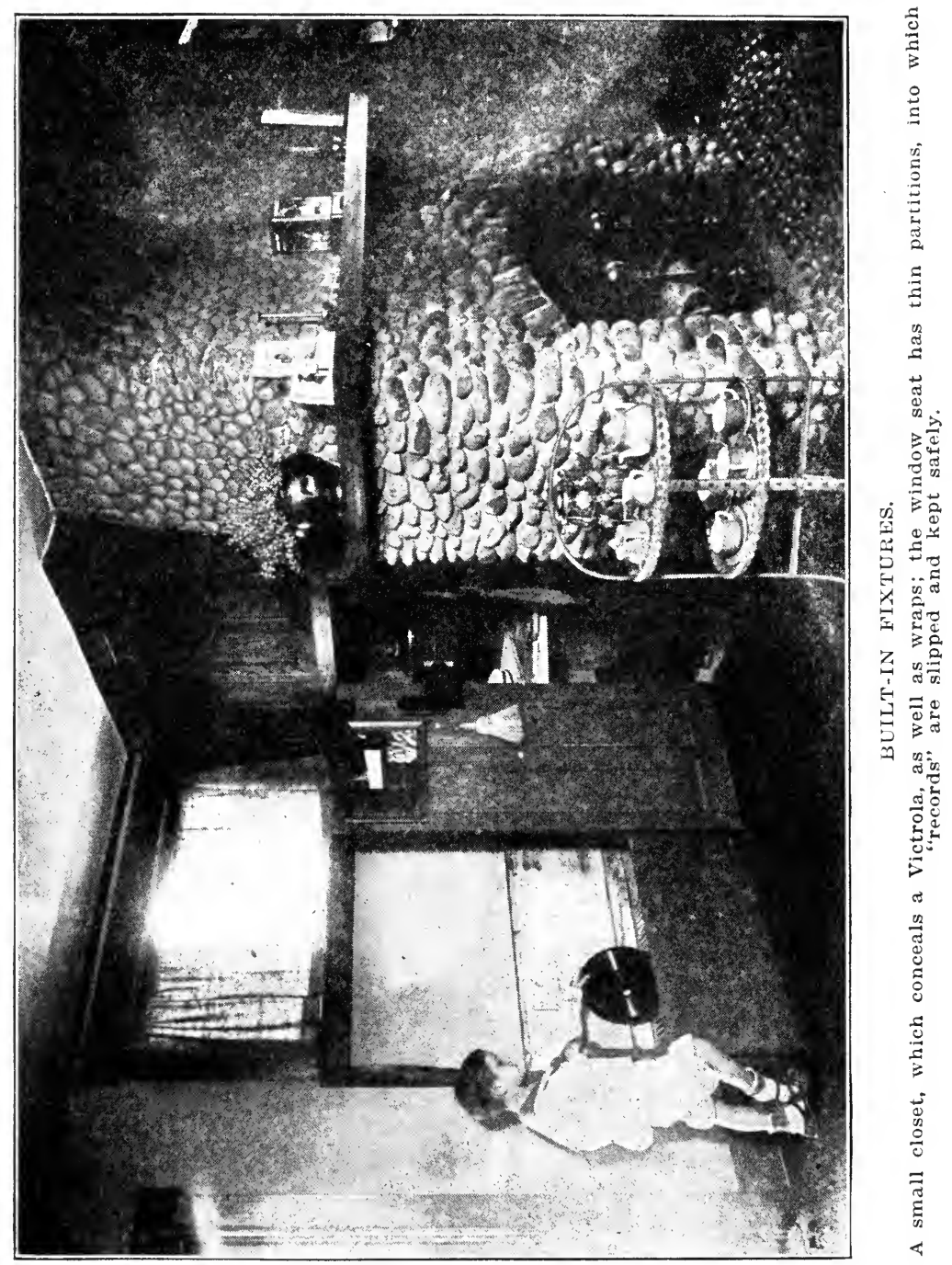




\section{HOUSEHOLD ENGINEERING}

\section{XI}

\section{PLANNING THE EFFICIENT HOME}

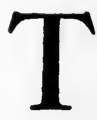

TO HAVE a "home of one's own" is the universal ideal, yet no two ideal homes would be identical. Just as was shown in consideration of the subject of BudgetMaking, how the "budget" must be adapted to the individual family, so the home must conform as nearly as possible to the needs and aims of the particular family dwelling in it.

The question "What is a Home?" may well be asked and answered before going on with definite suggestions for home planning. Is the home merely a shelter where the material needs of eating and sleeping can be satisfied? Or must not the home also provide for the educational, ethical and aesthetic needs of mind and spirit? Truly the efficient home must be built to cover the educational and spiritual demands equally with the practical demands of a house. To illustrate, the efficient home must be so built as to help people, especially children, develop the greatest health, and the best character and possibilities in them, i. e., provision for music, a play room, or work benches for a growing boy, are even more important than hardwood floors, laundry chutes or a tile bath. A "den" for father or a "business corner" for mother may conduce to broader development than the same amount of floor space added to the size of a parlor, unused except for company. 
There is then a sharp distinction between "home planning" and "house planning." The most up-to-date, most laborsaving and most beautiful house might truly be a failure as a "home" unless it provided also for the higher interest's of the family, and was especially adapted to the needs of the particular family using it.

\section{Requirements of the Efficient Home}

The requirements of a normal family of parents and several children for a truly efficient home may be summarized as follows:

I. Privacy given by adequate grounds, shrubbery and interior arrangement.

2. Desirable "exposure" for light and sunshine in the most important rooms.

3. Interior arrangement-compact, labor-saving, easy-tocare-for surfaces and furnishings; rooms for collective enjoyment and for individual privacy and work.

4. Pleasing exterior of harmonious color and line; material and construction maximum fireproof and weatherproof.

5. High-grade plumbing; restful lighting; adequate, clean heating.

6. Built-in conveniences; adequate storage for food, fuel, clothing, etc.

7. Low operating cost; low upkeep and repairs.

\section{NeEd of Privacy}

Privacy is one of the most desirable ends expected in the individual home, and the one quality that chiefly distinguishes the home from a hotel, boarding house or any coöperative living plan. Adequate lot or grounds and concealing shrubbery will partly secure this; but the house construction itself affects the amount of privacy or lack of it. By the use of many windows of the wide "plate glass" type it often happens that the occupants of rooms are always visible 
from the street. Now even more air and more light would be secured if the windows were placed closer to the ceiling with higher sills, which would at the same time insure more privacy. The function of windows is to let in light and air, but they should be so placed or grouped as to give seclusior to the inmates at the same time.

The common "double hung" plate glass window permits only half the window opening to be used for air, is often ugly in line, and prevents privacy. There should be a greater use of casement windows. These permit the whole opening to be used for air, have higher sills, and in many ways are more atractive. The casement-opening-out type is somewhat more weatherproof than the type opening in, and it does not take up room space when opened; its disadvantage is that the screens must be on the inside, but there are various fixtures on the market for opening the windows while the screens are in place; it is also more difficult to clean, as the washing must be done from the outside. Casements which open in should be used above the first floor.

When the climate is very warm or very cold, the windows must be small to protect against excessive heat or cold, but in the temperate climate of our country, and with modern efficient heating plants, the windows may be large or many. Indeed, the tendency of present American architecture is to let in more light and air by the use of numerous windows. The "sun porch" or "sun parlor" is usually the most popular and attractive room in the house.

Many windows increase the cost of heating somewhat but with later types of "window strips" or the less desirable double windows, comfort may be had even in cold weather, and health and cheer of sunshine retained.

Porches should be planned for privacy and preferably should not face directly on the street nor be connected with the main entrance. Any one who has noticed a typical 
"row" of American houses, with all porches adjacent, filled with rocking, gossiping people, will recognize that such an arrangement does not make for privacy.

\section{The Best Exposure}

The most desirable "exposure" or the relation of the principal rooms to the points of the compass and sunshine is the most important consideration in the arrangement of rooms in house planning. It often happens that a house plan is selected having attractive and convenient arrangement of rooms which was designed for an entirely different "exposure." The diagram showing the sun's path in summer and winter will make the following points clear: In the winter time in the northern hemisphere, the sun rises south of east and sets south of west, so that rooms having only north windows will get no sunshine whatever throughout the day. South windows will receive sunshine all day long, east windows sunshine in the morning and west windows in the afternoon. In midsummer the sun rises north of east and sets north of west so that all rooms will get sunshine part of the day. The hot afternoon sun of summer is not desirable.

In midwinter the sun at noon is only a third the way up from the horizon to the zenith and the slanting rays will not be cut off by overhanging eaves or projections. In midsummer the sun is nearly overhead at noon and eaves will protect the windows from the glare of the midday sun.

The prevailing cooling breezes in our eastern states on hot summer days are from the south and west, so that a southern exposure is warmest in winter and coolest in summer, and is altogether the most favorable exposure.

The following, then, are the best exposures for the various rooms :

1. Living room-south will give sunshine and warmth in winter and comfort in summer. 


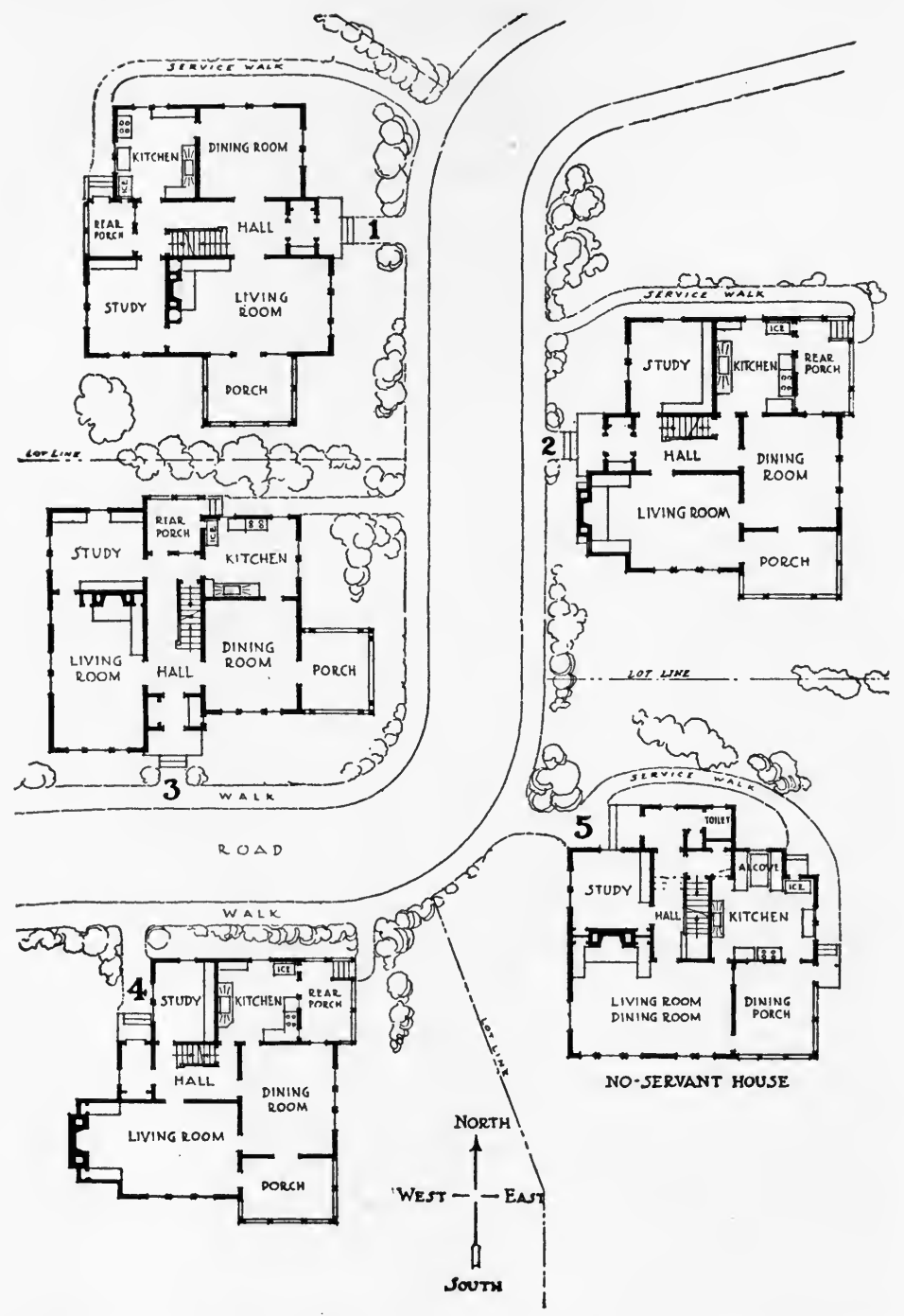

HOUSE PLANS WITH LIVING ROOM ON THE SOUTH, DINING-ROOM EAST, KITCHEN NORTH, PORCH EAST AND SOUTH 
2. Dining room-east will give cheery morning sun at breakfast, winter and summer. West undesirable, because the level rays of the setting sun give discomfort at the evening meal.

3. Kitchen-north and east will be cool, cheery and not too hot and sunny for afternoon work.

4. Living porch—east, southeast or northeast—not west, as that brings the full hot sun in the afternoon, just at the

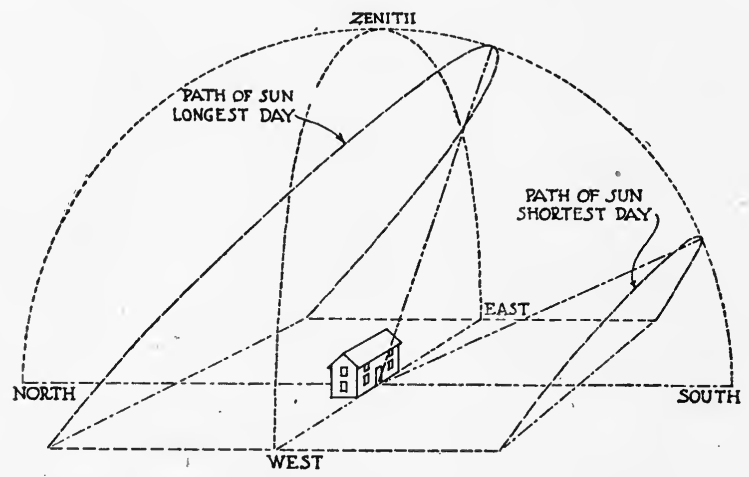

Diagram Showing Path of Sun in Summer and in Winter

SUN RISES SOUTH OF EAST AND SETS SOUTH OF WEST IN WINTER AND NORTH OF EAST AND WEST IN SUMMER

time when home maker and friends have leisure fo: porch recreation.

5. Bed rooms-any exposure except entirely west or full north, which are too hot, or too cheerless, respectively. 6. Sleeping porch-west or south, not east, as early morning sun makes sleeping after sunrise difficult; north, too much exposed to winter winds.

The ideal sun plan of a rectangular house with four rooms on the first floor will have the living room in the southwest 
corner, the dining room in the southeast corner, the kitchen in the northeast corner, and the den, library or parlor in the northwest corner. The natural tendency is to place the living rooms facing the street and kitchen and dining room in the rear, but this is not always desirable on a lot facing. north. The sketches of practically the same house show how the rooms should be arranged on lots facing the four points of the compass. It is not so simple to design an attractive

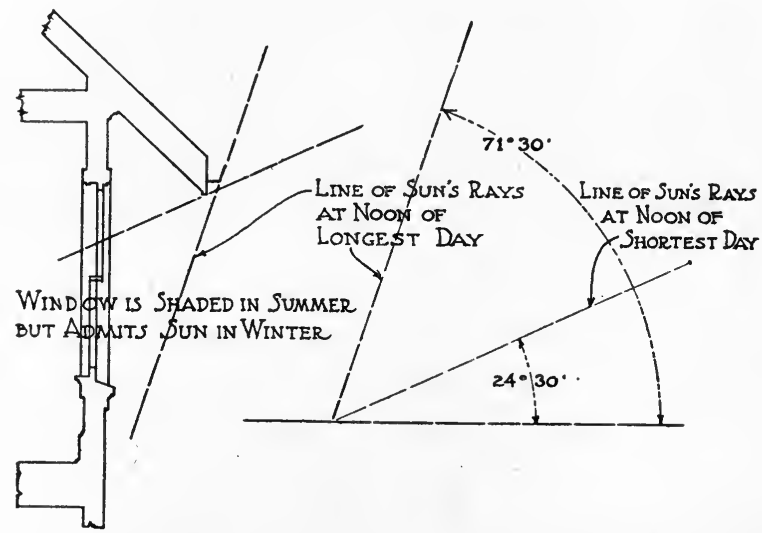

DIAGRAM SHOWING DIRECTION OF SUN'S RAYS IN SUMMER AND WINTER

house with the kitchen and dining room on the street, but it can be done.

When the ideal sun plan does not seem practicable, modifications can be made which will help; for example, a north dining room may have a projecting portion with a window which will catch the morning sun; west porches may be shaded by trees or awnings. Again, if the outlook or view is particularly attractive in any one direction, it may be better to modify the plan with this in mind. Houses on diagonal 
streets can have a more favorable sun plan than those placed straight north and south or east and west.

\section{The Materials of the Efficient Home}

From all practical points of view the house of masonry has advantages over other materials. Either brick, stone and stucco or "hollow tile" make the most permanent, satisfactory houses from the modern viewpoint. All of these materials are more fireproof, more sanitary and need less repair and upkeep than timber. Although the house built of timber may be attractive, today the cost of lumber makes the wood house nearly as expensive as that of more fireproof material, and the wood (particularly in the case of shingles) is not of so sturdy or lasting a quality as the wood which entered into the building of many houses still standing, built a century or more ago. The cement or stucco house on wood frame is not much more fireproof than an all-wood house, but has the advantage of permanent exterior finish.

From the appearance point of view the stucco house can be made more attractive by the quality or roughness and handling of the material as it is applied. It should also be so colored as to be more interesting and harmonious and less like stiff, gray cardboard.

The "style" or type of house can only be decided by personal taste, climate, etc. All that can be said here is that it would be much better to use a given sum of money in achieving a small house well than in attempting a larger or more elaborate house unsuccessfully. Generally speaking, these ideals should be realized in exterior construction:

I. The house should harmonize with the surroundings and seem to be an integral part of them; i. e., low, flat ground needs low roofed houses, not "castle" effects. The house must be "tied down" to the ground on which it rests first, by means of the right construction line of roof, etc., and 
second, by the means of shrubbery, lattice or other means to this end.

2. The type of house must be kept "true" throughout"English" type must not be confused with a "Swiss" porch, or an "Italian" villa with construction features which are clearly "Colonial"; otherwise the house will have a confused, inharmonious appearance. The advice of a good architect is needed here.

3. Absence of unnecessary juts, scroll or stone work, sparing of bays and angles. Straight walls are less expensive to build, appear better and enable the heating arrangement to be placed more satisfactorily.

4. The color plans, whether paint, masonry or stucco, should be pleasing, and as much as possible blended or in harmony with the adjoining houses and the feeling of the landscape.

\section{Efficient Interior Arrangements}

What needs must be met which affect the arrangement of the rooms in any house? There seems to be two broad demands: First, rooms large enough and suited to collective group-living and enjoyment; and second, rooms suited to private or individual comfort and development. With these two demands in view we see that every house should have at least one room large enough for a group of twelve people to be comfortably entertained. Such a room is the living room, which should be in size varying from I 2 by 18 to I 6 by 30 feet, which will give floor space sufficient for the dancing of young people, for a meeting or social gathering without crowding.

Having the second demand in view, we find there are many times when not all the family cares to be a part of the one large group, but some members would prefer to be quiet or by themselves. This brings up the need for a second or 

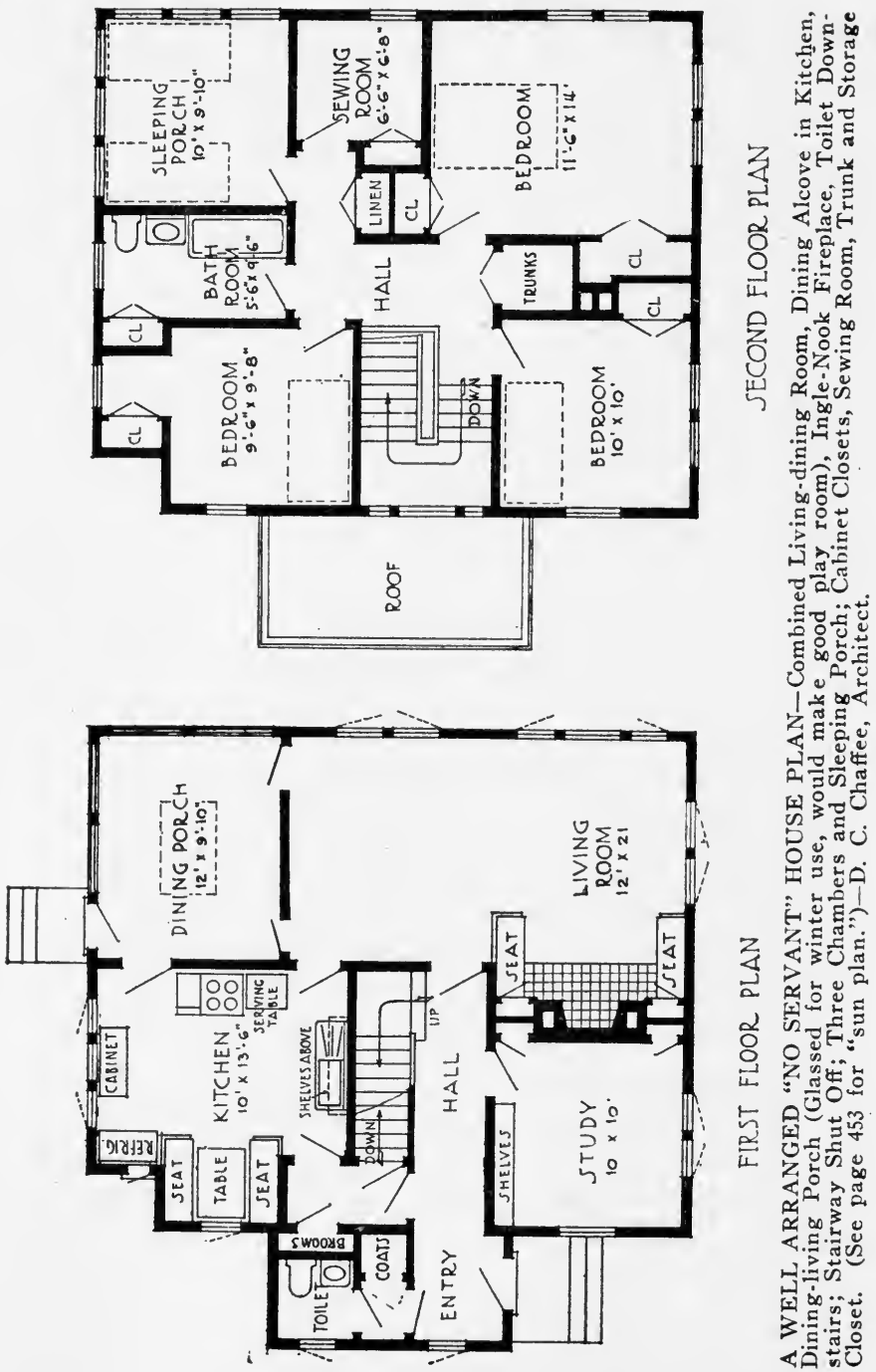
smaller room, which may be a "den," sitting room or library. Many are the occasions when a group of young folks wish to use the living room and when, if there were no extra small den on the first floor, the parents would have no alternative but share the noise of the young people or else.go upstairs to a bedroom. Again, there are many times when the home maker is entertaining guests in the living room when it would be convenient to have other members of the family, particularly the children, in a room by themselves. In families with small children this second room could be most conveniently a play room, or combined sewing room and play room. The one large living room and smaller sitting room are thus the two essential rooms which must be planned for in the efficient house.

\section{Is the Separate Dining Room Essential?}

Why a separate dining room in the small family home? Only about three hours of the day are spent here-does this amount of time justify the special room? If a family has plenty of building space and money, no fault can be found with the separate dining room; but a combination livingdining room seems the more efficient arrangement in the small, moderate cost home. The combined living-dining room is not a theory, but has been successfully and practically applied in many homes. The eating portion of the room can be separated by French or folding doors, or by a screen. A set of four doors instead of two, which fold back on themselves will give privacy to the dining portion and yet permit the dining and living room to be used as one. It is most delightful to have family meals in summer on screened porch, which may be glassed in for winter use. Then there is the popular dining alcove off the kitchen, which further lessens the need of a separate dining room. If there must be a choice between giving up the individual 


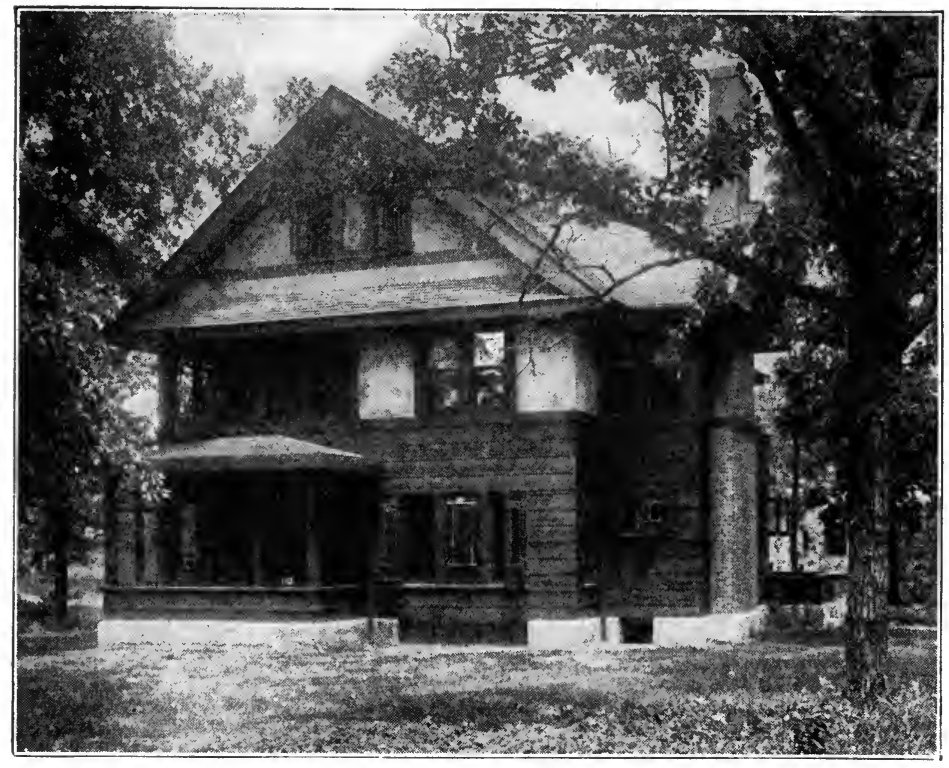

Rear of Hous? with Glazed and Screened Living Porch Below and Sleeping Porch Above

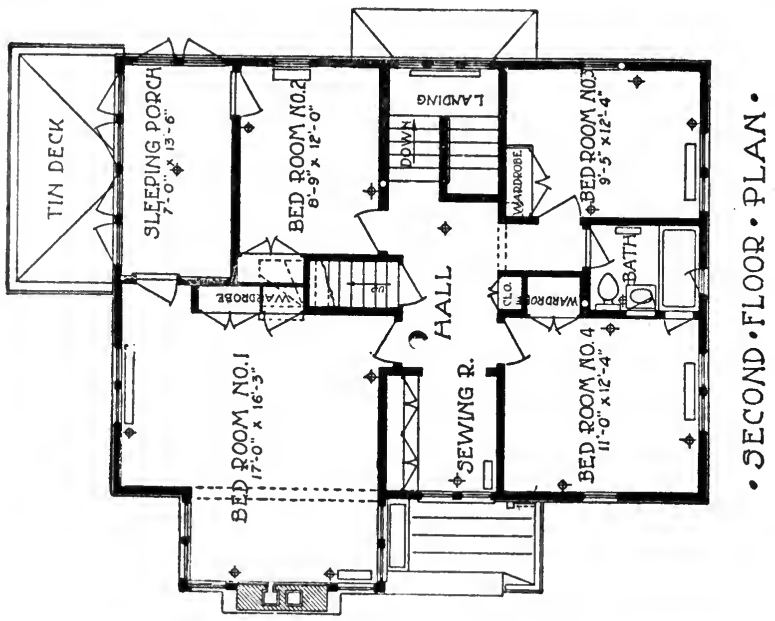




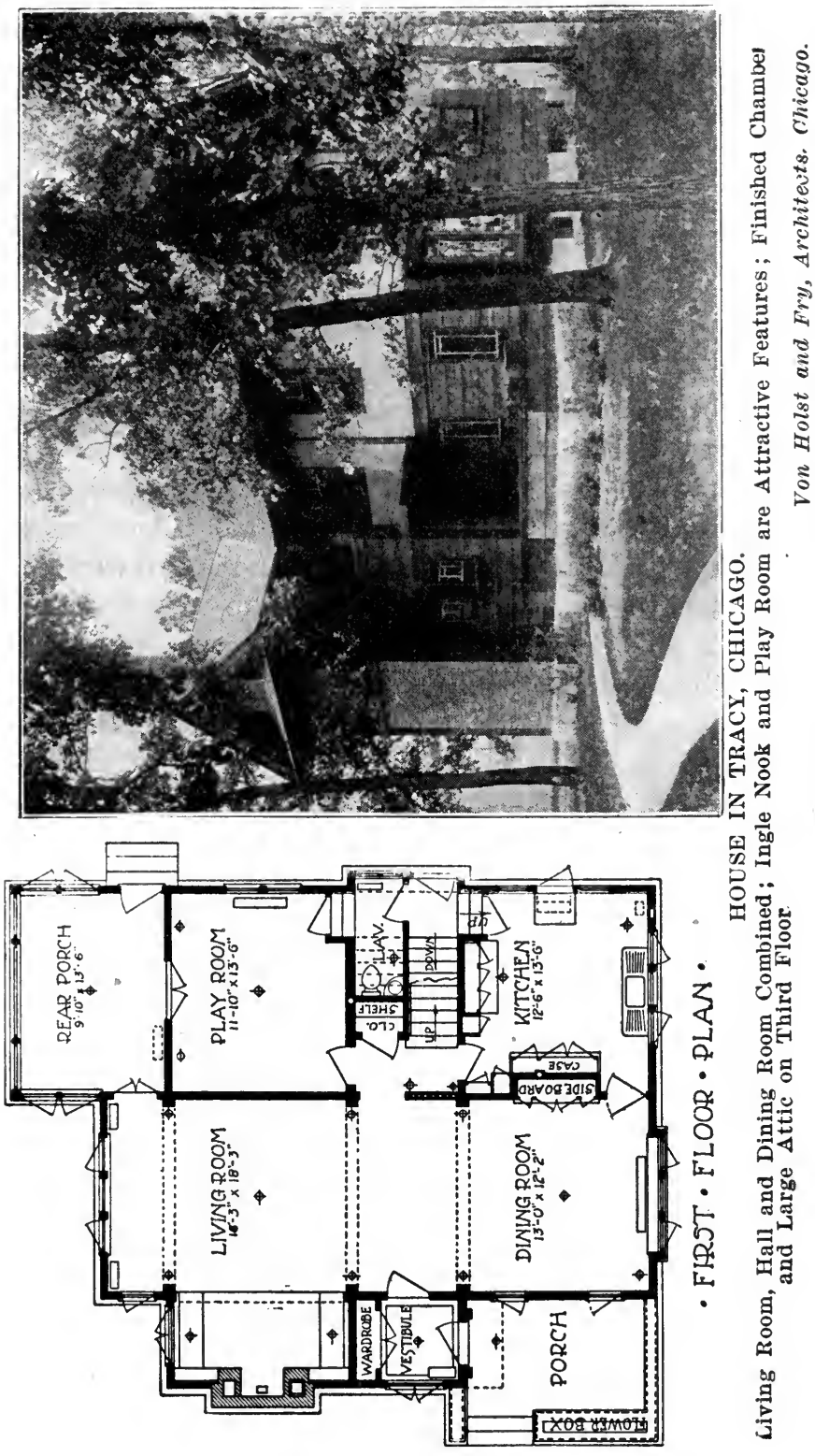


dining room and giving up the small den on the first floor, by all means let the dining room be omitted, rather than forego the small separate "withdrawing room."

\section{The Hall, Stairs and Entry}

Although the plan of the single large living room or livingdining room is excellent, it must be carried out with restrictions. For instance, many plans of inexpensive houses have the main staircase ascend directly from the living room and have the front door open directly into the living room. There are serious disadvantages to both these popular plans. In the no-vestibule plan, opening of the front door brings in a large quantity of cold air, slush and mud in bad weather; also a guest entering has no private place to remove wraps, or again, it often proves awkward or unpleasant to bring a stranger directly into a group in the living room.

The defense of the open stair is that it is picturesque and gives a more spacious appearance. The practical disadvantages, however, are that the open stairway (with no back stair) usually makes it necessary for every one wishing to go upstairs to cross the living room and ascend in full view of all; this is never pleasant, especially with children and servants. The second chief disadvantage is that the open stairway acts as a funnel to suck up heat from the first floor and often carry it wastefully upstairs.

Both front and rear hall should receive careful attention and not be altogether dispensed with. In general, means should be provided so that the front door may be answered without walking across the living room. There should be a back stairway, or the front stair be so placed that children and servants can go upstairs without disturbing persons in the living room or dining room. The rear entry should be so arranged that tradesmen need not enter into the kitchen, and also so that children have some other means of getting 
upstairs than continually tracking across the kitchen or other rooms. It is sometimes advisable to have a rear hall and here locate the telephone and a small seat and make of it a "business corner." It should connect with the rear entrance so that children can take wraps off here and tradesmen enter ; there could be a box for the storage of rubbers, toys, etc.

Rooms opening into each other may look more spacious, but they have two disadvantages-that of creating much harder work in cleaning and much greater possibilities for noise and lack of privacy. It is a poor arrangement where entrance is made directly into a living room and where the living room, dining room, and even den, are all open into each other with possible ineffective portieres. No privacy, no seclusion is possible in such rooms and no thorough cleaning except at a great amount of effort.

\section{Upstairs Interior Arrangement}

Privacy is essential upstairs, and every bedroom should have its own door and should be able to be reached without having to pass through some other room. The bathroom, particularly, should be located so that it can be reached from the hall direct. The only exception to this is where there is more than one bathroom and where the second bathroom belongs solely for the use of some individual bedroom or to two bedrooms.

There should be upstairs space provided to hold cleaning tools so that it will not be necessary to carry the tools from downstairs as mentioned in the chapter on Cleaning. Again, a linen and storage closet should be located on the hall so that it can be reached easily from all rooms.

\section{Built-in Furniture and Fixtures}

Many of us remember, and some of us still possess, the colossal wardrobes, bookcases and chests of past days. Some of these were both beautiful and useful; but from the point 
of view of convenience and modern housekeeping, they took up too much space, were almost impossible to move and seldom fitted in with the rest of the furniture. In the present lower ceilinged houses such immense pieces are highly impractical-the fewer the "movables" in any home the more harmonious the room and the easier to care for. Built-in fixtures need never be moved out to sweep the dust from under them, they take less floor space than movable pieces answering the same purpose, and they can be more successfully finished to match the trim and wall decoration. Also if plans are made for building-in when the house is being built, less portable furniture will be needed and hence more real economy practiced. For instance, a built-in closet for coat racks and a seat for overshoes is much more attractive and is easier to care for than a movable coat rack, settee and umbrella stand; or a built-in buffet will be more efficient and commodious than the usual "portable" sideboard. Here is a partial list of excellent built-in fixtures:

Kitchen cabinet recessed in wall.

Buffet and china closets.

Recessed iceboxes.

Medicine cabinet recessed.

Towel and linen closets in bathroom or hall.

Bookcases with open shelves.

"Pullman" ironing board fitting back into shallow closet.

Window seats which hold wood, magazines, etc.

Ingle-nook fireplace with seats.

Telephone table and seat.

Cedar closets for storage.

Broom or cleaning closet.

Built-in "victrola" cabinet, or cabinets for "records" or player-piano "rolls."

Recess for piano.

Built-in radiator covers.

Closet or recess for table-leaves.

Provision and milk receiver, built-in wall, locks when closed from outside. 
Another group of built-in fixtures is the chute which will save time and labor in house work. It is usual to connect fireplaces with ash chutes which run in the masonry work of the chimney to a hollow space in the base. Sometimes the kitchen range is connected with an ash chute of metal leading to metal ash can in the basement.

Then there is the laundry chute, which, if possible, should have opening in the upstairs hall near the bathroom and in the rear hall or in the kitchen, leading to the laundry. The laundry chute may be combined with a dust chute by a partition in the center or a dust chute may be made of oval furnace piping, run between the studding. The openings for dust should preferably be in the floor, closed by a small trap door. Such chutes are best made of or lined with galvanized iron and always should have self-closing doors at the lower outlet to prevent their acting as flues in case of fire and becoming a dangerous fire risk. Speaking of fire risk, every house should have a number of small fire extinguishers, located near the fireplace, in the kitchen, the laundry and near the heater, also in the upstairs hall.

\section{The Breakfast Alcove}

An especially interesting built-in fixture is the so-called "breakfast corner" or alcove in or adjoining the kitchen. The seats are permanent and the table may be fixed or movable for other uses in the kitchen. The corner should be lighted by one or more windows and a special lighting fixture. Such an alcove adds to the attractiveness of the kitchen and does away with the necessity of a maid eating in the kitchen or having a separate room for this purpose. The dining alcove may be located in the position of the usual butler's pantry, between kitchen and dining room, or in a corner of the kitchen where it may be backed up by the kitchen cabinet, china cabinet or sink without.loss of wall space. Often such a 
"breakfast" alcove becomes a breakfast, lunch and dinner alcove when the family is small, as it saves so many steps. Not infrequently it can be added to existing kitchen.

Still other built-in arrangements are "dumb waiters," best located near the rear hall, running from basement to the top floor and used for conveying food, laundry, waste baskets, etc., up and down, thus saving endless steps. In large houses a "lift" about four feet square is used for the same purposes and for carrying luggage or even one person. The kitchen elevator icebox spoken of before is a modified dumb waiter and is a manufactured article which can be duplicated by a competent carpenter. Another elevator which may be put in when building at small expense runs from the basement to the wood-box, near the fireplace.

Among the special fixtures are disappearing beds which fold in a special section of the wall made to receive them, and aired from outside. Or where great economy of space must be practiced there are other forms of "in-a-door beds" which fold up and can be swung into a ventilated closet or dressing room when not in use, and which are more desirable than the so-called "folding beds." These are particularly well adapted for use on sleeping porches and can be arranged to swing down into the chamber or sleeping porch as desired, being kept dry and warm in a closet between in the daytime. They are made by the Door-Bed Company of Chicago.

The more built-in fixtures the more floor space, the easier the cleaning and the more homelike and permanent the house.

\section{The Matter of Closets}

One of the most important of the group of built-in fixtures is the built-in or cabinet closet. Now, there are good closets and bad ones. The bad ones are those which are built too deep or particularly those which are so narrow and long that the entire front contents must, be brushed against to reach 
the articles furthest back. If the closet is intended for clothes only, and if garments are hung on a vertical rod, it should be built just a little deeper than the width of a dress hanger. Or, another good construction is to have such a system of garment hanging on an adjustable rod which will lift for.. ward, bringing with it five or six garments hung from front to back, after the manner of wardrobe trunks. In this case, a deeper closet would be more practical. In such closets

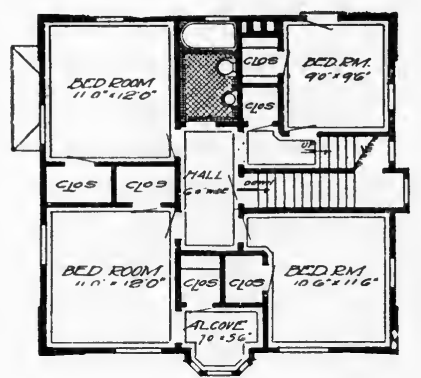

OLD STYLE CLOSET

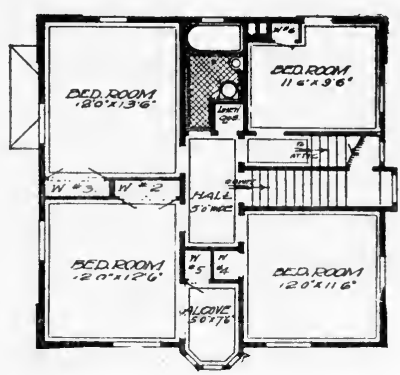

CABINET CLOSET

HOUSE PLANS, SHOWING THE SAVING OF FLOOR SPACE BY THE USE OF CABINET CLOSETS

From the Book of Designs of John Thomas Batts, manufacturer of Closet Furnishings, Grand Rapids, Mich.

the light doors open the entire width and disclose all the contents. Sometimes these cabinet closets are built six to ten inches off the floor and with or without drawer under. This adds to the cost but gives better protection against floor dust.

The same general rules apply to all closet construction. Do not have the shelves so broad that front articles have to be removed to get at those at the back. Do not have the shelves too high to reach, or too far separated, one from the other. High shelves are suitable only for storage, but not for everyday use.

A closet that is slightly oblong is the ideal, and two bedsooms can most conveniently have adjoining closets in the 
waste space between walls. An inclined board fitted with a cleat placed at the back wall of the closet floor will keep shoes in better shape and away from dust. No drawer in any builtin fixture should be too deep, or it will be too heavy to puii out when full; also a deep drawer makes it necessary to turn over the top contents in order to get at the lower layer. Avoid placing a heavy, deep drawer at the bottom of any closet, as this means an uncomfortable strain to bend over and pull it out.

An excellent linen closet is a great convenience. A very desirable one was built as follows: The lower part of the closet consists of different sized drawers to accommodate large and small sheets, towels, cases, etc. The upper part is divided into three sections or open shelves, each having a close-fitting, hinged door. These sections are to hold blankets and may be lined with tar paper or made of cedar wood. If more space is available, a linen room can be built with open shelves on three sides for the holding of blankets and large bedding. Then, some of the shelves can be partitioned off with shallow wooden uprights to fit exactly the size of towels, cases, etc. A drop-light fixture should be in such a room, also in every large closet to facilitate finding articles at night. It need hardly be emphasized that all such shelves and drawers should be labeled clearly for quick identification.

It is not advisable to place the linen closet in the bathroom, as is sometimes advocated, but preferably in the general hall where it will be more easy of access, though a small closet for clean towels is convenient.

In placing a small medicine or similar wall closet, it is much better to "recess" it so that it is flush with the wall, especially when located over a hand basin.

Kitchen and pantry shelves and closets have been spoken of in their respective places; the whole ideal in any closet building is to arrange its spaces so as to most conveniently 
fit the size and character of the contents which will be stored there, thus facilitating handling and preventing articles from being crushed.

\section{Efficient Plumbing}

One of the largest expenses in the American home is the plumbing. To save cost here, the plumbing fixtures should be kept near together to avoid excessive piping; i. e., bathroom should be over or at the same side of the house as the kitchen, and the laundry under the kitchen. The largest piping expense is the 4- to 6-inch "soil pipe" which runs practically straight from the basement through the roof and to which water closets must be connected closely; if toilets are not located near or over each other, separate soil pipes must be provided. If it is not desirable to put in all eventual fixtures at once, by all means plan the plumbing in advance so that the main piping can be put in when building; fixtures may then be put in later without great expense and without serious marring of floors and walls.

All home builders should know the difierence between "porcelain" and "enameled iron," which are often used as interchangeable terms in speaking of sinks, lavatories and tubs. Enameled iron ware is produced by a covering of enamel applied over cast iron. It must be fired in a kiln at a comparatively low temperature and its surface is softer and somewhat more porous than porcelain. The truly vitreous fixtures of porcelain are made of clay which undergoes a drying process of several weeks and then is subjected to a heat of about 2,500 degrees. This insures a high and impervious glaze which does not take stains so easily, cannot rust and is much easier to keep clean. Small sinks and lavatories of porcelain now cost hardly any more than a good grade of enameled iron and are altogether preferable.

Care should be taken to preserve the glazed surface of 
enamel or porcelain and consequently plumbing fixtures should not be scoured with gritty washing powders, nor should strong alkilies or acids be allowed to stay in them for any length of time.

Bathtubs should be of the built-in type, if possible, so as to avoid the cleaning bug-bear of "reaching under the tub." These are less expensive than when first introduced. An extra first floor or basement toilet is needful where there are small children or maids, and if one's pocket book permits, a small but individual bath adjoining the maid's room. A small lavatory or hand basin on the main floor, either adjoining the rear porch or connected with the central back hall, is also a great convenience. Such small lavatories may be located in a closet between two adjoining chambers at moderate expense.

In selecting the outlet traps of all lavatories, sinks and tubs, see that the trap can be detached easily or is provided with a screw cap which can be opened for the easy removal of clogged matter. There is usually a grease trap in the outlet of the kitchen sink to take care of stoppage.

Most of the exposed parts of the plumbing are nickeled; this is nickel plate over brass or bronze. Often the difference in price of two fixtures which look alike is owing to the better coating of nickel of one over the other. Poor nickeling quickly wears off, making the fixture unsightly. Porcelain and vitrified fittings for the bathroom are now replacing much of the nickeled ware formerly used.

The drain from the refrigerator should not be connected with the regular drainage of the house; there must be air space between so that there can be no possibility of sewer gas entering. Often the pipe from the refrigerator empties into the laundry tubs. This pipe is very apt to get clogged and it should be arranged so that it may be taken apart easily.

Other details of plumbing conveniences are "compression 
faucets," which save water, especially hot water; a white porcelain seat on the toilet instead of wood; noiseless tank attachments to the toilet; a hose connection for lawns on two sides of a large yard; a water connection on the porch for flushing; in large houses a small "slop sink" in the rear hall is an excellent addition to the cleaning closet.

The position of the main stop-cock for the water in the basement should be known to all members in the family so that it can be shut off instantly in case of an overflow or accident. It should be tested occasionally to see that it is not too stiff to turn easily. It is also a good plan to have water shut-offs in the principal lines of water supply so that the entire house need not be cut off from water when a new washer needs to be put in a faucet, or other repairs made.

When putting in the rough piping is the time to plan for a permanent vacuum cleaner; this should be located centrally in the basement with openings in each room. Or, if the house is small and compact, one opening in the hall on each floor will be sufficient with which to connect a long tubing. There is no doubt that the cost of such outfits will decrease in the near future, and it is now possible to secure an outfit for about \$I 50 for a small house.

\section{Adequate Heating Apparatus}

There is still difference of opinion as to the relative merits of hot air, steam and hot water for house heating. A hot air furnace is least expensive in initial cost; gives quick heat; provides ventilation when the cold air box is connected with the outside air; gives moist air when a special apparatus for furnishing water for evaporation is installed. The usual water tank situated in a cold part of the furnace is not sufficient to have much effect on the hot air supplied. The disadvantage of a furnace is that distant rooms requiring long runs of piping cannot be heated adequately and in a strong 
wind the rooms on the windward side are difficult to heat; also the pipes take up much space in the basement. A furnace is most suitable for a small, compact house. The furnace must be set deep enough so that there is sufficient angle to the hot air pipes to give a flow of hot air.

Steam heating is best for large houses and is used in nearly all large buildings and apartments. The piping and radiators can be much smaller than for hot water, making the cost of the plant less. No heat at all is given to the radiator until steam is formed so that the water must be kept at boiling - point or above to give heat; the circulation stops immediately if the fire goes down. The temperature of the radiators is over 212 degrees $\mathrm{F}$., which makes an uncomfortably intense heat. There are various systems of so-called "vapor heating," "modulated steam" and "vacuum" steam heating which in part overcome the disadvantages.

Hot water heating is usually considered the best for homes as the water in the radiators can be heated to any degree up to about 210 and the water will continue to circulate and give heat even after the fire gets low or goes out. The disadvantage of a hot water plant is that a considerable volume of water must be heated and it is thus not possible to modify the temperature of the rooms quickly for extreme changes in weather; also large pipes and radiators must be used to give good circulation and sufficient radiating surface. Hot water systems have an "expansion tank," located at the highest point, which is open to the air. If the water is heated much over the boiling point, the water will boil out of the system and cause all kinds of damage, consequently the "expansion tank" should always be provided with an overflow pipe leading to the basement or out of doors.

In the pressure hot water heating system a column of mercury or spring valve is introduced so that the water can be heated over the boiling point. This gives more rapid cir- 
culation and smaller pipes and radiators can be used, which saves somewhat in expense and makes less volume of water to heat.

Radiators should be placed under or near windows or near doors as this aids in the circulation of heated air and heats the cold parts of rooms. More artistic and low radiators are being manufactured; a combined radiator and warming closet arrangement is useful in the kitchen and dining room. Sometimes radiators are enclosed, with space for the air to enter at the bottom and space for the heated air to come out near a window, but it is not advisable to place shelves over radiators as this interferes with the circulation of air. Rooms are warmed by circulating warm air.

With moisture proof and cement lined basements, our American houses are very apt to be supplied with air that is much too dry for comfort or best health. Air at 70 degrees will take up five times as much moisture as air at o degrees F. ; consequently, in cold weather, even if the air outside is fairly moist, it will be very dry when heated to comfortable living room temperature. If no moisture is supplied, it will be drawn from the wood work, furniture and from our bodies. When coming from out of doors into warm, dry air, the perspiration in our clothing quickly evaporates, which produces a very considerable degree of cooling of the body. This is why a room with moist air at 65 degrees seems warmer than with dry air at 70 degrees.

There are a number of kinds of air moisteners on the market, the simplest being a flat corrugated pan which slips over each radiator at the back and is filled with water; others are placed on top of the radiator. For steam radiators there is a valve which lets out steam without noise, said to distribute the equivalent of five gallons of water per day. There is an arrangement for furnaces which supplies water automatically to a pan placed over the dome which will really 
supply the hot air with adequate moisture. Sprinkling the basement floor with five gallons of water night and morning will help somewhat.

Of furnaces, steam and hot water heaters there are types too numerous to mention, but by all means secure a heating plant with capacity sufficient to a little more than heat the house, for it is more economical of fuel to run a large heating plant slowly than to force a plant which is a little too small. The various magazine-feed heaters, like the Newport and Spencer, for hot water or steam, burn the smallest or buckwheat size of hard coal, which is cheaper and require fuel to be added only once in 24 hours in mild weather and twice in cold weather. A good draft is required for such heaters but is needed for efficiency in any heater.

There are several small devices which make the care of any system easier. One is a draft regulator with a dial located in any wall on the first floor, connected with chains to the damper and draft by which the degree of heat may be maintained with fair regularity. Another is an alarm clock arrangement which turns on the drafts at the desired time in the morning so that the house may be warm by breakfast time. The best arrangement is a thermostat which turns on the drafts when rooms go below the desired temperature and turns them off when the desired temperature is reached, thus automaticaily maintaining an even temperature in the house. This saves much coal, especially if from careless management the fire is allowed to burn until the house is uncomfortably hot and the coal only a bed of ashes, requiring a new fire to be built. These thermostatic heat regulators cost about $\$ 60$ installed by a plumber or electrician, but can be purchased from a mail order house for about $\$ 2.5$ and put in by the owner with very little trouble.

An efficient heater will not continue so unless kept clean. An eighth of an inch of soot or dust will reduce the con- 
ductivity of the heat absorbing surfaces to a very considerable extent. Steam boilers in factories are usually cleaned twice daily; house heating plants should be cleaned with a wire brush or otherwise daily or once or twice a week, depending on the fuel and heater. The heater should be thorougilly cleaned and overhauled in the spring and treated according to the directions of the manufacturers, not left to accumulate moisture and rust throughout the summer.

To save fuel, all hot water and steam pipes should be covered with asbestos pipe covering and the heater itself with asbestos cement; even furnace pipes should be covered with sheet asbestos.

\section{Tasteful, Restful Lighting}

Daylight comes chiefly from the sky, and is a perfectly diffused light, except direct sunshine, which is too intense for eye comfort. House lighting should approximate natural conditions and the best diffusion of the light for the general illumination of rooms is best obtained from the now common form of "indirect" lighting fixtures, in which all or nearly all of the light is thrown onto the ceiling, from whence it is diffused to all parts of the room. The "semi-indirect" fixtures, made with a bowl of thick opalescent glass, throw some of the light downward and are ideal for the general illumination of living rooms. The ceiling must be white or cream colored and the fixture suspended from the correct distance to obtain proper illumination without serious loss of lighting efficiency. Indirect lighting fixtures may now be had for gas as well as for electric lamps.

This soft, general illumination may be made sufficiently strong for reading in all parts of the room, but the better practice is to have individual portable lights or wall fixtures for close work like reading, sewing, music, etc.

All lights should be shaded so that the full glare of an 
intense light cannot fall directly in the eyes. This is especially needful with the high intensity of tungsten or "Mazda" lamps and Wellsbach mantles, which have many times the light intensity of the old-style carbon filament electric lamps and open flame gas burners. A softer effect may be secured by using frosted or ground glass globes instead of globes of clear glass. But even frosted globes should be shaded, preferably by shades which give an artistic color effect.

A point to remember in the installing of fixtures is the direction in which the greatest candle power occurs. In an electric bulb and in an upright gas mantle lamp the greatest intensity of light is directed horizontally ; in the inverted gas mantle the light is directed chiefly vertically downward. When it is desired to concentrate light downward, an inverted gas mantle or electric lamp with deflecting globe should be used, but when general illumination for room space is wanted then the upright gas mantle lamp or upright tungsten lamps should be used.

The worst possible light for a kitchen is the central drop electric light on a swinging cord, usually furnished without a reflector. It would be better to use wall fixtures over sink, stove and table or one indirect bowl to light the entire room.

In too many houses the details of artistic lighting are badly neglected and the discomfort of poor lighting not realized. The local electric light company will usually furnish information and booklets on the subject or very interesting material may be obtained by sending stamps to the Illuminating Engineering Society, 20 West 39th St., New York City.

\section{Drawing House Plans}

A truly efficient home must usually be "made to order," and the prospective house builder must have a fairly definite idea of what is wanted to be able to order it. This means furnishing the architect or builder with sketches or drawings 
made to scale. For preliminary sketches, a scale of $1 / 8$ inch in the drawing equals one foot in the house is commonly used. The working drawings are usually made with $1 / 4$ inch equal to a foot; larger for detailed drawings. Sketches are most easily made by the amateur housebuilder on cross section paper lightly ruled in eighth-inch squares. The first step in planning a house is to measure a few familiar rooms so as to have an idea of how large a room, say 12 by 16 feet, really is ; also to measure width of stairways, doors, windows, etc. Existing furniture should be measured so that it may fit wall spaces in the proposed house. It is usually easier to modify a plan found in magazines or house books which meets nearly all the requirements, rather than to start altogether anew.

The most difficult part of house planning is to arrange for the stairways; often not enough "head room" is provided for. The rule for stairs is that twice the "riser" plus the "tread" should equal 24 inches. Stairs with $61 / 2$-inch riser and I I-inch tread are very easy; a 7 -inch riser and Io-inch tread makes a comfortable flight; 8-inch riser with 8-inch tread makes a rather steep stairway but one often used for attic or cellar stairs.

The ceiling height is now from eight feet, in small-roomed houses, to ten feet in large houses with more spacious rooms. A house with a nine-foot ceiling will measure ten feet between floors, allowing a foot for floor joists, plaster and flooring. A stairway with 7 -inch risers will require $I 7$ steps from the first to the second floor in this house. If the stairway has a straight run and Io-inch treads, this would mean I6 times Io, or I60 inches, or 13 feet 4 inches from the edge of the first step to the edge of the landing floor. (Note that there will be one less treads than risers.) "Head room" under a landing would require a rise of at least 6 feet 2 inches, plus 8 inches for floor joists, etc., or 84 inches; this 
would be given by twelve 7 -inch risers, and with Io-inch tread there would be needed eleven times ten, or I Io inches, or 9 feet 2 inches. If the landing were 3 feet ro inches wide, the distance would be 13 feet from the beginning of the stairway to the wall side of the landing. From the landing to the second floor would require five steps more, but as there is one less tread than riser, this would make four times ten, or 40 inches.

The second floor can overhang the beginning of the stairway a certain amount, depending on the height of the ceiling and the steepness of the stairway. With the stairs we have been considering, three steps up make $2 \mathrm{I}$ inches rise, leaving "head room" of 6 feet 3 inches; these three steps would give 30 inches of "tread," so the second floor could project that much and still give sufficient head room. Too much projection is not desirable as it interferes with taking furniture up and down stairs and with the appearance and lighting. Winding stairs or stairs with "twisters" economize space but are somewhat dangerous, especially with the "twisters" at the top.

It is usual to place cellar stairs under, and attic stairs over, the main stairway to economize space, though if the roof lines would interfere with the attic stairs in such position, it may be better to run them parallel to the ridge pole, between chambers, and use the space underneath for closets.

As a general rule the partitions between rooms on the second floor should come directly over the partitions on the first floor, otherwise floor joists must be made extra strong, and even then ceilings are apt to crack from warping due to unequal drying out of the woodwork.

The least expensive house for the usable space is square or rectangular in outline, with one stairway, one chimney and one soil pipe. A bungalow costs somewhat more than the same floor space in a two-story house because the extra ex- 
pense for excavation, foundation and roof more than overbalance the saving in wall and stairs.

The planning of the kitchen should have special attention, but the subject has been well covered in Chapter I, pages I9 to 48 . The position of sink, range, refrigerator and cabinets should be clearly drawn in to scale. In the living room and chambers the position of couches, piano, beds, dressers, etc., should be carefully determined.

After the sketches are made they should be turned over to a good architect who may be able to suggest improvements and who will make the "working drawings" and the "elevations." He will be able to give the artistic roof lines, entrances and constructive details which go so far to make or mar the appearance of the house. He will also make the detailed drawings for built-in sideboards, bookcases, cabinets, as well as for the windows, doors and "trim," which is usually made at a mill and called "mill work." A good architect can easily add much more than the five or seven per cent of the cost charged for his services to the value of the property. In this country the sale value of the house always must be considered, for it is not often that a house is occupied by the same family for more than ten to twenty years.

\section{The Country Home}

$\Lambda$ farm house can now have all the modern conveniences of a city home and at a cost of less or no more than the difference in the price of the land. Even a small city "lot" costs $\$ 1,000$ or more and a good sized one from $\$ 3,000$ to $\$ 5,000$ or more; the farm house lot is usually worth perhaps $\$ 100$ at most.

Modern city improvements consist of (I) running water; (2) sewerage system for kitchen, laundry and bathroom;

(3) electric current for light and power; (4) gas. 
The best equipment for running water is a pneumatic tank, located in the basement, into which water and air are pumped under pressure. The compressed air in the top of the tank forces the water all over the house like any city supply. The pump is best operated by an electric motor which is automatically stared when the pressure becomes too low and stopped when the required pressure is reached. The cost of such an outfit is from $\$ 120$ to $\$ 150$. The pump may be operated also by a small gasoline engine which may be located at a distance from the tank, or by a windmill. The source of supply may be a well or cistern. If the well-water is very hard it may be advisable to have two tanks, one for cistern rain water and the other for the hard water. The hot water supply can be heated with a coal or kerosene heater as described on pages 52 and 53 .

A sewerage system usually involves the construction of a "sceptic tank," which is a modernized cesspool with overflow piping distributing the purified sewage underground in a safe and odorless manner. They work continuously summer and winter and require little attention or cleaning. About $\$$ Ioo would cover the cost.

A small automatic electric light plant operated with a gasoline motor may now be purchased for $\$ 200$ up. These produce current of 32 -volt intensity and special small motors, fans, vacuum cleaners, electric irons and toasters may be obtained at about the price of similar equipment for the usual I Io-volt current. Then there is the acetylene gas plant for light and for cooking, as well as plants for making gasoline gas. But it is. less expensive to use kerosene as fuel for cooking and the latest types of kerosene ranges are but little more trouble than a gas stove and cost less than a good coal range.

Ice can be harvested and stored on most farms; if not, then there are various "iceless" refrigators, consisting of a 
circular metal compartment with shelves which run through an 18-inch galvanized iron pipe from kitchen, pantry or porch through the basement floor, or about eight feet into the ground under the porch. The food compartment is raised or lowered by a crank and pulley arrangement. The cost is about $\$ 30$, or no more than an ice refrigerator. A temperature of between 55 and 60 degrees $F$. is said to be maintained.

A furnace or hot water heater is just as easily installed in the country as in the city, and indeed the farm house can be made as efficient and labor saving as any city house for the cost of an automobile, and any farmer who can afford an automobile, can afford to have an efficient house. A good way to start to investigate costs is to send for the special catalogs on plumbing, heating and electric lighting, of Sears, Roebuck \& Co., Chicago; then send for the catalogs of the Western Electric Co. and other specialty manufacturers.

\section{Sanitary Arrangements}

The kitchen garbage, waste paper, etc., can be disposed of in a convenient and sanitary way by an "incinerator," but these cost from \$75 up, which seems rather expensive. An arrangement which will do similar work is a so-called "kitchen heater," made to attach to a gas stove, which may be obtained from gas stove makers. These come with a gas burner "lighter" and cost only $\$$ I 2 to $\$$ I5. Some of them are furnished also with a hot water front. Another kitchen incinerator consists of a small brick chimney into which all garbage, waste paper and tin cans are put, the whole being burned once a week and the ashes taken out of the door in the basement.

A convenient garbage can is the Sanitas, which opens by foot pressure. The out-of-doors, underground garbage receptacles are also useful and keep the garbage away from 
dogs and flies as well as out of sight. In communities where there is no garbage collection it can be buried in a shallow trench or given fresh every day to chickens or stock.

Each community breeds its own flies and mosquitos, for neither pest can fly far. The flies propagate in horse manure and uncovered garbage; the mosquitos in stagnant pools of water, tin cans, cess pools and catch basins. It only means enlightened public sentiment and a comparatively small expenditure of money to rid any town of both these pests. Valuable bulletins may be obtained free on flies and mosquitoes from the Dept. of Agriculture, Washington, D. C.

Community housekeeping in keeping streets clean and abating the smoke nuisance affects materially the amount of cleaning and laundry work in each individual household. It is to be hoped that universal suffrage may help to do away with much of the dust and smoke now so common. The cost of community cleanliness would be, in all probability, less than the individual cost of extra cleaning and laundry work. Neighborhood clubs often can have streets "oiled" at very modest expense per family and do away with much of the street dust.

QUESTIONS ON HOUSEHOLD ENGINEERING, XI

Planning the Efficient Home

I. If you were planning to build, what would you include?

2. Would you favor a combined living-dining room?

3. What built-in fixtures would you have?

4. Could these be added to your present house:

(a) Sleeping porch (b) Dining or living porch

(c) Dining alcove? Show how by sketch.

5. What are the most serious faults in your present house? How might they be helped or remedied? 


\section{HOUSEHOLD ENGINEERING \\ XII}




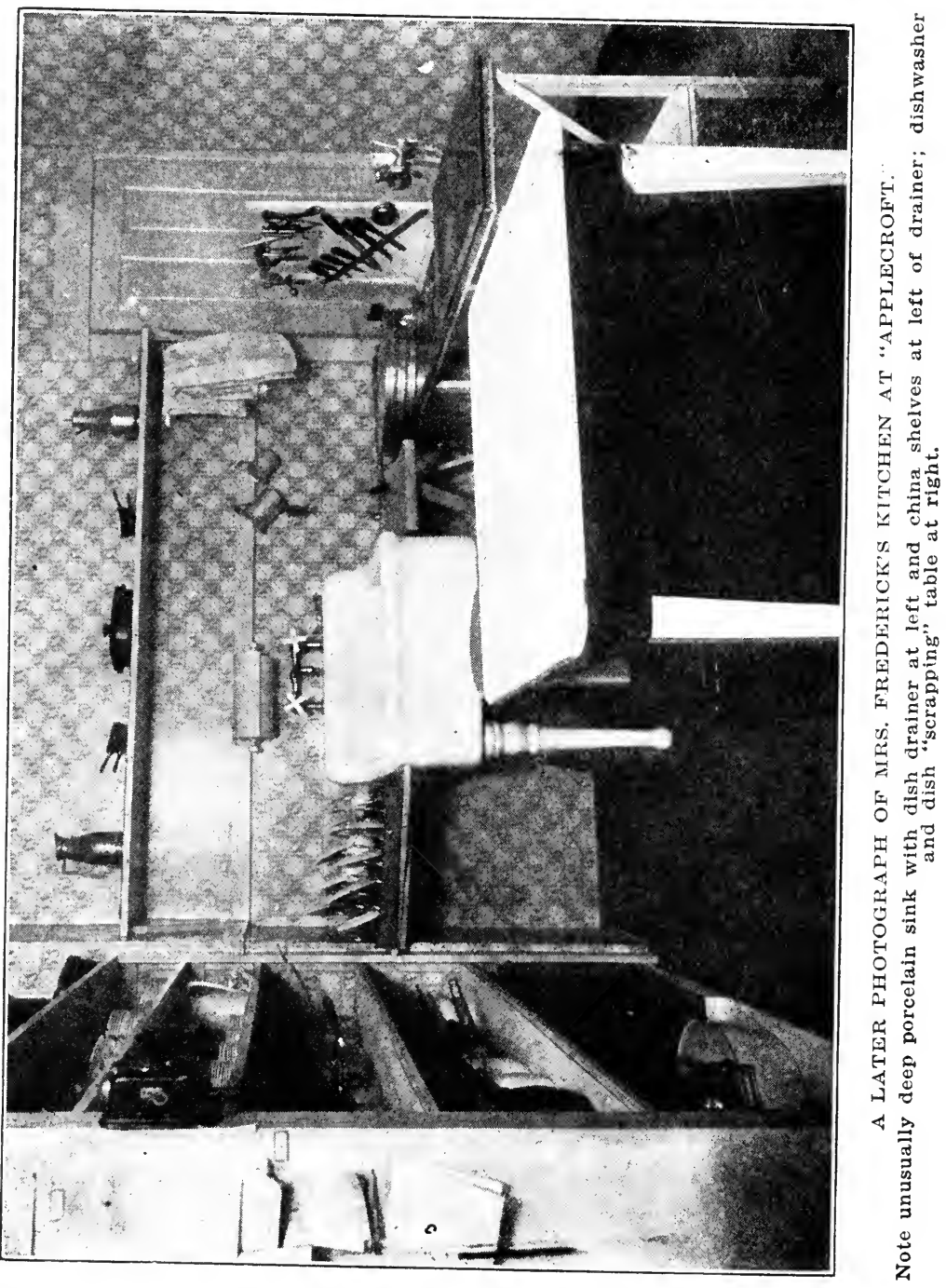




\section{HOUSEHOLD ENGINEERING}

\section{XII}

\section{HEALTH AND PERSONAL EFFICIENCY}

$7 \mathrm{HE}$ basis for all efficiency in work, whether in industry or the home, is health and controlled vitality. We may put a worker into the most efficient kitchen, we may hand her the most useful labor-savers, we may show her the easiest way to wash clothes, but all our teaching will be in vain if the worker is ill, if she "fags" easily, or if she is subject to headaches, "nerves," or other physical ailments. The woman with a headache cannot intelligently plan a family budget no matter how much she may know about one, nor can an "all-tired out" woman take enough interest in the subject of nutrition to enable her to feed her family a balanced ration. The important factor in doing work is the human factor-is the woman herself. Any improvement that can be made either in methods of work, or in the tools with which work is done, is important only in its effect on the welfare and health of the human worker-the woman in the home. Let it be repeated-household efficiency, and above all, personal efficiency, depend absolutely on the physical and mental health of the woman herself.

Although both men and women suffer from sickness and disease, the records of unbiased insurance companies assure us that women are considered the poorer "risks," and that 
women as a group are most subject to attacks of illness. This is probably due to the fact that until a few years ago it was considered "unwomanly" to indulge in active outdoor sports; it was thought "indelicate" to talk about and study the body and its functions; and there was a strong popular feeling against women wearing comfortable clothing or taking exercise which might make them "masculine."

We are fortunately entering a new period when it is decidedly "good form" for girls to be "athletic," and where, in schools, girls' camps, and in the popular mind, the close relationship between efficient work and good health is being daily more clearly recognized.

The homemaker of today must allow for definite health care in her daily schedule. Housekeeping will be drudgery or the reverse largely through her physical ability to meet the work-demands of house tasks, and her mental ability to withstand the tension of childbearing and child care. But the aim must be, not to see just how much strain they can endure, as much as to see how great they can make their strength to accomplish more work. Far too many women pride themselves on "what they can do," rather than on "what they are," and refuse to take time to build up their surplus vitality so that it shall be equal to the tasks and demands imposed by the three-fold service of housekeeper, wife, and mother.

There are several definite factors which the homemaiker must consider in her efforts to secure and retain health as a basis for personal efficiency:

I.-Air and sleeping conditions.

2.-Food and eating habits.

3.-Posture and comfort needs.

4.-Fatigue and balanced work conditions.

5.-Clothing. 


\section{6.-Exercise of the body for recuperation.}

7.-Exercise of the mind, emotion and aesthetic sense.

8.-Mental attitude toward her work.

\section{Importance of Fresh Air During Work Hours}

We can live for days without eating and drinking, but we cannot live without air for more than a few minutes. The homemaker, who spends possibly 60 to 80 per cent of her time indoors, must be sure to see that the air supply she breathes is adequate and changed sufficiently often. Moving air is of far more value than still air, for we take in about one-fifth the expired breath when there are no air currents; therefore windows should be opened from the top, and doors opened to give cross ventilation, or a horizontal windowventilating board inserted beneath the lower sash in winter. House temperature should be about $60-68$ degrees, not higher, since heat is debilitating, and overheated rooms are responsible for far more sickness than colder rooms which are more invigorating. It is excellent to accustom oneself to a low temperature and not "coddle" oneself as many homemakers do by becoming so used to indoors that they are not hardened, and are therefore more subject to cold, grippe, etc. House air should also be moist, since in cold dry weather it is estimated that the air supply of a house needs at least ten gallons of water every 24 hours.

The need for constantly changed air in the kitchen, where the homemaker spends so much time, bears repeating (see page 42). If the house is constantly permeated with cooking odors, then there is decidedly something wrong or lacking with the ventilation measures, which should be remedied. A professor friend of the author's constantly refers slightingly to, "The Home-the Museum of Smell and Taste." Certainly too many homes, and particularly kitchens, are 
so badly aired that the worker becomes overheated, faint, and enervated as she stands at stove or bends over sink.

Again, dust is a big enemy of the houseworker; house conditions should be such as to permit the least dust to collect; the carpet sweeper, the vacuum cleaner, oil dusters and small removable rugs are important not so much as laborsavers as because they minimize the number of impurities and disease germs, especially those which attack the nose and throat and come from expectorations and dirt tracked into the house.

Even if the homemaker finds it difficult to have a constant supply of fresh air in the house during the day, there is one time when she can control the air supply, and that is at night. The modern sleeping porch has come to stay, and outdoor sleeping is no longer a fad. Such outdoor sleeping should appeal to woman, so many of whose hours are spent in confining rooms, especially in apartment or small city houses. Night air is purer than day air, being free from smoke, rising dust, etc. Some women may say, "Oh, but I always sleep with both windows open." But there is no comparison between outdoor sleeping, and rest taken for $7-8$ hours in an indoor room, however well ventilated, which has been heated and occupied during the day and subject to house odors. Any porch, up or downstairs, can be screened, or be fitted with swinging windows at little expense; or a sleeping porch can be "built on," thus greatly enlarging the sleeping-room capacity of the house.

The secret of successful, comfortable, outdoor sleeping is to have as many blankets under as over the sleeper. The author has slept outdoors for five years with marked benefit to health. Two of her children have never slept indoors, and even as this chapter is being written, the baby four months old, has been sleeping outdoors in a temperature of 
2 below, with splendid results in uninterrupted night sleep from 9 p. $\mathrm{m}$. to $6 \mathrm{a}$. m., with no feedings between. Outdoor sleeping increases the supply of fresh air, which in turn stimulates digestion and gives additional repose to the nerves. Some families spend large sums spasmodically, going to expensive health resorts, when they might every night secure free, in their own locality, air just as pure and invigorating. Only one glance at the annual statistics showing the fearful mortality from tuberculosis-a "house disease"-throughout the nation, should induce every homemaker to urge outdoor sleeping on herself and her family, so that they can by this little at least, combat the increasing evils of confining business and industrial life.

Single beds always permit more comfort; and it is to be hoped that the sensible habit of having separate beds for each parent and member of the family will become even more popular. The homemaker, especially, should try by all means to secure private, uninterrupted sleep for $7-8$ hours to enable her to "carry on" to the end of the busy day which usually falls to her share.

\section{Eating Habits and Their Effect on Personal EFFICIENCY}

Much has been said about feeding the family and using the "balanced ration"; yet it appears that women, and particularly homemakers, do not give as much attention to their own eating habits as they should. It has been found that more women doing housework are either over, or under weight than women in outside occupations; large numbers of homemakers from 35-50 years of age are carrying excess fat. Tho this may be due to other contributing causes, it must be admitted that many women overeat, compared to their needs based on their height and the work that they do. 
Many women" say, "Oh, I know I'm fat, but I feel all right anyway." Nevertheless such women should practice those habits which will keep weight down automatically, no matter how well they feel, because (I) excess fat is unattractive from the appearance standpoint; (2) overweight after 35 years (according to the best insurance statistics) is closely associated with a high death rate; (3) and excess weight particularly handicaps efficiency in work or recreation.

Every homemaker, then, should closely estimate her own dietary. If she has servants and merely makes the beds or does light dusting, etc., then she needs only approximately I ,800-2,400 calories daily ; but if she does most of her housework, including the heavier work of room cleaning, laundry work, etc., then she will need more nearly 2,500-2,800 calories. (See page 33I.)

IDEAL WEIGHT TABLE FOR 'WOMEN AT AGE OF MATURITY (30 YEARS)

\section{Height Pounds}

$4 \mathrm{ft} .8 \mathrm{in} . .$. II 2

$4 \mathrm{ft} .9$ in.... II 4

$4 \mathrm{ft}$. Io in.... II6

$4 \mathrm{ft}$. II in.... II 8

$5 \mathrm{ft} . \quad \ldots$ I 20

$5 \mathrm{ft}$. I in.... I 22

Height Pounds

$5 \mathrm{ft.} 2$ in.... I 24

$5 \mathrm{ft} .3$ in.... 127

$5 \mathrm{ft} .4$ in.... I 3 I

$5 \mathrm{ft} .5$ in.... I 34

$5 \mathrm{ft} .6$ in.... 138

$5 \mathrm{ft} .7$ in.... I 42
Height Pounds $5 \mathrm{ft} .8$ in.... 146

$5 \mathrm{ft} .9$ in.... I 50

$5 \mathrm{ft}$. 10 in.... I54

$5 \mathrm{ft}$. II in.... I 57

$6 \mathrm{ft}$.

.... I6I

(From the tables compiled by forty insurance companies, and published by the Association of Life Insurance Medical Directors.)

\section{Evils of Constipation}

One of the chief evils of which women permit themselves to become victims, is the habit of constipation, and poor elimination of the colon and alimentary tract. Thousands and thousands of women habitually suffer from headaches, dizziness, fatigue and similar symptoms, which are directly tracable to an overstuffed and inadequately emptied colon, or 
lower bowel. Physicians call such conditions "auto-intoxication," meaning that the contents and poisons of the intestines which are not removed quickly enough, "back up" (just like an overful waste pipe or sewer) and thus poison the blood supply. It is this poisoning of the blood with waste and decaying food residues, that makes the "headaches," the "tired feeling" of which so many women constantly complain. Many women think they are "tired" from work, whereas the truth is that they are suffering from a slow poisoning of their systems due solely to inactive, sluggish elimination of food waste. Three times a day is the natural, normal evacuation of the bowels, or a movement after each meal to keep the system in the most healthful condition; once a day is the minimum.

Medicines and dangerous cathartics which irritate and often induce after effects of marked constipation should never be used. There are foods and diets which will normally gain the same results, and the homemaker, as a specialist in food values, should be the very woman to make intelligent use of such diets to improve her own health and thus retain and increase her personal efficiency. Anti-constipation measures are: (I) Much more "bulk" or "roughage" foods like the coarser vegetables, carrots, spinach, cabbage, celery (especially eaten raw), and the green foods like lettuce, chicory, romaine need be eaten; (2) "roughage" in the form of coarsely ground cereals, particularly bran, which may be eaten in some form at every meal, whole oatmeal, cracked wheat, and coarse flours and breads; (3) raw fruits like oranges, pears, grapes, berries, and such dried ones as figs, prunes, raisins; (4) avoidance of coffee and tea, which both stimulate and derange the liver and digestion; (5) minimum of meat, especially the red meats; (6) liberal drinking of water or fruit juices, from 2-3 quarts per day, between meals. 
Emphasis is here laid on this point of right feeding and adequate elimination, because no one other single health point is so vital to women's efficiency and so generally neglected, with the result of "nerves," irritability, and greatly impaired work power. As a class, too, women overeat of sweets and undereat on strengthening foods, as compared with men. They have delighted in the unhealthful fineness of bolted white flour, the excessive sweet of preserves, jams, and dessert fripperies. Women in the home, as well as in business, tend to prefer chocolate, puddings, and starchy foods, wrongly using a less quantity of protein, vegetables, and fresh fruits than they should. This excess sweet is favorable to teeth decay, "acid mouth," etc., and unfavorable to the strong muscle and blood development necessary if women are to work efficiently at maximum output of both their muscle and their mental power.

Other Mistaken Eating Habits of the Homemaker

Again, many women who do their own housework habitually undereat or have contracted wrong eating habits. Frequently such thin, nervous women and quick workers allow themselves to "eat any old time." Now, there is a bodily "rhythm" in every bodily act, and once the muscles have adjusted to a definite eating interval (or any other definite interval), that period should be observed, even tho we eat only a small quantity at a time. Many thin, nervous women are exceedingly rapid workers and can just "pitch in and do wonders" in a short time. Ofter they get started on a special stretch of work, key themselves up, and then refuse to sit down and eat until this long, badly planned work stretch is finished. The chances are that at the end of this prolonged strain, they either don't eat at all, or they eat hastily and ravenously. 
Another wrong habit practiced by women who are at home alone during the day is to "seize a snack" of cold stuff from the pantry and eat it hurriedly instead of taking time to prepare a warm dish. One should never eat when tired; never eat heartily before active physical work; never eat when the body feels "tense," or when suffering from excitement or anger. Definite regular meal periods must therefore be included in the daily work schedule, and work so planned as to avoid excessive long stretches of effort without adequate "rest periods."

\section{The Importance of Right Posture}

"Oh, but I get so tired doing housework," is the complaint of many women. Now, I ask fairly, is it the work itself which makes the "tired," or is it the way it is done? Is it dishwashing as a piece of manual work, or is it the stooping over a low sink? Is it the paring of vegetables, or is it the standing the slouchy way, weight on one hip? In other words, too often blame is laid upon the task itself, which should be put upon conditions or the manner under which the work is done. Right posture is an essential point in work efficiency. Frequently there is a slouchy poise, particularly while standing before table or stove; the weight may be balanced unevenly, or the shoulders allowed to stoop, thus keeping the chest in and not permitting proper breathing. Now a slouchy posture causes the blood to stagnate in the liver, and causes a feeling of mental confusion and despondency, and is often the cause of chronically cold hands and feet.

Again, if the abdomen is constantly held relaxed, the results are very bad, as this posture interferes with digestion, and is a contributing cause of many women's diseases. For these reasons some form of corset or abdominal belt or supporter should always be worn when doing the manual 
tasks of housework, especially by women who have had children and whose abdomen is unduly relaxed from childbearing.

Flat feet and broken arches are also more common in women than in men, largely due to faulty standing, which places unnecessary strain on the arches of the foot. More sensible shoes with broader heels will help, as well as always trying to stand in a poised symmetrical way with weight on balls of feet, not against the spine. Often the leg muscles are weak, which fault may be remedied by more vigorous exercise, running, tennis, etc. Many times wrong posture is held just because the matter is not given thought, as stooping over to hold a long-handle floor mop, or picking up a bucket with the weight thrown on the spine instead of on the shoulders, etc. Keep the chest up, and, above all, practice deep-breathing exercises, as it is practically impossible to breathe deeply and hold a very bad posture.

\section{Adopt Sitting Rather than Standing Postures}

If the bad posture results from wrong heights of working surfaces, a small change will usually remedy this (see pages 37-39). The sitting posture is always more efficient and less fatiguing than the standing position. It is quite easy to wash dishes, iron, make cake, pare vegetables, etc., while in a sitting posture, and this principle should be followed and not let it be regarded as "lazy to sit down." Do not make the mistake of thinking that standing is exercise, or that to sit down at work is a sign of weakness or inefficiency, as many women do so regard it. On the contrary, in factories and industries without number, work chairs and benches have been installed for tasks at which the worker formerly stood up, with the result that the workers have done the same or even a greater amount of work with far less fatigue. 
For some women, the typical office "high stool" is comfortable. For others, especially women of heavier weight and figure, it is most uncomfortable to thus perch on a small sitting surface with the legs drawn up or left hanging without foot rest. For such women some form of modified kitchen chair on 4-8 inch blocks of wood, with casters, to give additional height, will be found preferable. The author uses in her kitchen an adjustable, so-called "library" or office chair, with both adjustable back and seat, so that it may be raised or lowered to exactly fit the working surface at which it is temporarily used. Generally some kind of foot rest, such as a narrow board, should be nailed to the legs of the chair, as there is still less fatigue if the feet are supported; indeed, no sitting position with the legs hanging should ever be held for a long period.

\section{Relaxation Through Reclining Postures}

The power to relax is one habit which every homemaker must acquire if she does not know how already. Some people even sleep without relaxing! Relaxation consists in making the body "limp" and letting the tension of both muscles and nerves entirely disappear. While it can be practiced in a sitting position, it is always best to relax lying down. It may be put down as a rule that every homemaker should at least once a day lie down in a relaxed position from ten to twenty minutes. This is best taken by lying on a couch or bed flat on the stomach, arms hanging limp at sides. During such a horizontal position of the body, the blood pressure becomes less, and thus the cell walls are rested; also, it greatly strengthens the sex organs, many of whose ailments arise from too constant standing.

A noted woman, now elderly, and famous for the extent and variety of her interests, gives it as a positive injunction 
to women that they lie down a half hour each day if they would be free of "nerves" and have better health. Even a five-minute rest in a reclining posture gives much more rest than a much longer period taken in even a comfortable chair. It is a faulty American habit, and especially practiced by women, to use "rocking chairs." There is no rest in a "comfortable" rocking chair! It dissipates energy and makes for nerves. Try to use the Morris, wing-back, and square library types of chairs instead. The horizontal relaxing may often best be taken in the late afternoon before commencing the busy supper preparations necessitating standing; mothers with young children whose sleep is disturbed at night should by all means "make up" with a day nap, generally when the baby sleeps also.

Relation of Fatigue and Balanced Work Conditions To EFFICIENCY

One of the chief aims of household efficiency has thus been the elimination of fatigue. By standardizing dishwashing (see page 78 ), by using a power washer instead of the hands, by sitting down to work, etc., much of the fatigue accompanying work can be eliminated, as has been shown. The reason we wish to eliminate all possible fatigue is not only because waste time and effort result in actual money loss, but because fatigue has a direct relation to the personal efficiency of the worker. The "all-tired-out" woman isn't either a successful housekeeper, or a happy mother, or a helpful member of the community. It has been proved that the recovery from normal fatigue can be quickly made with a small amount of rest, but "That it takes more than twice the amount of rest to recuperate from twice the normal amount of fatigue." (Gilbreth.)

To prevent a more than normal amount of fatigue in 
household tasks, then, the homemaker should make a "survey" or study of all her work conditions and remedy them as much as possible either by different location of equipment (see pages 2I-37), by the use of improved tools (see Chapter III) or by different methods of work (see Chapter II), remembering that light, height of working surface, air, posture, heat, clothing are also factors which make for fatigue or the reverse. Next, after eliminating the unnecessary amount of fatigue, she must distribute the normal fatigue in a balanced way. Now, any period of work may be represented by a "graphic curve," which will vary at different periods. In business we frequently hear of "three-o'clock" fatigue common among office workers; in industries, the fatigue may be most marked at certain other hours of the day. We call this hour or time when the fatigue is most noticeable, the "peak-load."

Housework, like any other work, may be represented by a graphic curve. The author sent a questionnaire to Ioo women who did their own housework, and from their many replies prepared a graphic chart of housework for the period of one working day. This chart shows that in the period from 7 a. m. to 7 p. m. there were two distinct "peakloads"-one at about $2 \mathrm{p}$. m. and the other at $7 \mathrm{p}$. m. The location of these peak-loads in any individual case will, of course, depend on exactly what work is done in the periodthe "load" on a wash day coming perhaps at a different time from a day on which rooms were cleaned, or sewing done.

There are two objects which the housekeeper must strive to achieve in relation to these "loads" as they occur in her own individual housework:

I.-Prevent the "peak" from mounting so high by better planning of the work schedule. 
2.-Prepare and be ready to meet the "peak" when it occurs.

If, in your particular case, the "peak" rises at 2 p. m. and you find yourself unusually fatigued at this hour, you may prevent the "peak" very greatly by better scheduling of your work in the morning so that you are not so fatigued at this hour. If 7 o'clock, the after-supper hour, seems again to be the most wearisome, then this, too, may be changed if a rest is taken in the late afternoon, or if much of the supper preparation is made in advance. In other words, better scheduling (see Chapter II) so as to more evenly balance the workperiods will do much to lessen definite fatigue points of the working day.

Again, if a certain "peak" of the day must be necessarily high, and nothing you can do by planning will change it from that hour, then you must prepare to meet it by coming to it in a rested condition by making extra advance preparations, etc. For instance, $5: 30$ in the author's house is a very trying "peak-load" indeed. It is the children's supper hour; it is the time when the baby is undressed and bathed for the night; and the time when the other members of the family come home from business. No better scheduling will alter these facts. Therefore, they must be met as efficiently as possible. The table set in advance, several supper foods pre-cooked, the bath materials laid ready, the homemaker rested by a reclining nap, and refreshingly dressed, all help to meet this "peak-load" with the minimum of friction, hurry, and energy.

In all cases, heavy work like wall cleaning, sweeping, laundry, etc., should be followed by periods of sitting work. Again, excessive sitting should be interchanged with standing or walking around. It may be given as a rule, that the 
more strenuous the work, the longer should be the duration of the "rest" period (or whatever means is taken to counteract the fatigue) and the more frequent the rest periods should be made. It is a common mistake, especially of energetic women, to start a "spurt" of work, and "see it right through" without stopping. In general, it may be said that a two-hour interval of one kind of work is long enough, and that every two hours there should be even a slight rest, relaxation, change of posture, music, or mental interest.

When you think you just can't possibly wash another dish, go and put a stirring march record on the phonograph, or when your back gets tired at the stooping cleaning work, put on a polka or a schottisch and see what music does to sweep away fatigue! A face sponge, a clean waist, or a cup of malted milk are other first-aids to relaxation.

More work can be done if the worker applies herself steadily over short periods than if she works less steadily, or too steadily over prolonged periods.

\section{Confusion Creates Extreme Fatigue}

Another source of fatigue is found in disorderly, upset kitchens and overcrowded sinks, and rooms which have been allowed to "look like a sight." From the efficiency point of view such overcrowding should never be allowed to happen, not only because it causes excessive bodily fatigue to clean up such unusual confusion, but chiefly because disorder creates mental fatigue of a severe kind. "Clutter" causes the worker to become discouraged, and this in turn lowers her work efficiency. Therefore, those methods which prevent confusion, such as definite place for grouped equipment, the cleaning up of mixing bowls in cooking, the "pick-up-asyou-go" habit, are to be followed not only because they save time, but because they save emotional fatigue. 


\section{Clothing as an Aid to Personal Efficiency}

Clothing should serve these five purposes: (I) moderate warmth; (2) ventilation; (3) freedom from pressure; (4) cleanliness; (5) æsthetic appeal. Much fatigue (and thus lowered personal efficiency) is caused by too heavy underwear which does not permit ventilation to the skin. Fatigue is also caused by working in too tight clothing instead of doing such work as heavy cleaning in bloomers and middy blouse, thus freeing the legs from restriction.

(See pages 167-168.) Clean or new clothing also acts as an important antidote against fatigue by increasing the feelings of pleasure, change, and visual satisfaction in color, form, and texture which it gives the wearer.

A whole chapter could be written on the one point alone of women's general clothing and its relation to their personal efficiency. Most home women spend entirely too much time and emphasis on the subject of clothing; they have numerous changes and dresses which are too elaborate and which are made according to the arbitrary whims of fashion. It is a lamentable fact that while home women (more than business women) spend literally days of time thinking, discussing and making clothing, they spend almost no time in originating clothes to suit their personality, either in color and line or in developing such dresses that will make for more efficient work or greater asthetic appeal. Instead of studying their own body proportions and the colors that would enhance their good points, and making clothing to emphasize the beauty of these proportions and colors, they mistakenly follow "fashion." Thus the home woman is constantly "altering" this and that gown to adjust to fashion's vagaries, no matter whether they suit her or not, and if she has growing daughters, the appaling amount of time that this takes, and the inharmonious 
clothes that result, certainly detract from her personal efficiency -an amount of time and effort which could be devoted to other interests with far more profitable results.

Similarly, many women with children, especially girls, follow standards of dress for their children which are out of all proportion to their standards of living in other respects. They put lace-fringed white petticoats and embroidered white dresses on little girls, and make for them clothing which does not allow the highest comfort or make the child look its best. They make the mistake of thinking that "hand tucks" and embroidery-the details of clothing-give to dress that beauty which is based only on the selection of right colors and proportionate lines and form-if these be wrong and inartistic, no amount of needlework will cover it up! More time should be devoted to a study of the design and textures of clothing and less to the details of construction. Every woman should try and design for herself, or have designed for her, one work dress which best fits her needs and one house-gown for home wear which is artistic and brings out her "best points"-regardless of fashion. These two can then be copied in many materials to give variety, yet their making will always be more standardized and less timetaking than regular clothing, besides assuring that the design, line and form on which they are made needs no "altering" twice a year, but is always such as will best express the personality and emotion of the woman who wears them.

Further, the time consumed in the dressing process is often out of all proportion to its results or necessity. One of the minor but still important details in developing the woman's personal efficiency is to see how easily and quickly she can make her dressing schedule, and reduce such a mechanical process of repeated daily occurrence to the minimum. It may be interesting to know that in a series of time tests on 
dressing, it was found that a woman could easily dress for the street in ten minutes, or for a formal function in twenty minutes, if she followed a standard practice and kept her mind on what she was doing!

\section{Daily Physical Exercise Needed for Health EFFiciency}

"What, take more exercise when I do all my own housework ?" many women may exclaim in surprise. In general, all women in the home take too little exercise to keep them in good condition, and to enable them to store up surplus vitality. Most housework is done indoors, does not give a chance for increased air supply and invigoration; second, cooking, dusting and the like are all relatively slow work as compared with tennis, golf, basket-ball or such sports for women. It is seldom that housework makes a woman perspire and get into that "glow" which is so essential to perfect condition. And last, few women get into housework the "play" idea which the best forms of exercise give.

Therefore, the homemaker, above all other women, needs to indulge in daily outdoor exercise and sports, particularly such as develop the leg muscles. A woman thinks she is so tired from housework that she cannot take other exercise, and makes the mistake of thinking that "rest" always consists in lying down or taking a warm bath, etc. On the contrary, she should take that form of outdoor exercise which would not only offer her a change of scene, but which would so develop her as to enable her to stand the strain of the unusual standing and "tracking" she may have to do. A quick walk of at least two or three miles daily is an absolute necessity. Or if she can indulge weekly in tennis, golf, swimming, or plain gymnastics, she will receive great benefit.

Any woman who thinks she "doesn't need exercise" should 
watch her breathing when she runs for a car, or compare herself with women who are noted swimmers, golfers, etc. It is a peculiar delusion of many women to fail to see that exercise develops reserve nerve force as well as muscular resistance. Often they look at exercises as entirely unnecessary, thinking that the slow, and often puttery work of standing, sitting, tracking housework is genuine exercise. Isn't it unusual for the average married woman to take time for outdoor sports? Doesn't the term "housekeeper" usually carry with it a "settled" stay-in-the-kitchen sort of woman who no longer follows many of the sports in which, perhaps, as a girl she excelled? Yet need this be so? Real, vigorous daily outdoor exercise is demanded of every homemaker who wishes to keep her efficiency at the top notch.

Thousands of women think that as homemakers they are "overworked." Some, indeed, are working beyond their strength. But the plain facts are that most of these thousands of women who think they are being overworked are instead victims of bad air, wrong diet, poor elimination, body poisons, lack of exercise and worry and mental disquiet. They make the mistake of thinking that because they are "tired" it is work which is fatiguing them. They fail to see that most often it is not the work of the house, but these other wrong living habits that cause the fatigue and weakness. There are some women, perhaps, working beyond their capacity, but on the other hand their working capacity is only a fraction of what it would be if they took exercise, if they were not constipated, if they did not eat wrong foods, if they did not worry! There is no better economy in life than to keep up one's working power, and the homemaker on whom not only the work of the home depends, but also its guidance in educational and spiritual needs, must keep up her working power by strict attention to right living habits. 
Finally, it is an excellent plan to have a "survey" or diagnosis made annually of all body conditions and organs, even tho the woman may feel in perfect health. Never was the saying, "a stitch in time," more applicable. We all know the tooth which decays entirely, when a tiny filling the year previous might have saved it. So, too, such a "survey" of heart, blood pressure, sex organs, eyes, teeth, etc., may reveal slight indications of wrong which, taken in time, will never become the aggravated symptoms and illness they might develop into if unobserved at the start. One week spent in a sanitarium under the constant observation of trained health specialists will more than repay the cost, especially if the woman later follows the course of diet, or exercise, or hygiene planned for her special problems.

The Mind, Emotions and Senses Need Exercise Also!

There was a recent widespread discussion on the point of whether the home woman did not allow herself to stagnate mentally so that, say at 40 , she would be unable to successfully undertake a course of college work, and that she is always, as a matter of fact, less mentally alert than other women who have not been married or been homemakers for a number of years. It is often pointed out that as a class, homemakers are less interested in public affairs, less keenly alive than women who have made careers for themselves in other work or professions. Is this true, or need it be true?

It must be admitted, however regretfully, that far too many married women permit themselves to slip back into a slough of "mental inertia; they "lose interest"; they don't keep up with current events; and particularly they allow their minds to grow rusty and refuse to consider problems of beyond-the-home or of abstract interest. Here is, perhaps, the one crucial difference between men's work and 
homemaking as commonly practiced: men have had and do now follow tasks just as fatiguing, just as monotonous, just as limiting as any washing dishes or bathing babies could possibly be (let anyone who does not believe it think of men who run elevators, drive teams, add up figures, collect tickets, handle freight, dig sewers and the other jobs which make up the bulk of men's occupations) ; but men have, as a group, kept up their mental development because they separated their day's work from their after-work avocations and interests. Men hare not, nor do they have, necessarily more time than women, but they have utilized that spare time and afterwork-hours fipedom to better advantage.

Concretely, here is Mr. Smith, who works from 7:00 to $7: 00$ as an express agent-certainly a confining, monotonous kind of employment. Here is Mrs. Smith, who works from $7: 00$ to $7: 00$ at the confining, monotonous tasks so many women claim of the home. But-Mr. Smith reads a good daily paper; he bowls once a week; he spends Saturday afternoon at a free swimming tank; he votes; he belongs to a club of "fellers" who have great meetings solving problems for the president, the war, the railroads; he has a hobby for shooting galleries, or dogs; and, even if not a college professor, Mr. Smith keeps up his mental development outside of work hours. But Mrs. Smith-does she read a good paper; does she swim; does she belong to a club to discuss broad civic and abstract problems; does she have a wholesome hobby-or is it not too true that many and many a Mrs. Smith's whole universe is bounded by the price of butter, how Susie's dress is to be made, the baby's symptoms, and what Mrs. Jones told her about the Browns-does she not "talk shop" most of the time?

We must be fair and admit that the interests of many homemakers are bounded by the over-elaborate, petty details 
of their own individual housekeeping. We must admit this in order to clear homemaking of the many charges heaped against it by both men and women today; it is not homemaking that is narrow and limiting in mental discipline, but the fact that so many homemakers have neglected to have other definite, beyond-homemaking interests. Men have followed their work or occupation, and also an avocation-a spare-time set of interests; but many women have wrongly permitted homemaking to be both $a$ vocation and an avocation.

This is a serious point, because it has had so much effect on the popular idea of homemaking as "uninteresting," and why, particularly, numbers of young women think only of a "housekeeper" in terms of disparagement and reproach. Does Mr. Smith, the express agent, get his mental culture, such as it is, from his job itself? But Mrs. Smith has made the error of supposing that she could get all her mental culture from washing dishes. And often, because she didn't find it in washing dishes, she has wrongly, most wrongly, blamed dishwashing and wishes she were working at some other job. Now, the whole problem here is for every homemaker to find for herself extra homemaking avocations, interests, and pursuits, and to develop herself as a human being outside and beyond her housework and child-raising demands.

She can do this by:

1.-Reading newspapers, magazines and books of the better class, particularly books on civics, psychology, government, ethics, and critical reviews of more abstract interest.

2.-Having a hobby or fad far removed from housework, as music, sketching, garden, or flower collecting, care of pets, designing, civic improvement, suffrage, or some kind of reform work.

3.-Taking daily and weekly physical recreation in gymnastics, walking, tennis, or other sports. 
4.-Belonging to clubs or associations having broader interests or the wider interests of the home as objects, housewives and consumer leagues, civic clubs, art associations, etc.

5.-By giving even a short moment each day to abstract thinking, and cultivating a well thought out philosophy of life.

Women as a group, too, do not read enough worthwhile books. They overindulge in novels and fiction, but should also read books dealing with civics, labor problems, sociology, history, etc. It is even more necessary for the homemaker than for other women to include such reading in their plans for personal efficiency, since they are more closely confined and out of touch with world events. They should also read magazines other than the so-called "women's magazines," which unfortunately cater largely to the home in a narrow concrete way instead of to the larger human interests of the world of both men and women. Among the most profitable magazines are: The Literary Digest, The Review of Reviews, The New Republic, The Atlantic Monthly, World's Work, National Geographic, The Bookman, Good Health, The Woman Voter, Journal of Home Economics, etc. And there are many, many fine broadening books if the homemaker will but seek them.

\section{What Shall the Homemaker Do with Her} Leisure Time?

A reputedly brilliant man once said to the author: "Well, Mrs. Frederick, after a woman has found out how to save time and effort by following the ideas you give her, what is she going to do in all this spare time she has saved?" What, indeed, is the object of all the more efficient methods for doing work, the use of laborsavers, the time, effort and nerve-saving plans which this course on "Household Engineering" advocates so strongly to the homemaker? Is it 
only that she can do in ten minutes tasks which formerly took her twenty, or that she may find herself less tired by using a washing machine than by rubbing on a washboard?

No; far from it; the real object in saving time and effort in the work of the household is to enable the homemaker to have leisure time to devote to interests which are more important than the mere mechanics of living. Looked at closely, the homemaker must satisfy the following demands: (I) the manual or largely physical tasks of cooking, sewing, cleaning-what we call the "mechanics of living" which are involved in ordering and arranging that food, shelter, clothing, warmth be supplied as efficiently as possible to her family; (2) the mental and spiritual tasks of providing the "home atmosphere" of stimulation, sympathy, education, ethics, love to both husband and children; (3) the threefold physical, mental and spiritual demands of her own development as a woman and as a human being; (4) the mental and spiritual tasks of sharing in the larger home of the community and home of the nation by being a good neighbor, civic worker and patriotic and loyal citizen.

Now, looked at broadly, which set of demands are the most important? We can easily see that all demands should be satisfied in the ideal homemaker's life; but we can say truly that the first set of demands, the mechanics of living, important as they are, are not as important as any of the other three demands. In other words, the family, or men and women and children as individuals, are not getting all or even a full share of life if their food, shelter and clothing needs are completely satisfied, and nothing more. The most important needs are surely the mental and spiritual wellbeing which it is the homemaker's peculiar function to pour out to her family and the community. "Man cannot live by bread alone," it is written, and Jesus praised 
Mary, even though she only sat at his feet and listened, while Martha busied herself with household duties.

The real object, then, in all household efficiency, is to so lessen and reduce the time required and consumed by the demands of the mechanics of living - the dusting, and cooking, and washing, and mending of garments-that the homemaker shall have leisure time to devote to the three other and far more important ends of homemaking. "Household Engineering" is valuable only as a means to this end-it must never be regarded as an end in itself. Thousands of women have all their lives been nothing but housekeepers-they have fed their husbands three kinds of pie, but they have not fed them sympathy and interest in their business, or comradeship in work and recreations; they have spent hours seeing that their children were "properly" dressed, but they have not taken as many hours to see that the children were properly chaperoned, or trained, or brought in contact with stimulating and habit-forming persons, arts, or educational opportunities; they have broken their backs dusting and sweeping and scouring their own individual hearths and chairs and garbage pails-but they have neglected to keep the schools, and the amusements, and the city garbage pail up to a high and safe standard.

Or, again, thousands of married women have secretly or openly repined because they "never had the time" to follow up some cultural pursuit like music, and the arts, or indulge in some business which attracted them. Marriage and childbearing is thus commonly regarded as a barrier towards any outside "career" or cultural achievement; indeed, many brilliant women have refrained from marrying or refused at least to bear children because it has been so commonly accepted that housekeeping is so absorbing, so overwhelming that it does not leave any room to carry on other interests and pursuits at the same time. Is this, or need it be, true? 
If we sincerely study and face the facts, do we not find rather that many of these thousands of women do not value and make the most of the time and leisure they do have? Or, do they not waste hours in social chit-chat, in needless entertaining, in unnecessary thinking about clothes, in timewasting shopping, marketing, etc., instead of putting this valuable time to some definitely planned work or interest?

What is the value of one hour saved each day? Unless its use is planned for, this time is gone forever, and there is really no need or use in increasing the household efficiency which gives leisure unless it is planned to be utilized to the best advantage. Thirty minutes saved each day equals three hours per week of working days, or 156 hours a year. The Ioo best books of the world could be read in about 36 months of this time, saved at the rate of only a half hour per day!

But the homemaker's life is from twenty to thirty yearsthink of what could be accomplished over this period of years with only a saving of thirty minutes a day, that were planned for! One could perfect a modern language, learn a complete science, take a course of music, master a business, in the spare time of only thirty minutes per day distributed over the span of homemaking life. Think what you could do if you saved an hour, or an hour and a half-and then made the best use of it!

It is a mistake for women to think (as many do) that men have "more chance" to attain eminence or success in cultural pursuits than women, especially homemakers. Investigation proves that many, many men who have achieved notable success in some line have done it in after-work hours' leisure. So often women exclaim, "Oh, I had to give up my music," or, "I can't do any of these things because my housekeeping takes up all my time." Now, let it be repeated again that men have had to apply themselves at monotonous jobs and hard work, and that, all things considered, the responsibility 
of earning a living and supporting a family is as great a responsibility as that of managing a house and children. If more men have attained success, it has been in spite of family and work responsibility-it has been because men have organized themselves, organized their time and their leisure to make it count the most. Lincoln, Franklin, Carnegie all utilized their after-work hours and planned definitely to reach some desired goal; Lamb and Hawthorne and others were clerks tied to a most rigorous daily routine when they yet found time to write their beautiful fiction; and the example of such women as Harriet Beecher Stowe, who wrote Uncle Tom's Cabin in spite of poverty and care of small children, and Mme. Schumann-Heink and Louise Homer, who are great artists even though they have had six and eight children, and the lives of many other actresses, literary women, etc., all prove that homemakers can achieve a large share of personal efficiency and development, even world distinction, without sacrificing the emotional benefits of family life-if they organize themselves and plan the use of their leisure time.

\section{Organizing the Home}

Also, not only do few women plan to organize their time to the best purpose, but they do not organize their households as men do their business, into a going concern. For instance, it is a very poor strata of business man who, all himself answers the phone, writes his own letters, files them, gets the business, then comes back to the office and prepares it, etc., etc. He employs even a low-price assistant to do some of the work so that he can devote his own time to the more important phases of the business. In other words, he values his own time, and refuses to spend this high-price time at work which some other low-price assistant can do equally well. The idea of the modern, efficient "organization" is to 
have one acting head with co-assistants, helpers and others who are responsible for, and who do relieve the acting head of details, so that he will be able to put his entire energy into the more important work of an executive and manager. But few homemakers are willing to give over responsibility and to delegate authority to assistants - they still mistakenly cling to the old, inefficient conception of an "organization"that of having one head who has authority, and who must pass on every detail and practically carry the whole responsibility before anything is done.

Concretely, many women insist on doing "everything myself." They take hours doing the mending, or making baby dresses, when perhaps they were never trained for sewing, but can cook or do something else unusually well. Why not give over certain tasks about the home to others who are more experienced and fitted for them? Is it not a mistake to try and "do everything well"? The author knows a clever business girl who married and then entirely dropped her profession and started in to do every bit of her own work, including the sewing, which she found unusually difficult. After a few years she was an entirely different woman; she was exhausted from trying to learn and do several kinds of strange work-she scattered her efficiency. Would it not have been a great deal better to continue proficiency in her specialty in her spare time, and by means of it pay some woman who was an expert sewer and thus save har own energy at the same time she gave employment to another woman who needed it?

It is therefore far more important that the homemaker have a definite purpose and plan for the use of the leisure time which household efficiency will give her, than that it merely enables her to accomplish her present housework! with less effort. It is not lack of time which handicaps the homemaker, but lack of power, lack of will and a strong 
enough ideal to force her to subordinate housekeeping routine to the attainment of the higher ends of personal and family happiness and success. What shall the homemaker do with her leisure time? Here are some suggestions:

\section{Ten Things to Do in Leisure Time}

I.-Take time to read more about her own specialty, as government and state food bulletins, books on household management, child care, house planning, equipment, etc.

2.-Take time for actual correspondence course in homemaking subjects ; attend special classes in cooking, food conservation, budget making, which her community may offer.

3.- Take time to interest herself in and understand her husband's business, so that she can aid, sympathize and be a comrade in his work.

4.-Take time to supervise her children's school work, play, friendships; take them to museums, zoos and places of interest on their holidays.

5.-Take time for daily grooming, hygiene and physical exercise.

6.-Take time for reading and making personal thoughtout decisions on the problems of life, ethics, immortality, philosophy of life, etc.

7.- Take time for music, art, language, business, hobby or interest beyond housekeping.

8.-Take time to "keep up on" some specialty or pursuit in which she excelled before marriage, or by which she used to earn her living, so that she can relieve herself of some phases of housework for which she is not fitted, and so that, in case of death or disability, she would be more able to take upon herself the burdens of family support, if occasion required. 
9.-Take time for interest in local school, housing, sanitation, temperance and food conditions-belong to organizations having these aims as objecis.

I0.-Take time for active Red Cross or other war charity work ; act as census taker, soldier chaperone or other patriotic duty, either in war or peace.

The Homemaker's Mental Attitude and Its Relation to Her Efficiency

Sleep, air, posture, food, physical exercise, etc., have all been discussed in their relation to personal efficiency. We now come to the most important factor of all-the homemaker's mental attitude toward her work and its great influence on her personal efficiency.

In the first place, numbers of women constantly work under a sense of hurry, they seem constantly to be trying to "catch up," and this sense of tension and uneasiness enter into every smallest act. Now all such feelings of worry and tension are wasteful of energy and make for the nervous debility of the worker. It has been proved that even mild worry is more exhausting than hard work, and that this sense of hurry actually reduces the speed of their work. Indeed, much of the fatigue and depression that women blame on housework comes instead from this totally unnecessary nervous, wrong mental attitude which they permit to accompany their work.

Instead, it is vitally necessary that the homemaker develop a sense of equanimity, repose, calm, or inner spiritual poise which she may exert even in the most trying of situations. And this poise consists not so much in repressing anxiety as in ignoring it. To illustrate the difference, think of the mother who draws in her breath, who gets all "keyed up" and tense when Tommy comes in yelling from a slight fall, and the other, wiser mother, who calmly refuses to allow 
herself to be "worked up," and you have the difference between the inefficient, destroying mental attitude and the efficient and healthful mental one.

In the first case, the woman allows her mind to be swayed by her emotion and acts just as it wills; in the second case, she manages her mind and diverts and controls her attention. This "control of attention" is so important that every homemaker should practice it a few minutes daily, changing her attention from one thing which seems to absorb and master her, to some other thought, or even making her mind a blank nothingness. To do this easily is one of the greatest protections against tension, worry and fatigue. It will enable her to "see through" a huge pile of unwashed dishes, a serious illness of one of the children, or be of immense value in countless emergencies. Indeed, this attitude of mental control will, more than any one other thing, enable her to master housework and carry a heavy amount of work and strain without fatigue.

Again, so many women allow themselves to be needlessly affected by "interruptions" of one kind or another. The author has had women tell her over and over that the interruptions coincident with housekeeping work make them "nervous" and upset. Now, this need not be true. It is a psychical fact that monotonous work is far more fatiguing than interrupted work. The point is, not that the "interruption" makes the woman nervous, but that her own mental attiude towards the interruption is the real cause of her irritation. If she would relax and welcome the interruption instead of allowing herself to be irritated by it, each interruption would only serve as a rest and change of work, and means of lessening tension, instead of increasing it.

Second, women have differing mental attitudes toward their work, most of which greatly limit their efficiency in that work. From thousands of letters which have come to the 
writer, and also from talking to women all over the country, from farms, from small towns or large cities, I can say that women generally regard their work from some one of the following points of view:

I.-A widespread feeling that housework and child care is a kind of "trap" into which they have fallen because of their marriage, and from which they constantly struggle to escape, and yet which they cannot master or control.

2.-A clinging to the traditions and experience of the routine past, which makes them refuse to see the new modern conditions of homemaking, or to try and use improved methods or equipment because such practice was unknown to their mothers, or not included in their previous experience.

3.-An automatic, unthinking attitude, accompanied by unstandardized work, and lacking in any mental interest or imagito get finished as soon as possible in "any old way."

4.-A mania for some one phase of housework, as cooking, or sewing, on which all originality is spent to the neglect of an efficient management of the home as a whole.

5.-An excessive insistence on the details to the end that housework is exaggerated, elaborated and repeated, and the more important executive side of housework neglected.

6.-A mistaking of the physical, manual tasks of housekeeping to the almost total exclusion of time and attention upon the more important responsibilities of home-making; an emphasis which measures homemaking efficiency solely by the amount of and the exhaustiveness of the physical tasks accomplished and the time spent on the mechanics of living.

7.-A general fear, anxiety, lack of confidence and inability to control the situation; a refusal to make a survey of conditions, and knowing them, to apply definite, and even radical means of changing them; a lack of self-discipline and control of the will.

8.-A refusal to see in housework any opportunity for cultural development or self-improvement; a contempt for all housework, with a preference for teaching, business, or other career, and the hope to be relieved of it entirely some time and take up other, more "stimulating" work. 
9.-A failure to put into housework tasks the same interest, initiative and "punch" which are demanded to make any other occupation or business a success.

ro.-A refusal to see beyond individual housekeeping and its problems into the wider city and national housekeeping of which it forms a part, and which it so much influences in regard to national habits of thrift, conservation, character, stability and morale.

II.-The parasitic attitude-unfortunately rather common-a feeling that society, their husbands, parents or somebody, owes them a soft place; that they should not be expected to work, to give value received, but should be allowed to "adorn" society, amuse themselves, and cultivate their "higher faculties."

\section{What Is the Right Mental Attitude for} THE HOMEMAKER?

The right mental attitude involves the reverse of all the wrong attitudes listed above. The homemaker must look at her work as it is, as a problem which is interesting, which is stimulating, which will call into play all her highest training, and which, no matter how difficult, can be solved by the twelve principles of efficiency. (See page 9.)

That homemaker has the right mental attitude when she holds before herself a strong ideal of what she wishes to accomplish in her work; when she uses common sense in securing this ideal; when she standardizes her conditions of work so they save effort and time; when she standardizes operations in her work, and writes down standard practice instructions of the best, shortest, easiest way to do a certain task, and exactly what tools or equipment will give this; when she takes competent counsel from some person's experience, from a book, or a course of study; when she makes a plan or schedule not only for her daily housework, but a plan which shall provide for the larger interests and self development she is seeking; when, once having made such a 
plan, she dispatches it, or sees it through, against every obstacle and seeming difficulty; when she keeps reliable records of her business, so that she knows the cost, specifications, names, etc., required in that business; when she trains her will by discipline to accomplish what she sets out to do; when, finally, she gives herself and her family a fair deal and a true efficiency reward by achieving for herself and for them the largest measure of health, happiness, and success.

One means of retaining the right attitude is to bring the spirit of a game or competition into work. Try to make a "record" in some task, like bedmaking, or table setting. The next time try, with yourself, to better that record. Again, written standard practice instructions, such as may be hung in the kitchen or other room, are, in themselves, an inspiration to keep up the high standard of work.

Often "slogans" or mottoes hung up, even for a few days, have their effect in stimulating efficiency, as "Plan Your Work, then Work Your Plan." Always substitute science and tests for personal bias and tradition, and by this means keep up interest. How long does it take asparagus to steam -how long to boil? Try sweeping and cleaning a room in the efficient order, then the reverse-see if the work doesn't add in interest. Cultivate right feeling about work-be contented, enthusiastic, optimistic.

Above all, do not waste time and energy in worrying over conditions and circumstances-but expend the necessary mental energy to think them into better and more desirable conditions. Often women become discouraged solely because they don't take time and trouble to think clearly; because they don't adopt the scientific method of test, experiment and research in housework to replace the old traditional methods. Today we must relegate to the scrap-heap the traditional technical experiences of the past. For instance, 
in these times of conservation, who has use for recipes beginning with " 8 eggs and I quart of cream"? Or, who today is interested in roasting meat over a spit when the pressure cooker is at hand?

In other words, we must realize how rapid, how all-inclusive have been the changes in homemaking during the last fifty years-fifty years ago, even twenty-five years ago, there was no pressure cooker, no fireless, no insulated oven; there was no dishwasher, no washing machine, no heated mangle or electric utilities of any kind. Scarcely a can of food could be bought; clothing was all home-made. The homemaking that was fitted to those conditions is not adjusted to the conditions of today. All too often young women speak scornfully of "housekeeping," when they have in mind the old-fashioned kind of housekeeping. It is time to realize that the era of the "Household Engineering" has come!

\section{Conclusion}

"Household Engineering" has only tried to show the new, modern conception of homemaking, with its many possibilities for scientific work, for the use of improved machinery in the home, for less waste in materials, energy and timeto the end that the woman herself, and her family, and the nation be developed to the fullest power and vantage ground in health, happiness and true prosperity. If "Household Engineering" helps any homemakers and families do this, as it has so wonderfully helped the author, and if in any small measure it clears housekeeping from some of the slurs cast on it, and more rightfully places it in relation to the whole tremendous problem of woman, her work and development, and in relation to the business and industrial progress of the present era, the author will be well repaid for the labor it has cost her-a labor additional to the care of a country home and four young children-a labor made pos- 
sible only by well planned work and equally planned-for minutes of leisure time!

\section{QUESTIONS ON HOUSEHOLD ENGINEERING XII}

\section{Health and Personal Efficiency}

I. To what extent has your health affected your personal efficiency? What measures can you take to increase your degree of health and personal efficiency?

2. Are you conscious of a "peak-load" in your daily work and what can you do to lessen it?

3. To what extent and how has the study of Household Engineering saved you time in housekeeping?

4. Have you a well defined plan for use of your spare time? If so, what? If not, what appeals to you as worth working for?

5. What is your attitude towards homemaking as a profession? 


\section{BIBLIOGRAPHY}

\section{Cooking and Diet}

Lessons in Cooking Through Preparation of Meals-Robinson and Hammel. \$2.50.

Feeding the Family-Rose. \$2.50.

Low Cost Cooking-Nesbitt. \$1.00.

Successful Canning and Preserving-Powell. \$2.50.

Food and Dietetics-Norton. \$1.50.

How to Cook and Why-Condit and Long. \$1.50.

Principles of Cookery-Barrows. \$I.50.

Boston Cooking School Cook Book-Farmer. \$2.50.

A New Cookery Book-Farmer. \$2.50.

Mrs. Allen's Cookbook-Allen. \$2.35.

The New Cookery-Cooper. \$2.00.

How to Cook in Casserole Dishes-Neil. \$1.50.

Canning, Preserving and Pickling-Neil. \$1.50.

The Candy Cook Book-Bradley. \$1.50.

Candies and Bonbons and How to Make Them-Neil. \$I.50.

American Salad Book-DeLoup. \$1.25.

Fruit Recipes-Fletcher-Berry. $\$ 2.00$.

The Housekeeper's Apple Book-MacKay. \$1.25.

The Fireless Cookbook-Mitchell. \$1.50.

Book of Corn Cookery-Wade. \$1.oo.

Canning, Preserving and Jelly Making-Hill. \$2.00.

Cooking for Two-Hill. \$2.25.

Catering for Two-James. \$1.50.

Low Cost Recipes-Harbison. \$1.oo.

Economical Cooking for Two-Gibbs. \$1.00.

How to Cut Food Costs-Cooper. \$0.75.

Food and Flavor-Finck. \$2.00.

IOOI Food Tests-Wiley. \$1.50.

Principles of Nutrition-Jordan. \$2.50.

Food Products-Sherman. \$2.75.

American Home Diet-McCollum \& Simmonds. \$3.50.

\section{Management and Family Finance}

Household Management-Terrill. \$1.50.

The New Housekeeping-Frederick. \$1.75.

The Business of the Household-Taber. \$2.50.

Increasing Home Efficiency-Bruere. \$2.00.

Successful Family Life on a Moderate Income-Able. \$2.00.

Spending the Family Income-Donham. \$1.75. 
Save and Have-University Society. \$1.25.

Principles of Domestic Engineering-Patterson. \$2.00.

The Modern Household-Talbot and Breckenridge. \$I.00.

Cost of Food-Richards. \$1.00.

Cost of Living-Richards. \$1.00.

Marketing and Housework Manual-Donham. \$2.00.

The Woman Who Spends-Richardson. \$1.00.

The Up-to-Date Waitress-Hill. \$1.75.

The Expert Maid Servant-Herrick. \$I.00.

The Expert Waitress-Springstead. \$1.00.

The Duties of Servants (English). \$1.00.

Domestic Service-Salmon. \$2.00.

Wanted, A Young Woman to Do Housework-Barker. \$1.jo.

The Law of the Household-Beecher. \$1:25.

\section{Clothing and LAUndry}

Textiles and Clothing-Watson. \$I.50.

Dress Making-Fales. \$2.00.

Clothing for Women-Baldt. \$2.00.

Complete Course in Millinery-Bottomley. \$2.00.

Textiles-Woolman and McGowan. \$2.00.

Practical Sewing and Dressmaking-Allington. \$I.50.

Color Harmony in Dress-Andsley. \$1.25.

Sewing and Textiles-Turner. \$1.75.

A Guide to Laundry Work-Chambers. \$1.00.

Laundry Work-Sheppard. \$1.00.

Laundering-Balderson. \$1.25.

Chemistry of the Household-Dodd. \$1.50.

The Complete Housekeeper-Holt. \$2.00.

Housekeeper's Handbook of Cleaning-MacLeod. \$1.50.

Equipment, Building, Etc.

The Efficient Kitchen-Childs. \$1.50.

The Healthful Farmhouse-Dodd. \$0.60.

Home Labor Saving Devices-Scott. \$1.00.

The House: Its Plan, Decoration and Care-Bevier. \$I.50.

Modern American Homes-Von Holst. \$2.50.

A Book of House Plans-Butterfield. \$2.00.

Craftsman Homes-Stickley. \$2.00.

Bungalows and Camps-Comstock. \$2.00.

Distinctive Homes at Moderate Cost-Saylor. \$3.00.

The Family House-Osborne. \$1.00.

Care of a House-Clark. \$1.50.

The Book of Little Houses-Macmillan. \$0.75.

Low Cost Suburban Homes-Wright. \$1.25.

Inexpensive Homes of Individuality-Saylor. \$0.75.

Good Taste in House Furnishing-Sell: \$r.75.

Planning and Furnishing the Home-Quinn. \$1.50.

Designs for American Homes-Outwater. \$2.00. 
Interior Decoration-Parsons. \$3.50.

The Honest House-Goodnough. \$2.00.

The Lighting Book-Godinez. \$1.75.

Health, Child Care, Etc.

The Human Mechanism-Hough and Sedgwick. \$2.50.

Personal Hygiene-LeBosquet. \$1.50.

The Four Epochs of Woman's Life-Galbraith. \$2.00.

Exercise and Health-Hutchinson. \$o.6o.

Home and Community Hygiene-Broadhurst. \$2.00.

Household Hygiene-Elliott. \$1.50.

House Sanitation-Talbot. \$1.00.

Care of Children-Cotton. \$1.50.

Study of Child Life-Washburne. \$1.50.

\section{Education and Progress.}

Twelve Principles of Efficiency-Emerson. \$3.00.

Efficiency-Emerson. \$2.00.

Principles of Scientific Management-Taylor. \$1.50.

Fatigue Studies-Gilbreth. \$2.00.

Woman's Share in Primitive Culture-Mason. \$3.00.

Woman and Economics-Stetson. \$1.50.

Democracy and Social Ethics-Adams. \$1.50.

Progress in the Household-Salmon. \$1.50.

Home Problems from a New Standpoint-Hunt. \$1.50.

Blessed Be Drudgery-Gannett. \$1.25.

Family Secrets-Washburne. \$1.50.

Will the Home Survive?-W. L. George. \$2.00.

Fear and Conventionality-Clews-Parsons. $\$ 2.00$.

The Woman Voter-Austin. \$2.00.

Behind the Battle Lines-Doty. \$2.00.

The Ambitious Woman in Business-Gilbert. \$2.00.

Note. The foregoing books may be borrowed, one or two at a time by members of the School by sending postage (I2c to $24 \mathrm{c}$ ) with request. They will be sent to anyone on receipt of the price and postage by the American School of Home Economics, Chicago. 



\section{INDEX}

Accounting, Need of, 265

Accounts, Charge, Cost of, 323 Household, 286

Acetylene Gas, as Fuel, 122

Address File, 301

Advance Cooking, 197

Advancement, in Division of Income, 283

Advantages of Servantless Household, 380

Advertising and the Consumer, 357 for Houseworkers, 442

Adulteration of Foods, 341 of Textiles, 360

Air Moisteners, 471

Fresh, Need of, 483

Alcohol as Fuel, 118 Irons, 239

Alcove, Breakfast, 463

All-baked Dinner, 201

Allowance Plan, Objections to, 269

Aluminum Utensils, 58

Ampere, Meaning of, 125

Arrangement of Rooms, 401, 457

Artificial Silk, 362

Ash Shutes, 463

Avocations for Home-makers, 502

Baby Garments, Washing, 263

Babies, Schedules with, 76, 403

Baked Dinner, All, 201

Baking Temperatures, 207

Balanced Diet, Calories in, 331 Meals, 181,484

Balancing Household Accounts, 392

Bank Checks, Advantages of Using, 295

Bargains, Lure of, 358

Seasonal, in Clothing, 368

Bath Room, Care of, 153

Bath Rooms, 461, 468

Bath Tubs, 468

Bed Making, Time Study, 82

Bedroom Care, 152

Beds, Disappearing, 464

Bibliography, 517

Bins and Drawers, Kitchen, 48

Bluing, 220

Boiled Dinner, All, 201

Boiling Clothes, 219

Bonus for Houseworkers, 433

Borax, as Water Softener, 215

Breakfast Alcove, 463
Budget,Clothing, Typical, 366

for Irregular Income, 296

Food in Marketing, 327

How to Make, 285

Laws of, 278

Need of, 266

Plan in Spending, 271

Typical, 284

Variations in, 273

Buffet Meals, 382

Built-in Fixtures, 461

Fixtures, Kitchen, 40

Bulk in the Diet, 181

Bulk vs. Package Goods, 353

Bungalo Dress, 168

Bungalows, 477

Bushel, Legal Weights of, 348

Business Equipment, 63

Corner, 387, 449

Corner, Need of, 312

Butler's Pantry, 20

Buying Clothing, 359

Co-operative, 327, 406

Efficient, 317-367

Food, Refuse in, 336

Furniture, 373

House Furnishings, 372

Meats, 191, 236

Kitchen Equipment, 56

Parcel Post, 328

Seasonable, 327

Staples, 325

Cabinet Closets, 465

Kitchen, 28, 136

Calories, Composition of Food by, 334

of Food, by Measure, 335

of Food, Cost of 1000,338

per Pound, 331

Table of, Average, 332

Required, Daily, 330

Canned Goods, Purchasing, 352

Cans, Size of, 349

Carbohydrates, 180

Card Cookbook, 305

Cards, Household Record, 298

System of Household Account, 290

Care of House Heaters, 473

of Laundry Equipment, 253

Cash and Carry Stores, 324

Casserole Cooking, 202

Chairs, Kitchen, 39, 490 Rocking, 492 
Change of Shift, 148

Charge Accounts, Cost of, 323

Checks, Use of, 295

Children as an Investment, 282

Cost of, 282

Co-operation of in Servantless Household, 382, 400

What to do with, in Country Home, 404

Children's Clothing, 497

China Buying, 374

Cleaning Closet, 155

in Servantless Household, 398

Methods of, 146-167

of Equipment, 105

Outfit, List of, 171

Rooms, Order of, 74

Standard Practice in, 153

Standardizing, 81

Summary of, 172

Vacuum, 156

Walls, 175

Cleansers, List of, 174

Clearing away Processes, 21, 24

Clippings, Filing, 307

Closets, 464

Cabinet, 465

Cleaning, 155

Laundry, 232

Clothes Dryers, 231

Clothing and Efficiency, 495

Budget, 366

for Cleaning, 167

Home-Made, 368

Purchasing, 359

Ready-to-Wear, 367

Seasonal Reduction in, 368

Cold Mangles, 240

Color in Kitchen, 45

Plans of House, 457

Colored Clothes, Washing, 262

Combined Living-Dining Room, 459

Composition of Food by Calories, 334

Confusion and Fatigue, 495

Constipation, Evils of, 486

Construction of Equipment, 103

Consumer, Trained, 318

Woman as a, 166, 316

Contrast in Meals, 184

Cookbook, Card, 305

Cooking, 180

Balanced Meals, 183

Economies in, 207

Fireless, 203

in Advance, 197

in Casserole, 202

in Pressure Cookers, 134

Left-overs, 195, 206

Several Meals at One Time, 200 Steam, 203

Time Saving, 193

with Same Tools, 196

Cook's Rack, 34, 36

Co-operative Buying, 327

Living, Reason for Failure, 407

Schemes, 405
Corner, Business, 312 Sewing, 369

Corsets, 489

Cost of Children, 283

of Cleaning Outfit, 170

of Cooking Utensils, 60

of Distribution, 320

of Fuels, 124

of Laundry Equipment, 253

of Laundry Tubs, 230

of 1000 Calories, Table of, 338

of Operating Electrical Equipment, 129

of Operating Irons, 239

of Paper Products, 143

of Service, 413,431

Cotton Fiber, 212

Testing, 361

Country Home, What to Do Without Service, 403

Country Houses, Convenience for. 478

Country Laundry, 229

Country Schedules, 90

Cut-price, Lure, 358

Daily Schedule, The, 67

Deep Breathing, 490

Delivery, Cost of, 321

Den for Father, 499,459

Despatching, $11,66,398$

Dining Rooms, 459

Disappearing Beds, 464

Dishwashing, Hand, 112

in Servantless Household, 388

Machines, Tests of, 110

Standardizing, 79

Time Study of, 79

Dish Drainers, 109

Warmers, 51

Distribution, Cost of, 320

Division of Income, Typical, 273 Laws of, 278

Domestic Service, Objections to, 420

Draft Regulators, 472

Drawers, 466

Kitchen, 48

Drawing House-Plans, 474

Dress and Efficiency, 496 Budget, How to Make, 365

Standards of, 497

Working, 167

Dressing, Éfficiency in, 497

Dressmaking, Records, 370 Room or Corner, 369 Screen, 369

Standard Practice in, 371

Dryers, Clothes, 231

Dish, 109

Drying, Clothes, 221

Dumb Waiters, 464

Dusting, 154 Tools, 170

Dustless Duster, 156

Dust Pan, Long Handled. 165 Shute, 463 
Duties of Special Servants, 445

Eating Habits, Efficient, 485, 488 Economy of Labor-Saving Equipment, 392

Efficiency, Personal, 481-515

Reward, the, 431

Efficient Home, Planning, 449-480 Requirements of, 450

Electric Cooking, 393

Equipment, 123

Irons, 237

Light Plants, Individual, 478

Power, 127

Vacuum Cleaners, 158

Washing Machines, 249

Electrical Appliances in Servantless Household, 393

Devices, Cost of Operating, 129

Measurements, 124

Elevator Ice-box, 138

Elevators, 464

Employers, Women as, 435

Employment Agencies, 441

Enameled Iron Fixtures, 467

Engaging Houseworkers, 443

Entry, the, 460

Equipment and Family Needs, 102

as an Investment, 101

Business, 63

Cleaning, 170

Cleaning of, 105

Construction of, 103

Cooking, List, 60

for Home-Sewing, 370

Grouping of, 21, 29, 225

in Servantless Household, 391

Kitchen, 56

Laundry, Grouping, 225

Laundry, List of, 253

Relation of Schedules to, 88

Size of, 106

Exercise, Daily, 498

of Mind, 500

Expansion Tank, 470

Exposure, of the House, 452

Fair Deal for Houseworkers, 443

Family Financing and Record Keeping, 265-312

Farm House, Conveniences for, 477

Laundry in, 229

Father's Share in Servantless Household, 384

Fatigue, 492, 499 and Confusion, 495

in Cleaning, 148

Fibers, Textile, Testing, 360

in Laundry Work, 212

Financing, Family, 265

Financial Records, 304

Files, Card for Records, 298

Filing, Methods of, 308

Fireless Cooker, The, 130

Time-Saving With, 89
Fireless Cookers, Advantages and Disadvantages of, 132

Automatic, Electric, 123

Automatic, Gas, 131

Kerosene, 120

Fireless Cooking Methods, 203

Flat Feet, Cause of, 490

Flies and Mosquitoes, 480

Floors, Kitchen, 42

Cost of, 47

Cleaning, 174

Food Adulteration, 340

Buying, Refuse, in, 336

Buying by Nutritive Value, 330

Expense in Division of Income, 280

Habits, Efficient, 485

Planning, 179-208

Pure, Defined, 341

Sanitary Standards for, 339

Units per pound, Table of, 332

Units Required Daily, 331

Frying, Disadvantages of, 204

Fuel Saving Devices, 130

Saving in Cooking, 194, 206

Saving in House Heating, 471

Value of Food, 331

Value of Foods by Measure, 335

Fuels, Cost of, 124

Fuels, Time Saving, 117

Furnaces, 470

Furniture, Buying, 373

Furnishings, Simplifying, 398

Garbage Disposal, 55, 479

Gasoline, Irons, 239

Stoves, 121

Gloves for Cleaning, 169

Grouping Food Supplies, 31

Kitchen Equipment, 21, 29

Laundry Equipment, 225

Halls, 460

Hands, Care of, 169

Handkerchiefs, Washing, 219

Hard Water, 214

Harmony in Kitchen Equipment, 59

Heating Apparatus,

Heat Regulators, 472

Health of the Homemaker, 481-493

of the Mother, 104

Height, Correct, of Ironing Board, 237

of Laundry Tubs, 230

of Shelves, 3 ?

of Sink, 37

of Stool, 39

of Stove, 38

of Tables, 39

Home-Made Clothing, 368

Hot Water Supply, 51

Heating, 470

House Heating, 467 
House Planning, 449-480

Household Accounts, 286 Old Fashioned, 270

Card Files, 298

Household Tools, Helpful, 99-144

Housekeepers, Institutional, 447 Working, 439

Housekeeping Efficiency, Object of, 505

Housekeeping, Why Distasteful, 380

Houseworkers, Management of, 419-448

Book of Rules for, 444

How to Hold, 432 .

How to Obtain, 440

Incentive for, 434

Meals of, 437

Part-time, 408

Rights of, 425

Schedules for, 428

Social Life, for, 438

Understanding with, 443

Wages of, 431

Humidifiers, 483

\section{Ideals, 9}

in Budget-making, 275

of Servantless Household, 380

Iceless Refrigerator, 479

Improving Kitchen Arrangements, 28

Income, Division of, 273

Laws of Division of, 278

Index, Visible, 311

Indirect Lighting, 474

Interruptions, 84, 511

Institutional Housekeepers, 447

Inventory, House, 304

Investments, 282

Record, 304

Equipment, 101

Ironing, Machine, 239

Boards, 234

Irons, 237

Alcohol, 239

Cost of Operating, 239

Electric, 237

Gas, 238

Gasoline, 239

Iron Stains, Cause of, 214

Kerosene as a Fuel, 120

Hot Water Heater, 53

Stoves, 120

Kitchen, The Labor Saving, 19-64

Cabinets, 136

Chairs, 39, 491

Equipment, 56

Equipment, List of, 60

Floors and Walls, 43

Grouping Equipment in, 21

Improving the 28

Lighting the, 41,474

Table, Metal, 107

Table Tops, 45
Kitchen Ventilation of, 42

Knives, Kitchen, 139

Labels and the Consumer, 356

Labor Saving Equipment, 108

in Servantless, Household, 391

Kitchen, 19, 64

Tools, 99

Labor Savers, Classes of, 108

Laundry, The Practical, 211-264

Equipment, List of, 253

Grouping Equipment in, 225

Room, 223

Shute, 463

Soaps, 216

Stoves, 231

Work, Commercial, Cost of, 40:

Work, Routing, 227

Work, Saving, 401

Left Overs, 195, 206

Leisure Time, Use of, 503, 508

Lighting, House, 473

Kitchen, 41, 474

Lime Soap, 214

Lincrusta, 175

Linen Closets, 466

Fibers, 212, 359

Record, 303

Linoleum, 43

Cleaning, 172

Living Room, 457

Long-handled Tools, 164

Luxuries, 283

Management of $\mathbf{S}$ e $\mathbf{r}$ a $\mathrm{n}$ t l e s $\mathbf{s}$ Household, 386

Management of Houseworkers, 419-448

Mangles, 239

Market Days, 329

Reports, Use of, 324

Marketing for Meat, 191, 336 Money Saving, 121

Manual, Houseworkers, 444

Materials in House Building, 456

Meals, Balanced, 181, 485 Healthful, 488

Planned in Advance, 185

of Houseworkers, 437

Measurements; Electrical, 121

Measuring Devices, 141 Fraudulent, 343

Meats, Buying, 191, 336 Chopper, 107

Wholesale Prices of, 337

Medical Record File, 302

Medical Supplies, Buying, 37t

Medicine Closets, 466

Mental Attitude of Housekeepers, $15,504,513$

Menus for a Week, 187

Mercerized Cotton, 362

Milk, Comparative Price of, 333

Mineral Salts, 18

Mistress-Slave Attitude, 421

Moisture, Adding to Air, 471, 483 
Mopping Tools, 171

Mosquitoes and Flies, 480

Mothers' Helpers, 411

Municipal Housekeeping, 412

Naptha Soap, Washing with, 260

Nationality of Houseworkers, 447

Nerves, How to Prevent, 492

Net Weight Law, 347

Nutrients in Food, 180

Obtaining Household Help, 440

Office Work vs. Housework, 92

Operating Expenses, 281

Order of Housework, 66

Organizing the Home, 507

Oven Temperatures, 207

Ovens, Fireless Cooker, 120, 123, 131

Overlapping Meals, 205

Over Weight, Reducing, 486

Pantries, 20

Paper Products, 143

Paraffine Boil in Washing, 260

Parcel Post Buying, 328

Part-time Service, 408

Cost of, 412

Path of the Sum, 454

Peak-load in Housework, 493

Personal Efficiency, 481-515

Picking up, 383, 399

Planned-over Meals, 194, 205

Planning the Home, 449-480

Plans for Housework, 65-95

Plate Warmers, 51

Play-Room, 400, 449, 459

in Country Home, 403

Plumbing, 467

for Laundry, 227

Polish, Furniture, 174

Polishing Tools, 171

Porcelain, Fixtures, 467

Porches, 451

Posture in Working, 169, 489

Power in the Home, 394

Washers, 230

Preparing Processes, 21, 24

Pressure Cookers, 134

Price vs. Value, 319

Principles of Efficiency Engineering, 9

Privacy, Need of, 450

Privileges vs. Rights of Houseworkers, 425

Promotion of Houseworkers, 433

Protein, 180 Calories, 333

Pure Food, Definition of, 341

Purchasing Clothing, 359 Efficient Household, 315-376 Sheet, 186

Quantity Buying, 193, 324, 327
Radiators, 471

Record File, Divisions of, 300

Records, Address, 301

Clothes, 302

Dressmaking, 370

Financial, 304

Household, 296

Linen, 203

Medical, 302

of Sizes, 302

Reliable, 11

Receipts, Filing, 307

Recipes, Cooking, Card File of, 306

Reducing Overweight, 486

Refrigerator Drain, 468

Refrigerators, 54

Elevator, 138

Iceless, 479

Relaxation, 491

Rent, in Division of Income, 279

Rest Periods, 69, 494

for Servants, 425

Retailing, Cost of, 323

Rights of Houseworkers, 420

Rinsing Clothes, 220 Tray, 259

Rough-Dry Laundry Work, 402

Routing, Cleaning Work, 73

Kitchen Work, 21, 25

Laundry Work, 227

Rugs, Cleaning, 163

Sanitary Standards for Food, 339

Saving Laundry Work, 401

Saving Steps, 388

Savings, Proportion of, 281

Scales, 141 Faulty, 343

Schedule for a Week, 70

Minor Tasks in, 75

of Meals, 190

Timing in, 83

with Fireless Cooker, 89

Schedules, 65, 95

Advantages of, 83

Country, 90

Daily, 67

Examples of, 69

for Servants, 85, 428

Dressmaking, 371

in Servantless Household, 389

Records of, 94

Seasonable, 390

Special, 76

Scheduling, 11

Sclentific Management, 8

Seasonable Buying, 327

Seasonal Buying of Clothing, 368

Seconds, Buying, 59

Septic Tanks, 479

Servant Problem, the, 377, 419

Solution of, 422

Servantless Household, The, 377416

Servants, Cost of, 379

Disadvantages of, 378 
Servants, How to Hold, 433

How to Obtain, 440

Management of, $419-448$

Management of Several, 445

Schedule for, 85,430

"Servette," The, 137, 397

Service, Part-time, 408 Cost of Part-time, 412

Serving Meals, 396

Sunday Breakfast, 385

Set Tubs, Cost of, 230

Sewage Systems, for Country Houses, 478

Sewing Corner or Room, 369, 459 Screens, 369

Shelter, in Division of Income, 279

Shelves, Kitchen, 40

Shoddy, 363

Shoes for Housework, 490

Short Weights, 346

Shutes, 463

Silk Fibers, 212

Tests of, 362

Sinks, Kitchen, 49

Sitting at Work, 39, 490

Size of Cans, 349 of Equipment, 106

Sleeping Out of Doors, 484

Soaking Clothes, 218

Soap, Laundry, 216 Lime, 214

Naphtha, Washing with, 260

Solution, 263

Social Life of Servants, 438

Softening Water, 214

Sprinkling, 222

Square Deal for Houseworkers, 443

Stains, Removing, 216

Stairs, Planning, 460, 475

Standard Operations, 10

Standard Practice, 152

Manual, 94, 444

for Bathroom Care, 153

for Bedroom Care, 152

for Bread Making, 94

for Cleaning, 151

for Cleaning Living Rooms, 153

for Dish Washing, 79

for Dressmaking, 371

for Houseworkers, 444

for Washing, 255-262

Standardized Household Service, 423

Standardizing Cleaning, 81

Dishwashing, 79

in Servantless Household, 395 Special Tasks, 77

Standards in Servantless Household, 381

Starch Formula, 264

Starching, 221

Stationary Tubs, 228

Vacuum Cleaners, 162, 469

Steam Cooking, 132

Heating, 470

Step Savers, 135

Storage, Clothes, 302
Stoves, Laundry, 231

Kitchen, 50

Student Help, Part-time, 410

Style in Houses, 456

Sun Plan of the House, 454

Sunday as Day of Rest, 382, 385

Sweeper, Vacuum, 157, 160

Sweeping, 154

Tools, 170

Table Tops, 45

Metal Kitchen, 107

Taste of Meals, 103

Telephone, Use of in Buying, 329

Tests for Textile Adulterations, 361

Textile Fibers, 212, 359

Adulteration of, 360

Purchasing, 359

Test for, 362

The Efficient Buyer of, 363

Thermometer Cooking, 141

Oven, 207

Thermostat, 472

Time Saving, 395

Filing System, 310

Fuels, 117

in Cooking, 19, 196

Time Schedule of Meals, 170

Time Studies in Cooking, 197

of Cleaning, 150

of Cleaning Rugs, 163

of Dusting, 497

of Dishwashing, 78, 111

of Peeling Potatoes, 53

of Various Tasks, 81

Time off, Cooking Planned for, 198

Timing Special Tasks, 77

Tools, Cooking, 60

Cleaning, 170

Household, 99-144

Laundry, List of, 253

Trademarked Products, 353

Traps for Plumbing, 408

Trays, Serving Breakfast on, 385 Wheel, 137

Tubs, Height of, 230

Price of, 230

Vacations for Houseworkers, 433

Vacuum Cleaners, 156

Cleaners, Permanent, 162, 469

Fuel Saver, 135

Steam Heating, 470

Washers, 251

Value vs. Price, 319

Vegetable Preparation, 32

Preparation, Time Study of, 53

Preparing Table, 55

Ventilation, House, 483

Kitchen, 42

Volts, Meaning of, 124

Wages of Houseworkers, 432

Wall Covering, for Kitchen, 44, 47 
Walis, Cleaning, 175

Wash Boller Sprayer

Wash Day, Tuesday, 73

Washing Clothes, 217 Machines, 244

Planning the, 225

Powder, 216

Soda, Use of, 214

Waste in Food Buying, 330

Water in Laundry, 213

Softening, 214

Supply, Hot, 51

Systems for Country Houses, 478

Waste, Food, Preventing. 205

Watts, Meaning of, 124

Weekly Tasks, 66

Weight of Women, Average, Table of. 486
Weights and Measures, Faise, 343

Weights per Bushel, Table of, 348

Wet Wash, The, 402

Wheel Trays, 137

Wholesale Price, 324

Price of Beef, $\mathbf{3 3 7}$

Windows, 451

Cleanlng, 171

Women, as Purchasing Agents, 207

Wool Fibers. 212

Tests of, 362

Woolens, Washing, Standard Prac. tice, 261

Worsted Fabrics, 360

Working Housekeepers, 439

Worker's Manual, 444

Wringing Clothes, 217 


\section{HOME-STUDY COURSES IN HOME ECONOMICS}

For Home Makers, Mothers, Houseworkers, etc. Professional Courses, for Teachers, Dietitians, Institution Managers, Nurses, Demonstration Agents, etc. 100-page Bulletin on request.

\section{THE LIBRARY OF HOME ECONOMICS}

\section{Volumes $-3,000$ Pages-1,000 Illustrations}

FOOD SUBJECTS

PRINCIPLES OF COOKERY, by Anna Barrows, Columbia University.

FOOD AND DIETETICS, by Prof. Alice P. Norton, M. A., formerly University of Chicago, Editor Journal of Home Economics.

\section{HEALTH SUBJECTS}

HOUSEHOLD BACTERIOLOGY, by Prof. S. Maria Elliott, Simmons College.

HOUSEHOLD HYGIENE, by Prof. S. Maria Elliott, Simmons College.

\section{HOUSEH}

THE HOUSE : ITS PLAN, DECORATION AND CARE, by Prof. Isabel Bevier, Ph. M., University of Illinois.

\section{CHILDREN}

CARE OF CHILDREN, by Prof. A. C. Cotton, M. D., formerly Rush Medical College, University of Chlcago.

PERSONAL HYGIENE, by M. LeBosquet, S. B., Director A. S. H. F.

HOME CARE OF THE SICK, bY Amy E. Pope, Presbyterian Hospital, New York City.

\section{LD ARTS}

TEXTILES AND CLOTHING, by Kate M. Watson, formerly of Lewis Institute and University of Chicago.

STUDY OF CHILD LIFE, by Marion Foster Washburne, Editor Mothers' Magazine.

Price $\$ 1.50$ per Volume. Textbook edition, special rate to schools

\section{THE DOMESTIC SCIENCE LIBRARY}

\section{Volumes-4,000 Pages-1,200 Illustrations}

(1) Handbook of Food and Diet

Chemistry of the Household : Principles of Cookery; Food and Dietetics.

(3) Handbook of Health and Nursing

Household Bacteriology ; Personal Hygiene; Home Care of Sick.
(2) Handbook of Housekeeping

The House: Its Plan, Decoration and Care; Household Hyglene ; Household Management.

(4) Handbook of Dress and Childhood

Textiles and Clothing; Care of Children; Study of Child Life.

Price, 3/4 Leather Style, $\$ 3.00$ per Volume. Textbook edition, special rate (.5) Lessons in Cooking, Through Preparation of Meals

By PROF. Fiva R. RoBinsox, B.S., University of South Dakota, and Helex G. Hammel, Farmers' Institute, Lecturer, etc.

Price, $3 / 4$ Leather Style, \$3.00. Cloth, \$2.50. Textbook edition, special rate.

(6) Household Engineering, Scientific Management in the Home By Christine Frederick, Consulting Editor, Ladies' Ilome Journal.

Price, $3 / 4$ Leather Style, $\$ 3.00$. Cloth, $\$ 2.50$. Text edition, special rate。 COOKING FOR PROFIT-Bradley, \$3.00; LOW COST COOKING-Nesbit, $\$ 1.00$; HOUSEHOLD ACCOUNT BOOK, \$1.00 ; WEEKLY ALLOWANCE BOOK, 10c; HOUSEHOLD ACCOUNTING CHECK RECORD, $\$ 1.00$.

BULLETINS-Free-Hand Cooking, 10c; Food Values, 10c; Five-Cent Meals, $10 \mathrm{c}$; Wheatless-Meatless Meals, 10c: "The Art of Spending," $10 \mathrm{c}$; "How John and Mary Live and Save on $\$ 35$ a Week," 10c. American School of Home Economics, Chicago 
$$
\text { - }
$$ 


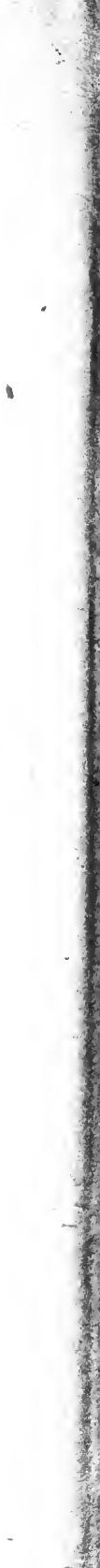




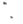

. 
$\lim _{x \rightarrow 0}$

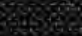

6.

F.

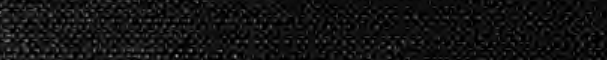

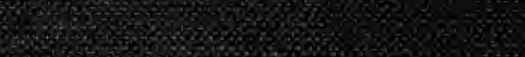

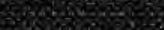

w.

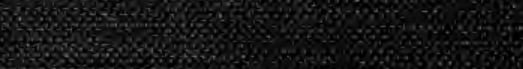
20

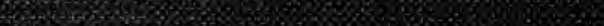

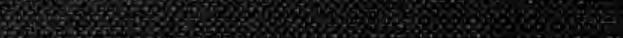

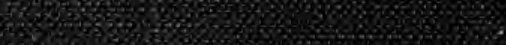

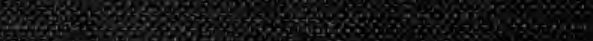

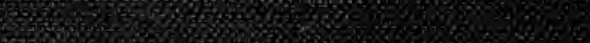

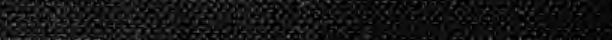

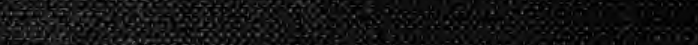

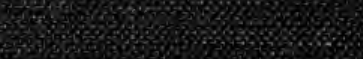

(2)

3.

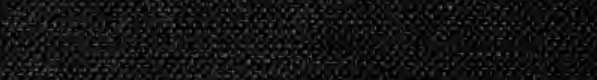

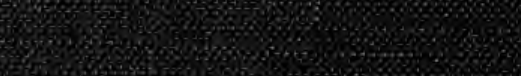

70 .

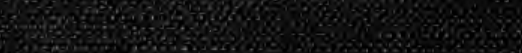

3.6.

H.

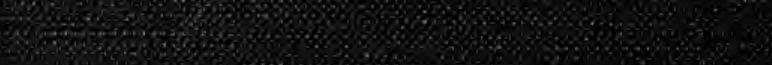

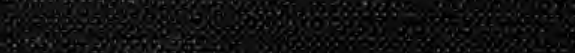

ing

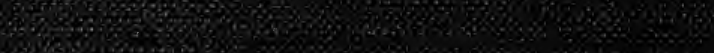

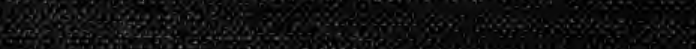

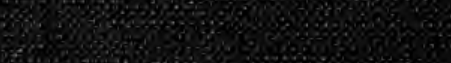

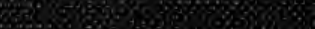

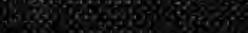

